\title{
Modelling the incubation microclimate to predict offspring sex ratios and hatching phenology in tuatara (Sphenodon punctatus)
}

\author{
Anna L. Carter
}

A thesis submitted to Victoria University of Wellington in fulfillment of the requirement for the degree of Doctor of Philosophy in Ecology and Biodiversity

Victoria University of Wellington

Te Whare Wānanga o te Ūpoko o te Ika a Māui 

Successful conservation of terrestrial biodiversity requires understanding and predicting the impacts of rapid climate warming on the suitability of both current and potential future habitats. Most predictions of range shifts and other population-scale effects of climate change rely to some extent on statistical links between a species' known geographical distribution and the suite of environmental conditions experienced within that space. However, species' responses to climate change are likely to be more complex than can be represented by the projection of current species-environment relationships into unknown environments. An important goal in biodiversity conservation is the development of quantitative tools with which to assess habitat suitability independently of distributions.

In populations of oviparous species, climate change and habitat modification may have distinct effects on different life stages. Temperatures that are well within the thermal tolerance range of adults, for example, may affect embryonic development rates, hatching phenology, or offspring survival and phenotype. I examined how environmental variation may affect the thermal suitability of habitat for facilitating embryonic development and maintaining balanced sex ratios in tuatara (Sphenodon punctatus), an endemic New Zealand reptile with temperature-dependent sex determination (TSD). Once widespread throughout New Zealand, populations are now restricted to offshore islands and fenced mainland sanctuaries, though establishment of additional populations via translocation is ongoing. Due to intensive conservation efforts, tuatara are not classified as an endangered species, but, like other species in which hatchling sex is determined by the incubation environment, populations are potentially at risk from the detrimental effects of sex-ratio bias. 
I conducted two seasons of field work on the island of Takapourewa to quantify the relationship between rapid vegetation succession and selection of nesting areas. I then used a variety of predictive models to link data on nesting behaviour collected in the field with the microclimate conditions experienced by nesting female tuatara and developing embryos. Using mechanistically modelled soil temperature data, I generated predictions of incubation temperatures, offspring sex ratios, and hatching dates for two populations of tuatara on environmentally distinct islands, Takapourewa and Hauturu, under current and projected future climate scenarios. Finally, I classified the thermal suitability of sites on Hauturu for facilitating successful embryonic development and created geospatial surfaces defining suitable nesting locations adjacent to tuatara habitats.

Offspring sex ratios on both islands are unlikely to become male-biased if the magnitude of climate warming observed over the next century more closely matches the minimum, rather than the maximum, projected warming scenario. On Takapourewa, the timing of nesting will be critical in determining whether sex ratios become male-biased under a scenario of maximum climate warming. Earlier nesting may also lead to shifts in hatching phenology under either scenario of climate warming. Warmer annual temperatures on Hauturu are more likely to lead to heavily male-biased offspring sex ratios under the maximum warming scenario. Female tuatara on Hauturu do not need to travel away from their current habitats to locate suitable nesting sites. Monitoring the population to quantify nesting behaviour on the island will be important for determining whether females' choices of incubation microclimates can compensate for the sex ratiobiasing effects of climate change. 


\section{Acknowledgements}

I would first like to thank my co-supervisors at Victoria University of Wellington, Nicky Nelson and Stephen Hartley, who provided me with many hours of advice and support from their unique perspectives. Nicki Mitchell, of the University of Western Australia, has been an excellent Honorary Research Associate; on top of her expertise in physiological ecology, she is a wonderful person to be around. I would not have been able to complete this project without Michael Kearney, of the University of Melbourne, who was beyond generous with his time and access to computing resources; his availability markedly improved the productivity and entertainment value of late-night debugging sessions. I would also like to thank Shirley Pledger, of the VUW School of Statistics and Operations Research, for her willingness to collaborate and her openness to my ideas. I would also like to acknowledge Warren Porter, of the University of Wisconsin, who wrote NicheMapper ${ }^{\mathrm{TM}}$ in the first place, and without whom none of us would be walking around campuses with our laptops open.

Many more people provided assistance or support at some stage of my research. I am grateful to Tim Jones, who generously shared his time and expertise in programming before I knew what I was doing. I would especially like to thank Sue Keall for her ability to answer endless, inane questions with a smile. Danielle Middleton and Ilse Corkery were enthusiastic purveyors of tuatara-related knowledge during my first year in New Zealand. The AWC Model Ecosystem project was a fortuitous tool in the first few months of my research, and I would like to thank Alexei Drummond, James Russell, and Richard Newcomb for facilitating my participation in that project. I would like to thank Simone Blomberg, of the University of Queensland, for good conversations that were mostly about statistics. I have been incredibly fortunate to share an office with people I can look forward to seeing- Helen Taylor, who read a chapter for me, and Lindsay Anderson, who 
let me tag along to Takapourewa once to sneak some extra data, and more recently, Steph Price and Patty Ramirez, who make me feel helpful. I would also like to thank Elizabeth Heeg and Rosalynn Anderson-Lederer for reading thesis chapters and Jamie Tam and Monica Awasthy, who would have done the same if I had asked them.

I would not have been able to undertake half the fieldwork required for my research without the willing participation of excellent field assistants, so I would like to thank Sylvia Ruarus and Rosie Rowe for their invaluable help on Hauturu and Zack Alexy, Elizabeth Jane Coombs, Ayla Murray, and Hanah Tamarua for being my unquestioning minions on Takapourewa. My research would not have been possible without the support of tangata whenua, particularly Ngāti Manuhiri, kaitiaki of Hauturu, and Ngāti Kōata, kaitiaki of Takapourewa. I would also like to thank the New Zealand Department of Conservation/Te Papa Atawhai for permission to conduct field research on both islands. DoC rangers Richard Walle, Leigh Joyce, and Nichy Brown on Hauturu and Tim Bacon, Frank Higgot, and Sue Caldwell on Takapourewa were consistently enthusiastic, interested, and generous with their time and assistance.

In addition, I would like to acknowledge the other members of DoC- Michelle Jenkinson, Mike Aviss, Bill Cash, Geraldine Moore, Peter Gaze, and Carol Knight- who provided me either with practical assistance or archived data at some point during my thesis research. Sean Doody, Jeanine Refsnider, and Danielle Shanahan also provided useful information for my field work on Takapourewa. I am eternally grateful to Jennifer Culbertson, who has more responsibility for this than she may realise. I would like to thank Grant Crosswell, of Marlborough District Council, who provided access to their GIS server and Chris Crook, of Land Information NZ, who solved a small yet amazingly persistent problem that, otherwise, I might still be trying to figure out. Thanks also go to António Gameiro Lopes, who wrote the software for WindStation and was always willing to answer questions and provide clarifications. I would also like to thank Kent Dalziel, of Geotechnics Ltd. in Auckland, for generously allowing me to avail myself of his 
technicians, testing equipment, and lab space for a week and then letting me return and do it again.

Finally, I would like to thank my parents for their editing help, especially my mom, who read through all my references voluntarily and more than once. I would also like to thank Soren, who has been an incredibly patient thesis companion, accepting near-complete loss of my attention with very little complaint. The final thanks go to Rosie, who is mentioned twice.

Funding for this thesis research was provided by Victoria University of Wellington and the Allan Wilson Centre and grants from the VUW Faculty of Science, the Society for Conservation Biology, the Australasian Society for the Study of Animal Behaviour, the VUW Centre for Biodiversity \& Restoration Ecology, the Society for Research on Amphibians \& Reptiles in New Zealand, the Royal Society of New Zealand Hutton Fund, and the British Ecological Society. This research was carried out with approval from the Victoria University of Wellington Animal Ethics Committee (Permit No. 2012R33) and the New Zealand Department of Conservation (Permit Nos. NM31919-FAU, 35087-FAU, AK-31086-FAU, AK-29386-FAU). 

This thesis is dedicated to my parents, who would never try to make me stay. 



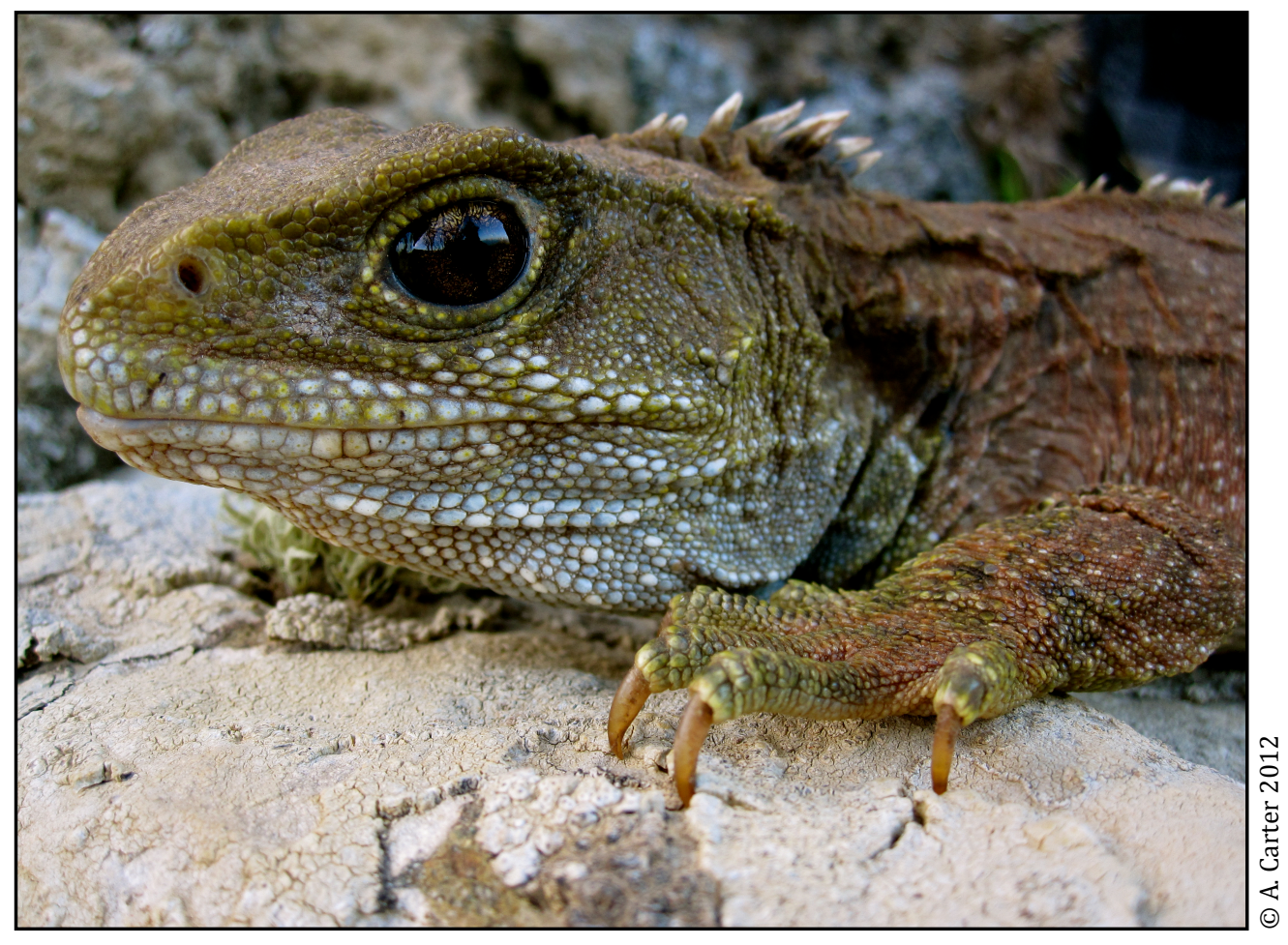

"There he goes. One of God's own prototypes. A high-powered mutant of some kind never even considered for mass production. Too weird to live, and too rare to die."

--Hunter S. Thompson, Fear and Loathing in Las Vegas 


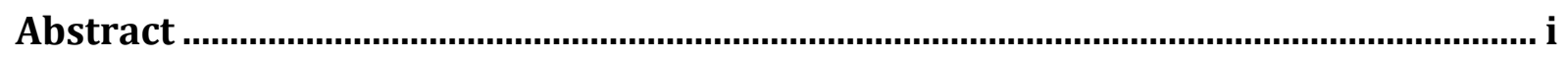

Acknowledgements ...........................................................................................................ii

Chapter 1: Modelling the incubation microclimate to predict offspring sex ratios and hatching phenology in tuatara (Sphenodon punctatus)

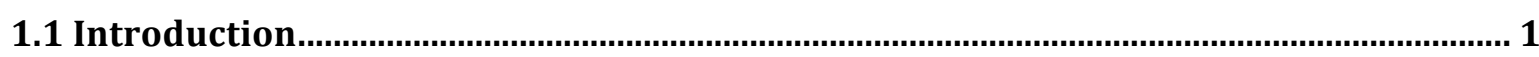

1.2 Incorporating the terrestrial microclimate into models of habitat suitability ............ 4

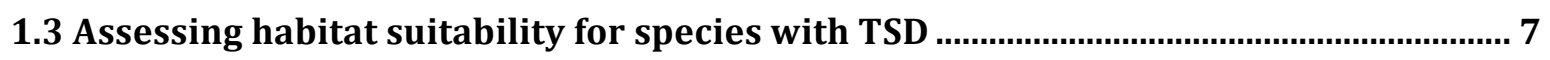

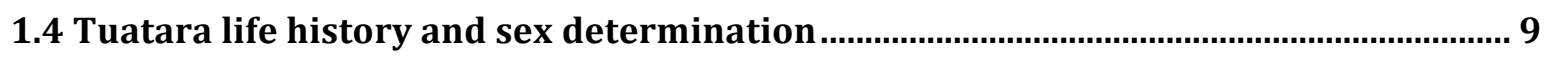

1.5 Thesis aim and organisation

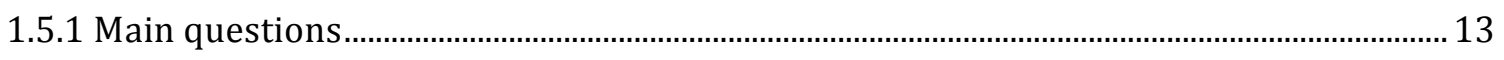

1.5.2 Thesis outline and style ................................................................................................. 14

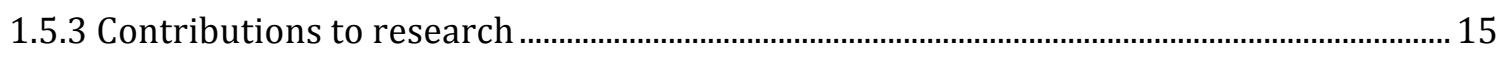

Chapter 2: Tuatara rookery selection responds to rapid vegetation change

\subsection{Introduction}

2.1.1 Variation in reproductive behaviour as a response to environmental change ............... 17

2.1.2 The role of nesting behaviour in maintaining balanced sex ratios ..................................... 19

2.1.3 Significance of nesting behaviour in New Zealand tuatara .................................................. 22

2.1.4 The role of vegetation composition in tuatara nest site selection ....................................... 24

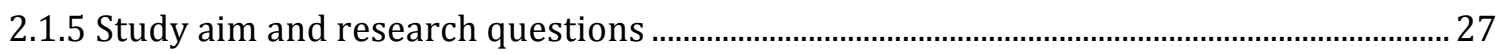

\subsection{Methods}

2.2.1 Remote classification of vegetation composition ................................................................ 27

2.2.2 Vegetation canopy shading in rookeries .............................................................................. 31

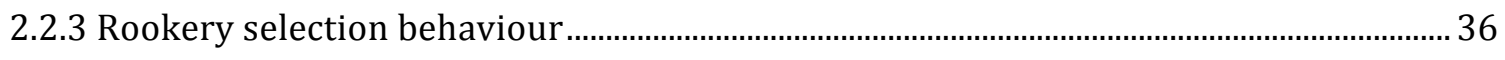

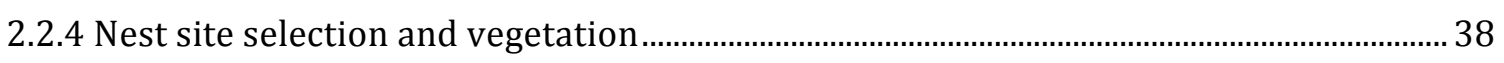

\subsection{Results}

2.3.1 Remote classification of vegetation composition .................................................................. 41

2.3.3 Vegetation canopy shading in rookeries ................................................................................ 44

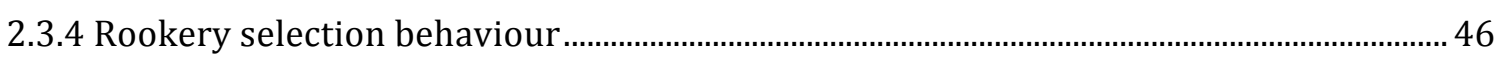

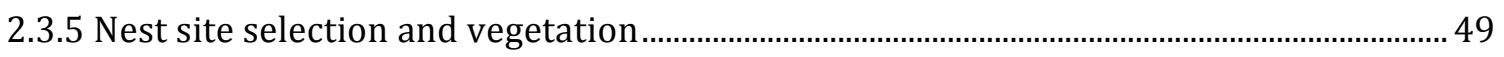

\subsection{Discussion}

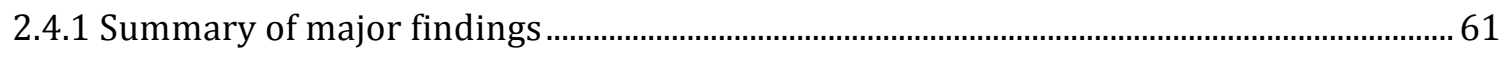

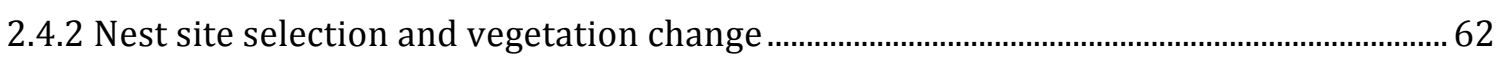

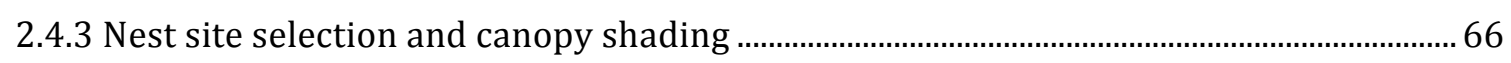

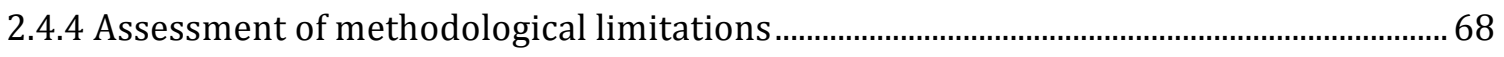

2.4.5 Implications for populations in modified habitats............................................................. 70 
Chapter 3: Local climate and high resolution geospatial data are required to model soil temperatures accurately within a small spatial extent

\subsection{Introduction}

3.1.1 Predictive niche models .75

3.1.2 Modelling the thermal environment of incubation .................................................................77

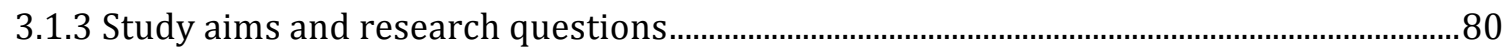

\subsection{Methods}

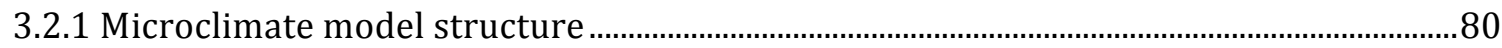

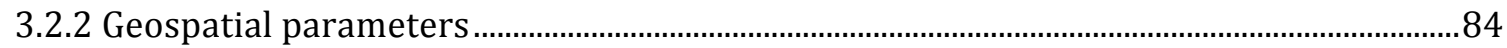

3.2.3 Soil physical and thermal properties......................................................................................

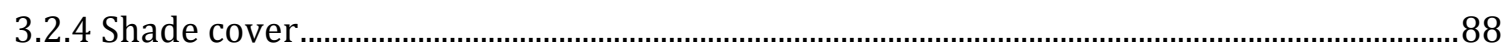

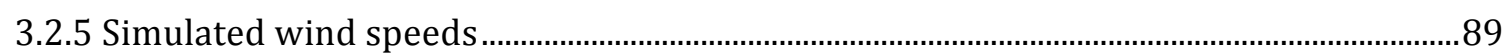

3.2.6 Soil temperature data................................................................................................................90

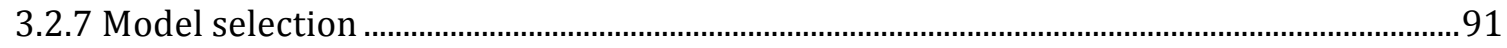

\subsection{Results}

3.3.1 Model selection

3.3.2 Effects of parameter values and resolution 98

\subsection{Discussion}

3.4.1 Summary of major findings

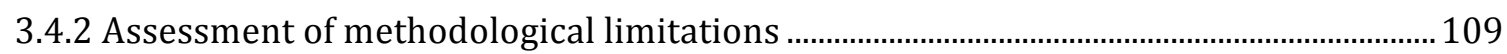

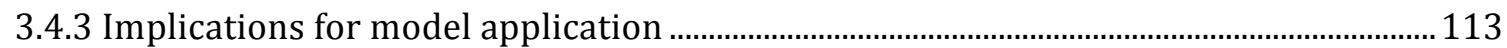

\section{Chapter 4: Predicting effects of climate change on offspring sex ratios and} hatching phenology in a population of tuatara

\subsection{Introduction}

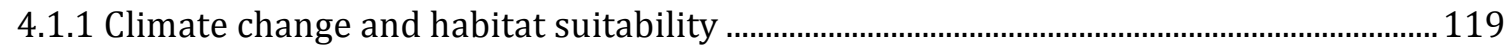

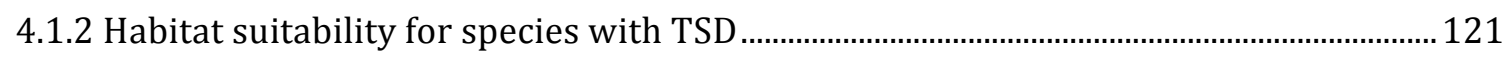

4.1.3 Predicting sex ratios in a population with TSD ................................................................. 123

4.1.4 Study aim and research questions..................................................................................... 127

\subsection{Methods}

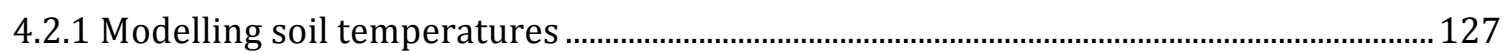

4.2.2 Calculating embryonic development rates and sex ratios ..............................................129

4.2.3 Sensitivity analysis ....................................................................................................................... 131

4.2.4 Simulating climate change ............................................................................................. 132

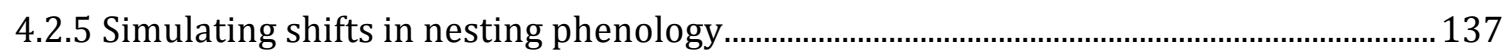

\subsection{Results}

4.3.1 Sensitivity analysis ........................................................................................................... 140

4.3.2 Incubation temperatures under the current climate ........................................................... 144

4.3.3 Effects of climate change on incubation temperatures and offspring sex ratios .......... 148

4.3.4 Effects of oviposition date on offspring sex ratios and hatching phenology................... 161 


\subsection{Discussion}

4.4.1 Summary of major findings ................................................................................................... 173

4.4.2 Assessment of methodology and future directions ........................................................... 180

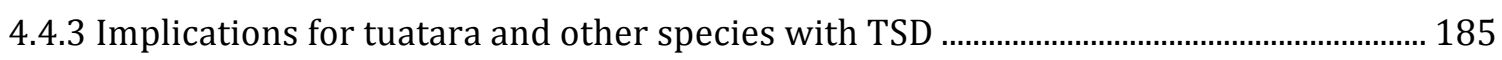

Chapter 5: Incubation suitability surfaces indicate hypothetical nesting locations and offspring sex ratios for a reintroduced population of tuatara

\subsection{Introduction}

5.1.1 Mechanistic modelling in conservation translocations. 191

5.1.2 Behavioural hypothesis-building using a nest site suitability surface ............................. 193

5.1.3 Reintroduction of tuatara to Hauturu...................................................................................... 196

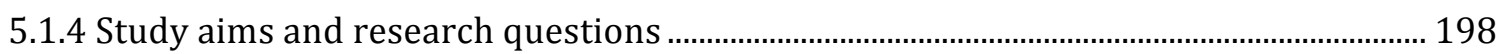

\subsection{Methods}

5.2.1 Developing the microclimate model ……..................................................................... 199

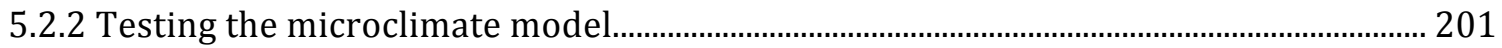

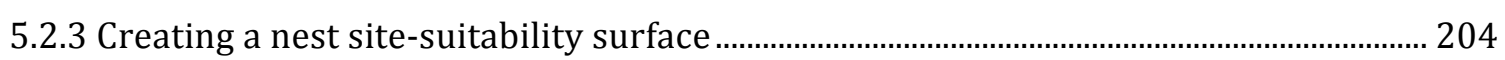

5.2.4 Modelling effects of climate change on nest site suitability .................................................. 206

\subsection{Results}

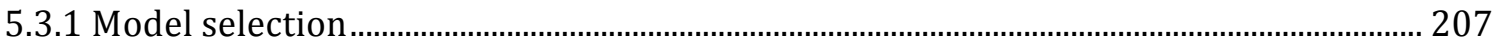

5.3.2 Nest site suitability and hatching phenology .................................................................. 214

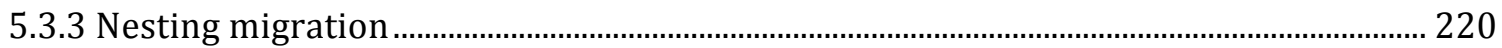

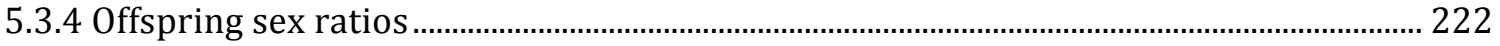

\subsection{Discussion}

5.4.1 Summary of major findings.

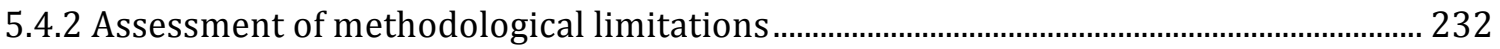

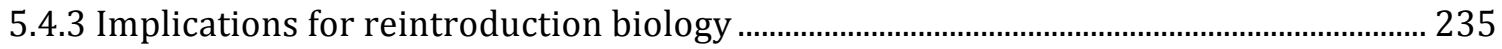

\section{Chapter 6: General discussion}

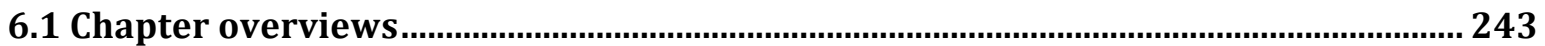

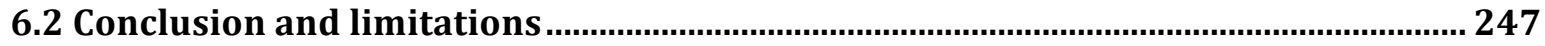

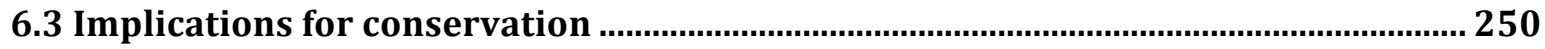

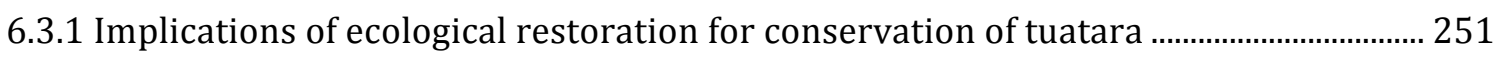

6.3.2 Reintroduction and monitoring of populations with TSD ………………………………... 252

6.4 Management recommendations and directions for future research........................ 253

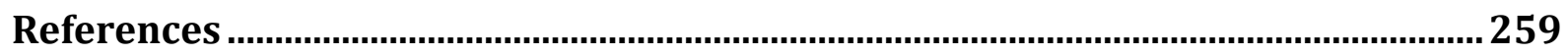

\section{Appendices}

Chapter 2

Chapter 3

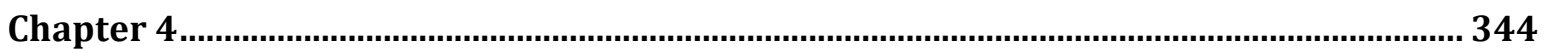

Chapter 5 


\section{Chapter 1}

\section{Modelling the incubation microclimate to predict offspring sex ratios and hatching phenology in tuatara (Sphenodon punctatus)}

\subsection{Introduction}

Of the more than 27,000 extant species currently classified as 'near threatened,' 'vulnerable,' 'endangered,' or 'critically endangered' on the IUCN Red List of Threatened Species, just under $11 \%$ are threatened by climate change (IUCN 2014). While few recent species extinctions are directly attributable to anthropogenic climate warming, shifts in species' ranges, phenology, and abundance are well-documented. In many ecosystems, the rate of environmental change may eventually outpace the ability of a population either to respond in-situ, whether evolutionarily or behaviourally, or to disperse to new sites. The risk of extinction for many species, therefore, increases with both the rate and the magnitude of projected climate change (Pimm et al. 1995; IPCC 2014). Consequently, a primary challenge for biodiversity conservation is the development of quantitative, spatially explicit tools with which to assess habitat suitability and, if possible, preemptively mitigate the effects of environmental change on vulnerable species (Pimm et al. 1995; Guisan \& Zimmerman 2000; Gillson et al. 2013; Mitchell et al. 2013a; Rout et al. 2013).

The theoretical basis of habitat suitability is the Hutchinson niche concept (Hutchinson 1957), which defines (i) the fundamental niche, the $n$-dimensional hypervolume of conditions under which a species can persist and (ii) the realised niche, the physical spaces within the fundamental niche that are currently being utilised by that species (Araújo \& Guisan 2006; Morin \& Lechowicz 2008; Kearney \& Porter 2009). Assessments of habitat suitability have typically been conducted using correlative species distribution models (SDMs), referred to here interchangeably as climate 
envelope models (CEMs), which link a species' distribution (i.e., its realised niche) with the in-situ environment (Guisan \& Zimmerman 2000; Pearson \& Dawson 2003; Austin 2007; Keith et al. 2008; Thuiller et al. 2008; Elith \& Leathwick 2009). Because CEMs rely on known distributions, they assume that (i) a population is in equilibrium with its environment (Guisan \& Theurillat 2000; Pearson \& Dawson 2003), (ii) interactions between a population and its environment do not change over time (Guisan \& Zimmerman 2000; Soberón \& Peterson 2005; Suttle et al. 2007), and (iii) a species' presence in a given location is indicative that the environment within that habitat is representative of its fundamental niche (Pearson \& Dawson 2003).

From a practical perspective, whether a CEM can successfully predict habitat suitability under scenarios of climate change depends on the extent to which the limited subset of environmental variables sampled within a species' current distribution actually represents the physiological processes that underlie the limits of its fundamental environmental niche. However, populations' responses to climate change are likely to be more complex than can be represented by the projection of current species-environment relationships into unknown climates (Hirzel et al. 2002; Brotons et al. 2004; Thuiller 2004; Keith et al. 2008; Lee \& Jetz 2008). The assumption of equilibrium required for making robust CEM predictions may be difficult to satisfy as species migrate to new habitats (Davis et al. 1998; Randin et al. 2006; Buckley 2008; Kearney et al. 2008, 2009a, 2009b; Bartelt et al. 2010; Hartley et al. 2010; Besson et al. 2011; Dormann et al. 2012) or otherwise respond to environmental change.

From an applied perspective, the utility of correlative SDMs is limited if distribution data available for a species are scant (Stockwell \& Peterson 2002; Wisz et al. 2008), depend on low-resolution historical records (Reside et al. 2011; Warren et al. 2014), or represent already-threatened populations that may be restricted to suboptimal habitat. The distribution of subfossil deposits of takahe (Porphyrio mantelli), a flightless rail endemic to New Zealand, for example, suggested first that the species was 
an alpine grassland specialist (Mills et al. 1984). The same data were later reinterpreted as evidence that takahe had originally inhabited lowland forests and became rangerestricted as a result of habitat modification and hunting (Beauchamp \& Worthy 1988). A predictive distribution model built using a grassland-based definition of habitat suitability for takahe would generate erroneous range estimations. Finally, even comprehensive distribution data are of limited use if the primary conservation concern for a species is indiscernible from observations of presence/absence (e.g., inbreeding depression (Jamieson et al. 2006) or shifting population demographics (Mitchell et al. 2009; Grayson et al. 2014)).

Compounding the uncertainty inherent in CEMs is the likelihood that global- or regional-scale predictions of climate change may not translate accurately to a spatial scale relevant for species with relatively small home ranges or otherwise restricted geographic distributions (Hutchinson \& MacArthur 1959; Porter et al. 2002, 2010; Geiger et al. 2003; Bennie et al. 2008; Ashcroft et al. 2009; Gillingham et al. 2012; Potter et al. 2013). A mean $2^{\circ} \mathrm{C}$ increase in surface temperatures within an area of $100 \mathrm{~km}^{2}$, for example, will not necessarily translate to a uniform $2^{\circ} \mathrm{C}$ increase in temperatures across each $1 \mathrm{~km}^{2}$ within the larger area (Bennie et al. 2008; Ashcroft et al. 2009; Gillingham et al. 2012). Few studies, however, have examined the potential effects of environmental change, in particular climate warming, on habitat suitability at biologically relevant spatial scales for terrestrial animals, especially those that are of conservation concern (Gillingham et al. 2012; Mitchell et al. 2008, 2009, 2013). 


\subsection{Incorporating the terrestrial microclimate into models of habitat suitability}

Climate change is modelled using surface air temperatures recorded at an approximate height of $2 \mathrm{~m}$ (IPCC 2013), the lower atmospheric bound of the macroclimate (Geiger et al. 2003). Most terrestrial organisms, however, interact directly with the microclimate (Kearney \& Porter 2004, 2009), or the 'climate near the ground,' the atmospheric layer below $2 \mathrm{~m}$ in which prevailing environmental conditions are modified by interactions between convection and friction (Geiger et al. 2003). Surface and sub-surface characteristics (e.g., complex topography, artificial structures, soil properties, near-ground vegetation composition) can dramatically alter microclimate conditions, compared with those measured at $2 \mathrm{~m}$ or higher (McCullough \& Porter 1971; Geiger et al. 2003).

While 'microclimate' was originally defined primarily to differentiate nearground environmental conditions from those in higher atmospheric layers (Geiger et al. 2003), the terms 'microclimate' and 'microenvironment' have consistently been used in reference to relatively small-scale sites that may provide unique microclimate conditions and result in climatic 'pockets' that are environmentally distinct from surrounding areas (Wilson 1998; Angiletta et al. 2009; Briscoe et al. 2014; Hannah et al. 2014). The microclimates of tree trunks in Australia, for example, grow well above the $2 \mathrm{~m}$ atmospheric boundary of the microclimate layer but are cooler than the surrounding air, providing a surface that koalas (Phascolarctos cinereus) can exploit to facilitate conductive heat loss during periods of extremely high air temperatures (Briscoe et al. 2014).

As a whole, the microclimate layer of the atmosphere may or may not provide environmental conditions that satisfy the fundamental niche of a given species. When defined as an environmentally distinct space, a particular microclimate can provide a refuge either from inhospitable conditions in the surrounding microclimate layer or 
from unfavourable macroclimate conditions (e.g. extreme temperatures or drought) (Geiger et al. 2003; Rull 2009; Mosblech et al. 2011; Hannah et al. 2014). Use of either 'microclimate' or 'microenvironment' as a noun in reference to location-specific environmental conditions carries the assumption that the climate space of interest is experienced by an organism at a relatively small scale. The use of 'microclimate' as an adjective in reference to the atmospheric microclimate layer is not strictly spatial scaledependent. A microclimate model, for instance, can be used to describe microclimate conditions on a global scale or within a single, small microclimate.

Recognition of the limitations of CEMs in capturing the environmental and physiological processes underlying a species' fundamental niche and, therefore, in predicting the realised niche under novel environmental scenarios (Hirzel et al. 2002; Brotons et al. 2004; Thuiller 2004; Keith et al. 2008; Lee \& Jetz 2008) is leading increasingly to the development of models that incorporate those processes (Kearney \& Porter 2004; Kearney et al. 2008; Keith et al. 2008; Williams et al. 2008; Kearney \& Porter 2009; Kearney et al. 2009; Moritz et al. 2012; Mitchell et al. 2008, 2013; Telemeco et al. 2013; Harfoot et al. 2014; Kearney et al. 2014a, b). In contrast to correlative approaches, process-explicit, or mechanistic, models do not rely on distribution data to define either current or future habitat suitability (Porter \& Tracy 1983; Porter et al. 2000; Buckley et al. 2008; Kearney \& Porter 2004, 2009; Monahan 2009). Instead, mechanistic models use the constraints imposed on an organism's energy budget by the exchanges of energy and mass between the macroclimate and the terrestrial microenvironment via radiation, convection, conduction, and evaporation (Porter \& Gates 1969; Porter et al. 1973; Tracy 1982) to define habitat suitability based on the fundamental niche.

Most mechanistic models used to describe distributional limits have focused primarily on the ways in which functional traits, especially thermodynamics, restrict organisms within particular microclimate spaces (Porter et al. 1973; Porter \& Tracy 
1983; Campbell \& Norman 1998; Gillooly et al. 2001; Williams et al. 2001; Bartelt et al. 2008; Kearney et al. 2008; Kearney \& Porter 2009). The fundamental physiological niche, therefore, defines the largest climate space in which an organism can survive (Porter \& Gates 1969). Mechanistic models of individual properties (e.g., behaviour, metabolism, life history) for diverse taxa have been extended to examine population dynamics (e.g., population growth, range expansion) (Dunham et al. 1989; Kearney \& Porter 2009) and community structure (e.g., predator-prey and other biotic interactions) (Porter et al. 1973; 2000, 2002). Mechanistic microclimate models can also be useful when micro-environmental variation can impact a population in ways that would not be apparent from distribution data or even, necessarily, from an processexplicit physiological model. In species with environmentally mediated forms of sex determination, for example, changing incubation conditions can lead to severely biased offspring and, ultimately, population sex ratios (Janzen 1994a; Hulin et al. 2006; Telemeco et al. 2013). Microclimate-mediated effects on population demographics can profoundly affect population viability (Janzen 1994a; Grayson et al. 2014) but would not be detectable using either an organism-level niche model or simple presence/absence data.

In nesting oviparous species, microclimate conditions can differentially affect adults and developing embryos. Incubation temperature can influence the rate of embryonic development (Kearney \& Shine 2004; Nelson et al. 2004b; Georges et al. 2005; Neuwald \& Valenzuela 2009), embryo survival (Burger \& Zappalorti 1988; Wilson 1998; Kolbe \& Janzen 2002; Refsnider \& Janzen 2010), hatching phenology (Mitchell et al. 2008; Cadby et al. 2010; Mitchell \& Janzen 2010; Nelson unpub. data), and offspring phenotype (Elphick \& Shine 1998; Downes \& Shine 1999; Shine 1999; Braña \& Ji 2000; Booth 2006; Woods \& Bonnecaze 2006; Hare et al. 2008; Amiel \& Shine 2012). Increases in air temperature can increase the number of basking and foraging opportunities for adult reptiles, for example (Porter et al. 1973; Porter \& Tracy 1983; Huang et al. 2013; 
Mitchell et al. 2013a), but may also lead to increases in the amount of thermal energy conducted to sub-surface nests, leading to more rapid embryonic development and earlier hatching (Mitchell et al. 2008; Mitchell \& Janzen 2010; Telemeco et al. 2013).

Many reptiles have evolved temperature-dependent sex determination (TSD), a suite of sex-determining systems in which the regime of incubation temperatures experienced by an embryo during a critical developmental window determines its sex (Bull 1983). In species with TSD, continually increasing incubation temperatures can lead to biased sex ratios with potentially detrimental consequences for population demographics and survival (Ewert et al. 2004; Harlow 2004; Janzen \& Phillips 2006; Mitchell et al. 2008, 2009; Schwanz et al. 2010). However, changes in offspring sex ratios are not likely to be detected using either distribution data or an adult-based mechanistic niche model. Assessing future habitat suitability for populations with TSD, therefore, requires predicting the impacts of micro-environmental variation on embryonic development, hatching phenology, and offspring sex, independently of changes in the microclimate space of adults.

\subsection{Assessing habitat suitability for species with TSD}

Because current rates of climate warming are higher than any recorded previously (Huntley et al. 1997), that TSD has been maintained as a suite of sexdetermining mechanisms through periods of prehistoric climatic upheaval (Janzen \& Paukstis 1988, 1991a, 1991b; Silber et al. 2010) is not assurance against population extinction. Habitat fragmentation has led to geographic isolation of small populations (Hughes 2000; Lacy 2000), reduced genetic variation (Crow \& Kimura 1970; Allendorf 1986; Lande et al. 1988) and dispersal capability (Gibbons 2000; Pearson \& Dawson 2003), and increased vulnerability to stochastic events (Lande 1993; Caughley 1994). From a conservation perspective, predicting offspring sex ratios for species with TSD is 
synonymous with determining the suitability of a microclimate for maintaining balanced sex ratios under both current and future climate scenarios. Few studies to date, however, have linked embryonic development and sex determination with spatiallyexplicit, mechanistic predictions of nest temperatures to examine habitat suitability for species with TSD (Mitchell et al. 2008, 2009).

In oviparous species with TSD, the sex of an embryo is determined by two linked organism-microclimate interactions: (1) the selection of a nest site by a reproductive female and (2) the subsequent facilitation of embryonic development and sex differentiation via the temperature regime experienced within the chosen microclimate. The nest environment itself is often explicitly studied as an interaction between a microclimate and organismal (i.e., embryonic) physiology in both birds (Swart et al. 1987; Hartman \& Oring 2003; Kim \& Monaghan 2005) and reptiles (Ratterman \& Ackerman 1989; Cagle et al. 1993; Shine 1999c). Embryos of Chinese pond turtles (Chinemys reevesii), for example, display active thermoregulatory behaviour in-ovo, shifting toward warmer areas inside their eggs but avoiding lethal temperatures (Zhao et al. 2013). Embryonic green turtles (Chelonia mydas) from a single island population display divergent thermal tolerances within a spatial extent of a few kilometers, based on maternal choice of nesting beach (Weber et al. 2012).

The evolution of nest site selection in most vertebrate species, especially reptiles, has been linked primarily to hatching success, rather than to hatchling phenotype (Warner \& Andrews 2002; Escalona et al. 2009; Refsnider \& Janzen 2010). Females choose nest sites that maintain suitable incubation conditions (Kamel \& Mrosovsky 2004; Ewert et al. 2005; Doody et al. 2009), minimise predation (Rand \& Dugan 1983; Madsen \& Shine 1999), or prevent competition with conspecifics (Williams \& Gilbert 1981; Matsushima \& Kawata 2005). The environment of nest site selection is most often examined implicitly, rather than explicitly, as a microclimate. Numerous studies have utilised either experimental manipulation of the nesting substrate, either in-situ (Wilson 
1998; Refsnider et al. 2010) or in a laboratory setting (Bragg et al. 2000; Warner and Andrews 2002), or in-situ observation (Spencer 2002; Ewert et al. 2005; Weber et al. 2012) to describe female preference for particular microclimate characteristics, both within and among populations.

While measured nest microclimate conditions can be linked statistically to offspring phenotypes, including sex, changing macroclimate conditions may create novel nesting microclimates that act differentially on offspring phenotype (Janzen \& Paukstis 1988, 1991; Ewert et al. 2004; Harlow 2004; Janzen \& Phillips 2006), fitness (Ewert \& Nelson 1991; Burke 1993; Janzen 1995; Shine 1999a, 1999b; Booth 2006; Janzen \& Phillips 2006), and survival (Burger \& Zappalorti 1988; Warner \& Andrews 2002; Escalona et al. 2009; Refsnider \& Janzen 2010). Since patterns of nest site selection in species with TSD have evolved primarily to maximise offspring survival, nesting behaviour that affects hatchling phenotype may become maladaptive if hatchling survival and offspring sex ratios become de-coupled, regardless of whether or not females modify their patterns of nest site choice. A spatially explicit, mechanistic microclimate model can be used in concert with either a biophysical or empirical model of embryonic development to link the nest microclimate with offspring sex ratios under hypothetical climate scenarios. Predictions of embryonic development rates and sex ratios have been modelled using a mechanistic model of soil microclimates for one population of tuatara, a reptile with TSD (Mitchell et al. 2008).

\subsection{Tuatara life history and sex determination}

The tuatara (Sphenodon punctatus) is a medium-sized, New Zealand-endemic reptile (Dawbin 1982; Cree 1992, 1994) and the only known extant species of Rynchocephalia (Fig. 1) (Hay et al. 2010). Though prehistorically widespread in New Zealand (Worthy \& Holdaway 2002; Wood 2009), wild populations are currently 
restricted to small offshore islands (Whitaker \& Daugherty 1991) and four protected mainland island sanctuaries (Miller et al. 2012). Translocations have increased the total number of populations from around 30 in the early 1990s (Cree \& Daugherty 1990) to 45 in 2013 (Gaze 2001; Miller et al. 2012; Nelson unpub. data).

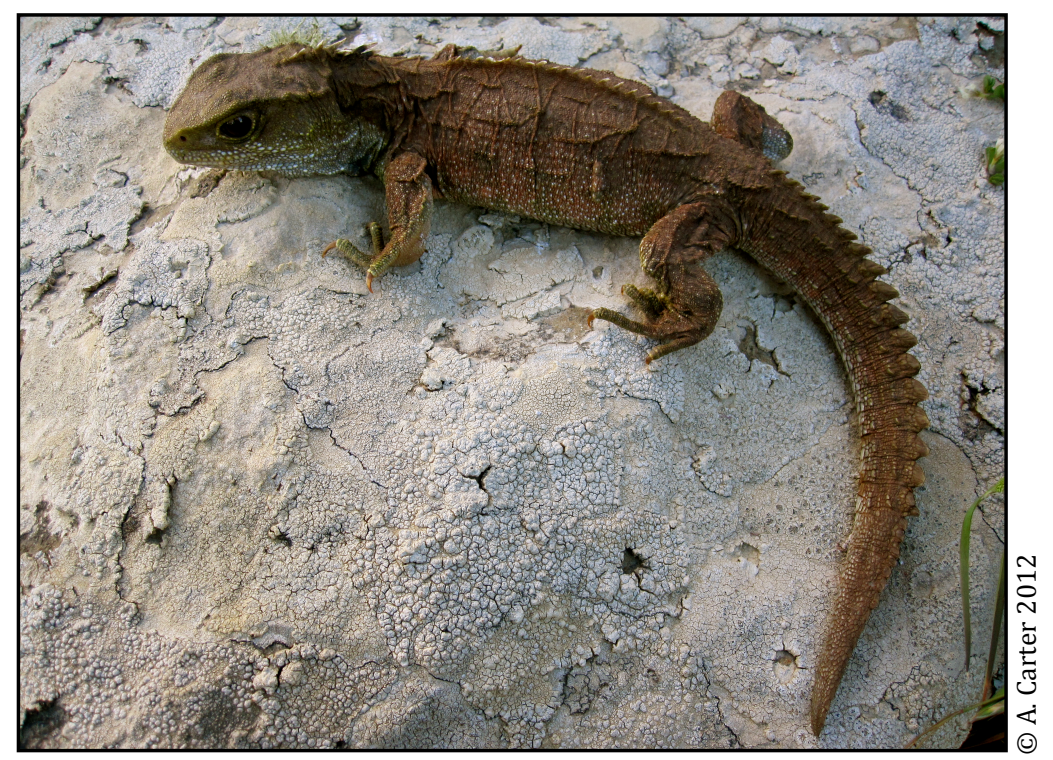

Figure 1. An adult female tuatara on the island of Takapourewa, New Zealand

Most populations of tuatara are small, consisting of no more than a few hundred individuals (Gaze 2001; Hoare et al. 2006; Mitchell et al. 2010), though Takapourewa (or Stephens Island), a 150-hectare island located in the Cook Strait, is home to at least 30,000 individuals (Newman 1982, 1987). As a result, the species is not immediately threatened with extinction and is classified as 'relict' under the New Zealand Threat Classification System (Hitchmough et al. 2012) and as 'least concern' by the IUCN Red List of Threatened Species (IUCN 2014). Nevertheless, compounding demographic effects of rapid climate warming have placed at least one population at risk of functional extinction within about 500 years, or around 12 generations (Mitchell et al. 2009; Grayson et al. 2014). 
Tuatara are slow-growing and long-lived, with an estimated life span of at least 100 years (Dawbin 1982), and are known to live to at least 91 years-old in the wild (N. Nelson pers. comm.). Females mature at around 11-13 years of age (Cree 1994) and, on Takapourewa, nest only every 2-5 years (Cree et al. 1992; Cree 1994; Newman et al. 1994). As a result, the generation interval within a population is approximately 40 years (Mitchell et al. 2009). Egg clutches are laid 50-250mm below the soil surface (Mitchell et al. 2008) in the austral spring and early summer and develop over 11-16 months (Cree et al. 1991; Thompson et al. 1996). Like many reptiles, tuatara have TSD; both the rate of embryonic development and the sex of hatchlings are determined by the incubation temperature (Georges 1989; Georges et al. 2005; Mitchell et al. 2006, 2008).

The tuatara is a cold-adapted reptile, emerging at air temperatures that are markedly lower than most other species of reptiles (Saint-Girons 1980), and is the only extant species known to have evolved the Type Ib pattern of TSD, in which incubation temperatures above a single threshold, around $22^{\circ} \mathrm{C}$, produce only male hatchlings (Nelson et al. 2004; Mitchell et al. 2006). In addition, the range of temperatures that produces both males and females within a single clutch, known as the transitional range of temperatures (TRT), is relatively narrow, only around $1^{\circ} \mathrm{C}$ (Mitchell et al. 2006). Uniquely even among species with TSD, populations of tuatara can become increasingly male-biased as a result of climate warming both directly, via production of increasing numbers of male offspring, and indirectly, via reduction of female fecundity, body condition, and survival (Mitchell et al. 2009; Miller et al. 2012; Grayson et al. 2014).

The lengthy life history of tuatara ensures that the species does not lend itself easily to generating climate change-based predictions within a timeframe relevant to populations. From a research perspective, monitoring of offspring sex ratios in wild tuatara populations would be costly in terms of both time and resources and impractical to attempt within the timeframe of a single research career. Intermittent assessment of adult sex ratios, the primary approach to studying population demographics (Grayson et 
al. 2014), is an important aspect of population monitoring but could be too little-too late for mitigating detrimental effects of climate warming on sex ratios. In addition, longterm monitoring of populations in protected Nature Reserves is impractical and somewhat antagonistic to the goal of minimising human impact on fragile or recovering ecosystems.

To date, the tuatara is the only species with TSD for which a spatially explicit mechanistic microclimate model has been used to predict offspring sex ratios under scenarios of climate change (Mitchell et al. 2008). The small population of tuatara on North Brother Island, a 4-ha island in the Cook Strait, is a potential worst-case scenario of the detrimental effects of changing microclimate conditions on population demographics. The population is currently around 70\% male and, if sex ratios become increasingly male-skewed, is at high risk of extinction within 500 years (Mitchell et al. 2009; Mitchell \& Janzen 2010; Grayson et al. 2014). The severe male bias is likely due at least in part to the relatively low variety of nesting microclimates available on the island, which results in an abundance of warm, male-producing nest sites, even under current macroclimate conditions (Nelson et al. 2004b; Mitchell et al. 2008). Additionally, females tend to nest preferentially in warmer areas (Mitchell et al. 2008), increasing the probability of successful hatching (Nelson unpub. data) but decreasing the numbers of female hatchlings produced.

Most other islands on which tuatara are resident provide a higher diversity of potential nesting microclimates than North Brother Island (Nelson et al. 2004b), though females in other populations have displayed a similar preference for relatively warm, open nesting areas (Nelson et al. 2004b; Refsnider et al. 2010). Predicting how macroclimate-scale changes will impact incubation microclimates and, in turn, how microclimate-scale changes in heterogeneous habitats will affect offspring sex ratios is crucial for conservation of tuatara and other species with TSD. In this thesis, I will examine how nesting females respond to rapid, microclimate-scale habitat change 
within the context of ecological restoration and test the ability of a mechanistic microclimate model to generate accurate, spatially explicit predictions of soil temperatures at the scale of individual nest sites. I will model embryonic development for two latitudinally distinct populations of tuatara under both current and potential future climate scenarios to increase knowledge of how climate change can affect nest site selection, hatching phenology, and offspring sex ratios in a species with TSD.

\subsection{Thesis aim and organisation}

The primary aim of this thesis is to advance understanding of how nesting behaviour and the microclimate of nesting interact to influence offspring sex ratios in wild populations of tuatara. A process-explicit microclimate model is used to examine how climate change affects the incubation micro-environment, allowing for prediction of outcomes within a timeframe relevant to an extremely long-lived reptile.

\subsubsection{Main questions}

- Do female tuatara display variation in nest site selection as a response to changes in the vegetation composition of their nesting habitat?

- Do local- or microsite-scale environmental data influence the accuracy of modelled incubation conditions?

- Do behavioural and environmental variation influence predicted rates of embryonic development or offspring sex ratios of tuatara under scenarios of climate change?

- Do modelled incubation conditions lead to testable hypotheses about nesting behaviour in a reintroduced population of tuatara? 


\subsubsection{Thesis outline and style}

This thesis includes four research-based chapters that are formatted for journal submission. As a result, while some repetition of information is necessary, particularly in the introductory sections, the structure is advantageous both for expediting publication and for maintaining coherence among separate studies. To provide insight into the effects of environmental change on a temporal scale relevant to conservation of tuatara, this study employs several computer-based modelling tools. In addition to developing two new island-wide models of offspring sex ratios in tuatara, this research is the first to test the effects of multi-scale input on accuracy of mechanistic microclimate predictions as well as the first to examine whether a high-resolution microclimate model can generate testable hypotheses for post-translocation population monitoring.

Chapter 2 examines in-situ nesting behaviour at both individual- and population levels, taking advantage of ongoing ecological restoration to assess the potential for variation in nest site selection in tuatara. This chapter includes the frameworks for two distinct publications: (1) a research paper discussing the novel use of remotely-sensed data to examine long-term nesting behaviour and (2) a methods-based paper describing

the development of a new mark-recapture model. Chapter 3 parameterises a mechanistic microclimate model to simulate sub-surface incubation conditions, examining the effects of local- and microsite-scale input variables on model accuracy. This study is the first to examine the effects of input parameter scale on the accuracy of microclimate predictions at a sub-metre resolution.

Chapter 4 uses the microclimate model developed in the previous chapter to examine effects of behavioural and environmental variation on embryonic development rates and offspring sex ratios under multiple climate scenarios and tests a method of geospatial analysis for interpolation of mechanistic predictions. Chapter 5 parameterises 
the microclimate model for a different study site to develop hypotheses about nesting behaviour and facilitate long-term monitoring in a small, reintroduced population of tuatara. The development rate and sex ratio functions programmed for Chapters 4 and 5 will be submitted as a methods-based paper and released as an R package, which will allow the user to predict offspring sex ratios for any species with TSD. Chapter 6 provides a summary of main research findings, discusses applications to conservation, and presents opportunities for future research.

\subsubsection{Contributions to research}

All study design, data collection and analyses, mapping, model testing, and writing were conducted by the author with advice from co-advisors. In addition, the following collaborations contributed to the completion of this thesis:

- Data collection: Field assistants contributed to the collection of in-situ data used in Chapters 2, 3, and 5. Data on tuatara nesting collected prior to 2011 were provided by Nicola Nelson, Associate Professor in the School of Biological Sciences at Victoria University of Wellington, NZ.

- Soil analyses: Textural classification of soil samples for Chapters 3 and 5 was conducted with the assistance of Sim Tirunahari, Laboratory Manager at Geotechnics Ltd. in Auckland, NZ.

- Mark-recapture modelling: The development of a new mark-recapture model used in Chapter 2 was undertaken as a collaboration with Shirley Pledger, Professor in the School of Mathematics, Statistics and Operations Research at Victoria University of Wellington.

- Predicting sex ratios: The beta version of the $\mathrm{R}$ function used for calculating development rates and sex ratios in Chapters 4 and 5 was programmed with the 
assistance of Tim Jones, then a PhD candidate in the School of Biological Sciences, Victoria University of Wellington.

- Microclimate modelling: The primary author of NicheMapR, Michael Kearney, Zoology Department at the University of Melbourne, provided regularly updated $\mathrm{R}$ scripts as well as Fortran code for microclimate modelling in Chapters 3, 4, and 5 well in advance of their public availability. He also provided access to the VLSCI high-performance computing facilities at the University of Melbourne, Australia for data processing in Chapters 4 and 5. Prior to the development of NicheMapR, the creator of the NicheMapper ${ }^{\mathrm{TM}}$ Fortran subroutines, Warren Porter, Professor at the University of Wisconsin, US provided access to his original modelling programme. 


\section{Chapter 2}

\section{Tuatara rookery selection responds to rapid vegetation change}

\subsection{Introduction}

\subsubsection{Variation in reproductive behaviour as a response to environmental change}

Phenotypic plasticity, the variations in morphology and behaviour that can be produced by single genotypes under different environmental conditions, is an important mechanism by which populations respond to environmental change and, consequently, either survive or become extinct (West-Eberhard 1989; Via et al. 1995; Pigliucci 2001; Sultan \& Spencer 2002; DeWitt \& Scheiner 2004; Pigliucci 2005). Behavioural variation is ubiquitous in nature; however, responses that confer an advantage in one set of environments (e.g., the conditions that have selected for a particular behavioural response) may be maladaptive under different conditions (Dochtermann \& Dingemanse 2013; Piper et al. 2013). The effects of environmental change on species' evolved behavioural patterns, particularly reproductive behaviour, may in some cases have a greater impact on the distributions and viability of species than the direct effects of abiotic factors (Visser \& Holleman 2001; Visser \& Both 2005; Parmesan 2007; Yang \& Rudolf 2010).

Reproductive events (e.g., nest construction and egg laying in oviparous species or tree masting) are often tightly linked with the abiotic environment, particularly with seasonal temperature cycles. Shifts in reproductive behaviours (e.g., phenology and nest site selection) correlated with climate fluctuations have been found in diverse taxa (Parmesan 2007; Both et al. 2009). Mismatched phenological shifts across trophic levels in a producer-herbivore-insectivore community may introduce additional constraints on offspring provisioning in insectivorous passerines, for example, if the caterpillars 
needed to feed nestlings are not available during peak hatching (Visser \& Both 2005; Gienapp \& Visser 2006; Both et al. 2009). Similarly, the link between temperature and breeding phenology is tighter near the current geographical limits of loggerhead sea turtles (Caretta caretta), and climate warming may allow for increased reproductive output or facilitate more rapid dispersal at higher latitudes (Mazaris et al. 2012).

The evolutionary underpinnings of nest site selection in many species are likely hierarchical, with females choosing nesting locations based on multiple factors (Refsnider \& Janzen 2010). Studies with insects (Courtney 1981; Scheirs et al. 2000) and at least one turtle species (Spencer 2002) have found evidence that females may choose oviposition sites to maximise maternal survival; however, females of most species likely select nesting locations primarily to increase offspring survival (Warner \& Andrews 2002; Escalona et al. 2009; Refsnider \& Janzen 2010), either by maintaining suitable incubation conditions (Kamel \& Mrosovsky 2004; Ewert et al. 2005; Doody et al. 2009) or by minimising predation (Rand \& Dugan 1983; Madsen \& Shine 1999) or conspecific competition (Williams \& Gilbert 1981; Matsushima \& Kawata 2005).

While offspring fitness is an important component of nest site selection in many species (Refsnider \& Janzen 2010), from evolutionary and practical perspectives, how the incubation environment affects offspring phenotype is secondary to survival (Escalona et al. 2009). The importance of nest site selection to offspring survival is wellsupported (Bragg et al. 2000; Nussey et al. 2007; Refsnider \& Janzen 2010). Nest site selection in both Australian freshwater turtles (Emydura macquarii) (Spencer 2002) and North American striped mud turtles (Kinosternum baurii) (Wilson 1998), for example, is a trade-off between optimal incubation conditions and reduced nest predation risk at lower-quality nest sites. Likewise, mortality of embryonic pine snakes (Pituophis melanoleucus) varies under incubation temperature regimes experienced in different nests (Burger \& Zappalorti 1988). 


\subsubsection{The role of nesting behaviour in maintaining balanced sex ratios}

Variations in reproductive behaviour have additional implications for species with environmentally mediated sex determination (ESD), in which offspring sex is partially or completely determined by the environment of embryonic development. For these species, reproductive phenology and nest site choice can affect not only parent and offspring survival but also population demographics. ESD species with two sexes, like those for which sex is determined genetically, have population sex ratios that approximate the 1:1 Fisherian optimum (Fisher 1930). Theoretically, if environmental conditions favour the over-production of one sex, the per capita reproductive success of individuals that produce the less common sex should increase. The genotype that produces the rare sex then increases in the population via frequency-dependent selection, and the sex ratio equalises (Fisher 1930; Bull \& Charnov 1988; Basolo 1994). Selection for plasticity in a behavioural trait that influences offspring sex would be favoured if environmental variation that can affect fitness occurs either (1) on a timescale shorter than the generation interval of a population or (2) on a small spatial scale (Bradshaw 1965). Controlling offspring sex ratios via manipulation of the developmental environment through varying nesting phenology or nest site selection, therefore, would be theoretically advantageous in a highly heterogeneous environment, particularly for long-lived species with ESD (Bull \& Bulmer 1989; Roosenburg \& Niewiarowski 1996; Janzen \& Krenz 2004; Schwanz \& Janzen 2008; Refsnider \& Janzen 2010).

Relatively rapid climate warming over the last few decades has focused attention on species with temperature-dependent sex determination (TSD) (Janzen 1994a; Hulin et al. 2006; Telemeco et al. 2013), a suite of ESD systems in which offspring sex is influenced or determined outright by the temperature of incubation (Bull 1983). Sex determination in these species depends on the incubation temperature within a 
particular window of embryonic development, the thermosensitive period (TSP), which varies among species but is usually around 25-35\% of total development (Mrosovsky \& Pieau 1991). Different systems of TSD are characterised by their transitional range of temperatures (TRT), the range of constant incubation temperatures that produces both sexes within the TSP (Mrosovsky \& Pieau 1991; Ewert et al. 2004). Incubation temperatures above or below the TRT produce single-sex nests.

In vertebrates, TSD has been found in all extant reptile lineages (Ewert et al. 2004; Harlow 2004) as well as seven orders of fish (Conover 2004). The evolution of TSD at multiple branches within diverse taxa and the maintenance of TSD throughout periods of climatic upheaval, particularly in reptile lineages, support the assertion that TSD is adaptive or, minimally but less likely, evolutionarily neutral (Janzen \& Paukstis 1988, 1991a, 1991b; Silber et al. 2010). While TSD has been found in lizards with life spans of 1-2 years (Harlow 2004), the evolution of TSD in reptiles is theoretically favoured for longer-lived species (Charnov \& Bull 1977; Bull \& Bulmer 1989; Resetarits 1996; van Dooren and Leimar 2003; Schwanz \& Proulx 2008; Schwanz et al. 2010).

Empirical evidence backs the theoretical assertion that TSD is adaptive in reptiles, and data from multiple species support an array of hypotheses of differential offspring fitness (Ewert \& Nelson 1991; Burke 1993; Janzen 1995; Shine 1999a, 1999b; Janzen \& Phillips 2006). Under differential fitness models for TSD, either (1) the incubation temperature itself influences phenotypes of male and female offspring differently or (2) some other environmental variable affects phenotypes of males and females differently, either during or after completion of development. In either generalised scenario, a developmentally plastic offspring phenotype is the connection between incubation temperature and fitness (Shine 1999a; Booth 2006). Juvenile eastern three-lined skinks (Bassiana duperreyi), for example, incubated in relatively warm nests display a higher capacity for learning in the short term than hatchlings incubated in cooler nests (Amiel \& Shine 2012), potentially improving their ability to 
avoid predation. Incubation temperature also affects morphology (i.e., mass and body length) and performance in the same species (Elphick \& Shine 1998) and in hatchling wall lizards (Podarcis muralis) (Braña \& Ji 2000) and egg-laying skinks (Oligosoma suteri) (Hare et al. 2008), as well as post-hatching growth of juvenile soft-shelled turtles (Pelodiscus sinensis) (Du \& Ji 2003). In oviparous species, exposure to different incubation temperature regimes and, therefore, offspring phenotypes can vary either with location or nest depth or through variation in the timing of nesting. From a conservation perspective, however, covariation of nesting phenology and temperature alone is unlikely to be sufficient for maintaining balanced sex ratios in TSD species under predicted maximum rates of climate warming (Morjan 2002; Hulin et al. 2006; Mitchell et al. 2008; Telemeco et al. 2009).

Evidence for differential offspring fitness supports the hypothesis that maternal control of nest temperature is an important component of maintaining optimal sex ratios in TSD species (Bull et al. 1982a, 1982b, 1988; Hughes \& Brooks 2006); however, nest site selection behaviour itself is not generally under high selection pressure. Research to date primarily has examined population-level differences in nest site selection, focusing on correlations between microhabitat characteristics and incubation temperatures. Comparisons of nest characteristics among multiple, geographically distinct populations have found that nest temperatures may be similar across habitats with significantly different environmental characteristics. Studies of nest site choice in leopard geckos (Eublepharis macularius) (Bull et al. 1988) and painted turtles (Chrysemys picta) (Janzen \& Morjan 2003a; McGaugh et al. 2010; McGaugh \& Janzen 2011) have estimated repeatability (Boake 1989; Arnold 1994; Dohm 2002) of nest site selection behaviour to be low, approximately 0.20 . Repeatability of nest site choice in painted turtles translates to an effective heritability of nest temperatures of only about 0.04 (Janzen \& Morjan 2003), even when females choose similar nest microhabitats across nesting seasons (Morjan 2003a, b). Nest-site temperature has been found to be 
female-specific in a preliminary study of three species of geckos, two of which are species with TSD (Bragg et al. 2000). Among populations of Australian water dragons (Physignathus lesueurii sp.) nest depth varies along an elevation gradient, maintaining similar nest temperatures and, potentially, hydric conditions (Doody et al. 2006; Doody 2009). Despite consistently low estimates of the repeatability of nest site choice, however, recent research has found that control of offspring sex ratios enhances maternal fitness in painted turtles, providing empirical support for an adaptive link between nest site selection behaviour and offspring sex ratios in a reptile with TSD (Mitchell et al. 2013b).

\subsubsection{Significance of nesting behaviour in New Zealand tuatara}

The tuatara (Sphenodon punctatus), the only known extant species of nonsquamate Rynchocephalia (Hay et al. 2010), is a long-lived, cold-adapted (Barwick 1982) reptile endemic to New Zealand (NZ). Wild populations of tuatara are currently confined to 45 islands, four of which are fenced mainland island sanctuaries, and the species is classified as 'relict' under the NZ Threat Classification System (Hitchmough et al. 2009, 2012). While not immediately threatened, they are still considered at-risk (Towns 2011), and they remain a national conservation priority (Gaze 2001; Towns et

al. 2001; Nelson et al. 2002b; Towns 2011; Hitchmough et al. 2012). The largest and most-studied population of tuatara (Tyrrell et al. 2000; Mitchell et al. 2009) resides on 150-hectare Takapourewa, an offshore Nature Reserve also known as Stephens Island.

Tuatara are slow-growing, with a generation interval of around 40 years (Mitchell et al. 2009) and a conservatively estimated lifespan of at least 100 years (Dawbin 1982), though they are known to live to at least 91 years in the wild (N. Nelson pers. comm.). Females reach sexual maturity at around 11-13 years of age (Cree 1994). Adult females on Takapourewa nest nocturnally every 2-5 years and deposit clutches of, 
on average, 9 eggs at the end of self-constructed nest tunnels (Cree et al. 1992; Cree 1994; Newman et al. 1994) that vary in depth from 50-250 mm (Mitchell et al. 2008). Eggs in natural nests require approximately 11-16 months for completion of development (Cree et al. 1991; Thompson et al. 1996), the rate of which is determined by the incubation temperature (Georges 1989; Georges et al. 2005; Mitchell et al. 2008).

The tuatara is the only species known to exhibit the TSD type Ib pattern, in which a mixed offspring sex ratio is produced within the pivotal range of incubation temperatures, with only males hatching above a single sex-determining threshold temperature (Cree et al 1995; Nelson et al. 2004; Mitchell et al. 2006). Consequently, the potential for a consistently warming climate to male-bias population sex ratios is a concern for long-term population viability (Nelson et al. 2002b, 2004b; Mitchell et al. 2008, 2010; Grayson et al. 2014). Previous research supports an offspring differential growth model for evolution of the type Ib TSD pattern in tuatara. Incubation temperature affects juvenile growth rates, and females incubated at temperatures just below the female/male threshold of $\sim 22^{\circ} \mathrm{C}$ (Mitchell et al. 2009; Nelson et al. 2010) are larger than those hatched from cooler nests (Thompson 1990; Nelson et al. 2004).

Following the offspring differential fitness model, an optimal tuatara nesting environment would, all else being equal, incubate eggs at a temperature near the upper threshold of the TRT for the duration of the TSP, thereby producing a mixed-sex cohort with 'large' females. Embryonic development is temperature-dependent, and embryos in warmer nests develop more rapidly (Georges 2005; Mitchell et al. 2008). Earlier hatching after a warmer incubation may increase the probability of offspring survival by decreasing the chance of a nest being excavated and unhatched eggs subsequently destroyed by females nesting the following season (Refsnider et al. 2009). In addition, offspring that hatch later, just prior to austral winter, may experience higher mortality as a result of decreased nutrient availability and subsequent lower growth rates (Mitchell et al. 2008; Nelson unpub. data). Timing of oviposition on Takapourewa has 
not been shown to vary among rookeries in different years. The onset and duration of the TSP, however, vary significantly among years and are correlated with oviposition date (Nelson unpub. data). As in other reptile species with TSD (Refsnider 2012), variation in nesting phenology alone is probably unable to compensate for extreme climate warming (Nelson et al. 2004b; Mitchell et al. 2008; 2009).

Female tuatara on Takapourewa primarily have been observed nesting in rookeries, colonial nesting areas (Doody et al. 2009) that are relatively un-vegetated and warm compared to non-rookery and forested areas (Cree 1994; Thompson et al. 1996; Refsnider et al. 2010). On a population level, female tuatara have shown high short-term fidelity to rookery areas and may, to some extent, select nest sites using conspecific cues (Refsnider et al. 2010). Nesting is not strictly colonial, however, and individuals have been observed nesting opportunistically in non-forested areas (Brown 2000). Selection of more shaded nest sites is a potential strategy for controlling offspring sex ratios (Mitchell et al. 2008; Telemeco et al. 2009). Experimental manipulation of vegetation density does not affect within-rookery distribution of tuatara nests, and females have shown no preference for artificially shaded versus bare-soil sites within a single rookery (Refsnider et al. 2010), though the potential for selection of naturally shaded nest sites is unknown. Relatively recent habitat change on Takapourewa provides an opportunity to examine the potential for variation in nest site choice in tuatara.

\subsubsection{The role of vegetation composition in tuatara nest site selection}

Studies of tuatara reproductive behaviour on Takapourewa have been constrained by extensive historical habitat modification. European settlement of the island commenced in 1892 with the beginning of lighthouse construction (Brown 2000). By 1915, approximately $90 \%$ of the low kohekohe (Dysoxylum spectablile) forest that had covered most of the island until at least the mid-1800s (Dieffenbach 1843; Walls 
1976; Walls et al. 1988) had been cleared and replaced by pasture and artificial structures deemed necessary for permanent human habitation. Remaining forest stands were further reduced by wind and salt exposure. Ecological restoration began in 1951 with the construction of a stock exclusion fence that prevented further grazing on the south-western part of the island and allowed for natural vegetation regeneration (Brown 2000). Multiple revegetation efforts have been undertaken by the Wildlife Service and the NZ Department of Conservation with varying levels of success (DOCa). Systematic, accelerated revegetation efforts began in 1992. Forest cover on the island was estimated at that time to be about 10 ha; the rest was comprised of approximately 20 ha of low scrub, 23 ha of remaining pasture, and 97 ha of tussock fields interspersed with other tall grasses and mixed herbs (DOCa). All grazing mammals were removed in mid-2004 (C. Knight [DOC] pers. comm.; Nelson unpub. data)

Population studies have previously identified pasture areas as important tuatara mating and nesting areas (Butler 1989; Thompson et al. 1989) and the forest edges as critical juvenile habitat (McIntyre 1988). Prior to commencement of extensive revegetation, the tuatara population on Takapourewa was estimated to contain between 30,000-50,000 individuals (Newman 1982, 1987) and was thought to be larger than would have occurred on the island naturally. Restoration was not immediately undertaken in known tuatara rookeries to prevent loss of nesting space (East et al. 1995), even though nesting areas would have been forested prior to the initiation of lighthouse construction. Studies of tuatara nesting behaviour on Takapourewa have been conducted only within the context of a highly modified habitat, however, and little quantitative information is available on the characteristics of 'natural' rookeries. Repeatability of behaviour tends to be higher over shorter periods of time, regardless of taxon or species longevity (Bell et al. 2009). High short-term fidelity of nesting females to modified rookery areas should not, therefore, be taken as evidence that nest site selection behaviour previously observed in tuatara is canalized. 
The importance of vegetation cover to nest site selection behaviour likely varies among reptile species. Female painted turtles display philopatry both to nest locations and to vegetation types in the wild (Valenzuela 2001) but compensate for temperature increases by selecting shaded sites under controlled conditions (Kolbe \& Janzen 2002; Refsnider \& Janzen 2012). Results conflict on whether hatching success and sex ratio are correlated with either variable (Janzen 1994b; Valenzuela 2001; Schwanz et al. 2010; Refsnider \& Janzen 2012). Vegetation canopy may also play a role in the tradeoff between predator avoidance and maintaining nest moisture levels observed in other turtle species (Spencer \& Thompson 2003). A relative lack of vegetation coupled with a shallow soil profile may have facilitated the highly male-biased population sex ratio on North Brother Island (Mitchell et al. 2008, 2009; Grayson et al. 2014), which is latitudinally similar to Takapourewa. Though tuatara nesting behaviour on Takapourewa has been well-documented since the 1980s, and individual nesting events have been recorded for multiple seasons since at least 2002, rookery-specific or nestsite specific information on vegetation composition had not been collected in concert. Likewise, few studies have examined the relationship between vegetation change and nest site selection in an oviparous species within the context of ecological restoration. Land cover change analysis, however, can be used to derive vegetation information from island-wide aerial photography that was collected during approximately the same timeframes as behavioural data.

Land cover change analysis, or land use change analysis, is a method of remote sensing most commonly used to model patterns of anthropogenic disturbance (e.g., agriculture or urbanisation) of a landscape and to predict future changes (Agarwal et al 2000; Gao 2009; Pontius \& Petrova 2010). Conversion of habitat for human use, as on Takapourewa, has been driven historically by short-term, largely economic considerations (Versace et al. 2008). From an ecological perspective, then, land cover change is often synonymous with habitat loss and associated deleterious effects (Huang 
et al. 2012). Processes of land use change also have been examined in order to plan for habitat restoration or to guide effective land management (Versace et al. 2008) as well as for studying a variety of ecological questions (Roughgarden et al. 1991). In this study, 'land cover change' does not necessarily suggest a loss of vegetation and is used interchangeably with 'vegetation change.'

\subsubsection{Study aim and research questions}

In order to determine whether nesting behaviour can influence offspring sex ratios in tuatara, the potential for behavioural variation must first be determined. The aim of this study was to quantify the relationship between vegetation composition and nest site choice by combining a longitudinal dataset of tuatara rookery selection behaviour with remotely-sensed vegetation data. Research questions included: (1) how have rookery areas changed in vegetation composition across nesting years? (2) Does rookery selection vary among nesting years? (3) Does vegetation composition influence rookery selection? (4) Does vegetation canopy cover influence nest site choice within rookeries?

\subsection{Methods}

\subsubsection{Remote classification of vegetation composition}

Historical vegetation maps for Takapourewa have only previously been produced by hand. Polygons drawn from human-eye level, however, are very coarse-scale compared to the microhabitats experienced by a nesting female tuatara and are likely to be spatially inaccurate. For this study, three-band colour aerial photographs were used to quantify changes in island vegetation at a resolution more relevant to nest site selection. Images from 2000, 2007, and 2011 (Aerial Surveys Limited, Auckland, NZ) 
were chosen for classification because they were the highest-resolution aerial photographs available for the study site, were not obscured by clouds, and were representative of island vegetation composition before, during, and after removal of grazing mammals (Fig. 1). The photo from 2000 was manually georeferenced to the 2011 image and transformed using a cubic spline, a smoothing function that optimises local accuracy by perfectly stacking congruent user-selected points in both images (de Smith et al. 2007). Quality of the transformation was continually assessed using root mean squared error (RMSE), calculated as the sum of residual errors across all control points. The transformation function was applied to additional control points until the RMSE $<0.0001 \mathrm{~m}$.

Photos were projected to the same coordinate system (2000 New Zealand Transverse Mercator/NZ Geodetic Datum 2000) and 'island-only' images were extracted from the surrounding ocean. The horizontal resolution of the coarsest image was approximately $1.97 \mathrm{~m}$. To be able to compare the final classifications directly, all three images were re-sampled to $2 \times 2 \mathrm{~m}$ pixels using bilinear interpolation, which determines pixel values based on a weighted-distance average of the four nearest input pixels (de Smith et al. 2007), minimising data loss. Pixels representing manmade structures were removed from the re-sampled images. Image manipulation and spatial analyses were conducted in ArcMap $^{\text {TM }}$ Desktop v 10.1 software (Environmental Systems Research Institute [Esri] ArcGIS: Redlands, CA, USA 2012).

Historical reports (Walls 1976; Walls et al 1988) and vegetation maps (DOCa) were used to define six broad land-cover classes (East et al. 1995): dense bush/forest $(F)$, scrub $(S)$, vineland $(V)$, tall mixed grasses $(G)$, maintained pasture $(P)$, and bare ground/rock (R). Defined classes were representative of habitats that (1) could be reliably defined on a per-pixel basis using the range of spectral signatures in the chosen aerial photographs and (2) were representative of the vegetation types encountered by a nesting female tuatara. Rocky coastal areas were spatially, but not statistically, 
distinguishable from small areas of un-vegetated soil (i.e., bare ground) and boulders that are known tuatara nesting habitat. As a result, both bare soil and hard rock have membership in class 'rock,' so nests located within a 'rock' pixel in this study may be constructed either directly in an area of bare soil or underneath a large boulder. The chosen classes likewise did not distinguish every vegetation type present on the island; tussock, for example, was not defined separately from other tall grasses even though substantial areas of tussock-dominated habitat exist on the island. Land cover classes were treated in subsequent analyses as non-ordinal; however, the transitions between some pairs of classes (e.g., especially from maintained pasture to tall grasses) could reasonably be considered ordered (East et al. 1995).

Between 10-25 rectangular training windows containing varying numbers of pixels were manually defined for each broad land cover class, depending on the total spatial area and variety of spectral signatures representing a given class (Gao 2009). An area that was in shadow, for example, would have a substantially different spectral signature in at least one colour band versus an area of the same class that was out of shadow, so training windows of both were needed to maximise classification accuracy. The definitions of land cover classes were modified until the distribution of spectral signature values of pixels in each training set was unimodal and similar sets (e.g., "pasture in sun" vs. "pasture in shadow") had non-overlapping standard deviations. Finally, a contingency table was used to test the ability of the training sets to successfully classify themselves, further indicating that chosen classes were statistically distinguishable (Congalton \& Green 1999; Gao 2009). Separate training sets were developed for each photo, since the signatures of identical land cover classes varied among images.

All land cover classes were to some extent patchy, and boundaries between adjacent types were not always clearly delineated in the photos, so supervised maximum likelihood classification was used to classify single pixels into their first and 
second most likely categories based on the training sets. A distance-weighted fuzzy classification algorithm (distance weight $=0.85$ ) was then used to differentiate $3 \times 3$-pixel $\left(36 \mathrm{~m}^{2}\right)$ windows into single classes by calculating the sum of weighted distances of all classes contained in a window. Land cover classifications were conducted with ERDAS Imagine® software (Intergraph Corporation: Madison, AL, USA 2013).

Classification accuracy was evaluated by comparing 500 stratified random pixels from each classified image against the original aerial images and historical maps (Gao 2009). In addition to overall agreement between original and classified images, quantity disagreement (incorrect numbers of pixels classified as each land cover type, irrespective of spatial arrangement of misclassified pixels) and allocation disagreement (incorrect spatial arrangement of land cover types, irrespective of the number of pixels misclassified) were calculated for each classification (Table 1, Appendix 2.1) (Pontius 2000, 2002; Pontius et al 2008) using cross-tabulated error matrices (Pontius \& Lippitt 2006; Pontius \& Millones 2011). Since the most recent available aerial imagery was from 2011, land cover change analysis was used to generate a land cover classification map for 2012, providing vegetation data across the entire study period (Appendix 2.2-3). 

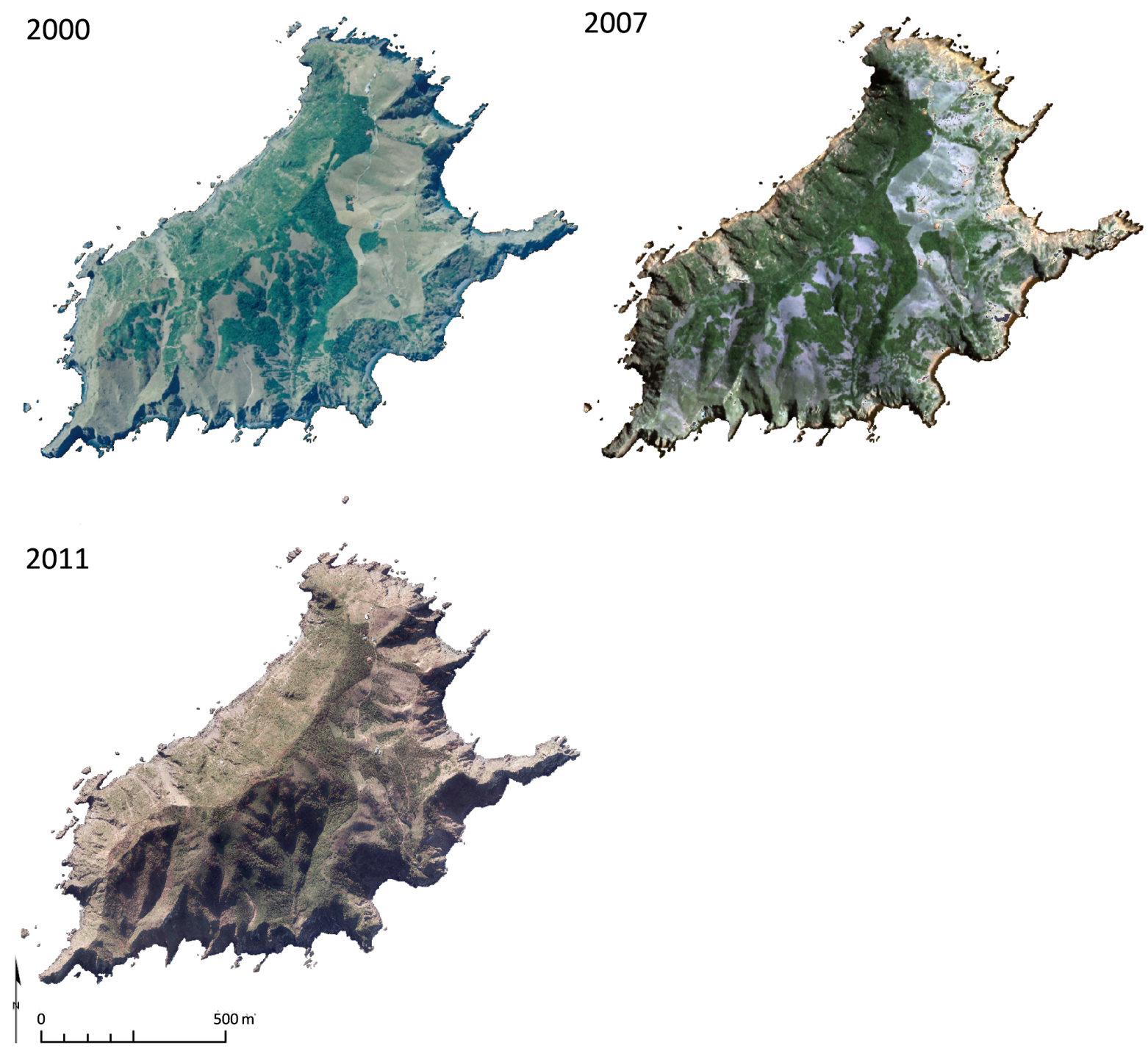

Figure 1. Colour aerial imagery of Takapourewa, New Zealand used for land cover classification. The edge effects of the fence line can be seen clearly, particularly along the eastern forest boundary.

\subsubsection{Vegetation canopy shading in rookeries}

Shade cover affects the amount of solar radiation that reaches a microsite and, consequently, the temperature at that location. Shade cover near ground-level on Takapourewa was investigated by analysing the gap light apparent in full-sky hemispherical photography at nest and random (i.e., non-nest) sites. Nest sites were selected at random within each rookery. To reduce the chance of artificial increases in variance at non-nest sites due to the varieties of vegetation types present in a given 
location (Doody et al. 2009), rookery areas were defined by generating a 5m-radius buffer around 2002-2005 nests using GIS. Additionally, a 2m-radius buffer was generated around nests observed in 2011 to reduce the chance of inadvertently defining a non-nest point at a location that would eventually become a nest. Random points were then generated inside the larger pre-defined rookery area but outside the individually buffered nest sites, and photos were collected as near to the end of the nesting season as practicable given time and weather constraints.

Photo points were marked, and photos were taken in 2011 and 2012 at the same 6 nest sites and 5 random points within each rookery. While lack of a nest at a randomly sampled point did not necessarily indicate lack of suitability as a nest site, no nesting activity was subsequently observed at non-nest locations in either year. In addition, 5 photos were taken at random points in areas formerly identified as rookeries in which no nests were observed in 2011 (Light House, Robyn's Tree) and under the forest canopy (Keeper's Bush) to provide a comparison with traditional nesting areas. Random points in former rookeries were generated using a single, 5m-radius buffer around all former nests. Shade cover at different locations under a forest canopy should be similar, so random points in Keeper's Bush were generated within $5 \mathrm{~m}$ of the access track to prevent damage to seabird burrows, which occur with high density in areas further off the track.

To provide accurate hemispherical photographs of vegetation cover, a digital camera (Nikon $\left.{ }^{\odot} 995\right)$ with circular $\left(180^{\circ}\right)$ fisheye lens (Nikon ${ }^{\circledR}$ FC-E8) was attached to a ball-tilt head and ground spike, which was then inserted into the soil to ground level. The camera was rotated using a compass so that the top of the lens was oriented to geographic north, and the lens was leveled using a double-axis bubble level. Photographs were taken within the hour prior to sunset in dry, still-air conditions to minimise error from lens reflectance, moisture, and vegetation movement. Photos at the same points were collected in the same order each year as weather permitted. The 
dominant vegetation type (of the six categories used in the remote sensing analysis) was recorded at each photo location. Each image was recorded as a GPS point and linked to the 2011 and 2012 land cover classification maps using GIS. Vegetation types recorded in-situ were used as ground-truthing, since the resolution of the land cover classification maps was low relative to single photo points.

Shade cover and mean daily clear-sky solar radiation were computed using Gap Light Analyzer ${ }^{\odot}$ (GLA) v 2.0 software (Frazer et al. 1999, 2000), which combines photographic analysis of site-specific vegetation cover with a global solar radiation model. Region-specific parameter values for cloudiness index, spectral fraction, and beam fraction were calculated to incorporate differences between maritime and mainland climate patterns to the extent possible with available climate data. The cloudiness index, $\mathrm{Kt}$, is the ratio of the amount of global solar radiation, $\mathrm{H}$, that reaches a horizontal location on the ground (i.e., transmitted through cloud cover but not necessarily vegetation) to the amount of extraterrestrial solar radiation, $\mathrm{H}_{0}$, incident on a horizontal surface outside the atmosphere (Lui \& Jordan 1960):

$$
K t=\frac{H}{H_{0}}
$$

Mean monthly Kt values for Takapourewa were computed using daily global solar radiation information obtained from the NIWA CliFlo database (http://www.cliflo.niwa.co.nz) for the nearest coastal meteorological station (Nelson Aws: $\left.\left[-41.302^{\circ} \mathrm{S}, 173.219^{\circ} \mathrm{E}\right]\right)$ and extraterrestrial radiation data included in the software (Appendix 2.4).

The spectral fraction of radiation is the ratio of photosynthetically active radiation, $R_{p}$, transmitted to a horizontal location on the ground to the total amount of shortwave radiation, $R_{s}$, transmitted to the same location. Daily spectral fraction values for Takapourewa were estimated using the known negative relationship between $R_{p} / R_{s}$ and daily Kt values $\left(\mathrm{R}^{2}=0.73\right)$ (Papaioannou et al. 1996): 


$$
\frac{R_{p}}{R_{S}}=1-\exp \left(-0.499 K t^{-0.219}\right)
$$

The beam fraction is the ratio of the amount of direct solar radiation, $\mathrm{H}_{\mathrm{b}}$, that is not absorbed or scattered by the atmosphere, and diffuse radiation, $\mathrm{H}_{\mathrm{d}}$, that is scattered by clouds. Daily beam fraction values were approximated for Takapourewa using daily Kt values by $\left(\mathrm{R}^{2}=0.77\right)$ (Frazer et al. 1999):

$$
\frac{H_{b}}{H_{d}}=1-\exp \left(-3.044 K t^{2.436}\right)
$$

Calculated values for cloudiness index, spectral fraction, and beam fraction values were converted to daily means for each month (Appendix 2.4).

The programme default solar constant value of $1367 \mathrm{~W} / \mathrm{m}^{2}$ (Iqbal 1983) was used, as total solar radiation output varies by approximately $0.1 \%$ over the course of an 11-year solar cycle (Willson \& Hudson 1991) and was not expected to impact local incident radiation calculations. The clear-sky transmission coefficient was set at 0.64 (Gates 2003). Sky-region brightness was calculated over one year (1 January - 31 December) using a solar time step of 2 minutes for 36 azimuth regions and 9 zenith regions. Calculations used the Universal Overcast Sky model (Hutchison et al. 1980), which assumes that all sky regions are equally bright (i.e., the zenith is no brighter than the horizon and vice versa).

Unique topographical information was input for each photo site. Since the amount of solar radiation incident at different points on a relatively small island should not differ due to planetary tilt (i.e., distance from the equator), the same latitude value [4040'00" S] (New et al. 2002) was used to define location for all images. The highly variable topography both within and among rookeries, however, could affect both the intensity and duration of radiation experienced at microsites. Values for elevation and slope, therefore, were generated by overlaying the unique GPS location of each photo with a high-resolution digital elevation model (Aerial Surveys Limited, Auckland, NZ) of the island. Photos were converted to binary images prior to analysis, with "sky" pixels 
represented by white and "shade" pixels represented by black (Fig. 2). Calculations were corrected for image orientation and polar lens distortion (Herbert 1987). Values for canopy openness were converted to shade [100 - canopy openness] to parameterise a microclimate model (Chapter 3) and to maintain consistency among studies.

A generalised linear model (GLM) with binomial error distribution and logit link was used to determine whether shade cover differentiated among nest and non-nest sites within rookeries in the first sampling year only $(\mathrm{N}=77)$, since locations initially were defined as 'nest' or 'non-nest' in 2011. A generalised least squares (GLS) model with 'year' as a fixed effect was used to determine whether shade cover was predicted by vegetation type and whether those relationships varied between sampling years or among rookeries. GLS models were fit using R package nlme (Pinheiro \& Bates 2013). All models were fit using maximum likelihood estimation. 

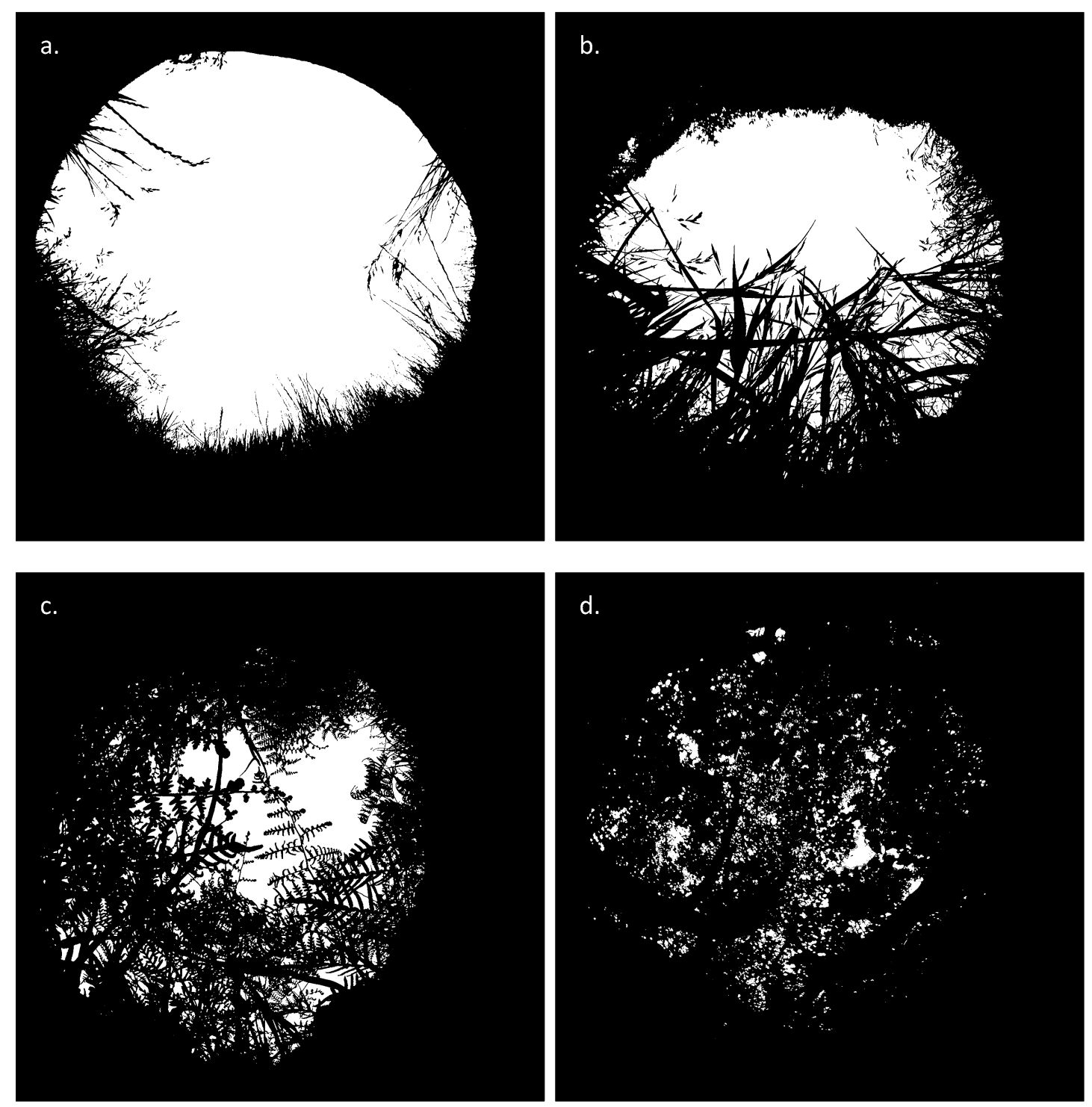

Figure 2. Examples of binary images generated from full-sky hemispherical photos collected on Takapourewa. The dominant vegetation class at photo locations $\mathrm{a}, \mathrm{b}$, and $\mathrm{c}$ is tall grasses, with shading due solely to vegetation, respectively, of $40.20 \%, 63.59 \%$, and $80.29 \%$. Image d was taken under forest canopy and has a percent shading of $91.59 \%$.

\subsubsection{Rookery selection behaviour}

Tuatara nesting activity was recorded on the island of Takapourewa, NZ throughout the spring nesting seasons (approximately 4-6 weeks centered around the month of November) in 2002-2005 and 2011-2012. Teams of 2-4 researchers surveyed known rookeries (eight in 2002-2005, ten in 2011-2012; Fig. 3) in 5m-wide transects 
positioned approximately perpendicular to the slope of the area. Rookeries were surveyed nightly in the same order from approximately one hour after sunset until all transects were searched once. Time spent in each rookery was a function of, primarily, nesting activity on any given night and, secondarily, rookery area.

Female tuatara were hand-captured on first observation, weighed and measured (snout-vent length, vent-tail length, and regenerated tail length), and individually numbered with a non-toxic black marker to prevent unnecessary recaptures. Individuals were abdominally palpated to assess reproductive condition in all years; however, observations of nesting behaviour (i.e., digging, ovipositing, nest backfilling, nest guarding) were considered more reliable than palpation in 2011-2012 and were used as proxies for reproductive condition. Gravid females captured in 2002-2005 were injected with passive integrated transponder (PIT) tags (Allflex ISO FDX-B) to facilitate identification in subsequent years (Nelson et al. 2004; Refsnider et al. 2009). PIT-tag numbers were recorded in 2011 and 2012; however, no additional females were tagged. Identification was also possible of females that had been toe-clipped during pre-2002 studies. Wild female tuatara rarely nest in consecutive years (Cree 1994), so pseudoreplication between 2011 and 2012 (i.e., inadvertently attributing nesting by a single female in consecutive years to different individuals) was unlikely. All observations of nesting females in rookery areas were recorded as GPS points in 2011 and 2012 (Garmin Oregon ${ }^{\odot}$ 550, Garmin Limited, USA). Numbers of females were compared within and among rookeries sampled in all study years using a loglinear analysis referenced to a chi-square distribution.

Nests and scrapes (i.e., nesting attempts with a tunnel length of at least $100 \mathrm{~mm}$ ) were individually numbered and recorded as GPS points. Nests and the identities of females associated with nests were monitored nightly over the course of each nesting season. Scrapes that showed no indication of activity after initial observation were considered abandoned and were not included in analyses. Scrapes in which no eggs 
were laid by the end of the study period, but that showed continued evidence of nesting, were included in analyses as nests. Only locations with evidence of recent activity (e.g., fresh digging) were recorded in any given year to prevent pseudo-replication in nest numbers among years. Nest locations were recorded in all systematically surveyed rookeries as well as opportunistically, since individuals occasionally nest in locations outside rookery boundaries. Vegetation type at each nest location was recorded in 2011 and 2012 to provide ground-truthing data for vegetation types generated using GIS. Numbers of nests were compared within and among the eight rookeries sampled in all six study years using a loglinear analysis referenced to a chi-square distribution.

\subsubsection{Nest site selection and vegetation}

Most nesting data were collected outside of the years for which reliable vegetation classifications could be generated. Examining nest site selection within the context of vegetation change required interpolation of land cover types for nesting events recorded in 2002-2005. All recorded nest locations ( $=1003)$ were linked with vegetation composition by overlaying nest site GPS coordinates with the four categorical land cover maps from 2000, 2007, 2011, and 2012. The published minimum distance root mean squared (2DRMS) accuracy for the GPS units used was $<10$ m (i.e., the probability that a point was recorded as within $10 \mathrm{~m}$ of its 'true' location was $\geq 0.95$ ). Since map resolution of land cover classes was $6 \times 6 \mathrm{~m}$, the vegetation types linked to a nest site using GIS could have been horizontally inaccurate by one pixel, so in-situ vegetation data were used to ground-truth the land cover classes generated using GIS. All map pixels that contained at least one nest during any sampling season were then extracted from each of the four vegetation maps $(\mathrm{N}=465)$.

To simplify interpolation, transitions between land cover types were assumed to be unidirectional (i.e., no pixel transitioned 'backwards'), singular (i.e., no pixel 
underwent unknown transitions), and smooth across the time interval (i.e., an equal proportion of a transition occurred in each year between known years). Land cover classes were not treated as ordinal, so any class was allowed to transition to any other class. For each nest-containing pixel that transitioned between two land cover types between years $\mathrm{y}_{1}$ and $\mathrm{y}_{2}$, the proportion, $\mathrm{c}_{\mathrm{i}}$, of each pixel classified as the initial class in each year, $\mathrm{y}_{\mathrm{i}}$, was

$$
c_{i}=\frac{y_{2}-y_{i}}{y_{2}-y_{1}}
$$

and the proportion, $\mathrm{c}_{\mathrm{f}}$, of each pixel classified as the final class in each year was

$$
c_{f}=\frac{y_{i}-y_{1}}{y_{2}-y_{1}}
$$

Given a pixel with an initial class of pasture in 2000 that was classified in 2007 as tall grasses, for example, the proportion of that pixel interpolated as pasture in 2003 was $4 / 7(\sim 0.57)$, and the proportion as tall grasses was $3 / 7(\sim 0.43)$. GLMs with Poisson error distribution and log link were used to examine whether the proportions of each land cover type predicted the density of nests in nest-containing pixels (i.e., whether vegetation could be used as a measure of rookery attractiveness/repulsiveness). Models were weighted by the total number of nests in each sampled location, since the numbers of nests varied significantly among rookeries. Model fit was examined using likelihood ratio tests referenced to a chi-square distribution. The Beach Ridge and Winch Track rookeries were not systematically sampled for nesting prior to 2011 so were excluded from the analysis.

Rookery selection was examined for the subset of identifiable (i.e., PIT-tagged) females for which at least two nesting events were recorded $(\mathrm{N}=429)$ during the study period. Because data linking identifiable female tuatara with their chosen nesting locations had been recorded primarily at the rookery level (i.e., GPS data linking females' IDs with their specific nest sites were not recorded systematically across all study years), analyses were restricted to examining categorical rookery choice; however, the 
terms 'nest site choice/selection,' and 'rookery choice/selection' are used interchangeably in this study.

Since female tuatara do not generally nest in adjacent years, the probability of capturing identifiable individuals was not equal across sampling years (e.g., females initially captured in 2002 or 2003 were more likely to nest in 2012 than females first captured in 2011). To determine whether vegetation change affected the probability that a female would be observed nesting in a particular rookery in her second year of observation, the probability of observation had to first be estimated independently of vegetation composition. A multistate capture-recapture analysis based on the CormackJolly-Seber model (Lebreton et al. 1992; Pledger et al. 2003) was used to estimate probabilities of survival, nesting, and capture in the sampled years between capture records. Probabilities of (1) survival and (2) nesting observation and subsequent capture (if nesting was observed) were assumed to be independent of rookery choice.

Each observed combination of sampling years and rookery choices in those years was classified as a unique nesting 'segment.' The influence of vegetation on the resultant maximum likelihoods of a rookery choice in the second sampling year for each segment (i.e., whether females nested in the same rookery or switched rookeries) was examined using GLMs with binomial error distribution and logit link, weighted by the number of segments of each type. Improvements in model fit were assessed using likelihood ratio tests. Relationships between rookery choice and vegetation were examined with multinomial GLM models using R package nnet (Venables \& Ripley 2002). Since some segment types were represented by very small sample sizes, improvements in model goodness-of-fit were assessed using second-order-corrected Akaike's Information Criterion (AICc) (Sakamoto et al. 1986), calculated using R package qpcR (Spiess 2013). All analyses were conducted in R v 3.0.1 (R Development Core Team 2008), with significance assessed at the 95\% level. Figures and maps were generated in, 
respectively, $\mathrm{R}$ and ArcMap and edited for publication using GNU image manipulation program (GIMP Development Team 2013).

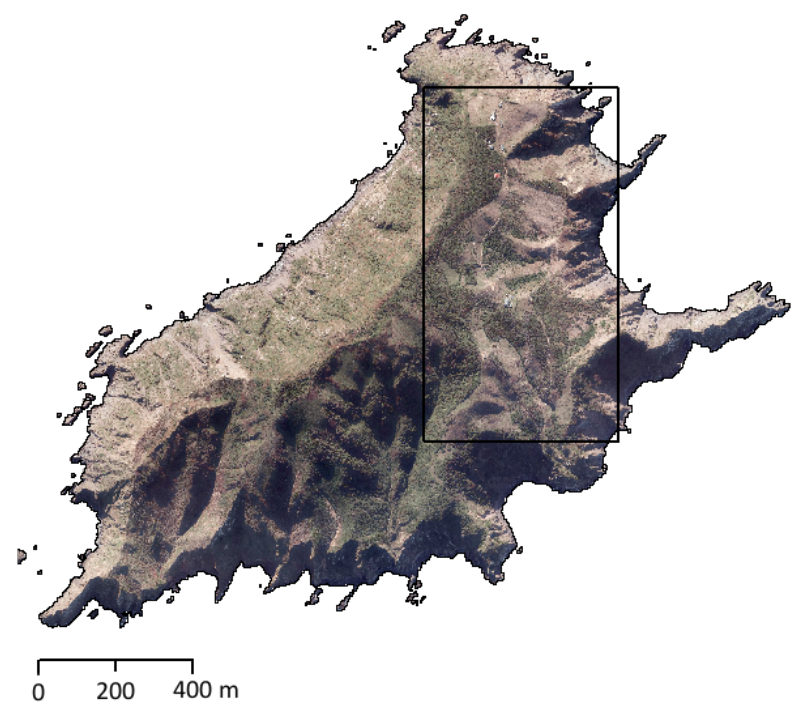

Years sampled | Years with nests
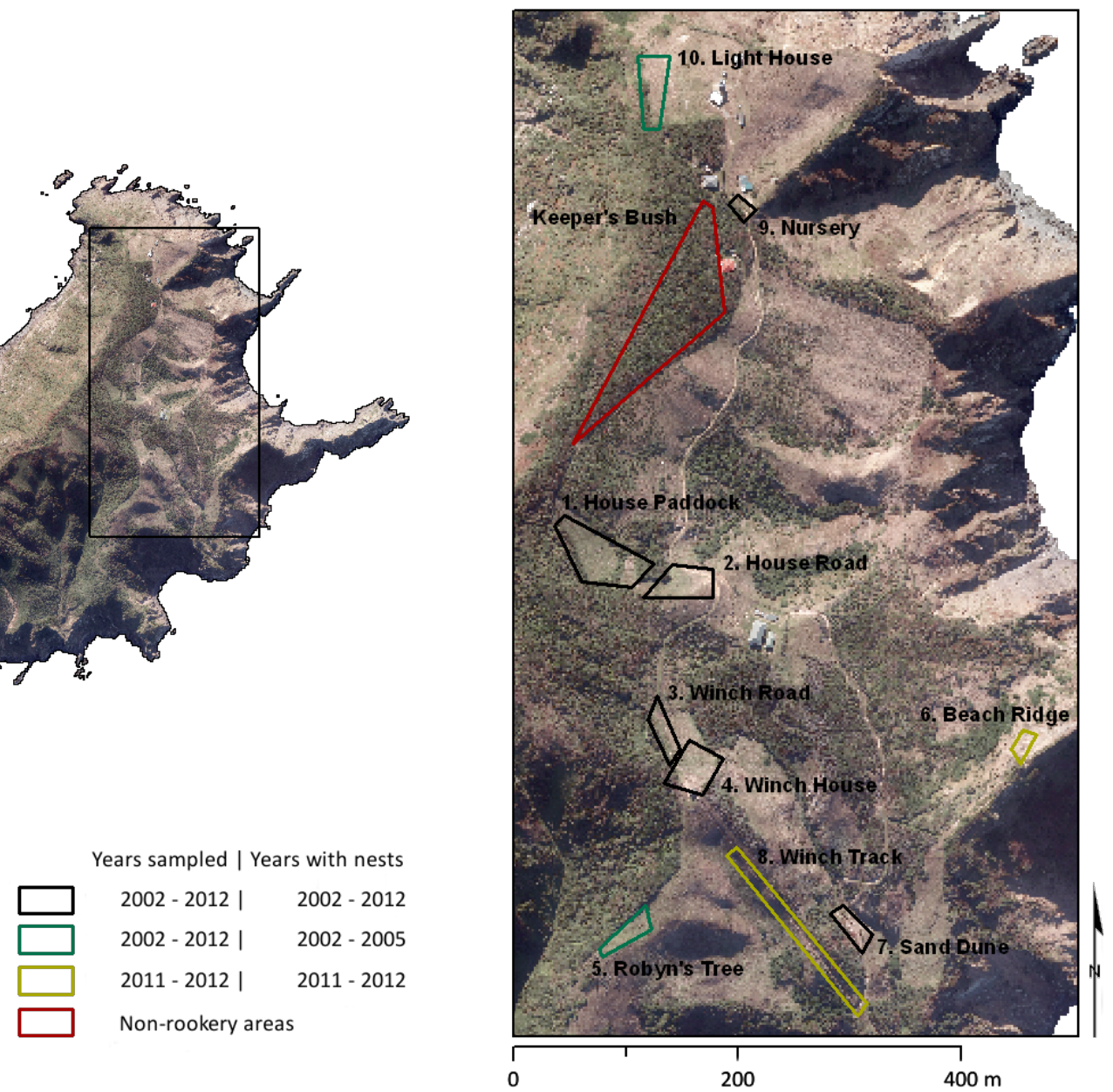

Figure 3. Aerial photo of Takapourewa from 2011, showing locations sampled throughout the study. Rookery areas are numbered in the order that they were searched nightly and colourcoded to differentiate between areas in which nesting was or was not observed.

\subsection{Results}

\subsubsection{Remote classification of vegetation composition}

Whether the accuracy of a land cover classification is sufficiently high for a given application is somewhat subjective; however, overall accuracy of $85-90 \%$ is regularly reported as acceptable for most applications (Gao 2009). Most error in the land cover 
classification maps generated for this study was due to quantity, rather than allocation, disagreement. Misclassification, therefore, was more likely to result in areas of adjacent land cover types (e.g., pasture and tall grasses) that were larger or smaller than what was represented in the original imagery (Appendix 2.1). The proportions of each land cover type in all three classifications, however, only differed from the original aerial images by $\leq 4 \%$ (Table 1 ). The land cover classifications generated for this study (Fig. 4) were realistic representations of the changing vegetation composition of the study site. A land cover map for 2012 was generated using the transitions observed between 2000 and 2011 (Fig. 5). Full results from the land cover change analysis are presented in Appendix 2.3.

Table 1. Accuracy assessment of classifications based on cross-tabulated error matrices, where a perfect classification would have proportion agreement $=1$ and quantity and allocation disagreement $=0$. Detailed error matrices for all three years are in Appendix 2.1 .

\begin{tabular}{rrrr}
\hline Year & proportion agreement & quantity disagreement & allocation disagreement \\
\hline $\mathbf{2 0 0 0}$ & 0.89 & 0.10 & 0.02 \\
$\mathbf{2 0 0 7}$ & 0.89 & 0.08 & 0.04 \\
$\mathbf{2 0 1 1}$ & 0.87 & 0.11 & 0.03 \\
\hline
\end{tabular}



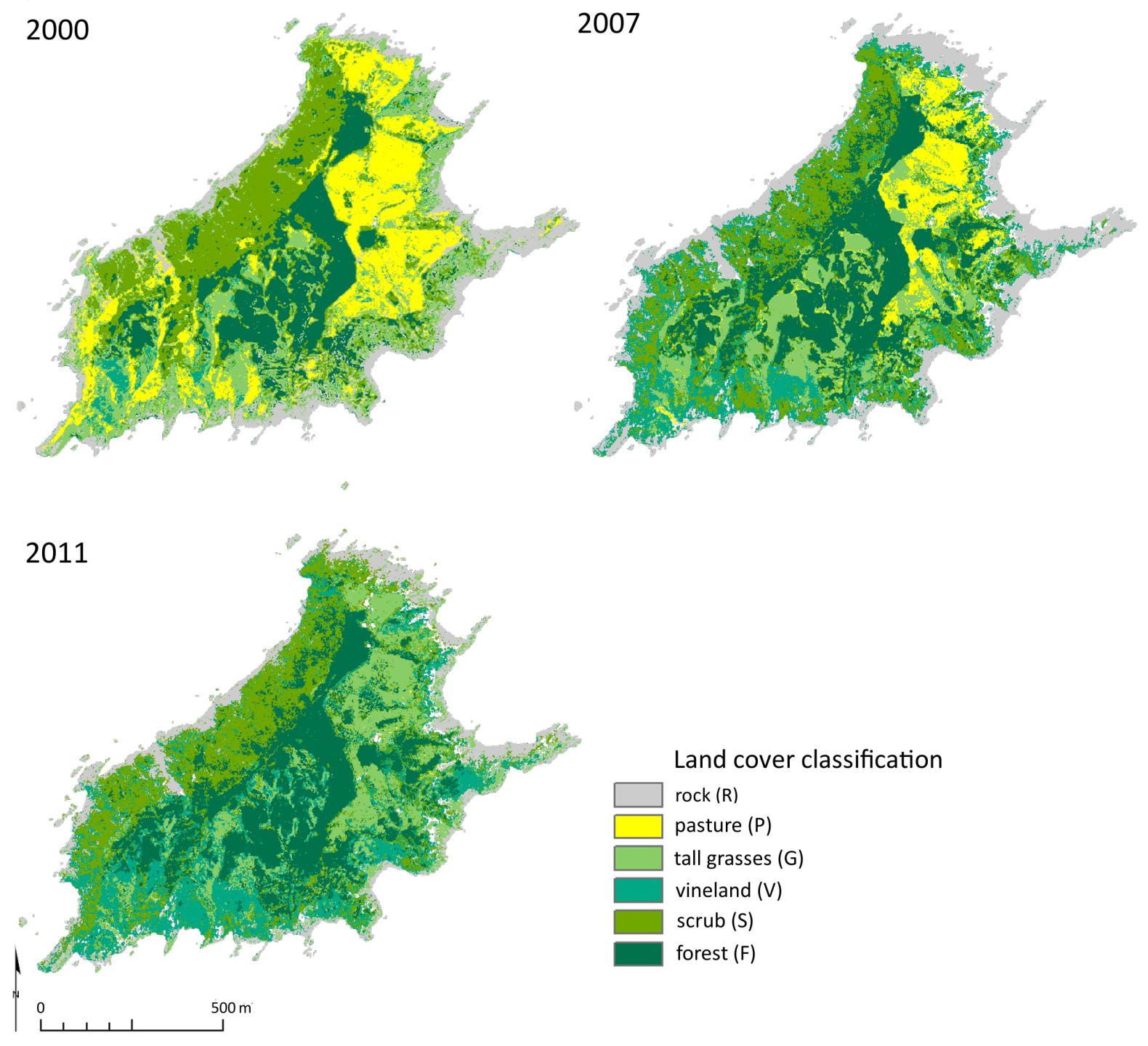

Land cover classification

rock (R)

pasture $(\mathrm{P})$

tall grasses $(G)$

vineland (V)

scrub (S)

forest (F)

Figure 4. Categorical land cover maps generated for Takapourewa from supervised maximum likelihood classification of aerial photography collected in three different years 


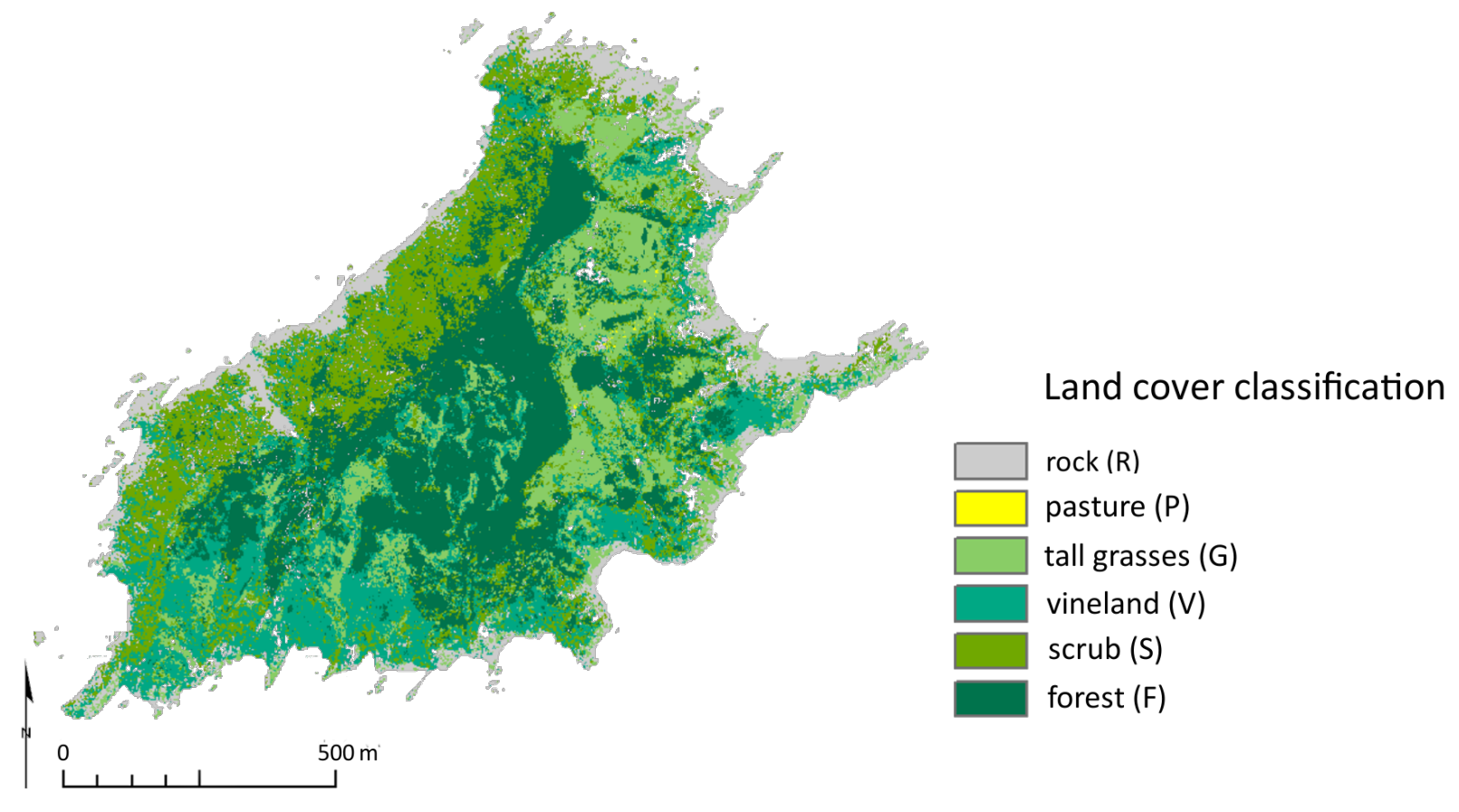

Figure 5. Land cover classification map predicted for Takapourewa for the year 2012 using the best-fit transition model from 2000/2011

\subsubsection{Vegetation canopy shading in rookeries}

The percent of canopy shading calculated across all sampled locations and both years $(\mathrm{N}=185)$ varied from $20.65 \%$ in the Winch Track rookery to $95.69 \%$ under the forest canopy in Keeper's Bush (Fig. 6). The maximum percent shading in non-forested areas was $94.10 \%$. The mean percent shading in forested and non-forested areas, respectively, was $94.15 \%$ and $43.93 \%$. Percent shading in most locations was between 20-60\%. Comparison of baseline GLS and LME models found no difference in shade cover at the sampled locations between 2011 and $2012\left(\mathrm{X}^{2}=1.58 \mathrm{e}^{-7}, \mathrm{df}=1, \mathrm{p}>0.99\right)$. LME models found that shading varied significantly, however, among locations $\left(\mathrm{X}^{2}=\right.$ 117.40, $\mathrm{df}=1, \mathrm{p}<0.0001)$. Removing the forested area of Keeper's Bush from the model did not eliminate the effect of location $\left(\mathrm{X}^{2}=44.62, \mathrm{df}=1, \mathrm{p}<0.0001\right)$. A binomial GLM found that canopy shading did not differentiate between nest and non-nest sites within the seven rookery areas containing both $\left(X^{2}=3.69, \mathrm{df}=1, \mathrm{p}=0.07\right)$. 
The primary goal of this portion of the study was to examine the differences among nest and non-nest locations in known rookeries, so the sampling design was biased towards areas that were classified as pasture prior to 2011. Of the 184 photos collected, 116 were classified as tall grasses in at least one sampling year. Of the remaining 68, 23 were classified as bare ground, 20 as scrub, 14 as vineland, and 11 as forest. An LME model with sampling location included as a random effect determined that vegetation type did not predict canopy shading in either rookery or non-rookery areas $(\mathrm{p}=0.85)$. Removing the forested area from the analysis, while still controlling for sampling location, did not expose a statistically significant effect of vegetation type on canopy shading $(\mathrm{p}=0.59)$.

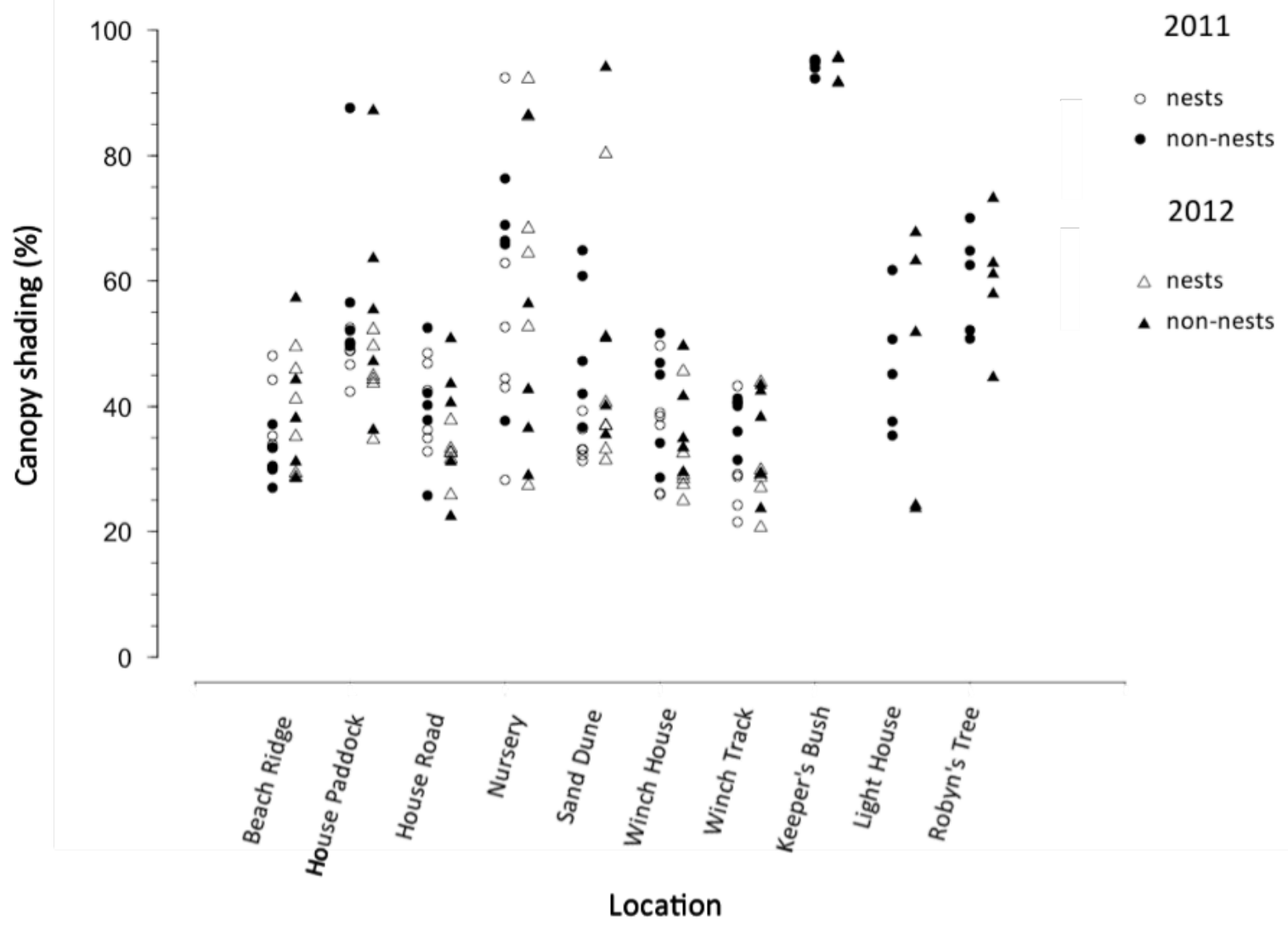

Figure 6. Percent of sky area shaded due to vegetation at all sampled nest and non-nest sites in 2011 and 2012. $\mathrm{N}=6$ for nests in rookery areas; $\mathrm{N}=5$ for non-nests in all areas. 
Locations of 1003 tuatara nests were recorded in ten rookeries throughout the course of the study, 895 of which were marked in the eight rookeries that were sampled across all six years of the study (Fig. 7). A saturated loglinear model was produced for rookeries sampled in all years by including the interaction between rookery and year $\left(X^{2}=0, p=1\right.$, deviance $\left.=0\right)$, indicating that the number of nests observed in each rookery deviated significantly from expected values among years $\left(X^{2}=154.81, d f=35, p\right.$ $=5.23 \mathrm{e}^{-17}$, deviance $\left.=174.46\right)$. The minimum expected frequency $=1.93$, and $14.58 \%$ of expected frequencies were $<5.00$ (Agresti 2002).

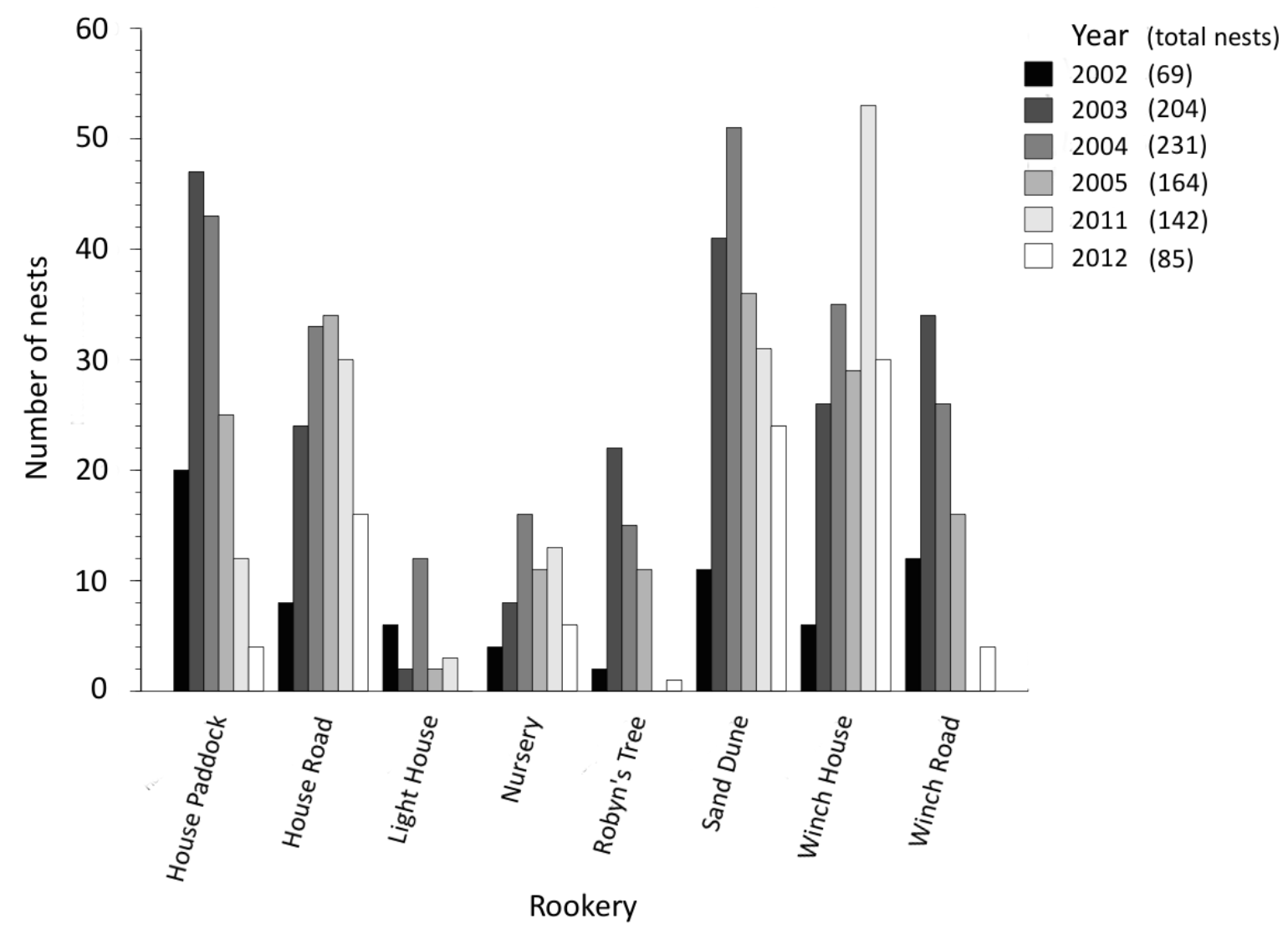

Figure 7. Bar plot showing the total number of nests $(\mathrm{N}=895)$ observed in each of the eight rookeries sampled across all six years of the study period 
A significant increase in nest numbers was observed in the Winch House rookery in 2011 and 2012, while a significant decrease in nest numbers was observed only in the House Paddock. Higher-than-expected nest numbers were observed in the Winch Road, Light House, and Robyn's Tree rookeries in at least one year between 2002-2004, with subsequent non-significant decreases observed in 2011 and 2012. No significant differences among years were observed in the House Road, Sand Dune, or Nursery rookeries (Fig. 8). 


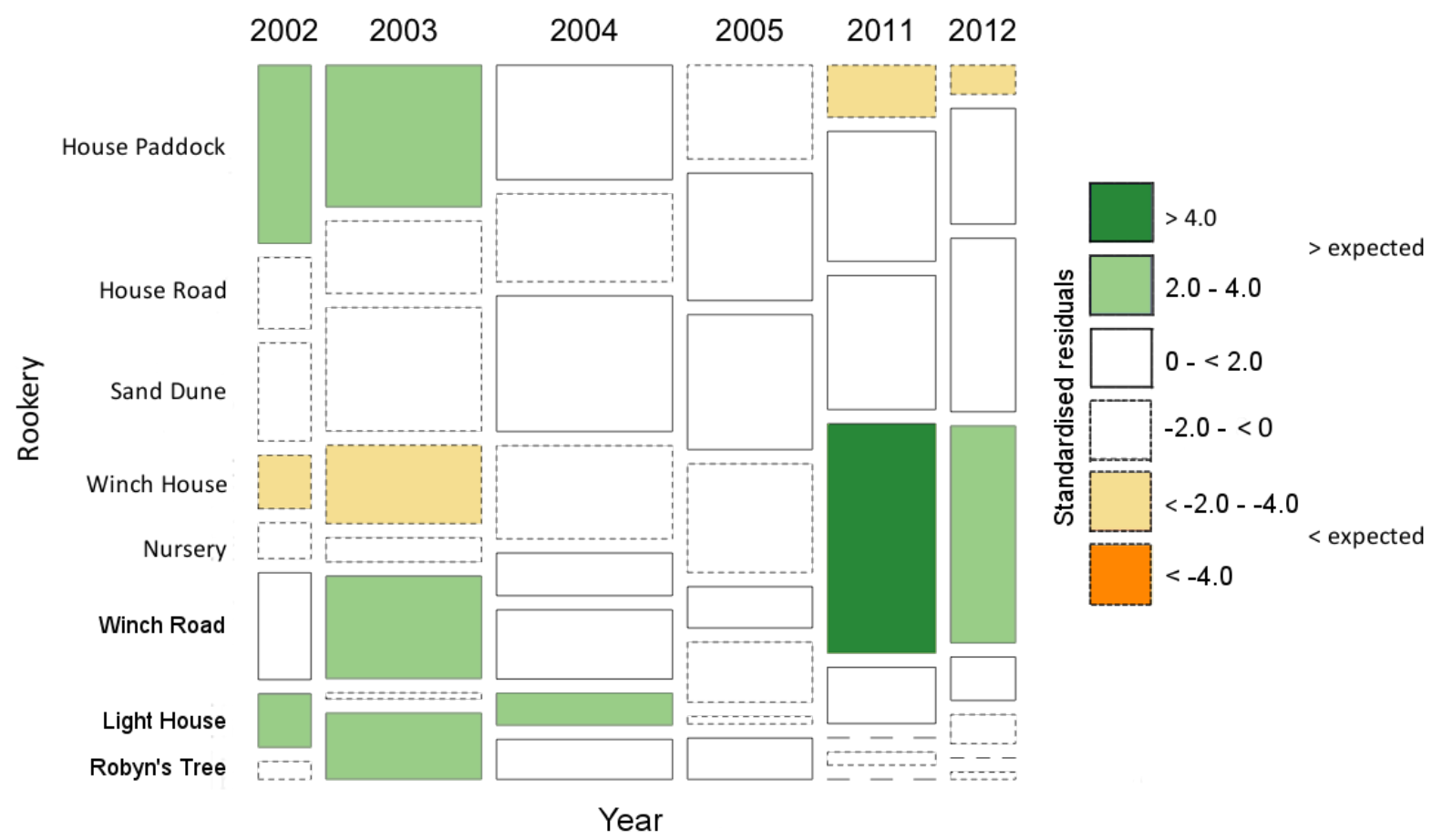

Figure 8. Two-way, area-proportional mosaic plot based on a loglinear model of nest numbers in the eight rookeries surveyed in all sampling years. Rectangle widths show the relative frequency of nests observed per year (e.g., more nests were observed in 2003 than in the previous year, so the 2003 column is wider than 2002). Rectangle heights show the relative frequencies of nests in each rookery per year (e.g., more nests were recorded in the House Paddock than in the Winch House in 2002, so the row is taller). Shading of standardised Pearson residuals indicates significantly higher (green) or lower (yellow) nest numbers than would be expected if the number of nests in any given rookery was independent of the nesting year. Borders of individual rectangles indicate whether model residuals are positive (solid) or negative (dashed). Degree of shading indicates significance of approximately $\mathrm{p}<0.05$ and $\mathrm{p}<0.001$, respectively, for lighter and darker shading (Friendly 1994, 2000). 


\subsubsection{Nest site selection and vegetation}

Overlaying all nest site GPS coordinates with the categorical vegetation maps generated a set of 465 pixels containing at least one nest during at least one year of the study period. Of nest-containing pixels, 399 were located in the eight rookeries sampled across all six years of the study. The remaining 66 were observed in either the Beach Ridge or Winch Track rookeries, which were sampled only in 2011 and 2012 and not included in subsequent analyses, or were located on Clare's Track or the Palace Road (in any year) and sampled opportunistically. Vegetation type was interpolated across the study period for all nest-containing pixels (Fig. 9). All locations underwent an expected rapid decrease in pasture between 2007 and 2011. Already-low proportions of rock (i.e., bare ground) likewise decreased across the study period. Forest was the only land cover class that acted as an absorbing state (i.e., no pixel transitioned from forest to any other class). 


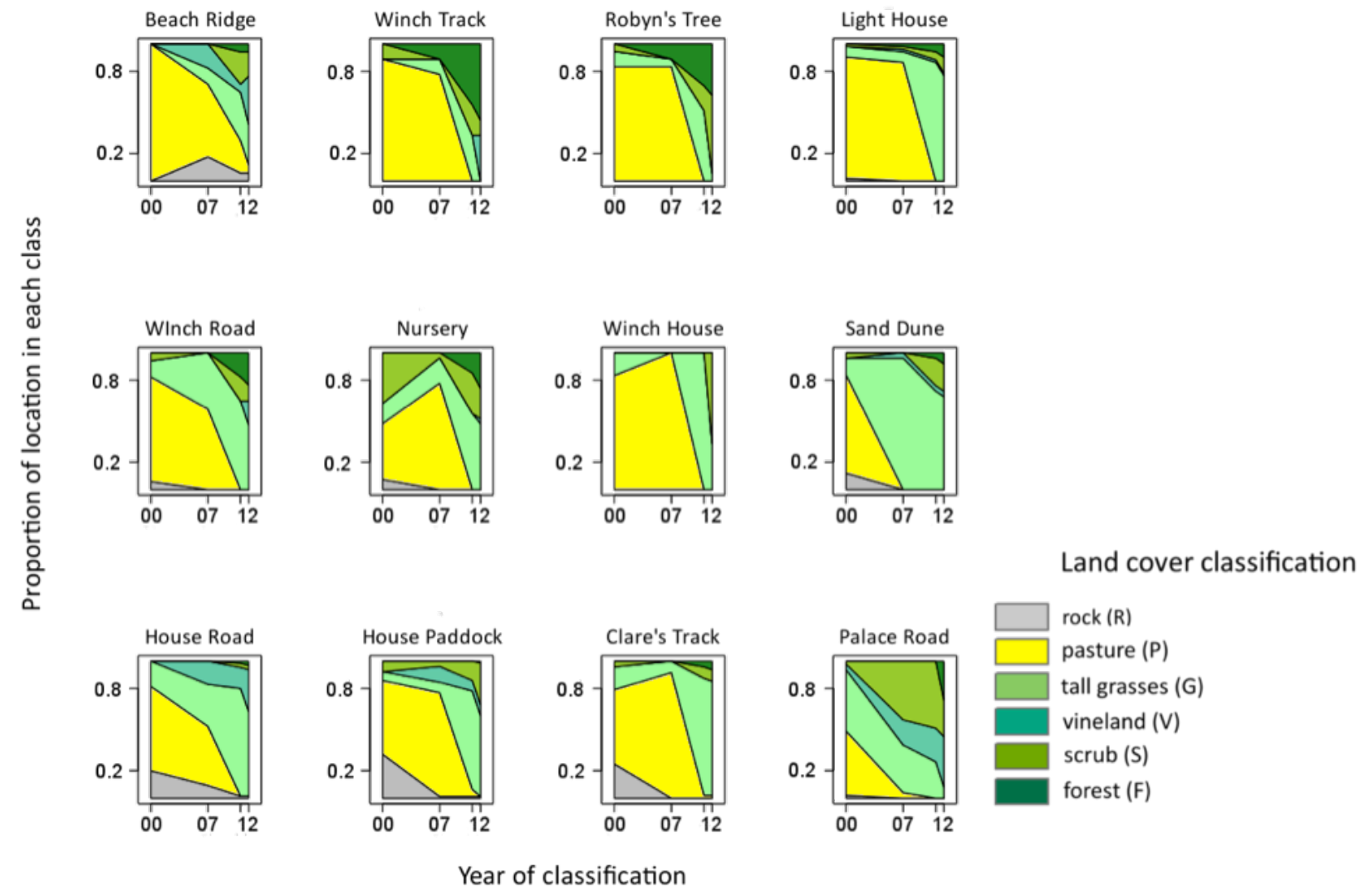

Figure 9. Polygon plots showing the proportion of each sampled nesting location in each land cover class, interpolated across seven years between 2000 and 2007, four years between 2007 and 2011, and one year between 2011 and 2012. Interpolated vegetation proportions were generated across all years using all nest-containing pixels $(\mathrm{N}=465)$, regardless of the year(s) in which nests were observed in each pixel. The plotted proportions of land cover classes are therefore independent of nest density in any given year. 'Rock' indicates areas of both unvegetated soil and solid rock, since nests may be constructed underneath large boulders. The Beach Ridge and Winch Track rookeries were not included in the analysis of nest density due to unequal sampling effort across study years. 
Poisson GLMs found that the interpolated proportions of every vegetation type predicted nest density (Fig. 10). Increasing proportions of all land cover types except vineland predicted a reduction in the number of nests within pixels. Pasture was the single best predictor of nest density, followed by the proportion of tall grasses (Table 2).

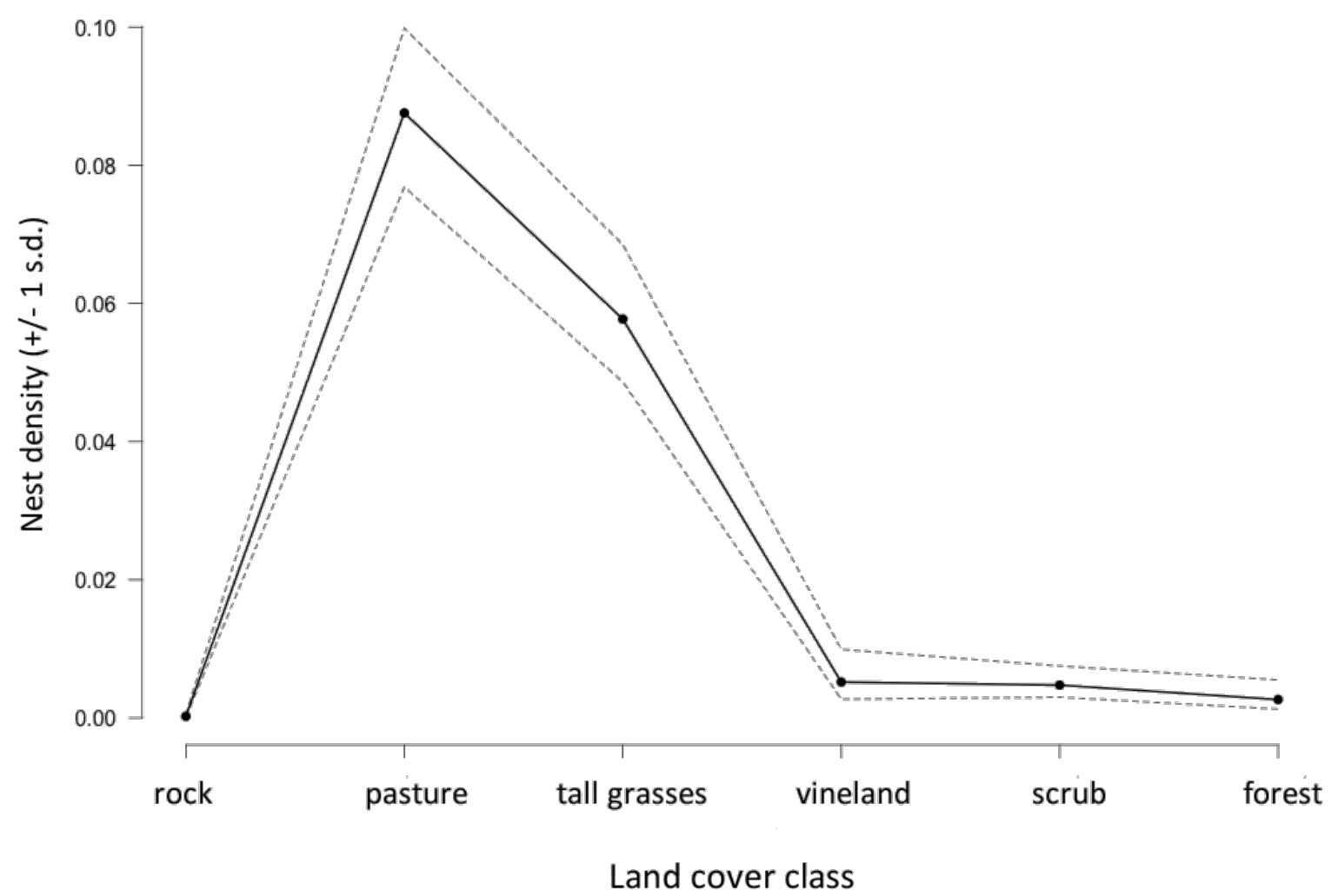

Figure 10. Line plot showing the effect of land cover class on mean nest density, with dotted lines showing 95\% confidence intervals 
Table 2. Summary of results of single-predictor Poisson GLMs and corresponding likelihood ratio tests examining effects of each vegetation type on nest density in areas sampled in all six study years. Rate ratios show change in mean nest density with increases of each vegetation type. Asterisks indicate the level of significance of $p$-values: $p \leq 0.05^{*}, p \leq 0.01^{* *}, p \leq 0.001^{* * *}$.

\begin{tabular}{rccccc}
\hline & & \multicolumn{3}{c}{$95 \%$ Cls for rate ratios } \\
Land cover type & 6-coeff & lower & rate ratio & upper & $\boldsymbol{X}^{2}$ \\
\hline rock & $-1.50^{* *}$ & 0.09 & 0.22 & 0.57 & $10.15^{* *}$ \\
pasture & $-1.12^{* * *}$ & 0.27 & 0.33 & 0.39 & $134.12^{* * *}$ \\
tall grasses & $-0.91^{* * *}$ & 0.32 & 0.40 & 0.51 & $59.12^{* * *}$ \\
vineland & $0.85^{* *}$ & 1.25 & 2.33 & 4.37 & $6.68^{* *}$ \\
scrub & $-1.16^{* * *}$ & 0.19 & 0.31 & 0.51 & $24.43^{* * *}$ \\
forest & $-1.38^{* * *}$ & 0.13 & 0.25 & 0.49 & $19.06^{* * *}$ \\
\hline
\end{tabular}

A total of 2202 female tuatara were observed nesting during the study period. Of all females sampled, 2119 were observed nesting in the eight rookeries surveyed in all six study years (Fig. 11). One female was observed in rookery areas annually from 20022005 but was not included in subsequent analyses, as her reproductive condition was unconfirmed (N. Nelson pers. comm.). A saturated loglinear model was generated by including the interaction between rookery and year $\left(X^{2}=0, p=1\right.$, deviance $\left.=0\right)$, indicating that the number of females observed in each rookery deviated significantly from expected values among years $\left(\mathrm{X}^{2}=347.37, \mathrm{df}=35, \mathrm{p}=4.32 \mathrm{e}^{-53}\right.$, deviance $=$ 361.99). The minimum expected frequency $=5.16$ (Agresti 2002). Either higher- or lower-than-expected numbers of nesting female tuatara were observed in at least one study year in every rookery except the Winch House (Fig. 12). 


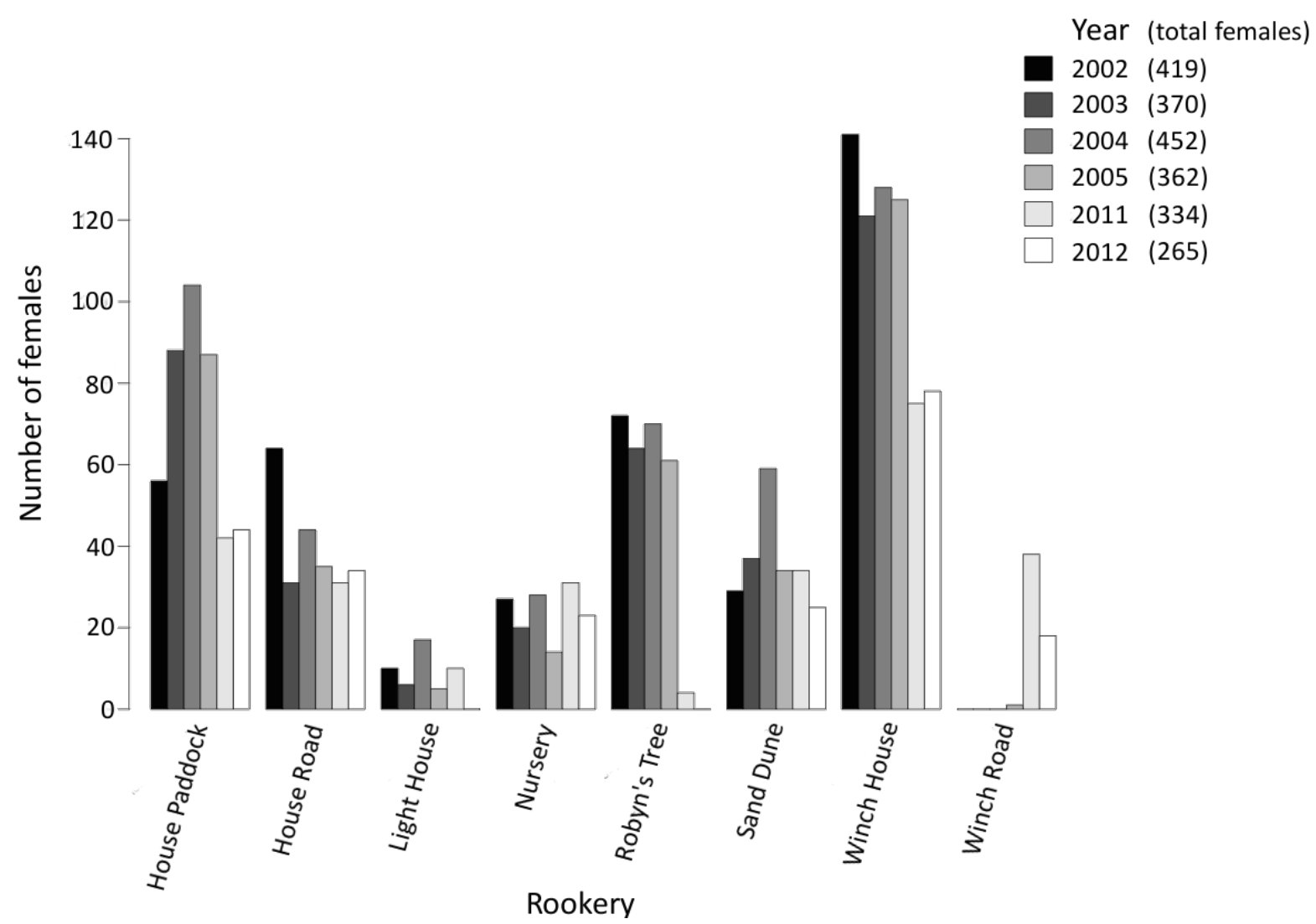

Figure 11. Bar plot showing the total number of nesting female tuatara $(\mathrm{N}=2119)$ observed in the eight rookeries sampled across all six years of the study period. 


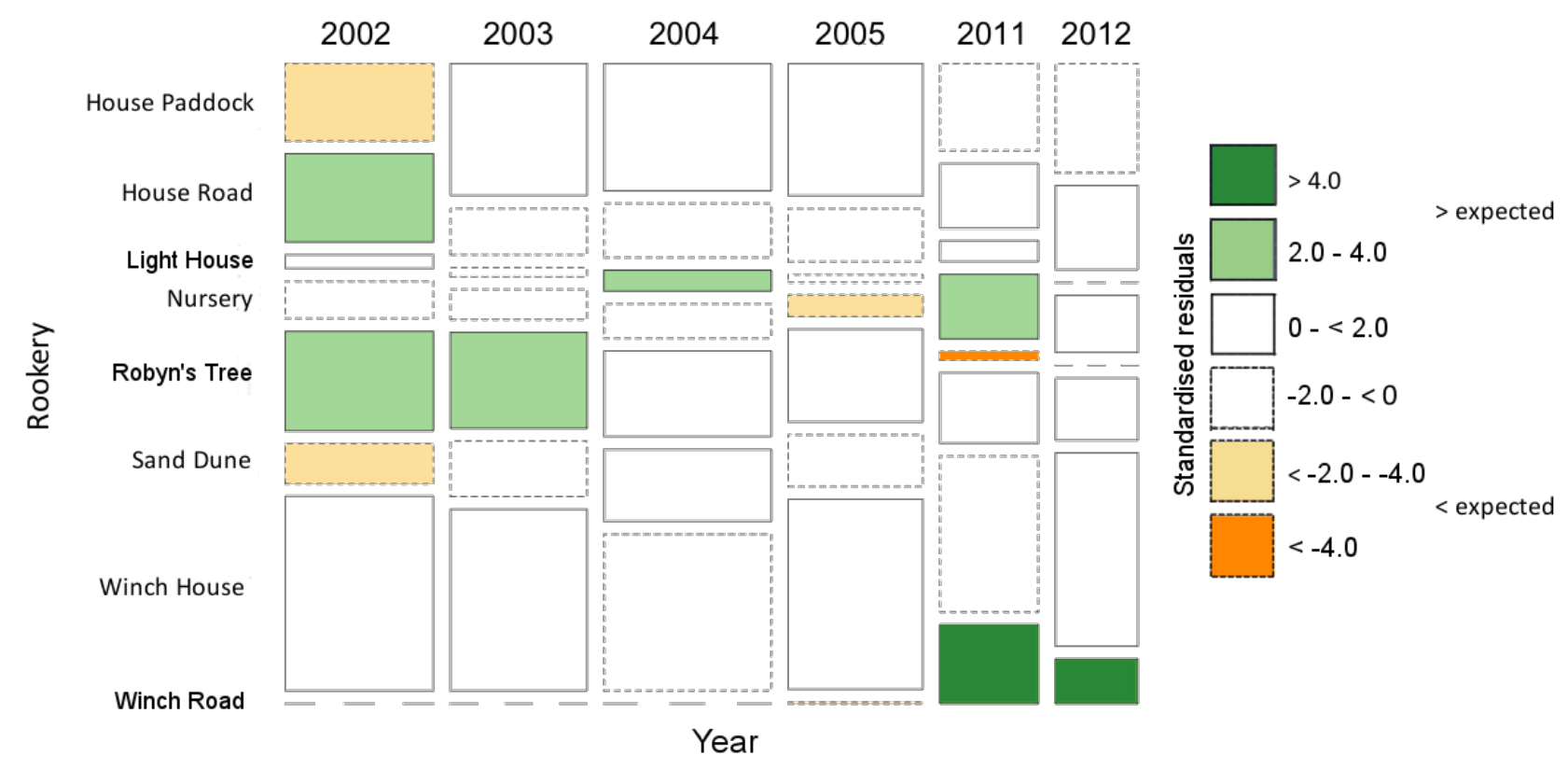

Figure 12. Two-way, area-proportional mosaic plot based on a loglinear model of females nesting in rookeries sampled in all study years. Rectangle widths show the relative frequency of females observed per year (e.g., fewer females were observed in 2011 than in 2004, so the 2004 column is wider). Rectangle heights show the relative frequencies of females in each rookery per year (e.g., more females were recorded in the House Paddock than in the Nursery in 2002, so the row is taller). Shading of standardised Pearson residuals indicates significantly higher (green) or lower (yellow) frequencies than would be expected if the number of females in any given rookery was independent of the nesting year. Borders of individual rectangles indicate whether model residuals are positive (solid) or negative (dashed). Degree of shading indicates significance of approximately $\mathrm{p}<0.05$ and approximately $\mathrm{p}<0.001$, respectively, for lighter and darker shading (Friendly 1994, 2000). 
Of the 2202 nesting females sampled during the study period, 1508 were individually identifiable via PIT-tags. Of those, 429 were observed nesting at least twice between 2002 and 2012. Each unique combination of sampling years and rookery choices generated 1224 possible segments, 740 of which were observed and modelled to examine whether vegetation affected the probability of switching rookeries. Females who nested three times $(\mathrm{N}=61)$ during the study period increased the count of two segment types (i.e., the second year of the first segment and first year of the second segment were equivalent). Of observed segments, 687 represented a switch in rookery from the first to the second year, and 53 represented choice of the same rookery in both years. The minimum proportion of rookery switches between years, 0.02 , was recorded in 2003-2005 segments (Fig. 13). The mean proportion of switches between segment years was 0.22 . The mean proportion of switches for segments in which the second year was either 2011 or 2012 was 0.28 . Each of the eight rookeries sampled in all six study years contained at least one segment representing a switch in rookery choice (Table 3).

Relative to their initial choices in 2002-2005, higher proportions of females switched rookeries in the final two study years. Binomial GLMs found that the first year of observation (i.e., 2002, 2003, etc.) affected whether females chose the same rookery in the second observed year $\left(\beta=0.04, X^{2}=6.06, \mathrm{df}=1, \mathrm{p}=0.02\right)$. Neither the second year $\left(\beta=0.03, X^{2}=1.48, d f=1, p=0.23\right)$ nor the number of years between observations $(\beta=$ $0.08, X^{2}=0.15, d f=1, p=0.70$ ) influenced rookery choice. 


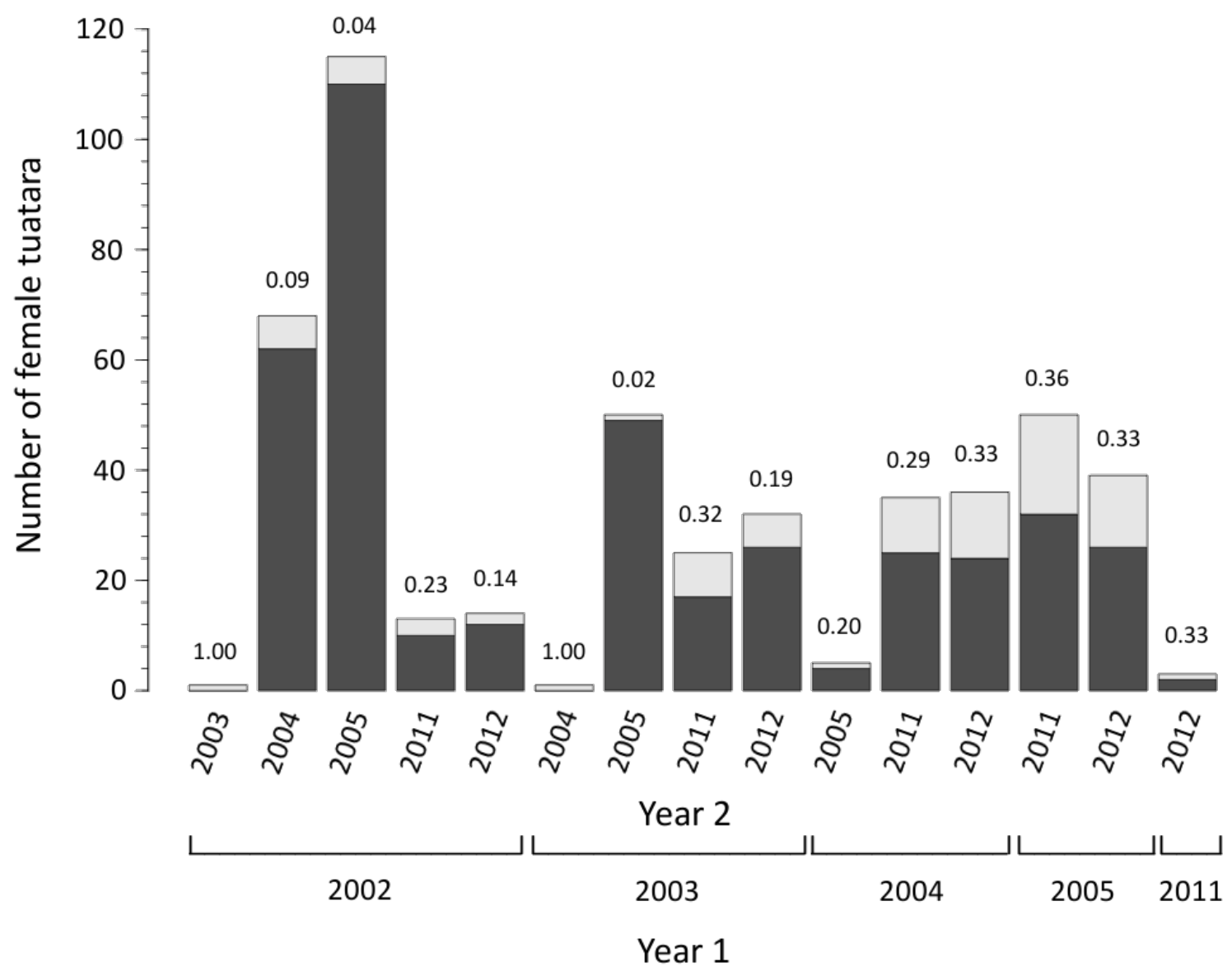

Figure 13. Bar plot showing same (dark gray) vs. different (light gray) rookery choices in year 2, relative to year 1 , made by individual female tuatara observed nesting multiple times during the study period ( $\mathrm{N}=492$ observations of 429 individuals). Labels on individual bars show the proportion of nesting females in each year 2 that chose a different rookery, relative to their choice in year 1 . The single individual observed annually from 2002-2005 was not included in analyses of rookery choice, as her reproductive status could not be confirmed (Nelson pers. comm.). 


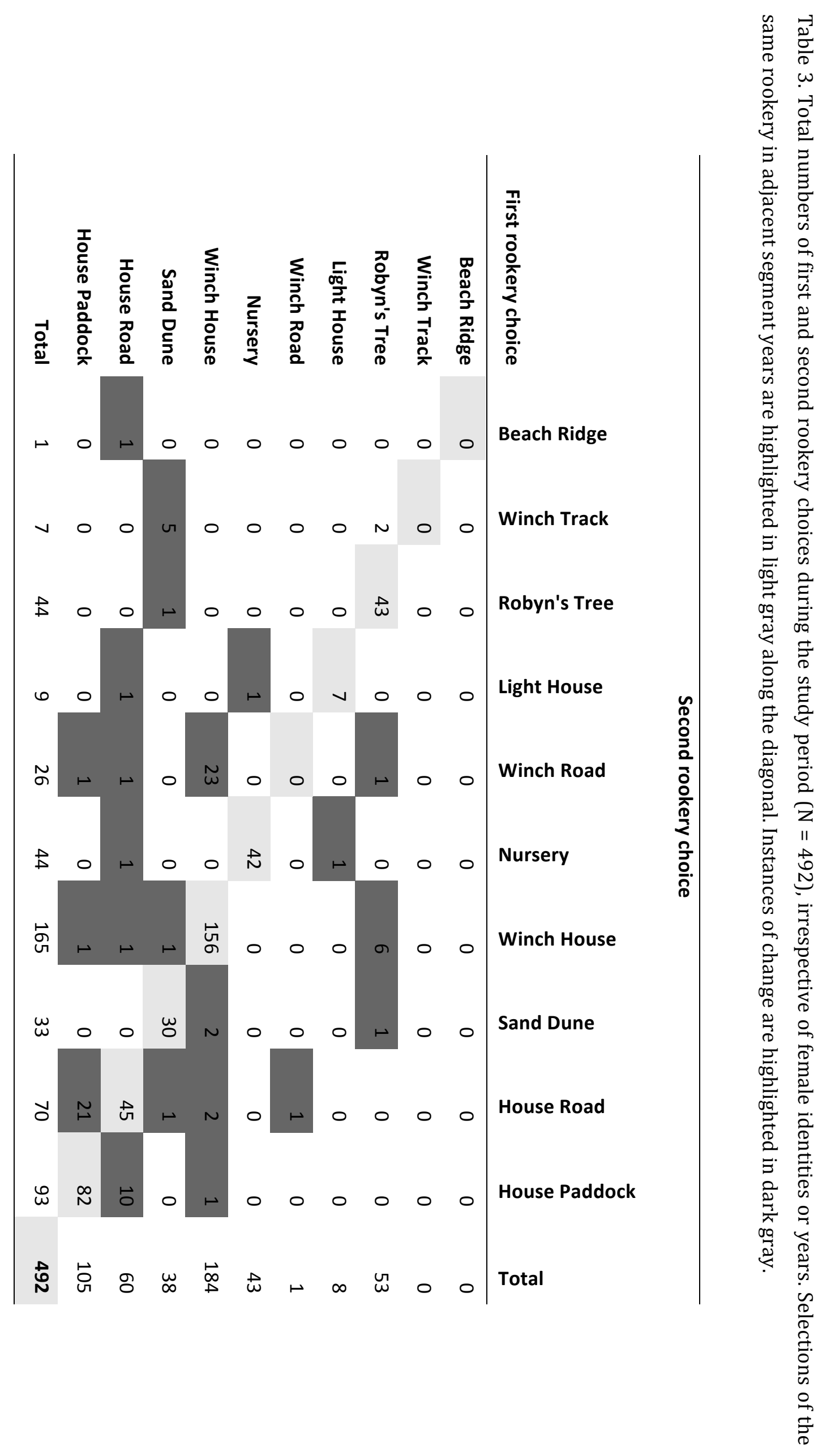


Vegetation cover affected rookery selection between segment years (Table 4). Binomial GLMs found that only the proportions of rock and tall grasses in a rookery in the first nesting year of a segment affected rookery choice in the second year of a segment. A higher proportion of rock in the first rookery increased the likelihood that a nesting female would choose the same rookery in the second year of nesting. A higher proportion of tall grasses decreased the probability that the second rookery choice would be the same. The proportions of all land cover types except tall grasses in the first rookery in the second year of a segment predicted whether the first and second rookery choices would be the same. A higher proportion of forest in the initial rookery in the second year was the single best predictor of rookery switching, while a higher proportion of rock in the first rookery in the second year increased the likelihood that the first and second rookery choices would be the same. The proportion of all land cover types except scrub in the second year of a segment in the rookery chosen in that year predicted whether the first and second rookery choices were the same. A higher proportion of forest in the second rookery was the single best predictor that rookery choices would differ, while higher proportions of rock and vineland increased the likelihood that rookery choices would be the same in both segment years. 
Table 4. Summary of results of binomial GLMs and corresponding likelihood ratio tests examining effects of land cover within rookeries in the first and second years (year1, year2) on the probability that first and second rookery choices (rook1, rook2) would be the same between years. Asterisks indicate the level of significance of $p$-values: $p \leq 0.05^{*}, \mathrm{p} \leq 0.01^{* *}, \mathrm{p} \leq 0.001^{* * *}$.

\begin{tabular}{|c|c|c|c|c|c|}
\hline \multicolumn{6}{|c|}{ Proportion of land cover type in the first rookery in the first year } \\
\hline \multirow[b]{2}{*}{ Land cover type } & \multicolumn{5}{|c|}{ 95\% Cls for odds ratios } \\
\hline & 6-coeff & lower & odds ratio & upper & $x^{2}$ \\
\hline rock & $28.45^{* * *}$ & $2.06 \mathrm{e}^{10}$ & $2.28 \mathrm{e}^{12}$ & $2.51 \mathrm{e}^{14}$ & $275.79 * * *$ \\
\hline pasture & 0.60 & 0.53 & 1.83 & 6.23 & 0.95 \\
\hline tall grasses & $-21.89 * * *$ & $6.15 \mathrm{e}^{-13}$ & $3.10 \mathrm{e}^{-10}$ & $1.56 \mathrm{e}^{-7}$ & $141.55^{* * *}$ \\
\hline vineland & -1.69 & $1.75 e^{-3}$ & 0.18 & 19.46 & 0.51 \\
\hline scrub & -2.35 & $6.38 \mathrm{e}^{-3}$ & 0.10 & 1.44 & 3.02 \\
\hline forest & -86.13 & 0 & $3.91 e^{-38}$ & $\infty$ & 2.36 \\
\hline \multicolumn{6}{|c|}{ Proportion of land cover type in the first rookery in the second year } \\
\hline rock & $10.06 * * *$ & 382.35 & $2.34 \mathrm{e}^{4}$ & $1.43 e^{6}$ & $23.57 * * *$ \\
\hline pasture & $0.77^{* *}$ & 1.21 & 2.16 & 3.87 & $6.86^{* *}$ \\
\hline tall grasses & 0.03 & 0.48 & 1.03 & 2.23 & $5.80 e^{-3}$ \\
\hline vineland & $-3.90 * * *$ & $2.02 e^{-3}$ & 0.02 & 0.20 & $12.64 * * *$ \\
\hline scrub & $-1.57^{*}$ & 0.04 & 0.21 & 0.96 & $4.21^{*}$ \\
\hline forest & $-35.88 * * *$ & $1.96 \mathrm{e}^{-22}$ & $2.61 \mathrm{e}^{-16}$ & $3.46 \mathrm{e}^{-10}$ & $86.46 * * *$ \\
\hline \multicolumn{6}{|c|}{ Proportion of land cover type in the second rookery in the second year } \\
\hline rock & $28.38 * * *$ & $9.25 e^{9}$ & $2.11 \mathrm{e}^{12}$ & $4.81 \mathrm{e}^{14}$ & $141.54 * * *$ \\
\hline pasture & $0.77^{* *}$ & 1.26 & 2.16 & 3.70 & $7.95^{* *}$ \\
\hline tall grasses & $-1.18 * * *$ & 0.16 & 0.31 & 0.57 & $13.98 * * *$ \\
\hline vineland & $11.24^{* * *}$ & $1.53 \mathrm{e}^{3}$ & $7.62 e^{4}$ & $3.79 e^{6}$ & $45.39 * * *$ \\
\hline scrub & 0.62 & 0.50 & 1.85 & 6.91 & 0.83 \\
\hline forest & $-71.47 * * *$ & $2.44 \mathrm{e}^{-40}$ & $9.14 e^{-32}$ & $3.43 e^{-23}$ & $218.15^{* * *}$ \\
\hline
\end{tabular}


Multinomial GLMs were used to examine the effects of observation year, initial rookery choice, and rookery vegetation composition on the second rookery choice. The two overall relatively best-fit models (by AICc value) included the initial rookery, proportions of both vineland and tall grasses in the second rookery in the second year of each segment and either the proportion of forest or scrub in the second rookery in the second segment year (Table 5; Appendix 2.5).

Table 5. Summary of model comparisons among top 12 (by AICc value) candidate multinomial GLMs and a null model (model 0) used to examine the effects of years, initial rookery choice, and interpolated proportions of land cover types in rookeries on subsequent rookery choice. Variable codes are first and second rookery choices (rook1, rook2); first and second segment years (year1, year2); a binary indicator of rookery choice between years (stay); the number of years between observations (interval); and the proportion of each land cover type $[\mathrm{X}]$ in the first rookery in the first year (r1y1.X), the first rookery in the second year (r1y2.X), and the second rookery in the second year (r2y2.X). Summaries of all 91 candidate models are in Appendix 2.5.

\begin{tabular}{|c|c|c|c|c|c|c|}
\hline model \# & model (rook2 ) & deviance & AICc & $\Delta_{\mathrm{i}} \mathrm{AIC}$ & $\omega_{\mathrm{i}} \mathrm{AIC}$ & rel.LL \\
\hline 85 & rook $1+r 2 y 2 . V+r 2 y 2 . G+r 2 y 2 . S$ & $<0.01$ & 200.71 & 0.00 & 0.34 & 1.00 \\
\hline 86 & rook $1+r 2 y 2 . V+r 2 y 2 . G+r 2 y 2 . F$ & $<0.01$ & 200.71 & 0.00 & 0.34 & 1.00 \\
\hline 70 & rook1 + r2y2.V + r2y2.G + interval & 0.08 & 200.80 & 0.09 & 0.32 & 0.96 \\
\hline 84 & rook $1+r 2 y 2 . V+r 2 y 2 . G+r 2 y 2 . P$ & 9.83 & 210.54 & 9.83 & $<0.01$ & $<0.01$ \\
\hline 64 & rook1 + r2y2.V +r2y2.G & 29.53 & 211.77 & 11.06 & $<0.01$ & $<0.01$ \\
\hline 69 & rook $1+r 2 y 2 . V+r 2 y 2 . G+$ stay & 12.06 & 212.77 & 12.06 & $<0.01$ & $<0.01$ \\
\hline 78 & rook $1+r 2 y 2 . V+r 2 y 2 . G+r 1 y 2 . P$ & 13.60 & 214.31 & 13.60 & $<0.01$ & $<0.01$ \\
\hline 87 & rook1 + r2y2.V + r2y2.G + r2y2.F + interval & $<0.01$ & 219.23 & 18.52 & $<0.01$ & $<0.01$ \\
\hline 88 & rook $1+r 2 y 2 . V+r 2 y 2 . G+r 2 y 2 . F+r 1 y 2 . P$ & $<0.01$ & 219.23 & 18.52 & $<0.01$ & $<0.01$ \\
\hline 90 & rook $1+r 2 y 2 . V+r 2 y 2 . G+r 2 y 2 . F+r 2 y 2 . S$ & $<0.01$ & 219.23 & 18.52 & $<0.01$ & $<0.01$ \\
\hline 89 & rook $1+r 2 y 2 . V+r 2 y 2 . G+r 2 y 2 . F+r 2 y 2 . P$ & 0.26 & 219.49 & 18.78 & $<0.01$ & $<0.01$ \\
\hline 0 & 1 & 1847.84 & 1865.87 & 1665.16 & 0 & 0 \\
\hline
\end{tabular}




\subsection{Discussion}

\subsubsection{Summary of major findings}

Understanding patterns of nest site selection, particularly how females respond to environmental variation, is critical for predicting the impacts of climate change on populations. In species with temperature-dependent sex determination, female nesting behaviour has the potential to affect population demographics in addition to individual fitness and survival. Detecting biologically meaningful behavioural variation in species that are long-lived or that reproduce intermittently may be constrained by life history as well as by the difficulty inherent in collecting long-term behavioural and environmental data. Using ongoing ecological restoration as context, this study quantified the relationships between vegetation change and nest site selection in a population of tuatara to determine whether female nesting behaviour responds in-situ to environmental variation.

Tuatara nesting areas on Takapourewa have undergone relatively rapid and significant changes in vegetation composition, experiencing an almost complete loss of maintained pasture between 2007 and 2011. Rookeries in 2011 and 2012 were comprised primarily of tall grasses, though the proportions of other vegetation types continue to increase. The inability of vegetation modelling to distinguish areas of Takapourewa that have been actively replanted from those that have undergone only 'natural' regrowth suggests that restoration efforts have accelerated replacement of modified land cover types with native vegetation in areas subject to relatively recent grazing pressure.

Both nest density and the numbers of nesting females observed in individual rookeries were significantly different among study years. Vegetation type, especially the proportion of pasture in a nesting location, affected nest density; however, all land cover 
types, including bare ground and pasture, were associated with a decrease in nest numbers. Canopy shading was not different between nest and non-nest sites within previously established rookery areas, confirming previous research that found no preference for 'open' versus artificially shaded locations in nesting female tuatara (Refsnider et al. 2010). Vegetation type did not predict microsite canopy shading.

At a broader scale, vegetation composition not only influenced rookery-switching behaviour but also predicted the specific rookery choices of nesting females. Proportions of bare ground and forest were the best individual predictors of rookery choice, supporting previous findings that tuatara likely do not construct nests in forest (Newman 1987; Cree et al. 1989) and prefer locations with minimal vegetation (Refsnider et al. 2010), at least on Takapourewa. Bare ground increased the likelihood that a female would nest in the same rookery in different nesting seasons, while forest increased the likelihood that a female would switch rookeries. For all females observed, the most likely second rookery choice during this study was always the initial rookery. Including proportions of land cover types in the second rookery, especially vineland and tall grasses, improved models of rookery selection, regardless of whether or not the second rookery was the same one chosen in the initial nesting season.

\subsubsection{Nest site selection and vegetation change}

Localisation of tuatara nest sites on Takapourewa is in transition. Research through 2005 found very low short-term levels of rookery-switching in tuatara and no significant response to experimentally manipulated vegetation density (Refsnider et al. 2010). Prior to anthropogenic modification, areas identified as tuatara rookeries in both the current study and in previous research were completely forested. Whether vegetation played a significant role in nest site selection or in controlling tuatara offspring sex ratios during prehistoric periods of fluctuating climate is unknown. 
Likewise, whether tuatara display natal philopatry (i.e., whether females tend to nest initially in the same locations from which they hatched) (Lindeman 1992; Reinhold 1998) has not been directly examined. Doing so would require monitoring individuals from known offspring cohorts for more than a decade. The increase in rookeryswitching behaviour observed in the current study, however, suggests that high levels of rookery fidelity in tuatara are likely an indicator of rookery suitability, itself an artefact of habitat modification via grazing. Philopatry to either rookeries or specific locations within a single rookery is probably not the primary driver of nest site selection in tuatara.

Rapid land cover change during the study period revealed a clear link between vegetation composition and nest site selection. Every land cover class defined for this study was a significant predictor of nest density, though vineland was the only vegetation type that predicted an increase in nest density. Transitions to vineland occurred from multiple initial vegetation classes but were spatially prevalent at forest edges, which provide habitat for juveniles (McIntyre 1988). The association of vineland with an increase in nest density may indicate a secondary preference for nesting in close proximity to home burrows (Refsnider et al. 2010) or adjacent to habitat suitable for offspring (Refsnider \& Janzen 2010).

That pasture was associated with a decrease in nest density was a counterintuitive result, given the previously reported importance of pasture areas as tuatara nesting habitat. An increase in any type of vegetation may artificially decrease observed nest numbers simply because nests are more difficult to see when obscured by vegetation. However, more nests were observed in both 2011 and 2012, after nesting areas had transitioned primarily to tall grasses, than in 2002, when the majority of utilised nesting space within rookeries was classified as pasture. Likewise, a decrease in per-pixel nest density does not necessarily indicate a decrease in overall nesting activity. 
Vegetation composition clearly impacts nest numbers; however, nest density alone is probably not a sufficient indicator of rookery suitability. Higher proportions of pasture in nesting locations predicted the highest overall nest density of any land cover type; however, nest density may have been lower in large pastures (e.g., the House Paddock rookery) simply because suitable nesting space was not a limiting factor in those areas. The number of nests in the House Paddock was greater than expected in both 2002 and 2003, even though the proportion of pasture remained similar through 2007. As a result, pasture was associated with significantly decreasing numbers of nests. The inability of the land cover classification methodology to capture fuzzy transitions between vegetation types, particularly from pasture to tall grasses (i.e., to differentiate among different 'pasture' heights), could also be responsible for the link between pasture and decreasing nest numbers.

Changes in land cover on Takapourewa have modified the observed density of tuatara nests as well as nest site selection itself. Females that nested in rookeries with a relatively high proportion of rock (i.e., bare ground) were more likely to choose the same rookery in subsequent years. Females that nested in rookeries with initially higher proportions of tall grasses were less likely to choose the same rookery in subsequent years. Increasing proportions of vineland, scrub, or forest in the first chosen rookery reduced the likelihood that a female would choose the same rookery in her second nesting season. Females were significantly more likely to switch rookeries if higher proportions of the initial rookery transitioned to forest between the first and second nesting seasons.

For future studies, accumulation of nest-site selection data across additional years would increase understanding of aspects of tuatara reproductive behaviour not examined here. Quantifying the contribution of phenotypic plasticity to nest site choice of individual females using multinomial longitudinal data would require a larger sample of known females with at least three nesting records. Additionally, while tuatara are 
thought to select a nest site based primarily on the likelihood of successful hatching (Nelson unpub. data), continued research within an increasingly heterogeneous environment as well as with additional populations may elucidate whether offspring sex ratios are controlled by some females indirectly via choice of nest site.

Continued restoration of vegetation on Takapourewa may have additional consequences for tuatara nesting. Regrowth of forest in particular may eventually lead to increased competition among female tuatara for a lower number of preferred nesting sites. As a result, the fitness of 'early' nesters could decrease, as more nests are excavated and eggs destroyed by 'late' nesters (Refsnider et al. 2009); the fitness of late nesters would subsequently increase. In addition, nesting areas may undergo a spatial shift if previously popular rookeries become unsuitable for incubation.

Female tuatara on Takapourewa have been observed migrating during nesting season from their home burrows to the cliffs that surround the island (Moore 2008). As forest cover increases, 'preferred' nesting sites may be increasingly defined by territorial proximity and the relative amount of energy expenditure required for undertaking nesting migration (Refsnider et al. 2010). A significant reduction in body condition of tuatara observed on Takapourewa from 1949 - 2003 suggests that the population may be experiencing delayed density-dependent effects of habitat modification (Moore et al. 2007). If nesting requires increasingly higher energy expenditure, the proportion of females nesting in any given season may decrease, reducing potential population growth. Finally, ecological restoration will likely lead to changes in the abiotic environment of incubation, regardless of nest site choice. In particular, increased vegetation can both directly and indirectly affect soil temperature cycles, consequently influencing hatching success, juvenile fitness and survival, sex ratios, and population size. 


\subsubsection{Nest site selection and canopy shading}

While vegetation clearly influences nest site selection, tuatara did not choose nest locations based on microsite-scale shade availability. With the exception of areas under the forest canopy, which were consistently $>90 \%$ shaded, vegetation type did not predictably influence the amount of shade available at any of the sampled locations. Likewise, shading did not determine whether a particular location within a rookery area was or was not a nest site, and every nest site sampled was partially shaded (20-60\%), regardless of predominant vegetation type.

Canopy shading should not by itself be considered a barrier to nest construction. Continued re-growth of vegetation will eventually reduce the proportion of bare soil on the island to near-zero and increase the proportion of forest to approximately prehuman levels. Previous research has found no evidence of tuatara nesting in the areas currently classified as forest on Takapourewa (Newman 1987; Cree et al. 1989; Nelson unpub. data). Whether forested areas were utilised as rookeries prior to the arrival of humans is unknown. Under current climate conditions, nests constructed in cooler locations have a lower probability of hatching, since completion of embryonic development is temperature-dependent. Even if tuatara do not choose to nest under the forest canopy under warmer climate scenarios, few locations on Takapourewa that are physically amenable to digging a nest are completely devoid of vegetation. Additional factors (e.g., vegetation height or soil characteristics) may cause soil temperature profiles under the forest canopy to be unsuitable for either nesting or embryonic development or both. Research on warmer-latitude islands (e.g., the northern island of Hauturu) is needed to determine whether soil temperatures under forest canopy are insufficient for completion of embryonic development in other populations of tuatara (Chapter 5). 
The primary concern for conservation of species with TSD is the effect of climate warming on population sex ratios. Offspring sex is determined in tuatara within the 25$35 \%$ window of total embryonic development (i.e., the thermo-sensitive period, or TSP) (Nelson et al. 2010). If increased vegetation shading reduces the amount of belowcanopy solar radiation that reaches ground level, tuatara nests could experience cooler temperatures in summer months (i.e., when the potential amount of transmitted solar radiation is highest due to planetary orientation) and a corresponding reduction of development rates. As a result, a higher proportion of embryos may reach the TSP later and, consequently, experience lower overall temperatures during the TSP, developing as females and, potentially, hatching later.

In addition to reflecting transmitted solar radiation above the ground, increased density of vegetation at ground level may provide an insulating effect against loss of heat energy from the soil. As a result, sub-surface nests could experience higher minimum temperatures during winter months and correspondingly higher rates of embryonic development, leading to earlier hatching. A previous study of tuatara (Mitchell et al. 2008) proposed installation of artificial shading as a means of manipulating offspring sex ratios in-situ for species conservation in locations with highly skewed sex ratios. Understanding how shading interacts with the near-ground and soil microenvironments (i.e., whether canopy provides an insulating or cooling effect within nests) in varying habitats and at different times during the incubation cycle is necessary for determining the shading configurations most likely to maintain balanced population sex ratios.

Vegetation re-growth has additional implications for the incubation microenvironment, as roots impact soil characteristics both directly and indirectly. Tuatara prefer nesting in loose, as opposed to compacted, soils (Refsnider et al. 2010) and dig deeper nests in areas of sandy soils versus areas comprised of clay (Nelson unpub. data). Both the diameter of individual roots and density of root masses vary with vegetation type; thick masses of grass roots, for example, may impede nest construction. Roots also 
influence soil moisture content, either directly through water uptake and accumulation (Zhuang et al. 2001) or indirectly by modifying soil porosity through growth (Kodesova et al. 2006). Land cover transitions on Takapourewa are roughly ordinal (East et al. 1995), particularly in areas of former pasture. Removal of grazing pressure has resulted in succession of pasture by tall grasses, which increased the probability of rookeryswitching behaviour. Replacement of tall grasses, which increase soil porosity but may impede nest construction in the short-term, with woody species that have larger, more sparsely distributed roots may increase availability of less-compacted soils and provide additional nesting habitat in the long-term.

\subsubsection{Assessment of methodological limitations}

The availability of high-resolution aerial photography allowed retrospective generation of vegetation data for this study. Using fuzzy classification reduced the likelihood of classification errors but decreased resolution of the vegetation data. The resolution of land cover information generated for this study, therefore, was much lower than what would be collected in-situ at individual nest sites. Since tuatara nest site choice has been recorded consistently on a rookery level, however, higher-resolution vegetation information for years prior to 2011 would have been of limited use in this study. The proportion of pixels classed incorrectly was less than 0.05 across all classifications, supporting the assertion that land cover classes chosen for this study were realistic and successfully differentiated using the available imagery.

The ability of the $2000 / 2007$ transition model to predict the observed vegetation change past 2007 was low. In particular, the transitions from pasture were conservatively predicted by both models, though the 2000/2011 model estimated a higher vulnerability to change in pasture areas. The lack of predictive ability in the $2000 / 2007$ model does not necessarily indicate that the driver variables chosen for this 
study had insufficient explanatory power, particularly given the high estimated relevance weights of geospatial indicators. Rather, inability to predict the near-total loss of pasture likely reflects the rapidity with which modified land covers are replaced by natural vegetation growth and, especially, active re-vegetation, when artificial effects (i.e., grazing and maintenance) are removed.

Areas of Takapourewa that have been actively re-planted could not be differentiated from un-planted areas. While grazing pressure could not be explicitly included in transition models, the relative unimportance of replanting for explaining vegetation change suggests that livestock removal has positively impacted ecological restoration at least as much as, if not more than, active revegetation. Future research may find that replanting accelerates the rate at which forest is restored to the areas which were still classified as pasture in 2007, relative to what might have otherwise occurred.

The predictive ability of the transition model for 2000/2011 could not be independently assessed due to lack of post-2011 aerial imagery. The high accuracy of the first-iteration land cover map approximating 2011 (i.e., the ability of the model to predict itself) suggests, however, that the 2000/2011 transition model accurately quantified the vegetation dynamics within the time period of interest. As with any transition model, predictions are likely to decrease in accuracy with increasing time from the last known state. The land cover map generated for 2012, though, did not contain any areas for which the predicted land cover composition was unexpected or otherwise suspect. At least in the short term, the land cover classifications and transition models developed for this study are representative of vegetation dynamics on Takapourewa.

The rapidly changing vegetation composition of the island, particularly loss of pasture, ultimately provided a means of determining whether nest site selection behaviour in female tuatara responds in-situ to environmental variation on a time scale 
relevant to the relatively long reproductive interval of the species. Future research, provided post-2012 remotely sensed data are available, should be able to take advantage of the methods used in this study to examine continuing impacts of ecological restoration on Takapourewa-resident populations, including tuatara. If feasible, future studies should take advantage of additional data types (e.g., multispectral satellite imagery or LiDAR height points) to provide an extended research timeframe as well as supplementary accuracy assessment for the land cover maps developed in this study.

\subsubsection{Implications for populations in modified habitats}

Rapid land cover change is ubiquitous in nature, whether caused by anthropogenic modification or 'natural' occurrences such as wildfires or prolonged weather systems. Gradual transitions among vegetation types are also facilitated by climate fluctuations (Kerhoulas et al. 2013) as well as agricultural and conservation practices (Millett \& Edmondson 2013; Shou-Li et al. 2013) that lead to shifts in the distribution, growth, and abundance of plant species. Unlike the vegetation transitions in progress on Takapourewa, however, short-term land cover changes are largely destructive of natural vegetation patterns (Didham et al. 2007; Huang et al. 2012). Few terrestrial animal populations occupy habitats that have not been, to some extent, adapted for human use. Understanding how land cover transitions influence nesting behaviour is therefore critical for predicting population-level effects of environmental variation in oviparous species.

Either destruction or, as in the case of this study, restoration of natural vegetation may equate to loss of favoured nesting habitat. While deforestation is, on the whole, accurately described as 'habitat loss,' individuals of species that prefer to nest in relatively un-vegetated areas may benefit from modification that creates additional nesting space, as long as the environmental correlates of nest site preference remain 
positive or neutral with respect to survival and fitness. The de-coupling of maternal preference and nest site suitability in modified areas could create ecological traps (Kolbe \& Janzen 2002). From the standpoint of species conservation, then, the direction of habitat change may be less important than the ways in which land cover transitions interact with evolved patterns of nesting behaviour.

The revegetation of Takapourewa was undertaken with the recommendation that small pasture areas should remain intact (DOCb), primarily to provide habitat for nesting female (Thompson et al. 1988) and juvenile (McIntyre 1988) tuatara. Rather than leading to a reduction in rates of nesting, however, successional revegetation is facilitating a spatial shift in nest site selection. While the availability of pasture areas as preferred nesting habitat was likely beneficial for the population in the short term, a burst of recruitment facilitated by the availability of additional nesting habitat may have increased the number of tuatara on Takapourewa above carrying capacity (Moore et al. 2007). Replacement of low-growing vegetation types by forest may reduce recruitment, while creating additional habitats for adult tuatara (Newman 1982). The diversity and abundance of invertebrates are higher in forested areas (Gaze 2001), so reforestation may provide additional energy resources for tuatara, eventually leading to improved body condition of adults (Moore et al. 2007).

Though subfossil deposits indicate tuatara were distributed historically throughout New Zealand (Cree \& Butler 1993; Jones et al. 2009; Hay et al. 2010), wild populations are currently restricted primarily to offshore islands and, as a result, are delineated into two main latitudinal groups (Nelson et al. 2002a; Hay et al. 2010). Northern populations (e.g., Hauturu) are subject to mean annual temperatures that are approximately $4^{\circ} \mathrm{C}$ higher (NIWA 2013) than those experienced by Cook Strait populations (e.g., Takapourewa). Vegetation may therefore play an important role in maintaining balanced sex ratios on northern islands. Hauturu, for example, is almost completely forested, and little is known about nest site selection behaviour in the 
resident wild tuatara population or in other populations that inhabit heavily vegetated environments (Tyrrell et al. 2000). Understanding the effects of vegetation composition on tuatara nesting behaviour will be important for predicting the impacts of climate change on sex ratios in both Northern and Cook Strait populations. Additionally, both current land cover composition and potential future transitions among vegetation types should be considered when examining the suitability of translocation sites for tuatara.

Overall, species that inhabit latitudinal or vegetation gradients may offer the best opportunities for examining the implications of vegetation change and other mechanisms of environmental variation for nesting behaviour and offspring phenotypes. Warmer-latitude populations of Australian agamid lizards (Physignathus lesueurii), for example, currently select more shaded nest sites than populations in cooler climates, effectively maintaining similar nest temperatures across sites (Doody et al. 2006). Loss of shade with no behavioural compensation (e.g., digging deeper nests) could lead to skewed hatchling sex ratios in northern populations. North American snapping turtles (Chelydra serpentina) prefer to nest in relatively open areas and, in de-vegetated areas, tend to select patches of bare sand that are uncommon in unmodified habitats, influencing nest temperatures and offspring sex ratios (Kolbe \& Janzen 2002). Hatchling sex ratios in green sea turtles (Chelonia mydas) are highly correlated with shade cover (Standora \& Spotila 1985), and de-vegetation of coastal nesting areas could reduce the proportion of male sea turtle hatchlings to near zero.

Most species choose nesting locations primarily to maximise offspring survival (Escalona et al. 2009; Refsnider \& Janzen 2010). If vegetation change creates a novel nesting environment that acts differentially on the survival and phenotype of hatchlings, however, nesting behaviour that affects offspring phenotype may become maladaptive in the new habitat, regardless of whether females alter their choice of nesting sites. Additional research is needed to determine how female preferences for vegetation composition translate into suitability of chosen nest sites for facilitating embryonic 
development and affecting offspring phenotype. Quantifying patterns of nest site selection relative to transitions in land cover is a critical first step in understanding how the effects of a changing environment can interact with reproductive behaviour to impact populations. 



\section{Chapter 3}

\section{Local climate and high resolution geospatial data are required to model soil temperatures accurately within a small spatial extent}

\subsection{Introduction}

\subsubsection{Predictive niche models}

Predicting the impacts of environmental variation on species is of primary concern to ecology, most recently for examining population viability and predicting range shifts within the context of modern climate change and habitat modification (Porter et al. 2000; Pearson \& Dawson 2003; Thuiller 2004; Guisan \& Thuiller 2005; Kearney \& Porter 2004). Understanding and, crucially, predicting the future consequences of those impacts require accurate representation of an organism's environmental niche. Current approaches to niche modeling are based largely on the Hutchinson niche concept (Hutchinson 1957), which differentiates between (i) the fundamental niche, the range of environmental conditions under which a species can persist (described by an $n$-dimensional hyper-volume), and (ii) the realised niche, the spaces within the potential range actually occupied by that species (Araújo \& Guisan 2006; Morin \& Lechowicz 2008; Kearney \& Porter 2009).

Most predictions of climate-induced range shifts are made using correlative methods (also labeled 'climate envelope models' or CEMs) (Guisan \& Zimmerman 2000; Thuiller et al. 2008). Correlative distribution models rely on presence/absence data to describe the realised niche using statistical relationships between observed species distributions and environmental variables (Elith \& Leathwick 2009) but assume that (i) a population is in equilibrium with its environment (Pearson \& Dawson 2003), (ii) the relationships between a population and its environment are static (Guisan \& Zimmerman 2000; Soberón \& Peterson 2005; Suttle et al. 2007), and (iii) the 
environment of the realised niche is an accurate reflection of at least one dimension of the fundamental niche (Pearson \& Dawson 2003). The physiological processes that constrain organisms within their 'climate envelope' are only implicitly captured by statistical models (Porter et al. 2002; Pearson \& Dawson 2003; Kearney 2006; Kearney \& Porter 2009).

The low spatial resolution at which environmental variables are typically sampled in-situ (Kearney \& Porter 2004; Kearney et al. 2014b), relative to the microenvironment actually experienced by an organism (Geiger et al. 2003), is an additional limitation on the predictive capacity of a niche model (Dormann et al. 2012). At best, environmental data are simultaneously sourced from (1) weather stations, which collect multiple data types (e.g., air temperature and humidity, wind speed, rainfall) long-term at a single point that is geographically near to a population of interest and (2) measurements collected instantaneously or over a pre-determined time period (e.g., with dataloggers) at statistically chosen points within the known habitat of a population (Porter et al. 2002; Kearney \& Porter 2004; Austin 2007).

The highest-resolution interpolated (i.e., statistically modelled), gridded climate data currently available for a global extent are the WorldClim climate layers, which are freely available at horizontal spatial resolutions of 30" and 10' (approximately $1 \mathrm{~km}$ and $20 \mathrm{~km}$ ) and contain current mean monthly terrestrial rainfall and mean, minimum, and maximum monthly air temperature data (Hijmans et al. 2005). In addition, the datasets most recently developed by the Climatic Research Unit (CRU CL 2.0) include monthly precipitation, mean temperature, relative humidity, sunshine hours, ground frost, and $10 \mathrm{~m}$ mean monthly wind speed data for the 1961-1990 normal period at a $10^{\prime}$ spatial resolution (New et al. 2002).

Both the WorldClim and CRU CL 2.0 datasets are widely used in the development of CEMs (Guisan \& Zimmerman 2000; Guisan \& Thuiller 2005; Elith et al. 2006) and predictive climate models (Jeffrey et al. 2001; Wood et al. 2004; Tait et al. 2006; Smith et 
al. 2007; Tait et al. 2012), which may subsequently be used to inform conservationfocused research and applications in biodiversity management (Loiselle et al. 2003; Brooks et al. 2006; Kerhoulas et al. 2013). A global-extent set of gridded microclimate surfaces, 'microclim,' is also available at an approximately $15 \mathrm{~km}$ horizontal resolution (Kearney et al. 2014a). Most organisms, however, experience their environment within a geographical extent much finer than that defined using global climate surfaces (Hutchinson \& MacArthur 1959; Porter et al. 2002, 2010; Geiger et al. 2003; Potter et al. 2013).

Populations of oviparous species, in particular, may be differentially affected by the environmental profiles of two distinct, but nested, fundamental niches: the adult niche and the niche of embryonic development. Especially in species that display little or no parental care, the abiotic environment of incubation must remain within the physiological tolerance limits of an embryo to maximise the probability of offspring survival (Escalona et al. 2009; Huey et al. 2012). In addition to impacting survival, the incubation environment can have important phenotypic and fitness consequences for juveniles (Shine 1999; Mitchell \& Seymour 2003; Booth 2006; Woods \& Bonnecaze 2006; Andrewartha et al. 2008, 2010; Eads et al. 2012). Statistical models of the nest environment, however, do not explicitly capture developmental processes with potential population-level impacts, particularly if, as in most oviparous species, nest site choice is more tightly correlated with offspring survival than with hatchling phenotype (Escalona et al. 2009; Refsnider \& Janzen 2010).

\subsubsection{Modelling the thermal environment of incubation}

In contrast with climate surfaces interpolated from direct measurements of the abiotic environment, mechanistic climate models define environmental variables as the outcomes of atmospheric and soil thermodynamic processes (Porter et al. 1973, 2002). 
The temperature profile of a below-ground nest is not measured in-situ but is calculated as the quantity of energy conducted below the soil surface, after emitted solar radiation that reaches the outer atmosphere is reduced by atmospheric scattering and absorption (e.g., by clouds and greenhouse gasses) and by low-altitude reflection (e.g., by vegetation and the soil surface) (McCullough \& Porter 1971; Porter et al. 1973; Geiger et al. 2003). Soil temperature is a model output rather than an input and, thus, is independent of and can be validated against field measurements.

Additionally, the spatial resolution of temperature output is limited by the resolution of input GIS layers, rather than that of an in-situ temperature sampling regime. Increasing the resolution of a derived climate surface requires either development of improved interpolation methods or collection of additional field measurements, the latter of which may be constrained by practical considerations (e.g., restricted site access or limited availability of research funding, sampling equipment, or personnel). The growing availability of high-resolution, remotely-sensed data that can increase the resolution of mechanistic model outputs is an additional argument for choosing a process-explicit method when high resolution, microclimate-scale predictions are required.

In species with environmentally mediated sex determination (ESD), the indirect effects of the environment on population sex ratios may be a more pressing concern than the direct effects of climate change on individual adults (Janzen 1994; Nelson et al. 2004; Mitchell et al. 2008; Grayson et al. 2014). Especially under consistently warming climatic conditions, populations that have temperature-dependent sex determination (TSD) (Bull 1983) are at increased risk of skewed sex ratios and potentially harmful demographic effects (Janzen 1994; Mitchell et al. 2008; Mitchell \& Janzen 2010; Wright et al. 2012). In species that have TSD, both embryonic development rates and offspring sex are functions of the incubation temperature regime inside nests. The ability to accurately predict incubation conditions is therefore critical for conservation of species 
with TSD, both in current and potential future habitats. Predictions that link embryonic development and sex determination directly with the incubation micro-environment (Mitchell et al. 2008) can provide a more reliable means of explaining and predicting population-level effects at fine-scale spatial and temporal resolutions under novel environmental scenarios (Woods \& Bonnecaze 2006; Kearney \& Porter 2009; Kearney et al. 2009a, b; Kearney et al. 2014a, b).

The relationship between fluctuating temperatures and embryonic development in vertebrates with TSD is best modelled by a non-linear function (Sharpe \& DeMichele 1977; Girondot 1999; Godfrey et al. 2003; Georges et al. 2004, 2005; Neuwald \& Valenzuela 2009; Woolgar et al. 2013). Accurately predicting offspring sex ratios of species with TSD, therefore, requires hourly or, at a minimum, daily soil temperature data (Georges et al. 2005; Mitchell et al. 2008), preferably at a very high spatial resolution (i.e., the scale of single nests or colonial nesting areas) and for multiple climate scenarios (Cagle et al. 1993; Mitchell et al. 2008; Kearney et al. 2014b).

Mechanistically derived climate surfaces have the potential to inform sex ratio predictions in addition to describing the microclimate of adult organisms, but few studies have developed soil temperature data at a biologically meaningful spatial resolution. The accuracy of one process-explicit model, NicheMapR, has been tested at a 5km spatial resolution, continent-wide for Australia (Kearney et al. 2014b) and for regions of North America (Kearney et al. 2014a). A model of hourly soil temperatures generated using NicheMapper ${ }^{\mathrm{TM}}$ (Porter et al. 1973; Porter \& Mitchell 2006) at a 0.5m resolution was used to predict hatchling sex ratios in a population of tuatara (Sphenodon punctatus), a long-lived reptile with TSD, on North Brother Island, New Zealand (Mitchell et al. 2008), but the study did not explicitly test the effects of microsite variation (e.g., in soil properties) on model accuracy. A spatially explicit model of soil temperature has been tested at a horizontal resolution of $5 \mathrm{~m}$ but is currently limited to generating predictions for a single, topsoil layer (Bennie et al. 2008). Developing and 
applying mechanistic models to predict the effects of environmental change, including climate warming, on populations requires both an accurate description of the microclimate experienced by organisms and an understanding of how variation in model input affects model accuracy.

\subsubsection{Study aims and research questions}

The primary goals of this study were to (i) examine how the resolution of microclimate parameters affects the accuracy of modelled soil temperatures within a small spatial extent and (ii) develop a model of soil temperatures for the island of Takapourewa, New Zealand with which to, ultimately, predict embryonic development rates and offspring sex ratios for tuatara at the spatial scale of individual nests (Chapter 4). The research questions asked during this study were: (1) how do topography, soil thermal properties, shade availability, and wind speed affect accuracy of microsite soil temperature predictions? (2) Do microclimate-level parameter values improve model performance over those generated using broad-scale, mean values? (3) Do local, temporal weather data improve model performance compared to predictions generated using periodic climate means?

\subsection{Methods}

\subsubsection{Microclimate model structure}

Soil temperatures were modelled for the island of Takapourewa (also known as Stephens Island), an offshore Nature Reserve located in Cook Strait, New Zealand [approx. 4040'00" S 17400'00" E], using NicheMapR v 2011b (Kearney et al. 2014a, b), a global-extent R-implementation of the NicheMapper microclimate model (McCullough \& Porter 1971; Porter \& Mitchell 2006). To model soil temperatures, NicheMapR uses a 
one-dimensional, finite-distance algorithm (Carslaw \& Jaeger 1959; Porter et al. 1973) to solve heat-mass balance equations for ten specified soil depths (McCullough \& Porter 1971) at any given location and time. The input data include geospatial parameters (e.g., elevation, slope, aspect, horizon) for calculating clear-sky solar radiation using the integrated SOLRAD radiation model (McCullough \& Porter 1971; Porter et al. 2002); daily maximum and minimum values of seasonally dynamic climate variables (e.g., wind speed, air temperature, humidity, rainfall, cloud cover); and values for relatively constant, site-specific environmental variables (e.g., physical properties of soils, shade availability). Values of soil properties can optionally be modified to simulate a layer of organic topsoil, or 'organic cap,' which reduces conduction of thermal energy. The programme also incorporates scattering of solar radiation due to atmospheric aerosols using a modified version of the Global Aerosol Data Set (GADS) (Koepke et al. 1997; Kearney et al. 2014a, b) (Fig. 1).

For this study, the NicheMapR global functions were modified to integrate usersupplied input of values for local environmental variables at any available spatial and temporal resolution. Daily observations of rainfall (resolution $0.2 \mathrm{~mm}$ ), relative humidity (resolution 1\%), minimum and maximum air temperatures (resolution $0.1^{\circ} \mathrm{C}$ ), and minimum and maximum wind speeds (resolution $0.1 \mathrm{~m} / \mathrm{s}$ ) for the study period were obtained from the NIWA CliFlo database (http://www.cliflo.niwa.co.nz) for the weather station on Takapourewa [Station No. 26169]. Daily observations of relative humidity were assumed to represent daily maxima. Minimum daily values were estimated by splining minimum monthly humidity data across 365 days. Cloud cover for the study period was calculated as the difference between maximum possible (i.e., out of 24 hours) and recorded daily sunshine hours (resolution $0.1 \mathrm{hr}$ ), expressed as a percentage, using sunshine data from the Blenheim Research weather station [NIWA Station No. 12430], which was the nearest station at which data on sunshine hours were recorded in 2011. 
To test whether local weather data improved microclimate model accuracy over that obtained using global-scale climate information, monthly climate data interpolated to a 10' latitude/longitude resolution for the World Meteorological Organization standard normal period 1961-1990 (New et al. 2002) were extracted from the University of East Anglia Climatic Research Unit CL 2.0 database (http://www.cru.uea.ac.uk/cru/data) (Appendix 3.1). Minimum and maximum relative humidity; minimum and maximum air temperatures; and minimum and maximum wind speeds extracted from the global database were splined to provide 365 daily climate records. Total monthly rainfall was spread evenly across the number of rainy days (i.e., the number of days with $>0.1 \mathrm{~mm}$ of rainfall) per month. Daily maximum air temperature and wind speed and minimum relative humidity and cloud cover were assumed to occur one hour after local solar noon. Daily minimum air temperature and wind speed and maximum relative humidity and cloud cover were assumed to occur at local sunrise (Kearney et al. 2014a, b). 


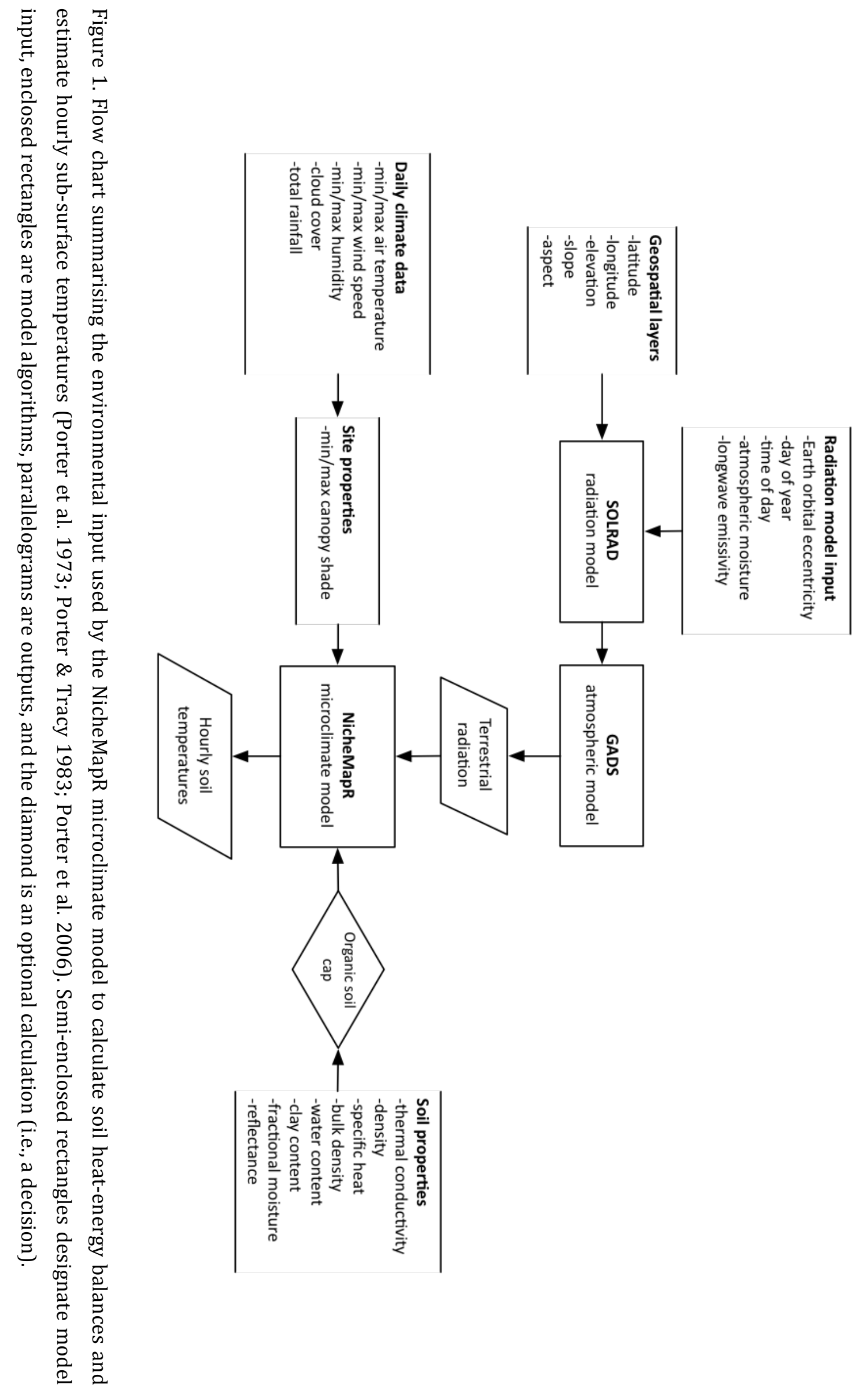




\subsubsection{Geospatial parameters}

Geospatial data were generated in $\operatorname{ArcMap}^{\mathrm{T}}$ Desktop v 10.1 software (Environmental Systems Research Institute [Esri] ArcGIS: Redlands, CA, USA 2012) using a $0.5 \mathrm{~m}$ resolution gridded digital elevation map (DEM) of the study site (Aerial Surveys Ltd., Auckland, NZ 2011). Values for latitude, longitude, and elevation were extracted directly from the DEM. Slope was calculated as the ratio of maximum change in elevation to horizontal distance from each pixel to its eight nearest neighbours. Aspect was calculated as the maximum change in slope from each pixel to its eight nearest neighbours (Appendix 3.2). To correct solar radiation values for complex topography, twenty-four horizon angles were calculated for each pixel of the DEM using the r.horizon function in GRASS v 6.4 (GRASS Development Team 2012).

In this study, the term 'spatial resolution' refers to the area represented by a single pixel of gridded climate or geospatial data and is distinct from 'spatial scale,' which refers to both (i) the area of a terrestrial microclimate of interest (e.g., the home range of an organism) and (ii) the area over which microclimates are aggregated (e.g., the distribution of a species or a study area). The terms 'spatial extent' and 'geographical extent' refer to the entire area represented by a gridded climate or geospatial dataset and is independent of both spatial resolution and scale (Whittaker et al. 2001). Whether a microclimate surface can be reasonably labeled as 'high resolution' is relative to the geographic extent of an area of interest. A horizontal spatial resolution of 30", or approximately $1 \mathrm{~km}$, can be considered very high resolution for a global or continental model (Hijmans et al. 2005; Kearney et al. 2014a, b) but would be extremely lowresolution if the area being represented is a small island. 


\subsubsection{Soil physical and thermal properties}

The physical and thermal properties of soils influence the amount of energy that is transferred and the rate of transfer from the surface to subsequent depths (i.e., the soil heat flux). Heat conduction through soils is described primarily by thermal conductivity $(\lambda)$ and specific heat capacity (pc) (de Vries 1963). While soil properties are relatively stable across temperature changes (de Vries 1963), both physical and thermal properties of soils and, especially, moisture content can vary significantly with space and depth and affect heat transfer (Campbell 1985; Ochsner et al. 2001; Kearney et al. 2014b). The inclusion of local soil bulk density and moisture content in an Australiawide test of NicheMapR did not significantly improve model performance (Kearney et al. 2014a, b); however, the effects of site-specific soil thermal properties on prediction accuracy have not been examined. For this study, thermal conductivity, bulk density, and fractional soil moisture and density of soil minerals were determined experimentally (details below) for the soil types and type composites $(\mathrm{N}=10)$ present on Takapourewa (Ward 1961). Specific heat of soil minerals, soil reflectance, and clay content were estimated from other soil properties. Soil properties were adjusted with depth inside the model subroutine (Kearney et al. 2014a, b).

To define locations for soil sampling, a hard-copy map of soil types (Ward 1961) was manually georeferenced to a high-resolution aerial photo of the study location (Aerial Surveys 2011) and transformed to the 2000 New Zealand Transverse Mercator/NZ Geodetic Datum 2000 coordinate system in $\operatorname{ArcMap}^{\text {TM }}$ using a cubic spline. Control points were defined for the transformation until the root mean squared error (RMSE) $<0.0001 \mathrm{~m}$. The quality of the original map was low relative to the aerial photo, so locations for soil sampling were defined by generating a random point at least $20 \mathrm{~m}$ from the edges of each stratified soil polygon to minimise the potential for confusing soil 
types. Sampling locations were also restricted to within $20 \mathrm{~m}$ of the access track to avoid unnecessary damage to island vegetation.

Soil cores were collected from 15-20 November, 2012 and at least 12 hours following rainfall to avoid inflation of soil moisture content. Surface vegetation was removed, and one core sample (volume $\approx 1455 \mathrm{ml}$ ) was collected from $20 \mathrm{~mm}$ below the surface to a depth of around $200 \mathrm{~mm}$ (i.e., the approximate depth distribution of tuatara nests) at each sampling location ( $\mathrm{N}=10)$ using a hand-held stainless steel soil corer and a striking plate (Materials Advisory Testing Service, Stokes Valley, NZ) to avoid compression of samples used to determine soil density. An additional sample of approximately $1000 \mathrm{ml}$ was obtained for each soil type using a hand trowel. Samples were double-bagged on collection and stored out of direct sunlight until testing (Sheppard \& Addison 2006).

Soil testing was conducted in a climate-controlled laboratory (Geotechnics Ltd., Auckland, NZ) from 2-8 December, 2012. Thermal conductivity was determined using a calibrated non-steady-state thermal probe (Bristow 1998) and digital thermal resistivity meter (TP09/MTN01; Hukeseflux Thermal Sensors, Delft, The Netherlands), following a modified testing protocol (ASTM 2008). Samples were passed through a $10 \mathrm{~mm}$ sieve and homogenised (Sheppard \& Addison 2006), and the bulk density of each soil type was then calculated as the ratio of the total mass $(\mathrm{g})$ and volume ( $\mathrm{ml}$ ) of the moist core sample. The mass of soil needed to attain each calculated bulk density was then remoulded into a concrete cylinder of known volume in five $36 \mathrm{~mm}$ layers of equal mass to achieve a consistent density throughout the sample. Once re-moulded, samples were removed from the concrete mould, weighed to confirm sample mass, sealed in a plastic bag to minimise moisture loss, and stored overnight in a climate-controlled lab to allow the temperature throughout each sample to equalise prior to testing.

The testing method (ASTM 2008) was modified in two ways to allow for determination of $\lambda$ for very low-density soils: (1) re-moulded samples were wrapped 
with an A4-size sheet of transparent polyethylene terephthalate (PET) and supported by a circle of fine steel mesh to maintain sample integrity and (2) the thermal probe was suspended above soils that were of sufficiently low density to cause the needle to sink into the sample. To prevent excessive temperature increases during testing of relatively dry or low-density soils, the thermal probe heating voltage was set at $2 \mathrm{~V}$ for samples with calculated density $\leq 1.2 \mathrm{t} / \mathrm{m}^{3}$ and at $2.5 \mathrm{~V}$ for samples with density $>1.2 \mathrm{t} / \mathrm{m}^{3}$. The $\lambda$ $(\mathrm{W} / \mathrm{mK})$ of each sample was measured over 600 seconds to a standard deviation of < 0.1. Two fractions of each soil sample, one sieved and one un-sieved, were also weighed, oven-dried for 24 hours at $108^{\circ} \mathrm{C}$ (Contherm Scientific Thermotec ${ }^{\mathrm{TM}} 2000$ Series drying oven), and re-weighed to determine moisture content and confirm calculated dry densities (Sheppard \& Addison 2006). Fractional soil moisture was calculated as the ratio of the mass of water ( $\mathrm{g}$ ) in each sample to the sample dry mass.

The density of the mineral component of soils $\left(\mathrm{kg} / \mathrm{m}^{3}\right)$ was calculated as the ratio of sample mass to the sample volume, with sample mass corrected for the masses of water, carbon (Ward 1961), and organics. The organic component was estimated using a ratio of carbon to organic matter of 0.5 (de Vries 1963; Campbell 1985). Specific heat $\left(\mathrm{MJ} / \mathrm{m}^{3}-\mathrm{K}\right)$ of the mineral component of soil types was estimated by calculating the volumetric specific heat of each soil sample and solving for the mineral component, given the volume fractions of each component (de Vries 1963; Campbell 1985; Campbell \& Norman 1998):

$$
p c_{s}=p c_{m} x_{m}+p c_{w} x_{w}+p c_{a} x_{a}+p c_{o} x_{o}
$$

where pc is the specific heat of, respectively, the whole soil (s) and the mineral (m), water (w), air (a), and organic (o) components; $x$ is the volume fraction of each component; and $\mathrm{pc}_{\mathrm{a}} \approx 1.00, \mathrm{pc}_{\mathrm{w}} \approx 4.18$, and $\mathrm{p} \mathrm{c}_{\mathrm{o}} \approx 2.50$ (de Vries 1963; Campbell 1985). Soil reflectance was estimated using the Munsell colour values (Escadafal 1989; Post et al. 2000) previously reported for soils on Takapourewa (Ward 1961). Clay content was estimated as $20 \%$ for all soil types following textural classification (Shirazi \& Boersma 
1984; Hendrickx et al. 2003; FAO 2006). Cliffs surrounding the island, which were not included in historical surveys (Ward 1961) or sampled for this study, were assigned mean values of soil properties (Appendix 3.3). Volumetric water content at saturation was estimated as $0.26 \mathrm{~m}^{3} / \mathrm{m}^{3}$, and surface roughness was estimated as $0.004 \mathrm{~m}$ for all simulations (Kearney et al. 2014a). Whether inclusion of spatially-explicit, experimentally-derived soil properties improved model accuracy was examined using parameter values for the mineral fraction of a generalised soil with a bulk density of $1300 \mathrm{~kg} / \mathrm{m}^{3}$, mineral density of $2560 \mathrm{~kg} / \mathrm{m}^{3}$, thermal conductivity of $2.5 \mathrm{~W} / \mathrm{mK}$, and specific heat of 870 J/kg-K (de Vries 1963; Campbell 1985; Campbell \& Norman 1998; Kearney et al. 2014a, b).

\subsubsection{Shade cover}

Shading by vegetation affects the proportion of total solar radiation that reaches the soil surface and, consequently, soil temperature (Geiger et al. 2003). Using gap-light analysis of hemispherical photography, microsite shade availability on Takapourewa was estimated at around 95\% under the forest canopy and $20-60 \%$ at most non-forested sample locations (Chapter 2 Fig. 6), regardless of vegetation type. While a microsite could be classified remotely or in-situ as non-vegetated or as dominated by a particular vegetation type (e.g., tall grasses vs. forest), classifications did not reliably predict the amount of shade available at the soil surface (Chapter 2). As a consequence, microsite shade values could not be generalised to non-sampled points based on vegetation class, and temperature predictions were instead generated using shade values of $0 \%, 20 \%$ (the approximate minimum calculated shade), 45\% (the approximate mean non-forest shading), and $60 \%$ (the approximate upper range of shade at non-forested locations) for all sites. Temperatures were also generated for each site using a random integer value for canopy shading of between $20-60 \%$. 


\subsubsection{Simulated wind speeds}

The exposed maritime environment of the study site increased the likelihood that wind would significantly impact soil temperatures. Single-point wind station data were not expected to reflect the effects of highly variable local topography on wind (Mitchell et al. 2008), so a separate turbulence velocity model was used to simulate wind speeds across the island. The goal of this study was not to generate accurate, micrositeresolution values for wind speed, which would have been an unrealistic expectation of the simulator (Ross et al. 1988; Lopes 2003), but to determine whether modelled temperature predictions were sensitive to the effects of wind speed at a microsite scale. Wind speeds on Takapourewa were modelled using WindStation $^{\text {TM }}$ v 4.0.1 software (Lopes 2003), which incorporates a Navier-Stokes turbulence equation solver (CANYON) (Lopes et al. 1995) and a kinematic model (NUATMOS) (Ross et al. 1988) to simulate wind flow over complex terrain.

Development of turbulent velocity profiles required wind data input for two altitudes. A logging anemometer (Inspeed Vortex Wind Sensor and Madgetech Pulse 101A datalogger) was installed in November 2011 at a height of $2 \mathrm{~m}$ directly below the permanent weather station at $180 \mathrm{~m}$ asl (mast height $10 \mathrm{~m}$ ), and wind speed was recorded at two-minute intervals for approximately one year. Wind speed and direction data recorded at hourly intervals during the same period by the permanent weather station were obtained from the NIWA climate database (http://cliflo.niwa.co.nz/). The permanent station at the study site is located in a relatively open, exposed area, so the wind direction at a height of $2 \mathrm{~m}$ was assumed to be equal to the direction at $10 \mathrm{~m}$.

Wind flow simulations required two terrain-defining geospatial datasets: elevation and roughness, which includes both the 'bumpiness' inherent in local topography and the heights of above-ground elements such as vegetation, rocks, and artificial structures (Lopes 2003, 2011). Elevation data were extracted at a horizontal 
resolution of $2 \mathrm{~m}$ directly from a re-sampled, gridded DEM of the study site (Appendix 3.2). Approximate maximum vegetation height measurements were recorded in November 2011 and 2012 at the same locations at which hemispherical photographs were collected (Chapter 2), and broad roughness values were subsequently assigned to a categorical land cover classification map of the island generated using an aerial photograph captured in 2011 (Chapter 2 Fig. 4). Values for roughness height based on land cover class were $0.5 \mathrm{~m}, 1 \mathrm{~m}$, and $4 \mathrm{~m}$ for, respectively, rock, pasture, and scrub; tall grasses and vinelands; and forest. Hourly wind field simulations were run inside a gridded bounding box with a horizontal resolution of approximately $25 \mathrm{~m}$. Gridded wind speeds were converted to daily maximum and minimum values (Appendices 3.4-5).

\subsubsection{Soil temperature data}

The mean depth of tuatara nests on Takapourewa is about $110 \mathrm{~mm}$ (Cree et al. 1992); however, nest depths range between 50 and 250mm (Cree et al. 1991; Thompson et al. 1996). To maintain consistency between studies (Mitchell et al. 2008) and, as most temporal variation in soil temperature dissipates at around $200 \mathrm{~mm}$ below the soil surface (Geiger et al. 2003), validation temperatures for this study were collected at $100 \mathrm{~mm}$ and $200 \mathrm{~mm}$. To provide field data for comparison with NicheMapR soil temperature predictions, 44 temperature dataloggers (34x Thermochron ${ }^{\mathrm{TM}}$ DS1921G/H iButtons and 8x Onset HOBO ${ }^{\text {TM }}$ Pro v2 U23-002) were used to collect hourly temperature data at the study site.

Since the primary interest was in accurately predicting temperatures within tuatara nests, four dataloggers were installed in November 2011 at approximately $100 \mathrm{~mm}$ below the soil surface in each of ten known tuatara rookeries (Chapter 2 Fig. 3), regardless of whether or not nests were observed that year. Not all rookeries remained active in 2011, so rookery boundaries were defined by generating a $5 \mathrm{~m}$-radius buffer 
around all nests observed between 2002-2011 using GIS software. Dataloggers were installed at random points inside each defined rookery. In addition, two temperaturehumidity dataloggers (Pro v2s) were installed at $100 \mathrm{~mm}$ and $200 \mathrm{~mm}$ below the soil surface at the geometric centre of the House Road, Winch House, Winch Track, and Sand Dune rookeries, as they were the most consistently active nesting areas during the study period (Chapter 2 Figs. 7, 11).

The resolution of model output could never be higher than the resolution of the DEM used to derive geospatial input (i.e., sub-grid datalogger redundancy could only provide information on measurement precision not model accuracy). All randomly placed dataloggers, therefore, were separated by at least $5 \mathrm{~m}$ of horizontal distance to ensure multiple sets of temperature data were not accidentally linked to a single prediction due to GPS error. All datalogger locations were recorded as GPS points (Garmin Oregon ${ }^{\odot}$ 550, Garmin Limited, USA). Eleven of the iButton dataloggers either failed or were otherwise unrecoverable, so hourly soil temperature data were successfully collected from 31 dataloggers at 27 different microsites. Each iButton collected approximately 1,400 observations across three months, and each Pro v2 datalogger collected approximately 8,350 observations across approximately one year.

\subsubsection{Model selection}

Experimentally determined soil properties and modelled wind data were extracted to the resolution of the geospatial data by using a distance-minimising algorithm to link each microsite (i.e., latitude and longitude) with site-specific microclimate data. Regardless of the resolution of different input data types, each microsite was defined by, at a minimum, a unique combination of geospatial parameters. Three sets of sites were generated: (1) a set of points at which in-situ temperature data were obtained for model validation ( $\mathrm{N}=27)$; (2) a whole-island set of points derived at 
maximum DEM resolution $(\mathrm{N}=481,967)$ for predicting tuatara offspring sex ratios (Chapter 4); and (3) a set of points randomly sub-sampled from the whole island $(\mathrm{N}=$ 500) for examining the sensitivity of sex ratio predictions to error in modelled soil temperatures (Chapter 4). Hourly temperature predictions were generated using seven different baseline models, each parameterized to increasing complexity. With the exception of the 'global' implementation, models were run using unique geospatial data for all datalogger sites (Table 1).

Table 1. Seven basic microclimate model parameterisations and corresponding sources of input data: 'global' mean data were downloaded from the CRU 2.0 global climate database and represent the 1961-1990 standard normal period; 'local daily' climate data were obtained from weather stations for the year 2011; 'gridded' wind data were generated using a turbulence velocity model; 'local' soil properties were determined empirically; and 'generalised' soil properties were based on published values.

\begin{tabular}{rrrrrr}
\hline model \# & model name & geospatial data & climate data & soil data & wind data \\
\hline 1 & global & global database & global mean & generalised & global mean \\
2 & high-res & microsite & global mean & generalised & global mean \\
3 & weather_1 & microsite & local daily & generalised & local daily \\
4 & weather_2 & microsite & local daily & generalised & gridded \\
5 & soils_1 & microsite & global mean & local & global mean \\
6 & soils_2 & microsite & local daily & local & local daily \\
7 & micro & microsite & local daily & local & gridded \\
\hline
\end{tabular}

All seven models were run using the five scenarios of percent-shading: $0,20,45$, 60, and random [20:60] to generate 35 baseline models. Each of the 35 models was modified and re-run to examine the effects of key soil properties on the accuracy of temperature predictions. The effects of moisture on soil temperature were examined for 
all models by simulating evaporative cooling of the soil surface on days that received at least $1.5 \mathrm{~mm}$ of rainfall (Kearney et al. 2014b). In addition, because the presence of vegetation and bioturbation by burrowing organisms can reduce the ability of soils to transfer thermal energy, all models were modified to include a 50mm 'organic soil cap' (Kearney et al. 2014b). As the soils on Takapourewa are relatively shallow (Ward 1961), models run using generalised soil physical and thermal properties (soils_1, soils_2, micro) were modified to simulate a rock substrate with bulk and mineral densities of $2640 \mathrm{~kg} / \mathrm{m}^{3}$, thermal conductivity of $3.0 \mathrm{~W} / \mathrm{mK}$, and specific heat of $820 \mathrm{~J} / \mathrm{kg}-\mathrm{K}$ (Kearney et al. 2014a). Likewise, models parameterised with experimentally derived soil properties were run using generalised values for thermal conductivity and density of soil minerals. Finally, because data on local cloud cover were obtained from a nearby weather station, not the weather station on Takapourewa, models parameterised with local weather data (i.e., weather_1, weather_2, soils_2, micro) were run using both daily observations and splined mean monthly cloud cover data.

Tuatara development in-situ routinely requires more than 12 months for completion (Cree et al. 1992; Nelson unpub. data). Since the goal of this study was to develop an accurate temperature model, hourly predictions were generated for a single year. The ability of each model to predict realistic soil temperatures was assessed using summary statistics. Values for the coefficient of determination $\left(\mathrm{R}^{2}\right)$, root-mean-squared deviation (RMSD), and normalised RMSD (nRMSD) were calculated for each comparison between predicted hourly soil temperatures and corresponding observed values at each datalogger location, with lower values indicating better agreement between observed and modelled soil temperatures. RMSD was calculated using the 'rmse' function in R package hydroGOF (Zambrano-Bigiarini 2014). Normalised RMSD (nRMSD) was calculated as the RMSD value divided by the range of observed hourly soil temperatures (i.e., maximum temperature - minimum temperature) for each comparison between predicted and corresponding observed values as well as modelled and observed daily 
maxima and minima (Horton \& Corkrey 2011; Kearney et al. 2014b). The accuracy of lower-resolution, larger-extent implementations of NicheMapR, within around $2-3^{\circ} \mathrm{C}$ of observed soil temperatures (Kearney et al. 2014a, b), was used as a benchmark for assessing the quality of models developed in this study. Figures were generated in R and edited using GNU image manipulation program (GIMP Development Team 2013).

\subsection{Results}

\subsubsection{Model selection}

Neither simulation of evaporative cooling nor inclusion of a $50 \mathrm{~mm}$ organic soil cap increased accuracy from the baseline set of models, and inclusion of either decreased accuracy of the models with the lowest relative RMSD values. Simulating a rock instead of soil substrate had no effect on either RMSD or $\mathrm{R}^{2}$ values. In contrast, models run using generalised values for soil thermal conductivity and mineral density and experimentally-derived values for other soil properties generated more accurate temperature predictions than those run using only experimentally-derived values. Substitution of statistically smoothed, mean monthly cloud cover data for daily observed values decreased RMSD values across all models parameterised using local weather data.

The range of RMSD values among all sites was $1.45-10.11^{\circ} \mathrm{C}$ at $100 \mathrm{~mm}$ and 1.63 - $5.77^{\circ} \mathrm{C}$ at $200 \mathrm{~mm}$ depth. Mean RMSD across all models varied from $2.63-6.34^{\circ} \mathrm{C}$ at $100 \mathrm{~mm}$ and from $1.94-5.38^{\circ} \mathrm{C}$ at $200 \mathrm{~mm}$ depth. Except for the $20 \%, 45 \%$, and $60 \%$ shading scenarios of 'high-res' and 'soils_1,' RMSD values were lower at $200 \mathrm{~mm}$ compared to $100 \mathrm{~mm}$ for the same models. Only the $0 \%$ and $20 \%$ shading scenarios of 'soils_2' and the $20 \%$ shading scenario of 'micro' had RMSD values $<3.0^{\circ} \mathrm{C}$. Normalised RMSD values varied from $19.10-54.51 \%$ at $100 \mathrm{~mm}$ and from $10.87-30.43 \%$ at $200 \mathrm{~mm}$. $\mathrm{R}^{2}$ varied from $0.36-0.56$ at $100 \mathrm{~mm}$ and from $0.76-0.81$ at $200 \mathrm{~mm}$ depth. Of all models 
tested, the $0 \%$ shading scenario of the 'soils_2' model had the lowest RMSD and nRMSD values at both $100 \mathrm{~mm}$ and $200 \mathrm{~mm}$ depths. The 'weather_1' models had the highest $\mathrm{R}^{2}$ values ( $>0.54$ ) at $100 \mathrm{~mm}$. The $0 \%$ and $20 \%$ shading scenarios of the 'weather_1' and 'soils_2' models had the highest $\mathrm{R}^{2}$ values (>0.80) at 200mm depth.

The accuracy of site-specific, daily minimum soil temperature predictions was similar across models; however, statistical values at $100 \mathrm{~mm}$ and $200 \mathrm{~mm}$ were reversed, relative to those calculated for hourly predictions. RMSD values varied from 2.78 $2.99^{\circ} \mathrm{C}$ at $100 \mathrm{~mm}$ and from $3.00-3.36^{\circ} \mathrm{C}$ at $200 \mathrm{~mm}$. Normalised RMSD values varied from $9.52-10.23 \%$ for predictions of minimum temperatures at $100 \mathrm{~mm}$ and from 18.29 - $20.49 \%$ at $200 \mathrm{~mm} . \mathrm{R}^{2}$ of minimum predicted temperatures was $0.66-0.67$ at $100 \mathrm{~mm}$ and $0.76-0.77$ at $200 \mathrm{~mm}$. The ability of any model to predict site-specific, daily maximum soil temperatures was lower; RMSD values varied from $6.31-7.70^{\circ} \mathrm{C}$ at $100 \mathrm{~mm}$ and from $3.51-4.13^{\circ} \mathrm{C}$ at $200 \mathrm{~mm}$. Normalised RMSD values were similar to the lowest nRMSD values calculated across all soil temperature predictions, varying from $21.60-26.34 \%$ at $100 \mathrm{~mm}$ and $21.41-25.24 \%$ at $200 \mathrm{~mm}$. $\mathrm{R}^{2}$ values were 0.42 at $100 \mathrm{~mm}$ and between $0.76-0.77$ at $200 \mathrm{~mm}$ for predictions of maximum soil temperatures.

Summary statistics were also calculated between site-specific maximum and minimum soil temperatures and corresponding maximum and minimum air temperatures recorded by the weather station on Takapourewa The RMSD and nRMSD values between observed maximum soil temperatures at $100 \mathrm{~mm}$ and maximum air temperatures were, respectively, $4.81^{\circ} \mathrm{C}$ and $17.34 \%$, and the $\mathrm{R}^{2}$ value was 0.17 . The RMSD and nRMSD values between observed minimum soil temperatures and minimum air temperatures were, respectively, $3.40^{\circ} \mathrm{C}$ and $21.65 \%$, and the $\mathrm{R}^{2}$ value was 0.58 . At a depth of $200 \mathrm{~mm}$, the RMSD and nRMSD values between observed maximum soil temperatures and maximum air temperatures were, respectively, $3.63^{\circ} \mathrm{C}$ and $4.15 \%$, and the $\mathrm{R}^{2}$ value was 0.41 . The RMSD and nRMSD values between observed minimum soil 
temperatures and minimum air temperatures at $200 \mathrm{~mm}$ were, respectively, $5.83^{\circ} \mathrm{C}$ and $5.46 \%$, and the $\mathrm{R}^{2}$ value was 0.34 .

Since the primary goals of this study were to examine the effects of input parameter resolution on model accuracy as well as to develop the most accurate model of soil temperatures possible with available environmental data, complete summary statistics are presented for all shading scenarios of the set of models with the lowest RMSD: the 'soils_2' model, simplified with smoothed cloud cover data and generalised values for soil minerals density and thermal conductivity but parameterised with experimentally-derived values for other soil properties (Fig. 2). 


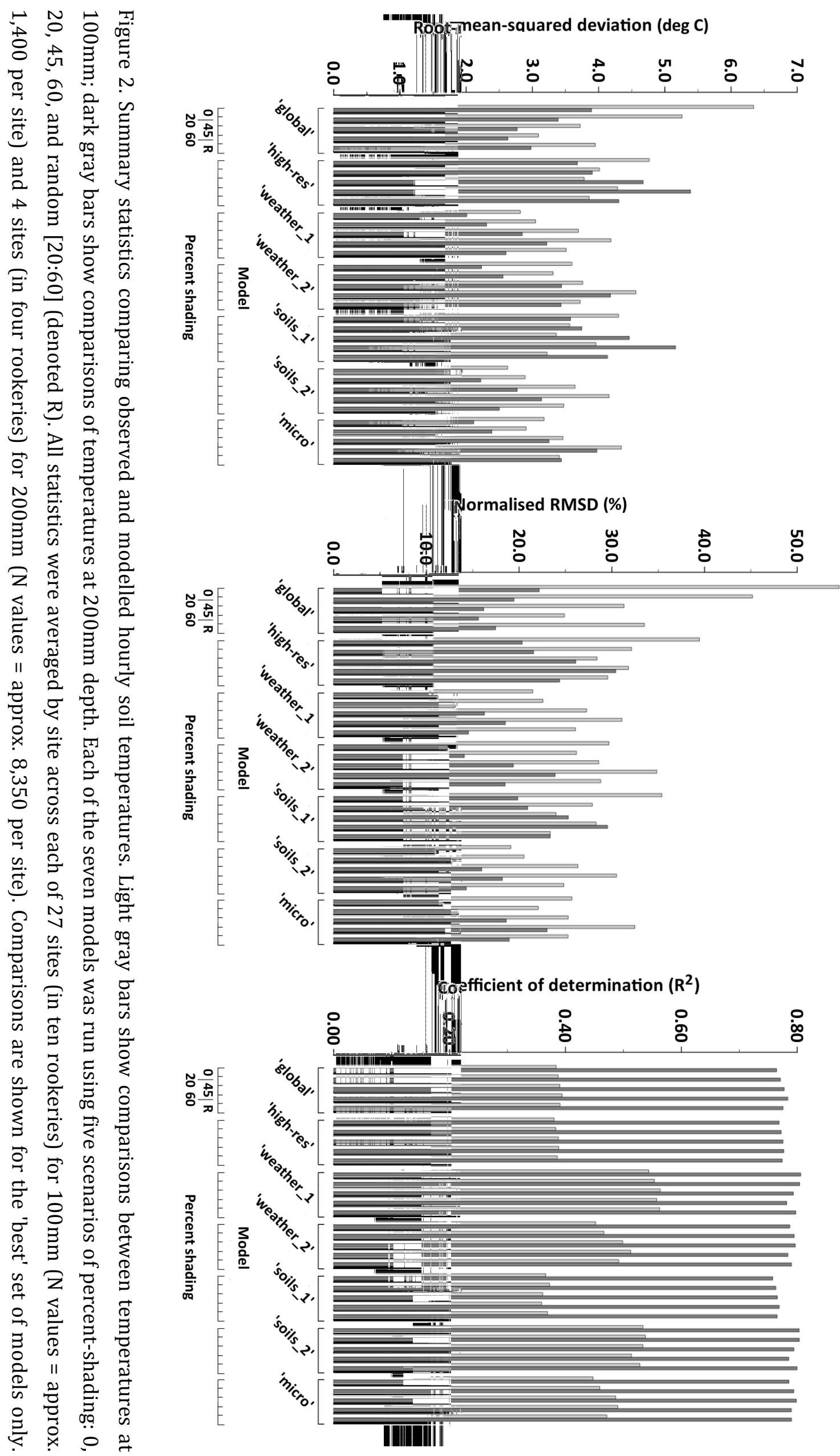




\subsubsection{Effects of parameter values and resolution}

Inclusion of high-resolution geospatial parameters (i.e., elevation, slope, aspect, and horizon angles) in 'high-res' improved accuracy of modelled soil temperatures over predictions generated with 'global' only when simulated shade at microsites was relatively low. RMSD values decreased by $0.09-1.58^{\circ} \mathrm{C}$ for the $0 \%, 20 \%$, and random [20:60] shading scenarios at $100 \mathrm{~mm}$ and the $0 \%$ shading scenario at $200 \mathrm{~mm}$. RMSD values increased by 0.06 $-1.20^{\circ} \mathrm{C}$ at $100 \mathrm{~mm}$ and by $0.51-2.78^{\circ} \mathrm{C}$ at $200 \mathrm{~mm}$ for other shading scenarios. Inclusion of local, daily climate data (i.e., maximum/minimum air temperatures, maximum relative humidity, maximum and minimum wind speeds, daily rainfall) in 'weather_1' improved the accuracy of predictions generated with 'high-res' for all shading scenarios, decreasing RMSD by $0.09-1.95^{\circ} \mathrm{C}$ at $100 \mathrm{~mm}$ and $1.60-2.17^{\circ} \mathrm{C}$ at $200 \mathrm{~mm}$. Inclusion of experimentally-derived, local soil properties (i.e., specific heat, bulk density, fractional soil moisture, reflectance) in 'soils_1' decreased RMSD values for soil temperatures modelled using 'high-res' by 0.33 $0.63^{\circ} \mathrm{C}$ at $100 \mathrm{~mm}$ and by $0.10-0.23^{\circ} \mathrm{C}$ at $200 \mathrm{~mm}$.

Parameterisation of 'weather_1' with modelled wind speed data decreased the accuracy of soil temperature predictions, increasing RMSD values by $0.06-0.78^{\circ} \mathrm{C}$ at $100 \mathrm{~mm}$ and by $0.22-0.96^{\circ} \mathrm{C}$ at $200 \mathrm{~mm}$. Overall, inclusion of both daily weather data and experimentally-derived soil properties in 'soils_2' increased the accuracy of modelled soil temperatures generated using 'soils_1.' RMSD values decreased by $0.67-1.67^{\circ} \mathrm{C}$ under the 0 and $20 \%$ shading scenarios but increased by $0.19-0.28^{\circ} \mathrm{C}$ under $45 \%, 60 \%$, and random [20:60] shading scenarios at $100 \mathrm{~cm}$. RMSD values decreased by $1.52-2.02^{\circ} \mathrm{C}$ under all shading scenarios at $200 \mathrm{~mm}$. Overall, inclusion of modelled wind data in 'micro' decreased the accuracy of soil temperature predictions. With the exception of the $45 \%$ shading scenario, under which the RMSD value decreased by $0.19^{\circ} \mathrm{C}$, RMSD values increased by 0.02 $0.56^{\circ} \mathrm{C}$ at $100 \mathrm{~mm}$. RMSD values increased by $0.17-0.94^{\circ} \mathrm{C}$ at $200 \mathrm{~mm}$ under all shading scenarios for 'micro,' compared with 'soils_2' (Figs. 3, 4). 

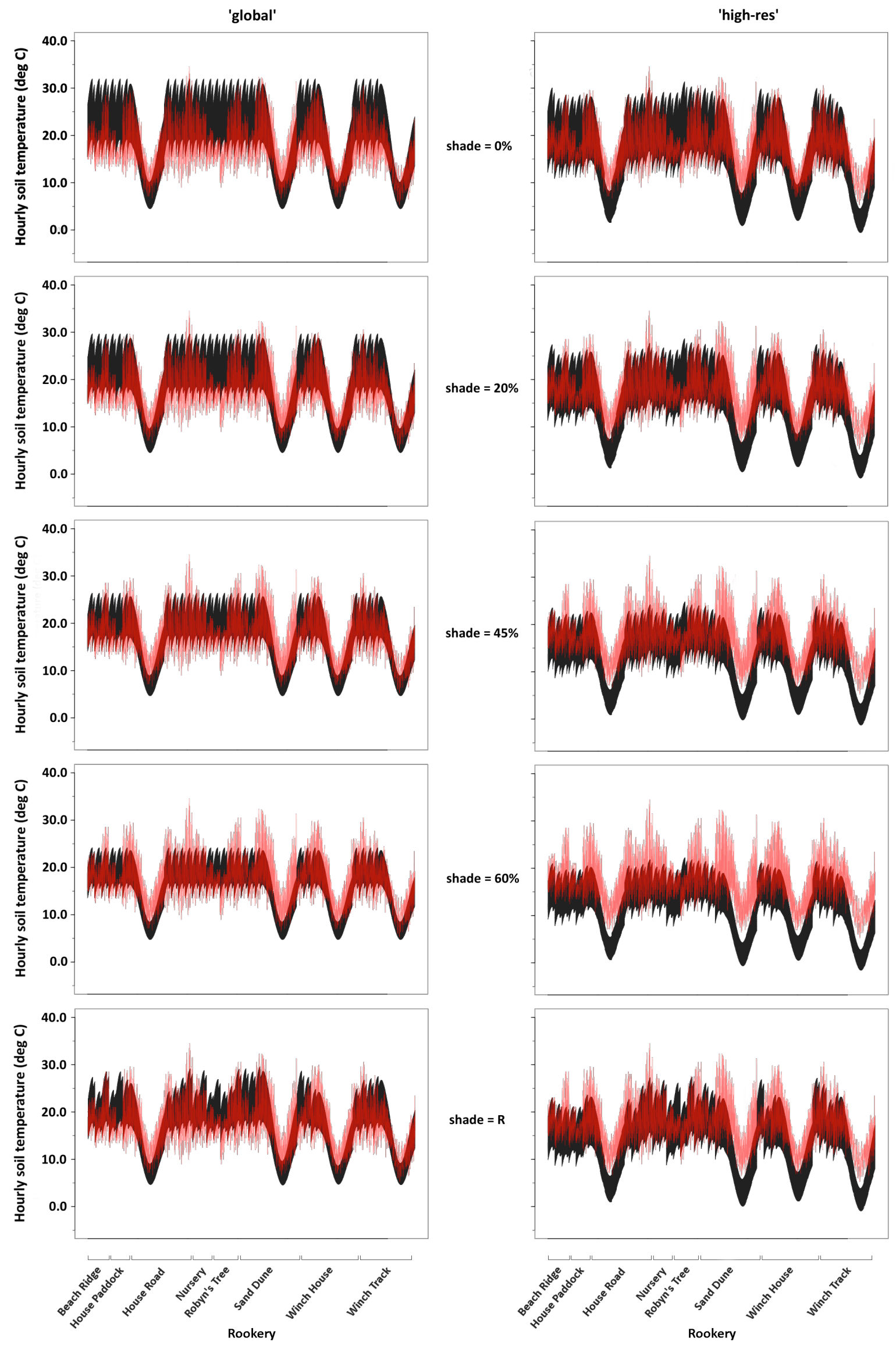

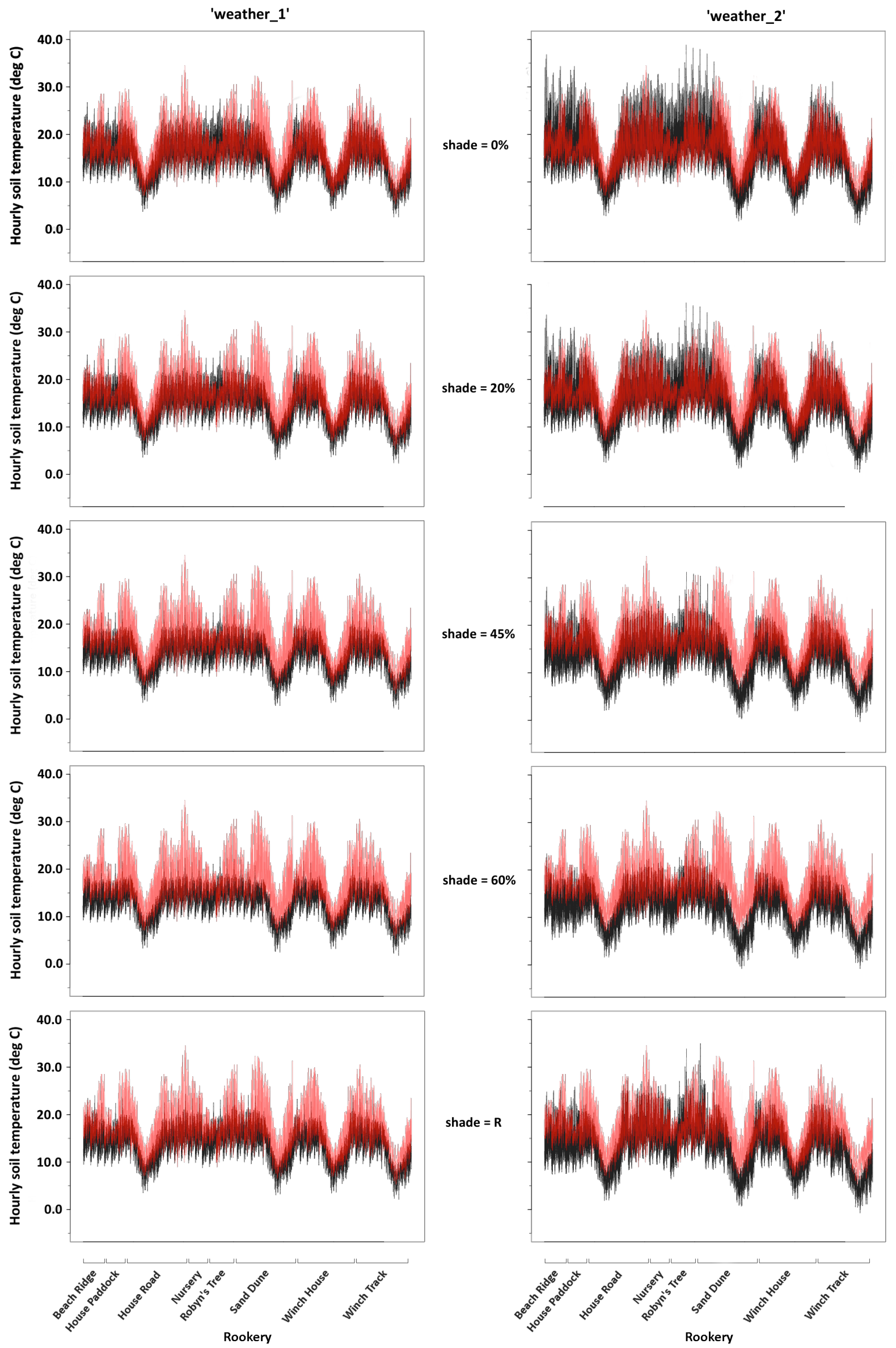


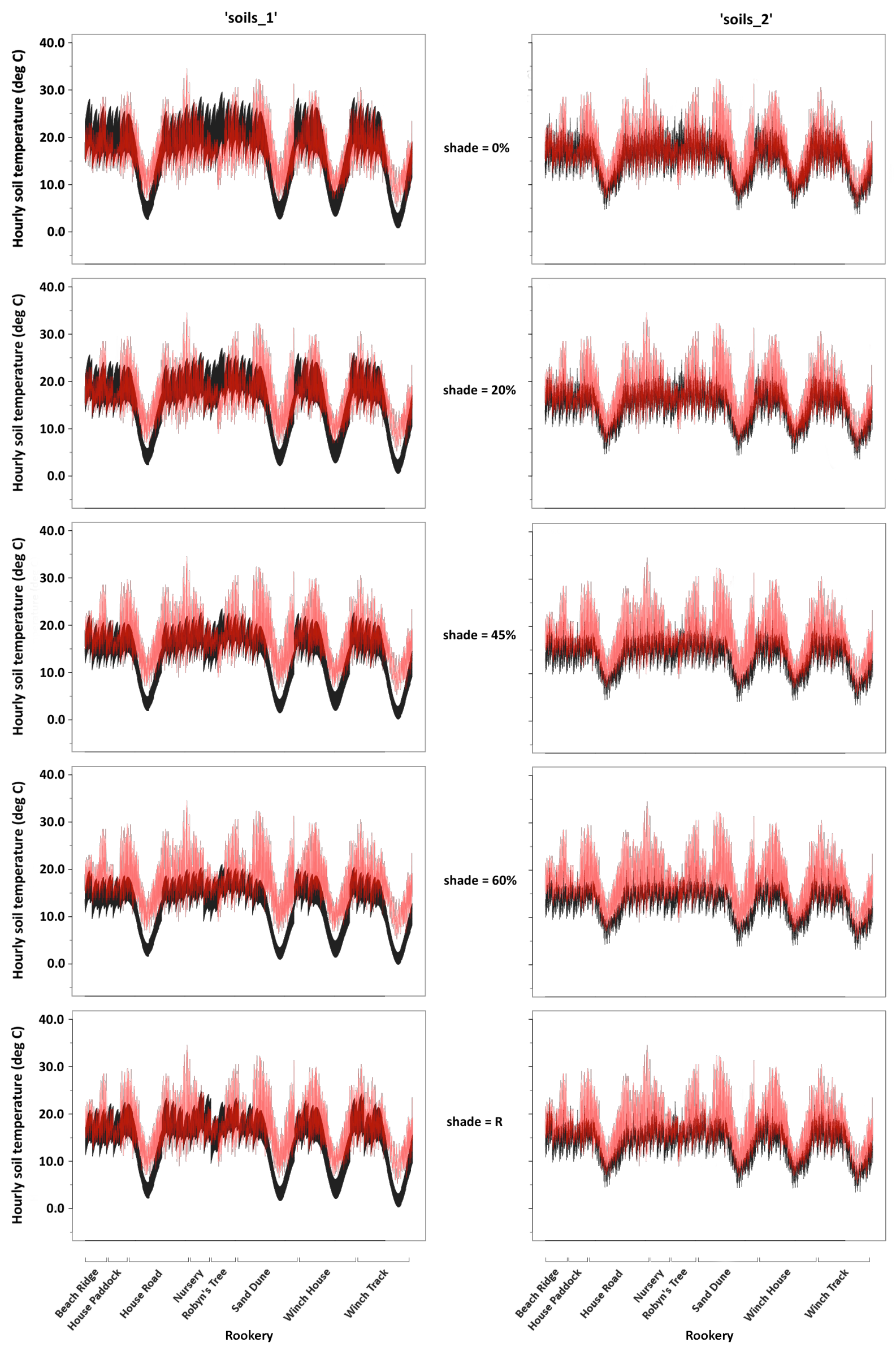



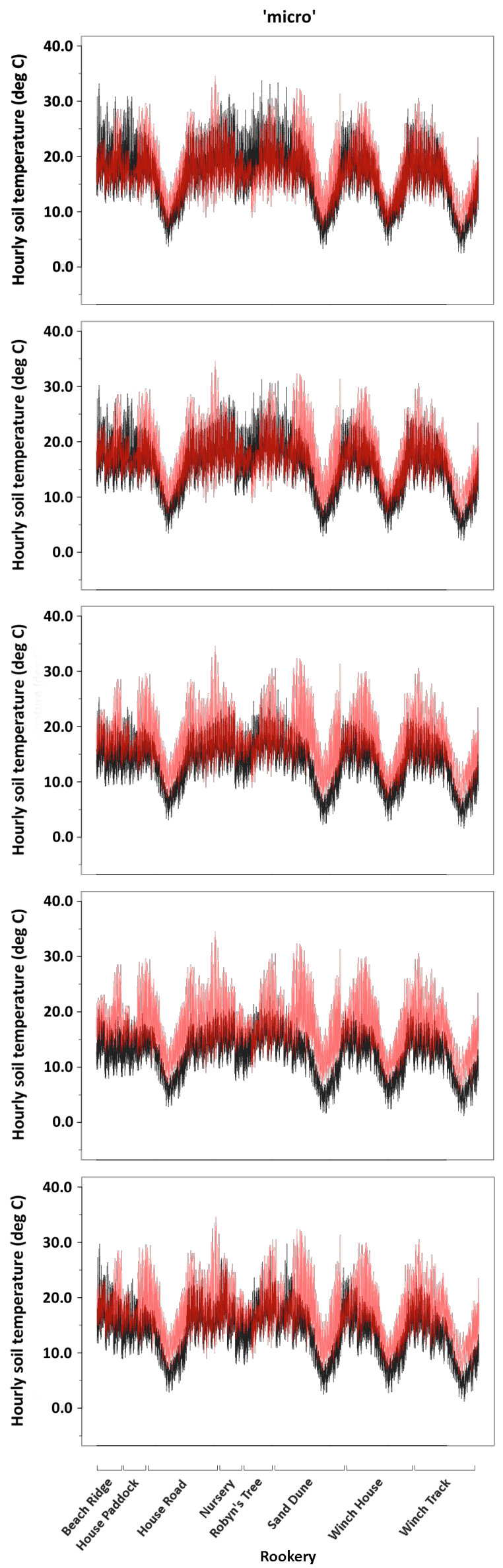

shade $=0 \%$

shade $=20 \%$

shade $=45 \%$

shade $=60 \%$

Figure 3. Line plots showing hourly soil temperature records at $100 \mathrm{~mm}$ (red), ordered by time within each sampling location and overlaid onto modelled data (black). In-situ values appear darker where they overlap with predicted values. The $0 \%$ shading scenario of 'soils_2' was, overall, the most accurate model examined $\left(\mathrm{RMSD}=2.63^{\circ} \mathrm{C}\right)$. Model output is shown for the 'best' set of models only. 


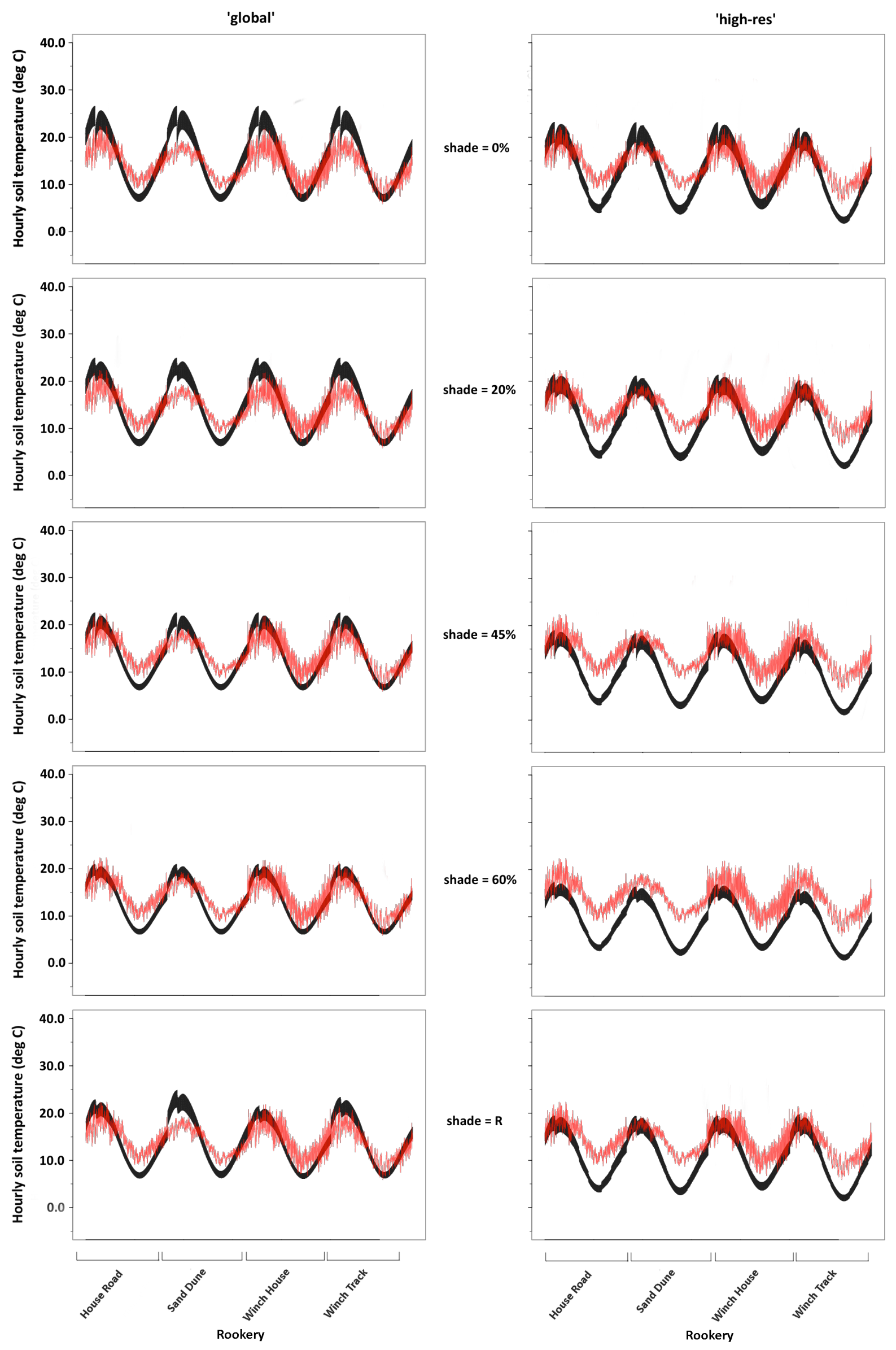



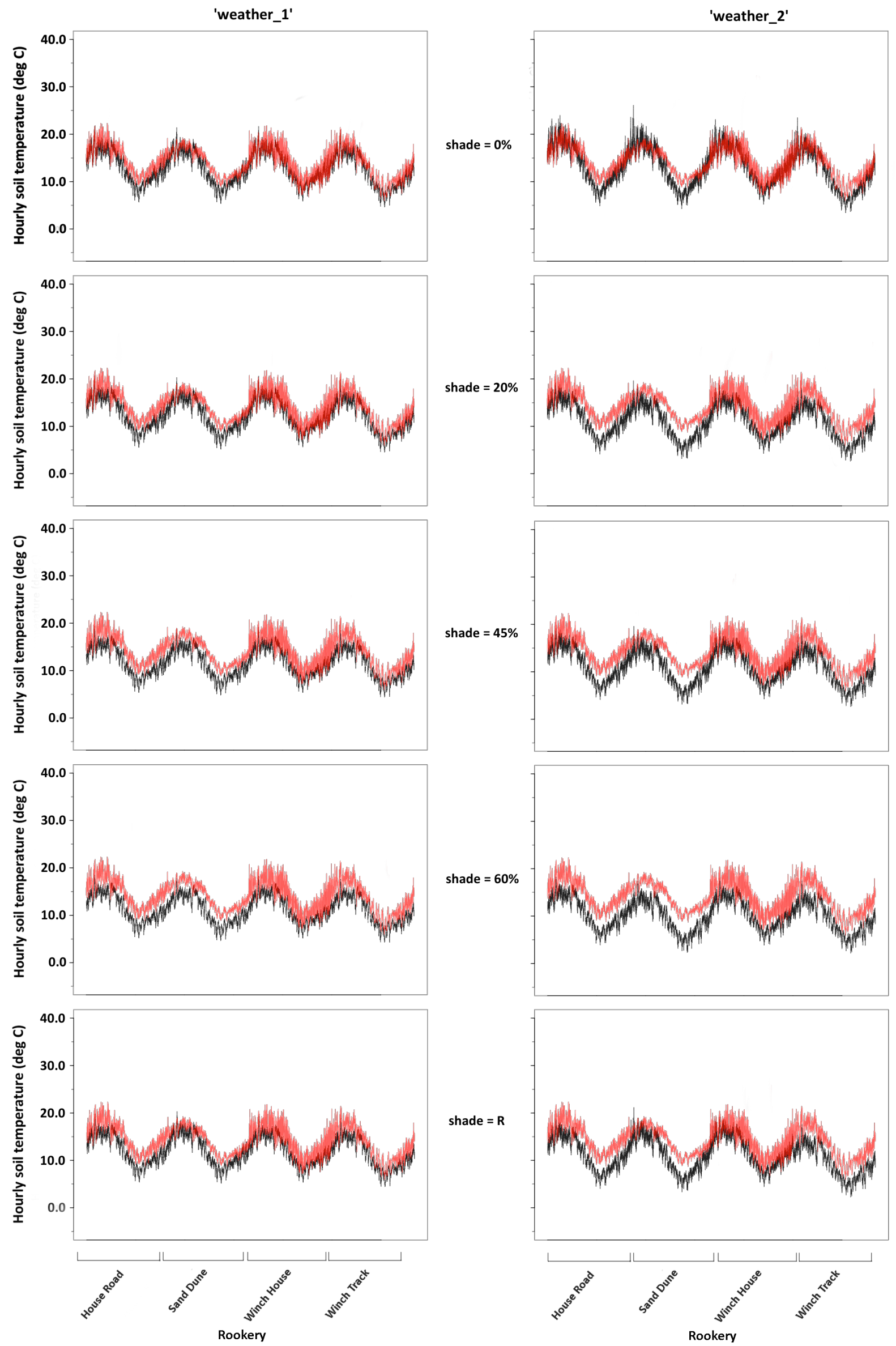

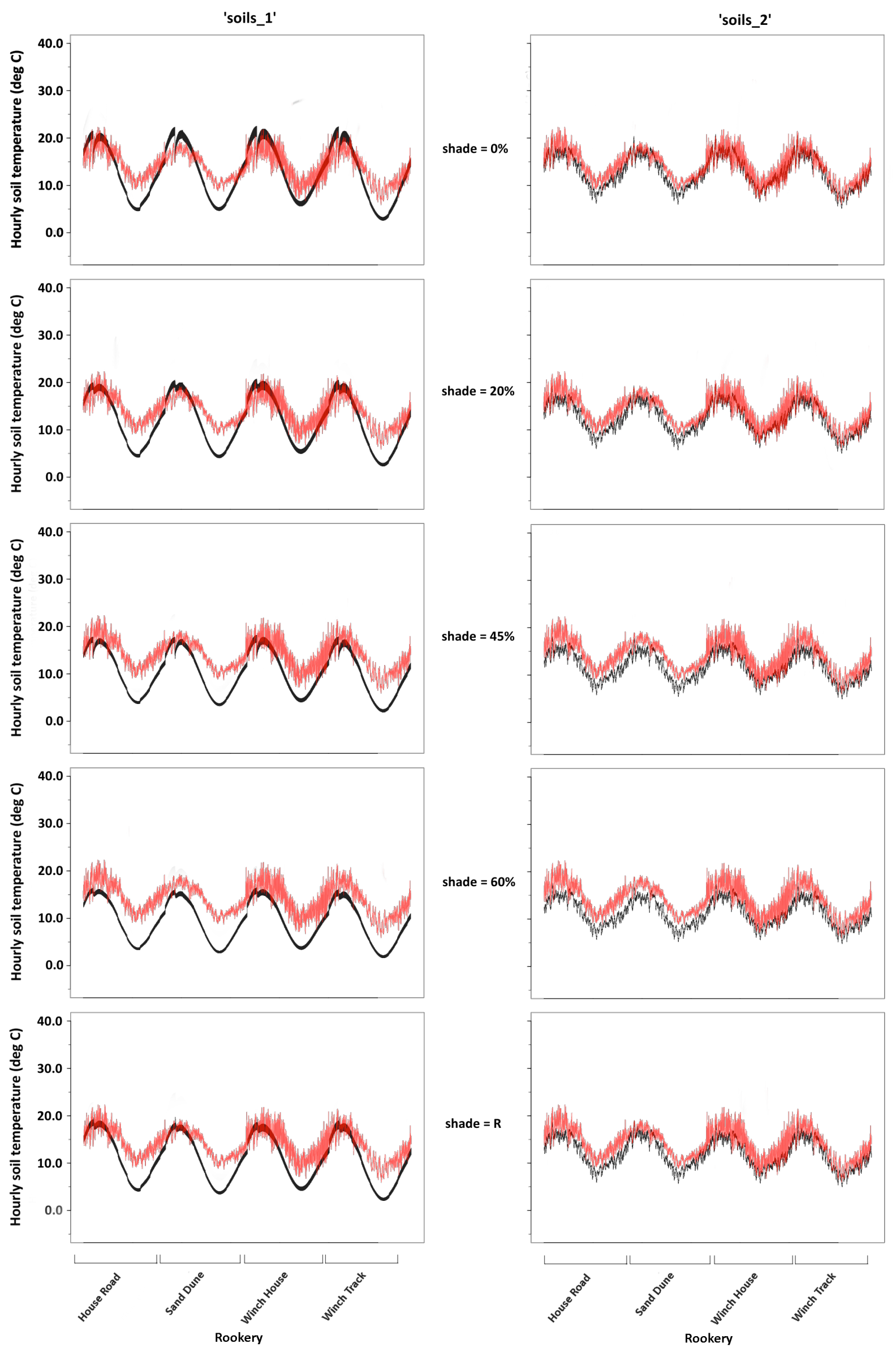


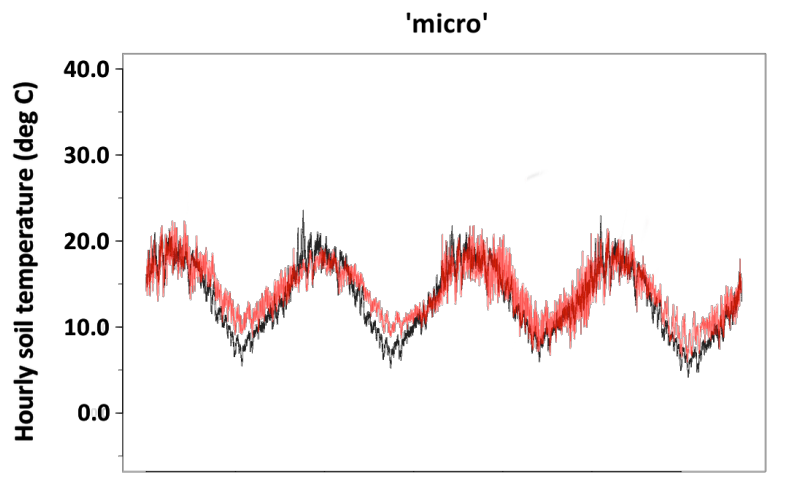

shade $=0 \%$

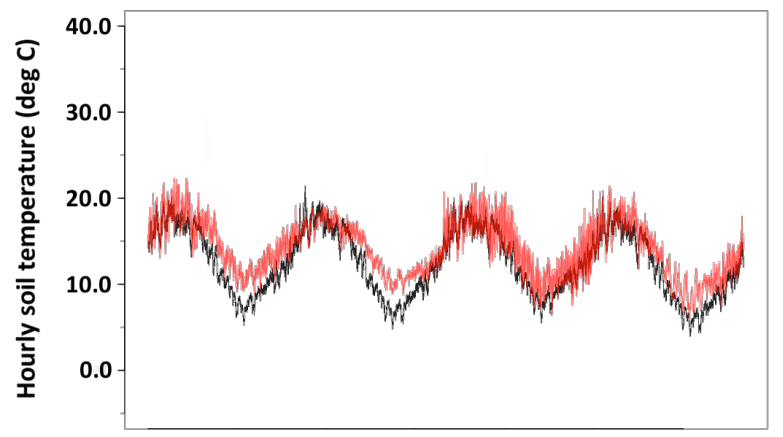

shade $=\mathbf{2 0} \%$

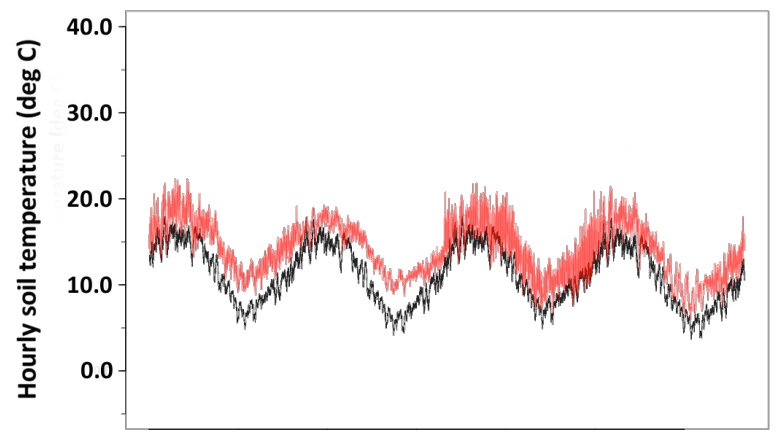

shade $=45 \%$

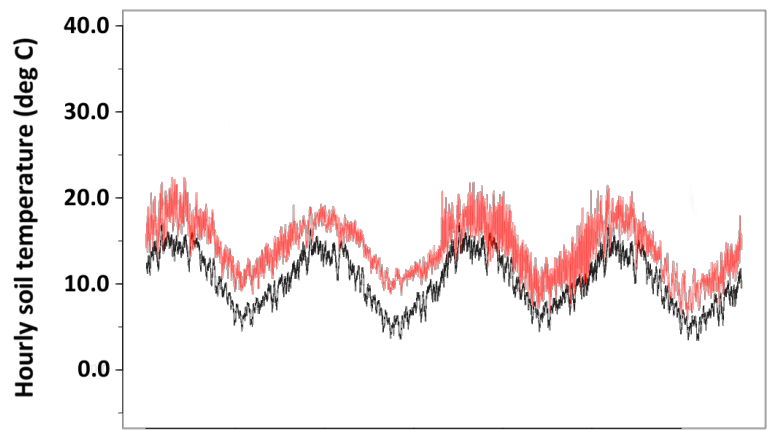

shade $=60 \%$

Figure 4. Hourly soil temperature observations at $200 \mathrm{~mm}$ (red), ordered by time (over one year) within each sampling location and overlaid onto modelled data (black).

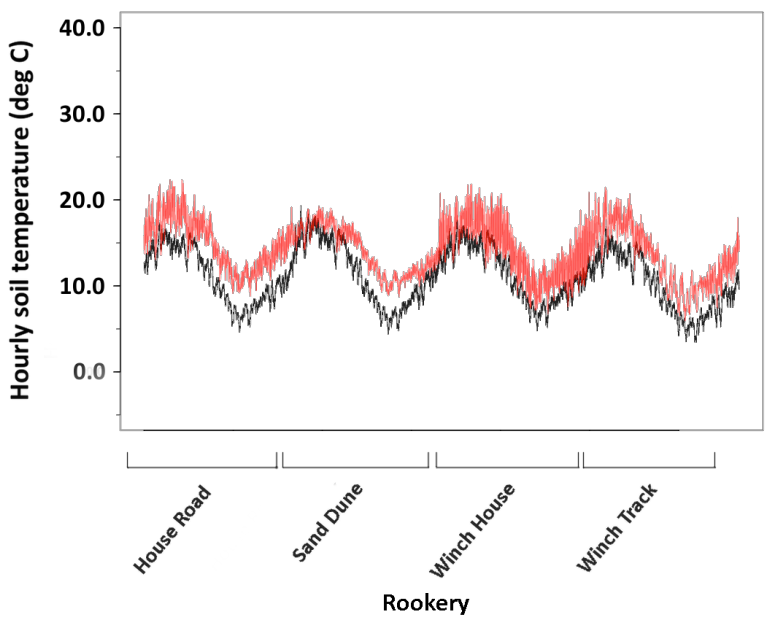
In-situ data appear darker where they overlap with predicted values. The $0 \%$ shading scenario of 'soils_2' was, overall, the most accurate model examined $\left(\mathrm{RMSD}=1.94^{\circ} \mathrm{C}\right)$. Model output is shown for the 'best' set of models only. 


\subsection{Discussion}

\subsubsection{Summary of major findings}

This study modified a mechanistic microclimate model, NicheMapR (Kearney et al. 2014a, b), to examine the effects of multi-resolution, multi-extent input data on the accuracy of hourly soil temperatures simulated at a sub-metre spatial resolution. While comparisons between empirical and mechanistic predictions have previously been undertaken (Kearney \& Porter 2004; Kearney et al. 2014a, b), the effects of parameter resolution on prediction accuracy have only been extensively examined via comparison with in-situ data at spatial resolutions similar to those of large-scale, empirical climate models (Porter et al. 1973; Kearney et al. 2014a, b). This research was the first to explicitly test the accuracy of a mechanistic microclimate model within a small spatial extent, and findings suggest that site-specific, microsite-resolution climate and soil properties matter little to predictions of soil temperatures, even at an extremely high spatial resolution. Further research is needed to determine whether decreasing the resolution of geospatial inputs (e.g., from $<1 \mathrm{~m}$ to $10 \mathrm{~m}$ ), thus reducing computation time, affects the accuracy of model predictions. In addition, the results of this study should be validated at additional locations that are environmentally distinct from the island examined here. Nevertheless, from the perspective of predictive ecology, obtaining local, daily weather data and high resolution geospatial information is likely to improve model accuracy more than sampling of microsites.

Overall, the models examined in this study were better predictors of soil temperatures at $200 \mathrm{~mm}$ than at $100 \mathrm{~mm}$, reflecting the reduction in temperature variance that accompanies decreasing conduction of heat energy with soil depth. Hourly soil temperatures generated using the models with the three lowest values of the rootmean-squared deviation (RMSD) were within approximately $3^{\circ} \mathrm{C}$ of temperatures 
observed at $100 \mathrm{~mm}$, a value comparable or superior to the accuracy of previous model implementations at horizontal spatial resolutions of 5km (Kearney et al. 2014b) and 15km (Kearney et al. 2014a). Modelled temperatures were consistently lower than observed values, regardless of the shading scenario. Data collected over a full year revealed a seasonal pattern at three of the four sites in which higher-capacity dataloggers were installed, with soil temperatures slightly under-predicted in the austral winter, compared to other seasons. All but two of the modelled scenarios generated hourly soil temperatures within $5^{\circ} \mathrm{C}$ of observed values, and most were accurate to within $3-4^{\circ} \mathrm{C}$ of soil temperatures observed at $100 \mathrm{~mm}$ depth. Normalised RMSD (nRMSD) values of the two most accurate models (i.e., the $0 \%$ shading scenarios of 'weather_1' and 'soils_2') indicated that the minimum residual variance across all sites was within only about $20 \%$ of observed values at $100 \mathrm{~mm}$ and within about $10 \%$ of observed values at $200 \mathrm{~mm}$. Similarly, $\mathrm{R}^{2}$ values across all models suggested that the ability to explain variance in observed values at $100 \mathrm{~mm}$ compared to $200 \mathrm{~mm}$ was reduced by almost 30\% (Fig. 2).

Maximum daily air temperature records were better predictors of maximum daily soil temperatures at $100 \mathrm{~mm}$, but not at $200 \mathrm{~mm}$, when compared with modelled values. The accuracy of predicted maxima was much lower at $100 \mathrm{~mm}$, within a mean of only around $6.5^{\circ} \mathrm{C}$ of observed values, than at $200 \mathrm{~mm}$, which was within approximately $3.5^{\circ} \mathrm{C}$ of observed values. Predicted minimum daily soil temperatures were more accurate than air temperature minima, within a mean of $3^{\circ} \mathrm{C}$ of observations at both $100 \mathrm{~mm}$ and $200 \mathrm{~mm}$ for most of the models examined.

Hourly soil temperatures were generated at a sub-metre spatial resolution with an accuracy comparable to lower-resolution implementations of NicheMapR, although marked improvements in accuracy were only facilitated by inclusion of high resolution geospatial information and local weather data. The simulation of surface-level vegetation shading improved model accuracy when climate input were sourced from the 
global database. Only the $60 \%$ shading scenario of the 'global' model, however, generated soil temperatures within approximately $3^{\circ} \mathrm{C}$ of observed values. Inclusion of experimentally derived soil properties did not markedly improve the accuracy of models parameterised with local weather data. Inclusion of high-resolution, modelled wind speed data slightly decreased model accuracy relative to scenarios driven by local, daily minimum and maximum wind speeds.

The most accurate model developed in this study, 'soils_2,' only improved upon the second-best model, 'weather_1,' by a mean of $0.2^{\circ} \mathrm{C}$, an increase in accuracy that is unlikely to impact the outcomes of biophysical models (e.g., predictions of offspring sex ratios in species with temperature-dependent sex determination, sensu Mitchell et al. 2008). In addition, parameterisation of 'weather_1' requires no in-situ data collection, which reduces the time and financial resources required for model implementation and largely eliminates the environmental impacts of habitat access. Future application of either parameterisation should assess the effects of model error by testing the sensitivity of biophysical models to variations in soil temperatures of $3^{\circ} \mathrm{C}$ at $100 \mathrm{~mm}$ and $2^{\circ} \mathrm{C}$ at $200 \mathrm{~mm}$ (Chapter 4$)$.

\subsubsection{Assessment of methodological limitations}

Simulation of site-specific soil properties only slightly improved accuracy of predicted soil temperatures compared to that of models parameterised with generalised values (Figs. 3, 4). The spatial resolution of experimentally determined values of soil physical and thermal properties was coarse, relative to the resolution of geospatial data. The values of soil parameters used in this study may not accurately represent the properties of soils on Takapourewa. Determination of soil properties in-situ along a higher-resolution, stratified sampling grid might improve model predictions. Physical and thermal properties vary little within broad soil classifications, however, and 
collecting empirical measurements of soil thermal properties is costly and timeconsuming. An implementation of NicheMapper ${ }^{\mathrm{TM}}$ (Porter \& Mitchell 2006) developed to predict soil temperatures on North Brother Island (Mitchell et al. 2008) was parameterised using published properties of Takapourewa hill soils (DSIR 1968), which were geographically and physically similar to those on North Brother Island (N. Mitchell pers. comm.). Moreover, the accuracy of models parameterised using location-specific soil values in this and previous studies (Kearney et al. 2014a, b) was not improved over that of models that simulated a generalised sandy soil. More research is needed to determine if different patterns might occur at a site with highly organic soils, which are much less thermally conductive than sandy soils (de Vries 1963; O'Donnell et al. 2009) (Chapter 5).

Increasing the simulated canopy shading to levels representative of mean shade provided by ground-level vegetation cover on Takapourewa reduced accuracy of predicted soil temperatures relative to that of temperatures modelled under the assumption of $0 \%$ shade. The current implementation of NicheMapR does not incorporate the insulating effect (Oliver et al. 1987) of low-growing plants (e.g., thick grasses), so vegetation can only reduce modelled soil temperatures via a shading mechanism. At lower spatial resolutions (e.g., if the pixels of a microclimate surface represent square-kilometers), canopy shading greater than $0 \%$ may accurately represent the mean shading over the area represented by each pixel. As the spatial resolution of a microclimate surface increases, however, the shading of a single pixel is less likely to be accurately represented by a mean value. Rather than specifying 'true' shade at ground-level, therefore, canopy shading for very high-resolution microclimate surfaces may be more accurately characterised as a binary variable signifying whether each site is or is not shaded.

While topography affected the wind profiles experienced at different points on Takapourewa, the accuracy of modelled soil temperatures in this study was not 
improved by inclusion of gridded wind speed data. This result is unlikely to be an artefact of the soil temperature sampling design (limited to the eastern one-third of the island by access restrictions), as modelled maximum and minimum wind speeds varied considerably among sampling sites. Strong effects of wind speed on soil temperatures might be more apparent on the access-restricted northwestern face of Takapourewa, which is the least-sheltered area of the island and regularly exposed to gale-force winds from the west and northwest (Ward 1961). Wind modelling in this study likewise showed that the topography of the western portion of the island facilitates the overall highest wind speeds in this location. Wind speed had a significant effect on the accuracy of modelled soil temperatures on North Brother Island (Mitchell et al. 2008), and gridded wind speed data may improve accuracy of soil temperature predictions in locations that are similarly steep and exposed.

Mean seasonal easterly-westerly wind speeds are predicted to vary in New Zealand by about -2.5 to $+3.6 \mathrm{~m} / \mathrm{s}$ under climate change models for the next century (http://ww.mfe.govt.nz). Changes in mean wind speeds may have a small effect on air temperatures near the soil surface and, consequently, on soil temperatures in the future; however, the accuracy of a mechanistic microclimate model should not be affected by changes in macroclimate conditions. The location of the weather station on Takapourewa may affect the accuracy of modelled wind speeds, as westerly and southerly winds may be dampened topographically prior to sampling. Southerly winds are typically associated with reduced air temperatures in the southern hemisphere, though the current implementation of NicheMapR does not account for the effects of wind direction on air temperatures. Installation of multiple dual-height anemometers for provision of additional points of input to the turbulence velocity model could be undertaken to fine-tune a wind speed model. A more efficient means of examining the effects of both wind speed and direction on accuracy of soil temperatures would be to 
collect air temperature data at multiple points on the island to provide higher-resolution climate input to the microclimate model.

As one goal of this study was to develop a model of soil temperatures specifically for predicting site-specific offspring sex ratios in tuatara, the in-situ temperature sampling regime was stratified among known nesting areas. In addition, access restrictions limited installation of dataloggers to the eastern one-third of the island, so the observed temperatures were not necessarily representative of the variety of microenvironments on Takapourewa. However, because soil temperatures were predicted using a process-explicit model, rather than by extrapolating data from environmentally similar locations (i.e., via a correlative climate model), accuracy of predictions generated for un-sampled faces would only be reduced if the climate on the western side of Takapourewa is not represented by conditions observed at the permanent weather station near the northeastern point of the island.

Sampling of daily weather patterns (i.e., model input) at environmentally stratified points might be beneficial for predicting soil temperatures across the whole island. Properties of the soil types present on the western portion of Takapourewa, primarily Takapourewa steepland soils, were quantified in this study, and inclusion of experimentally-derived soil properties had a negligible effect on the accuracy of predicted hourly soil temperatures. Ecological restoration of Takapourewa (Chapter 2) may necessitate future re-parameterisation of the model developed in this study, as increases in forest cover can affect available canopy shading and the organic content of soils, both of which decrease the amount of heat energy transferred below the soil surface (de Vries 1963; Geiger et al. 2003; O'Donnell et al. 2009). 


\subsubsection{Implications for model application}

This study has shown that soil temperatures modelled mechanistically at an extremely high spatial resolution (i.e., $<1 \mathrm{~m}$ ) have an accuracy comparable to that of lower-resolution (i.e., 5-15km) continental- or global-extent models (Kearney et al. 2014a, b). More importantly, predicting soil temperatures on Takapourewa to within 2$3^{\circ} \mathrm{C}$ of observed values required the microclimate model to be parameterised both with microsite-resolution geospatial data and local, daily weather information. Hourly soil temperatures predicted using the 'global' model, which was parameterised with geospatial and climate input of approximately the same spatial resolution as the globalextent 'microclim' terrestrial climate layers (Kearney et al. 2014a), were only accurate to within about $6^{\circ} \mathrm{C}$ of observed values.

Continental- and global-extent microclimate layers are unlikely to provide accurate estimates of microclimate conditions when the spatial scale of a study area is smaller than the resolution of the climate layer itself. Because the 'global' model in this study was parameterised using climate and geospatial layers at a spatial resolution of 10' (approximately 20km), all 27 sampling sites on Takapourewa were represented by a single pixel. The highest-resolution interpolated climate layers currently available (i.e., 30 " or approximately $1 \mathrm{~km}$ ) represent Takapourewa as three pixels. Very high-resolution geospatial layers are less likely to be readily available for study areas that are relatively isolated or uninhabited (e.g., protected wildlife reserves), especially those with a small spatial extent (e.g., small islands). For example, the highest-resolution digital elevation model for Takapourewa that is publicly available has a horizontal resolution of $20 \mathrm{~m}$, while the best model for the metropolitan area of Wellington, NZ has a resolution of $1 \mathrm{~m}$ (http://www.koordinates.com).

This study did not explicitly examine whether reducing the spatial resolution of geospatial input (e.g., from $0.5 \mathrm{~m}$ to $10 \mathrm{~m}$ or $20 \mathrm{~m}$ ) would affect accuracy of soil 
temperature predictions. Increasing either the resolution or the extent of geospatial input increases the number of individual sites for which microclimate conditions are output and, as a result, markedly increases computation time. Determining the minimum resolution of geospatial data required to accurately predict microclimate conditions at small scales or within a small spatial extent would be beneficial for minimising the resources necessary for developing very high-resolution microclimate surfaces.

From the perspective of predictive ecology, the selection of an appropriate model resolution is critical for examining the potential for range shifts and for identifying barriers to population dispersal, particularly under novel environmental conditions (Bean et al. 2014). The lower the spatial resolution of a microclimate surface, the more likely that a biophysical model would over-estimate the availability of suitable habitat for a species of interest. Likewise, biologically significant sources of environmental variation (e.g., relatively narrow dispersal barriers) can be masked by low-resolution microclimate surfaces. Barring practical limitations (e.g., the availability of geospatial layers, high-performance computing capability), the spatial resolution of a gridded microclimate surface should be informed both by the extent of the study area and by the size of an individual microclimate of interest.

If the goal of a study is to predict range shifts by modelling changes in the spatial extent of an organism's fundamental niche, the pixels that comprise a microclimate surface should be smaller than the total area of habitat currently used by the organism. A higher-resolution microclimate surface is also more likely to predict expansion or contraction of a home range, if climate change affects the ability of an organism to meet its metabolic needs within its current habitat (McNab 1963; Gittleman \& Harvey 1982; Mace et al. 1983). Since producing a mechanistic microclimate surface is computationally intensive (Kearney et al. 2014a, b), a low resolution layer could be used to delineate areas for which a higher-resolution surface should be generated. For 
example, potential locations for translocation of the Critically Endangered Australian western swamp tortoise (Psuedemydura umbrina) could be defined, first, using a 10' surface (Mitchell et al. 2013a). A management decision could then be informed by generating a very high-resolution surface to provide a detailed assessment of the subset of areas identified as suitable future habitat.

For oviparous species, modelling the sub-surface microenvironment experienced by developing embryos separately from the conditions experienced by adults is necessary for predicting the effects of climate change and other novel environmental scenarios on offspring survival and phenotype (Shine 1999; Booth 2006; Woods \& Bonnecaze 2006). High-resolution microclimate data can facilitate greater understanding of embryonic development by linking the abiotic incubation environment with physiological processes at the scale of individual nest sites (i.e., $<1 \mathrm{~m}$ ). If the aim of developing a microclimate surface is to locate areas within a habitat that are suitable for nesting (i.e., sites that facilitate completion of embryonic development), generating a nest-scale microclimate surface is probably unnecessary. However, if key aspects of offspring phenotype depend directly on microclimate conditions (e.g., in species with TSD) the sensitivity of predicted total development to model error should ideally be examined at a high spatial resolution to quantify uncertainty in the boundaries between suitable and unsuitable sites.

Both the mean and variance of incubation temperatures can affect hatchling phenology and phenotype (Shine \& Harlow 1996; Ashmore \& Janzen 2003; Georges et al. 2004; Andrewartha et al. 2010; Du \& Shine 2010; Georges 2013). Error of $2-3^{\circ} \mathrm{C}$ in a soil temperature surface may affect predictions of offspring traits generated by a temperature-dependent development model, particularly if modelled incubation temperatures regularly fluctuate below the point at which development stops. Controlled incubation at between $26-30^{\circ} \mathrm{C}$ of Australian pig-nosed turtles (Carettochelys inscupta) (Georges et al. 1994), marine turtles (Caretta caretta), and alpine skinks 
(Bassiana duperreyi) (Shine \& Harlow 1996) found that total observed embryonic development was higher than expected if mean daily incubation temperatures were low, relative to the maximum temperature at which development occurs (Georges et al 2005). When mean daily incubation temperatures are high, relative to the developmental maximum, fluctuations tend to reduce the total amount of embryonic development observed, compared with modelled values (Georges et al 2005).

Whether similar effects of fluctuating incubation temperatures, either daily or hourly, would be observed in cold-adapted reptiles has not been extensively examined. Tuatara, for example, have developmental minimum and maximum values of, respectively, around $10^{\circ} \mathrm{C}$ and $30^{\circ} \mathrm{C}$ (Mitchell et al. 2006; Nelson et al. 2010) and are incubated artificially at temperatures of $20-23^{\circ} \mathrm{C}$ (Keall et al. 2010; Nelson et al. 2010). The error in hourly soil temperatures modelled in this study and with lower-resolution implementations of NicheMapR is approximately $2-3^{\circ} \mathrm{C}$. Overall development and incubation periods modelled for pig-nosed turtles (Georges et al. 2005) using the Dallwitz-Higgins empirical development rate function (Dallwitz \& Higgins 1992), which has also been used to generate predictions for tuatara, are robust to fluctuations of up to $5^{\circ} \mathrm{C}$ from daily mean incubation temperatures, as long as observed temperatures do not drop below the developmental minimum (Georges et al 2005).

Skewed sex ratios can profoundly influence the viability of a population (Janzen 1994a; Mitchell et al. 2010; Grayson et al. 2014), and microclimate surfaces have been used to provide spatially explicit predictions of hatching phenology and offspring sex ratios in species with TSD (Mitchell et al. 2008; Kearney et al. unpub. data; Chapters 4, 5). Given a model that perfectly predicts soil temperatures, the resolution of a microclimate surface used for predicting offspring sex ratios in species with TSD should be informed by the transitional range of temperatures (TRT), the range of constant incubation temperatures that produces both males and females within a nest. Since the width of spatial boundaries between $100 \%$ male-producing and $100 \%$ female-producing 
habitats will increase as the width of the TRT increases, predictions of sex ratios should be generated at higher spatial resolutions for species, such as tuatara, with very narrow TRTs (i.e., $<1.5^{\circ} \mathrm{C}$ separating male-producing and female-producing CTEs) (Ewert et al. 2004).

The spatial resolution of a microclimate surface can differentially affect the usefulness of modelled offspring sex ratios, depending on the scale of the study area. Predictions of sex ratios generated at a spatial resolution that is high, relative to the size of a habitat, can be used to delineate between areas that are more likely to be either male-producing or female-producing (Mitchell et al. 2008, Fig. S3), even if modelled soil temperatures contain error. In contrast, if the spatial resolution of a microclimate surface is low, compared to the size of the study area, predictions of offspring sex ratios could be uninformative. Under-prediction of $2-3^{\circ} \mathrm{C}$ in a high-resolution surface of soil temperatures for Takapourewa, for example, may lead to female-biased estimates of offspring sex ratios but can still differentiate areas that may to produce high proportions of male offspring under scenarios of climate warming (Chapter 4). Similarly, a 5kmresolution surface of soil temperatures may provide useful estimates of sex ratios across the main North and South Islands of New Zealand but, even with no error, would be uninformative for the small islands to which tuatara are currently restricted. 



\section{Chapter 4}

\section{Predicting effects of climate change on offspring sex ratios and hatching phenology in a population of tuatara}

\subsection{Introduction}

\subsubsection{Climate change and habitat suitability}

Successful conservation of terrestrial biodiversity requires understanding and prediction of the impacts of rapid climate warming on the suitability of both current and potential future habitats (Keith et al. 2008; Gillson et al. 2013; Mitchell et al. 2013a; Reside et al. 2013). Models of habitat suitability have primarily been developed using bioclimatic envelopes and various statistical relationships between the environment and species' known distributions (Guisan \& Zimmerman 2000; Guisan \& Thuiller 2005; Keith et al. 2008; Thuiller et al. 2008). The ability of a species to persist in a given location, however, depends on complex interactions of the abiotic environment with processes at both population (Porter 2000; Akçakaya et al 2004; Wintle et al. 2005; Maschinski et al. 2006; Araújo \& Luoto 2007; Lavergne et al. 2010) and individual (Porter et al 2000; Geiger et al. 2003; Kearney \& Porter 2004; Kearney 2006; Porter et al 2010) levels.

Increasing recognition of the inability of statistical models to reliably predict habitat suitability under future climate scenarios (Hirzel et al. 2002; Brotons et al. 2004; Thuiller 2004; Keith et al. 2008; Lee \& Jetz 2008) is leading to explicit incorporation of the processes underlying species' distributions into predictive models (Kearney \& Porter 2004; Kearney et al. 2008; Keith et al. 2008; Williams et al. 2008; Kearney \& Porter 2009; Kearney et al. 2009; Moritz et al. 2012; Mitchell et al. 2013; Telemeco et al. 2013). Process-explicit, or mechanistic, models can also be useful when environmental change can affect a population's micro-environment in ways that are not apparent from analyses of distribution data (Hartley et al. 2010). 
In oviparous species, shifts in the environment with little or no impact on adults may dramatically affect more vulnerable developing embryos. Environmental variation that is neutral or even beneficial for adult individuals (Deutsch et al. 2007; Mitchell et al. 2013a; Vasseur et al. 2014) can influence the rate of embryonic development (Kearney \& Shine 2004; Nelson et al. 2004b; Georges et al. 2005; Neuwald \& Valenzuela 2009), embryo survival (Kolbe \& Janzen 2002; Refsnider \& Janzen 2010), hatching phenology (Mitchell et al. 2008; Mitchell \& Janzen 2010; Nelson unpub. data), and offspring phenotype (Elphick \& Shine 1998; Downes \& Shine 1999; Shine 1999; Braña \& Ji 2000; Booth 2006; Woods \& Bonnecaze 2006; Hare et al. 2008; Amiel \& Shine 2012) in ways that affect both offspring fitness in the short term and, in the longer-term, population health (Mitchell \& Janzen 2010; Mitchell et al. 2009, 2012, 2013).

Ectotherms, especially reptiles, are vulnerable to detrimental effects of climate warming (Moritz et al. 2012; Walters et al. 2012; Cabrelli 2013; Kingsolver et al. 2013), particularly shifts in temperature ranges and interactions between temperature and other climate variables (Clusella-Trullas et al. 2011). Many reptile species have evolved temperature-dependent sex determination (TSD), a suite of sex-determining mechanisms in which the temperature of incubation determines offspring sex (Bull 1983; Janzen \& Paukstis 1988, 1991; Ewert et al. 2004; Harlow 2004; Janzen \& Phillips 2006). While higher air temperatures can create additional basking and foraging opportunities for adult reptiles (Porter et al. 1973; Porter \& Tracy 1983; Mitchell et al. 2013a), an increase in the amount of heat energy conducted to sub-surface nests of oviparous species can lead to more rapid embryonic development and earlier hatching as well as skewed offspring sex ratios (Mitchell et al. 2008, 2009; Mitchell \& Janzen 2010; Telemeco et al. 2013).

Suitable nesting habitat can be statistically defined by examining the environmental correlates of nest site selection (i.e., nest, as opposed to adult, presence/absence); however, nest site choice in species with TSD is typically only 
indirectly correlated with hatchling phenotype (Escalona et al. 2009; Refsnider \& Janzen 2010). As a result, preference for nesting habitat and suitability of the preferred nesting habitat for maintaining balanced sex ratios may become de-coupled under novel climate conditions, masking indirect effects of climate change on population demographics. Populations that are adapted to relatively cool environments (e.g., those at high altitudes

or latitudes), for example, may be at increased risk of severely skewed sex ratios, as females tend to prefer warm nesting locations that maximise the probability of successful hatching (Thompson et al. 1996; Nelson et al. 2004b; Ewert et al. 2005; Mitchell et al. 2008). Nest site selection alone, therefore, may not provide a reliable indicator of the suitability of a habitat for maintaining balanced hatchling sex ratios. Assessing future habitat suitability for populations with TSD, therefore, requires predicting the impacts of environmental variation on embryonic development, hatching phenology, and offspring sex, independently of direct effects on adults.

\subsubsection{Habitat suitability for species with TSD}

Although TSD has evolved multiple times in diverse vertebrate taxa (Janzen 1995; Janzen \& Krenz 2004; Janzen \& Phillips 2006; Quinn et al. 2011) and has been maintained through periods of prehistoric climate change (Janzen \& Paukstis 1988, 1991; Silber et al. 2010), current rates of climate warming are higher than any previously recorded (Huntley 1997; IPCC 2013). In addition, habitat fragmentation has led to geographic isolation of small populations (Hughes 2000; Lacy 2000) and, as a consequence, reduction in genetic variation (Crow \& Kimura 1970; Allendorf 1986; Lande et al. 1988) and dispersal capability (Gibbons 2000; Pearson \& Dawson 2003) as well as increased vulnerability to stochastic events (Caughley 1994). Under extreme climate scenarios, many species with TSD are unlikely to maintain balanced population sex ratios via evolutionary adaptation (Morjan 2003; Mitchell et al. 2009) or through 
plasticity in either nesting behaviour (Nelson et al. 2004b; Schwanz \& Janzen 2008; Telemeco et al. 2009) or embryonic development (McGaugh \& Janzen 2011).

Predicting offspring sex ratios for future climate scenarios is a priority for conservation of species with TSD (Janzen 1994b; Hulin et al. 2009; Neuwald \& Valenzuela 2009; Mitchell \& Janzen 2010; Telemeco et al. 2013), particularly if the sex ratio of a population is likely to become male-biased in warmer climates (Tucker et al. 2008; Mitchell et al. 2009; Mitchell \& Janzen 2010; Boyle et al. 2014; Grayson et al. 2014). Research to date has been limited primarily to either examining the effects of temperature on embryonic development and hatchling sex in a controlled laboratory setting (Georges et al. 2005; Neuwald \& Valenzuela 2009) or evaluating correlations between hatchling sex ratios and directly measured soil temperatures or mean air temperatures (Janzen 1994b; Hays et al. 2003; Nelson et al. 2004b; Doody et al. 2006; Hawkes et al. 2007; Telemeco et al. 2009; Besson et al. 2012). While these research methods increase understanding of the distribution of TSD patterns across species, they can only broadly address the microsite-scale environmental processes that drive embryonic development and hatchling sex in-situ. Likewise, while a predictive link has been established between sexes of released hatchlings and adults in at least one species with TSD, the painted turtle (Chrysemys picta) (Schwanz et al. 2010), monitoring offspring sex ratios in wild populations, especially for species in which juveniles are highly cryptic or display no sexual dimorphism, may be impractical.

From the perspective of biodiversity conservation, predicting near-future operational sex ratios solely via establishment of current offspring sex ratios limits opportunities for examining preemptive population management strategies. Few studies to date have linked empirical models of embryonic development and spatially-explicit predictions of nest temperatures to examine habitat suitability for species with TSD (Mitchell et al. 2008, 2009; Monasterio et al. 2011). As a result, despite the necessity of defining habitat suitability to include long-term maintenance of balanced sex ratios, 
particularly for reptiles with TSD, conservation planning efforts have yet to regularly incorporate the effects of climate change on embryonic development in vulnerable populations (Cabrelli 2013).

\subsubsection{Predicting sex ratios in a population with TSD}

The relationship between incubation temperature and offspring sex in reptiles with TSD is described by a non-linear function encompassing the critical window of embryonic development, the thermosensitive period (TSP), during which sex is determined (Mrosovsky \& Pieau 1991; Ewert et al. 2004). The timing and length of the TSP vary among species, but sex determination occurs typically during the first half of incubation and encompasses around $10-20 \%$ of total development (Mrosovsky \& Pieau 1991; Valenzuela 2001). Patterns of TSD are differentiated by the range of constant incubation temperatures that produces both males and females in a clutch, known as the transitional range of temperatures (TRT). The point within the TRT at which offspring sex ratios are exactly 1:1 is the pivotal temperature (Mrosovsky \& Pieau 1991). Whether both sexes are produced within one or two TRTs determines the shape of the sex ratio function (i.e., TSD type I or II) for a species (Mrosovsky \& Pieau 1991). Three distinct patterns of TSD are currently recognised. In TSD type Ia, incubation temperatures above the TRT produce female offspring and, in TSD type Ib, male. In TSD type II, only female offspring are produced at incubation temperatures above the 'warm' TRT and below the 'cool' TRT, while primarily males are produced at temperatures that fall between both TRTs (Valenzuela 2001).

The rate of embryonic development in vertebrates with TSD is also a non-linear function of incubation temperature, with higher temperatures yielding, in general, more rapid rates of development (Sharpe \& DeMichele 1977; Dallwitz \& Higgins 1992; Girondot 1999; Godfrey et al. 2003; Georges et al. 2004, 2005; Neuwald \& Valenzuela 
2009). Thus, the experienced temperature regime influences the duration of the incubation period (i.e., hatching phenology), but incubation temperature only affects offspring sex during the TSP. Developing embryos regularly experience temperature fluctuations throughout their full range of physiological tolerance (Georges et al. 2004, 2005; Mitchell et al. 2006). Thus, obtaining the best possible estimates of offspring sex ratios requires predicting both the timing of the TSP and the laboratory-equivalent incubation temperature, known as the constant temperature equivalent, or CTE (Georges et al. 2004, 2005), experienced during that particular developmental window. Accurately predicting sex ratios and hatching phenology for a wild population within a particular habitat, therefore, requires spatially-explicit, preferably hourly temperature data that captures the variation experienced in natural nests.

Most reptile species with TSD have evolved either type Ia or type II patterns (Deeming 2004; Ewert et al. 2004; Harlow 2004); however, the New Zealand-endemic tuatara (Sphenodon punctatus) is both the only known extant species of Rynchocephalia (Hay et al. 2010) and the only extant species known to have evolved TSD type Ib (Nelson et al. 2004a; Mitchell et al. 2006). Tuatara may survive more than 100 years in the wild (Dawbin 1982; Cree 1992, 1994) and have been confirmed to live to at least 91 years (Nelson pers. comm.). In addition, females require 11-13 years to reach sexual maturity and then reproduce only every 2-5 years (Cree et al. 1992; Cree 1994). As a result, tuatara have a generation interval of around 40 years (Mitchell et al. 2009) and are unlikely to respond evolutionarily to rapid climate change.

Tuatara are cold-adapted reptiles (Saint-Girons 1980; Barwick 1982; Cree 1994; Thompson \& Daugherty 1998), and populations have evolved extremely low pivotal temperatures, around $22^{\circ} \mathrm{C}$, and a relatively narrow TRT (Ewert et al. 2004) of around $1^{\circ} \mathrm{C}$ (Mitchell et al. 2006). Constant incubation at $25^{\circ} \mathrm{C}$ increases embryo mortality to nearly 100\% (Thompson 1990). In contrast, constant incubation temperatures below $30^{\circ} \mathrm{C}$ and above approximately $32^{\circ} \mathrm{C}$ produce all female hatchlings in crocodilians, while 
males and mixed-sex nests are produced at intermediate temperatures (Deeming 2004). In most species of lizards, mixed-sex nests are produced at constant incubation temperatures of around $26-28^{\circ} \mathrm{C}$ (Harlow 2004). Temperatures of up to $32^{\circ} \mathrm{C}$ are known to produce both sexes in some Australian agamids (Harlow 2004), though constant incubation at temperatures above $31^{\circ} \mathrm{C}$ increases embryo mortality (Harlow 2001). In turtles that have evolved TSD type II, mixed-sex nests result from constant incubation at a broader range of temperatures, around $22-27^{\circ} \mathrm{C}$, but the upper limit of male-producing temperatures approaches $29^{\circ} \mathrm{C}$ in some Kinosternon sp. (Ewert et al. 2004). In most turtle species with TSD type Ia, incubation temperatures of up to $27^{\circ} \mathrm{C}$ typically produce $100 \%$ male offspring, and temperatures above 29 or $30^{\circ} \mathrm{C}$ generally result in $100 \%$ females (Ewert et al. 2004). Incubation temperatures that produce females or mixed-sex nests in other species can easily lead to highly male-biased offspring sex ratios in tuatara. Defining habitat suitability for tuatara to include maintenance of balanced or female-biased offspring sex ratios or, minimally, the prevention of extreme male bias can provide a template for conservation of other species with TSD that are at long-term risk of demographic effects of climate warming.

Dispersal is limited in populations of tuatara both because individuals tend to maintain very small, long-term home ranges (Gans et al. 1984; Newman 1987; Moore et al. 2009) and because populations are currently restricted to relatively small offshore islands and fenced mainland sanctuaries. On at least one island, Takapourewa, female tuatara migrate from their home territories to nest (Refsnider et al. 2010, 2013), and nest site selection responds rapidly to land cover change (Chapter 2). The extent to which current habitats and, specifically, preferred nesting habitat are suitable for maintaining balanced offspring sex ratios long-term under extreme climate warming has only been investigated for a single population to date (Mitchell et al. 2008, 2009).

The relict population of tuatara on 4-ha North Brother Island $\left[41^{\circ} 07^{\prime} \mathrm{S} 174^{\circ} 27^{\prime}\right.$ E] is currently about 70\% male (Mitchell et al. 2008, 2009; Grayson et al. 2014) and is 
one of the few populations of any species for which high-resolution, spatially-explicit soil temperatures have been used to predict hatchling sex ratios and inform extinction probabilities (Mitchell et al. 2008, 2009; Grayson et al. 2014). Due to the open, unvegetated homogeneity and consistent relative warmth of available nesting habitat (Nelson et al. 2004b; Mitchell et al. 2008), which are likely responsible for the severe male bias, tuatara on North Brother Island are an example of the worst-case scenario of habitat-specific effects of climate warming in a population already at high risk of sexratio bias (Mitchell et al. 2009; Mitchell \& Janzen 2010; Grayson et al. 2014).

Most other islands occupied by tuatara, while relatively small, provide a higher diversity of potential nesting habitats than North Brother Island (Nelson et al. 2004b). The largest population, at least 30,000 individuals (Newman 1987) compared with about 500 on North Brother Island (Thompson et al. 1992; Nelson et al. 2002a, 2002b; Hoare et al. 2006; Mitchell et al. 2010), and most well-studied population of tuatara resides on Takapourewa, a 150-ha Nature Reserve also known as Stephens Island (Tyrrell et al. 2000; Mitchell et al. 2009). Short-term research has estimated the current population sex ratio to be approximately 1:1 (Nelson et al. 2004b; Moore et al. 2009). While the population of tuatara on Takapourewa is likely more resilient to short-term environmental stochasticity and long-term demographic effects of climate warming than much smaller populations, the island provides an opportunity to examine the suitability of a heterogeneous habitat for maintaining balanced offspring sex ratios. Long-term conservation of existing populations as well as establishment of new populations via translocation requires mapping the suitability of both current and potential future habitats. 


\subsubsection{Study aim and research questions}

Assessing habitat suitability for oviparous species with TSD requires understanding the effects of behavioural and environmental variation on sex ratios. The aim of this study was to develop spatially explicit predictions of in-situ incubation temperatures and corresponding hatchling sex ratios for a population of tuatara. Research questions included: (1) do predicted scenarios of climate warming influence incubation temperatures, hatchling sex ratios, or hatching phenology? (2) Does variation in nesting phenology affect hatchling sex ratios or hatching phenology? (3) Does variation in nest location or nest depth affect hatchling sex ratios or hatching phenology?

\subsection{Methods}

\subsubsection{Modelling soil temperatures}

This study was conducted on the island of Takapourewa/Stephens Island [4040'00" S $174^{\circ} 00^{\prime} 00^{\prime \prime}$ E] (Fig. 1), an offshore Nature Reserve at the northernmost point of the Marlborough Sounds in New Zealand. Cyclical soil temperatures were predicted using the NicheMapR v 2011b global microclimate model (Kearney et al. 2014b), which was modified to allow input of high-resolution geospatial data and local climate information and validated against hourly soil temperature data collected at the study site (Chapter 3). Geospatial data (i.e., latitude, longitude, elevation, slope, aspect, horizon angles) were extracted from a sub-metre resolution gridded digital elevation model of the island for 481,967 unique points. Observations of rainfall (total $\mathrm{mm}$ ), relative humidity $(\%)$, minimum and maximum air temperatures $\left({ }^{\circ} \mathrm{C}\right)$, and minimum and maximum wind speeds (m/s) were obtained from the NIWA CliFlo database (http://www.cliflo.niwa.co.nz) from the weather station on Takapourewa, New Zealand 
[Station No. 26169]. Observations of relative humidity were recorded at either 08:00 or 09:00 and were assumed to represent daily maxima. Minimum daily values were estimated by splining minimum monthly humidity values across 365 days. Daily air temperature and wind speed minima and maxima were calculated from hourly data.

Because completion of embryonic development in tuatara generally requires between 11-16 months in natural nests (Cree et al. 1991, 1992; Thompson et al. 1996), hourly soil temperature predictions were generated for two years, with an initial oviposition date of 30 November, the approximate mean lay date observed in 2011 and 2012 (Carter unpub. data). The mean nest depth on Takapourewa is about $110 \mathrm{~mm}$ (Cree et al. 1992), though nest depth ranges from 50 to 250mm (Cree et al. 1991; Thompson et al. 1996). Temperatures were modelled island-wide for ten soil depths: $0,15,50,100$, $150,200,300,500,1000$, and 2000mm. Definitions for ten soil depths were required to run the model, but temperatures at depths above $50 \mathrm{~mm}$ or below $300 \mathrm{~mm}$ were not used in this study. 


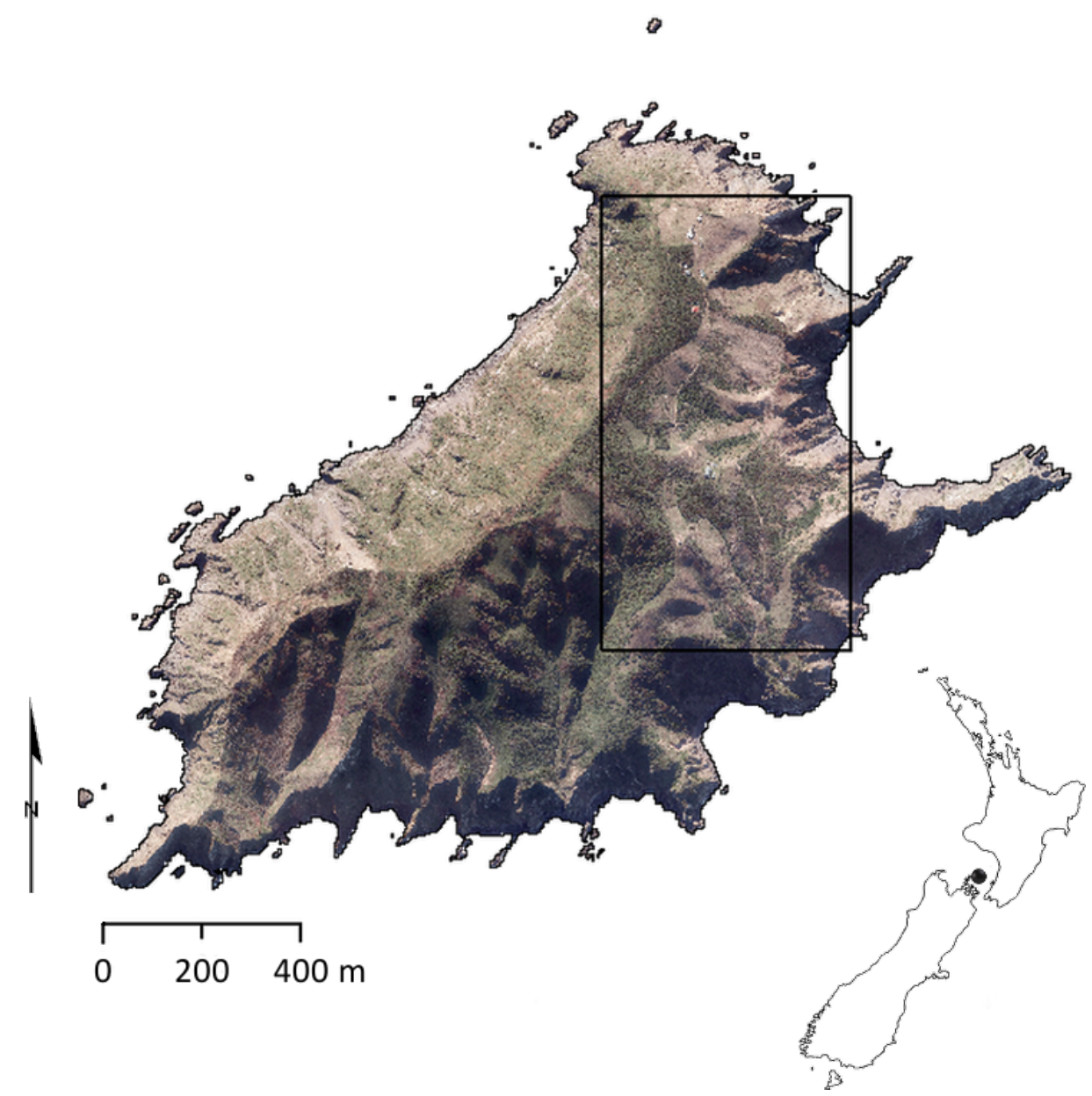

Figure 1. Aerial photograph from 2011 showing Takapourewa. The area of the island accessible to researchers is denoted approximately by the black box. The black dot approximates the island's location relative to the North and South Islands of New Zealand.

\subsubsection{Calculating embryonic development rates and sex ratios}

The percentage of embryonic development per hour, ds/dt, was calculated from all soil temperature predictions using the Dallwitz-Higgins non-linear development rate function (Dallwitz \& Higgins 1992; Georges et al. 2005):

$$
\begin{gathered}
\frac{d s}{d t}=\frac{\left\{b_{1}^{\left.10^{\left[-v^{2}\left(1-b_{5}+b_{5} v^{2}\right)\right]}\right\}}\right.}{24} \\
v=\frac{\left(u+e^{b_{4} u}\right)}{c_{2}} \\
u=\left(\frac{T-b_{3}}{b_{3}-b_{2}}\right)-c_{1}
\end{gathered}
$$




$$
\begin{gathered}
c_{1}=\frac{1}{\left[1+0.28 b_{4}+0.72 \ln \left(1+b_{4}\right)\right]} \\
c_{2}=\frac{1+b_{4}}{1+1.5 b_{4}+0.39 b_{4}{ }^{2}}
\end{gathered}
$$

where ' $b_{1}$ ' is the maximum development rate; ' $b_{2}$ ' is the temperature at which development is approximately $\mathrm{b}_{1}{ }^{*} 0.10$; $\mathrm{b}_{3}$ ' is the temperature $\left({ }^{\circ} \mathrm{C}\right)$ at which the maximum development rate is reached; ' $\mathrm{b}_{4}$ ' is the shape of the curve as the development rate approaches 0 at very low temperatures; ' $b_{5}$ ' is a measure of the asymmetry of the development rate; ' $\mathrm{c}_{1}$ ' and ' $\mathrm{c}_{2}$ ' are location-specific constants; and ' $\mathrm{T}$ ' is the temperature for which the development rate is calculated (Dallwitz \& Higgins 1992). Parameter values used in this study; $b_{1}=0.98545, b_{2}=13.78641, b_{3}=30, b_{4}=60, b_{5}=0.40, c_{1} \approx$ $0.24503, \mathrm{c}_{2} \approx 1.24958$; were previously derived for tuatara on Takapourewa (Mitchell et al. 2008).

Calculated hourly development was accumulated over time until hatching (i.e., the first hour in which total development $\geq 100 \%$ ). Locations at which development did not reach $100 \%$ within 24 months (Cree et al. 1989) were classified as non-viable nesting locations (Mitchell et al. 2006). The possibility of embryo mortality was assessed by calculating development conditional on the requirement that a location could not exceed a conservative maximum thermal tolerance of $38^{\circ} \mathrm{C}$ (Nelson et al. 2004b; Mitchell et al. 2006, 2008) in any single hour prior to hatching. To determine the constant incubation temperature equivalent (CTE) for each location, soil temperatures within the TSP for tuatara (i.e., the 25-35\% developmental window) (Nelson et al. 2010) were extracted and re-ordered by ascending hourly development rate. The CTE was taken to be the soil temperature corresponding to the developmental median of the TSP (i.e., the point at which $50 \%$ of the total development during the TSP had occurred).

Each CTE was converted to a spatially-explicit estimate of offspring sex ratio, sr, using the A-logistic function (Girondot 1999; Godfrey et al. 2003): 


$$
s r(t)=\left[1+\left(2^{e^{k}}-1\right) e^{\left(\frac{1}{s}\right)(p-t)}\right]^{-\frac{1}{e^{k}}}
$$

where ' $t$ ' is the CTE calculated for a given point; ' $p$ ' is the species- or population-specific pivotal temperature $\left({ }^{\circ} \mathrm{C}\right)$; 's' describes the shape of the sex ratio function, either positive or negative, as temperatures transition from male-producing to female-producing; and ' $\mathrm{k}$ ' is a numeric constant equal to $2 * \ln (\mathrm{x} / 1-\mathrm{x})$ that allows for asymmetry around $\mathrm{p}$, where the lower and upper boundaries of the TRT, respectively, produce sex ratios of $\mathrm{x}$ and 1-x. Parameter values used in this study; $\mathrm{p}=22.03, \mathrm{~s}=0.0096, \mathrm{k}=3.60$; were previously derived for tuatara on Takapourewa (Mitchell et al. 2006) and provided conservative estimates of male hatchlings in the population of tuatara on North Brother Is. (Mitchell et al. 2008).

\subsubsection{Sensitivity analysis}

Sensitivity of total embryonic development, CTE values, hatching dates, and timing of the TSP to microclimate model error was assessed by increasing hourly soil temperatures by the root-mean-squared error of the NicheMapR implementation (i.e., 'weather_1') used in this study (Chapter 3). Since the microclimate model was only tested against temperature data at two depths, $100 \mathrm{~mm}$ and $200 \mathrm{~mm}$, the distribution of model error across 50-300mm was estimated based on a linear reduction in error with depth. Hourly soil temperatures were generated at 500 random points, sampled from the whole-island dataset. Modelled soil temperatures were increased by $2^{\circ} \mathrm{C}$ at $200 \mathrm{~mm}$ (Chapter 3 ) and $+/-0.5^{\circ} \mathrm{C}$ for, respectively, every $50 \mathrm{~mm}$ reduction or increase in soil depth. Hourly soil temperatures generated using NicheMapR and to which no correction for model error was added are hereafter referred to as 'modelled' values, while those increased to account for model error are referred to as 'error-corrected' values. Incubation temperatures, sex ratios, total embryonic development, and hatching dates 
were predicted for the set of 500 test points using both modelled and error-corrected temperatures for the median oviposition date of 30 November.

\subsubsection{Simulating climate change}

Scenarios of climate change were chosen based on the most recently available minimum and maximum seasonal warming projections for the Marlborough region of New Zealand from the reference period 1980-1999 to projections for 2080-2099 (MFE 2008), generated by down-scaling global predictions (IPCC 2007) for the same period (Mpelasoka et al. 2001). The most recent NZ climate change models use the period of 1989 - 1999 to define the 'normal' climate for projections; however, air temperature data recorded on Takapourewa in 2000 [NIWA Station No. 4153] were used to model the 'current climate' scenario in this study for two reasons. First, air temperatures in NZ were the second-highest on record in 1999; air temperatures recorded in 2000 were less anomalous, compared to the 'normal' period (http://www.niwa.co.nz). Second, data on vegetation cover used to define shade for the current climate scenario were generated using aerial photography collected in the year 2000 (see below and Chapter 2). To simulate the minimum projected warming scenario, minimum and maximum air temperatures were increased seasonally by the predicted range of $0.3-0.9^{\circ} \mathrm{C}$, with an increase of $0.3^{\circ} \mathrm{C}$ in the meteorological austral spring, a $0.6^{\circ} \mathrm{C}$ increase in autumn and winter, and an increase of $0.9^{\circ} \mathrm{C}$ in summer. To model maximum warming, minimum and maximum temperatures were increased by the predicted range of $4.8-5.6^{\circ} \mathrm{C}$, with an increase of $4.8^{\circ} \mathrm{C}$ in the austral spring, a $5.0^{\circ} \mathrm{C}$ increase in autumn and winter, and a $5.6^{\circ} \mathrm{C}$ increase in summer (MFE 2008).

Ongoing revegetation of Takapourewa will increase the proportion of forested habitat that, under current climate conditions, does not facilitate completion of embryonic development (Thompson et al. 1996). Mean microsite-scale shading is 
around 95\% under the forest canopy (Chapter 2), and soil temperatures in non-forested locations were most accurately modelled under the assumption of $0 \%$ shading, regardless of the predominant vegetation type (Chapter 3). To simulate the effects of ongoing ecological restoration on soil temperatures under climate change scenarios, points were overlaid onto a categorical land cover map generated for the year 2000 using aerial photography and a map of the distribution of forest predicted for the year 2100 (Fig. 2) using land cover change analysis (Chapter 2). The available shading was increased from $0 \%$ to $95 \%$ in locations classified as forest, regardless of year. 


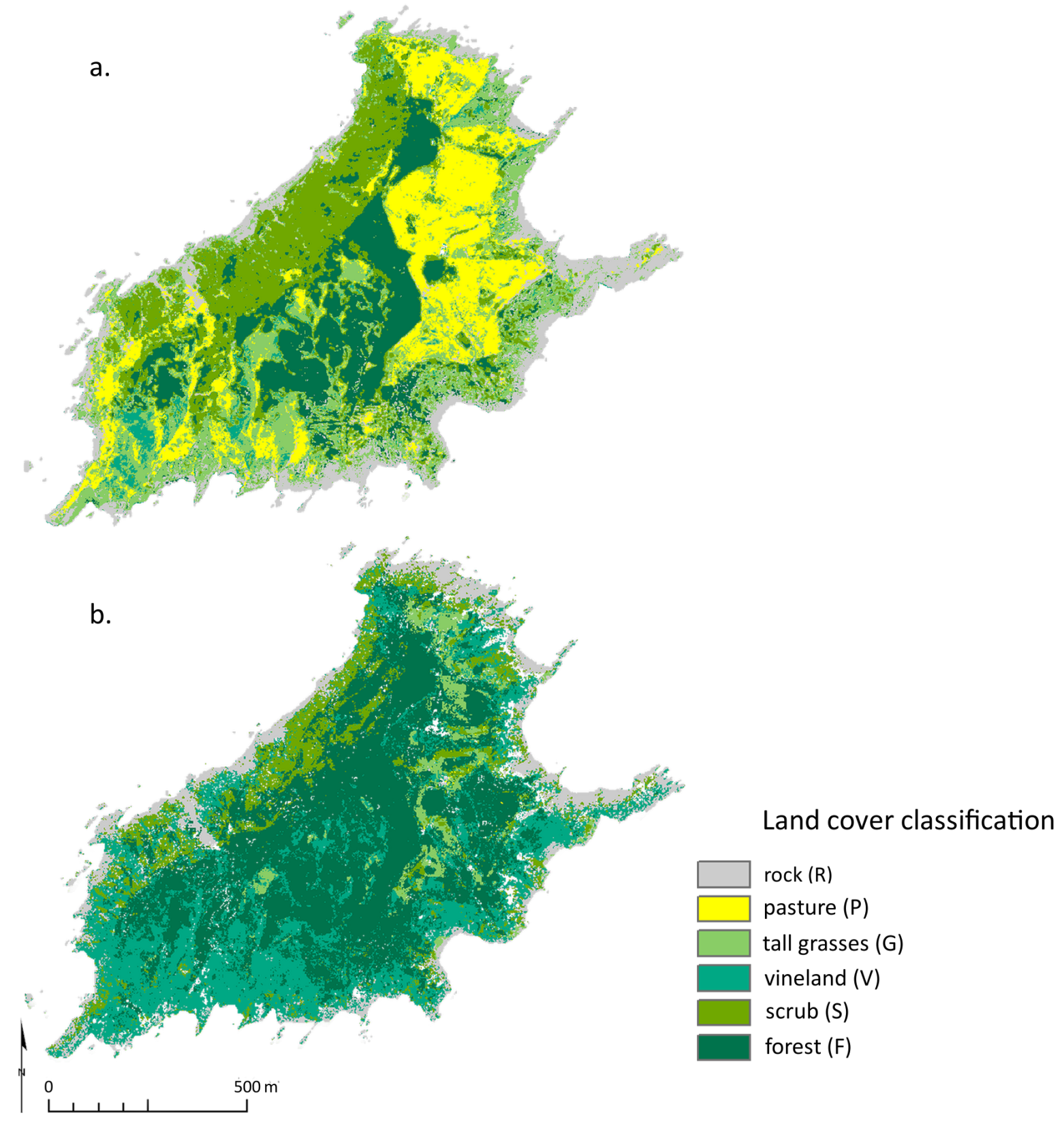

Figure 2. Land cover classification maps (a) developed for the year 2000 (i.e., the current climate) using aerial photography and (b) predicted for the year 2100 (Chapter 2) and used to simulate shade for both modelled scenarios of climate change. Available shade was increased to 95\% in areas classified as forest under all three climate scenarios. Soil temperatures were simulated using $0 \%$ shade for all other land cover types. 
Running the NicheMapR microclimate model for the high resolution, wholeisland dataset used for Takapourewa was computationally intensive, so geostatistical models were used to simulate climate change. Mechanistic developmental predictions for climate change scenarios were generated for a subset of 500 points (Voltz \& Webster 2001), sampled randomly at a minimum separating distance of $2 \mathrm{~m}$ from the wholeisland dataset. Predictions under the current climate and minimum warming scenarios were nearly identical (see section 4.3.1), so the spatial extent of models used to simulate the minimum climate warming scenario was constrained to sites for which a CTE value was calculated (i.e., points at which development reached at least 35\%) at each soil depth under the current climate scenario. Thus, sites that were unlikely to facilitate development under the scenario of minimum warming would not be erroneously modelled. Reducing the spatial extent decreased the number of mechanistically modelled points from 500 to 472 for the minimum warming scenario. The scenario of maximum climate warming was simulated within the full spatial extent of the island using all 500 points. Because hourly soil temperatures generated conservative estimates of male hatchlings, even when corrected for microclimate model error, development models were run for both climate change scenarios using error-corrected hourly soil temperatures (see Section 4.3.1). Mechanistic predictions of CTEs were then interpolated across the whole island using geostatistical models.

Mechanistically modelled CTE values displayed a slight degree of spatial autocorrelation. Thus, predicted CTEs under scenarios of climate change were modelled for Takapourewa using ordinary kriging, a robust method of geostatistical analysis that fits a semivariogram between pairs of points within a defined radius to quantify autocorrelation as a function of distance (Cressie 1985, 1988, 1990; Wackemagel 1998). Like radial basis functions (e.g., thin-plate smoothing splines) that have been frequently used to interpolate climate data across geospatial surfaces (Hutchinson 1998a, b; Boer et al. 2001; Jarvis \& Stuart 2001a, b; Apaydin et al. 2004; Hofierka et al. 2002; Hijmans et 
al. 2005; Hancock \& Hutchinson 2006; Hutchinson et al. 2009), kriging can be considered an exact interpolator under the assumption of no measurement error (i.e., the prediction surface passes exactly through the values of the training data, and the resultant prediction standard error at those points is zero) (ESRI 2003).

Kriging models for Takapourewa were optimised to the training points using leave-one-out cross validation and validated independently using developmental predictions modelled with NicheMapR for a second set of 500 randomly sampled points. Accuracy of the interpolated surfaces was assessed using the root-mean-squared deviation (RMSD) between mechanistically modelled and statistically interpolated predictions, calculated using the 'rmse' function in R package hydroGOF (ZambranoBigiarini 2014). Final interpolated CTE values were converted to hatchling sex ratios (Girondot 1999; Godfrey et al. 2003). To create spatially explicit surfaces of interpolated developmental predictions, sex ratios were binned into ten categories by proportion of male hatchlings (e.g., sex ratios from 0.0-0.1 were classified as 0.1 ; sex ratios $>0.9-1.0$ were classified as 1.0; etc.).

All three climate scenarios were also simulated using error-corrected soil temperature values for known nesting locations of tuatara. A subset of 200 nest sites was randomly selected from a dataset of 814 nesting locations for which GPS points had been collected in either 2002-2005 or 2011-2012 (Chapter 2). Incubation temperatures, hatchling sex ratios, and hatch dates were predicted for soil depths from $50-300 \mathrm{~mm}$ using the median oviposition date of 30 Nov and tested against results of identical simulations using a set of 200 points selected randomly from the whole-island dataset. The distribution of CTEs at nest sites versus random locations were compared using two-sample Kolmogorov-Smirnov tests, bootstrapped using 1000 permutations with the 'ks.boot' function in R package Matching (Sekhon 2011, 2013). 


\subsubsection{Simulating shifts in nesting phenology}

To examine whether shifts in oviposition date influence incubation temperatures, sex ratios or hatching phenology under scenarios of climate change, developmental models were run for the whole-island dataset for oviposition dates +/- 30 days from the median date of 30 November in 15-day increments for all three climate scenarios. Since onset of the nesting season tends to occur earlier in warmer years on Takapourewa (Nelson unpub. data), additional developmental models simulating oviposition dates of 1 and 15 October were run for the scenario of maximum climate warming. All models simulating shifts in oviposition phenology were run using error-corrected hourly soil temperatures (see Section 4.3.1) for a subset of 200 sites, sampled randomly from the whole-island dataset. Generalised linear models were used to examine the interactive effects of oviposition date and depth on calculated CTEs and predicted hatchling sex ratios. Periodic data on hatching days were converted to an angular scale by:

$$
x=2 \pi y k^{-1}
$$

where ' $\mathrm{y}$ ' is the predicted Julian day of hatching, ' $\mathrm{k}$ ' is the total number of possible hatching days $=365$, and ' $\mathrm{x}$ ' is the resultant angular equivalent of the Julian day in radians (Gumiaux et al. 2003; Berens \& Velasco 2009; Gill \& Hangartner 2010). Predicted angular hatching dates were transformed into planar coordinates defined by $\cos (\mathrm{x}) \sin (\mathrm{x})$ for analysis. Data on hatching dates were multimodal under most simulations, so Rao's test (Rao 1969, 1972) was used to determine whether apparent mean hatching dates were significantly different from what would be observed given a uniform circular distribution (Jammalamadaka \& Sengupta 2001; Berens \& Velasco 2009) at each depth and oviposition date under all three modelled climate change scenarios. Total embryonic development, CTEs, and offspring sex ratios were also modelled island-wide for Takapourewa under the current climate scenario for five simulated oviposition dates using mechanistically modelled, non error-corrected soil 
temperatures. The empirical model of temperature-dependent embryonic development used in this study does not include mortality as a function of incubation time. Thus, the probability of hatching was assumed to be 1.00 for all dates within two years from each simulated oviposition date. Since differential mortality has not been quantified between hatching in, for example, December after 12 months versus after 24 months in-ovo, total development times were not included in analyses (see Section 4.4.2).

All microclimate and developmental modelling was conducted in $\mathrm{R} v$ 3.0.3 software (R Development Core Team 2008) (Fig. 3). Whole-island models were run using the Victorian Life Sciences Computation Initiative (VLSCI) Peak Computing Facility at the University of Melbourne, Australia. Geostatistical modelling was conducted using the Geospatial Analyst extension (ESRI 2003) in $\operatorname{ArcMap}^{T M}$ Desktop v 10.1 software (Environmental Systems Research Institute [Esri] ArcGIS: Redlands, CA, USA 2012). Data on hatching phenology were summarised using the 'mean.circular' and 'rao.spacing.test' functions in R package circular (Lund \& Agostinelli 2013) and plotted as polar coordinates using R package ggplot2 (Wickham \& Chang 2014). Figures and maps were generated in, respectively, $\mathrm{R}$ and $\operatorname{ArcMap}^{\mathrm{T}}$ and edited using GNU image manipulation program (GIMP Development Team 2013). 


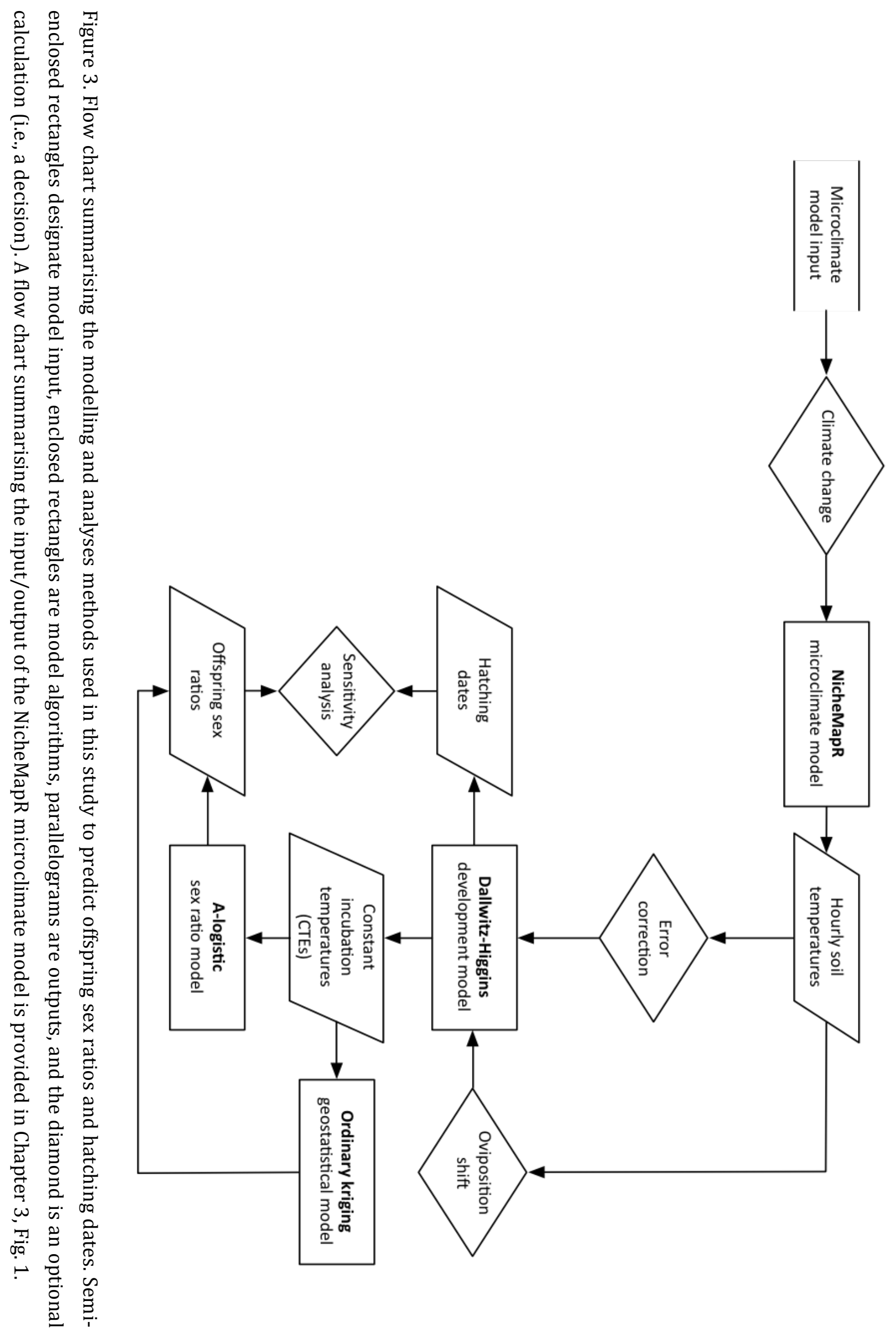




\subsection{Results}

\subsubsection{Sensitivity analysis}

Error-corrected values for constant incubation temperature equivalents (CTEs) differed from modelled values by a mean of $0.55^{\circ} \mathrm{C}$ at a depth of $50 \mathrm{~mm}$ to a mean of $0.29^{\circ} \mathrm{C}$ at a soil depth of $200 \mathrm{~mm}$ under the current climate scenario. Under the scenario of minimum climate warming, error-corrected CTEs differed from modelled temperatures by between a mean $0.03^{\circ} \mathrm{C}$ to a mean $-0.18^{\circ} \mathrm{C}$ from, respectively, $50 \mathrm{~mm}$ to $200 \mathrm{~mm}$. Under both the current climate and minimum warming scenarios, errorcorrected CTE values at depths of $150 \mathrm{~mm}$ and $200 \mathrm{~mm}$ were slightly lower than modelled values, while corrected values were higher than modelled values at a depth of $300 \mathrm{~mm}$. The variance of error-corrected CTE values was greater under both the current climate and minimum warming scenarios, with minimum CTEs lower and maximum CTEs higher at all depths. Under either climate scenario, predicted CTE values decreased little below a soil depth of $100 \mathrm{~mm}$. Under the scenario of maximum predicted regional climate warming, CTE values that were corrected for error differed from modelled values by between $3.94^{\circ} \mathrm{C}$ at $50 \mathrm{~mm}$ to $1.72^{\circ} \mathrm{C}$ at a depth of $300 \mathrm{~mm}$. The difference between mean error-corrected and mean modelled CTEs decreased with depth under the scenario of maximum climate warming. Minimum error-corrected CTE values were a mean $6.6^{\circ} \mathrm{C}$ higher than modelled values, reducing variance in the error-corrected values compared to modelled values under the maximum warming scenario (Fig. 4).

Mean offspring sex ratios predicted using error-corrected versus modelled CTEs were similar under the current climate and minimum warming scenario. Under both scenarios, minimum and maximum predicted proportions of males offspring were 0.00 for all soil depths from $100 \mathrm{~mm}$ to $300 \mathrm{~mm}$. The maximum predicted sex ratio at a depth of $50 \mathrm{~mm}$ was estimated as 0.00 using modelled incubation temperature values and as 
0.28 using error-corrected CTEs under the scenario of minimum climate warming. Under the maximum climate warming scenario, minimum and maximum predicted sex ratios varied from 0.00 to 1.00 at all soil depths, using both modelled and errorcorrected CTEs. Mean offspring sex ratios predicted using error-corrected CTEs were higher than those predicted using modelled values by between 0.63 and 0.02 from, respectively, soil depths of $50 \mathrm{~mm}$ to $300 \mathrm{~mm}$ (Fig. 4).

Under both the current climate and minimum warming scenarios, embryonic development was not predicted to reach $100 \%$ at depths below $50 \mathrm{~mm}$, and only $2 \%$ of sites were predicted to facilitate hatching at $50 \mathrm{~mm}$. Using error-corrected hourly soil temperatures increased the percentage of sites that were predicted to facilitate hatching to between $49 \%$ and $2 \%$ at, respectively, $50 \mathrm{~mm}$ and $300 \mathrm{~m}$ under the current climate scenario. The number of sites for which hatching was predicted using error-corrected hourly soil temperatures was slightly lower under the scenario of minimum climate warming, compared with values modelled under the current climate, from $38 \%$ of sites at $50 \mathrm{~mm}$ to approximately $2 \%$ of sites at $300 \mathrm{~mm}$. Hatching was not predicted for any of the 500 test sites at a soil depth of $300 \mathrm{~mm}$ under the minimum warming scenario, regardless of whether modelled or error-corrected soil temperatures were used. Under the scenario of maximum predicted climate warming, from between $82 \%$ of sites at $50 \mathrm{~mm}$ to $73 \%$ of sites at $300 \mathrm{~mm}$ were predicted to reach $100 \%$ development using modelled hourly soil temperatures. Using error-corrected values, all 500 sites were predicted to facilitate hatching at depths from $50 \mathrm{~mm}$ to $200 \mathrm{~mm}$, and $94 \%$ of sites were predicted to hatch at a depth of $300 \mathrm{~mm}$ (Fig. 4).

The single site predicted to reach $100 \%$ of development using modelled temperature values at $50 \mathrm{~mm}$ under the current climate and minimum warming scenarios was predicted to hatch in early November, austral spring. Using errorcorrected values, the greatest percentage of nests was predicted to hatch in November under both the current climate and minimum warming scenarios. Mean hatch dates 
were significantly different from what would be expected under a uniform distribution at depths of $50 \mathrm{~mm}\left(\mathrm{X}^{2}=181.04, \mathrm{df}=1, \mathrm{p}<0.001\right), 100 \mathrm{~mm}\left(\mathrm{X}^{2}=160.14, \mathrm{df}=1, \mathrm{p}<\right.$ 0.001), and $150 \mathrm{~mm}\left(\mathrm{X}^{2}=159.76, \mathrm{df}=1, \mathrm{p}<0.05\right)$ under the current climate scenario, when error-corrected values were used. Under the scenario of maximum climate warming, the greatest percentage of sites was predicted to hatch in austral summer, regardless of whether modelled or error-corrected values were used. However, mean predicted hatching dates were significantly different from a uniform distribution only at depths of $50 \mathrm{~mm}\left(\mathrm{X}^{2}=173.04, \mathrm{df}=1, \mathrm{p}<0.001\right)$ and $100 \mathrm{~mm}\left(\mathrm{X}^{2}=160.75\right.$, df $=1, \mathrm{p}<$ 0.001) when modelled hourly soil temperatures were used. Mean hatch dates calculated with error-corrected soil temperatures were significantly different from uniform at all soil depths, varying from late January at $50 \mathrm{~mm}\left(\mathrm{X}^{2}=182.20, \mathrm{df}=1, \mathrm{p}<0.001\right)$ and early February at depths of $100 \mathrm{~mm}\left(\mathrm{X}^{2}=172.54, \mathrm{df}=1, \mathrm{p}<0.001\right), 150 \mathrm{~mm}\left(\mathrm{X}^{2}=161.28, \mathrm{df}=\right.$ $1, \mathrm{p}<0.001), 200 \mathrm{~mm}\left(\mathrm{X}^{2}=160.54, \mathrm{df}=1, \mathrm{p}<0.001\right)$, and $300 \mathrm{~mm}\left(\mathrm{X}^{2}=158.79, \mathrm{df}=1, \mathrm{p}\right.$ $<0.001$ ) (Fig. 5).

The proportion of male hatchlings predicted on Takapourewa from 2002-2005 varied from 0.22-0.66 using soil temperature data recorded in-situ, under the assumption of $100 \%$ hatching success (Nelson unpub. data). Observations of males in the same years varied from $0.30-0.64$ of offspring that successfully hatched (Nelson unpub. data). The proportions of male hatchlings predicted using both modelled and error-corrected hourly soil temperatures in this study were lower than those observed or predicted in previous studies. Since the mechanistic model developed for this study was a conservative predictor of male hatchlings, subsequent analyses were conducted for all depths and under all three simulated climate scenarios using only error-corrected soil temperature values. 


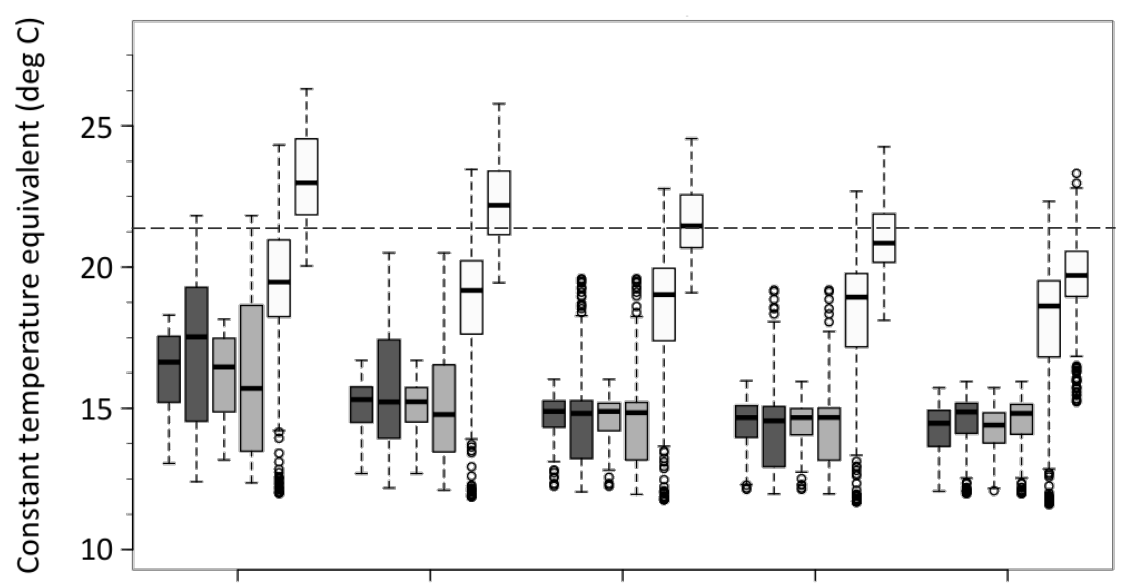

Climate scenario

current climate

minimum warming

$\checkmark$ maximum warming
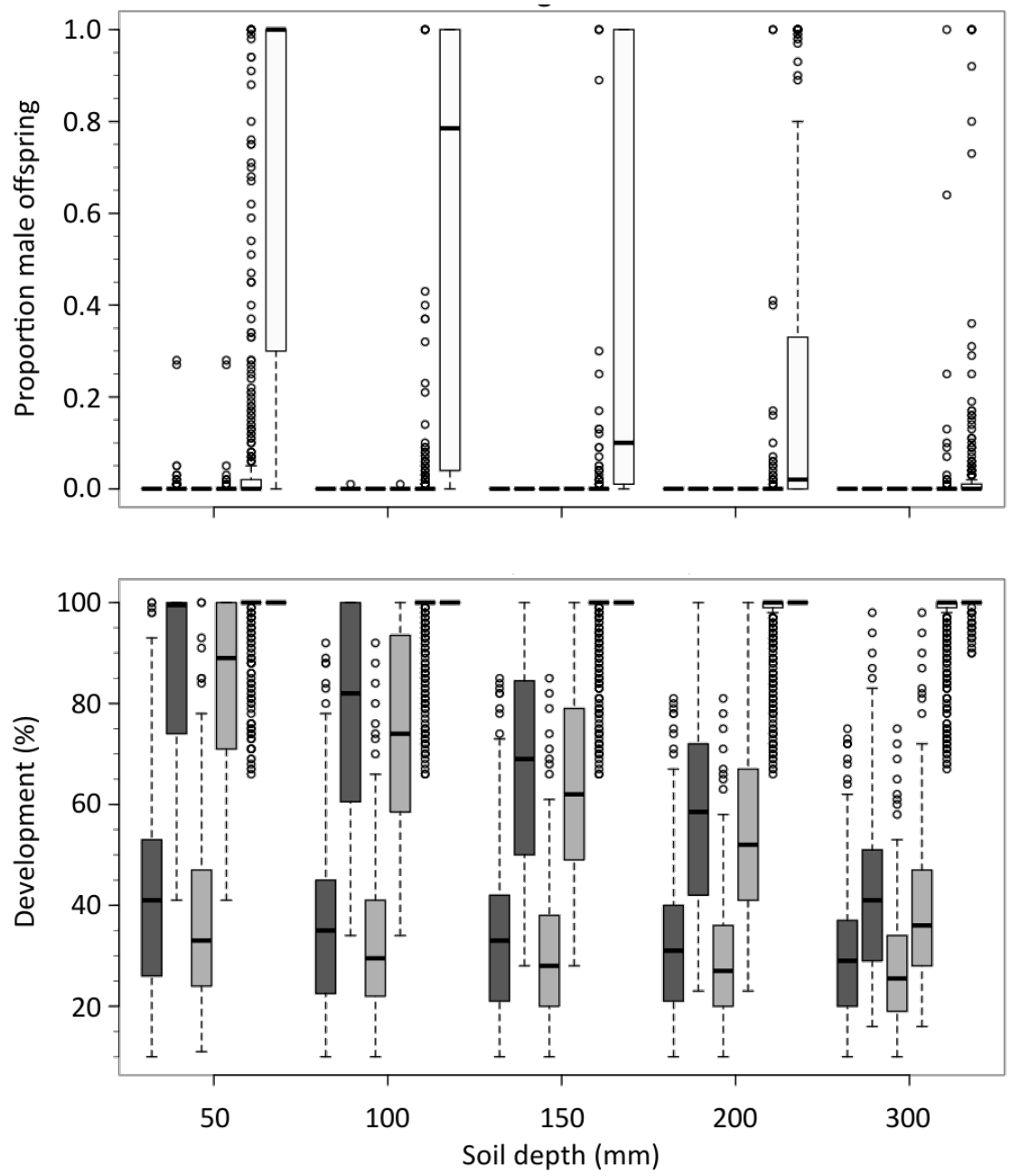

Figure 4. Boxplots summarising the sensitivity of CTEs, hatchling sex ratios (i.e., proportion of male offspring), and total embryonic development to model error for tuatara on Takapourewa, paired by climate scenario, for all modelled soil depths. In each shaded pair, the left-hand box shows the distribution of values generated using modelled hourly soil temperatures, and the right-hand box shows values predicted using error-corrected hourly soil temperatures. The dashed line on the plot of CTEs approximates the pivotal temperature. 

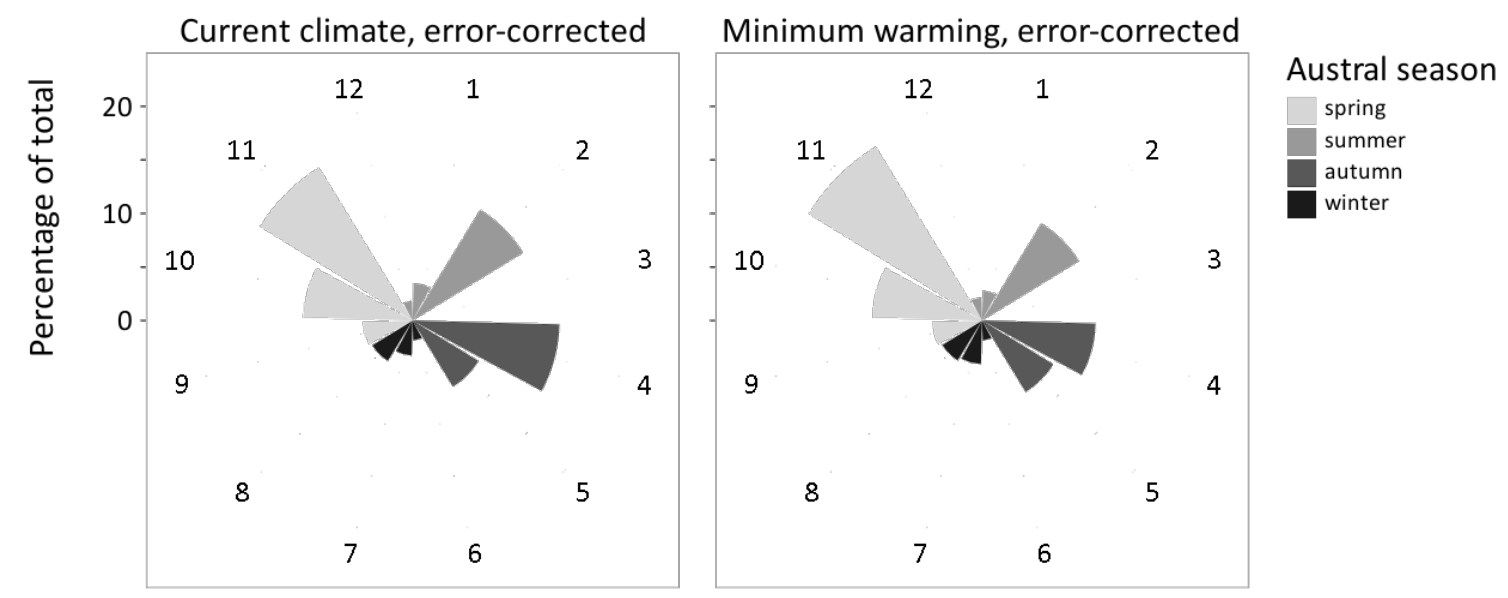

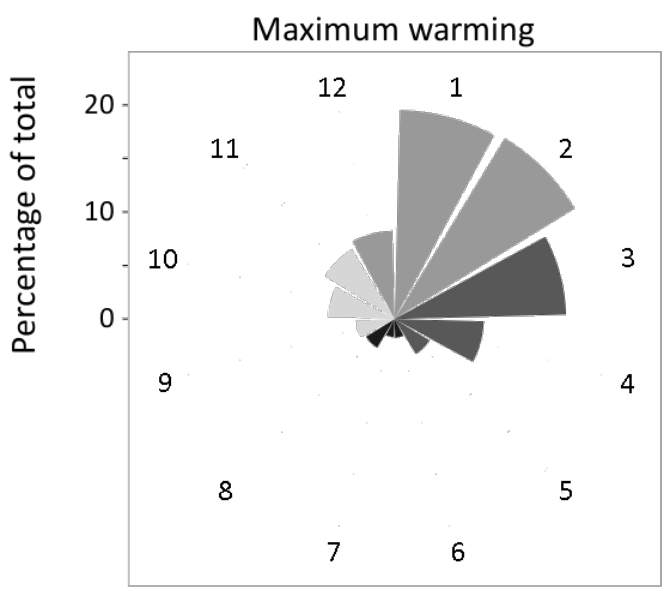

Month of hatching
Maximum warming, error-corrected

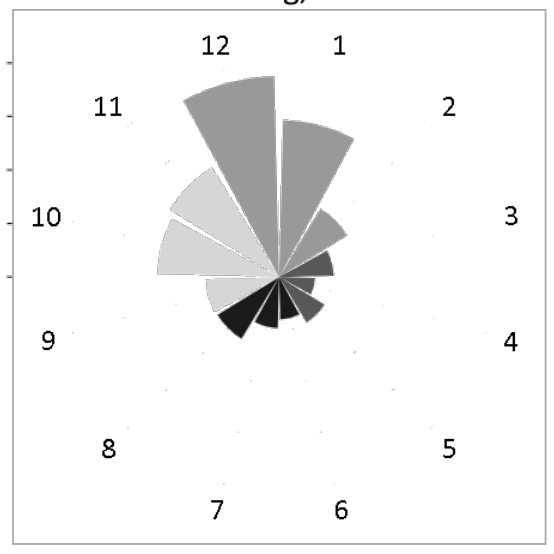

Month of hatching

Figure 5. Polar plots summarising the effects of microclimate model error on the monthly and seasonal distributions of hatch dates predicted for tuatara on Takapourewa, pooled across depths. Hatching was only predicted at one sampled site under both the current climate and minimum warming scenarios using modelled (i.e., non error-corrected) values, so plots are shown for error-corrected results only. Data are displayed as the percentage of sites predicted to hatch in each month, numbered from 1 (January) to 12 (December) out of the total predicted to hatch within 24 months from the median oviposition date of 30 November under each climate scenario.

\subsubsection{Incubation temperatures under the current climate}

When developmental models were run using island-wide, non error-corrected hourly soil temperatures for an oviposition date of 30 Nov, embryonic development was not predicted to reach completion for soil depths below $100 \mathrm{~mm}$ at any site, and less than $1 \%$ of sites were predicted to reach hatching at either $50 \mathrm{~mm}$ or $100 \mathrm{~mm}$. Less than $1 \%$ of sites were predicted to reach between $90-99 \%$ of embryonic development at 
either $50 \mathrm{~mm}$ or $100 \mathrm{~mm}$. The maximum percentage of development predicted at soil depths of $150 \mathrm{~mm}, 200 \mathrm{~mm}$, and $300 \mathrm{~mm}$ was, respectively, $89 \%, 85 \%$, and $78 \%$. CTE values were only calculated for sites at which embryonic development was predicted to reach at least 35\%, the upper limit of the thermal sensitive period (TSP) estimated for tuatara. At soil depths of $50 \mathrm{~mm}, 58 \%$ of sites were predicted to reach at least $35 \%$ embryonic development. The CTE values calculated at those sites varied from $12.98^{\circ} \mathrm{C}$ to $18.49^{\circ} \mathrm{C}$. CTE values calculated for sites at $100 \mathrm{~mm}$ varied from $12.46^{\circ} \mathrm{C}$ to $16.79^{\circ} \mathrm{C}$ and were predicted for $48 \%$ of sites. At a depth of $150 \mathrm{~mm}$, CTE values were calculated for $42 \%$ of sites and varied from $12.27^{\circ} \mathrm{C}$ to $16.48^{\circ} \mathrm{C}$. CTE values were calculated for $37 \%$ of sites at a soil depth of $200 \mathrm{~mm}$ and varied from $12.09^{\circ} \mathrm{C}$ to $16.12^{\circ} \mathrm{C}$. At a soil depth of $300 \mathrm{~mm}$, CTEs were calculated for $30 \%$ of sites and varied from $11.96^{\circ} \mathrm{C}$ to $15.93^{\circ} \mathrm{C}$.

Similar patterns in CTE values were observed at all sites and for all modelled soil depths, regardless of the oviposition date. However, development reached at least 35\%, and CTEs were predicted island-wide, when the oviposition date was either 15 Nov or 30 Dec. If development were to reach completion at any site for which a CTE was successfully calculated using non error-corrected hourly soil temperatures under the current climate scenario, only female hatchlings would be produced at any depth from $50 \mathrm{~mm}$ to $300 \mathrm{~mm}$, regardless of the oviposition date. Few CTE values that were predicted using mechanistically modelled hourly soil temperatures under the current climate scenario were $>16^{\circ} \mathrm{C}$ below a depth of $50 \mathrm{~mm}$, so spatial distributions of CTEs are presented graphically for five simulated oviposition dates at soil depths of $50 \mathrm{~mm}$ and $100 \mathrm{~mm}$ only (Fig. 6). 


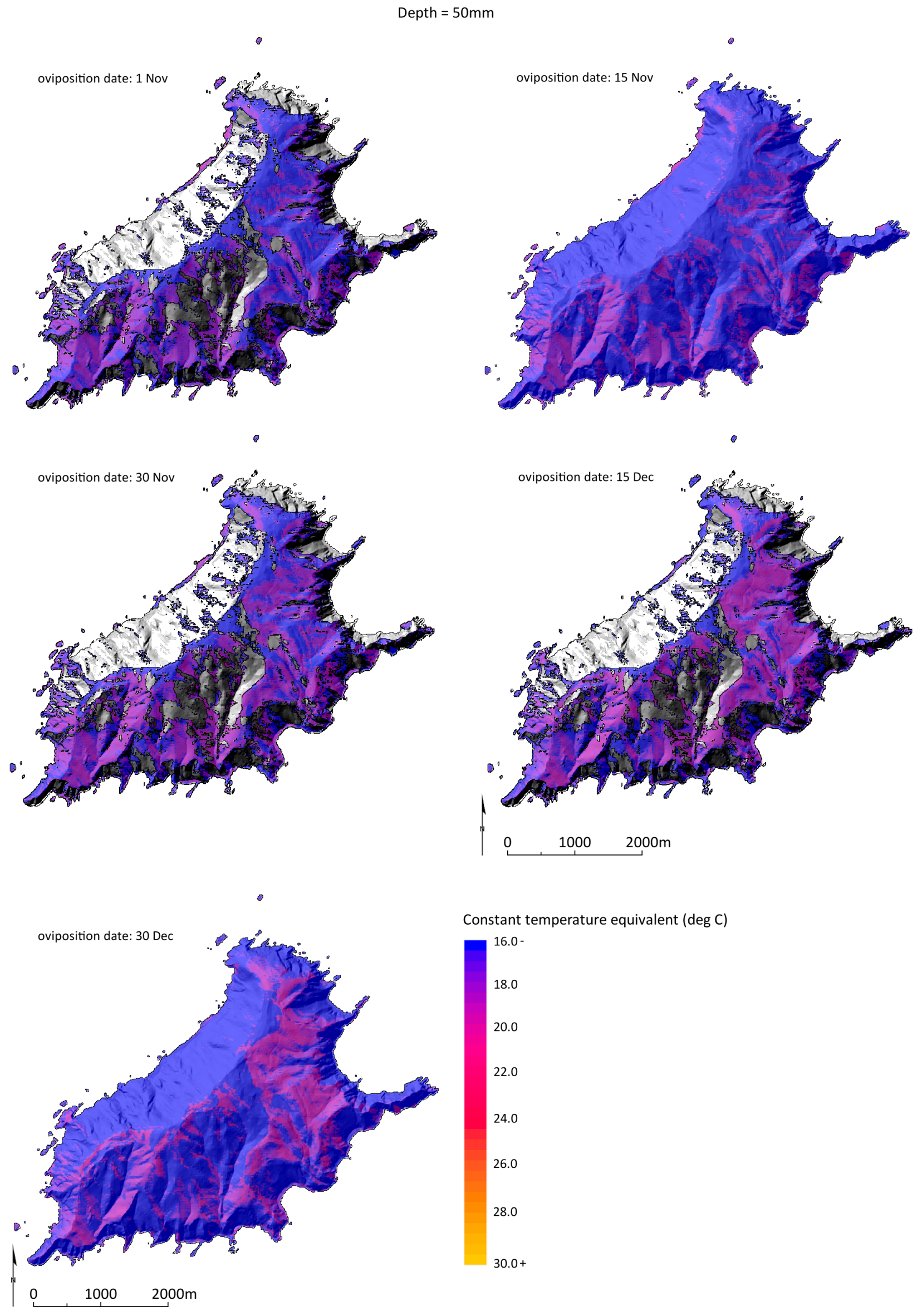



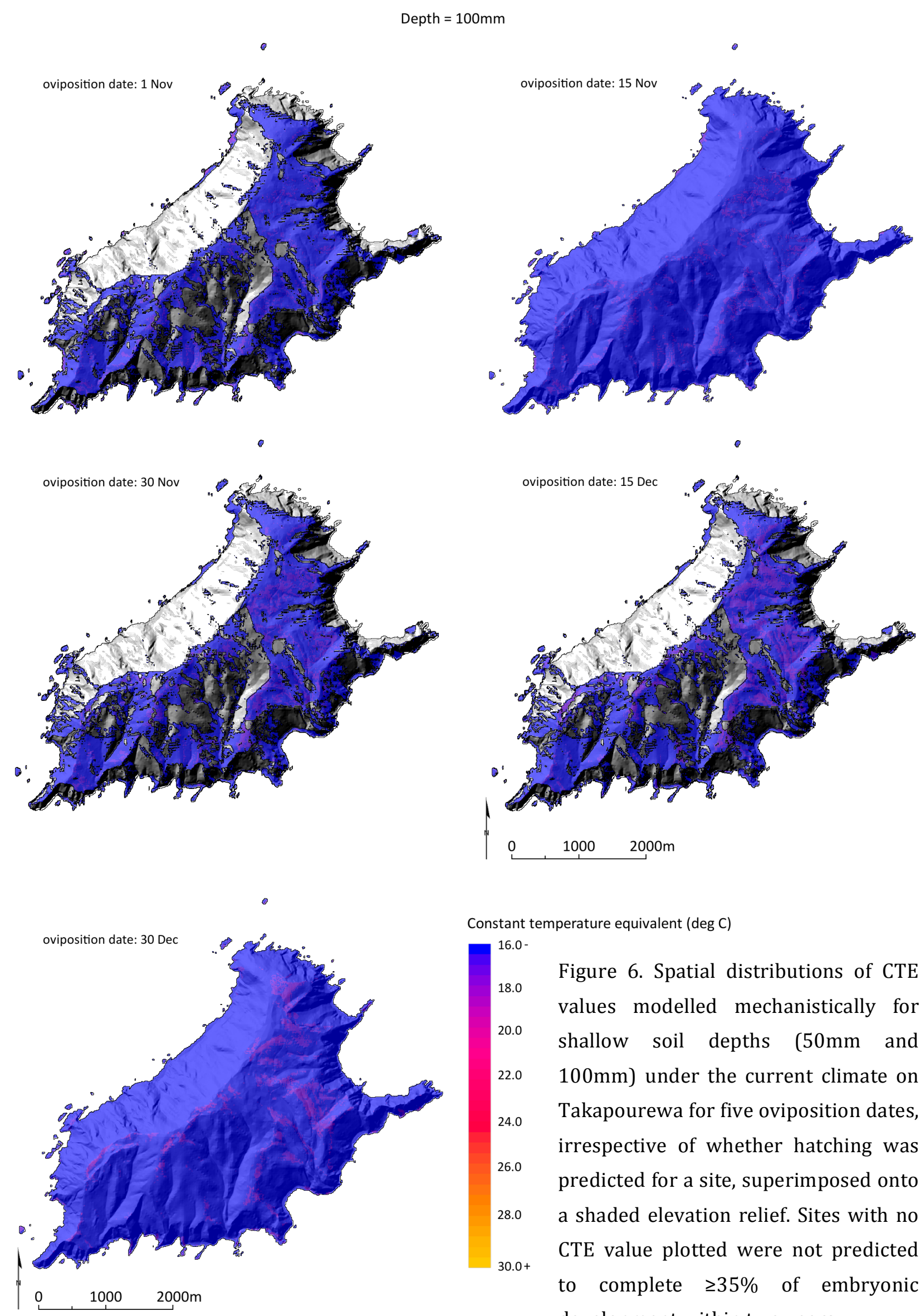

Constant temperature equivalent $(\operatorname{deg} \mathrm{C})$

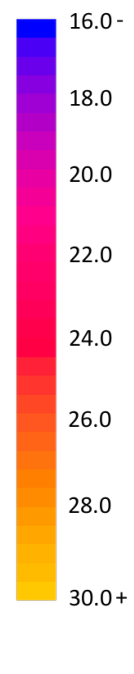

Figure 6. Spatial distributions of CTE values modelled mechanistically for shallow soil depths $150 \mathrm{~mm}$ and $100 \mathrm{~mm}$ ) under the current climate on Takapourewa for five oviposition dates, irrespective of whether hatching was predicted for a site, superimposed onto a shaded elevation relief. Sites with no CTE value plotted were not predicted to complete $\geq 35 \%$ of embryonic development within two years. 


\subsubsection{Effects of climate change on incubation temperatures and offspring sex ratios}

CTEs were predicted for both the minimum and maximum climate warming scenarios using ordinary kriging models, optimised using leave-one-out cross validation to a training set of CTEs modelled using NicheMapR for 500 random points on Takapourewa. Both the mean prediction errors and mean standardised prediction errors were near zero for all modelled soil depths under both the minimum and maximum climate warming scenarios, suggesting that predictions generated using the training data were unbiased. Root-mean-squared standardised errors of the predictions were close to 1.00 at all depths, suggesting that the kriging models accurately predicted variability in CTE values.

Model predictions were also independently tested against a second set of mechanistically modelled values. Calculated root-mean-squared deviation (RMSD) values were higher for the independent validation of interpolated CTEs under the minimum warming scenario than under the scenario of maximum climate warming. The RMSD in model predictions decreased with increasing soil depth and was lower for the maximum warming scenario at all soil depths except $300 \mathrm{~mm}$, compared with the scenario of minimum warming. The RMSD in predicted proportions of male offspring was high at soil depths from 50-200mm under the maximum climate warming scenario, although mean predicted CTEs and sex ratios were similar between modelling methods. This finding may reflect an inability to accurately model offspring sex ratios when the CTE is within the narrow transitional range of incubation temperatures, especially if model error shifts the CTE from one (Table 1). 


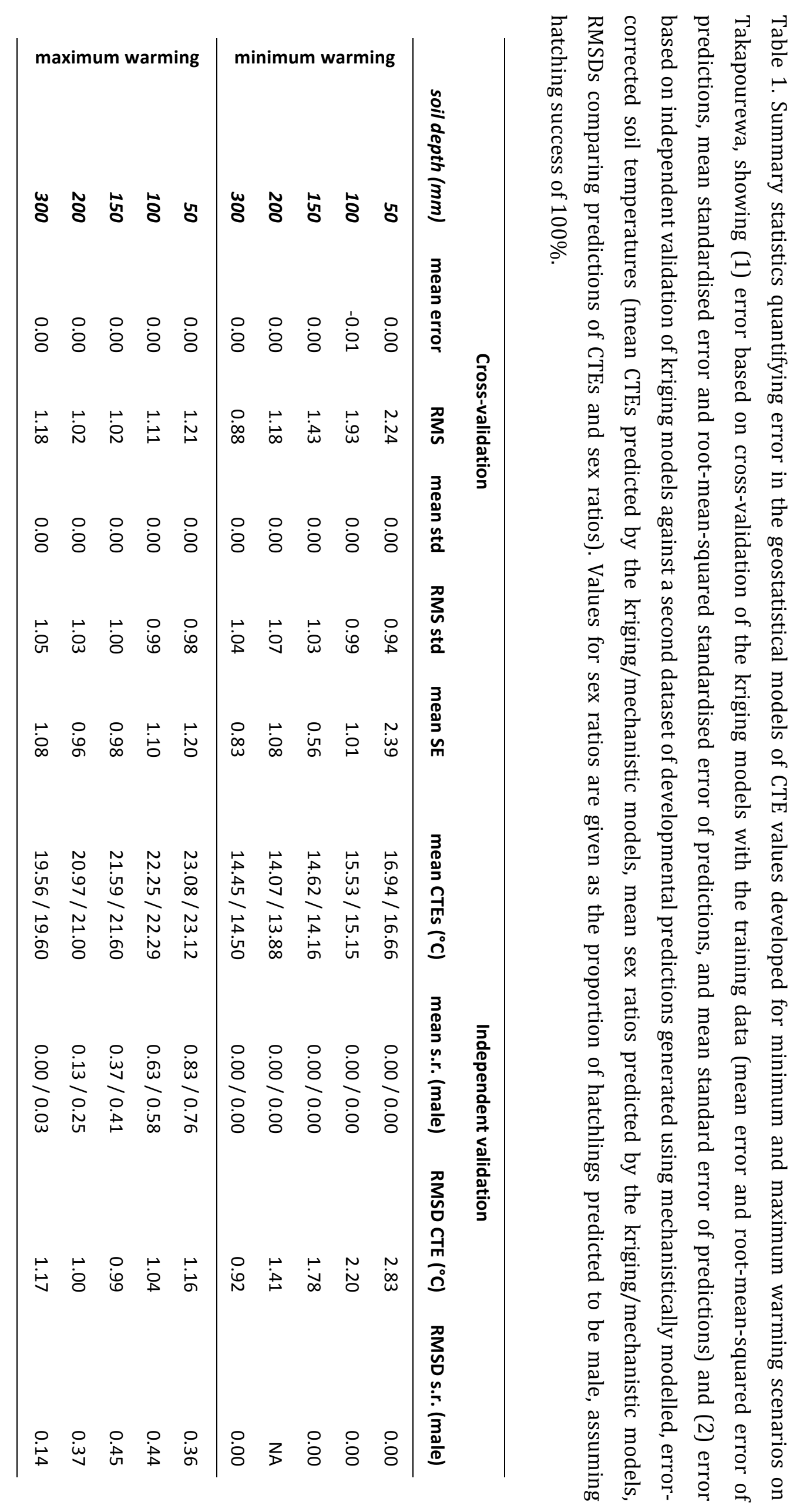


A two-sample Kolmogorov-Smirnov test found that the distributions of modelled CTEs for all depths and under both simulated climate warming scenarios were significantly different between modelling methods $(0.14 \leq \mathrm{D} \leq 0.37, \mathrm{p}<0.001)$. Under the scenario of minimum climate warming, most sites were predicted to produce allfemale nests at all depths; hence, the distributions of modelled sex ratios were not different between modelling methods $(\mathrm{D} \leq 0.03, \mathrm{p}=1.00)$. Distributions of predicted sex ratios were different between modelling methods under the maximum climate warming scenario at all depths $(0.12 \leq \mathrm{D} \leq 0.15,0.01 \leq \mathrm{p} \leq 0.001)$. Under the minimum climate warming scenario, mean CTEs predicted using ordinary kriging were lower than those generated using mechanistically modelled hourly soil temperatures at depths of $100 \mathrm{~mm}$, $150 \mathrm{~mm}$, and $200 \mathrm{~mm}$ and higher than mechanistically predicted values at soil depths of $50 \mathrm{~mm}$ and $300 \mathrm{~mm}$. Mean CTEs and sex ratios modelled using geostatistical methods were, on the whole, similar to or slightly higher than mechanistically predicted values under the scenario of maximum climate warming.

A Mann-Whitney-Wilcoxon test found that the CTEs predicted for the minimum climate warming scenario were not significantly different between modelling methods at soil depths of $50 \mathrm{~mm}(\mathrm{~W}=33029, \mathrm{p}=0.51)$ or $200 \mathrm{~mm}(\mathrm{~W}=19983, \mathrm{p}=0.36)$. However, CTEs were significantly different between methods at depths of $100 \mathrm{~mm}$ (W = 29013, $\mathrm{p}<0.05), 150 \mathrm{~mm}(\mathrm{~W}=26622, \mathrm{p}<0.001)$, and 300mm $(\mathrm{W}=7220, \mathrm{p}<0.001)$ under the scenario of minimum climate warming. Under the maximum warming scenario, predicted CTEs were not different between modelling methods at depths from $50 \mathrm{~mm}$ to $200 \mathrm{~mm}(120662 \leq \mathrm{W} \leq 124919,0.34 \leq \mathrm{p} \leq 0.99)$ but differed at a depth of 300mm ( $\mathrm{W}=115037, \mathrm{p}<0.05)$. Because most predictions of hatchling sex ratios under the minimum climate warming scenario were 0.00 , statistical significance could not be calculated for results at depths lower than $50 \mathrm{~mm}$. At a soil depth of $50 \mathrm{~mm}$, however, predicted offspring sex ratios differed significantly between modelling methods $(\mathrm{W}=$ $30915, \mathrm{p}<0.01)$. Under the scenario of maximum climate warming, predicted hatchling 
sex ratios were different at soil depths of 50mm $(\mathrm{W}=136815, \mathrm{p}<0.01), 100 \mathrm{~mm}(\mathrm{~W}=$ 133672, $\mathrm{p}<0.05)$, and 300mm (W = 104163, $\mathrm{p}<0.001)$. However, predicted offspring sex ratios did not different significantly between modelling methods at depths of $150 \mathrm{~mm}(\mathrm{~W}=127672, \mathrm{p}=0.55)$ or $200 \mathrm{~mm}(\mathrm{~W}=121237, \mathrm{p}=0.40)($ Fig. 7$)$. 

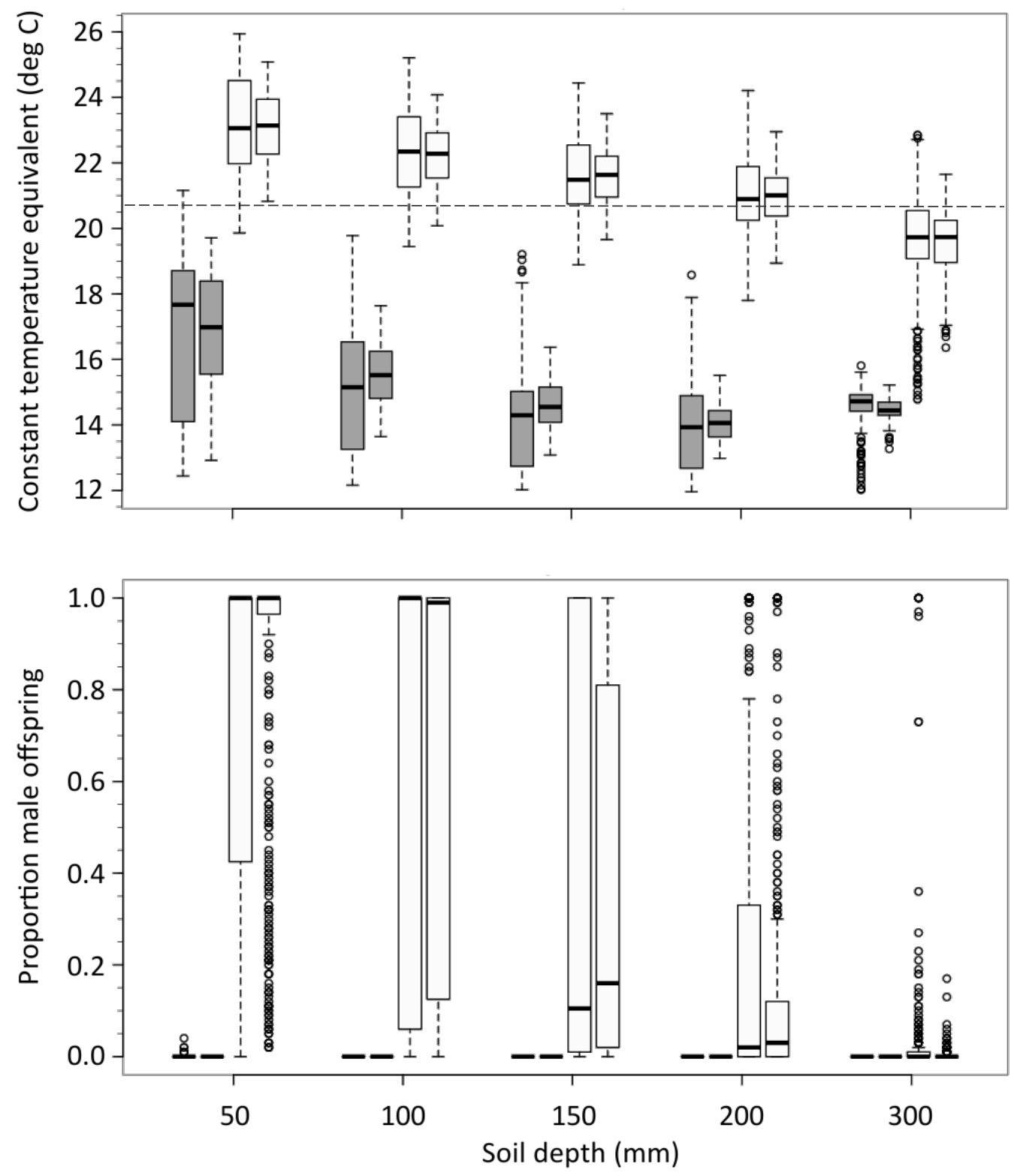

Figure 7. Boxplots showing the distributions of CTEs and proportions of male offspring predicted using mechanistic and geostatistical methods for scenarios of minimum (gray) and maximum (white) climate warming. In each shaded pair, the left-hand box shows the distribution of values generated using mechanistically modelled hourly soil temperatures, and the right-hand box shows values predicted using geostatistical models. The dashed line on the plot of CTE values approximates the pivotal temperature of tuatara. Despite the statistical differences between distributions of CTEs and offspring sex ratios predicted for tuatara by the different methods in this study, ordinary kriging is unlikely to under-predict proportions of male hatchlings, compared with the mechanistic model. Thus, ordinary kriging may be considered a useful tool for examining offspring sex ratios at a very high spatial resolution, particularly when computing resources are limited. 
Predictions of incubation temperatures and offspring sex ratios were modelled for the scenario of minimum climate warming only within the areas of Takapourewa that were predicted to reach at least $35 \%$ of total embryonic development under the non error-corrected current climate scenario (see Section 4.3.2). At a soil depth of $50 \mathrm{~mm}$, calculated CTEs varied from $14.23^{\circ} \mathrm{C}$ to $19.80^{\circ} \mathrm{C}$. CTEs varied from $13.06^{\circ} \mathrm{C}$ to $18.60^{\circ} \mathrm{C}$ at a soil depth of $100 \mathrm{~mm}$ and from $12.91^{\circ} \mathrm{C}$ to $17.40^{\circ} \mathrm{C}$ at a depth of $150 \mathrm{~mm}$. At a soil depth of $200 \mathrm{~mm}$, modelled CTEs varied from $11.97^{\circ} \mathrm{C}$ to $19.19^{\circ} \mathrm{C}$, and CTEs varied from $11.98^{\circ} \mathrm{C}$ to $15.95^{\circ} \mathrm{C}$ at a soil depth of $300 \mathrm{~mm}$. For sites that reached $100 \%$ of embryonic development, the CTE values modelled under the scenario of minimum climate warming using error-corrected soil temperatures would produce all-female hatchlings, regardless of soil depth, for an oviposition date of 30 Nov.

Incubation temperatures and offspring sex ratios were modelled under the scenario of maximum climate warming for all sites on Takapourewa, using an oviposition date of 30 Nov. Predicted offspring sex ratios varied from 0.00 (i.e., $100 \%$ female) to 1.00 (i.e., 100\% male) at all modelled soil depths. At a depth of 50mm, CTEs varied from $20.08^{\circ} \mathrm{C}$ to $26.24^{\circ} \mathrm{C}$, and the mean sex ratio of 0.85 . CTEs varied from $19.48^{\circ} \mathrm{C}$ to $25.60^{\circ} \mathrm{C}$ at a depth of $100 \mathrm{~mm}$, and the mean predicted hatchling sex ratio of 0.65. At a soil depth of $150 \mathrm{~mm}$, CTEs varied from $19.13^{\circ} \mathrm{C}$ to $24.49^{\circ} \mathrm{C}$, and the mean predicted hatchling sex ratio was 0.38 . CTEs predicted for a soil depth of $200 \mathrm{~mm}$ varied from $18.19^{\circ} \mathrm{C}$ to $24.20^{\circ} \mathrm{C}$, with a mean predicted sex ratio of 0.13 . At a soil depth of $300 \mathrm{~mm}$, modelled CTEs varied from $15.33^{\circ} \mathrm{C}$ to $23.23^{\circ} \mathrm{C}$, and, while all-male clutches were predicted under the maximum climate warming scenario, the mean predicted hatchling sex ratio was 0.00 (Fig. 8, 9). 


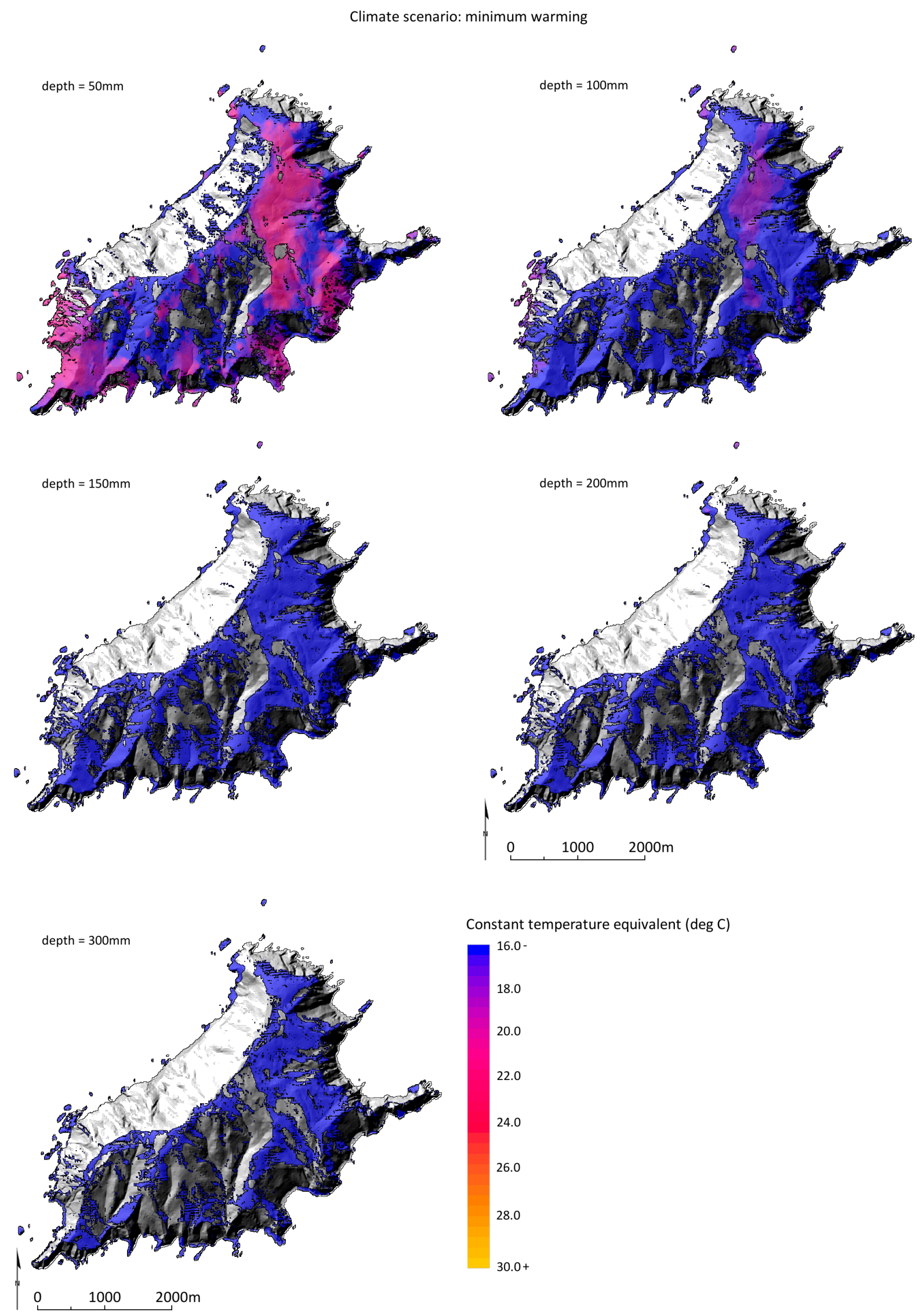

Constant temperature equivalent $(\operatorname{deg} \mathrm{C})$

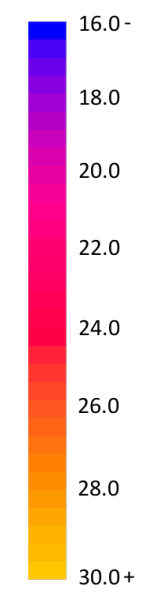



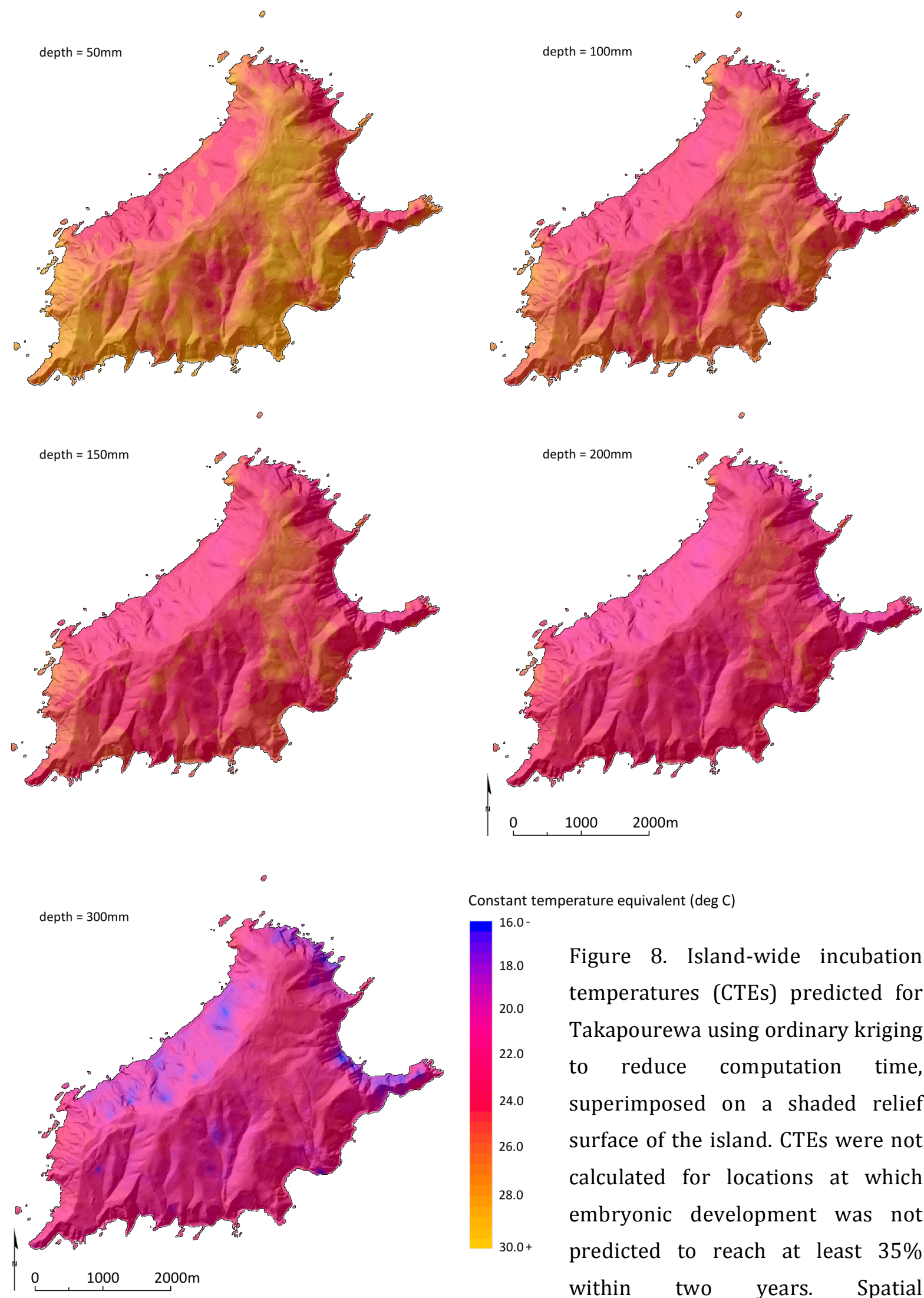

Constant temperature equivalent $(\operatorname{deg} C)$

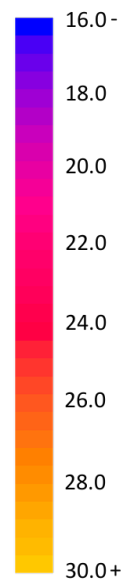

Figure 8. Island-wide incubation temperatures (CTEs) predicted for Takapourewa using ordinary kriging to reduce computation time, superimposed on a shaded relief surface of the island. CTEs were not calculated for locations at which embryonic development was not predicted to reach at least $35 \%$ within two years. Spatial distributions of model standard errors are in Appendix 4.1. 

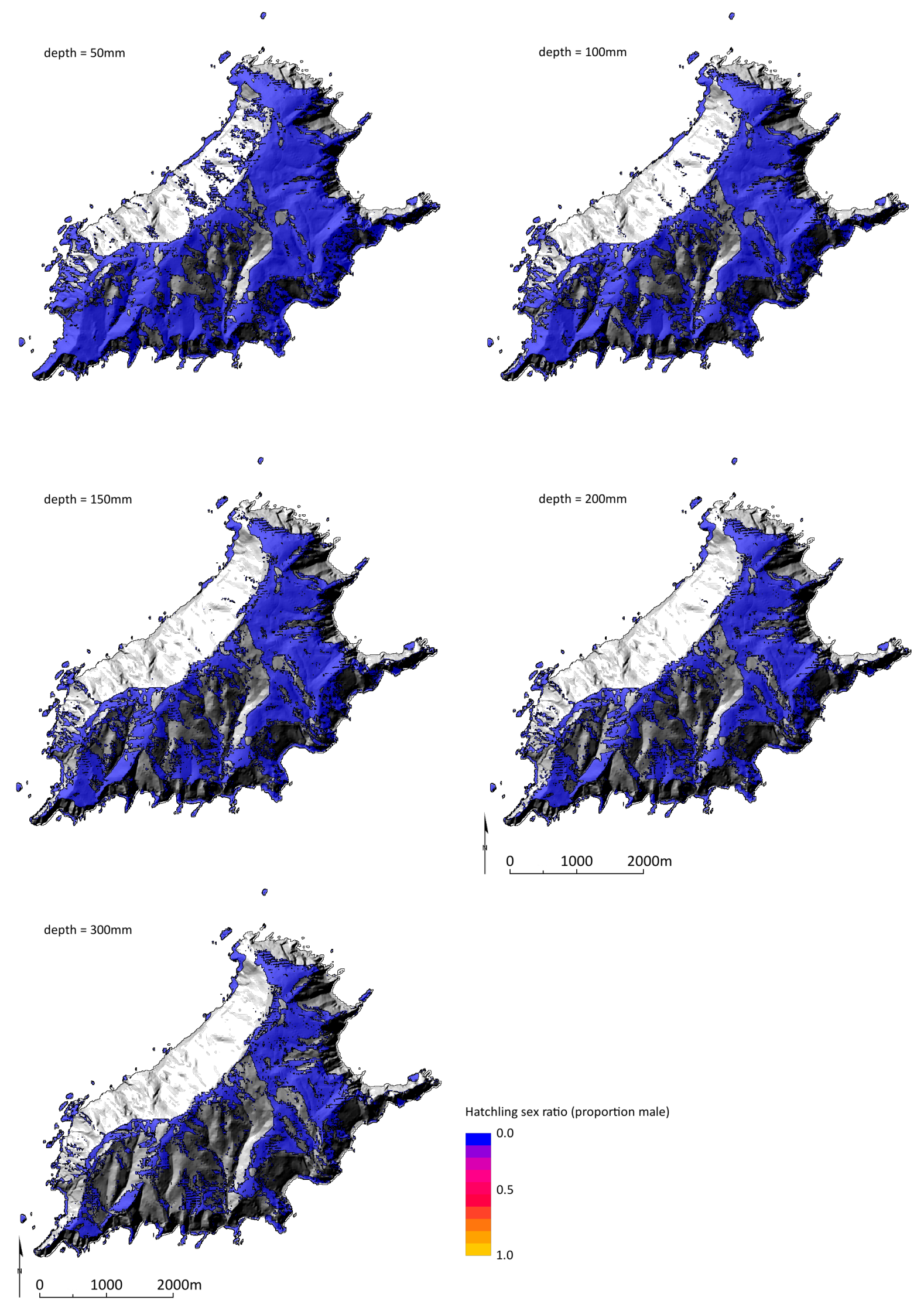
Climate scenario: maximum warming
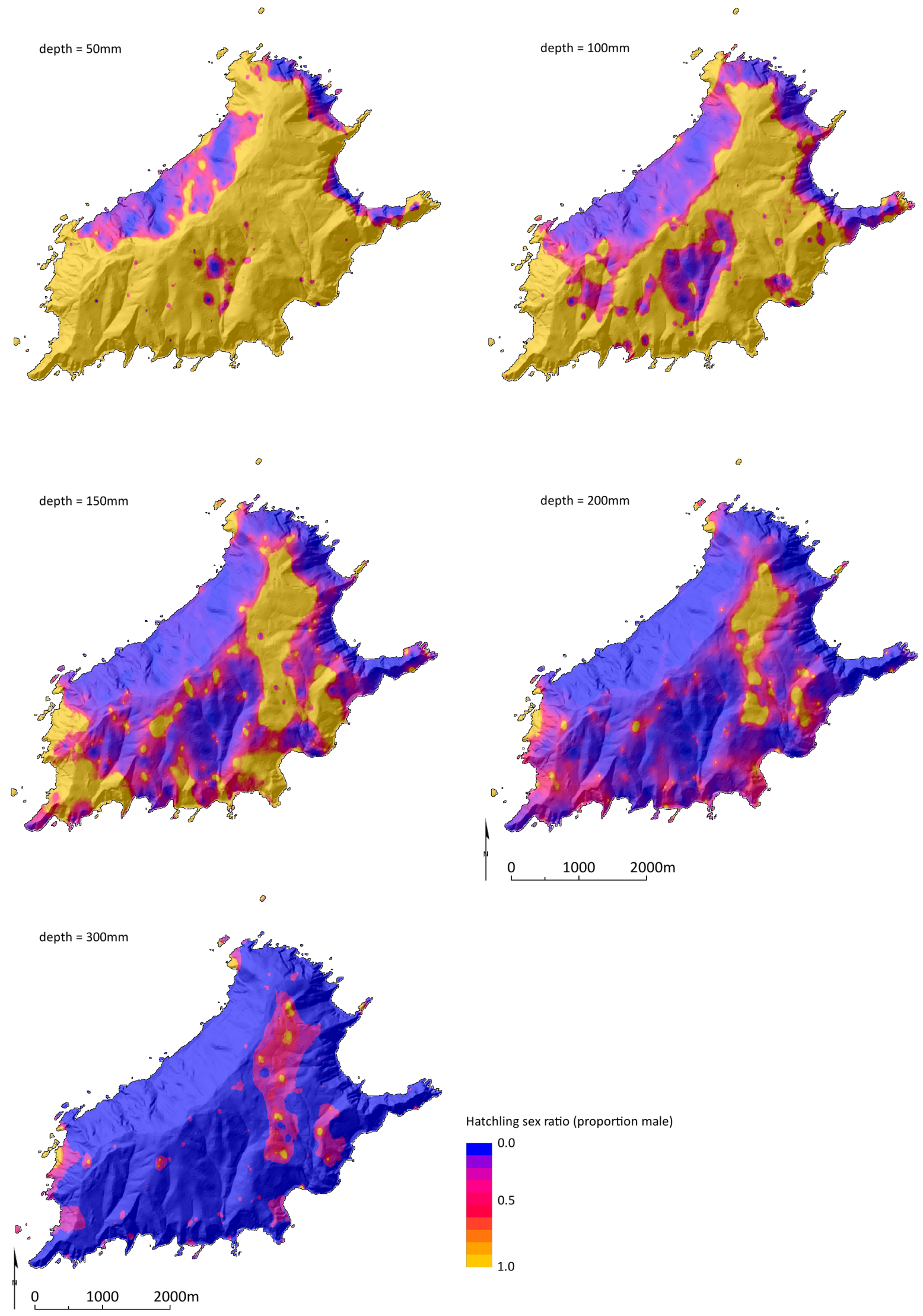

Hatchling sex ratio (proportion male)

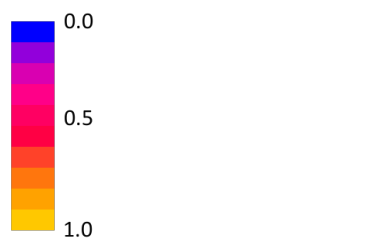


Figure 9. Spatial distributions of the proportions of male offspring predicted for Takapourewa under scenarios of climate change using CTEs modelled using ordinary kriging to reduce computation time. Sex ratios were not calculated for locations at which embryonic development was not predicted to reach at least $35 \%$ within two years. All predicted sex ratios were binned by the proportion of male hatchlings (i.e., $\leq 0.1=0.1 ; 0.1-\leq 0.2=0.2 ; 0.9-1.0=1.0$; etc.).

Predicted CTEs at known nesting locations varied under the current climate scenario from $12.26^{\circ} \mathrm{C}$ to $20.41^{\circ} \mathrm{C}$ across all depths, producing all-female hatchlings at all sites. A Kolmogorov-Smirnov (K-S) test found that the distributions of CTEs were significantly higher at nest sites, compared to random locations, at soil depths of $50 \mathrm{~mm}$ $(D=0.25, p<0.001), 100 \mathrm{~mm}(\mathrm{D}=0.17, \mathrm{p}<0.01), 150 \mathrm{~mm}(\mathrm{D}=0.37, \mathrm{p}<0.001), 200 \mathrm{~mm}$ $(\mathrm{D}=0.30, \mathrm{p}<0.001)$, and $300 \mathrm{~mm}(\mathrm{D}=0.61, \mathrm{p}<0.001)$. Likewise, under the scenario of minimum warming, CTEs varied from $12.18^{\circ} \mathrm{C}$ to $20.41^{\circ} \mathrm{C}$ across all depths and produced all-female hatchlings at all nest sites. A K-S test found that the distributions of CTEs were higher at nest sites than at random locations at soil depths of $50 \mathrm{~mm}(\mathrm{D}=0.39, \mathrm{p}<$ $0.001), 100 \mathrm{~mm}(\mathrm{D}=0.21, \mathrm{p}<0.001), 150 \mathrm{~mm}(\mathrm{D}=0.41, \mathrm{p}<0.001), 200 \mathrm{~mm}(\mathrm{D}=0.35, \mathrm{p}<$ $0.001)$, and $300 \mathrm{~mm}(\mathrm{D}=0.65, \mathrm{p}<0.001)$ under the scenario of minimum climate warming.

Under the maximum climate warming scenario, CTEs varied from $17.16^{\circ} \mathrm{C}$ to $26.38^{\circ} \mathrm{C}$ across all depths, and most nest sites produced all-male nests. The mean predicted hatchling sex ratio varied between $95-99 \%$ male at soil depths from $50 \mathrm{~mm}$ to $150 \mathrm{~mm}$, and no all-female nests were produced at those depths. At a soil depth of $200 \mathrm{~mm}$, the mean predicted hatchling sex ratio was 0.6 , and sex ratios varied from 0 $100 \%$ male. At a soil depth of $300 \mathrm{~mm}$, the mean predicted hatchling sex ratio was 0.08 , and the maximum percentage of males predicted was $48 \%$ under the scenario of maximum climate warming. A K-S test found that the distribution of CTE values was higher at nest sites than at random locations at soil depths of $50 \mathrm{~mm}(\mathrm{D}=0.58, \mathrm{p}<$ 
0.001), 100mm (D = 0.61, p < 0.001), 150mm $(\mathrm{D}=0.58, \mathrm{p}<0.001), 200 \mathrm{~mm}(\mathrm{D}=0.57, \mathrm{p}<$ $0.001)$, and $300 \mathrm{~mm}(\mathrm{D}=0.57, \mathrm{p}<0.001)$ under the maximum warming scenario.

Under the current climate scenario, $90 \%$ of the 200 randomly selected nesting locations were predicted to hatch at a depth of $50 \mathrm{~m}$. Predicted hatching success decreased with increasing depth to $79 \%$ at $100 \mathrm{~mm}, 41 \%$ at $150 \mathrm{~mm}$, and $3 \%$ at $200 \mathrm{~mm}$. No nest sites were predicted to hatch at a soil depth of $300 \mathrm{~mm}$ under the current climate scenario. Under the scenario of minimum warming, 93\% of nest sites were predicted to hatch at a soil depth of $50 \mathrm{~mm}$. Predicted hatching success decreased with increasing soil depth to $83 \%$ at $100 \mathrm{~mm}$, to $44 \%$ at $150 \mathrm{~mm}$, and to $3 \%$ at $200 \mathrm{~mm}$. No nesting locations were predicted to facilitate successful hatching at a soil depth of $300 \mathrm{~mm}$. Under the scenario of maximum climate warming, all 200 nest sites were predicted to hatch successfully at all soil depths.

Mean hatching dates for nest sites were similar under the current climate and minimum warming scenarios and were predicted to occur in late March, early austral autumn, at a soil depth of $50 \mathrm{~mm}$. Mean hatching dates were predicted in mid October, mid austral spring, at a depth of $100 \mathrm{~mm}$ and in austral summer at depths of $150 \mathrm{~mm}$ and $200 \mathrm{~mm}$. Under the scenario of maximum climate warming, mean hatching dates were predicted to occur in late austral winter at a depth of $50 \mathrm{~mm}$ and throughout austral spring at depths from $100 \mathrm{~mm}$ to $200 \mathrm{~mm}$. The mean hatching date was predicted for austral summer at a soil depth of $300 \mathrm{~mm}$ under the maximum warming scenario. All mean hatching dates calculated for known nest sites by depth and climate scenario were statistically significant (Table 2). 
Table 2. Mean hatching dates predicted for sampled nest sites on Takapourewa under three climate scenarios. The significance of the Rao's test statistic was $\mathrm{p} \leq 0.001$ under all three modelled climate scenarios for all soil depths at which sites were predicted to hatch. A mean hatch date marked as '-' indicates that no sites were predicted to hatch under the given scenario.

\begin{tabular}{rrrrrrr}
\hline & \multicolumn{2}{c}{ Current climate } & \multicolumn{2}{c}{ Minimum warming } & \multicolumn{2}{c}{ Maximum warming } \\
soil depth $(\mathbf{m m})$ & hatch date & \multicolumn{1}{c}{$\boldsymbol{x}^{\mathbf{2}}$} & hatch date & \multicolumn{1}{c}{$\boldsymbol{x}^{2}$} & hatch date & $\boldsymbol{x}^{\mathbf{2}}$ \\
\hline $\mathbf{5 0}$ & $24 \mathrm{Mar}$ & 222.08 & $25 \mathrm{Mar}$ & 225.09 & $20 \mathrm{Aug}$ & 243.05 \\
$\mathbf{1 0 0}$ & $24 \mathrm{Oct}$ & 191.25 & $16 \mathrm{Oct}$ & 190.86 & $17 \mathrm{Sep}$ & 242.41 \\
$\mathbf{1 5 0}$ & $26 \mathrm{Nov}$ & 263.78 & $28 \mathrm{Nov}$ & 270.68 & 16 Oct & 245.84 \\
$\mathbf{2 0 0}$ & $14 \mathrm{Dec}$ & 278.30 & $14 \mathrm{Dec}$ & 266.30 & $13 \mathrm{Nov}$ & 257.33 \\
$\mathbf{3 0 0}$ & - & NA & - & NA & 2 Jan & 269.75 \\
\hline
\end{tabular}

\subsubsection{Effects of oviposition date on offspring sex ratios and hatching phenology}

Development models were run for +/- 15 and +/- 30 days from the median oviposition date of 30 November (i.e., Julian day 335) using error-corrected hourly soil temperatures for 200 randomly sampled sites. Predictions were generated for oviposition dates of 1 and 15 November (i.e., Julian days 305 and 320) and 15 and 30 December (i.e., Julian days 350 and 365) for all three climate scenarios and, additionally, for 1 and 15 October (i.e., Julian days 274 and 288) for the maximum warming scenario.

Constant incubation temperatures (CTEs) were calculated for sites that were predicted to reach at least $35 \%$ of embryonic development, the upper bound of the simulated thermosensitive period (TSP), within two years. Under the current climate scenario, CTEs were lowest when the simulated oviposition date was either 1 Nov or 30 Nov. The mean CTE was lowest at all depths when the oviposition date was 30 Nov and varied from $16.76^{\circ} \mathrm{C}$ at a soil depth of $50 \mathrm{~mm}$ to $14.46^{\circ} \mathrm{C}$ at a depth of $300 \mathrm{~mm}$. Mean CTE values were highest when the simulated oviposition date was 15 Nov and varied from $22.97^{\circ} \mathrm{C}$ at a soil depth of $50 \mathrm{~mm}$ to $19.60^{\circ} \mathrm{C}$ at a depth of $30 \mathrm{~mm}$ under the current climate scenario. Under the scenario of minimum climate warming, a clear reduction 
with depth of CTE values was only observed when the simulated oviposition date was 15 Dec, compared with other dates. Mean CTE values calculated for all other oviposition dates were similar to the relatively low values observed under the current climate scenario for the median oviposition date of 30 Nov. Under the scenario of minimum warming, CTEs varied from $16.11^{\circ} \mathrm{C}$ at $50 \mathrm{~mm}$ to $14.50^{\circ} \mathrm{C}$ at $300 \mathrm{~mm}$ when the simulated oviposition date was 30 Nov. Under the scenario of maximum climate warming, a reduction with depth in CTEs was apparent for all seven simulated oviposition dates, with no single date generating predicted CTE values that were consistently low, relative to other dates.

A generalised linear model found that oviposition dates of 1 and 15 Nov and 15 and 30 Dec significantly affected predicted CTEs at all depths under the current climate scenario ( $p<0.001)$; however, a simulated oviposition date of 30 Nov did not affect CTE values differentially at different soil depths. Under the scenario of minimum climate warming, predicted CTE values varied significantly by depth but were affected differentially by depth only when the simulated oviposition date was $15(\mathrm{p}<0.01)$ or 30 Dec $(\mathrm{p}<0.001)$. Similarly, under the maximum climate warming scenario, CTEs varied significantly by depth; however, only oviposition dates of 15 Dec $(p<0.05)$ or 30 Dec (p $<0.001$ ) significantly affected CTE values, and differential effects by soil depth were only observed at $150 \mathrm{~mm}$ for $15 \mathrm{Dec}$ and $300 \mathrm{~mm}$ for 30 Dec (Fig. 10). 


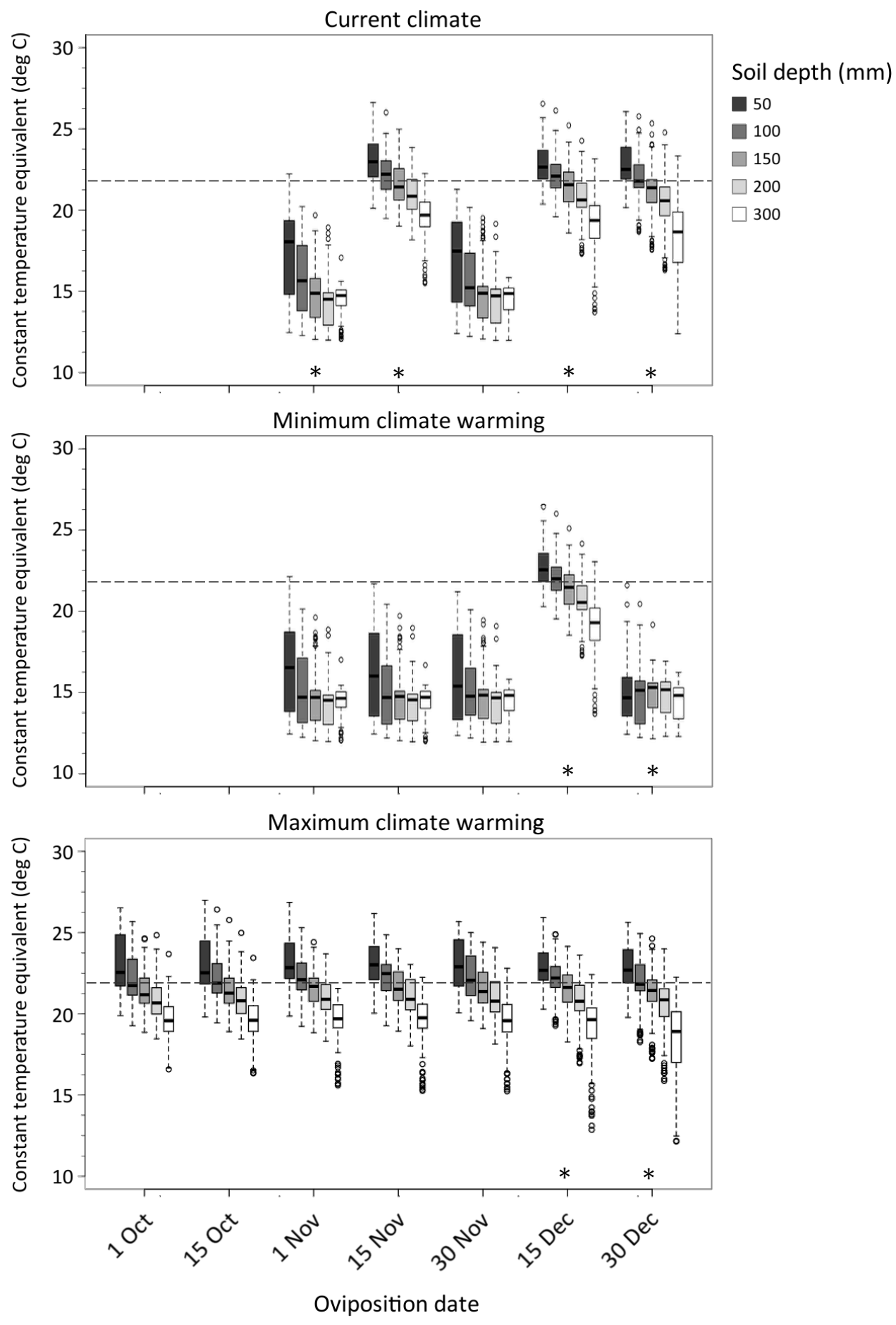

Figure 10. Boxplots showing the effects of shifting oviposition dates on the distribution by depth of CTE values under the three modelled climate scenarios for sites on Takapourewa at which the total embryonic development was predicted to reach at least 35\% within two years. Onset of nesting tends to occur earlier in warmer years (Nelson unpub. data), so two additional oviposition dates were simulated for the maximum warming scenario. An asterisk indicates that the CTEs predicted for a given oviposition date were significantly affected by an interaction between date and at least one soil depth under each climate scenario ( $p \leq 0.05)$. The dashed lines approximate the pivotal temperature. 
The effects of simulated oviposition dates on CTE values resulted in datedependent patterns in predicted hatchling sex ratios. Under the current climate scenario, mixed-sex nests were only predicted at a soil depth of $50 \mathrm{~mm}$ when the simulated oviposition date was either 1 Nov or 30 Nov, though the mean predicted proportion of male hatchlings predicted for both dates was still $<0.01$ at that depth. Mean proportions of male hatchlings predicted for other oviposition dates were similar and decreased with increasing soil depth, varying from $0.69-0.76$ at a soil depth of $50 \mathrm{~mm}$ and from $0.16-0.22$ at a soil depth of $300 \mathrm{~mm}$ under the current climate scenario. Under the scenario of minimum climate warming, mean predicted proportions of male offspring $>0.01$ were only predicted to hatch below depths of $100 \mathrm{~mm}$ when the simulated oviposition date was 15 Dec. The mean predicted proportion of male hatchlings varied from 0.76 at a depth of $50 \mathrm{~mm}$ to 0.02 at a depth of $300 \mathrm{~mm}$ under the minimum warming scenario when the simulated oviposition date was 15 Dec. Under the scenario of maximum climate warming, predicted proportions of male offspring varied from 0.00-1.00 for all simulated oviposition dates, with nearly all sites predicted to produce all-male nests at a soil depth of $50 \mathrm{~mm}$ and primarily all-female nests at a depth of $300 \mathrm{~mm}$.

A generalised linear model found that oviposition dates of 15 Nov, 15 Dec, and 30 Dec significantly affected offspring sex ratios under the current climate scenario $(p<$ 0.001). Depth by itself did not significantly affect predicted sex ratios; however, oviposition dates affected sex ratios differentially by depth. Under the scenario of minimum climate warming, only an oviposition date of 15 Dec affected predictions of offspring sex ratios $(\mathrm{p}<0.001)$, and no independent effects of depth were observed. Oviposition dates of 1 Nov ( $p<0.001)$, 15 Nov ( $p<0.01)$, and 15 of Dec $(p<0.01)$ significantly affected predicted hatchling sex ratios under the scenario of maximum climate warming. However, a significant interaction between oviposition date and depth was only observed for predictions at soil depths of $200 \mathrm{~mm}$ and $300 \mathrm{~mm}$, when the 
simulated oviposition date was $1(0.01<\mathrm{p}<0.05)$ or 15 Nov $(\mathrm{p}<0.05)$. In contrast with the current climate and minimum warming scenarios, significant effects of soil depth were observed independently of oviposition date under the scenario of maximum climate warming for all depths ( $\mathrm{p}<0.001)$ (Fig. 11).

Tuatara generally require $11-16$ months to reach $100 \%$ of embryonic development (Cree et al. 1991, 1992; Thompson et al. 1996), and longer incubation times are probably associated with an increased probability of mortality (Thompson et al. 1996; Refsnider et al. 2009). However, because the empirical developmental models used in this study do not explicitly include embryonic mortality, the probability of hatching was assumed to be 1.00 for all sites at which development reached $100 \%$ within two years. Thus, hatching dates are considered equivalent between years (e.g., given an oviposition date of 30 Nov, a site predicted to hatch in Nov could have undergone either approximately 12 or 24 months of incubation).

Under the current climate scenario, mean hatching occurred in late spring or summer for most simulated oviposition dates and soil depths for which hatching was predicted. The mean hatch date at $50 \mathrm{~mm}$, given an oviposition date of 15 Nov, was predicted for 9 Mar, early austral autumn. Predicted mean hatching dates were shifted backwards by around three weeks with each simulated increase in soil depth. For an oviposition date of $1 \mathrm{Nov}$, mean hatching dates predicted for sites below $50 \mathrm{~mm}$ were not significantly different from a uniform distribution. Similar patterns were observed under the scenario of minimum warming, though mean hatching dates were only significantly different from uniform at all soil depths when the simulated oviposition date was 15 Dec. Under the scenario of maximum climate warming, nests were predicted to hatch later (i.e., mean hatching dates shifted backward) both as simulated oviposition dates were shifted later and as soil depths increased. Mean hatching dates at $50 \mathrm{~mm}$ and $100 \mathrm{~mm}$ were predicted from 17 of May, late austral autumn, to mid-late 
austral winter when the oviposition date was 1 or 15 of Oct or 1 or 15 of Nov (Fig. 12;

Table 3). 

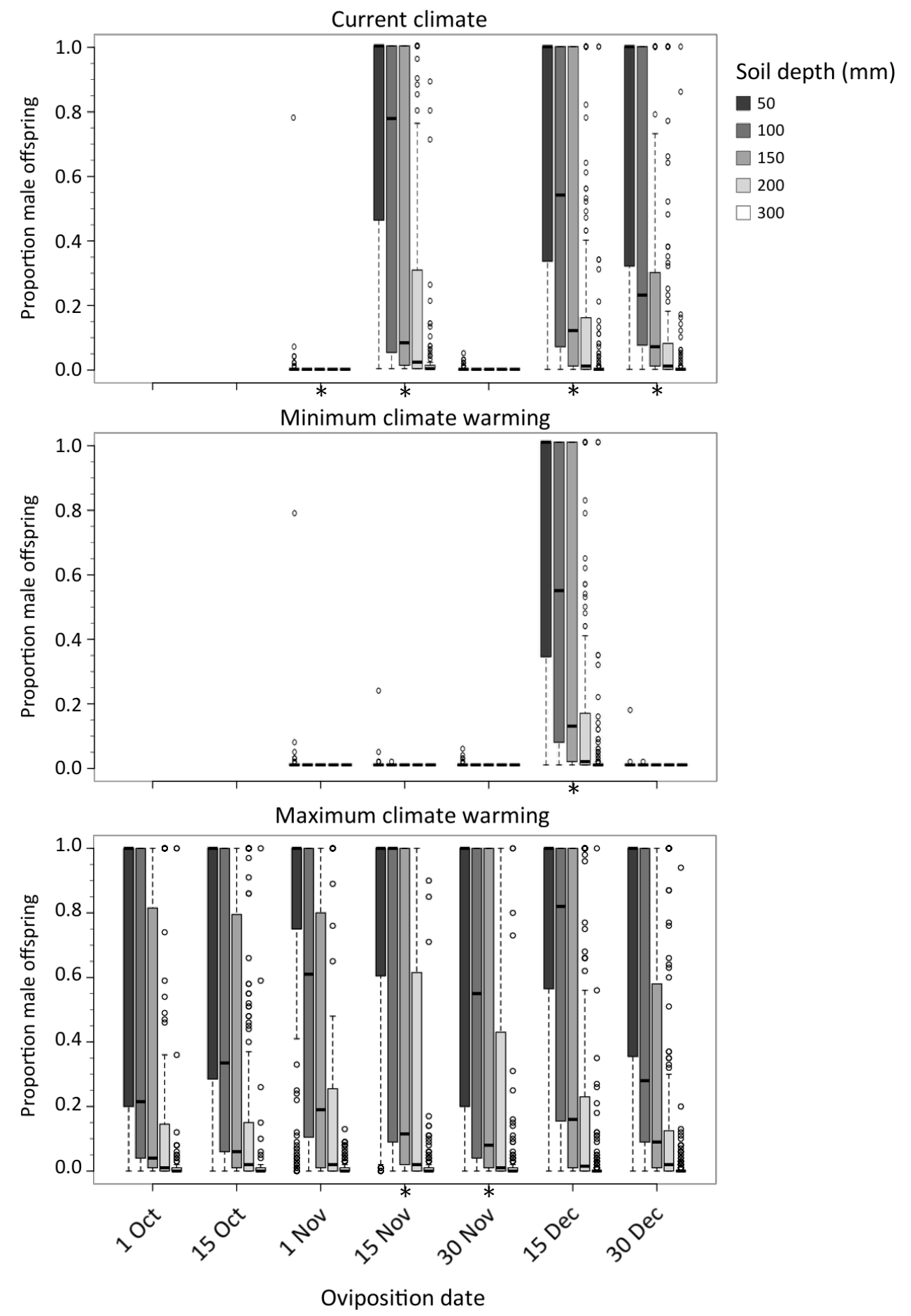

Figure 11. Boxplots showing the effects of shifting oviposition dates on the distribution by depth of predicted proportions of male tuatara hatchlings under the three modelled climate scenarios for sites at which total embryonic development was predicted to reach at least $35 \%$ within two years. Onset of nesting tends to occur earlier in warmer years (Nelson unpub. data), so two additional oviposition dates were simulated for the maximum warming scenario. An asterisk below the plot indicates that the sex ratios, given here as the proportion of male hatchlings, predicted for a given oviposition date were significantly affected by an interaction between date and at least one soil depth under each climate scenario $(\mathrm{p} \leq 0.05)$. 


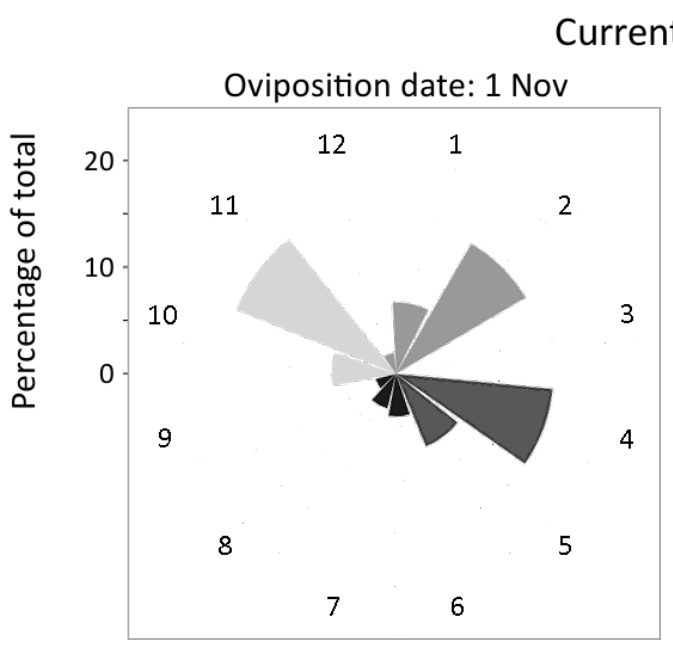

t climate

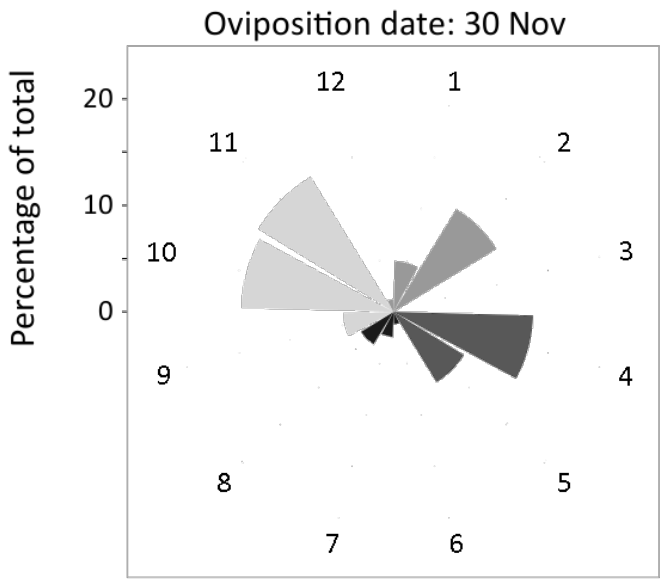

Oviposition date: $30 \mathrm{Dec}$

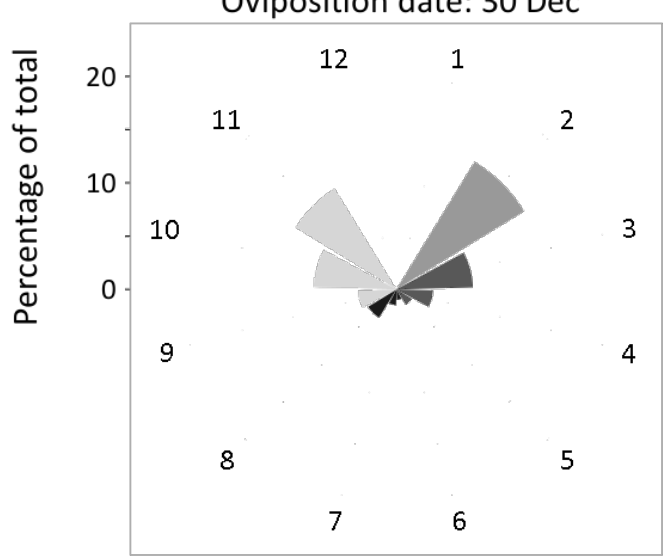

Month of hatching
Oviposition date: $15 \mathrm{Nov}$

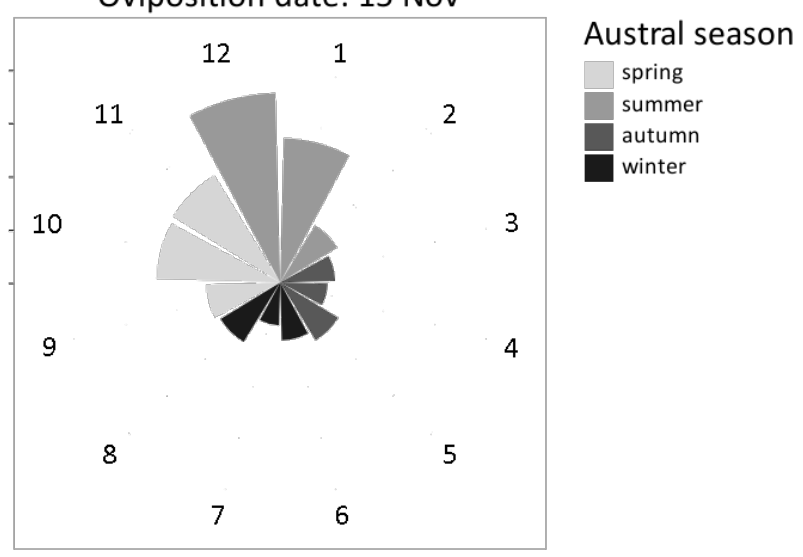

Oviposition date: $15 \mathrm{Dec}$

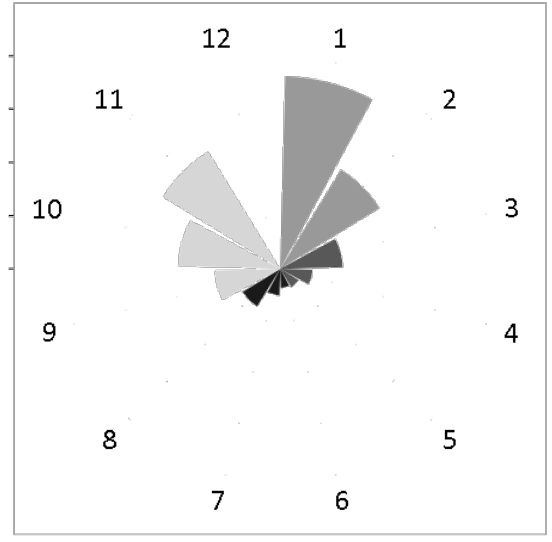

Month of hatching 
Minimum warming

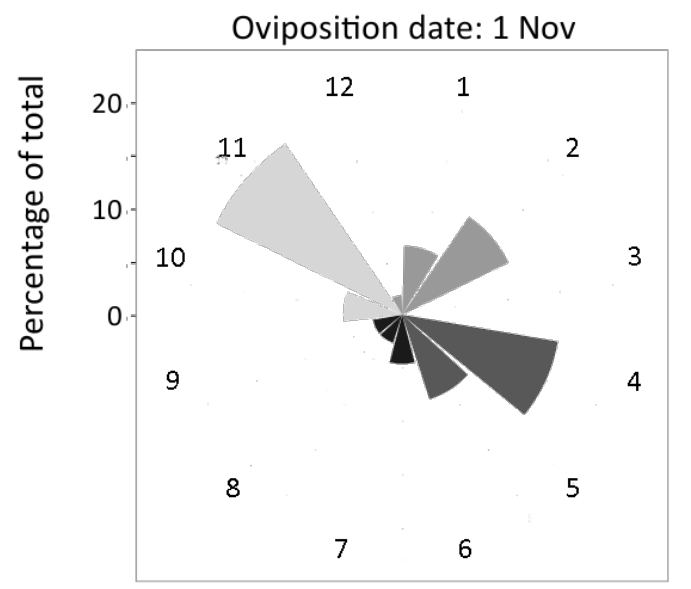

Oviposition date: 15 Nov
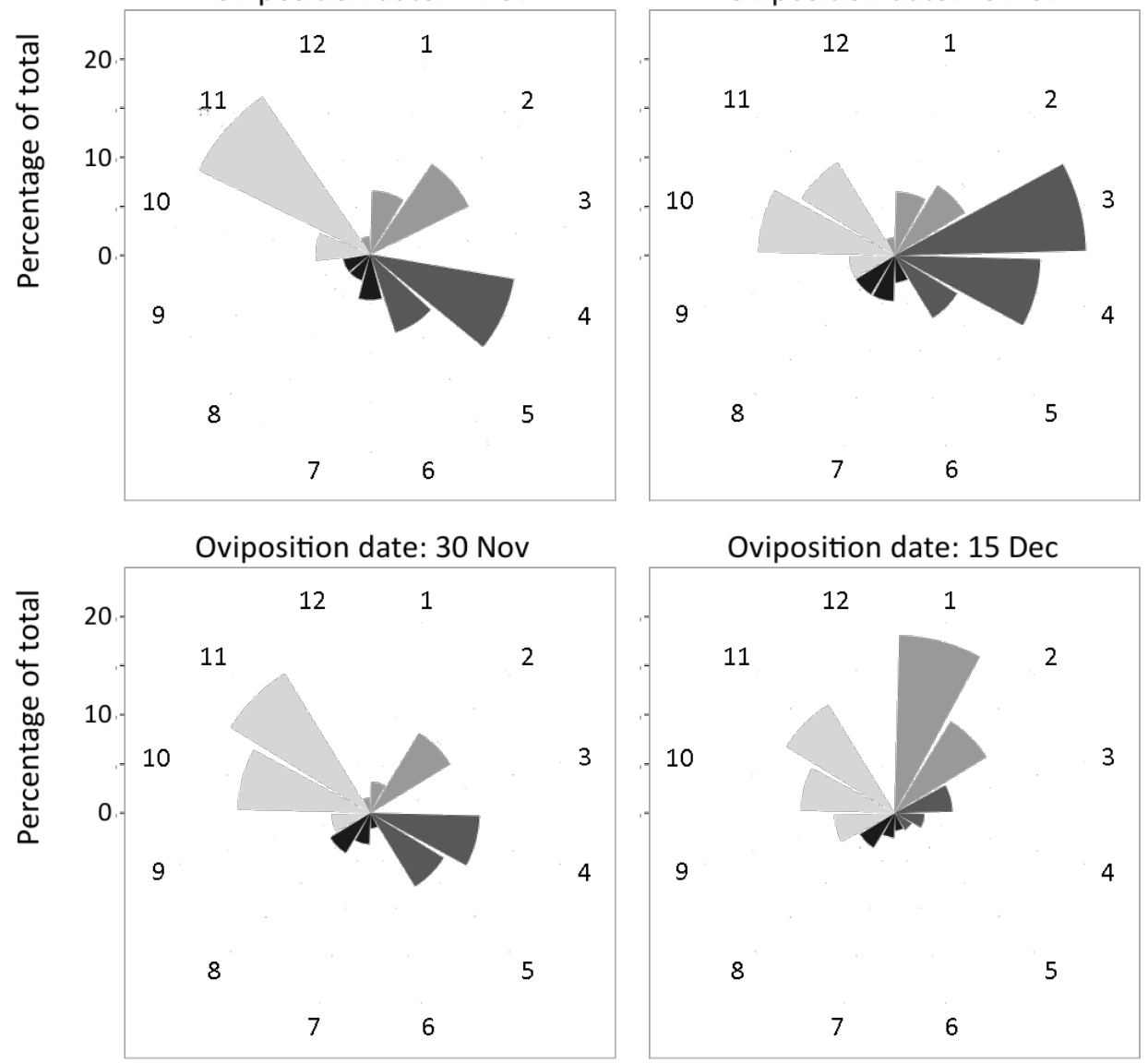

Austral season

spring

summer

autumn

winter

Oviposition date: 15 Dec

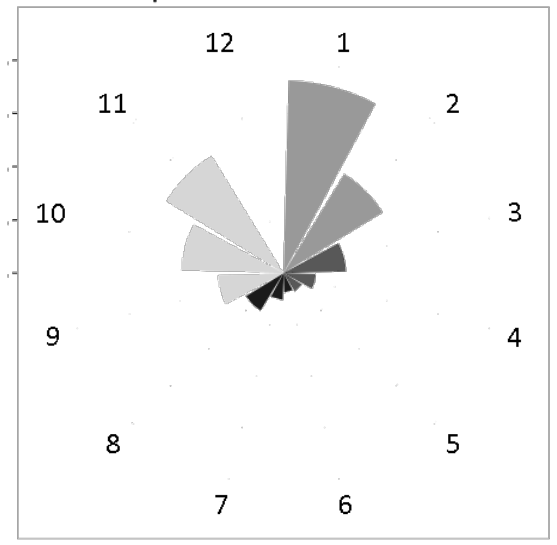

Month of hatching

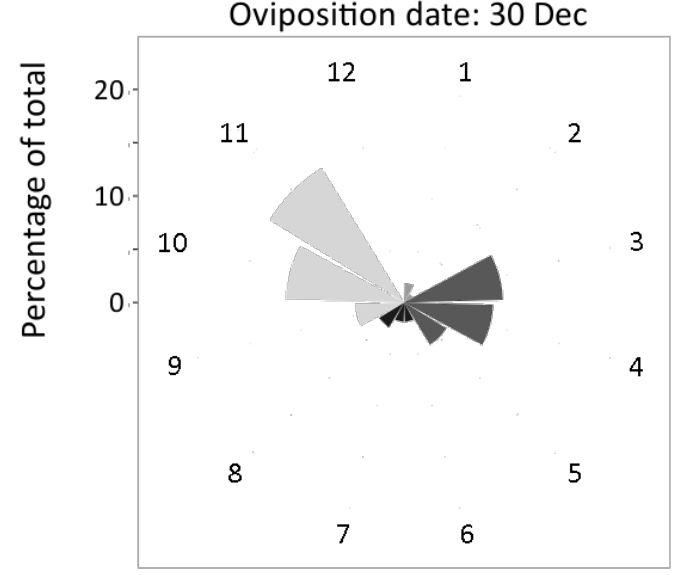

Month of hatching 
Maximum warming
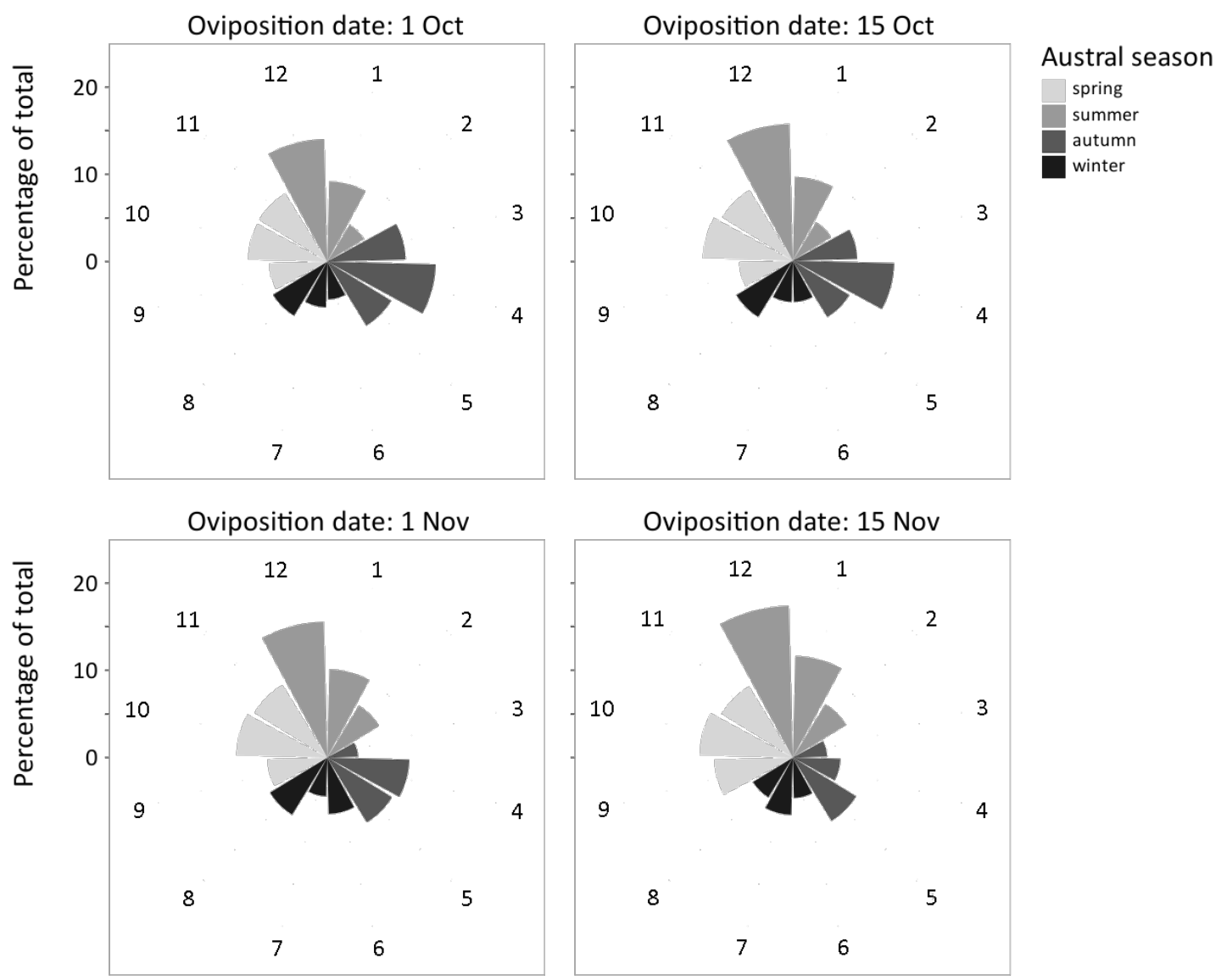

Oviposition date: 15 Nov
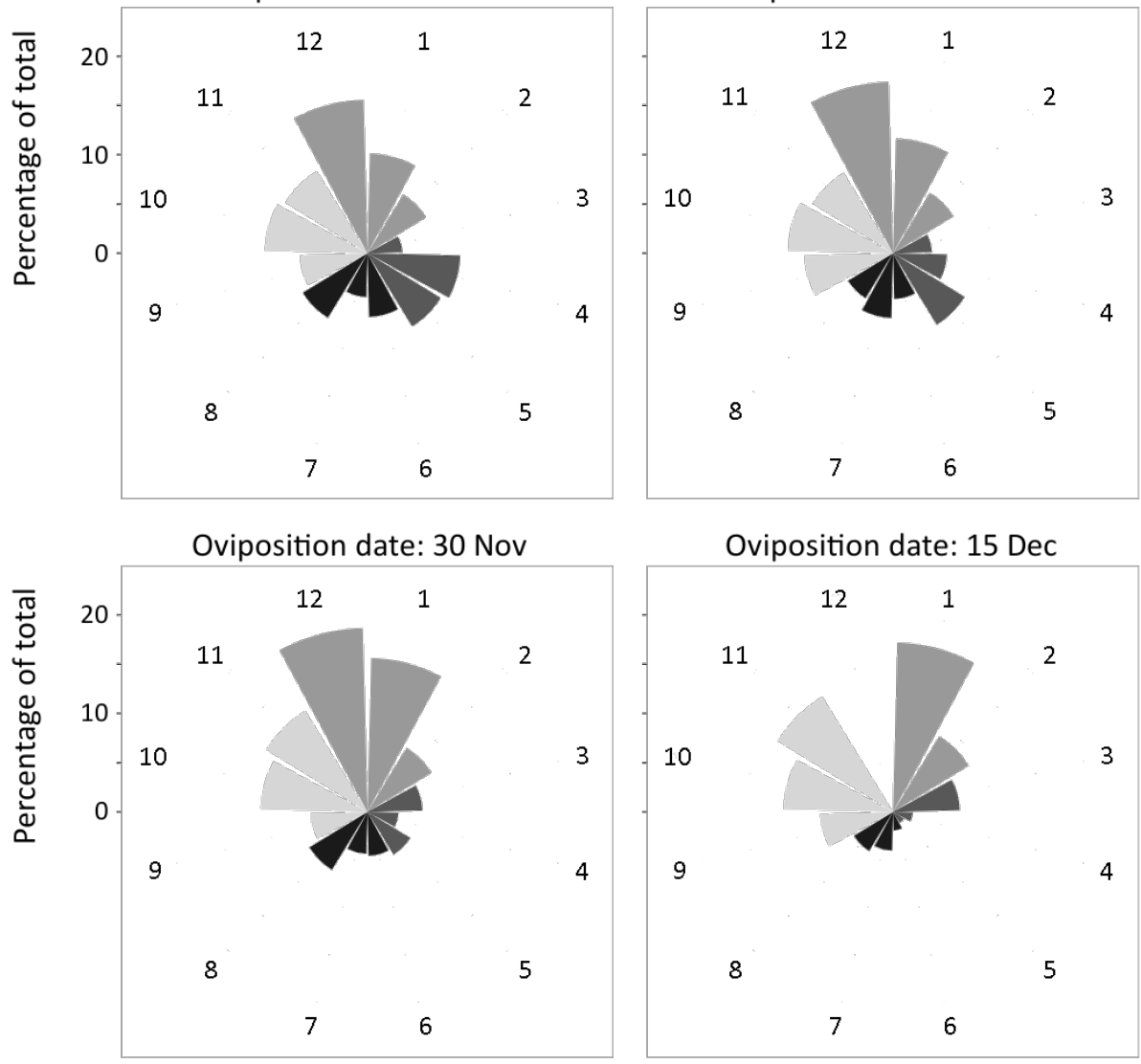

Oviposition date: 15 Dec
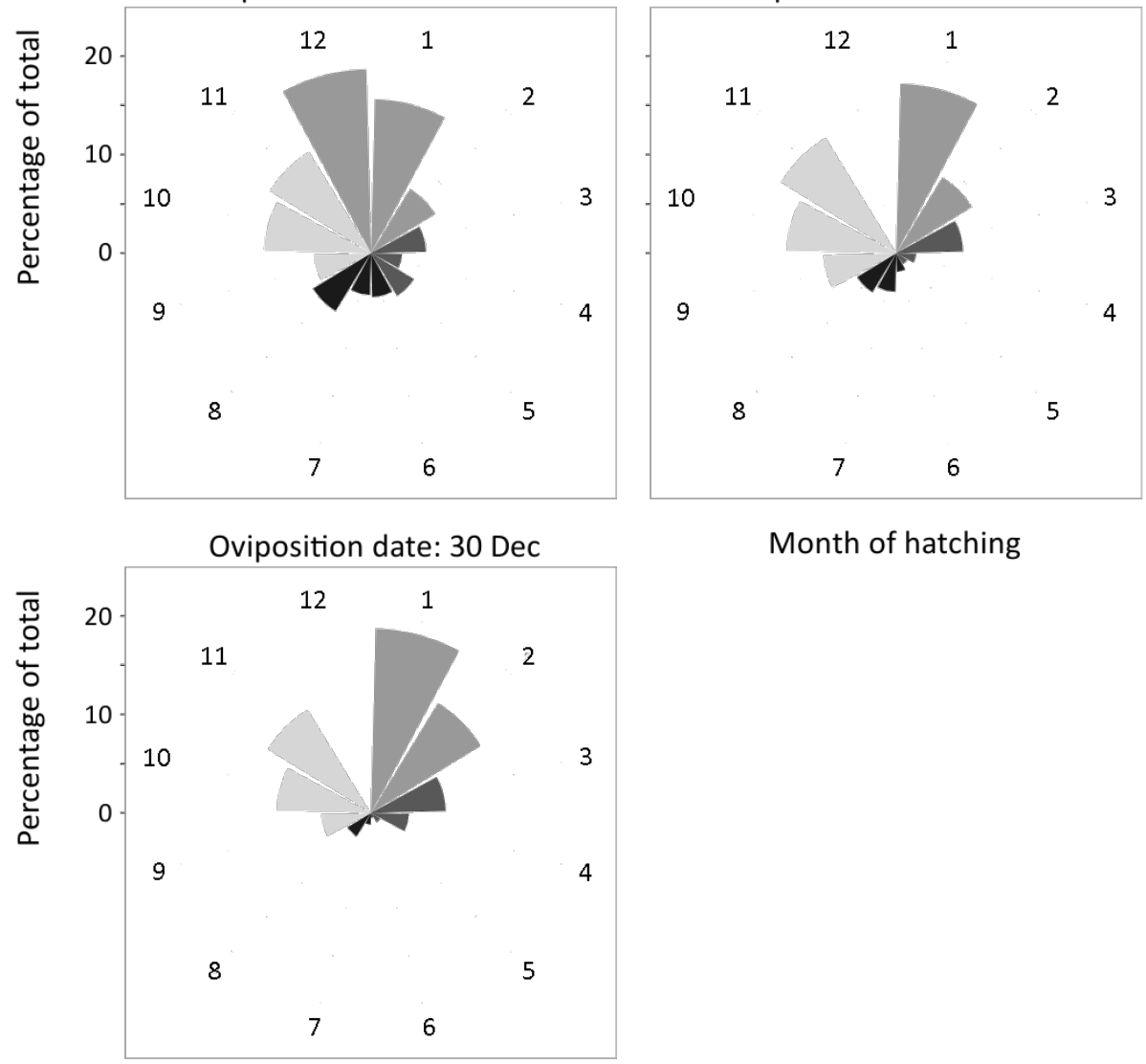

Month of hatching

Month of hatching 
Figure 12. Polar plots summarizing the effects of shifting oviposition dates on the monthly and seasonal distributions of hatch dates predicted for tuatara on Takapourewa, pooled across depths, for all three simulated climate scenarios. Onset of nesting tends to occur earlier in warmer years (Nelson unpub. data), so two additional oviposition dates were simulated for the maximum warming scenario. Data are displayed as the percentage of sites predicted to hatch in each month, numbered from 1 (January) to 12 (December), out of the total number of sites predicted to hatch within two years from oviposition. 


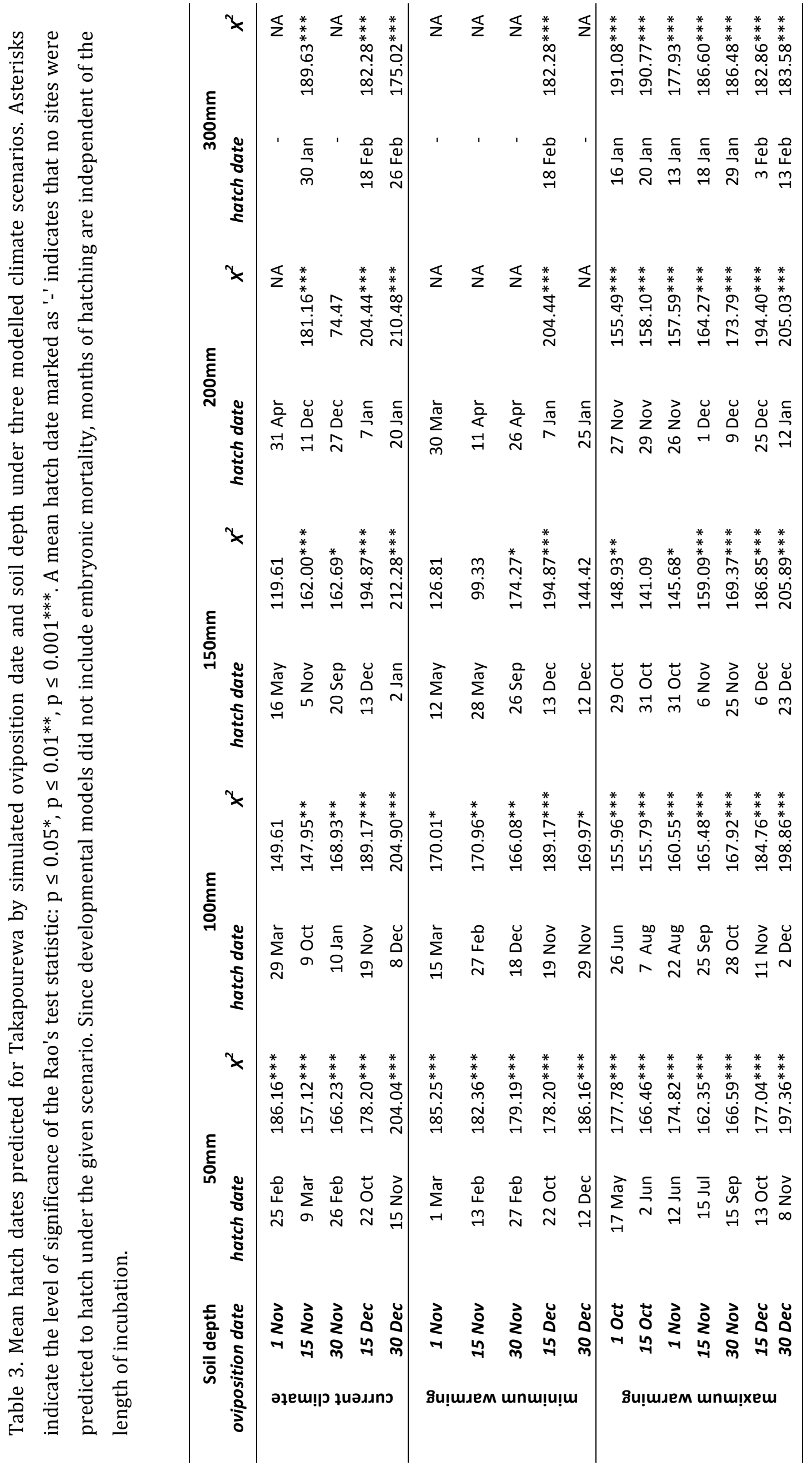




\subsection{Discussion}

\subsubsection{Summary of major findings}

This study used mechanistic and geostatistical modelling methods to develop spatially explicit, microclimate-scale predictions of incubation temperatures, hatchling sex ratios, and hatching phenology for the population of tuatara on the island of Takapourewa, New Zealand under three simulated climate scenarios. In addition, this research examined the effects of known microclimate model error on physiological predictions and used mechanistically modelled data to test the efficacy of geostatistical analyses as a predictive tool for conservation of a population with temperaturedependent sex determination (TSD).

The results of multiple models suggest that the scenario of minimum climate warming projected regionally for Takapourewa (MFE 2008) would be unlikely to result in male-biased hatchling sex ratios in the resident population of tuatara within the next hundred years. The minimum climate warming scenario may actually facilitate incubation at additional 'cool' sites, leading to an increase in proportions of hatchlings that are female, particularly if nesting phenology shifts forward to mid-November. Realisation of the maximum projected climate warming scenario could lead to malebiased sex ratios if the mean nest depth remains at $110 \mathrm{~mm}$. Population viability analyses carried out for the small population of tuatara on North Brother Island found that increasing hatchling sex ratios to $65 \%$ male dramatically increased extinction risk (Grayson et al. 2014). However, only oviposition dates of 15 Nov and 15 Dec were predicted to produce $\geq 65 \%$ male hatchlings at a soil depth of $100 \mathrm{~mm}$ on Takapourewa, and the population is likely more robust to the detrimental effects of sex ratio bias than tuatara on North Brother Island. 
While a scenario of minimum climate warming is unlikely to lead to male-biased offspring sex ratios via increasing incubation temperatures, earlier nesting could lead to a shift in hatching phenology. In particular, an oviposition date of 15 Nov led to an increase in the proportion of sites that were predicted to hatch in austral autumn. Fitness of juveniles that hatch later (i.e., post-summer) may be reduced, compared to that of earlier-hatching individuals that are larger and, thus, faster at the beginning of winter (Nelson et al. 2006). Mean hatch dates predicted under the scenario of maximum warming varied from late austral winter to spring. While post-hatching mortality might be initially higher, particularly in winter, juveniles that hatch faster due to higher incubation temperatures (i.e., more rapid rates of development) might have sufficient yolk reserves at hatching (Angiletta et al. 2000; Mitchell \& Seymour 2000; Mitchell et al 2008) to survive.

Under the current climate and minimum warming scenarios, oviposition phenology was key to determining whether constant incubation temperature equivalents (CTEs) were predicted to produce mixed sexes or nearly $100 \%$ female hatchlings. The date-dependent patterns of CTEs and sex ratios observed under both scenarios may be explained by the timing of the thermosensitive period (TSP), the developmental window during which sex is determined in-ovo. Earlier nesting may facilitate slower rates of embryonic development via cooler temperatures; however, the accumulation of development occurs over a longer period of time prior to the warmest weeks, and the TSP may have already passed by the time soil temperatures reach a maximum in summer. Later nesting may facilitate more rapid rates of development initially but, again, lead to the occurrence of the TSP after summer temperatures have peaked. The CTEs predicted for the current climate and minimum warming scenarios in this study could also be specific to the macroclimate data used to model soil temperatures. The unique pattern of peaks and troughs in air temperatures observed in 
any given year may interact with nesting phenology to influence the timing of the TSP and, consequently, hatchling sex ratios.

Hourly soil temperatures generated using the mechanistic model parameterised for Takapourewa were a mean approximately $2-3^{\circ} \mathrm{C}$ lower than values measured, respectively, at soil depths of $200 \mathrm{~mm}$ and $100 \mathrm{~mm}$ (Chapter 3 ). This study tested the impacts of that error on predictions of incubation temperatures, hatchling sex ratios, and hatching phenology, given an oviposition date of 30 Nov. Correcting for error in the microclimate model increased variance in CTE values, compared to those predicted using non error-corrected values, but had little effect on hatchling sex ratios under either the current climate or minimum warming scenario. Regardless of whether hourly soil temperatures used to generate developmental predictions were error-corrected or not, all predicted CTEs were well below the pivotal temperature for tuatara under both climate scenarios.

Counterintuitively, mean CTEs predicted under the scenario of minimum climate warming were slightly lower than those predicted for the current climate, particularly at soil depths of $50 \mathrm{~mm}$ and $100 \mathrm{~mm}$, when the oviposition date was 30 Nov. However, under the minimum warming scenario, the variance of CTE values was greater and, thus, minimum CTE values lower than those predicted under the current climate. Mean CTEs may have been lower under the minimum warming scenario simply because embryonic development reached at least 35\% within two years at sites for which a CTE value could not be calculated (i.e., did not reach $35 \%$ within two years) under the current climate. Under either scenario, mixed-sex nests were only predicted using error-corrected soil temperatures and only at the shallowest soil depth of $50 \mathrm{~mm}$ under the scenario of minimum warming scenario. All sites were predicted to produce $100 \%$ female nests at depths from $100 \mathrm{~mm}$ to $300 \mathrm{~mm}$ under both the current climate and minimum warming scenarios, and no site was predicted to produce more than $28 \%$ male hatchlings at a depth of 50mm under the minimum warming climate scenario. 
The primary effect of error correction under both the current climate and minimum warming scenarios was observed not in the prediction of offspring sex ratios but in the prediction of successful hatching. On the whole, only error-corrected hourly soil temperatures predicted successful hatching under either the current climate or the minimum warming scenario, given an oviposition date of 30 Nov. Increasing hourly soil temperatures to account for microclimate model error allowed for identification of additional sites that were more likely to facilitate successful completion of embryonic development. Approximately $48 \%$ of the total area of Takapourewa was identified as suitable for incubation under the current climate at a depth of $50 \mathrm{~mm}$ using errorcorrected soil temperature values, while only $2 \%$ of sites were predicted to facilitate hatching at the shallowest soil depth when non error-corrected values were used as input to the developmental model. Under the scenario of minimum climate warming, the percentage of sites predicted to facilitate hatching was reduced to around $40 \%$ at a soil depth of 50mm, when error-corrected soil temperatures were used.

Correcting for error in hourly soil temperatures differentially affected developmental predictions generated under the scenario of maximum climate warming, compared with the other climate scenarios. A positive linear error correction of $1-3.5^{\circ} \mathrm{C}$ across modelled soil depths facilitated increases in CTEs of $1.72-3.94^{\circ} \mathrm{C}$, and the variance in CTE values was reduced, compared with predictions generated using non errorcorrected hourly soil temperatures. Hatchling sex ratios varied from $0 \%$ to $100 \%$ male when either modelled or error-corrected soil temperatures were used, but predictions of male hatchlings were increased by between $63 \%$ and $2 \%$ at soil depths from, respectively, $50 \mathrm{~mm}$ to $300 \mathrm{~mm}$ when microclimate model error was included. Under the scenario of maximum climate warming, error-corrected hourly soil temperatures increased hatching success from $72-83 \%$ to $94-100 \%$ of sites from soil depths of, respectively $300 \mathrm{~mm}$ to $50 \mathrm{~mm}$. 
Since few sites were predicted to hatch successfully under either the current climate or minimum warming scenario when non error-corrected hourly soil temperatures were used as input to the developmental model, whether microclimate model error would affect hatching phenology under either scenario could not be quantified. Mean hatching dates were predicted to occur in late austral spring at depths from $50 \mathrm{~mm}$ to $150 \mathrm{~mm}$ under both scenarios, while mean hatching dates at soil depths of $200 \mathrm{~mm}$ and $300 \mathrm{~mm}$ were not significantly different from what would be expected under a uniform distribution. Under the scenario of maximum climate warming, most hatching was predicted to occur in austral summer when either modelled or error-corrected soil temperatures were used. Mean hatching dates were only significantly different from what would be expected under a uniform distribution at depths below $100 \mathrm{~mm}$ under the scenario of maximum climate warming.

Developmental models run island-wide and using non error-corrected hourly soil temperatures as input predicted that approximately $58 \%$ of sites on Takapourewa would reach at least $35 \%$ of embryonic development, the upper limit of the thermosensitive period (TSP) estimated for tuatara, at a soil depth of $50 \mathrm{~mm}$. The percentage of sites predicted to develop through the TSP decreased with depth to a minimum of $30 \%$ at a depth of $300 \mathrm{~mm}$. CTE values calculated across all depths varied from a minimum of $11.96^{\circ} \mathrm{C}$ at $300 \mathrm{~mm}$ to a maximum of $18.49^{\circ} \mathrm{C}$ at $50 \mathrm{~mm}$. Based on the island-wide mechanistic model, an oviposition date of 30 Nov would produce primarily female hatchlings, regardless of site. However, the current population sex ratio on the island is approximately 1:1 (Nelson et al. 2004b; Moore et al. 2009), and the lowest percentage of male hatchlings observed between 2002-2005 was 22\% (Nelson unpub. data). Thus, the mechanistic predictions generated using the single oviposition date of 30 Nov are highly conservative and likely do not realistically reflect offspring sex ratios on Takapourewa. Of the oviposition dates modelled mechanistically in this study, 15 of Dec may provide the most accurate predictions of hatchling sex ratios for both the 
current climate and minimum warming scenarios. Because of the high computational requirements of modelling development at an extremely high spatial resolution, future research should compare observed and modelled hatchling sex ratios to identify a single oviposition date that can be used as a representative 'nesting onset' for the full spatial extent of the island.

Due to the computational requirements of the microclimate and developmental models used in this study, climate change scenarios were modelled for Takapourewa using geostatistical analysis. A random subset of mechanistically modelled CTEs was interpolated island-wide using ordinary kriging, and predictions were tested independently against a second set of CTEs generated using mechanistically modelled hourly soil temperatures. The interpolated predictions were unbiased and accurately represented variance of CTE values. The RMSD between geostatistically and mechanistically modelled CTEs decreased with increasing soil depth, varying from $2.83^{\circ} \mathrm{C}$ to $0.93^{\circ} \mathrm{C}$ from, respectively, depths of $50 \mathrm{~mm}$ to $300 \mathrm{~mm}$ under the minimum warming scenario. Under the scenario of maximum warming, RMSD values were lower overall, decreasing from $1.16^{\circ} \mathrm{C}$ at a depth of $50 \mathrm{~mm}$ to $0.99^{\circ} \mathrm{C}$ at a depth of $150 \mathrm{~mm}$, but increased to $1.16^{\circ} \mathrm{C}$ at a depth of $300 \mathrm{~mm}$.

The RMSD values between predicted proportions of male hatchlings using mechanistically versus geostatistically modelled CTEs were 0.00 at all soil depths for which a sufficient number of samples was available under the scenario of minimum climate warming, reflecting the finding that few sites were predicted to produce male hatchlings under the minimum warming scenario. Under the scenario of maximum climate warming, the RMSD of predicted sex ratios was minimised at a soil depth of $300 \mathrm{~mm}$ to 0.14 and maximised at a soil depth of $150 \mathrm{~mm}$ to 0.45 . Predicted CTEs and resultant hatchling sex ratios decreased with increasing soil depth from $85 \%$ male at a depth of $50 \mathrm{~mm}$ to $13 \%$ male at $200 \mathrm{~mm}$. The mean proportion of male hatchlings predicted at a soil depth of $300 \mathrm{~mm}$ was 0.00 under the maximum warming scenario. 
The distributions of CTE values predicted mechanistically and geostatistically were significantly different under both minimum and maximum climate warming scenarios. However, the distributions of hatchling sex ratios predicted using separate methods were only different under the scenario of maximum climate warming, again reflecting the dominance of female-producing sites under the minimum warming scenario. Under the scenario of maximum climate warming, predicted hatchling sex ratios were significantly different at soil depths of $50 \mathrm{~mm}, 100 \mathrm{~mm}$, and $300 \mathrm{~mm}$ but not at depths of $150 \mathrm{~mm}$ or $200 \mathrm{~mm}$. Importantly, mean CTEs and sex ratios modelled using geostatistical methods were, on the whole, similar to or slightly higher than mechanistically predicted values under the scenario of maximum climate warming. Thus, the error in geostatistically modelled predictions would likely lead to overestimation, rather than under-estimation, of proportions of male hatchlings in the population of tuatara on Takapourewa

The effects of predicted climate warming were also modelled mechanistically for a subset of 200 known nesting locations and compared with predictions for a set of 200 random points on Takapourewa. Under all three climate scenarios, the distributions of CTEs predicted at nest sites differed significantly from those predicted at random locations at all soil depths. All nest sites were predicted to produce $100 \%$ female hatchlings under both the current and minimum warming scenarios. However, this result may reflect the effects of an oviposition date of $30 \mathrm{Nov}$. Under the scenario of maximum climate warming, all nest sites were predicted to produce between $95-99 \%$ male offspring at depths from $50 \mathrm{~mm}$ to $150 \mathrm{~mm}$, and no sites were predicted to produce all-female hatchlings. At a soil depth of $200 \mathrm{~mm}$, a mean $66 \%$ of hatchlings were predicted to be male, and predicted percentages of males varied from $0-100 \%$. At a soil depth of $300 \mathrm{~mm}$, a mean $8 \%$ of hatchlings were predicted to be male, with between 0 $48 \%$ of hatchlings predicted to be male under the scenario of maximum climate warming. 
Under the current climate and minimum warming scenarios, predicted hatching success decreased with increasing soil depth, and no nesting locations were predicted to facilitate successful hatching at a soil depth of $300 \mathrm{~mm}$. Under the scenario of maximum climate warming, all 200 nest sites were predicted to hatch successfully at all soil depths. Mean hatching dates predicted for nest sites were similar under the current climate and minimum warming scenarios and varied by depth from early austral autumn at a soil depth of $50 \mathrm{~mm}$ to mid austral spring at a depth of $100 \mathrm{~mm}$ and austral summer at depths of $150 \mathrm{~mm}$ and $200 \mathrm{~mm}$. Under the scenario of maximum climate warming, mean hatching dates were predicted to occur in late austral winter at a depth of $50 \mathrm{~mm}$ and throughout austral spring at depths from $100 \mathrm{~mm}$ to $200 \mathrm{~mm}$. The mean hatching date was predicted for austral summer at a soil depth of $300 \mathrm{~mm}$ under the maximum warming scenario.

The effects of shifting oviposition phenology on developmental predictions were examined for a subset of 200 sites for all three modelled climate scenarios. Mean predicted CTEs decreased with depth and were lowest at all depths when the oviposition date was 30 Nov and highest when the simulated oviposition date was 15 Nov. Under the scenario of minimum climate warming, a clear reduction with depth of CTE values was only observed when the oviposition date was 15 Dec. Mean CTE values calculated for all other oviposition dates were similar to values observed under the current climate scenario for the median oviposition date of 30 Nov. Under the scenario of maximum climate warming, a reduction with depth in CTEs was apparent for all seven simulated oviposition dates.

\subsubsection{Assessment of methodology and future directions}

The primary constraint on this study was the computational capacity required to generate hourly microclimate-scale predictions of soil temperatures using NicheMapR at 
an extremely high spatial resolution. Mechanistic predictions could only be generated island-wide for Takapourewa for the current climate scenario. Because predicted incubation temperatures were almost entirely female-producing under both the current climate and minimum warming scenarios, the effects of a scenario of maximum climate warming on incubation temperatures, offspring sex ratios, and hatching phenology could only be examined mechanistically for a small subset of sites. Nevertheless, the combination of mechanistic and statistical modelling methods used in this study provide information on the potential interactive effects of nesting behaviour and changing macro- and microclimate conditions.

Mean CTEs calculated for known nest sites in this study were lower than those reported for natural nests in both 1998-1999 and 2002-2003 (Nelson et al. 2004a); however, previous research has calculated CTE values based on a statistically estimated TSP of 45-55\% (Nelson et al. 2004a), rather than the experimentally determined period of 25-35\% (Mitchell et al. 2009), of total embryonic development, which would result in higher CTEs in warm summers. Likewise, CTEs predicted for both the current climate and minimum warming scenarios were almost entirely female-producing, even at the shallowest depths. In contrast, offspring sex ratios reported in 1999 from nests laid in 1998 on Takapourewa were about $64 \%$ male, and no nests from which hatchlings were sexed were female-biased (Nelson et al. 2004a). Furthermore, the current population sex ratio on Takapourewa is approximately 1:1 (Nelson et al. 2004b; Moore et al. 2009).

The discrepancy between in-situ observations of sex ratios and the island-wide mechanistic predictions generated in this study has several potential, non-mutually exclusive explanations. First, an unknown source of error in the microclimate model may be leading to erroneous predictions at nest sites. However, this possibility seems unlikely, as the microclimate model was parameterised using soil temperature data recorded in historic nesting areas at the approximate mean depth of tuatara nests (Chapter 3). Correcting hourly soil temperatures for known microclimate model error 
increased variance in CTEs under the current climate scenario but had little effect on sex ratio predictions, as predicted CTEs at most locations remained well within the femaleproducing range. Since only the sensitivity of CTEs and sex ratios to negative mean error was examined in this study, the possibility remains that an increase in the variance of soil temperatures might affect physiological predictions.

Alternatively, the nesting season observed in 1998-99 could have been significantly warmer than the macroclimate conditions simulated in this study. Mean annual temperatures in New Zealand in 1998-1999 were the highest observed between $1910-2010$ and were approximately $0.5^{\circ} \mathrm{C}$ higher than the mean air temperature in 2000 (http://www.niwa.co.nz). However, the similarity between modelled CTE values between the current climate and minimum warming scenarios examined in this study suggests that an annual increase in mean air temperatures of $0.5^{\circ} \mathrm{C}$ would not increase predicted CTEs to the extent that hatchling sex ratios would increase from $0 \%$ to $>50 \%$ male. The microclimate model used in this study was parameterised to hourly soil temperature data collected from late 2011, a warmer-than-average year for New Zealand, through 2012 (Chapter 3), which was characterised by relatively mild climatic conditions (http://www.niwa.co.nz). The year 2000, the representative 'current climate' year, was relatively warm as well (http://www.niwa.co.nz); however, the ability of a mechanistic microclimate model to predict soil temperatures in 'warm' versus 'cool' years should not be different.

The method of geostatistical analysis used in this study, ordinary kriging, was chosen for its broad applicability, robustness, (Boer et al. 2001; Apaydin et al. 2004) and ability to account for spatial autocorrelation within a dataset (Cressie 1985, 1988, 1990; Wackemagel 1998; ESRI 2003). A key difference between the variable of interest in this research- CTE values- and climate data (e.g., daily temperature minima and maxima) interpolated via kriging or thin-plate splines in other studies (Hutchinson 1998a ,b; Boer et al. 2001; Hofierka et al. 2002; Apaydin et al. 2004; Hijmans et al. 2005; Perry \& Hollis 
2005; Hutchinson et al. 2009) is that CTEs are not continuous measurements, but single values that act as proxies for a physiological process (i.e., sex determination). Thus, the effects of error on a predictive surface of offspring sex ratios are sensitive to the CTE value itself and will become more severe as the CTE approaches the pivotal temperature(s) of a species, around $22^{\circ} \mathrm{C}$ in tuatara (Mitchell et al. 2006). In addition, predictions of hatchling sex ratios for a species with a very narrow (Ewert et al. 2004) transitional range of temperatures (TRT), about $1^{\circ} \mathrm{C}$ in tuatara (Mitchell et al. 2006), are more likely to be over- or underestimated when the CTE is near or within the TRT.

Error of $-2.0^{\circ} \mathrm{C}$ in interpolated CTE values, for example, would not affect predicted offspring sex ratios in tuatara if the CTE is $17.5^{\circ} \mathrm{C}$, but error of just $-0.5^{\circ} \mathrm{C}$ could shift the predicted sex ratio of a site from primarily male to primarily female if the predicted CTE value is $21.5^{\circ} \mathrm{C}$. For tuatara, because kriging tends to under-predict large values and over-predict small values (ESRI 2003), the number of sites predicted as 'male-producing' (i.e., large CTE values) may be underestimated. For species with TSD type Ia, the number of male-producing sites may be overestimated. An extremely high degree of accuracy may be required to predict hatchling sex ratios in species with TSD type II vie geostatistical interpolation, since either positive or negative error could markedly under-predict proportions of male hatchlings. For this study, the most likely implication of error in CTE values is that the widths of areas predicted to produce mixed-sex nests are narrower than in reality. Rigorous comparison of the abilities of different methods of deterministic or geostatistical analysis to accurately predict CTEs was outside the scope of this study. However, future research would benefit from examining methods of maximising the accuracy of interpolated values, in addition to improving the mechanistic predictions from which geostatistical models are built.

Geostatistical analyses were limited in this study to interpolation of CTE values and were not used to examine hatching phenology. Like microclimate information, spatially explicit data on hatching dates could be interpolated using either geostatistical 
(Gumiaux et al. 2003) or deterministic methods. The error inherent in any surface-fitting function, however, has additional implications for circular data. Interpolation of hatching dates would require development of two models, each trained to a different trigonometric function of the dataset, for every scenario of interest. Separate kriging models could, for example, be fit to the sine and cosine of angular hatching dates. The respective signs of each location in the interpolated surfaces could be used to place the polar coordinates correctly in Cartesian space. Then, the cosine surface could be backtransformed to angular hatching dates and, finally, to days. However, even very small amounts of error (e.g., on the scale of 0.001 ) introduced into polar coordinates during interpolation could lead to error on the scale of months in predictions of hatching phenology (Carter unpub. data). Thus, examining the effects of climate warming on hatching dates was limited to a subset of sites in this study. Nevertheless, this research revealed patterns in the timing of hatching that may be affected by interactions between oviposition phenology and the incubation microenvironment.

This study tested the sensitivity of developmental predictions to mean error (i.e., RMSD) in hourly soil temperatures generated by the microclimate model but did not examine whether predicted sex ratios would be affected by variance in incubation temperatures. However, overall development and incubation periods modelled using the Dallwitz-Higgins development rate function (Dallwitz \& Higgins 1992), used here for tuatara, have been shown to be robust to fluctuations of up to $5^{\circ} \mathrm{C}$ from daily mean incubation temperatures, as long as temperatures do not drop below the developmental minimum (Georges et al 2005). Testing whether variance in hourly soil temperatures affects offspring sex ratios would be beneficial for reducing uncertainty in predictions for future climate scenarios. Depending on the magnitude of climate warming realised over the next few decades, the spatial boundaries between male-producing and femaleproducing areas predicted via geospatial analysis (i.e., areas that produce mixed-sex 
nests) may need to be expanded toward cooler sites to account for error in interpolated CTE values.

Finally, as time spent in-ovo increases, the probability of embryonic mortality may become higher due to temperature-mediated effects on hydric conditions in the egg (Thompson et al. 1996; Refsnider et al. 2009); reduction of energy reserves in the yolk (Angiletta et al. 2000; Mitchell \& Seymour 2000; Mitchell et al 2008); and, especially for tuatara on Takapourewa, to disturbance by nesting females (Refsnider et al. 2009). Success of tuatara nests on Takapourewa is typically < 50\% (Refsnider et al. 2009) and may be even lower at a relatively shallow soil depth of 50mm (Nelson et al 2004b). However, the time-dependent probability of mortality either before or after hatching has not been explicitly examined. Future research should endeavour to quantify how development time affects the probability of mortality both during incubation and after hatching, as total time spent in-ovo may interact with hatching phenology to affect juvenile survival and, potentially, sex ratios.

\subsubsection{Implications for tuatara and other species with TSD}

Predicting hatchling sex ratios is a priority for conservation of species with TSD, particular under possible scenarios of climate change (Janzen 1994b; Hulin et al. 2009; Neuwald \& Valenzuela 2009; Mitchell \& Janzen 2010; Telemeco et al. 2013). The results presented here for the population of tuatara on Takapourewa support the finding for North Brother Island (Mitchell et al. 2008) and the island of Hauturu (Chapter 5) that a scenario of maximum regional climate warming is likely to increase proportions of male hatchlings to levels that threaten population viability. In particular, the differences in offspring sex ratios predicted by the maximum, compared with the minimum, warming scenario in this study are similar to those modelled for other locations. Thus, the magnitude of climate warming observed over the next century will be critical in 
determining the long-term viability of tuatara populations in New Zealand and in guiding future translocations.

Crucially, shifts in nesting phenology (i.e., earlier nesting) are unlikely to affect hatchling sex ratios under a scenario of maximum climate warming. However, offspring sex ratios predicted for Takapourewa in this study decreased with depth, regardless of oviposition date. The mean depth of nests on Takapourewa is $110 \mathrm{~mm}$, though females construct nests at depths of 50-250mm (Cree et al. 1992; Cree 1994; Newman et al. 1994; Nelson et al. 2004a), and nest depth tends to increase with later oviposition dates (Nelson et al. 2004a). Thus, while population sex ratios may become male-biased as air temperatures increase, the magnitude of that bias can be moderated via nesting behaviour, if females select nest locations with friable soils (Mitchell et al. 2008; Refsnider et al. 2010) that are amenable to digging nests to depths $>100 \mathrm{~mm}$. Under a scenario of maximum climate warming, increasing nest depth can reduce proportions of male offspring, even if females nest earlier in the year. In addition, the probability of embryonic mortality is higher in nests at soil depths of $50 \mathrm{~mm}$ or less (Nelson et al. 2004a), so sex-biased survival could reduce numbers of male hatchlings.

The relatively high CTEs and hatchling sex ratios observed in-situ on Takapourewa between 1998-2005 may result from combined effects of topography and habitat modification. The areas utilised as nesting rookeries that are accessible to researchers were comprised primarily of pasture through at least 2007 (Chapter 2), and female tuatara preferentially nested in areas of pasture through at least 2005 (Butler 1989; Thompson et al. 1989; Nelson et al. 2004a; Chapter 2). However, succession of pasture with other vegetation types may have an insulating, rather than a cooling, effect on sub-surface temperatures. The CTEs calculated for known nests in this study were higher than values predicted for random locations, supporting previous findings that females select relatively warm nesting locations (Cree 1994), likely to maximise the probability of successful hatching (Nelson unpub. data). The tendency to select warm 
nesting locations (i.e., male-producing sites) may have also been favoured prehistorically by frequency-dependent selection (Fisher 1930; Bull \& Charnov 1988; Basolo 1994) in cool macroclimates.

In addition, the overall spatial distributions of CTEs and sex ratios predicted for all climate scenarios in this study show that the areas that have been historically utilised heavily as nesting sites by tuatara and that are accessible to researchers also happen to be the warmest areas on the island. However, the relative warmth both of known nest locations and of their surrounding areas found in this study is due not to vegetation but to topography. Thus, relatively higher proportions of male offspring may be produced in those areas, independently of vegetation composition. The estimated adult sex ratio may be similarly biased by the spatial limitations on population sampling. In-situ determination of hatchling sex ratios from more recently constructed nests (i.e., in any nesting season after the succession of heterogeneous vegetation types into former pasture areas) would help elucidate the source of the incongruity between offspring sex ratios modelled in this study and those reported previously. In addition, mark-recapture sampling within areas not currently accessible by researchers would be beneficial for determining whether the female-biased offspring sex ratios predicted in this study more closely reflect adult sex ratios in other areas of the island.

The reported in-situ hatching success of about $48 \%$ on Takapourewa has been attributed to nest disturbance and sub-optimal hydric conditions in nests (Thompson et al. 1996; Nelson et al. 2004a; Refsnider et al. 2009), and completion of embryonic development is known to be restricted to non-forested sites (Thompson et al. 1996). A maximum of $49 \%$ of sites (i.e., at a soil depth of $50 \mathrm{~mm}$ ) were predicted in this study to be thermally suitable for embryonic development under the current climate scenario, suggesting that incubation temperatures themselves may spatially constrain hatching success more than previously thought. Under either the current climate or minimum warming scenarios, forested areas of Takapourewa are likely to remain unsuitable for 
nesting. However, under maximum climate warming, incubation temperatures would not limit successful hatching to non-forested areas. That relatively large areas of Takapourewa are predicted to produce all-female and mixed-sex nests under the scenario of maximum climate warming highlights the importance of considering topographic effects, in addition to soil and vegetation composition, in definitions of habitat suitability for species with TSD.

Tuatara on Takapourewa tend to nest earlier in warmer years (Nelson et al. unpub. data), but the results of this study show that earlier nesting is unlikely to affect hatchling sex ratios under the maximum climate warming scenario. Oviposition is often tightly linked with the abiotic environment, particularly with seasonal temperature cycles, and climate-linked shifts in nesting phenology have been noted in diverse taxa (Visser \& Both 2005; Gienapp \& Visser 2006; Nussey et al. 2007; Parmesan 2007; Both et al. 2009; Mazaris et al. 2012). As has been observed in avian species in the northern hemisphere (Visser \& Both 2005; Gienapp \& Visser 2006; Both et al. 2009), shifts in hatching times, especially to late autumn or winter, could result in a phenological mismatch between tuatara and invertebrate prey abundance (Walls 1983), which could subsequently reduce juvenile growth and survival rates (Mitchell et al. 2008). More work is needed to determine how climate warming is likely to affect seasonal abundance and distribution of invertebrates in New Zealand (Watt et al. 2008). However, both experimental and model-based studies have concluded that variation in oviposition phenology is unlikely to prevent biased sex ratios in species with TSD under a scenario of maximum climate warming (Morjan 2002; Hulin et al. 2006; Mitchell et al. 2008; Schwanz \& Janzen 2008; Telemeco et al. 2009). Elucidating patterns of nesting phenology will likely be more important for monitoring and predicting population sex ratios if the magnitude of climate change observed over the next several decades more closely matches the minimum, rather than the maximum, predicted warming scenario. 
As a set of sex-determining systems, TSD may have been robust to prehistoric climate change (Silber et al. 2010). Theoretically, assuming a thermally heterogeneous environment, if climate warming leads to male-biased population sex ratios, then the fitness of females that tend to produce females; whether by location, depth, or timing; should increase (Fisher 1930; Bull \& Charnov 1988; Basolo 1994), and vice versa. However, if the magnitude of climate change observed over the next century more closely matches the maximum, rather than the minimum, predicted warming scenario, nest site selection may be the primary determinant of population demographics in species with TSD, particularly those that are long-lived (Bradshaw 1965). The results of this study suggest that topographic heterogeneity has important implications for microclimate incubation conditions, independently of either vegetation or soil composition. Indeed, the accuracy of the microclimate model used in this study was increased by inclusion of high resolution geospatial data but not by simulation of spatial variation in soil thermal properties (Chapter 3).

The interactive effects of nesting behaviour and micro-topography have not been well-examined in oviparous species, relative to other behaviour-environment interactions. Limited data on elevation have been collected with a focus on variation among, rather than within, populations. Female Australian water dragons (Physignathus lesueurii) that inhabit an approximately $700 \mathrm{~m}$ elevational gradient, for example, construct deeper nests at lower elevations, likely to maintain similar thermal microclimates in nests (Doody et al. 2006; Doody 2009). Elevation has been examined in patterns of nest site choice in loggerhead (Caretta caretta) and green sea turtles (Chelonia mydas), but only to the extent that increasing elevation from sea level is linked with tidal patterns and, indirectly, with vegetation composition (Hays et al. 1995).

Definitions of habitat suitability for species with ESD may benefit from explicit inclusion of micro-topographic heterogeneity, especially if latitudinal climate shifts are likely to facilitate increasingly biased sex ratios in a species of interest. Understanding 
the geographic boundaries of micro-topographic effects would be particularly beneficial for decision making in conservation-based translocations of species, such as those with ESD, that experience life stage-dependent effects of their environments. Under a maximum warming scenario of climate change, any potential translocation site, even those within a species' former range, may be a 'new' habitat due to climate-mediated shifts in available micro-environments. Growth and, consequently, survival of juvenile Australian western swamp tortoises (Pseudemydura umbrina), for example, increases with hydroperiod length (Mitchell et al. 2013a). Warming macroclimate conditions may provide additional basking opportunities for adults but will likely reduce the length of hydroperiods and detrimentally impact recruitment (Mitchell et al. 2013a). Thus, modelling variation both in temperature and water availability (e.g., rainfall, absorption, runoff) at a micro-environmental spatial scale is critical for optimising habitat selection for conservation of the species under climate change.

For species in which the microenvironment can differentially affect adults and developing embryos, neither the adult fundamental niche nor data on nest site selection may be a reliable predictor of offspring phenotype, including hatchling sex, or fitness. At relatively low latitudes within the geographic limits of a species' fundamental niche, for example, macroclimate effects may outweigh the ability of microhabitat characteristics (e.g., canopy shading) to mediate the incubation and post-hatching micro-environments, especially temperature and hydric conditions. However, at slightly higher latitudes, complex topography may produce a greater variety of potential incubation environments than would otherwise be facilitated by vegetational heterogeneity alone. Spatially explicit microclimate models, especially mechanistic models that are implemented at a high spatial resolution throughout a species' potential geographic range, can be used to define the latitudinal limits of suitable future habitats for species that are affected by distinct, life stage-dependent niches. 


\section{Chapter 5}

\section{Incubation suitability surfaces indicate hypothetical nesting locations and offspring sex ratios for a reintroduced population of tuatara}

\subsection{Introduction}

\subsubsection{Mechanistic modelling in conservation translocations}

Assessment of habitat suitability is a key component of reintroduction biology (Lomolino \& Channell 1998; Armstrong \& Seddon 2008; Osborne \& Seddon 2012; IUCN 2013), especially if the principal goal of a translocation is species-targeted conservation (i.e., either reintroduction or assisted colonisation) (Seddon 1999, 2010; HoeghGuldberg et al. 2008; Seddon et al. 2012). Models of habitat suitability, whether quantitative (Keith et al. 2008; Thuiller et al. 2008; Willis et al. 2009; Fouquet et al. 2010; Seddon 2010; Ledig et al. 2012) or qualitative (Kreyling et al. 2011; Osborne \& Seddon 2012), have largely been synonymous with bioclimatic envelopes (i.e., correlative species distribution or niche models) that are built from presence/absence data used to define whether a location is suitable or unsuitable for a population. Species for which translocation is a feasible conservation strategy, however, are likely to be rare or geographically restricted (Porter et al. 2006; Seddon et al. 2007; Miller et al. 2012; Osborne \& Seddon 2012; Mitchell et al. 2013a), limiting the amount of presence/absence data that can be collected.

Regardless of the availability of distribution data, whether a species can persist in any location (Seddon 1999) cannot be reliably inferred from its presence or absence in either past or current habitats (Seddon 2010; Osborne \& Seddon 2012), especially since rapid climate change and habitat modification are likely to facilitate shifts in species' ranges (Porter et al. 2000; Kearney \& Porter 2004; Loss et al. 2011; Regan et al. 2012; Gillson et al. 2013). Models based on organisms' physiological and behavioural 
constraints (i.e., mechanistic or process-explicit niche models) are increasingly being used to examine habitat suitability under climate change scenarios, independently of past or present distributions (Porter et al. 2000; Kearney \& Porter 2004, 2009; Buckley et al. 2008, 2010). Process-explicit niche models have also been used to describe hypothetical distributions of a presumed-extinct Hawaiian bird (Melamprosops phaeosoma) (Porter et al. 2006). Similarly, a recent study combined mechanistic microclimate, eco-hydrological, and biophysical models to examine locations for assisted colonisation of the critically-endangered Australian western swamp tortoise (Pseudemydura umbrina) under predicted scenarios of climate change (Mitchell et al. 2013a).

Quantitative data on habitat suitability are becoming progressively more important, not only for identification of appropriate release sites for translocations (Ledig et al. 2012; Osborne \& Seddon 2012; IUCN 2013; Mitchell et al. 2013a) but also for post-release monitoring of translocated populations (Walters 1986; Williams 2001; Carrete et al. 2006; Lyons et al. 2008; Cook et al. 2010; Armstrong \& Reynolds 2012; Osborne \& Seddon 2012; IUCN 2013). When a habitat is 'new,' relative to a population's current habitat, whether because a translocation has been undertaken outside of a species' previous range or because of environmental shifts within the current range, or due to a lack of fundamental physiological data, post-release monitoring can benefit from descriptions of an organism's niche that do not rely on presence/absence data. Mapping habitat suitability independently of known distributions can provide a means for examining the spatial-environmental constraints on translocations, including the potential for post-release dispersal (Le Gouar et al. 2012) and, in oviparous species, nesting migration and selection of suitable nest sites. 


\subsubsection{Behavioural hypothesis-building using a nest site suitability surface}

For translocated populations of species with temperature-dependent sex determination (TSD), predicting the ability of a new site to support embryonic development and maintain balanced hatchling sex ratios is as important as modelling suitability of habitat for adult individuals. Development of accurate, species- or population-specific predictions of sex ratios requires (1) understanding of the basic functional relationship between temperature and embryonic development (e.g., the timing of gonadal differentiation), which links developmental physiology with the incubation environment; (2) spatially-explicit soil temperature data, which link development functions to a particular habitat; and (3) knowledge of the spatial patterns of nest site selection, which determine which set of incubation conditions are actually experienced by embryos in the wild.

Mathematical functions describing the effects of incubation temperature on the rate of embryonic development and hatchling sex ratios are derived for a species using controlled incubation experiments carried out in a laboratory environment (Mrosovsky \& Pieau 1991; Dallwitz \& Higgins 1992; Girondot 1999; Godfrey et al. 2003; Georges et al. 2005). Accurate (i.e., within $1-3^{\circ} \mathrm{C}$ ) soil temperature data can be generated at multiple spatial scales using either empirical (Horton \& Corkrey 2011) or mechanistic (Kearney et al. 2014a, b; Chapter 3) climate models, which reduces the dependence of population monitoring on the capacity for collection of in-situ environmental measurements. In contrast, while the proximate causes of nest site selection can be examined in a controlled environment, linking nest site selection and soil temperatures in wild populations requires in-situ observation, either of nesting adult females or of nests. Conservation of species with TSD need not rely solely on in-situ monitoring of nesting behaviour and hatchling sex ratios, particularly if no preliminary data are available for a particular population. If no reliable data on nesting are available for a translocation site, 
a mechanistic description of nest-site suitability may provide either (1) a preliminary means of assessing the ability of a proposed translocation site to support embryonic development and to maintain balanced offspring sex ratios or (2) an hypothesis-based means of locating nesting habitat post-translocation and, subsequently, monitoring reproduction in a translocated population.

Reintroduction has been relatively successful in the establishment of new populations of tuatara (Sphenodon punctatus), a cold-adapted (Barwick 1982), New Zealand-endemic reptile with TSD (Gaze 2001; Blanchard 2002; Towns et al. 2007; Nelson et al. 2002; Miller et al. 2012). Ensuring long-term success of translocations using standard methods of population monitoring, however, is problematic. First, tuatara are extremely long-lived (Dawbin 1982) and have a generation interval of around 40 years (Mitchell et al. 2009). Breeding and, ultimately, persistence of a translocated population of tuatara (i.e., a 'successful' translocation) occurs on a protracted temporal scale (Miller et al. 2012). Second, sufficient latitudinal data on nesting phenology in wild populations are not currently available, and the timing of nesting tends to shift in response to shortterm climate patterns (Nelson unpub. data). Contrary to expectations, for example, female tuatara on northern islands have tended to nest later than those resident on islands in the Cook Strait (Tyrrell et al. 2000).

The proximate causes of nest site selection (i.e., specific nesting 'cues') in tuatara are largely unknown, though female tuatara on one island in the Cook Strait; Takapourewa, or Stephens Is.; choose nest sites based at least partially on conspecific cues (Refsnider et al. 2010). In addition, females on Takapourewa are known to migrate from their home territories to preferred nesting locations (Cree 1994; Thompson et al. 1996; Doody et al. 2009) and tend to travel laterally or downslope, presumably minimising energy expenditure (Refsnider et al. 2010). The density of home burrows in forested areas on Takapourewa/Stephens Is. is extremely high, though adjacent burrows may be nearly adjoining or separated by as much as $15 \mathrm{~m}$ (Newman 1987). While male 
tuatara, especially, maintain and defend small territories (Newman et al. 1987), the high density of burrows on Takapourewa likely results both from the large population size and the limited availability of the forested habitat preferred by adults (Newman 1982, 1987).

Though female tuatara on Takapourewa migrate to locate suitable nest sites, whether adult habitat in latitudinally dissimilar locations is suitable for nesting has only been studied experimentally at one location on the South Island of New Zealand, though research so far has been limited to recording of in-situ soil temperatures within a small spatial extent (Besson et al. 2012). Suitability of a habitat for adult individuals may not be a reliable proxy for either nest site suitability or the production of sufficient numbers of both male and female offspring. Ultimately, neither the timing of nesting nor, especially, the probable location of nests on can be reliably predicted for translocated or otherwise under-studied populations with the data currently available.

The ability to predict whether balanced population sex ratios can be maintained in the face of climate change is of paramount importance to conservation of species with TSD (Janzen 1994a; Hulin et al. 2006; Mitchell et al. 2009; Telemeco et al. 2013), including tuatara. Sphenodon were prehistorically widespread in New Zealand (Jones et al. 2009), and the evolutionary ancestor of tuatara likely survived in New Zealand throughout periods of substantial climatic upheaval (Jones et al. 2009), including a global $7^{\circ} \mathrm{C}$ increase and $8^{\circ} \mathrm{C}$ reduction in air temperatures, shifts that occurred over intervals of $\sim 15$ million years during the Miocene period (Zachos et al. 2001). Uniquely among species with TSD, incubation temperatures above around $22^{\circ} \mathrm{C}$ produce $100 \%$ male offspring in tuatara (Nelson et al. 2004; Mitchell et al. 2006). As a result, the unprecedented magnitude and rate of climate warming predicted for the next 50-100 years place populations of tuatara at direct risk of severe male bias. 


\subsubsection{Reintroduction of tuatara to Hauturu}

Te Hauturu-o-Toi $\left[36^{\circ} 12^{\prime} \mathrm{S} 175^{\circ} 07^{\prime} \mathrm{E}\right]$, also known as Little Barrier Island or simply Hauturu, is a 3100-ha island located at the northern boundary of the Hauraki Gulf in New Zealand (Fig. 1) (Hamilton 1961). Hauturu became the first Nature Reserve in New Zealand in 1978, and the island has been utilised as a sanctuary for endangered native birds, including North Island brown kiwi (Apteryx mantelli), kākāpō (Strigops habroptilus), and North Island saddleback (Philesturnus carunculatus) since at least 1881, despite the presence of feral cats through 1980 and Pacific rats (Rattus exulans, also known as kiore or Polynesian rats) through 2004 (Dodd 2007; Hamilton 1961). The southern one-third of the island was selectively logged for kauri (Agathis australis) in the late 1800s, though deforested areas had been colonised with young forest (e.g., manuka, Leptospermum scoparium) by at least the 1960s (Hamilton 1961). Exceptionally among the offshore islands of New Zealand, Hauturu has not been extensively modified by grazing mammals and, as a result, is the only large forested area in New Zealand that remains relatively undisturbed. Compared with that on other islands in New Zealand, the forest environment of Hauturu is also unusually complex (Hamilton 1961; Dodd 2007), due at least in part to the relatively steep altitudinal and, thus, environmental, gradient from sea-level to the island's summit at $722 \mathrm{~m}$.

The small population of tuatara on Hauturu, likewise, has a complex history. Hauturu may have been connected to other offshore islands or the New Zealand mainland during the last glaciation (Godwin et al. 1958; Hamilton 1961). If so, tuatara could have dispersed to Hauturu as recently as 13,000 years ago. Infrequent observations of tuatara have been documented on the island from the 1880s (Reischek 1885); however, due to the presence of invasive mammals, especially Pacific rats, the population was presumed extinct from the early 1980s until 1990 (McCallum \& Harker 1982; Daugherty et al. 1990). In 1991 - 1992, two surveys undertaken prior to a rat- 
eradication operation located and captured four adult male and four adult female tuatara. The captured individuals were subsequently housed in a semi-natural, predator-resistant enclosure at Te Maraeroa (Whitaker \& Daugherty 1991; Whitaker 1993; Keall et al. 2010), a relatively flat, low-lying area in the southwestern part of the island (Hamilton 1961).

Egg clutches laid inside the enclosure between 1992 - 2007 were removed and incubated at Victoria University of Wellington at constant temperatures of $20^{\circ} \mathrm{C}$ and $23^{\circ} \mathrm{C}$ to produce approximately equal numbers of, respectively, female and male hatchlings $(\mathrm{N}=152)$ (Keall 2010). Small differences in early incubation regimes and hatchling survival among years led to a slight female bias (Moore et al 2008). Juveniles were initially released into the captive enclosure on Hauturu (Keall et al. 2010), then reintroduced to locations near the adult capture sites after reaching a minimum snoutvent length of 120mm (Nelson et al. 2002, 2004a; Keall et al. 2010). Tuatara reach sexual maturity at 11-13 years of age on Takapourewa/Stephens Is., though individuals only nest every 2-5 years, on average (Cree et al. 1992; Cree 1994); however, tuatara from at least one northern population reach sexual maturity earlier, at 8-10 years of age (Newman et al. 1994). Nesting of the F1 generation has been recorded in the captive enclosure on Hauturu six times between 2003-2012 for females ranging in age from 916 years, though maternal identity has only been confirmed for one 16-yr-old female observed nesting in 2010 (S. Keall pers. comm.). In addition, surveys in the early 1990s may not have successfully captured every remaining adult tuatara (N. Nelson pers. comm.). Nesting in the wild population on Hauturu, therefore, is probably occurring. Compared to the relatively well-studied populations in the Cook Strait, however, seasonal monitoring of nesting in the wild population of tuatara on Hauturu would be costly in terms of both time and resources.

The scant historical records of tuatara on Hauturu include no information on nesting locations or on the 'natural' sex ratio on the island. While equal numbers of adult 
male and female tuatara were captured prior to the rat eradication, the sex ratio of the population prior to mammal invasion cannot be reliably inferred from eight individuals. Likewise, the sex ratio of the released juveniles was manipulated through artificial incubation. Because tuatara have not been observed nesting outside of the enclosure on Hauturu, both the preference of females for specific nesting habitats and soil temperatures in those habitats are unknown. Although juvenile tuatara were released on Hauturu near the capture sites of adults, cues (e.g., eggshell fragments or other evidence of previous nest building) are unlikely to be present in historic nesting locations. Any wild adult females that evaded capture in the 1990s may be highly dispersed, and would be unlikely to provide active nesting cues for the released females. The lack of un-forested areas on Hauturu also reduces any expectation that females would nest colonially. Finding locations in which females are likely to construct nests and, following, the sex ratios of offspring hatched in those sites, requires information on the suitability of microsites for embryonic development.

Female tuatara on smaller, higher-latitude islands migrate to relatively unvegetated areas to nest (Thompson et al. 1989; Butler 1989; East et al. 1995; Chapter 2). Notably, soil temperatures in forested areas on the island of Takapourewa are not warm enough to facilitate completion of embryonic development (Cree et al. 1989) under the current climate (Chapter 4). While few areas of Hauturu are un-forested, air temperatures on the island are approximately $4^{\circ} \mathrm{C}$ warmer, on average, than air temperatures recorded on Takapourewa (http://www.cliflo.niwa.co.nz).

\subsubsection{Study aims and research questions}

The aim of this study was to develop a microclimate-scale map of nest site suitability for a reintroduced population of tuatara to answer the questions: (1) what locations are likely to facilitate completion of embryonic development and maintain 
balanced sex ratios under the current climate? (2) How far away from release sites will female tuatara have to migrate to ensure eggs hatch? (3) Is climate warming likely to affect suitability of locations for nesting or the maintenance of balanced offspring sex ratios in the population of tuatara on Hauturu?

\subsection{Methods}

\subsubsection{Developing the microclimate model}

Soil temperatures were modelled for the island of Hauturu using the NicheMapR v 2011b global microclimate model (Kearney et al. 2014), which was modified to allow input of local, high-resolution geospatial and climate information and validated against hourly temperature data collected at the study site. Parameterisation of the model required site-specific geospatial data, values of soil physical and thermal properties, availability of shade, and daily climate information. The programme also incorporates scattering of solar radiation by atmospheric aerosols using a modified version of the Global Aerosol Data Set (GADS) (Koepke et al. 1997; Kearney et al. 2014).

Values for latitude, longitude, and geospatial parameters (i.e., elevation, slope, aspect) (Appendix 5.1) were extracted to points $(\mathrm{N}=1,102,342)$ using a digital elevation map (DEM) interpolated at a $5 \mathrm{~m}$ horizontal resolution from LiDAR points (Auckland Regional Council, Auckland, NZ) using ArcMap ${ }^{\text {TM }}$ Desktop v 10.1 software (Environmental Systems Research Institute [Esri] ArcGIS: Redlands, CA, USA 2012). To correct solar radiation calculations for the topographic shading, twenty-four horizon angles were calculated for each pixel of the DEM using the r.horizon function in GRASS v 7.0 (GRASS Development Team 2013). Two sets of sites were generated: (1) a set of points at which in-situ temperature data were collected for model validation $(\mathrm{N}=21)$ and $(2)$ a set of points sampled across the whole island on a $10 \times 10 \mathrm{~m}$ grid $(\mathrm{N}=307,801)$ for creation of an incubation suitability surface. 
Hauturu is an extinct andesite volcano, and the steep topography of the island facilitates a high rate of soil erosion (Hamilton 1961). In addition, stable soils are covered by a layer of decomposing organics (Hamilton 1961), which are less conductive of heat energy than mineral soils (de Vries 1963; O'Donnell et al. 2009). Soil physical and thermal properties were determined empirically for ten soil types and type composites present on Hauturu (Hamilton 1961). Locations for soil sampling were defined by manually georeferencing a hard-copy map of soil types (Hamilton 1961) to a $0.5 \mathrm{~m}$ resolution aerial photo of the study location (Land Information New Zealand Data Service, http://www.data.linz.govt.nz). While soils on Hauturu are classified into 22 types (Hamilton 1961), the size of Hauturu and difficulty of the terrain limited this study to ten composite steepland soils and brown granular clays accessible on the southern third of the island (Appendix 5.2). One sample of each type was collected during 24 April - 5 May 2012 at a random point at least 20m inside each soil polygon and within 50m of an access track to avoid unnecessary damage to island vegetation. Values for bulk density, specific heat, and fractional soil moisture were determined in a climatecontrolled laboratory (Geotechnics Ltd., Auckland, NZ) from 7-11 May 2012. Clay content was estimated as $20 \%$ for all soil types using textural classification (Shirazi \& Boersma 1984; Hendrickx et al. 2003; FAO 2006).

The organic component of each sample was estimated based on similarity to empirical values of percent-carbon obtained from temperature-sampling locations (Landcare Research, Palmerston North, NZ). Specific heat $\left(\mathrm{MJ} / \mathrm{m}^{3}-\mathrm{K}\right)$ of the mineral component of soil types was estimated by calculating the volumetric specific heat of each soil sample and solving for the mineral component, given the volume fractions of organics and water (de Vries 1963; Campbell 1985; Campbell \& Norman 1998). Soil reflectance was estimated using the Munsell colour values (Escadafal 1989; Post 2000) previously reported for soils on Hauturu (Hamilton 1961) (Appendix 5.2). Soil properties were averaged across sampling locations to generate a single set of values 
representing Hauturu soils with a bulk density of $1090 \mathrm{~kg} / \mathrm{m}^{3}$, mineral specific heat of $904 \mathrm{~J} / \mathrm{kg}-\mathrm{K}$, and fractional soil moisture content of $0.62 \mathrm{~m}^{3} / \mathrm{m}^{-3}$. Whether inclusion of local soil properties improved model accuracy was examined by simulating a generalised organic soil with a bulk density of $1400 \mathrm{~kg} / \mathrm{m}^{3}$, specific heat of $870 \mathrm{~J} / \mathrm{kg}-\mathrm{K}$, and soil fractional moisture content of $0.30 \mathrm{~m}^{3} / \mathrm{m}^{-3}$ (de Vries 1963; Campbell 1985; Campbell \& Norman 1998; Kearney et al. 2014). Volumetric water content at saturation was estimated as $0.26 \mathrm{~m}^{3} / \mathrm{m}^{3}$; surface roughness was estimated as $0.004 \mathrm{~m}$ for all simulations (Kearney et al. 2014a).

Daily observations of rainfall (resolution $0.1 \mathrm{~mm}$ ), relative humidity (resolution $1 \%$ ), minimum and maximum air temperatures (resolution $0.1^{\circ} \mathrm{C}$ ), and minimum and maximum wind speeds (resolution $0.1 \mathrm{~m} / \mathrm{s}$ ) for the study period were obtained from the NIWA CliFlo database (http://www.cliflo.niwa.co.nz) for the weather station at Warkworth, New Zealand [Station No. 17838], approximately 42km SW of Hauturu, the nearest station from which the required data types were available for 2011. Cloud cover for the study period was calculated as the difference between maximum possible (i.e., out of 24 hours) and observed daily sunshine hours, expressed as a percentage, using sunshine data from the weather station at North Shore, Albany, Auckland [NIWA Station No. 37852], about 66km SSW of Hauturu (Appendix 5.3).

\subsubsection{Testing the microclimate model}

Temperatures were collected hourly from 1 January to 31 December 2011 using 21 temperature dataloggers (Onset $\mathrm{HOBO}^{\mathrm{TM}}$ Pro v2 U23-002) installed at $0 \mathrm{~mm}$ and $100 \mathrm{~mm}$ below the soil surface at two random points within each of ten random plots stratified by elevation and from one site at Te Maraeroa (Fig. 1). One year of hourly soil temperatures were generated for the validation set using two different model configurations, both of which were parameterised using microsite-scale geospatial data 
and daily climate information for 2011: (1) 'soils_1' - a set of models run using generalised soil parameters; (2) 'soils_2' - a set of models run using local soil parameters. Both baseline models were run with and without simulation of a $50 \mathrm{~mm}$ organic soil cap with a lower thermal conductivity and higher specific heat ('cap') and with and without simulation of evaporative surface cooling ('pctwet'). Hauturu is almost entirely forested (Hamilton 1961); however, the accuracy of a previous high-resolution estimates of soil temperatures generated using NicheMapR was higher under the assumption of $0 \%$ shade than under realistic, ground-level shade (Chapter 3 ). Analysis of hemispherical photography collected at the site of each datalogger (see Chapter 2) found that ground-level shade was approximately 95\% at all sites. Thus, both sets of models were run for two scenarios of simulated canopy shading: 0\% ('open') and 95\% ('forest').

The ability of each model to predict observed values was assessed using summary statistics. Values for the coefficient of determination $\left(\mathrm{R}^{2}\right)$, root-mean-squared deviation (RMSD), and normalised root-mean-squared deviation (nRMSD) were calculated for each comparison between predicted hourly soil temperatures and corresponding observed values at each datalogger location. RMSD was calculated using the 'rmse' function in $\mathrm{R}$ package hydroGOF (Zambrano-Bigiarini 2014). Normalised RMSD was calculated as the RMSD value divided by the range of observed hourly soil temperatures (i.e., maximum temperature - minimum temperature) for each comparison between predicted and corresponding observed values (Horton \& Corkrey 2011; Kearney et al. 2014a, b). The accuracy of previous implementations of NicheMapR, all within around $2-3^{\circ} \mathrm{C}$ of observed soil temperatures (Kearney et al. 2014a, b; Chapter 3), was used as a benchmark for assessing the quality of models developed in this study. The most accurate microclimate model was used to develop gridded surfaces of nest site suitability and offspring sex ratios. 


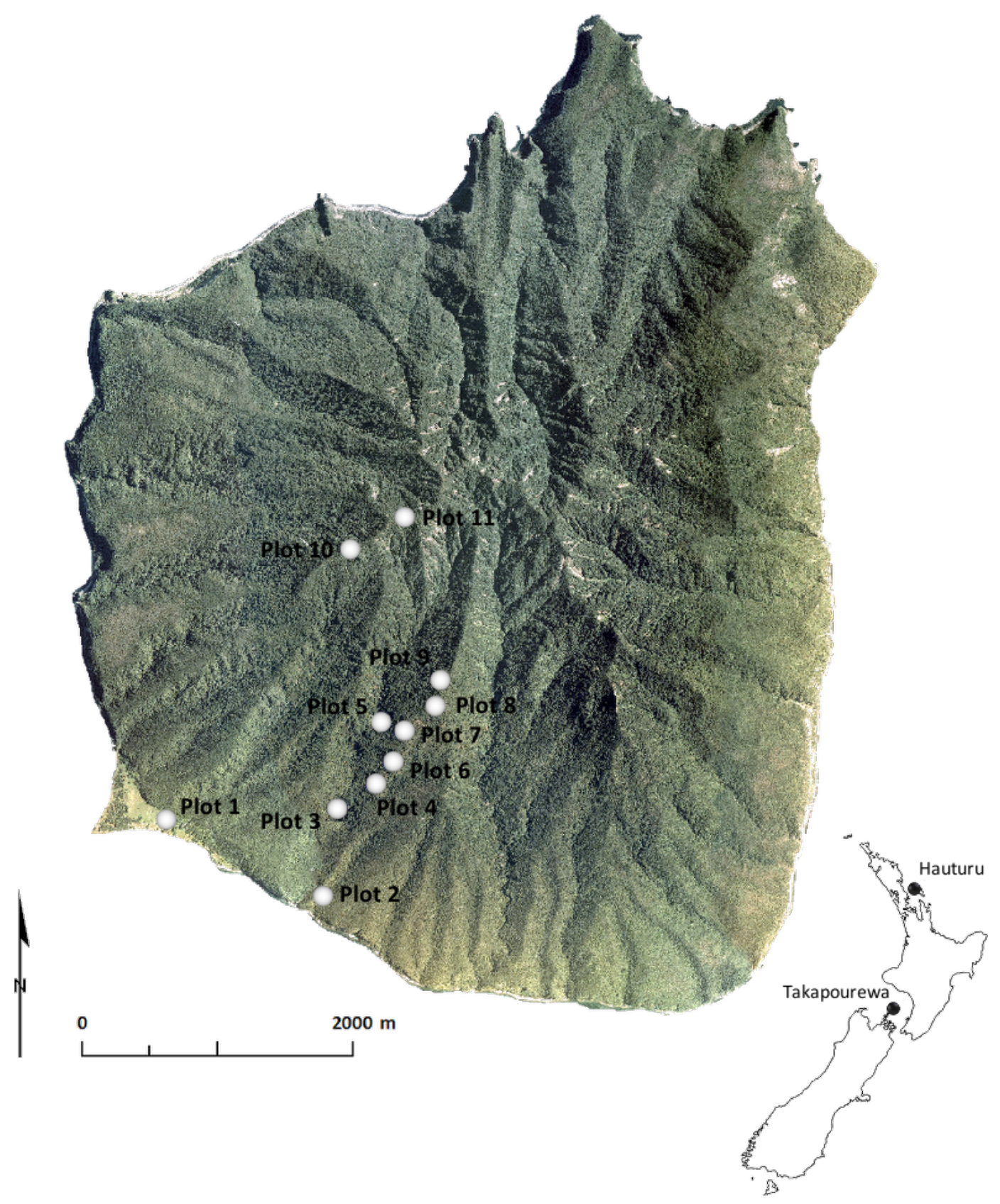

Figure 1. Aerial photograph (LINZ) showing locations of soil temperature sampling on Hauturu, numbered by ascending elevation. Plot 1 is located on Te Maraeroa flat. Black dots on the map of NZ show the locations of Hauturu and the island of Takapourewa, which is home to a large, wellstudied population of tuatara. 


\subsubsection{Creating a nest site-suitability surface}

The relationship between incubation temperature and embryonic development has not been directly examined for the population of tuatara on Hauturu; however, constant incubation of eggs from Hauturu alongside clutches from two well-studied populations suggests temperature-dependent embryonic development and sex determination in tuatara from Hauturu are similar to patterns observed in the population on the island of Takapourewa in the Cook Strait (Mitchell et al. 2006). Female tuatara on Takapourewa nest at depths from 50-250mm (Cree et al. 1991; Thompson et al. 1996). Hourly development rates were estimated from soil temperatures at depths of $50,100,150,200$, and $300 \mathrm{~mm}$ using the Dallwitz-Higgins development rate function (Dallwitz \& Higgins 1991; Georges et al. 2005) defined for tuatara on Takapourewa (Mitchell et al. 2008; Chapter 4). Oviposition has occurred in the captive enclosure on Hauturu as early as 11 November and as late as 1 April, though most dates have varied from late November to early December (Keall unpub. data). An oviposition date of 30 November was used in this study to provide a direct comparison with the median oviposition date simulated on Takapourewa (Chapter 4). Calculated hourly development was accumulated from oviposition until hatching, defined as the first day on which total development $\geq 100 \%$. A point was classified as suitable for nesting if (1) development reached $100 \%$ within 24 months (Cree et al. 1989) and (2) no hourly temperature prediction exceeded a conservative maximum thermal tolerance of $38^{\circ} \mathrm{C}$ (Nelson et al. 2004; Mitchell et al. 2006, 2008). Any point not meeting both suitability criteria was classified as a non-viable nesting location.

The constant-incubation temperature equivalent (CTE) was calculated at each suitable nesting location for the window encompassing $25-35 \%$ of total embryonic development, the approximate period during which sex is determined in tuatara (Nelson et al. 2010). Soil temperatures within the TSP were re-ordered by ascending hourly 
development rate, with the CTE defined as the developmental median during that window. Each CTE was converted to an estimate of offspring sex ratio using the Alogistic function (Girondot 1999; Godfrey et al. 2003) derived for the population of tuatara on Takapourewa/Stephens Is. (Mitchell et al. 2006, 2008). All points on Hauturu were defined by thermal suitability for embryonic development (i.e., 'hatched' vs. 'not hatched' within 24 months). Predicted hatching dates were categorised into months, regardless of whether hatching was predicted to occur in the first or second year of incubation. The probability of hatching was assumed to be equal across days and in both incubation years. Rao's test (Rao 1969, 1972) was used to determine whether mean hatching dates were significantly different from what would be observed under a uniform circular distribution (Jammalamadaka \& Sengupta 2001; Berens \& Velasco 2009) at each depth and under all three modelled climate change scenarios. Sites at which hatching was predicted to occur were further described by the predicted offspring sex ratio.

Juvenile tuatara were reintroduced into the wild on Hauturu into preconstructed, marked burrows that are still being utilised by released individuals (pers. obs). Release sites were visited in 2011 and 2012, and locations of burrows were marked as GPS points (Garmin Oregon ${ }^{\circledR}$ 550, Garmin Limited, USA). To determine whether migration would be necessary for selection of suitable nest sites on Hauturu, the two areas containing release burrows were overlaid onto the GIS surface of nest site suitability and buffered by a $20 \mathrm{~m}$-radius polygon (i.e., equivalent to 2 pixels outside of the burrows furthest from the centre of a release area) to provide a rough representation of the home territories of released individuals, including allowance for some dispersal.

The mean surface-corrected distance that female tuatara migrate to locate nesting sites on Takapourewa (Stephens Is.) is $86 \mathrm{~m}$, though females have been observed traveling nearly 400m (Refsnider et al. 2012). From the perspective of field research, the 
difficulty of navigating the steep, densely vegetated terrain of the study site, especially at night, could reduce the chance of locating nesting females within such a large area. To increase the prospect of observing nesting female tuatara, search areas were restricted to within a more realistic $200 \mathrm{~m}$ of release sites. Buffers of $50 \mathrm{~m}, 100 \mathrm{~m}$, and $200 \mathrm{~m}$ were generated around each home territory polygon to provide a guide for search area perimeters. To account for the difference in effort required to travel up- versus downslope, path distance analysis was used to generate a surface of topographically corrected and, thus, minimum travel distances between the two tuatara release sites and all suitable nesting locations within the $200 \mathrm{~m}$ polygon. The mean depth of nests on Takapourewa/Stephens Is. is about $110 \mathrm{~mm}$ (Cree et al. 1992), so suitability was defined based on successful hatching at the most similar modelled soil depth of $100 \mathrm{~mm}$.

\subsubsection{Modelling effects of climate change on nest site suitability}

Climate change scenarios were chosen based on the most recently available minimum and maximum seasonal warming projections for the Hauraki Gulf from the period 1980-1999 to 2080-2099 (MFE 2008), generated by down-scaling global predictions for the same period (IPCC 2007). To model the minimum projected warming scenario, input monthly minimum and maximum air temperatures were increased seasonally by the predicted range of $0.4-0.8^{\circ} \mathrm{C}$, with an increase of $0.4^{\circ} \mathrm{C}$ in the meteorological austral spring, a $0.6^{\circ} \mathrm{C}$ increase in autumn, a $0.5^{\circ} \mathrm{C}$ increase in winter, and an increase of $0.8^{\circ} \mathrm{C}$ in summer. To model maximum warming, monthly minimum and maximum temperatures were increased by the predicted range of $5.4-6.5^{\circ} \mathrm{C}$, with an increase of $5.4^{\circ} \mathrm{C}$ in the austral spring, a $5.9^{\circ} \mathrm{C}$ increase in autumn, a $5.5^{\circ} \mathrm{C}$ increase in winter, and a $6.5^{\circ} \mathrm{C}$ increase in summer (MFE 2008). Climate change scenarios were modelled for the entire island. 
All modelling was conducted in R v 3.0.3 software (R Development Core Team 2008), and run using the Victorian Life Sciences Computation Initiative (VLSCI) Peak Computing Facility at the University of Melbourne, Australia. Geospatial analyses were conducted in $\operatorname{ArcMap}^{\mathrm{Tm}}$ Desktop v 10.2 software (Environmental Systems Research Institute [Esri] ArcGIS: Redlands, CA, USA 2013) and GRASS v 6.4 (GRASS Development Team 2012). Data on hatching phenology were summarised using the 'mean.circular' and 'rao.spacing.test' functions in R package circular (Lund \& Agostinelli 2013). Figures and maps were generated in, respectively, $\mathrm{R}$ and ArcMap and edited using GNU image manipulation program (GIMP Development Team 2013).

\subsection{Results}

\subsubsection{Model selection}

The most accurate predictions of hourly soil temperatures at a depth of $100 \mathrm{~mm}$ and at the soil surface were obtained using the 'soils_2' model that included simulation of 95\% canopy shading and an organic soil cap. Overall, including local soil properties in the microclimate model ('soils_2') improved the accuracy of hourly soil temperatures over those generated using values for a generalised soil ('soils_1'). Predicted surface temperatures (i.e., at $0 \mathrm{~mm}$ ) were less accurate than those modelled at a depth of $100 \mathrm{~mm}$ for all models. Simulation of 95\% canopy shading ('forest') generated more accurate predictions than the 'open' models at both $0 \mathrm{~mm}$ and $100 \mathrm{~mm}$. Simulation of evaporative surface cooling ('pctwet') improved the accuracy of temperature predictions generated at the soil surface but not at $100 \mathrm{~mm}$. Inclusion of a $50 \mathrm{~mm}$ organic soil cap ('cap') improved the accuracy of predicted soil temperatures compared to those that did not include 'cap'; however, including both 'pctwet' and 'cap' in a single model decreased accuracy compared to that of models including only 'cap' (Fig. 2). 
The range of RMSD values among all sites was $0.76-6.99^{\circ} \mathrm{C}$ at $100 \mathrm{~mm}$ and 3.18 $12.84^{\circ} \mathrm{C}$ at $0 \mathrm{~mm}$. Mean RMSD values between observed and predicted hourly soil temperatures across all sites varied between $1.63-4.51^{\circ} \mathrm{C}$ at $100 \mathrm{~mm}$ and between 3.84 $9.16^{\circ} \mathrm{C}$ at $0 \mathrm{~mm}$. The normalised RMSD of the most accurate model, the implementation of 'soils_2' with 'cap,' was within a mean $15 \%$ of observed values at $100 \mathrm{~mm}$ and within approximately $30 \%$ of observed values at $0 \mathrm{~mm}$. The $\mathrm{R}^{2}$ of the best overall model was 0.85 at $100 \mathrm{~mm}$ and 0.36 at $0 \mathrm{~mm}$ (Fig. 2). The 95\% shading scenario of the 'soils_2 + cap' implementation was used to predict soil temperatures for the whole-island set of points. 


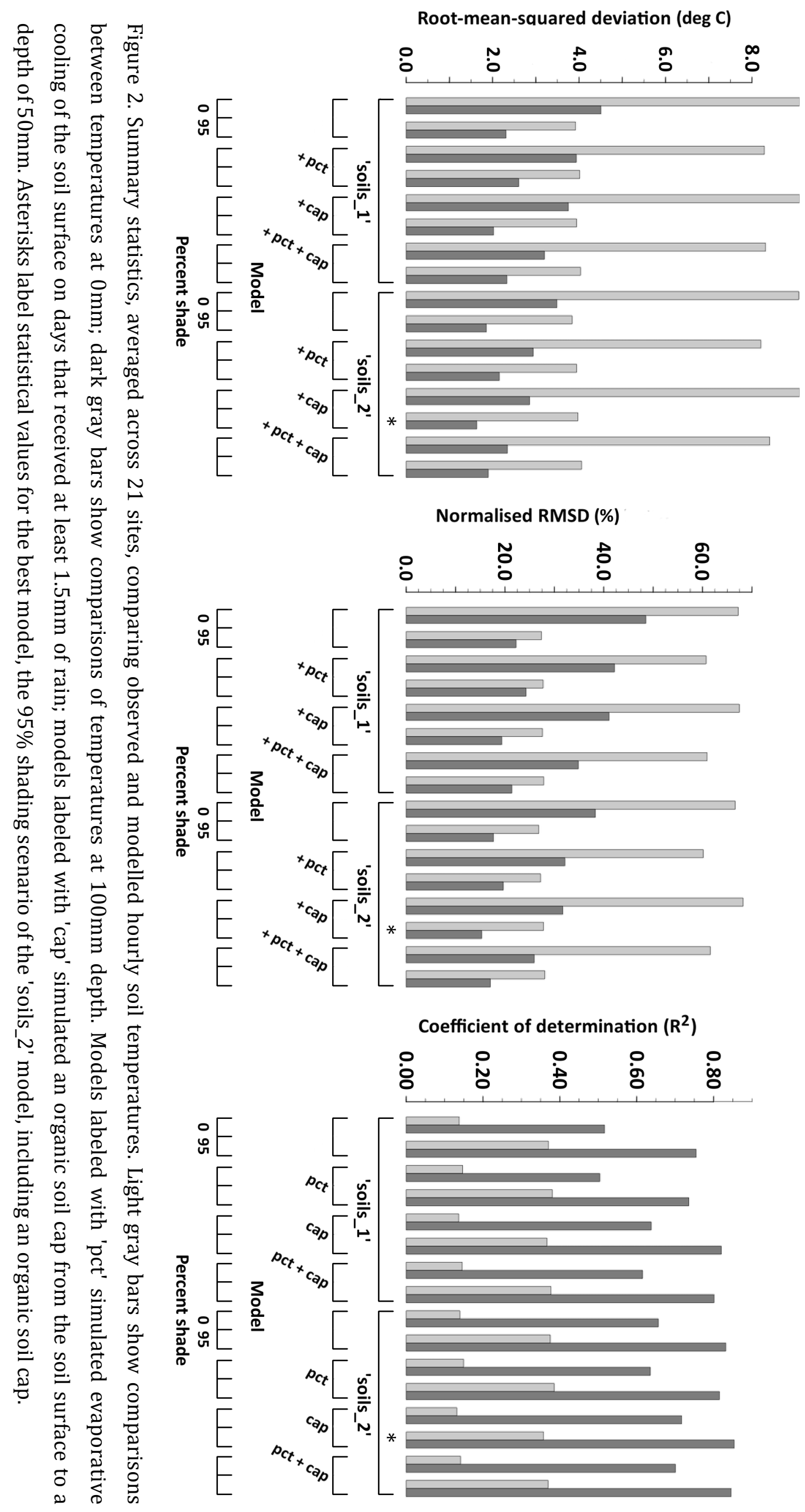




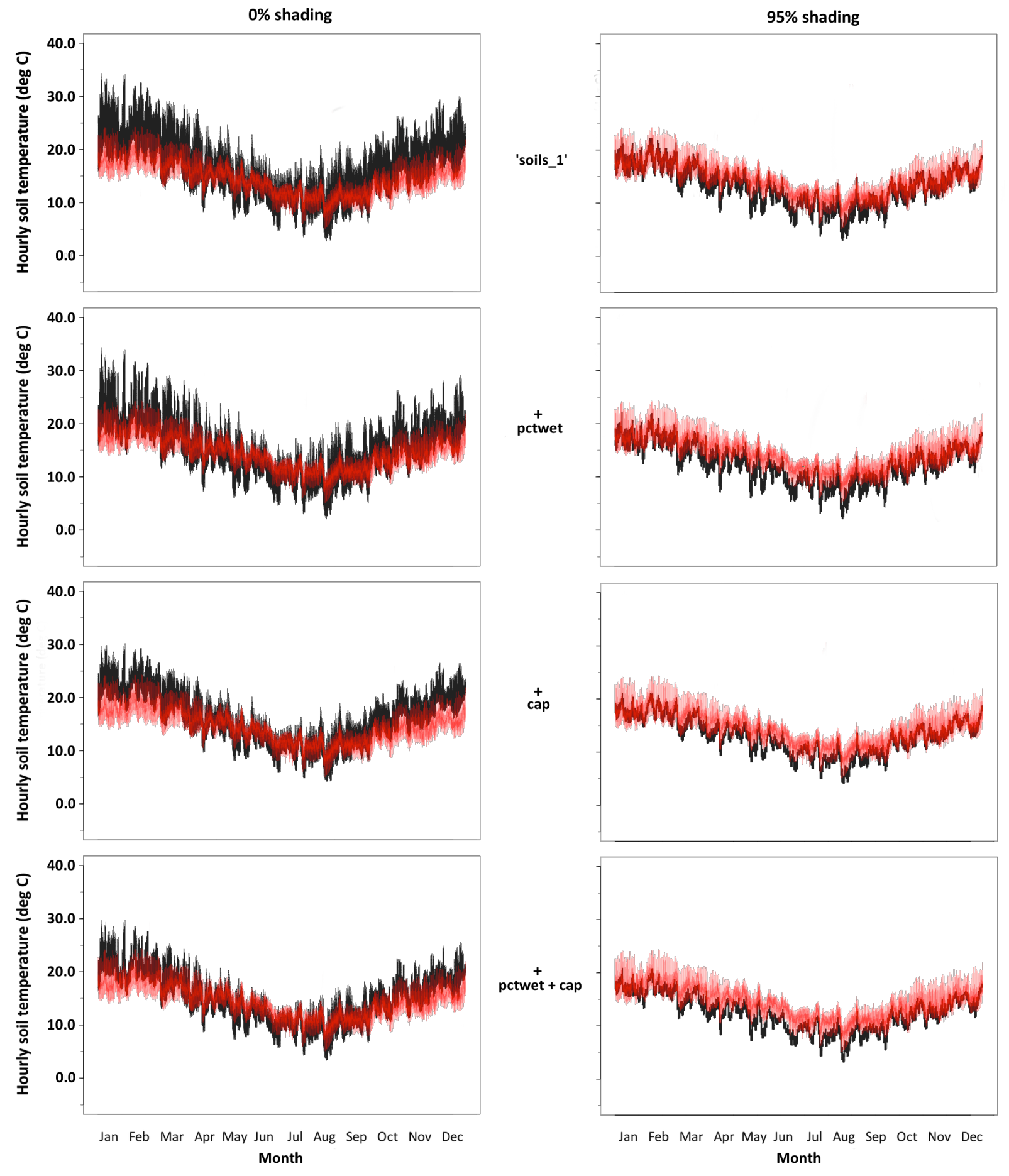



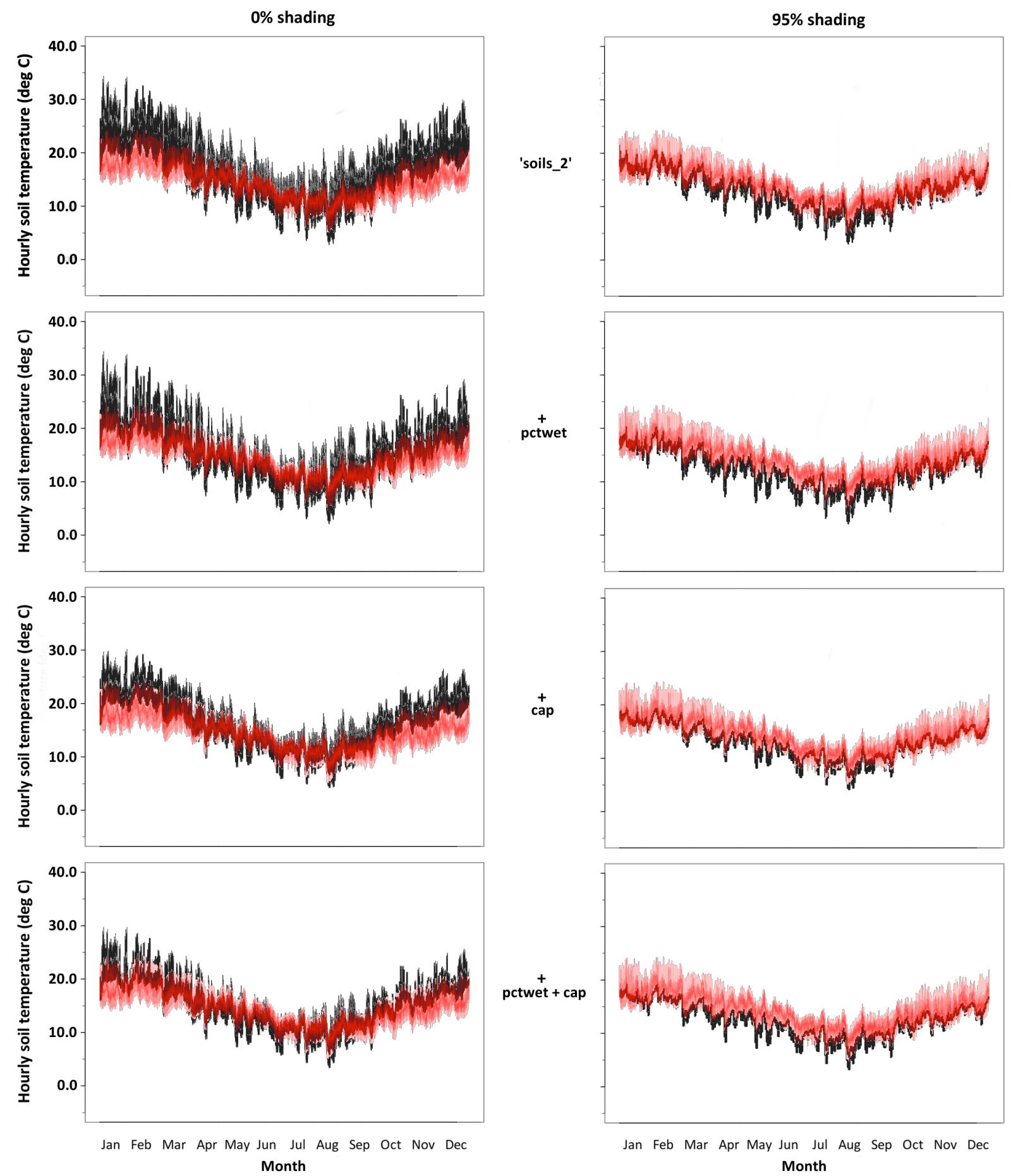

Figure 3. Line plots showing all hourly soil temperature observations at $100 \mathrm{~mm}$ (in red), ordered by time and overlaid onto modelled data (in black). Models labeled with 'pctwet' simulated evaporative cooling of the soil surface on days that received at least $1.5 \mathrm{~mm}$ of rain; models labeled with 'cap' simulated an organic soil to $50 \mathrm{~mm}$ depth. The $95 \%$ shading scenario of 'soils_2 + cap' $\left(\mathrm{RMSD}=1.63^{\circ} \mathrm{C}\right)$ was used to generate a surface of incubation suitability for Hauturu. 


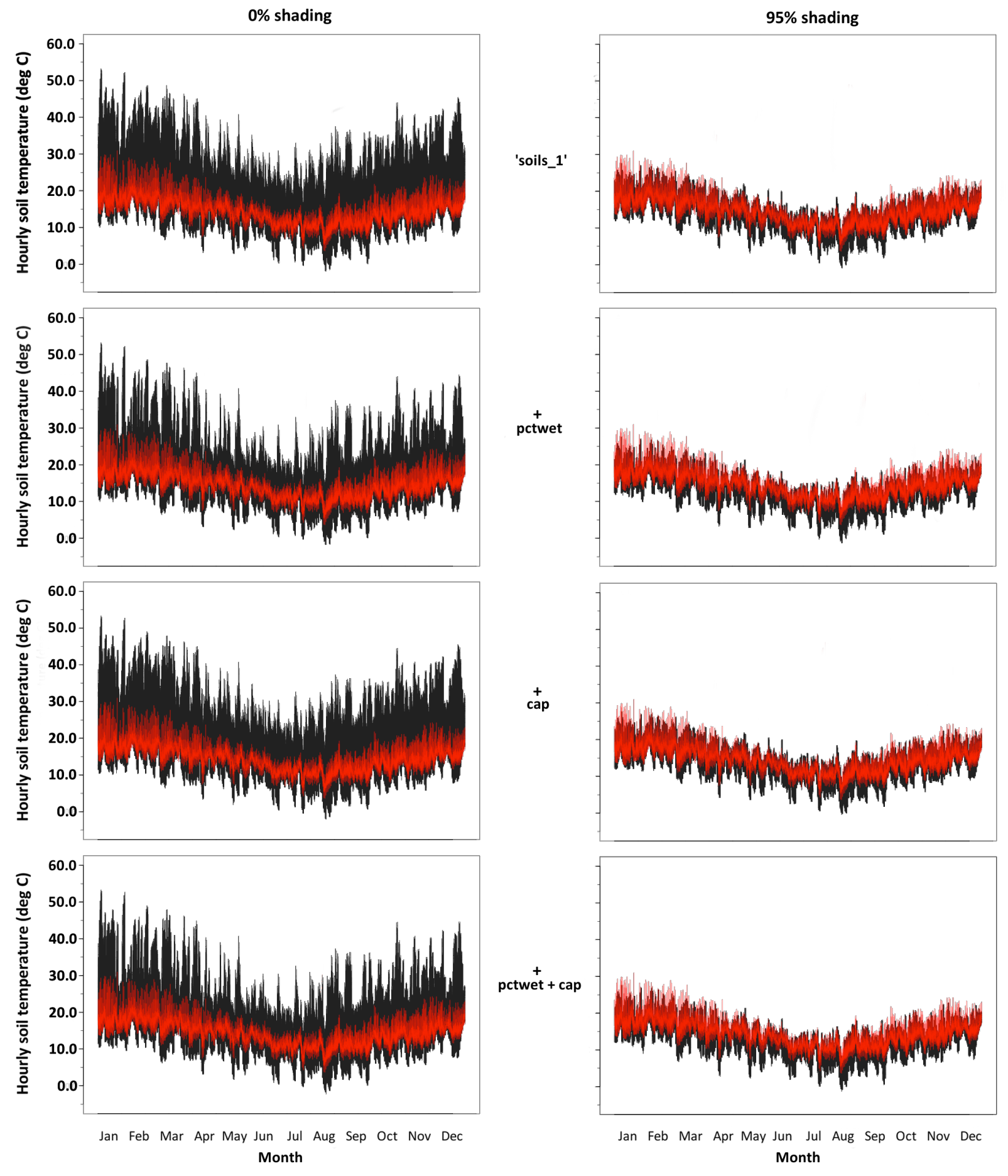



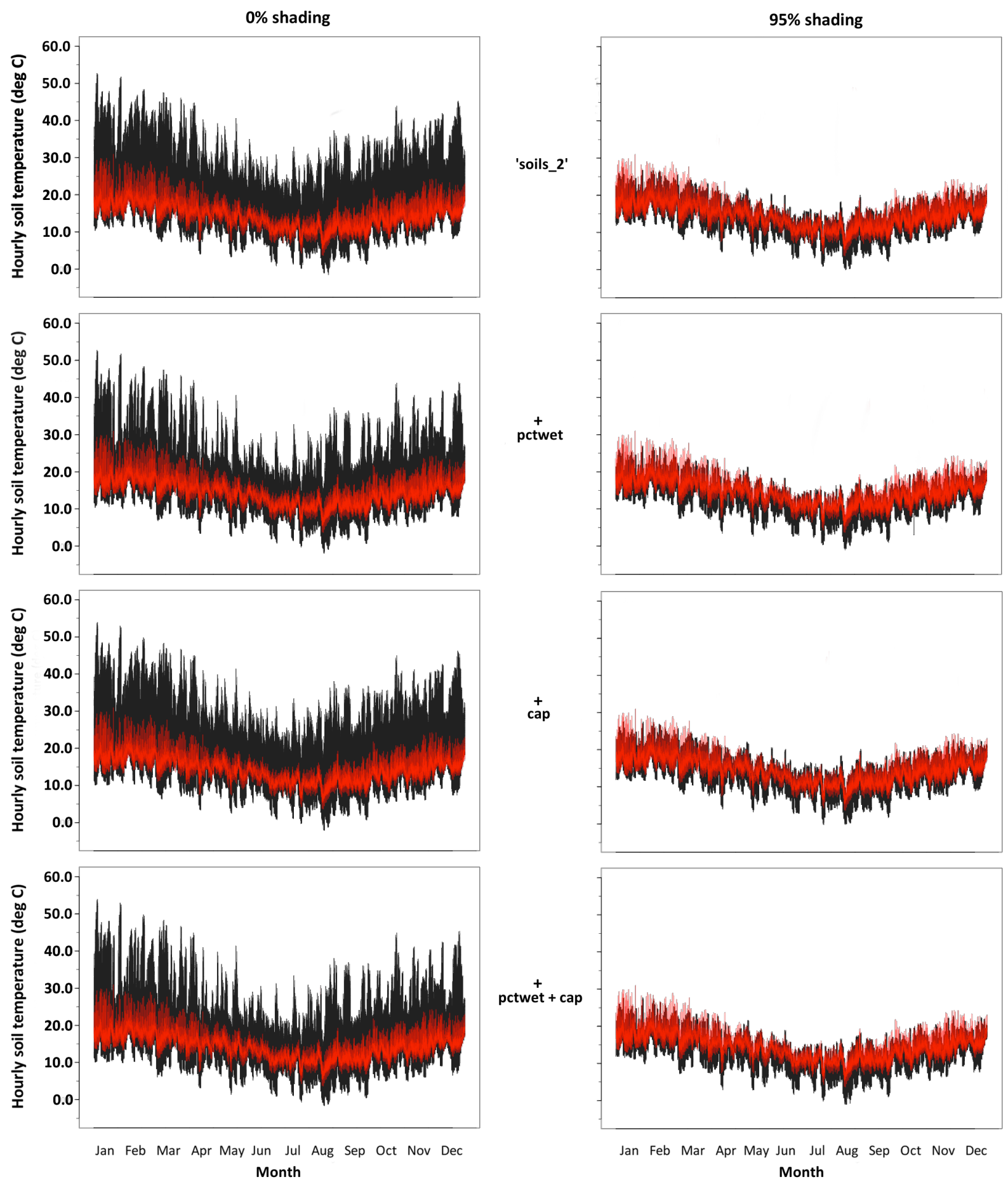

Figure 4. Line plots showing all hourly soil temperature observations at $0 \mathrm{~mm}$ (in red), ordered by time and overlaid onto modelled data (in black). Models labeled with 'pctwet' simulated evaporative cooling of the soil surface on days that received at least $1.5 \mathrm{~mm}$ of rain; models labeled with 'cap' simulated an organic soil to $50 \mathrm{~mm}$ depth. The $95 \%$ shading scenario of 'soils_2 + cap' $\left(\mathrm{RMSD}=3.84^{\circ} \mathrm{C}\right)$ was used to generate a surface of incubation suitability for Hauturu. 


\subsubsection{Nest site suitability and hatching phenology}

A site was classified as suitable for nesting if (1) embryonic development reached 100\% within 24 months (Cree et al. 1989) of oviposition and (2) no hourly temperature prediction exceeded $38^{\circ} \mathrm{C}$ (Nelson et al. 2004; Mitchell et al. 2006, 2008). No site was excluded based on the second criterion under any of the three modelled climate scenarios. The percentage of sites on Hauturu classified as suitable nesting locations for tuatara was similar for the current climate and minimum warming scenarios, varying from around $40 \%$ of sites at a soil depth of $50 \mathrm{~mm}$ to $30 \%$ at $300 \mathrm{~mm}$. Nests at an additional $13 \%$ of sites were predicted to reach at least $90 \%$ of total development across depths from $50-300 \mathrm{~mm}$. Under the maximum warming scenario, $100 \%$ of sites were predicted to facilitate completion of embryonic development at depths from $50-300 \mathrm{~mm}$ (Fig. 5).

Given the simulated oviposition date of 30 Nov, mean hatch dates predicted for suitable nest sites on Hauturu were similar for the current climate and minimum warming scenarios. All sites were predicted to hatch in December, austral summer, at all depths under the current climate and at depths of $50 \mathrm{~mm}$ to $150 \mathrm{~mm}$ under the minimum warming scenario. Sites were predicted to hatch in November and January at depths of, respectively, $200 \mathrm{~mm}$ and $300 \mathrm{~mm}$ under the scenario of minimum warming. Under the scenario of maximum climate warming, more sites were predicted to hatch in autumn at all depths than in other seasons; however, mean hatch dates predicted under the maximum warming varied from September through October, austral spring, at depths from $50 \mathrm{~m}$ to $200 \mathrm{~mm}$. The mean hatching date predicted for a soil depth of $300 \mathrm{~mm}$ under the scenario of maximum warming was mid-August, late austral winter. All calculated mean hatch dates were statistically significant at all depths and under all three modelled climate scenarios $\left(356.67 \leq X^{2} \leq 358.76, p<0.001\right)$. The total number of sites predicted to hatch in winter increased slightly with depth under the maximum 
warming scenario. The numbers of sites predicted to hatch were similar among all soil depths in spring, autumn, and winter but decreased with depth in summer under the current climate and minimum warming scenarios. Summer was also the only season in which fewer sites were predicted to hatch under maximum climate warming, compared with the current climate and minimum warming scenarios (Fig. 6). 

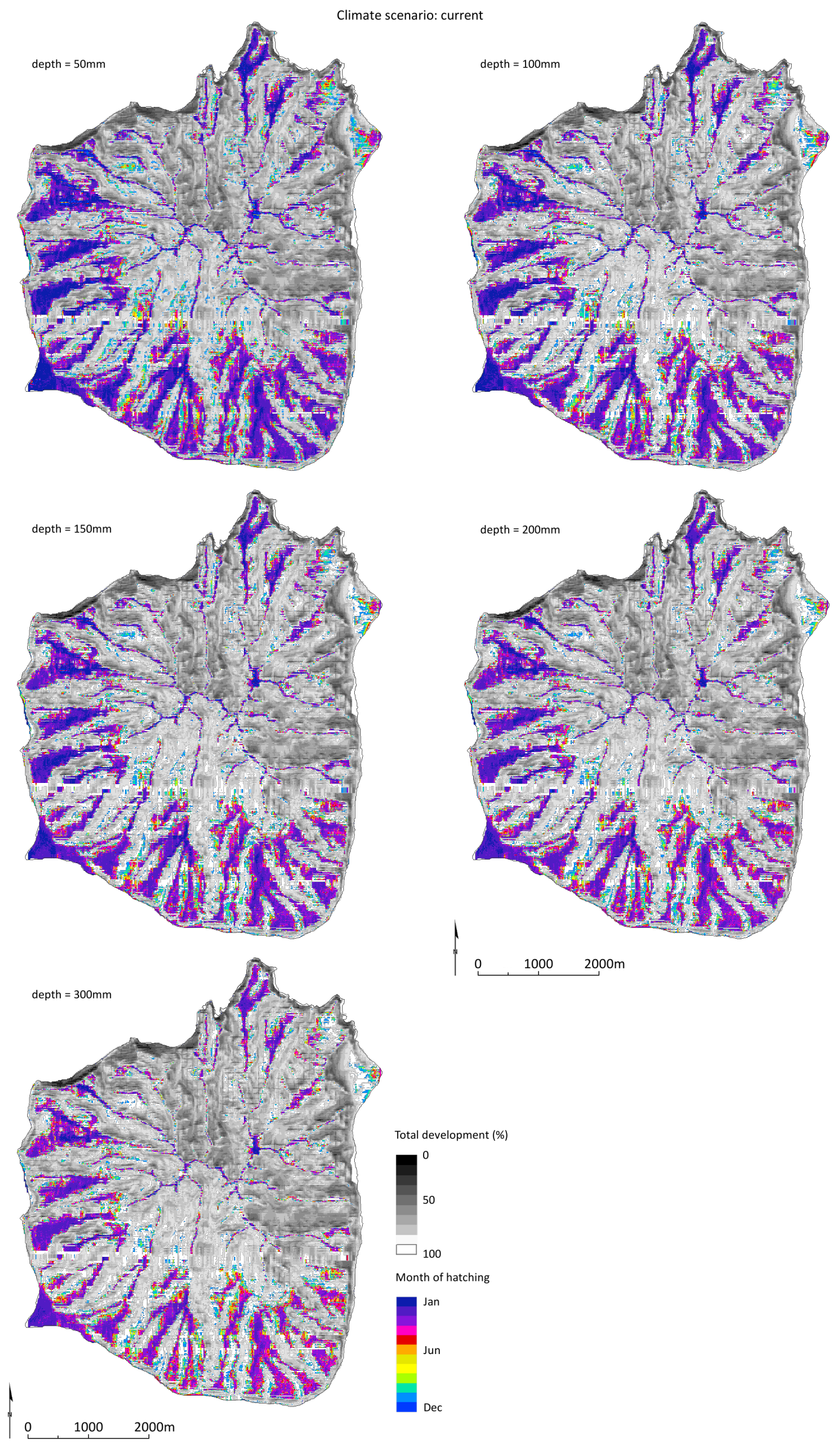

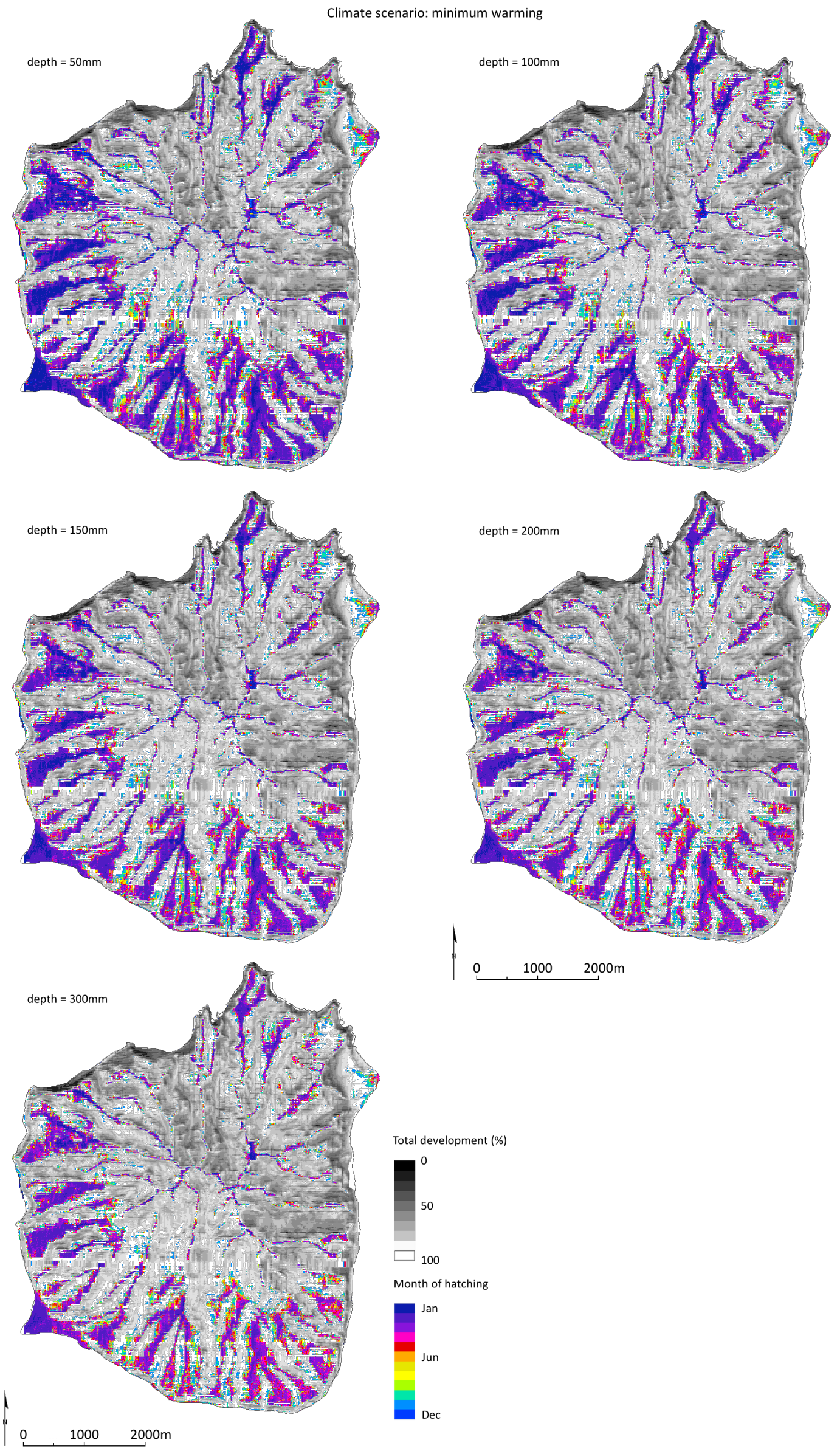

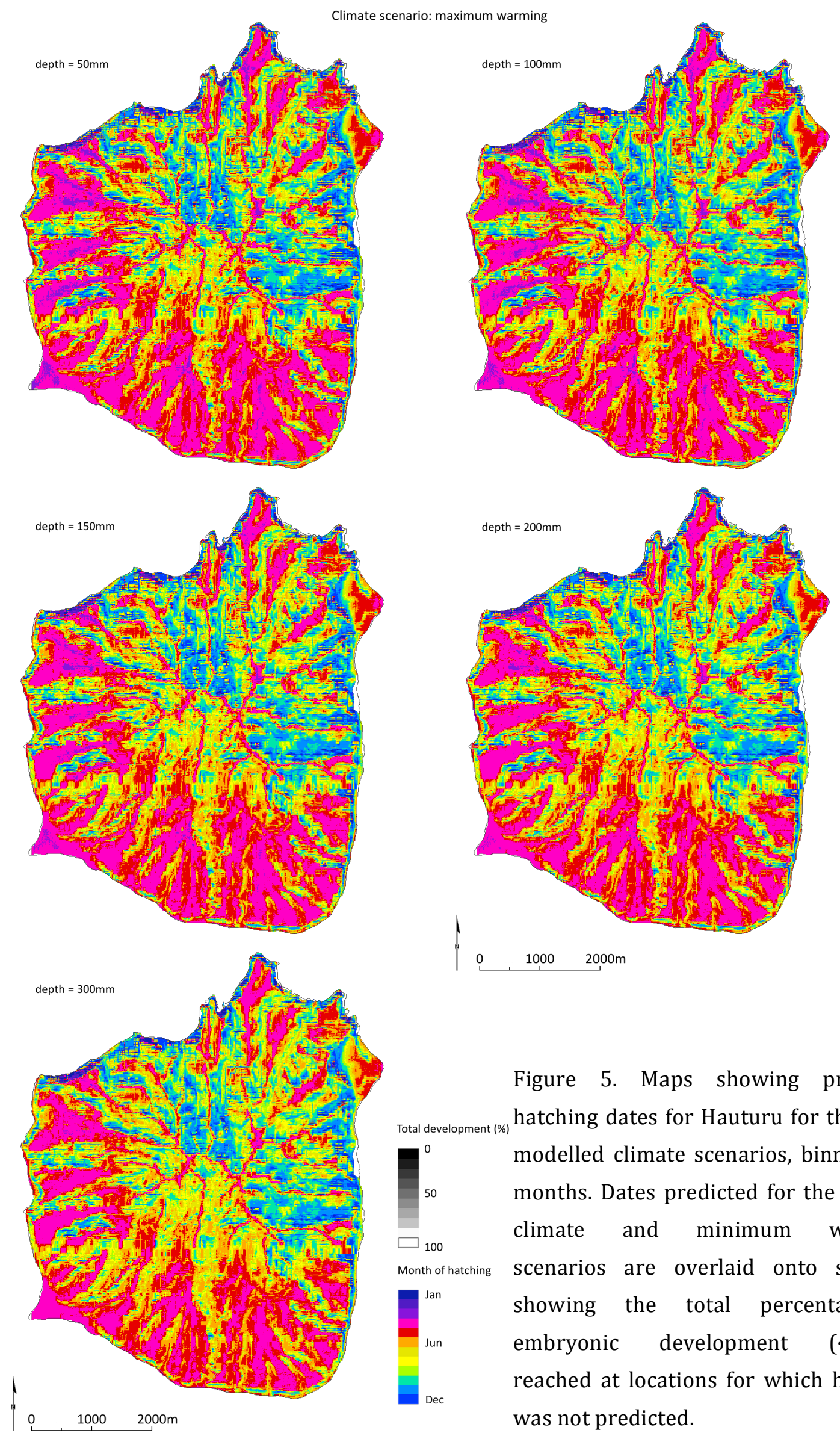

Figure 5. Maps showing predicted hatching dates for Hauturu for the three modelled climate scenarios, binned into months. Dates predicted for the current climate and minimum warming scenarios are overlaid onto surfaces showing the total percentage of embryonic development $(<100 \%)$ reached at locations for which hatching was not predicted. 


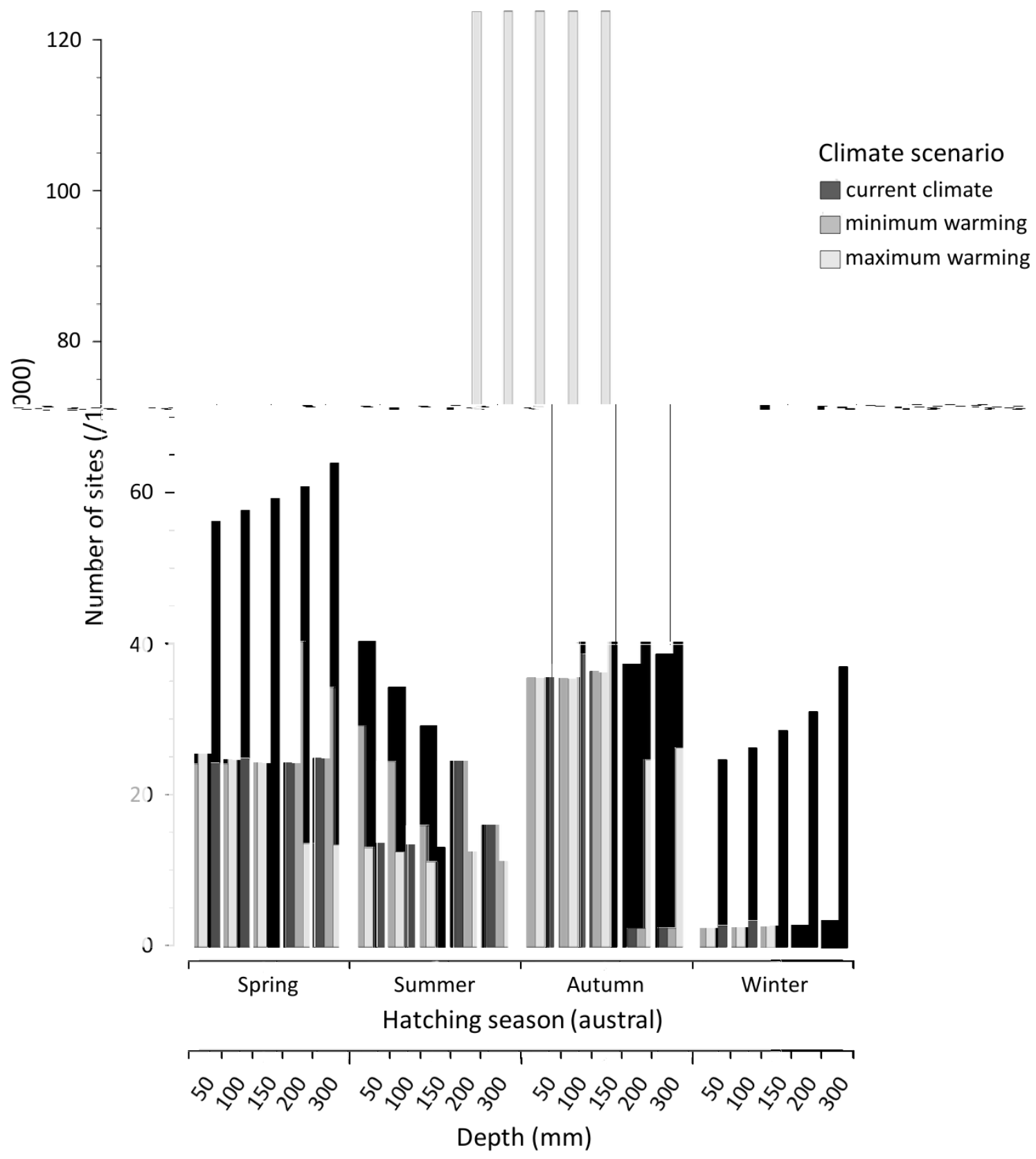

Figure 6. Bar plot showing the seasonal frequency of hatching dates predicted under the three modelled climate scenarios across all sites and for all modelled soil depths from 50-300 mm. 


\subsubsection{Nesting migration}

Under all three modelled climate scenarios, suitable nesting sites were located immediately adjacent to both tuatara release areas on Hauturu as well as within the $20 \mathrm{~m}$-radius release area polygons themselves. Thus, female tuatara that reside within one of the two release sites examined in this study do not need to undertake migration away from the release area to locate suitable nesting locations. Release areas are referred to as Site 1 and Site 2 to maintain the security of translocation sites. Under the current climate and minimum warming scenarios, the mean path distance to suitable nest locations within Site 1 was $18 \mathrm{~m}$ and, within Site 2, approximately $30 \mathrm{~m}$. Maximum path distances estimated for Site 1 and Site 2 were, respectively, 59m and 74m. All sites within the $200 \mathrm{~m}$-radius polygons were classified as suitable nesting areas under the maximum climate warming scenario. Thus, calculated path distances were representative of the minimum-distance travel paths, not only from the release areas to suitable adjacent nesting sites but also from release sites throughout the entire surrounding 200m-radius area. Under the scenario of maximum climate warming, the mean path distance within Site 1 was $17 \mathrm{~m}$ and, within Site 2, about $55 \mathrm{~m}$. Maximum path distances estimated for Site 1 and Site 2 were, respectively, 60m and 199m under the maximum warming scenario (Fig. 7). 


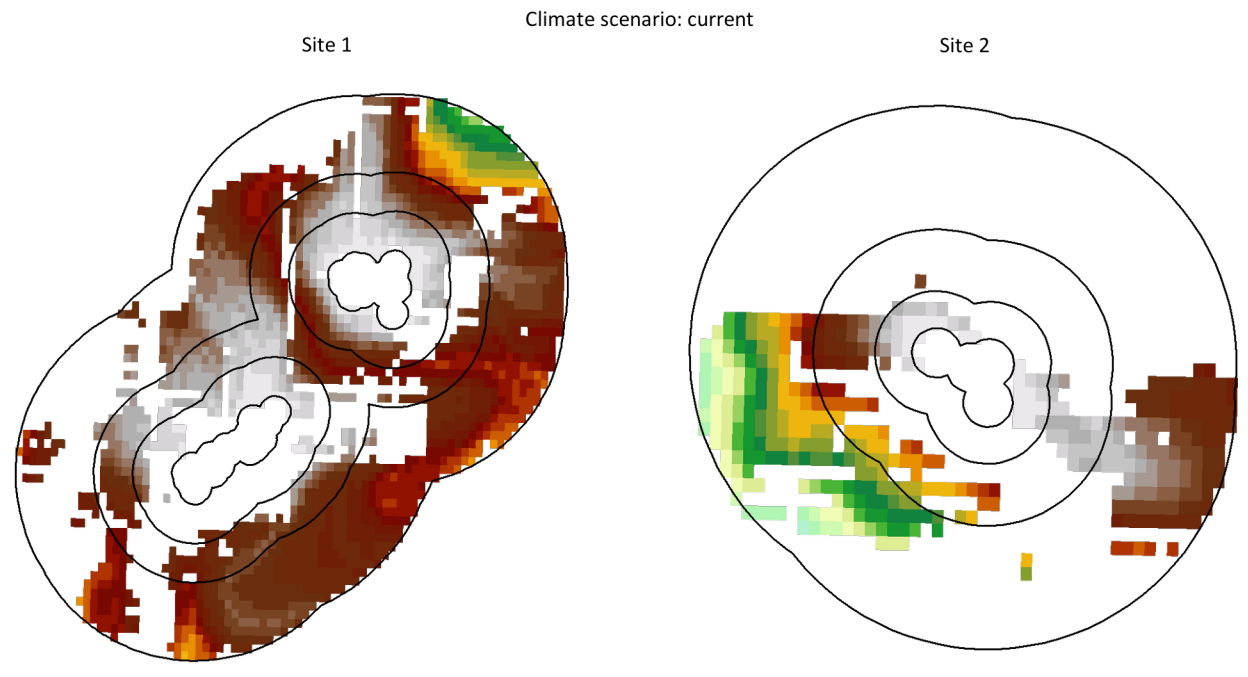

Climate scenario: minimum warming
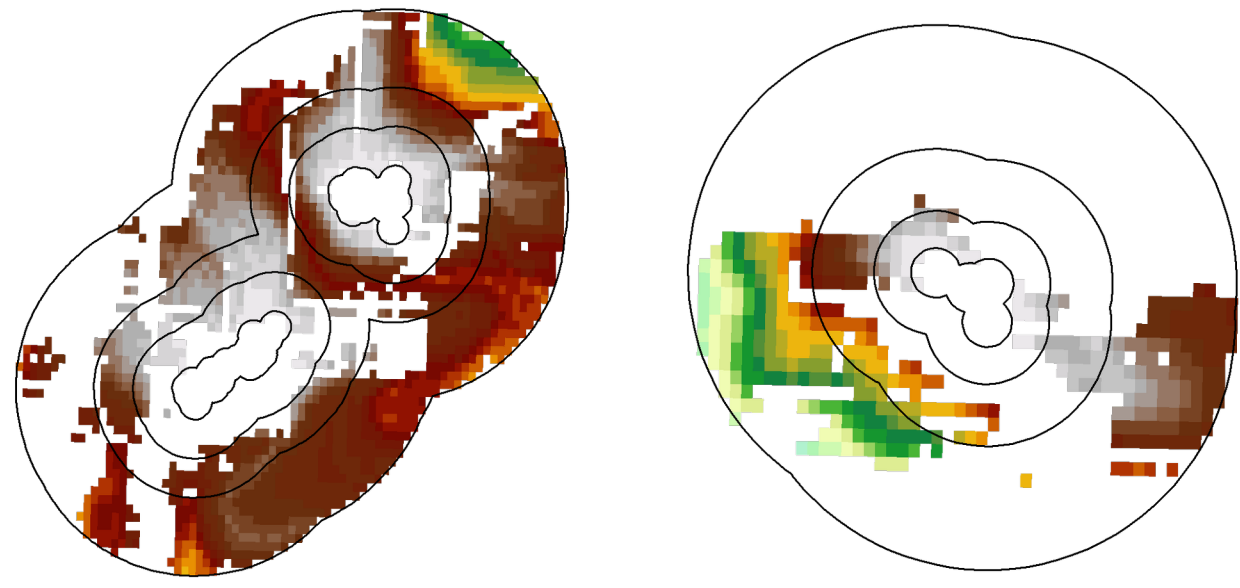

Climate scenario: maximum warming
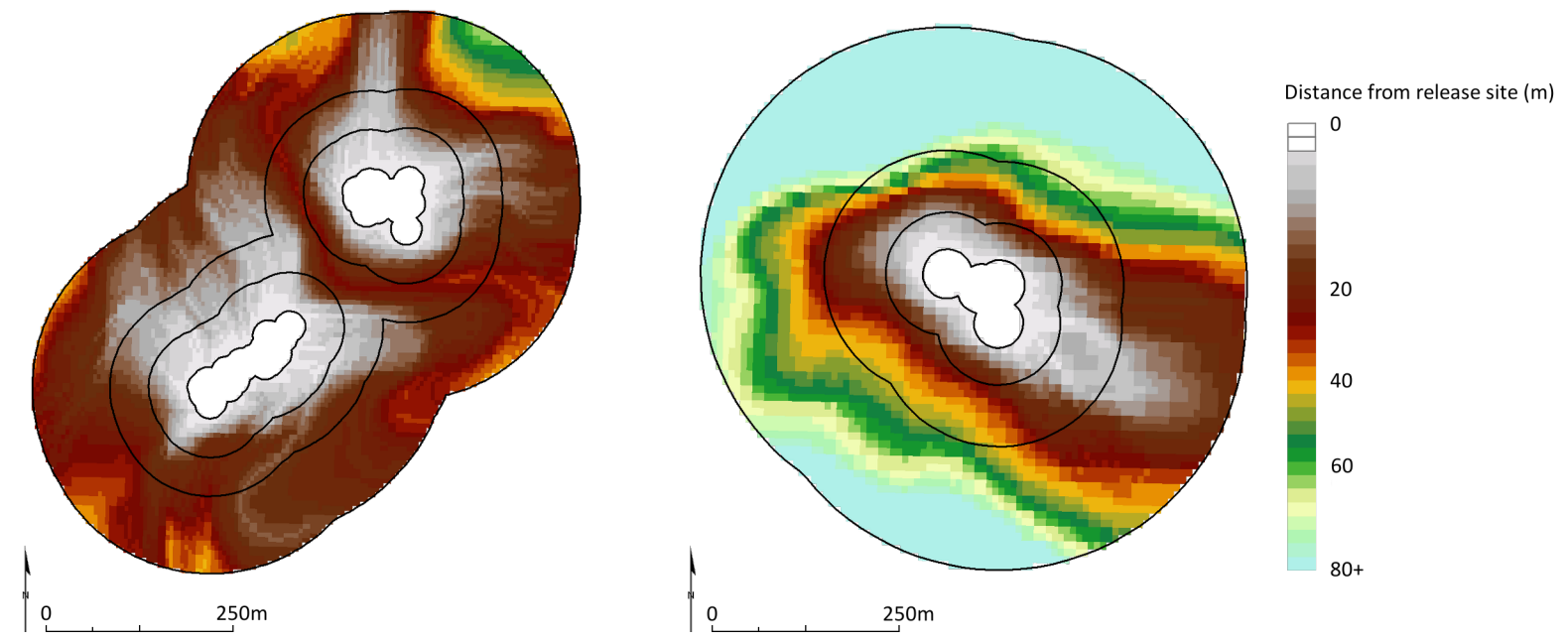

Figure 7. Polygons showing topographically corrected path distances to suitable nesting locations, referenced to true north, within 200 linear metres of two tuatara release sites (central polygons). White areas outside the release-site polygons in the 'current' and 'minimum warming' climate scenarios are unsuitable for nesting. 


\subsubsection{Offspring sex ratios}

The proportion of hatchlings predicted as male varied from 0.00 to 1.00 at suitable incubation sites under all three climate scenarios. Mean predicted offspring sex ratios (i.e., the proportion of male hatchlings) varied from 0.04 at a soil depth of $50 \mathrm{~mm}$ to 0.00 at a depth of $300 \mathrm{~mm}$ under both the current climate and minimum warming scenarios. Maximum sex ratios varied from 1.00 at $50 \mathrm{~mm}$ to 0.38 at $300 \mathrm{~mm}$ under the current climate and minimum warming scenarios. All male hatchlings were only predicted to develop under the current climate and minimum warming scenarios at a depth of $50 \mathrm{~mm}$, and modelled maximum proportions of male hatchlings decreased to 0.68 at $100 \mathrm{~mm}$. Predicted hatchling sex ratios decreased with increasing depth under both the current and minimum warming scenarios. Under the scenario of maximum climate warming, mean predicted proportions of male hatchlings varied from 0.99 at $50 \mathrm{~mm}$ to 0.95 at $300 \mathrm{~mm}$, and the maximum predicted sex ratio at all modelled soil depths was 1.00 .

Under both the current climate and minimum warming scenarios, the percentage of suitable nesting locations predicted to hatch all female tuatara varied from $53 \%$ at $50 \mathrm{~mm}$ to $87 \%$ at $300 \mathrm{~mm}$. The percentage of sites predicted to produce mixed-sex nests (i.e., a sex ratio $<1.00$ and $>0.00$ ) varied from $47 \%$ at $50 \mathrm{~mm}$ to $13 \%$ at $300 \mathrm{~mm}$. Less than $1 \%$ of suitable nesting locations were predicted to hatch all male offspring under either the current climate or minimum warming scenarios. Under simulated maximum climate warming, less than $1 \%$ of the island of Hauturu was predicted to hatch all female offspring at soil depths between $50-300 \mathrm{~mm}$. The percentage of locations predicted to produce all male offspring varied from $97 \%$ at $50 \mathrm{~mm}$ to $91 \%$ at $300 \mathrm{~mm}$. Mixed-sex nests were predicted to hatch from between $3 \%$ of sites at $50 \mathrm{~mm}$ and $8 \%$ of sites at $300 \mathrm{~mm}$ under the scenario of maximum climate warming (Fig. 8). 
Mean constant incubation temperature equivalents (CTEs) predicted for suitable incubation sites under the current climate and minimum warming scenarios varied from $20.11^{\circ} \mathrm{C}$ at $50 \mathrm{~mm}$ to $18.95^{\circ} \mathrm{C}$ at $300 \mathrm{~mm}$. Minimum CTE values varied from $17.32^{\circ} \mathrm{C}$ at $50 \mathrm{~mm}$ to $16.44^{\circ} \mathrm{C}$ at $300 \mathrm{~mm}$, and maximum CTE values varied from $22.30^{\circ} \mathrm{C}$ to $21.95^{\circ} \mathrm{C}$ at, respectively, $50 \mathrm{~mm}$ and $300 \mathrm{~mm}$. Under the maximum warming scenario, mean CTE values varied from 25.30 at $50 \mathrm{~mm}$ to 24.42 at $300 \mathrm{~mm}$. Minimum CTE values varied from $19.06^{\circ} \mathrm{C}$ at $50 \mathrm{~mm}$ to $19.21^{\circ} \mathrm{C}$ at $300 \mathrm{~mm}$, increasing slightly with decreasing soil depth. Maximum CTE values varied from $28.71^{\circ} \mathrm{C}$ at $50 \mathrm{~mm}$ to $27.93^{\circ} \mathrm{C}$ at $300 \mathrm{~mm}$ under the scenario of maximum climate warming (Appendix 5.4). 

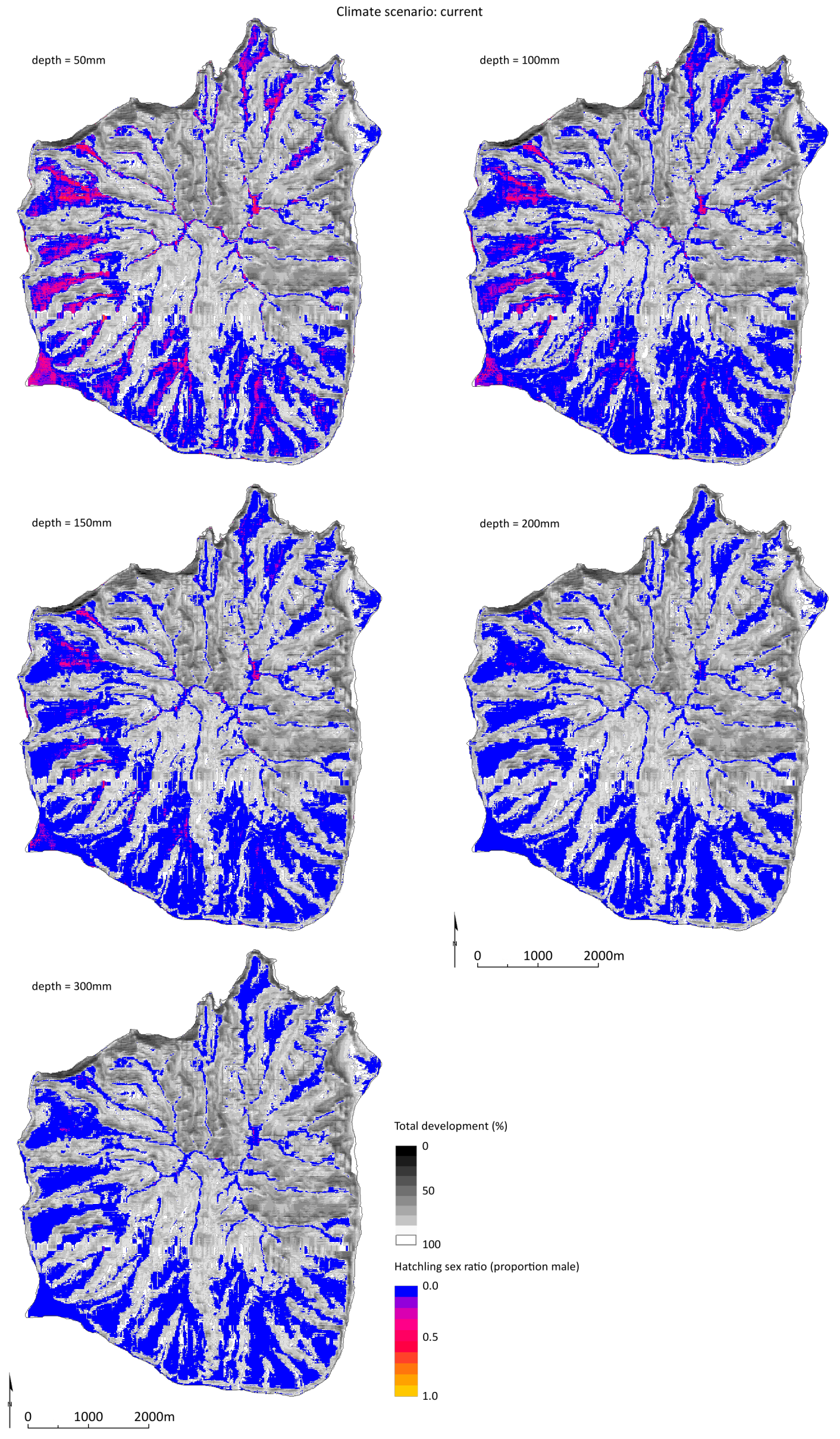

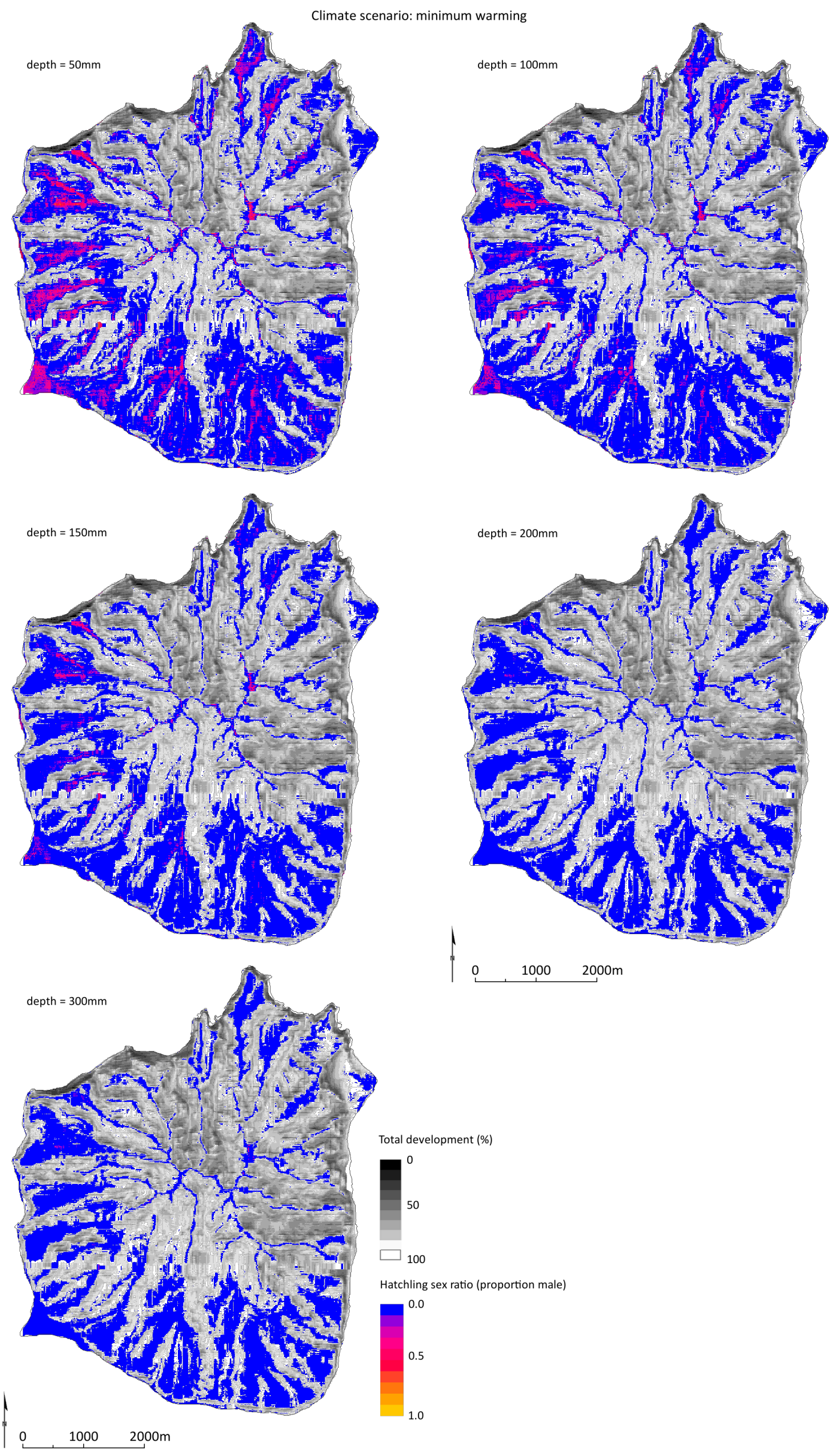

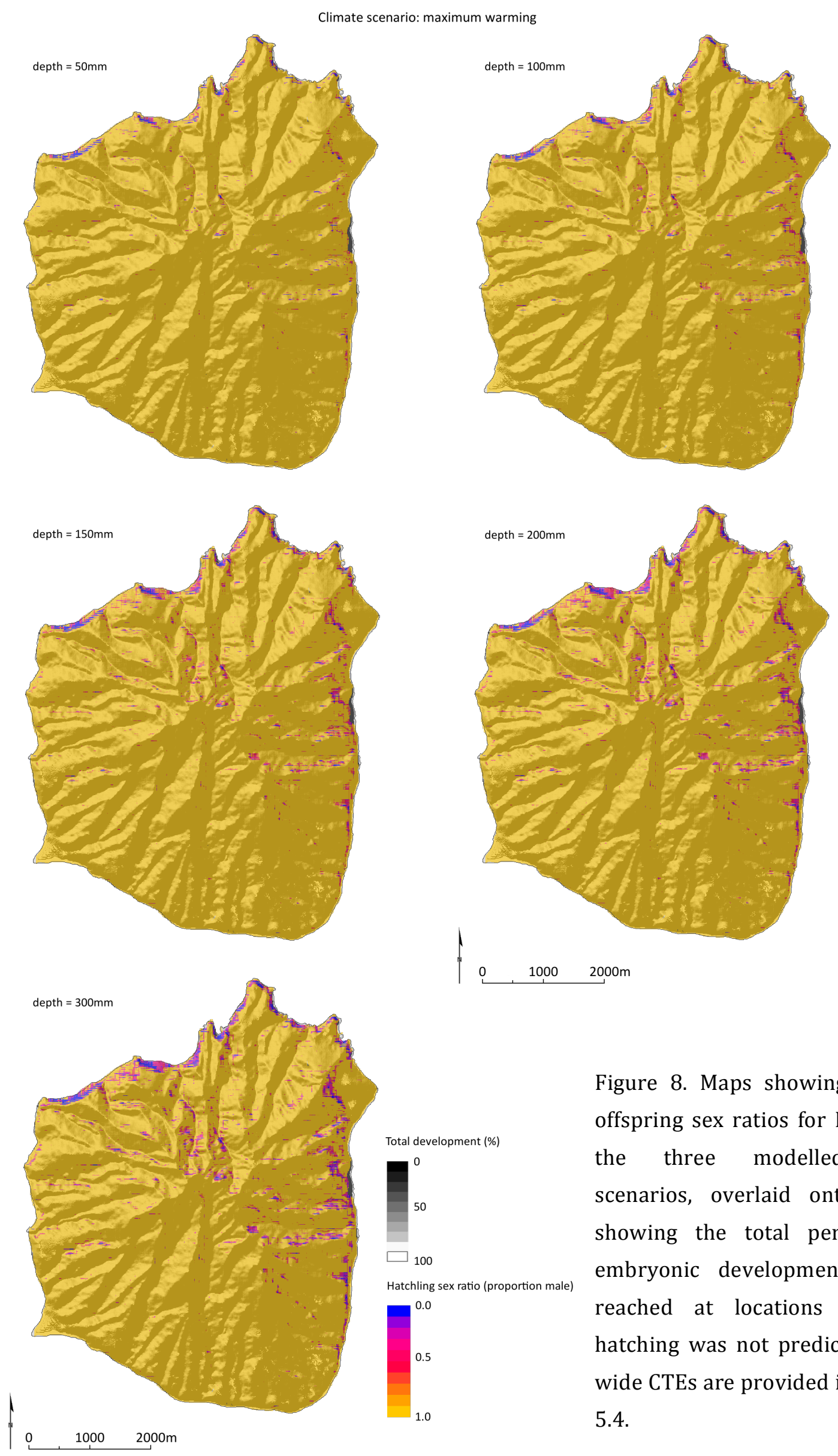

Figure 8. Maps showing predicted offspring sex ratios for Hauturu for the three modelled climate scenarios, overlaid onto surfaces showing the total percentage of embryonic development ( $<100 \%)$ reached at locations for which hatching was not predicted. Islandwide CTEs are provided in Appendix 5.4 . 
Under both the current climate and minimum warming scenarios, most suitable nest sites within $200 \mathrm{~m}$ of the two tuatara release areas produced all female offspring at a soil depth of $100 \mathrm{~mm}$. The mean offspring sex ratio predicted for Site 1 was 0.03 and, for Site 2, 0.02. The maximum sex ratios predicted for Site 1 and Site 2 were, respectively, 0.31 and 0.32 under the current climate and minimum warming scenarios. All but a few sites within $200 \mathrm{~m}$ of either release area were predicted to produce all male hatchlings (i.e., an offspring sex ratio of 1.00 ) at a depth of $100 \mathrm{~mm}$ under the maximum warming scenario. The minimum offspring sex ratio predicted for Site 1 was 0.51 . However, a few locations within $200 \mathrm{~m}$ of Site 2 were predicted to produce mixed-sex nests at $100 \mathrm{~mm}$, with a minimum predicted offspring sex ratio of 0.03 under the scenario of maximum climate warming (Fig. 9). 

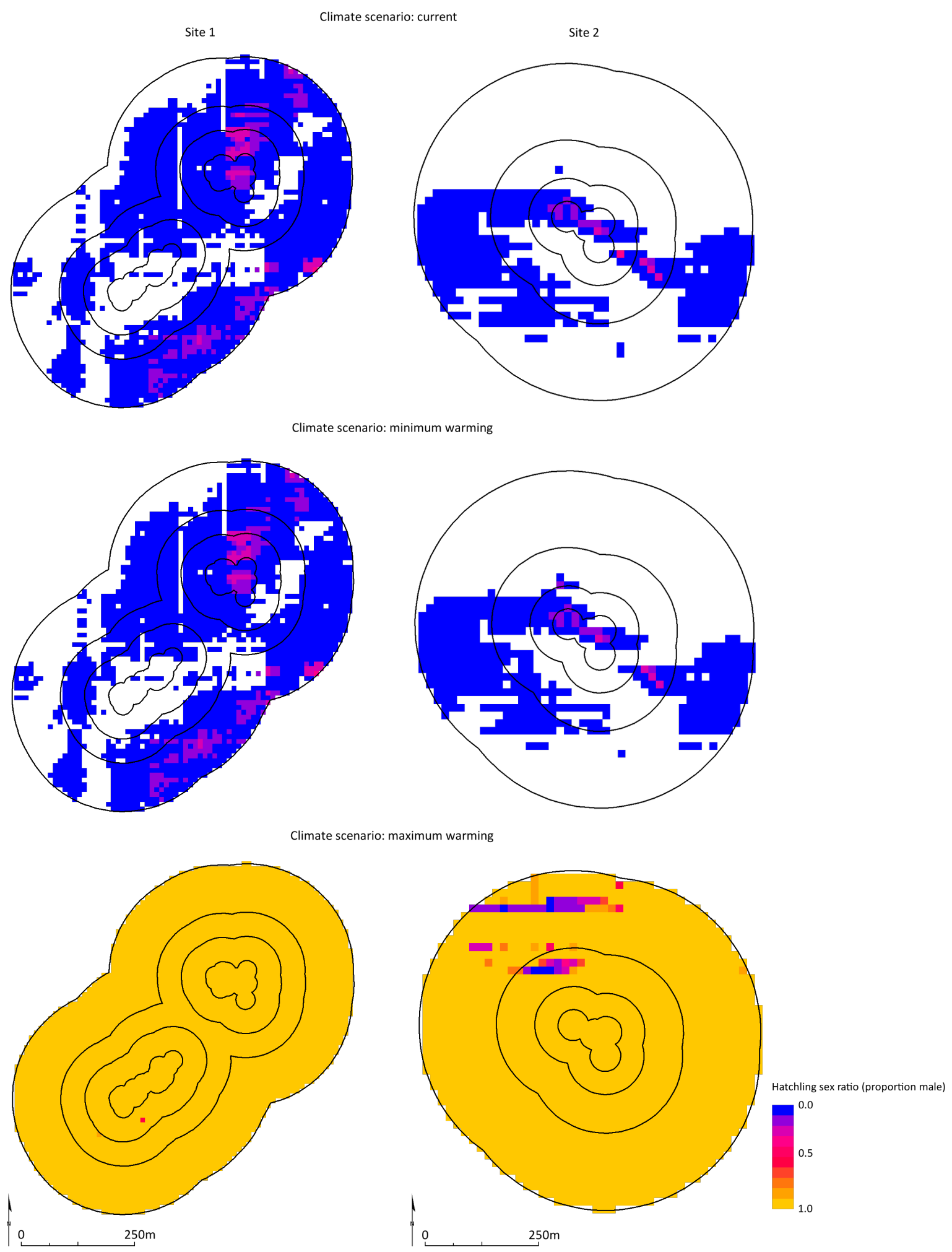

Figure 9. Polygons showing offspring sex ratios predicted for suitable nesting sites within 200 linear metres of tuatara release sites (central polygons). White areas in the 'current' and 'minimum warming' climate scenarios were not predicted to reach $100 \%$ embryonic development within 2 years of oviposition. 


\subsection{Discussion}

\subsubsection{Summary of major findings}

This study parameterised a mechanistic microclimate model, NicheMapR (Kearney et al. 2014a, b), to examine thermal suitability of habitat for supporting embryonic development and maintaining balanced sex ratios in the small, reintroduced population of tuatara on the island of Hauturu. Spatially explicit predictions of offspring sex ratios and hatching phenology have been developed previously for two other populations of tuatara in which nest site selection has been extensively studied (Mitchell et al. 2008, Chapter 4). In this study, however, modelling of embryonic development and hatchling sex ratios was explicitly approached from the perspective of applied conservation. This research translated spatially explicit predictions of microclimatescale soil temperatures into testable hypotheses of nesting behaviour in a population for which patterns of nest site choice are unknown.

The best model was the implementation of 'soils_2' that simulated 95\% canopy shading and an organic soil cap. The candidate model parameterisations examined in this study were consistently better predictors of hourly temperatures at a depth $100 \mathrm{~mm}$ than at the soil surface (i.e., at $0 \mathrm{~mm}$ ), reflecting the reduction in temperature variance that results from decreased conduction of heat energy with soil depth. In contrast with a previous high-resolution implementation of NicheMapR (Chapter 3), simulation of soil properties (i.e., bulk density, specific heat, soil moisture) specific to Hauturu ('soils_2') increased model accuracy over that obtained using generalised soil parameters ('soils_1'). Hourly soil temperatures generated using the best model were well within a mean $2^{\circ} \mathrm{C}$ of observed values at $100 \mathrm{~mm}$ but only within a mean $4^{\circ} \mathrm{C}$ of values observed at $0 \mathrm{~mm}$ and slightly under-predicted at both depths. Values for root-mean-squared deviation (RMSD) were similar to previous implementations of NicheMapR (Kearney et 
al. 2014a, b; Chapter 3). Normalised RMSD values of 'soils_2' indicated that the minimum residual variance across all sites was within $15 \%$ of observed values at $100 \mathrm{~mm}$ and $30 \%$ of observed values at the soil surface. The 'soils_2' implementation explained $85 \%$ of variance in hourly soil temperatures at $100 \mathrm{~mm}$. However, $\mathrm{R}^{2}$ values indicated that ability of the most accurate model to explain variance in observed temperatures at the soil surface compared to a depth of $100 \mathrm{~mm}$ was reduced by about $42 \%$, from around 0.85 to 0.35 .

Hourly soil temperature predictions were used to model development rates of tuatara embryos at soil depths of $50,100,150,200$, and $300 \mathrm{~mm}$ for the island of Hauturu under both the current climate and regional predicted minimum and maximum warming scenarios for the period 2080-2099. Sites were classified as suitable nesting habitat if embryonic development reached $100 \%$ within two years of the modelled oviposition date of 30 November. Predictions generated for the current climate and minimum warming scenarios were nearly identical. Between $30-40 \%$ of Hauturu was classified as thermally suitable for nesting under both scenarios. Another $13 \%$ of unsuitable sites were predicted to develop to at least $90 \%$ completion at $50 \mathrm{~mm}$, so incubation temperatures experienced during slightly warmer years might facilitate hatching over half of the island.

Hatching was predicted to occur throughout the austral spring, summer, and autumn at most sites, though mean hatching was predicted for autumn. Few sites were predicted to hatch in winter under the current climate and minimum warming scenarios. Predictions generated under the scenario of maximum regional warming varied considerably from those developed under the current climate and minimum warming scenarios. All locations on Hauturu were predicted to provide thermally suitable nesting habitat at soil depths from $50-300 \mathrm{~mm}$. The mean hatch date predicted under the maximum warming scenario was in early austral winter, and fewer sites were 
predicted to hatch in summer, compared with the current climate and minimum warming scenarios.

Predicted hatchling sex ratios varied from $100 \%$ female to $100 \%$ male under both the current climate and minimum warming scenarios. The percentage of sites predicted to produce all-female hatchlings increased with depth from $53 \%$ to $87 \%$. The percentage of locations predicted to produce mixed-sex nests decreased with depth from $47 \%$ to $13 \%$. All-male hatchlings were predicted at less than $1 \%$ of sites and only at a soil depth of $50 \mathrm{~mm}$. Under the scenario of maximum climate warming, mean predicted offspring sex ratios decreased with depth from $99 \%$ male at $50 \mathrm{~mm}$ to $95 \%$ male at $300 \mathrm{~mm}$, and all-male hatchlings were predicted for all soil depths. A mean $5 \%$ of sites across all modelled soil depths were predicted to produce both male and female hatchlings under the maximum warming scenario.

Thermally suitable nesting locations were identified immediately adjacent to both reintroduction sites on Hauturu under all modelled climate scenarios. Thus, female tuatara do not need to travel away from their release areas to locate suitable nesting habitat. Most of the locations identified within a 200m radius of release sites were predicted to produce $100 \%$ female hatchlings at $100 \mathrm{~mm}$ under both the current climate and minimum warming scenarios. The few relatively warm sites were predicted to hatch about $30 \%$ males at $100 \mathrm{~mm}$. Under the scenario of maximum predicted climate warming, all but a few sites within a $200 \mathrm{~m}$ radius of the two release sites were predicted to hatch $100 \%$ males at $100 \mathrm{~mm}$. Barring heavily female-biased mortality, under both current climate conditions and a scenario of minimum warming, offspring sex ratios on Hauturu are unlikely to become male-biased within the next 100 years. However, under a scenario of maximum climate warming, most of the area of Hauturu will likely become unsuitable for maintaining balanced offspring sex ratios, placing tuatara populations at increased risk of extinction (Mitchell et al. 2009; Grayson et al. 2014). 


\subsubsection{Assessment of methodological limitations}

Daily climate records were not available from the permanent weather station on Hauturu for 2011, the year during which in-situ soil temperature data were collected for this study, so local climate data were sourced from the nearest weather stations for which data were available. Even if site-specific climate data would have improved the 'soils_2' model parameterisation, the accuracy of hourly soil temperatures generated for this study was similar to that of global- and continental-scale model implementations (Kearney et al. 2014a, b). The model developed for Hauturu was slightly more accurate than an island-scale implementation developed for Takapourewa (Chapter 3). This result may partially reflect the availability of field data. The higher memory capacity of the dataloggers used on Hauturu allowed for collection of hourly soil temperature data for a full year at all 21 sampling locations, providing a more representative set of validation data against which to test model predictions. In addition, the need for sitespecific climate data may become less stringent as the spatial extent of a study area increases. However, whether site-specific climate data would improve the model developed in this study cannot be tested. Regardless, the results of this study show that lack of an on-site weather station is not necessarily a barrier to development of an accurate model of soil temperatures.

One aim of this research was to develop hypotheses about nest site selection in the reintroduced populations of tuatara on Hauturu by identifying sites within defined search radii that were predicted to be thermally suitable for embryonic development. Whether the results of this study can be applied in the field depends largely on the ability to observe females undertaking nest construction, which requires an accurate prediction of the timing of nesting. Against expectations (Nelson unpub. data), female tuatara on northern islands have tended to nest later than those on islands in the Cook Strait, with onset of nesting occurring in December (Tyrell et al. 2000). Oviposition has 
occurred in the captive enclosure on Hauturu as early as 11 November and as late as 1 April, though most dates have varied from late November through December (Keall unpub. data). Whether the patterns of nesting phenology observed in captive females have been maintained in the individuals released into the wild is unknown. The relatively high variability in oviposition dates on Hauturu suggests that the timing of nesting may be less constrained in a warmer climate, although multiple years of data will be required to quantify the relationship between macroclimate conditions and nesting phenology in the wild. Establishing even preliminary data on nesting in the reintroduced populations may require several consecutive seasons of fieldwork, even with knowledge of the thermal suitability of release sites for nesting.

This study did not examine total development times, so did not differentiate between hatching during the first versus the second year of development under any modelled climate scenario. However, the probability of mortality for embryonic tuatara may increase with time spent in-ovo. As development time increases, the amount of yolk remaining in an egg and, thus, energy reserves available at hatching decrease (Angiletta et al. 2000; Mitchell \& Seymour 2000; Mitchell et al 2008). Integrating the timedependent probability of mortality both prior to and post-hatching into developmental predictions is a crucial next step in improving the accuracy of models of temperaturedependent embryonic development and sex determination.

While this study did not test the sensitivity of developmental predictions to error in modelled soil temperatures, research on the island of Takapourewa found little effect of error on predicted CTEs and offspring sex ratios under the current climate and minimum warming scenarios. Under the scenario of maximum climate warming, CTEs and predicted proportions of male hatchlings were markedly higher when hourly soil temperatures were adjusted for error in the microclimate model, compared to values generated using non error-corrected soil temperatures (Chapter 4). Similar patterns would likely be observed on Hauturu. Thus, the percentage of sites likely to produce 
mixed-sex nests under the current climate and minimum warming scenarios may be higher in reality. However, since so few sites on Hauturu were predicted to produce female hatchlings under the maximum warming scenario, increasing hourly soil temperatures to account for error in the microclimate model is unlikely to have a biologically meaningful effect on predictions of CTEs or hatchling sex ratios on the island. From the perspective of reintroduction biology, the effects of model error on rates of embryonic development are more immediately important. The percentage of sites predicted to facilitate successful hatching under the current climate and minimum warming scenarios may increase dramatically if error-corrected hourly soil temperatures are used to predict rates of embryonic development.

Finally, predictions of embryonic development times and offspring sex ratios were generated using model parameters, derived via controlled and natural incubation of tuatara eggs from Takapourewa (Mitchell et al 2006, 2008), which estimate slightly conservative proportions of male offspring on North Brother Island (Mitchell et al. 2008). Parameter values have not been derived explicitly for Hauturu or other northern islands. However, because the annual macroclimate of Hauturu is, on average, around $4^{\circ} \mathrm{C}$ warmer than that of islands in the Cook Strait (http://www.cliflo.niwa.co.nz), the population of tuatara on Hauturu may be adapted to the warmer climate and may have evolved a slightly higher pivotal temperature or developmental maximum temperature or a higher thermal tolerance (Miller et al. 2012). Either (1) conducting controlled incubation experiments, including back-switching, with eggs sourced from Hauturu or (2) testing the sensitivity of the development and sex ratio functions to realistic variations in parameter values would be useful for confirming the validity of the models utilised in this study. 


\subsubsection{Implications for reintroduction biology}

The thermal suitability of a location for facilitating embryonic development is a critical ecophysiological constraint on the identification of habitat that will allow populations of oviparous reptiles, especially those with TSD, to persist (Shine 1999c; Shine 2002; Monasterio et al. 2011; Besson et al. 2012). The results presented here for a reintroduced population of tuatara show that a process-explicit microclimate model can be used to inform translocations of oviparous species by quantifying incubation suitability at a biologically relevant spatial resolution under multiple climate scenarios. In addition, the modelled microclimate surfaces developed for Hauturu show the importance of using microclimate-scale predictions in assessments of habitat suitability when planning translocations, both for reintroductions and assisted colonisations.

The releases of tuatara to Hauturu were classified as reintroductions, translocations to within the known former geographic range of a species (Seddon et al. 2010; IUCN 2013). Juvenile tuatara were released onto the island at two spatially disparate locations that approximate the sites at which eight adults were captured in the early 1990s (Keall et al. 2010). Whether those release areas contained or were adjacent to suitable nesting habitat was unknown prior to this study, including at the time of release. That the modelled microclimate surfaces generated in this study identified suitable nesting habitat within both release areas on Hauturu is fortunate, as the reintroduction relied on the implicit assumptions that both release areas would: (1) provide suitable resources to support the survival of the released juveniles to sexual maturity and facilitate population growth through the survival of subsequent generations, (2) either encompass or be within attainable migration distance of suitable nesting habitat for females, and (3) contain nesting habitat that would produce approximately equal or slightly female-biased offspring sex ratios in the long-term, including under predicted scenarios of climate warming. 
A comprehensive mark-recapture study is needed to quantify survival rates of the juvenile tuatara released into the wild on Hauturu and determine whether recruitment has occurred. However, the results of this study show that, under both the current climate and the scenario of minimum regional warming predicted for 20802099, both release sites on Hauturu are capable of supporting embryonic development and, overall, are likely to produce female-biased hatchling sex ratios. In addition to confirming that the reintroduction sites on Hauturu should be able to support successful embryonic development, this study demonstrated that both release areas on Hauturu encompass soil microclimates that can facilitate hatching under the current climate, an outcome that is immediately beneficial for two main reasons. First, inexperienced females may be more likely to locate suitable nesting sites, increasing the chances that eggs will hatch successfully and contribute to a more rapid rate of population growth under current climatic conditions than if extensive searching was required. Second, if females select oviposition sites within the areas identified as suitable in this study, monitoring for nesting behaviour is more likely to be successful within a shorter timeframe, since females may nest within close proximity to marked burrow sites.

Female tuatara are more likely to travel downhill or laterally to nest (Refsnider et al. 2013), probably at least in part to minimise energetic costs of nesting, so patterns of post-translocation nesting could potentially be controlled by releasing females into burrows that minimise migration distances to thermally suitable sites. If no thermally suitable locations are identified at what is otherwise a preferred translocation site, artificial nesting sites could be created adjacent to a release area via vegetation removal. A similar strategy could be adopted as a means of establishing populations of tuatara at sites that, as a result of being heavily shaded, are currently too cold for facilitating embryonic development but that are predicted to maintain balanced offspring sex ratios under scenarios of extreme climate warming. Maintenance of slightly female-biased sex ratios, predicted for the current a minimum warming scenarios on Hauturu, may also 
facilitate a more rapid rate of population growth (Freedberg \& Taylor 2007), key for establishment and persistence of a reintroduced population. Relatively rapid growth of the two released populations may also provide an opportunity to examine whether sexbiased dispersal affects population persistence (Doody \& Moore 2010; Boyle et al. 2014).

The dramatic differences in predicted offspring sex ratios and, to a lesser extent, hatching phenology between the minimum and maximum warming scenarios are similar to those previously modelled for North Brother Island (Mitchell et al. 2008) and support the conclusion that the magnitude of climate warming observed over the next century will be critical in determining the future viability of tuatara populations in New Zealand. Offspring sex ratios on Hauturu are likely to become extremely male-biased if climate change observed over the next century more closely matches the maximum, rather than the minimum, projected warming scenario, easily surpassing the $65 \%$ threshold for increased extinction risk that was determined for the population of around 500 tuatara on North Brother Island (Mitchell et al. 2009; Grayson et al. 2014).

Tuatara on Takapourewa typically nest well within the $50-300 \mathrm{~mm}$ range of depths examined in this study (Cree et al. 1992; Cree 1994; Newman et al. 1994), and hatchling sex ratios decrease with increasing depth (Nelson et al. 2004a; Chapter 4). On Hauturu, constructing deeper nests is not likely to reduce offspring sex ratios under maximum warming conditions. Nest site selection is more likely to be linked with offspring survival (i.e., successful incubation) than with hatchling sex (Nelson unpub. data), and the paucity of female-producing sites predicted for Hauturu at any depth under the scenario of maximum climate warming reduces the chance that females would successfully locate a relatively cool nesting location.

Shifting oviposition phenology is another means by which maternal nesting behaviour can, theoretically, manipulate offspring sex ratios in species with TSD. Tuatara on Takapourewa/Stephens Is. tend to nest earlier in warmer years (Nelson 
unpub. data), though oviposition date is not likely to affect hatchling sex ratios on that island under the scenario of maximum climate warming (Chapter 4). Given that offspring sex ratios are predicted as $>90 \%$ male at all depths under the scenario of maximum climate warming on Hauturu, onset of nesting would probably have to shift far enough, whether earlier or later, to time the thermosensitive period outside of summer.

In addition to quantifying the suitability of the two initial release sites on Hauturu for providing nesting habitat, the microclimate surfaces developed in this study can be used to inform any future translocations of tuatara that occur outside the capture sites of adults. Current guidelines for conservation-based translocations differentiate between reintroduction, the release of organisms to locations within the indigenous range of the species, and assisted colonisation, the introduction of organisms outside their known range (IUCN 2013). Despite the lack of historical distribution data for tuatara on Hauturu, any future translocations could, in a geographic sense, realistically be considered reintroductions. Prehistoric dispersal of tuatara across Hauturu would have been limited by the relatively large size of the island, rather than by topographic or environmental barriers. Nevertheless, the selection of optimal release sites is limited by the proximity of suitable nesting habitat.

Under the current climate and minimum warming scenarios, any further releases should be restricted to the $40 \%$ of the island that, according to the predictions of this study, can support embryonic development of tuatara. Under a scenario of maximum climate warming, targeting translocations to the $8 \%$ of Hauturu predicted to produce mixed-sex nests with the expectation that females would preferentially select the coolest nesting locations within those areas is unrealistic. In-situ manipulation of offspring sex ratios via installation of shade cloth over nest sites, which has been proposed as a management option for sparsely vegetated North Brother Island (Mitchell et al. 2008), would likely be ineffective on Hauturu and other forested islands. Whether Hauturu and 
other northern locations will remain suitable habitat for tuatara in the future will depend on the magnitude of climate change observed over the next few decades.

The threat of climate change is one of the primary motivations for the consideration of assisted colonisation as a conservation tool (Seddon et al. 2009; Fouquet et al. 2010; Loss et al. 2010; IUCN 2013; Mitchell et al. 2013a; Rout et al. 2013). If the rate of macro-scale climate change observed over the next few decades more closely matches the maximum, rather than the minimum, predicted warming scenario, conservation of species with TSD may rely on assisted colonisation to cooler microclimates, some of which are currently unsuitable for supporting embryonic development (Besson et al. 2012). Rather than attempting to monitor site-specific temperatures via in-situ sampling (Besson et al. 2012), the methods used in this study can be translated to any proposed translocation site to examine suitability of habitat for temperature-dependent development and sex determination, regardless of whether data on nest site selection are available.

In addition to informing the pre-translocation decision process, spatially explicit data on incubation suitability may be useful for facilitation of post-translocation monitoring, since the probability of observing nesting behaviour should be increased if suitable and unsuitable incubation microclimates are known, especially in species known to undertake nesting migration. The primary goal of a translocation is population growth (Armstrong \& Seddon 2008); however, successful reproduction in species with TSD does not guarantee persistence if offspring sex ratios are unknown. Spatially explicit, predictive data on hatchling sex ratios can reduce uncertainty in posttranslocation monitoring if data on nest site selection are not immediately forthcoming.

Post-release dispersal, in general, is a major barrier to monitoring of translocated populations (Armstrong \& Seddon 2008). While this study only examined micro-habitat suitability in terms of temperature-dependent incubation, the same principles could also be applied to translocations, more generally. Spatially explicit, mechanistic predictions 
have already been used to examine habitat suitability for species for which assisted colonisation has been proposed and, to a limited extent, utilised as a conservation tool (Mitchell et al. 2013a). Spatially explicit, statistical models of dispersal have been developed to examine home ranges for reintroductions of beavers (Castor fiber) (MacDonald et al. 2000; South et al. 2000) and Eurasian lynx (Lynx lynx) (Kramer-Schadt et al. 2004). Suitability surfaces based on an organism's fundamental niche could be used to predict species- or population-specific patterns of dispersal from release sites and reduce uncertainty in post-translocation monitoring in novel environments for which no dispersal data are available. Predictive models of habitat suitability can also facilitate an experimental approach to translocations (Seddon et al. 2007) by defining multiple release sites along a suitability gradient.

Finally, surfaces that predict population dispersal based on a species' fundamental niche can facilitate pre-translocation risk assessment (Ricciardi \& Simberloff 2009 b) by informing the probability that a translocated population would be capable of invasion-scale dispersal. Much of the debate surrounding the use of assisted colonisation as a conservation strategy has focused on the not unfounded risk that the intentional release of a 'new' species into an ecosystem may become an unintentional biological invasion (Hoegh-Guldberg et al. 2008; Mueller \& Hellmann 2008; Ricciardi and Simberloff 2009a, b; Sax et al. 2009; Seddon et al. 2009; Hewitt et al. 2011; Lawler \& Olden 2011; Lunt et al. 2013). At a fundamental level, this criticism is grounded in a paradigm that defines habitat based on a geographical, rather than an environmental, delineation between suitability and non-suitability.

Reintroductions may be less likely to present the ecosystem-level risks that have made assisted colonisation a subject of controversy. However, a consequence of the range-based classification of translocations is that reintroductions carry the implicit assumption that, even if the previous geographic range is no longer environmentally suitable, the bioclimatic envelope of the former range can be used to infer the future 
suitability of translocation sites (Ledig et al. 2012; IUCN 2013). The features that lead to the consideration of translocation as a potential conservation tool for a given species are the same characteristics that introduce uncertainty into distribution-based models of habitat suitability: small, isolated populations; limited dispersal capability; and habitation of highly fragmented landscapes (Loss et al. 2010). A model of habitat suitability based on the known former range of a species is almost certain to be incomplete. 



\section{Chapter 6}

\section{General discussion}

Long-term persistence of species with temperature-dependent sex determination (TSD) depends on the suitability of both current and future habitats for maintaining balanced population sex ratios. The central aim of this thesis was to examine how rapid environmental change, primarily climate warming, may affect hatching phenology and offspring sex ratios in two populations of tuatara (Sphenodon punctatus). The four studies comprising this research provide insight into how variation in the macroclimate can affect microclimate-scale incubation conditions in wild populations and highlight the importance of considering spatial resolution and scale when developing predictive

microclimate surfaces. Here, I provide a summary of the findings of this research and discuss the implications for conservation and management of tuatara and other species with TSD.

\subsection{Chapter overviews}

\section{Chapter 2}

\section{Tuatara rookery selection behaviour responds to rapid vegetation change}

Understanding nest site selection behaviour is crucial for predicting the impacts of habitat modification and other sources of environmental variation on oviparous species, especially those with TSD. In Chapter 2, a longitudinal dataset of rookery selection behaviour linked with remotely-sensed land cover data revealed a clear relationship between nest site selection and vegetation composition for tuatara on the island of Takapourewa, New Zealand. While nesting females tended to choose the same rookery in multiple nesting seasons, successional change in vegetation explained observed increases in rookery-switching behaviour. Previous reports of rookery fidelity 
in tuatara are likely an artefact of arrested succession maintained by continual management on Takapourewa and preference of females for relatively open nest sites. The effects of land cover change should be accounted for in assessments of habitat suitability for tuatara. Modelled vegetation cover was used to inform a mechanistic microclimate model in Chapters 3 and 4. The methodology of this study also tested a novel method for retroactively quantifying behavioural observations using remotely sensed data.

\section{Chapter 3}

\section{Local climate and high-resolution geospatial data are required to model soil temperatures mechanistically at a small spatial scale}

Since most terrestrial organisms experience their environment within a relatively small spatial extent, the predictive capacity of modelled climate surfaces can be limited by the spatial resolution of data used for model parameterisation. For oviparous species, modelling the sub-surface microenvironment experienced by developing embryos at the resolution of nests or nesting areas may be useful for predicting the effects of climate change and other novel environmental scenarios on offspring survival and phenotype. In Chapter 3, the effects of multi-resolution, multiextent input data on the accuracy of mechanistic soil temperature predictions were examined within a small spatial extent at high temporal and spatial resolutions. Soil temperatures were modelled for the island of Takapourewa at an accuracy comparable to lower-resolution, large-extent microclimate models, within $2-3^{\circ} \mathrm{C}$ of observed values. However, the small extent of the study area required that the model be parameterised with high-resolution geospatial data and local, daily climate data. Importantly for populations restricted within relatively small areas, large-extent microclimate layers are unlikely to provide accurate estimates of local conditions when the spatial scale of a 
habitat is smaller than the resolution of the climate layers. The most accurate microclimate model parameterisation developed in this study was used to predict hatching phenology and incubation temperatures in Chapter 4 and modified to examine nest site suitability in a different study location in Chapter 5.

\section{Chapter 4}

\section{Predicting effects of climate change on offspring sex ratios and hatching phenology in a population of tuatara}

The ability of a population to persist in a given location depends on complex, organism-microclimate interactions. In species with TSD, females' preference for nesting habitat and the suitability of chosen nesting sites for maintaining balanced sex ratios may become de-coupled under novel climate conditions, masking indirect effects of climate change on population demographics. In Chapter 4, a mechanistic microclimate model was used to predict incubation temperatures, offspring sex ratios, and hatching dates for tuatara on the island of Takapourewa under the current climate and two hypothetical warming scenarios. Mechanistic and geostatistical models were used to examine the effects of climate warming projected for the next century on embryonic development, hatching phenology, and offspring sex ratios.

If the magnitude of climate change realised over the next one hundred years more closely matches the minimum, rather than the maximum, projected scenario of regional warming, tuatara hatchling sex ratios on Takapourewa are unlikely to become male-biased. Under a scenario of maximum climate warming, offspring sex ratios could become male-biased if females continue to nest at a mean depth of $110 \mathrm{~mm}$. Oviposition phenology was key to determining both the overall patterns of sex ratios predicted and whether, under the maximum warming scenario, nests at $100 \mathrm{~mm}$ would be more likely 
to produce proportions of male hatchlings that could threaten future population viability.

\section{Chapter 5}

Incubation suitability surfaces indicate hypothetical nesting locations for a reintroduced population of tuatara

Quantitative data on habitat suitability are becoming progressively more important to reintroduction biology, both for identification of appropriate release sites for translocations and for post-translocation monitoring. For oviparous species with TSD, an effective definition of habitat suitability necessitates both that females can locate nest sites and that those locations support embryonic development and, over the next century, maintain balanced offspring sex ratios. In Chapter 5, a spatially explicit, mechanistic microclimate model was used to define hypothetical nesting locations and to predict hatching phenology and offspring sex ratios for a small, reintroduced population of tuatara on the island of Hauturu under current and projected future climate scenarios.

Between $40-50 \%$ of the area of Hauturu can facilitate successful hatching under the current climate or a scenario of minimum climate warming, and both previous release sites are adjacent to suitable nesting habitat. Thus, females do not have to migrate more than a few metres away from their home burrows to nest. Offspring sex ratios on Hauturu are unlikely to become male-biased over the next century, unless the magnitude of climate change observed more closely matches the scenario of maximum projected warming. In that case, $100 \%$ of sites on Hauturu would facilitate completion of embryonic development, but sex ratios may become highly male-biased. Mean hatching was predicted to occur in austral summer under the current climate and minimum 
warming scenarios but shifted to spring under the scenario of maximum climate warming.

\subsection{Conclusion and limitations}

Determining how macro-scale climate change will affect microclimate conditions and, in turn, how changing microclimate conditions will impact populations is challenging due to the uncertainty inherent in predictive models. One key advantage of process-explicit models is that predictions generated at a microclimate scale under novel macroclimates do not rely on a forced statistical relationship between conditions at the two spatial scales. Both the microclimate model (Kearney et al. 2014a, b) and development rate and sex ratio functions (Mitchell et al. 2006, 2008) utilised here have been tested previously against in-situ data. Chapter 3 and, to a lesser extent, Chapter 5 of this thesis sought to further reduce uncertainty by (1) examining the accuracy of the microclimate model at an extremely high spatial resolution and within a small spatial extent and (2) testing the sensitivity of the empirical development rate and sex ratio functions to residual error in the modelled microclimate surfaces. Nevertheless, a few caveats should be noted in consideration of the results presented here.

Given that female tuatara on Takapourewa may nest as often as every two years for several decades, the data presented in Chapter 2 provide only a snapshot of nesting patterns on the island. While this research revealed a clear link between vegetation succession and shifting nest site choice in tuatara, the specific characteristics that signal the suitability of a non-forested microsite for nest construction remain unquantified. The incubation conditions experienced by developing embryos depend on the microclimate that a nesting female selects, not on the mechanisms underlying the choice. Thus, from a conservation perspective, knowledge of specific nest site cues is less important than spatially explicit data on nest site choice in species with TSD. One 
advantage of using a process-explicit microclimate model for describing changing incubation conditions is that, provided spatial data on nesting patterns are available, nest sites do not need to be sampled repeatedly. Nevertheless, the relationship between nest site choice and vegetation observed on Takapourewa revealed a potential limitation in the modelling programme used in subsequent chapters.

Female tuatara differentiated among vegetation types but, corresponding with the results of a previous study (Refsnider et al. 2010), did not select only locations that were completely devoid of vegetation. This outcome is not unexpected, as few locations on Takapourewa remain bare following the removal of grazing pressure from the former pasture areas. Likewise, nesting has been taking place on Takapourewa within a humanmodified landscape for just over three generations of tuatara, and the use of pasture areas as rookeries is more than likely a behavioural, rather than evolutionary, response to environmental change. Vegetation may have complex effects on soil temperatures that are not accounted for in the current implementation of NicheMapR and, thus, remain indiscernible to this research. Especially under scenarios of climate warming, insulating effects of low-growing vegetation may be important to predicting incubation conditions in females' chosen nest sites. Future work may benefit from linking observed and modelled soil temperatures with a vegetation growth model (e.g., LPJ-Guess) (Smith 2001; Smith et al. 2001) to examine whether incorporating effects of near-ground vegetation on soil heat transfer would improve model accuracy.

Even given hourly soil temperature data modelled with $100 \%$ accuracy, calculations of embryonic development rates and offspring sex ratios are still subject to bias (Mitchell et al 2008; Nelson et al. 2010). Parameter values derived from constant incubation experiments may not reflect the variation experienced by embryos in natural nests or, in particular, on different islands. The parameters used here were derived from both experimental data and field studies on Takapourewa (Mitchell et al. 2008; Nelson unpub. data), but model predictions have not been independently tested against in-situ 
hatchling sex ratios on that island under different macroclimate conditions (i.e., in different years). The sex ratio function parameters used in this study provided slightly conservative estimates of male hatchlings on North Brother Island in a previous study (Mitchell et al. 2008). Sampling in-situ hatchling sex ratios on Takapourewa was outside the scope of this research; however, testing the predictions provided in Chapter 4, particularly in a relatively 'warm' year, would be beneficial for examining whether modelled predictions are the same as observed values. Likewise, parameterising the development rate and sex ratio functions for tuatara on Hauturu would help determine whether modelled predictions are sensitive to physiological differences between populations (e.g., pivotal temperatures, developmental minimums and maximums).

The primary constraint on this research was the computation time required to run the microclimate model and development rate functions for microsites at the extremely high spatial resolution used in Chapter 4. With any spatially explicit model, the number of sites for which predictions are generated and, thus, the computing capability required to do so, increases if either the spatial resolution of model input or the spatial extent of the study area increases. Generating predictions for more than approximately 1000 sites without access to either a supercomputer or performanceoptimised cloud computing platform is currently impractical (pers. obs.). Since memory capacities and processor speeds double approximately every two years (Intel 2005), the ability to model hatching phenology and predict offspring sex ratios at the scale of individual nest sites using only consumer-level computing will probably be limited for the foreseeable future to habitats contained within a small spatial extent. Alternatively, incubation microclimates could be modelled mechanistically at a lower spatial resolution (e.g., nesting areas rather than individual sites). The geostatistical model used in Chapter 4 should be further explored as a method for circumventing the computational requirements of mechanistic modelling at very high spatial resolutions. 
Due to computational limits, the effects of input resolution on accuracy of soil temperatures could not be examined for every possible combination of parameters. Nor could the sensitivity of physiological predictions to error in predicted temperatures be examined for the full extent of Takapourewa. Though Chapter 3 sought to reduce uncertainty in model predictions through rigorous examination of multiple model parameterisations, that the most accurate model parameterisation included a mean 2$3^{\circ} \mathrm{C}$ of error suggests that additional work may be able to improve upon the model parameterisation used in this research. Despite these limitations, however, the conservative predictions of hatching phenology and offspring sex ratios provided in Chapter 4 may be an outcome of oviposition date-dependent effects, rather than error in the microclimate model.

\subsection{Implications for conservation}

The results presented here for tuatara emphasise several points relevant to the conservation of populations with temperature-dependent sex determination and, more generally, to the use of predictive microclimate surfaces in conservation ecology: (1) spatially explicit, in-situ data on patterns of nest site choice can link predictions of embryonic development rates and offspring sex ratios to changing macroclimate conditions, even if specific nesting cues are unknown. (2) Definitions of habitat suitability for species with TSD should include spatially explicit predictions of offspring sex ratios, informed by data on nesting patterns, if the latter are available. However, the suitability of habitat for supporting embryonic development and maintaining balanced offspring sex ratios should not be inferred either from nest site selection or historic adult presence. (3) Whether the spatial resolution of a given macro- or microclimate surface is 'high' should be considered relative to the spatial extent of an area of interest, not to the resolution of previous models. Published 'high-resolution' climate and 
microclimate surfaces, while invaluable for examining global- or regional-scale patterns of change, may contain unacceptable error if applied within relatively small areas. (4) In many cases, a mechanistic microclimate model will be no more data-hungry than a correlative distribution model, while offering several key advantages over statistical methods. The development of high-resolution mechanistic microclimate surfaces is limited more by computational capability than by the availability of input data.

\subsubsection{Implications of ecological restoration for conservation of tuatara}

The preference of female tuatara for un-vegetated nesting areas could be adopted as a tool for conservation and management of populations in the Cook Strait, used either in tandem with or in lieu of artificial incubation. The revegetation of Takapourewa was undertaken with the recommendation that small pasture areas should remain intact (DOCb) to provide habitat for nesting female (Thompson et al. 1988) and juvenile (McIntyre 1988) tuatara. Given that the population may be above carrying capacity (Moore et al. 2007), facilitation of increased nesting would probably not be beneficial for the population on Takapourewa. However, this advice is not unfounded for much smaller populations or those that have been recently translocated.

Establishment of a self-sustaining population via translocation requires that introduced individuals reproduce in their new habitat and, for species with TSD, that offspring sex ratios remain approximately 1:1. Female tuatara in wild populations nest only every few years (Cree et al. 1992; Cree 1994; Newman et al. 1994). In addition, because females camouflage nest openings, locating nests in the wild without directly observing nesting behaviour is extremely difficult (Tyrrell et al. 2000). The creation of artificial rookeries (i.e., a site that would facilitate successful embryonic development and maintain balanced sex ratios) adjacent to release sites, via removal of canopy vegetation, could be used to facilitate the observation of nesting behaviour in wild 
populations and provide confirmation that females are reproducing. The optimal location(s) for an artificial rookery could be determined using spatially explicit models of embryonic development to prevent over-production of one sex in modified sites. Habitat modification is, to some extent, incompatible with restoring and protecting native ecosystems on protected islands. However, the efficacy of the technique could be tested at translocation sites on the New Zealand mainland, such as Maungatautari Ecological Island on the North Island or Orokonui Ecosanctuary on the South Island, which are protected sanctuaries but not restricted Nature Reserves.

\subsubsection{Reintroduction and monitoring of populations with TSD}

Current guidelines for conservation-based translocations differentiate between reintroduction, the release of organisms to locations within the indigenous range of the species, and assisted colonisation, the introduction of organisms outside their known range (IUCN 2013). However, the thermal suitability of a proposed translocation site for facilitating embryonic development and maintaining balanced hatchling sex ratios in species with TSD cannot be reliably inferred from either past or present distributions. A mechanistic model of soil temperatures can be used to rigorously assess the suitability of potential translocation sites under current and projected future climate scenarios, independently of distribution data.

The use of process-explicit microclimate and niche models may also reduce the time and financial investments required for translocation planning. Geospatial and climate data required for model parameterisation are increasingly available via free download at multiple spatial and temporal resolutions. In contrast with correlative distribution models, updating a mechanistic model for a 'new' climate scenario does not require repeated collection of in-situ microclimate data. Since running the models used

here at a very high spatial resolution is computationally intensive, relatively low 
resolution microclimate surfaces could be used to identify the specific areas within potential translocation sites for which higher resolution models should be generated.

The implications of temperature-dependent embryonic development for hatching phenology may be as important for conservation of species with TSD as the effects of climate change on offspring sex ratios. Given appropriate physiological data, a microclimate surface used to model embryonic development could, for example, also be extended to predict invertebrate phenology in the same habitat and determine whether juveniles would have sufficient prey available after hatching. Using the same microclimate surfaces, the life cycles and activity patterns of multiple species can be modelled to examine the potential for interspecific competition at potential translocation sites.

\subsection{Management recommendations and directions for future research}

Translocation has so far been an effective tool in the conservation of tuatara (Keall et al. 2010; Miller et al. 2012), increasing the total number of populations from a minimum of 30 in the 1990s (Cree \& Daugherty 1990) to 45 in 2013 (Gaze 2001; Miller et al. 2012; Nelson unpub.). As funding and other logistical considerations permit, tuatara populations are being translocated not only to offshore islands but also to enclosed (i.e., rodent-protected) sanctuaries on the New Zealand mainland (Miller et al. 2012; Nelson unpub. data). Ultimately, tuatara could be reintroduced to their former range throughout mainland New Zealand (Gaze 2001; Miller et al. 2012). Given the findings of this and previous research (Mitchell et al. 2008, 2009), translocations of tuatara, even to sites within their known prehistoric range, should not be undertaken without quantifying location-specific habitat suitability to include the effects of both current and future climate scenarios on offspring sex ratios. 
Recommendation I: Planning for conservation translocations should include the implications of temperature-dependent development and sex determination for assessments of habitat suitability.

Established conservation guidelines (Gaze 2001) do not account for the potential effects of climate warming on demographics of either relict or translocated populations of tuatara, a key concern for species with TSD (Mitchell et al. 2008). Notably, the current definition of optimal nesting habitat is based primarily on the observation that females prefer to nest in relatively warm, open areas and secondarily on the requirement that soils in nesting areas are sufficiently moist to prevent desiccation of eggs (Gaze 2001). Minimally, long-term viability of a tuatara population requires that offspring sex ratios do not increase beyond 65\% male (Mitchell et al. 2009; Grayson et al. 2014). Planning for translocations, however, should strive to identify locations that will maintain balanced offspring sex ratios, preferably without artificial manipulation of incubation microclimates (e.g., via in-situ shading or ex-situ incubation) (Seddon 1999), even under a scenario of maximum climate warming.

The definition of suitable nesting habitat for tuatara, or any species with TSD, should be based not merely on the preferences of females for particular microclimate characteristics but on how the various microclimates within a given area would impact offspring sex ratios if utilised as a nesting habitat. Identifying locations that are relatively warm under the current climate as suitable nesting habitat for Cook Strait tuatara could inadvertently lead to male-biased offspring sex ratios in translocated populations under climate warming scenarios. Future studies should endeavour to overlay spatially explicit predictions of offspring sex ratios with locations that are thermally suitable for adults, thereby limiting the definition of habitat suitability to include only sites that will support both the survival of individuals and maintenance of viable populations via in-situ reproduction over at least the next 100 years. In addition, 
more research is needed to define habitat suitability for northern tuatara without relying on data collected for Cook Strait populations.

Recommendation II. Pre-translocation planning should include a predictive, micrositeresolution assessment of habitat suitability.

Because macro-scale climate warming is likely to facilitate changes in microclimate conditions, translocations of tuatara and other species with TSD should be approached as assisted colonisations, rather than reintroductions, even if a proposed translocation site is known to be within a species' historic distribution. That the characteristics of a particular habitat are correlated with a species' current realised niche is insufficient evidence that either current or future microclimate conditions will maintain balanced offspring sex ratios. Pre-translocation planning should include a spatially explicit assessment of habitat suitability, using the aforementioned criteria, for both current and future climate scenarios. Since the development of microclimate surfaces at the spatial resolution of nest sites is computationally intensive, future research should examine how the resolution of geospatial data, which determines the number of sites for which predictions are generated, affects model accuracy. Running NicheMapR with the minimum spatial resolution of input data required to generate accurate predictions of soil temperatures would reduce computation time, increasing the feasibility of using a process-explicit microclimate model within a context of applied conservation and management.

If high-performance computing is needed due to the number of sites for which predictions are required, increasing availability of low-cost cloud computing via web services such the Amazon EC2 platform could be used by managers to access highperformance computing at any time and without reliance on a larger research project. Computing costs, which range from 0.02 to around 2.20 NZD per core hour 
(http://aws.amazon.com/ec2), should be included by default in pre-translocation planning budgets. While predicting offspring sex ratios for proposed translocation sites may increase management costs initially, pre-translocation modelling is justified by (1) the gain in knowledge of the relative suitability of potential translocation sites and (2) the reduction in potential for inadvertent translocation of a population to habitat that is currently or will likely become unsuitable for maintaining balanced offspring sex ratios.

Recommendation III. Spatially explicit predictions of offspring sex ratios for tuatara should be viewed as a vital means of remote population monitoring and revised as global climate change models are updated.

Modelling offspring sex ratios in species with TSD cannot completely substitute for in-situ sampling of population sex ratios, since post-hatching conditions may differentially affect male and female survival (Mitchell et al 2008). Even more importantly, balanced adult sex ratios should not be considered evidence that current incubation conditions are producing similar numbers of males and females. Since the age of a wild tuatara cannot be reliably determined without some means of permanent identification (e.g., PIT-tagging), the current sex ratio of an established population reflects decades of past incubation conditions. Even severe bias in offspring sex ratios would not be reflected in a population until successive generations of hatchlings reach sexual maturity. In small populations, contiguous male-producing years could result in relatively sudden skewing of adult sex ratios once hatchlings from those years enter the reproductive population. Development of a non-invasive method with which to determine sex of juvenile tuatara would be beneficial for determining whether predicted offspring sex ratios are similar to those observed in the wild. Future research should also thoroughly examine whether causes of mortality in juvenile tuatara differentially 
affect male and female offspring and determine the extent to which predicted hatchling sex ratios are likely to reflect future adult sex ratios.

As the number of wild tuatara populations increases (Miller et al. 2012), in-situ sampling of either offspring or adult sex ratios will become increasingly impractical. The microclimate and embryonic development models used in this research can be used not only to inform assessments of habitat suitability but also as a method of remotely monitoring offspring sex ratios. High-resolution GIS layers and yearly climate data needed to parameterise the microclimate model are increasingly freely available in New Zealand from online data repositories (e.g., LINZ, NIWA), and downscaled global climate change scenarios are updated every few years (MFE). Since tuatara embryos may take up to two years to fully develop (Cree et al. 1991, 1992), a microclimate model can be used to predict soil temperatures and resultant embryonic development rates and offspring sex ratios yearly, either for every population or a representative subset of populations (e.g., from ecoregions) using the previous two years of local climate data. Future research should determine whether sex ratios of populations in environmentally distinct habitats are subject to bias at different minimum thresholds of climate warming.

Whether sex ratios can remain balanced in tuatara populations without management intervention will depend to a large extent on the magnitude of climate warming observed over the next century. Updated assessments of global climate change are released by the IPCC about every six years (http://www.ipcc.ch). Predictions of future hatchling sex ratios can be improved for tuatara populations as global predictions are downscaled for New Zealand (http://www.mfe.govt.nz). Predictive, spatially explicit models provide the means with which conservation and management of tuatara and other species with TSD can become preemptive of, rather than reactive to, the microenvironmental effects of global climate change. 



\section{References}

Agarwal, C., G.L. Green, M. Grove, T. Evans, \& C. Schweik. 2000. A review and assessment of landuse change models: dynamics of space, time, and human choice. Center for the Study of Institutions, Population, and Environmental Change, Bloomington, Indiana, USA: Indiana University, 76 pp.

Agresti, A. 2002. Categorical analysis. 2nd Ed. Hoboken, New Jersey, USA: Wiley, 710 pp.

Akçakaya, H.R., V.C. Radeloff, D.J. Mladenoff, \& H.S. He. 2004. Integrating landscape and metapopulation modeling approaches: viability of the sharp-tailed grouse in a dynamic landscape. Conservation Biology 18: 526-37.

Allendorf, F.W. 1986. Genetic drift and the loss of alleles versus heterozygosity. Zoo Biology 5(2): 181-90.

[ASTM] American Society for Testing and Materials International. 2008. ASTM D5334-08: Standard test method for determination of thermal conductivity of soil and soft rock by thermal needle probe procedure.

Amiel, J.J. \& R. Shine. 2012. Hotter nests produce smarter young lizards. Biology Letters 8: 372-4.

Andrewartha, S.J., N.J. Mitchell, \& P.B. Frappell. 2008. Phenotypic differences in terrestrial frog embryos: effect of water potential and phase. Journal of Experimental Biology 211: 3800-7.

Andrewartha, S.J., N.J. Mitchell, \& P.B. Frappell. 2010. Does incubation temperature fluctuation influence hatchling phenotypes in reptiles? A test using parthenogenetic geckos. Physiological and Biochemical Zoology 83: 597-607.

Angilletta, M.J.J., R.S. Winters, \& A.E. Dunham. 2000 Thermal effects on the energetics of lizard embryos: implications for hatchling phenotypes. Ecology 81: 2957-68.

Apaydin, H. F.K. Sonmez, \& Y.E. Yildirim. 2004. Spatial interpolation techniques for climate data in the GAP region in Turkey. Climate Research 28: 31-40. 
Araújo, M.B. \& A. Guisan. 2006. Five (or so) challenges for species distribution modeling. Journal of Biogeography 33: 1677-88.

Araújo, M.B. \& M. Luoto. 2007. The importance of biotic interactions for modelling species distributions under climate change. Global Ecology and Biogeography 16: 743-53.

Armstrong, D.P. \& M.H. Reynolds. 2012. Modelling reintroduced populations: the state of the art and future directions. In: J.G. Ewen, D.P. Armstrong, K.A. Parker, \& P.J. Seddon, Eds. Reintroduction biology: integrating science and management. West Sussex, UK: WileyBlackwell, pp. 165-222.

Armstrong, D.P. \& P.J. Seddon. 2008. Directions in reintroduction biology. Trends in Ecology and Evolution 23(1): 20-5.

Arnold, S.J. 1994. Multivariate inheritance and evolution: a review of concepts. In: C.R.B. Boake, Ed. Quantitative genetic studies of behavioral evolution. Chicago, Illinois, USA: University of Chicago Press. pp. 17-48.

Ashcroft, M.B., L.A. Chisholm, \& K.O. French. 2009. Climate change at the landscape scale: predicting fine-grained spatial heterogeneity in warming and potential refugia for vegetation. Global Change Biology 15: 656-67.

Ashmore, G.M. \& F.J. Janzen. 2003. Phenotypic variation in smooth softshell turtles (Apalone mutica) from eggs incubated in constant versus fluctuating temperatures. Oecologia 134: 182-8.

Austin, M. 2007. Species distribution models and ecological theory: a critical assessment and some possible new approaches. Ecological Modelling 200: 1-19.

Bartelt, P.E., R.W. Klaver, \& W.P. Porter. 2010. Modeling amphibian energetics, habitat suitability, and movements of western toads, Anaxyrus (=Bufo) boreas, across present and future landscapes. Ecological Modelling 221: 2675-86. 
Barwick, R.E. 1982. Observations on active thermoregulation in the tuatara, Sphenodon punctatus (Reptilia: Rynchocephalia). In: Newman, D.G., Ed., New Zealand Herpetology. New Zealand Wildlife Service Occasional Publication 2: 377-91.

Basolo, A. 1994. The dynamics of Fisherian sex-ratio evolution: theoretical and experimental investigations. The American Naturalist 144(3): 473-90.

Bean, W.T., L.R. Prugh, R. Stafford, H.S. Butterfield, M. Westphal, \& J.S. Brashares. 2014. Species distribution models of an endangered rodent offer conflicting measures of habitat quality at multiple scales. Journal of Applied Ecology 51: 1116-25.

Beauchamp, A.J. \& T.H. Worthy. 1988. Decline in distribution of the takahe Porphyrio (= Notornis) mantelli: a re-examination. Journal of the Royal Society of New Zealand 18(1): 103-18.

Bell, A.M., S.J. Hankison, \& K.L. Laskowski. 2009. The repeatability of behaviour: a meta-analysis. Animal Behaviour 77: 771-83.

Bennie, J., B. Huntley, A. Wiltshire, M.O. Hill, \& R. Baxter. 2008. Slope, aspect and climate: spatially explicit and implicit models of topographic microclimate in chalk grassland. Ecological Modelling 216(10): 47-59.

Berens, P. \& M.J. Velasco. 2009. The circular statistics toolbox for Matlab. Technical Report No. 184, Max Planck Institute for Biological Cybernetics. 9 pp.

Besson, A.A. \& A. Cree. 2011. Integrating physiology into conservation: an approach to help guide translocations of a rare reptile in a warming environment. Animal Conservation 14: 28-37.

Besson, A.A., N.J. Nelson, C.M. Nottingham, \& A. Cree. 2012. Is cool egg incubation temperature a limiting factor for the translocation of tuatara to southern New Zealand? New Zealand Journal of Ecology 36(1): 90-9.

Blanchard, B. 2002. Tuatara captive management plan. Threatened species occasional publication 21A, Tuatara Recovery Group, Department of Conservation [DOC], Wellington, New Zealand. 76 pp. 
Boake, C.R.B. 1989. Repeatability: its role in evolutionary studies of mating behavior. Evolutionary Ecology 3: 173-82.

Boer, E.P.J., K.M. de Beurs, \& A.D. Hartkamp. 2001. Kriging and thin-plate splines for mapping climate variables. International Journal of Applied Earth Observation and Geoinformation 3(2): 146-54.

Booth, D.T. 2006. Influence of incubation temperature on hatchling phenotype in reptiles. Physiological and Biochemical Zoology 79(2): 274-81.

Both, C., M. van Asch, R.G. Bijlsma, A.B. van den Burg, \& M.E. Visser. 2009. Climate change and unequal phenological changes across four trophic levels: constraints or adaptations? Journal of Animal Ecology 78: 73-83.

Boyle, M., L.E. Schwanz, J. Hone, \& A. Georges. 2014. How do climate-linked sex ratios and dispersal limit range boundaries? BMC Ecology 14:19, doi:10.1186/1472-6785-14-19.

Bradshaw, A.D. 1965. Evolutionary significance of phenotypic plasticity in plants. Advances in Genetics 13: 115-55.

Bragg, W.K., J.D. Fawcett, \& T.B. Bragg. 2000. Nest-site selection in two eublepharid gecko species with temperature-dependent sex determination and one with genotypic sex determination. Biological Journal of the Linnean Society 69(3): 319-32.

Braña, F. \& X. Ji. 2000. Influence of incubation temperature on morphology, locomotor performance, and early growth of hatchling wall lizards (Podarcis muralis). Journal of Experimental Zoology 286: 422-33.

Briscoe, N.J., K.A. Handasyde, S.R. Griffiths, W.P. Porter, A. Krockenberger, \& M.R. Kearney. 2014. Tree-hugging koalas demonstrate a novel thermoregulatory mechanism for arboreal mammals. Biology Letters 10: 20140235.

Bristow, K.L. 1998. Measurement of thermal properties and water content of unsaturated sandy soil using dual-probe heat-pulse probes. Agriculture and Forest Meteorology 89: 75-84. 
Brooks, T.M., R.A. Mittermeier, G.A.B. da Fonseca, J. Gerlach, M. Hoffman, J.F. Lamoreux, C.G. Mittermeier, J.D. Pilgrim, \& A.S.L. Rodrigues. 2006. Global biodiversity conservation priorities. Science 313(58): 58-61.

Brotons, L., W. Thuiller, M.B. Araújo, \& A.H. Hirzel. 2004. Presence-absence versus presence-only modelling methods for predicting bird habitat suitability. Ecography 27: 437-48.

Brown, D. 2000. Stephens Island: ark of the light. Blenheim, NZ. 248 pp.

Buckley, L. 2008. Linking traits to energetics and population dynamics to predict lizard ranges in changing environments. American Naturalist 171: E1-19.

Buckley, L.B., G.H. Rodda, \& W. Jetz. 2008. Thermal and energetic constraints on ectotherm abundance: a global test using lizards. Ecology 89(1): 48-55.

Buckley, L.B., M.C. Urban, M.J. Angilletta, L.G. Crozier, L.J. Rissler, \& M.W. Sears. 2010. Can mechanism inform species' distribution models? Ecology Letters 13: 1041-54.

Bull, J.J. 1983. Evolution of sex determining mechanisms. Menlo Park, CA, USA: Benjamin/Cummings. 316 pp.

Bull, J.J. \& M.G. Bulmer. 1989. Longevity enhances selection of environmental sex determination. Heredity 63: 315-20.

Bull, J.J. \& E.L. Charnov. 1988. How fundamental are Fisherian sex ratios? Oxford Surveys in Evolutionary Biology 5: 96-135.

Bull, J.J., W.H.N. Gutzke, \& M.G. Bulmer. 1988. Nest choice in a captive lizard with temperaturedependent sex determination. Journal of Evolutionary Biology 2: 177-84.

Bull, J.J., R.C. Vogt, \& M.G. Bulmer. 1982a. Heritability of sex ratio in turtles with environmental sex determination. Evolution 36: 333-41.

Bull, J.J., R.C. Vogt, \& C.J. McCoy. 1982b. Sex determining temperatures in turtles: a geographic comparison. Evolution 36: 326-32.

Burger, J. \& Zappalorti, R.T. 1988. Effects of incubation temperature on sex-ratios in pine snakes: differential vulnerability of males and females. American Naturalist 132: 492-505. 
Burke, R.L. 1993. Adaptive value of sex determination mode and hatchling sex-ratio bias in reptiles. Copeia: $854-9$.

Burrough, P.A. \& McDonell, R.A. 1998. Principles of Geographical Information Systems. New York: Oxford University Press, 190 pp.

Butler, D.J. 1989. Stephens Island resource document. NZ Department of Conservation: Unpublished report.

Cabrelli, A. 2013. The impacts of climate change on Australian reptiles. Unpublished Ph.D. thesis. Macquarie University, NSW, Australia. 266 pp.

Cadby, C.D., G.M. While, A.J. Hobday, T. Uller, \& E. Wapstra. 2010. Mulsti-scale approach to understanding climate effects on offspring size at birth and date of birth in a reptile. Integrative Zoology 5: 164-75.

Cagle, K.D., G.C. Packard, K. Miller, \& M.J. Packard. 1993. Effects of the microclimate in natural nests on development of embryonic painted turtles, Chrysemys picta. Functional Ecology 7(6): 653-60.

Campbell, G.S. 1985. Soil physics with BASIC: transport models for soil-plant systems. Amsterdam, The Netherlands: Elsevier. 150 pp.

Campbell, G.S. \& J.M. Norman. 1998. An introduction to environmental biophysics. 2nd Ed. New York, NY, USA: Springer-Verlag.

Carrete, M., J.A. Donazar, \& A. Margalida. 2006. Density-dependent productivity depression in Pyrenean bearded vultures: implications for conservation. Ecological Applications 16: 1674-82.

Carslaw, H.S. \& J.C. Jaeger. 1959. Conduction of heat in solids. London: Oxford Univerity Press. $520 \mathrm{pp}$

Caughley, G. 1994. Directions in conservation biology. Journal of Animal Ecology 63: 215-44.

Charnov, E.L. \& J. Bull. 1977. When is sex environmentally determined? Nature 266: 828-30. 
Clusella-Trullas, S., T.M. Blackburn, \& S.L Chown. Climatic predictors of temperature performance curve parameters in ectotherms imply complex responses to climate change. The American Naturalist 177(6): 738-51.

Congalton, R.G. \& K. Green. 1999. Assessing the accuracy of remotely sensed data: principles and practices. New York: Lewis Publishers, 137 pp.

Conover, D.O. 2004. Temperature-dependent sex determination in fishes. In: N. Valenzuela \& V. Lance, Eds. Temperature-dependent sex determination in vertebrates, Washington, DC, USA: Smithsonian, pp 11-20.

Cook, C.N., D.G. Morgan, \& D.J. Marshall. 2010. Re-evaluating suitable habitat for reintroductions: lessons learnt from the eastern barred bandicoot recovery program. Animal Conservation 13: $184-95$.

Courtney, S.P. 1981. Coevolution of Pierid butterflies and their cruciferous foodplants III. Anthocharis cardamines (L.) Survival, development and oviposition on different hostplants. Oecologia 51: 91-6.

Cree, A. 1994. Low annual reproductive output in female reptiles from New Zealand. New Zealand Journal of Zoology 21: 351-372.

Cree, A. \& D. Butler. 1993. Tuatara recovery plan (Sphenodon spp.). Threatened Species Recovery Plan Series no. 9. Department of Conservation, New Zealand.

Cree, A., J.F. Cockrem, \& L.J. Guillette. 1992. Reproductive cycles of male and female tuatara (Sphenodon punctatus) on Stephens Island, New Zealand. Journal of Zoology London 226: 199-217.

Cree, A. \& C.H. Daugherty. 1990. Captive breeding of the New Zealand tuatara: past results and future directions. 5th World Conference on Breeding Endangered Species in Captivity. Cincinnati, OH, USA, 1988. pp. 477-90. 
Cree, A., C.H. Daugherty, \& J.M. Hay. 1995. Reproduction of a rare reptile, the tuatara Sphenodon punctatus, on rat-free and rat-inhabited islands in New Zealand. Conservation Biology 9: 373-83.

Cree, A., C.H. Daugherty, S.F. Schafer, \& D. Brown. 1991. Nesting and clutch size of tuatara (Sphenodon guntheri) on North Brother Island, Cook Strait. Tuatara 31: 9-16.

Cree, A., M.B. Thompson, \& C.H. Daugherty. 1995. Tuatara sex determination. Nature 375: 543.

Cree, A., M.B. Thompson, L.J. Guillette Jr., J.M. Hay, \& M.E. McIntyre. 1989. Embryonic development of tuatara in forested and open habitats on Stephens Island, New Zealand. Proceedings of the 2nd Annual Conference of the Society for Research on Amphibians and Reptiles in New Zealand [SRARNZ]. Middlemarch, New Zealand. New Zealand Journal of Zoology 16: 270, abstract.

Cressie, N. 1985. Fitting variogram models by weighted least squares. Journal of the International Association for Mathematical Geology 17: 653-702.

Cressie, N. 1988. Spatial prediction and ordinary kriging. Mathematical Geology 20: 405-21. (Erratum, Mathematical Geology 21: 493-4).

Cressie, N. 1990. The origins of kriging. Mathematical Geology 22: 239-52.

Crow, J.F. \& M. Kimura. 1970. An introduction to population genetics theory. Caldwell, New Jersey, USA: Blackburn Press. 608 pp.

Dallwitz, M.J. \& J.P. Higgins. 1992. User's guide to DEVAR: a computer program for estimating development rate as a function of temperature. $2^{\text {nd }}$ Ed. Division of Entomology Report No. 2, Commonwealth Scientific and Industrial Research Organisation [CSIRO], Australia. 27 pp.

Daugherty, C.H., A. Cree, J.M. Hay, and M.B. Thompson. 1990. Neglected taxonomy and continuing extinctions of tuatara (Sphenodon). Nature 347: 177-9.

Davis, S.K. 2005. Nest-site selection patterns and the influence of vegetation on nest survival of mixed-grass prairie passerines. Condor 107: 605-16. 
Davis, A.J., L.S. Jenkinson, J.H. Lawton, B. Shorrocks, \& S. Wood. 1998. Making mistakes when predicting shifts in species range in response to global warming. Nature 391: 783-6.

Dawbin, W.H. 1982. The tuatara Sphenodon punctatus: aspects of life history, growth, and longevity. In: D.G. Newman, Ed. New Zealand Herpetology, New Zealand Wildlife Occasional Publication 2: 237-50.

Deeming, D.C. 2004. Prevalence of TSD in crocodilians. In: Temperature-dependent sex determination in vertebrates. N. Valenzuela \& V. Lance, Eds. Smithsonian Institution Press. pp 33-41.

[DSIR] Department of Scientific and Industrial Research (New Zealand), NZ Soil Bureau. 1968. Soils of New Zealand, Part 3. Soil Bureau Bulletin, 127 pp.

de Smith, M.J., M.F. Goodchild, \& P.A. Longley. 2007. Geospatial analysis: a comprehensive guide to principles, techniques and software tools. Leicester, U.K.: Matador. 760 pp.

de Vries, D.A. 1963. Thermal properties of soils. In: Physics of plant environment. van Wijk, W.R., Ed. Amsterdam: North-Holland. pp 210-35.

Deutsch, C.A., J.J. Tewksbury, R.B. Huey, K.S. Sheldon, C.K. Ghalambor, D.C. Haak, \& P.R. Martin. 2007. Impacts of climate warming on terrestrial ectotherms across latitude. Proceedings of the National Academy of Sciences U.S. 105(18): 6668-72.

DeWitt, T.J. \& S.M. Scheiner. 2004. Phenotypic plasticity: functional and conceptual approaches. New York: Oxford University Press, 247 pp.

Didham, R.K., J.M. Tylianakis, N.J. Gemmell, T.A. Rand, \& R.M. Ewers. 2007. Interactive effects of habitat modification and species invasion on native species decline. Trends in Ecology and Evolution 22(9): 489-96.

Dieffenbach, E. 1843. Travels in New Zealand; with contributions to the geography, geology, botany and natural history of that country. vol. 1. London: John Murray.

[DOC]a Department of Conservation (New Zealand). n.d. Takapourewa fence/revegetation plan: 1998-2003 and beyond. Unpublished report, 3 pp. 
[DOC]b Department of Conservation (New Zealand). n.d. Stephens Island (Takapourewa) revegetation plan (untitled). Unpublished report, $46 \mathrm{pp}$.

[DOC]c Department of Conservation (New Zealand). n.d. Maps of Stephens Island (Takapourewa) replanting, 1992-2011 (untitled). Unpublished report, 3 pp.

Dochtermann, N.A. \& N.J. Dingemanse. 2013. Behavioral syndromes as evolutionary constraints. Behavioral Ecology, doi:10.1093/beheco/art002.

Dodd, A. 2007. Te Maraeroa Flat, Little Barrier Island: heritage assessment. New Zealand Department of Conservation [DOC]. 26 pp.

Dohm, M.R. 2002. Repeatability estimates do not always set an upper limit to heritability. Functional Ecology 16: 273-80.

Doody, J.S. 2009. Superficial lizards in cold climates: Nest site choice along an elevational gradient. Austral Ecology 34: 773-9.

Doody, J.S., S. Freedberg, \& J.S. Keogh. 2009. Communal egg-laying in reptiles and amphibians: evolutionary patterns and hypotheses. The Quarterly Review of Biology 84(3): 229-52.

Doody, J.S., E. Guarino, A. Georges, B. Corey, G. Murray, \& M. Ewert. 2006. Nest site choice compensates for climate effects on sex ratios in a lizard with environmental sex determination. Evolutionary Ecology 20: 307-30.

Doody, J.S. \& J.A. Moore. 2010. Conceptual model for thermal limits on the distribution of reptiles. Herpetological Conservation and Biology 5(2): 283-9.

Dormann, C.F., S.J. Schymanski, J. Cabral, L. Chuine, C. Graham, F. Hartig, M. Kearney, X. Morin, C. Römermann, B. Schröder, \& A. Singer. 2012. Correlation and process in species distribution models: bridging a dichotomy. Journal of Biogeography 39: 2119- 31.

Downes, S.J. \& R. Shine. 1999. Do incubation-induced changes in a lizard's phenotype influence its vulnerability to predators? Oecologia 120: 9-18. 
Du, W. \& X. Ji. 2003. The effects of incubation thermal environments on size, locomotor performance and early growth of hatchling soft-shelled turtles, Pelodiscus sinensis. Journal of Thermal Biology 28: 279-86.

Du, W.-G. \& R. Shine. 2010. Why do the eggs of lizards (Bassiana dupereyi: Scincidae) hatch sooner if incubated at fluctuating rather than constant temperatures? Biological Journal of the Linnean Society 101: 642-50.

Dunham, A.E., B.W. Grant, \& K.L. Overall. 1989. Interfaces between biophysical and physiological ecology and the population ecology of terrestrial vertebrate ectotherms. Physiological Zoology 62(2): 335-55.

Eads A., N.J. Mitchell, \& J. Evans. 2012. Patterns of genetic variation in desiccation tolerance in embryos of the terrestrial-breeding frog, Pseudophryne guentheri. Evolution 66: 2865-77.

East, K.T., M.R. East, \& C.H. Daugherty. 1995. Ecological restoration and habitat relationships of reptiles on Stephens Island, New Zealand. New Zealand Journal of Ecology 22: 249-61.

Eastman, J.R. 2012. Idrisi Selva manual. Version 17.01. Worcester, MA, USA: Clark Labs. 324 pp.

Eastman, J.R., L. Solorzano, \& M. Van Fossen. 2005. Transition potential modeling for land-cover change. In: GIS, spatial analysis, and modeling. D.J. Maguire, M. Batty, \& M.F. Goodchild, Eds. Redlands, CA: ESRI Press. pp. 357-85.

Elith, J., C.H. Graham, R.P. Anderson, M. Dudý'k, S. Ferrier, A. Guisan, R.J. Hijmans, F. Huettmann, J.R. Leathwick, A. Lehmann, J. Li, L.G. Lohmann, B.A. Loiselle, G. Manion, C. Moritz, M. Nakamura, Y. Nakazawa, J.M. Overton, A.T. Peterson, S.J. Phillips, K. Richardson, R. Scachetti-Pereira, R.E. Schapire, J. Sobero'n, S. Williams, M.S. Wisz, \& N.E. Zimmermann. 2006. Novel methods improve prediction of species' distributions from occurrence data. Ecography 29: 129-51.

Elith, J. \& J.R. Leathwick. 2009. Species distribution models: ecological explanation and prediction across space and time. The Annual Review of Ecology, Evolution, and Systematics 40: 677-97. 
Elphick, M.J. \& R. Shine. 1998. Long-term effects of incubation temperature on the morphology and locomotor performance of hatchling lizards (Bassiana duperreyi, Scincidae). Biological Journal of the Linnean Society 63: 429-47.

[ESRI] Environmental Systems Research Institute. 2003. ArcGIS 9: Using ArcGIS Geostatistical Analyst. Redlands, California, USA: ESRI Press. 306 pp.

Escadafal, R. 1989. Munsell soil color and soil reflectance in the visible spectral bands of landsat MSS and TM data. Remote Sensing of Environment 27(1): 37-46.

Escalona, T., N. Valenzuela, \& D.C. Adams. 2009. Nesting ecology in the freshwater turtle Podocnemis unifilis: spatiotemporal patterns and inferred explanations. Functional Ecology 23: 826-35.

Ewert, M.A., C.R. Etchberger, \& C.E. Nelson. 2004. Turtle sex-determining modes and TSD patterns, and some TSD pattern correlates. In: Temperature-dependent sex determination in vertebrates. N. Valenzuela \& V. Lance, Eds. Smithsonian Institution Press. pp 21-32.

Ewert, M.A., J.W. Lang, \& C.E. Nelson. 2005. Geographic variation in the pattern of temperaturedependent sex determination in the American snapping turtle (Chelydra serpentina). Journal of Zoology 265:81-95.

Ewert, M.A. \& C.E. Nelson. 1991. Sex determination in turtles: diverse patterns and some possible adaptive values. Copeia: 50-69.

Fisher, R.A. 1930. The genetical theory of natural selection: a complete variorum edition. Oxford University Press, 356 pp.

Fix, E. \& J.L. Hodges. 1951. Discriminatory analysis, nonparametric discrimination: consistency properties. Report No. 4, Project No. 21-49-004, USAF School of Aviation Medicine.

[FAO] Food and Agricultural Organization of the United Nations. 2006. Guidelines for soil description. 4th Ed. Rome, Italy. 109 pp. 
Fouquet, A., G.F. Ficelota, A. Haigh, \& N. Gemmell. 2010. Using ecological niche modelling to infer past, present, and future environmental suitability for Leiopelma hochstetteri, an endangered New Zealand native frog. Biological Conservation 143: 1375-84.

Frazer, G.W., C.D. Canham, \& K.P. Lertzman. 1999. Gap Light Analyzer (GLA), Version 2.0: Imaging software to extract canopy structure and gap light transmission indices from truecolour fisheye photographs, users manual and program documentation. Simon Fraser University, Burnaby, BC, Canada \& The Institute of Ecosystem Studies, Millbrook, NY, USA.

Frazer, G.W., J.A. Trofymow, \& K.P. Lertzman. 2000. Canopy openness and leaf area index in chronosequences of coastal temperate rainforests. Canadian Journal of Forest Research 30(2): 239-56.

Freedberg, S. \& D.R. Taylor. Sex ratio variance and the maintenance of environmental sex determination. Journal of Evolutionary Biology 20(1): 213-20.

Friendly, M. 1994. Mosaic displays for multi-way contingency tables. Journal of the American Statistical Association 89: 190-200.

Friendly, M. 2000. Visualizing categorical data. Cary, NC, USA: SAS Institute, 451 pp.

Gans, C., J.C. Gillingham, \& D.L. Clark. 1984. Courtship, mating and male combat in tuatara, Sphenodon punctatus. Journal of Herpetology 18: 194-7.

Gao, J. 2009. Digital analysis of remotely sensed imagery. New York: McGraw-Hill, 645 pp.

Gates, D.M. 2003. Biophysical ecology. New York, NY, USA: Springer-Verlag, 635 pp.

Gaze, P. 2001. Tuatara recovery plan 2001-2011. Wellington, NZ: Biodiversity Recovery Unit, NZ Department of Conservation. 38 pp.

Geiger, R., R.H. Aron, \& P. Todhunter. 2003. The climate near the ground. 6th Ed. New York, NY, USA: Rowman \& Littlefield. 584 pp.

Georges, A. 1989. Female turtles from hot nests: is it duration of incubation of proportion of development that matters? Oecologia: 81: 323-8. 
Georges, A. 2013. For reptiles with temperature-dependent sex determination, thermal variability may be as important as thermal averages. Animal Conservation 16: 493-4.

Georges, A., K. Beggs, J.E. Young, \& J.S. Doody. 2005. Modelling development of reptile embryos under fluctuating temperature regimes. Physiological and Biochemical Zoology 78(1): 1830.

Georges, A., S. Doody, K. Beggs, \& J. Young. 2004. Thermal models of TSD under laboratory and field conditions. In: N. Valenzuela \& V. Lance, Eds. Temperature-dependent sex determination in vertebrates. Washington, DC, USA: Smithsonian, pp. 79-89.

Georges, A., C. Limpus, \& R. Stoutjesdijk. 1994. Hatchling sex in the marine turtle Caretta caretta is determined by proportion of development at a temperature, not daily duration of exposure. Journal of Experimental Zoology 270: 432-4.

Gibbons, J.W., D.E. Scott, T.J. Ryan, K.A. Buhlmann, T.D. Tuberville, B.S. Metts, J.L. Greene, T. Mills, Y. Leiden, S. Poppy, \& C.T. Winne. 2000. The global decline of reptiles, deja vu amphibians. Bioscience 50: 653-66.

Gienapp, P. \& M.E. Visser. 2006. Possible fitness consequences of experimentally advanced laying dates in great tits: differences between populations in different habitats. Functional Ecology 20: 180-5.

Gill, J. \& D. Hangartner. 2010. Circular data in political science and how to handle it. Political Analysis 18(3): 316-36.

Gillingham, P.K., B. Huntley, W.E. Kunin, \& C.D. Thomas. 2012. The effect of spatial resolution on projected responses to climate warming. Diversity and Distributions 18(10): 990-1000.

Gillooly J.F., J.H. Brown, G.B. West, V.M. Savage, \& E.L. Charnov. 2001. Effects of size and temperature on metabolic rate. Science 293(5538): 2248-51.

Gillson, L., T.P. Dawson, S. Jack, \& M.A. McGeoch. 2013. Accommodating climate change contingencies in conservation strategy. Trends in Ecology and Evolution 28(3): 135-42. 
GIMP Development Team. 2013. GNU Image Manipulation Program. Version 2.8. http://www.gimp.org.

Girondot, M. 1999. Statistical description of temperature-dependent sex determination using maximum likelihood. Evolutionary Ecology Research 1: 479-86.

Gittleman, J.L. \& P.H. Harvey. 1982. Carnivore home-range size, metabolic needs, and ecology. Behavioural Ecology and Sociobiology 10: 57-63.

Godfrey, M.H., V. Delmas, \& M. Girondot. 2003. Assessment of patterns of temperaturedependent sex determination using maximum likelihood model selection. Ecoscience 10(3): 265-72.

Godwin, H., R.P. Suggate, \& E.H. Willis. 1958. Radiocarbon dating of the eustatic rise in ocean level. Nature London 181: 1518-9.

Götmark, F., D. Blomqvist, O.C. Johansson, \& J. Bergkvist. 1995. Nest site selection: a trade-off between concealment and view of the surroundings? Journal of Avian Biology 26:305-12.

GRASS Development Team. 2013. Geographic Resources Analysis Support System (GRASS) Software. Open Source Geospatial Foundation Project. http://grass.osgeo.org.

Grayson, K.L., N.J. Mitchell, J.M. Monks, S.N. Keall, J.N. Wilson, \& N.J. Nelson. 2014. Sex ratio bias drives conditions for extinction in an isolated tuatara (Sphenodon punctatus) population. PloS One, in press.

Green, D.R. \& S. Hartley. 2000. Integrating photointerpretation and GIS for vegetation mapping: some issues of error. In: Vegetation mapping: from patch to planet. Alexander, R. \& A.C. Millington, Eds. New York, NY, USA: Wiley. pp. 103-34.

Gumiaux, C., D. Gapais, \& J.P. Brun. 2003. Geostatistics applied to best-fit interpolation of orientation data. Tectonophysics 376: 241-59.

Guisan, A. \& J.-P. Theurillat. 2000. Equilibrium modeling of alpine plant distribution: how far can we go. Phytocoenologia 30: 353-84. 
Guisan, A. \& W. Thuiller. 2005. Predicting species distribution: offering more than simple habitat models. Ecology Letters 8: 993-1009.

Guisan, A. \& N.E. Zimmerman. 2000. Predictive habitat distribution models in ecology. Ecological Modelling 135: 147-86.

Hamilton, W.M. 1961. Little Barrier Island (Hauturu). 2nd Ed. Bulletin 137, Department of Scientific and Industrial Research [DSIR]. 103 pp.

Hancock, P.A. \& M.F. Hutchinson. 2006. Spatial interpolation of large climate data sets using bivariate thin-plate smoothing splines. Environmental Modelling and Software 21(12): 1684-94.

Hannah, L., L. Flint, A.D. Syphard, M.A. Moritz, L.B. Buckley, \& I.M. McCullough. 2014. Fine-grain modeling of species' response to climate change: holdouts, stepping-stones, and microrefugia. Trends in Ecology and Evolution 29(7): 390-7.

Hare, K.M., S. Pledger, \& C.H. Daugherty. 2008. Low incubation temperatures negatively influence locomotor performance and behaviour of the nocturnal lizard Oligosoma suteri (Lacertidae: Scincidae). Copeia 2008:16-22.

Harfoot, M.B., T. Newbold, D.P. Tittensor, S. Emmott, J. Hutton, V. Lyutsarev, M.J. Smith, J.P.W. Scharlemann, \& D.W. Purves. 2014. Emergent global patterns of ecosystem structure and function from a mechanistic general ecosystem model. PLoS Biology 12(4): e1001841.

Harlow, P.S. 2001. Ecology of sex-determining mechanisms in Australian agamid lizards. Unpublished Ph.D. thesis. Macquarie University, NSW, Australia. 258 pp.

Harlow, P.S. 2004. Temperature-dependent sex determination in lizards. In: N. Valenzuela \& V. Lance, Eds. Temperature-dependent sex determination in vertebrates. Washington, D.C.: Smithsonian, $194 \mathrm{pp}$.

Hartley, S., P.D. Krushelnycky, \& P.J. Lester. 2010. Integrating physiology, population dynamics and climate to make multi-scale predictions for the spread of an invasive insect: the Argentine ant at Haleakala National Park, Hawaii. Ecography 33: 83-94. 
Hartman, C.A. \& L.W. Oring. 2003. Orientation and microclimate of horned lark nests: the importance of shade. The Condor 105(1): 158-63.

Hawkes, L.A., A.C. Broderick, M.H. Godfrey, \& B.J. Godley. 2007. Investigating the potential impacts of climate change on a marine turtle population. Global Change Biology 13: 92332.

Hay, J., S. Sarre, D. Lambert, F. Allendorf, \& C. Daugherty. 2010. Genetic diversity and taxonomy: a reassessment of species designation in tuatara (Sphenodon: Reptilia). Conservation Genetics 11(3): 1063-81.

Hays, G.C., A.C. Broderick, F. Glen, \& B.J. Godley. 2003. Climate change and sea turtles: a 150-year reconstruction of incubation temperatures at a major marine turtle rookery. Global Change Biology 9: 642-6.

Hays, G.C., A. Mackay, C.R. Adams, J.A. Mortimer, J.R. Speakman, \& M. Boerema. 1995. Nest site selection by sea turtles. Journal of the Marine Biological Association of the U.K. 75: 667-74.

Hendrickx, J.M.H., R.L. van Dam, B. Borchers, J.O. Curtis, H.A. Lensen, \& R.S. Harmon. 2003. Worldwide distribution of soil dielectric and thermal properties. Proceedings SPIE 5089, Detection and Remediation Technologies for Mines and Minelike Targets VIII 1158: doi:10.1117/12.487116.

Herbert, T.J. 1987. Area projections of fisheye photographic lens. Agricultural and Forest Meteorology 39: 215-23.

Hewitt, N., N. Klenk, A.L. Smith, D.R. Bazely, N. Yan, S. Wood, J.I. MacLellan, C. Lipsig-Mumme, \& I. Henriques. 2011. Taking stock of the assisted migration debate. Biological Conservation 144: $2560-72$.

Hijmans, R.J., S.E. Cameron, J.L. Parra, P.G. Jones \& A. Jarvis. 2005. Very high resolution interpolated climate surfaces for global land areas. International Journal of Climatology 25: 1965-78. 
Hirzel, A.H., J. Hausser, D. Chessel, \& N. Perrin. 2002. Ecological-niche factor analysis: how to compute habitat-suitability maps without absence data? Ecology 83(7): 2027-36.

Hitchmough, R.A., J.M. Hoare, H. Jamieson, D. Newman, M.D. Tocher, P.J. Anderson, M. Lettink, \& A.H. Whittaker. 2009. Conservation status of New Zealand reptiles, 2009. New Zealand Journal of Zoology 37(3): 203-24.

Hitchmough, R.A., P. Anderson, B. Barr, J. Monks, M. Lettink, J. Reardon, M. Tocher, \& T. Whittaker. 2012. Conservation status of New Zealand reptiles, 2012. New Zealand Threat Classification, Series 2. Wellington, NZ: DOC. 16 pp.

Hoare, J.M., S. Pledger, S.N. Keall, N.J. Nelson, N.J. Mitchell, \& C.H. Daugherty. 2006. Conservation implications of a long-term decline in body condition of the Brothers Island tuatara (Sphenodon guntheri). Animal Conservation 9: 456-62.

Hoegh-Guldberg, O., L. Hughes, S. McIntyre, D.B. Lindenmayer, C. Parmesan, H.P. Possingham, \& C.D. Thomas. 2008. Assisted colonization and rapid climate change. Science 321: 345-6.

Hofierka, J. J. Parajka, H. Mitasova, \& L. Mitas. 2002. Multivariate interpolation of precipitation using regularized spline with tension. Transactions in GIS 6(2): 135-50.

Holway, D.A. 1991. Nest-site selection and the importance of nest concealment in the blackthroated blue warbler. The Condor 93(3): 575-81.

Horton, B. \& R. Corkrey. 2011. A weighted coefficient model for estimation of Australian daily soil temperature at depths of $5 \mathrm{~cm}$ to $100 \mathrm{~cm}$ based on air temperature and rainfall. Soil Research 49: 305-14.

Huang, S.-P., C.-R. Chiou, T.-E. Lin, M.-C. Tu, C.-C. Lin, \& W.P. Porter. 2013. Future advantages in energetics, activity time, and habitats predicted in a high-altitude pit viper with climate warming. Functional Ecology 27: 446-58.

Huang, J., R.G. Pontius Jr., L. Qingsheng, \& Z. Yujia. 2012. Use of intensity analysis to link patterns with processes of land change from 1986 to 2007 in a coastal watershed of southeast China. Applied Geography 34: 371- 84. 
Huey, R.B., M.R. Kearney, A. Krockenberger, J.A. Holtum, M. Jess, \& S.E. Williams. 2012. Predicting organismal vulnerability to climate warming: roles of behaviour, physiology and adaptation. Philosophical Transactions of the Royal Society B: Biological Sciences 367: 166579.

Hughes, L. 2000. Biological consequences of global warming: is the signal already apparent? Trends in Ecology and Evolution 15: 56-61.

Hughes, E.J. \& R.J. Brooks. 2006. The good mother: does nest-site selection constitute parental investment in turtles? Canadian Journal of Zoology 84: 1545- 54.

Hulin, V., V. Delmas, M. Girondot, M.H. Godfrey, \& J.-M. Guillon. 2009. Temperature-dependent sex determination and global change: are some species at greater risk? Oecologia 160: 493-506.

Huntley B., W. Cramer, A.V. Morgan, H.C. Prentice, \& J.R.M. Allen. 1997. Predicting the response of terrestrial biota to future environmental changes. In: Past and future rapid environmental changes: the spatial and evolutionary responses of terrestrial biota. Huntley B., W. Cramer, A.V. Morgan, H.C. Prentice, \& J.R.M. Allen, Eds. Berlin: Springer-Verlag. pp. 487-504.

Hutchinson, M.F. 1957. Concluding remarks. In: Cold Spring Harbour symposia on quantitative biology: population studies: animal ecology and demography 22: 415-27. New York, NY, USA: Cold Spring Harbor.

Hutchinson, M.F. 1998a. Interpolation of rainfall data with thin plate smoothing splines I: two dimensional smoothing of data with short range correlation. Journal of Geographic Information and Decision Analysis 2: 139-51.

Hutchinson, M.F. 1998b. Interpolation of rainfall data with thin plate smoothing splines II: analysis of topographic dependence. Journal of Geographic Information and Decision Analysis 2: 168-85.

Hutchinson, M.F., D.W. McKenney, K. Lawrence, J.H. Pedlar, R.F. Hopkinson, E. Milewska, \& P. Papadopol. 2009. Development and testing of Canada-wide interpolated spatial models of 
daily minimum-maximum temperature and precipitation for 1961-2003. Journal of Applied Meteorology and Climatology 48: 725-41.

Hutchinson, G.E. \& R.H. MacArthur. 1959. A theoretical ecological model of size distributions among species of animals. The American Naturalist 93(869): 117-25.

Hutchison, B.A., D.R. Matt, \& R.T. McMillen, 1980. Effects of sky brightness distribution upon penetration of diffuse radiation through canopy gaps in a deciduous forest. Agricultural and Forest Meteorology 22: 137-47.

Intel. 2005. Excerpts from a conversation with Gordon Moore: Moore's Law. Video transcript. Retrieved from http://large.stanford.edu/courses/2012/ph250/lee1/ docs/Excepts_A_Conversation_with_Gordon_Moore.pdf.

[IPCC] Intergovernmental Panel on Climate Change. 2007. Climate change 2007: the physical science basis. Contribution of Working Group I to the Fourth Assessment Report of the IPCC. Solomon, S., D. Qin, M. Manning, Z. Chen, M. Marquis, K.B. Averyt, M. Tignor, \& H.L. Miller, Eds. Cambridge, UK and New York, NY, USA: Cambridge University Press. 996 pp.

[IPCC] Intergovernmental Panel on Climate Change. 2013. Climate change 2013: the physical science basis. Contribution of Working Group I to the Fifth Assessment Report of the IPCC. Stocker, T.F., D. Qin, G.-K. Plattner, M. Tignor, S.K. Allen, J. Boschung, A. Nauels, Y. Xia, V. Bex, \& P.M. Midgley, Eds. Cambridge, UK and New York, NY, USA: Cambridge University Press. 1535 pp.

[IPCC] Intergovernmental Panel on Climate Change. 2014. Summary for policymakers. In: Climate Change 2014: Impacts, Adaptation, and Vulnerability. Part A: Global and Sectoral Aspects. Contribution of Working Group II to the Fifth Assessment Report of the IPCC. Field, C.B., V.R. Barros, D.J. Dokken, K.J. Mach, M.D. Mastrandrea, T.E. Bilir, M. Chatterjee, K.L. Ebi, Y.O. Estrada, R.C. Genova, B. Girma, E.S. Kissel, A.N. Levy, S. MacCracken, P.R. Mastrandrea, \& L.L. White, Eds. Cambridge, UK and New York, NY, USA: Cambridge University Press. pp. 1-32. 
[IUCN] International Union for Conservation of Nature / Species Survival Commission. 2013. Guidelines for reintroductions and other conservation translocations. Version 1.0. Gland, Switzerland: IUCN/SSC, 72 pp.

[IUCN] International Union for Conservation of Nature. 2014. The IUCN Red List of Threatened Species. Version 2014.1. http://www.iucnredlist.org. Accessed 19 July 2014.

Iqbal, M. 1983. An introduction to solar radiation. New York: Academic Press, 416 pp.

Jamieson, I.G., G.P. Wallis, \& J.V. Briskie. 2006. Inbreeding and endangered species management: is New Zealand out of step with the rest of the world? Conservation Biology 20(1): 39-47.

Jammalamadaka, S.R. \& A. Sengupta. 2001. Topics in circular statistics. Singapore: World Scientific Press. 336 pp.

Janzen, F.J. 1994a. Climate change and temperature-dependent sex determination in reptiles. Proceedings of the National Academy of Sciences USA 91: 7487-90.

Janzen, F.J. 1994b. Vegetational cover predicts the sex ratios of hatchling turtles in natural nests. Ecology 75(6): 1593-9.

Janzen, F.J. 1995. Experimental evidence for the evolutionary significance of temperaturedependent sex determination. Evolution 49(5): 864-73.

Janzen, F.J. \& J.G. Krenz. 2004. Phylogenetics: which was first, TSD or GSD? In Temperaturedependent sex determination in vertebrates. In: N. Valenzuela \& V.A. Lance, Eds. Washington, DC, U.S.A: Smithsonian, pp. 121-130.

Janzen, F.J. \& C.L. Morjan. 2003. Repeatability of microenvironment-specific nesting behaviour in a turtle with environmental sex determination. Animal Behaviour 62: 73-82.

Janzen, F.J., \& G.L. Paukstis. 1988. Environmental sex determination in reptiles. Nature 332:790.

Janzen, F.J., \& G.L. Paukstis. 1991a. Environmental sex determination in reptiles: ecology, evolution, and experimental design. Quarterly Review of Biology 66:149-79.

Janzen, F.J., \& G.L. Paukstis. 1991b. A preliminary test of the adaptive significance of environmental sex determination in reptiles. Evolution 45:435-40. 
Janzen, F.J. \& P.C. Phillips. 2006. Exploring the evolution of environmental sex determination, especially in reptiles. Journal of Evolutionary Biology 19: 1775-84.

Jarvis, C.H. \& N. Stuart. 2001a. A comparison among strategies for interpolating maximum and minimum temperatures. Part I: the selection of 'guiding' topographic and land cover variables. Journal of Applied Meteorology 40: 1060-74.

Jarvis, C.H. \& N. Stuart. 2001b. A comparison among strategies for interpolating maximum and minimum temperatures. Part II: the interaction between number of guiding variables and the type of interpolation. Journal of Applied Meteorology 40: 1075-84.

Jeffrey S.J., J.O. Carter, K.B. Moodie, \& A.R. Beswick. 2001. Using spatial interpolation to construct a comprehensive archive of Australian climate data. Environmental Modelling and Software 16(4): 309-30.

Jones, D.N. 1988. Selection of incubation mound sites by the Australian brush-turkey Alectura lathami. Ibis 130: 251-60.

Jones, M.E.H., A.J.D. Tennyson, J.P. Worthy, S.E. Evans, \& T.H. Worthy. 2009. A sphenodontine (Rhynchocephalia) from the Miocene of New Zealand and palaeobiogeography of the tuatara (Sphenodon). Proceedings of the Royal Society B 276: 1385-90.

Kamel S.J. \& N. Mrosovsky. 2004. Nest site selection in leatherbacks, Dermochelys coriacea: individual patterns and their consequences. Animal Behavior 68: 357-66.

Keall, S.N., N.J. Nelson, \& C.H. Daugherty. 2010. Securing the future of threatened tuatara populations with artificial incubation. Herpetological Conservation and Biology 5(3): 55562.

Kearney, M.R. 2006. Habitat, environment and niche: what are we modelling? Oikos 115(1): 18691.

Kearney, M.R. 2012. Metabolic theory, life history and the distribution of a terrestrial ectotherm. Functional Ecology 26: 167-79. 
Kearney, M.R., A.P. Isaac, \& W.P. Porter. 2014a. microclim: global estimates of hourly microclimate based on long-term monthly climate averages. Scientific Data 1: doi:10.1038/sdata.2014.6.

Kearney, M.R., B.L. Phillips, C.R. Tracy, K.A. Christian, G. Betts, \& W.P. Porter. 2008. Modelling species distributions without using species distributions: the cane toad in Australia under current and future climates. Ecography, doi:10.1111/j.2008.0906-7590-05457.x.

Kearney, M.R. \& W.P. Porter. 2004. Mapping the fundamental niche: physiology, climate, and the distribution of a nocturnal lizard. Ecology 85(11): 3119-31.

Kearney, M.R. \& W.P. Porter. 2009. Mechanistic niche modelling: combining physiological and spatial data to predict species' ranges. Ecology Letters 12: 334-50.

Kearney, M.R., W.P. Porter, C. Williams, S. Ritchie, \& A.A. Hoffmann. 2009a. Integrating biophysical models and evolutionary theory to predict climatic impacts on species' ranges: the dengue mosquito Aedes aegypti in Australia. Functional Ecology, doi:10.1111/j.13652435.2008.01538.x.

Kearney, M.R., A. Shamakhy, R. Tingley, D.J. Karoly, A.A. Hoffman, P.R. Briggs, \& W.P. Porter. 2014b. Microclimate modelling at macro scales: a test of a general microclimate model integrated with gridded continental-scale soil and weather data. Methods in Ecology and Evolution, doi: 10.1111/2041-210X.12148.

Kearney, M. \& R. Shine. 2004. Developmental success, stability, and plasticity in closely related parthenogenetic and sexual lizards (Heteronotia, Gekkonidae). Evolution 58(7): 1560-72.

Kearney, M.R., R. Shine, \& W.P. Porter. 2009b. The potential for behavioral thermoregulation to buffer "cold-blooded" animals against climate warming. Proceedings of the National Academy of Sciences 106(10): 3835-40.

Kearney M.R., B.A. Wintle, \& W.P. Porter. 2008. Climate change and the distribution limits of folivorous possums from Australia: predictions from a biophysical model. Fourth 
International Conference in Africa for Comparative Physiology and Biochemistry, "Molecules to Migration," Maasai Mara Reserve, Kenya, July 19-25, abstract.

Kearney, M.R., B.A. Wintle, \& W.P. Porter. 2010. Correlative and mechanistic models of species distribution provide congruent forecasts under climate change. Conservation Letters 3: 203-13.

Keith, D.A., H. Resit, H.R. Akçakaya, W. Thuiller, G.F. Midgley, R.G. Pearson, S.J. Phillips, H.M. Regan, M.B. Ara jo, \& T.G. Rebelo. 2008. Predicting extinction risks under climate change: coupling stochastic population models with dynamic bioclimatic habitat. Biology Letters 4: $560-3$.

Kerhoulas, L.P., T.E. Kolb, M.D. Hurteau, \& G.W. Kosh. 2013. Managing climate change adaptation in forests: a case study from the U.S. Southwest. Journal of Applied Ecology 50: 1311-20.

Kim, S.-Y. \& P. Monaghan. 2005. Effects of vegetation on nest microclimate and breeding performance of lesser black-backed gulls (Larus fuscus). Journal of Ornithology 146(2): 176-83.

Kingsolver, J.G., S.E. Diamond, \& L.B. Buckley. 2013. Heat stress and the fitness consequences of climate change for terrestrial ectotherms. Animal Physiological Ecology, doi: $10.1111 / 1365-2435.12145$.

Kodesova, R., V. Kodes, A. Zigova, \& J. Simunek. 2006. Impact of plant roots and soil organisms on soil micromorphology and hydraulic properties. Biologia 61(19): 339-43.

Koepke, P., M. Hess, I. Schult, \& E.P. Shettle. 1997. Global Aerosol Data Set. Report No. 243, MaxPlanck-Institut für Meteorologie, Hamburg. ISSN 0937-1060.

Kolbe, J.J. \& F.J. Janzen. 2002. Impact of nest-site selection on nest success and nest temperature in natural and disturbed habitats. Ecology 83(1): 269-81.

Kramer-Schadt, S., E. Revilla, T. Wiegand, \& U. Breitenmoser. 2004. Fragmented landscapes, road mortality and patch connectivity: modelling influences on the dispersal of Eurasian lynx. Journal of Applied Ecology 41: 711-23. 
Kreyling, J., T. Bittner, A. Jaeschke, A. Jentsch, M.J. Steinbauer, D. Thiel, \& C. Beierkuhnlein. 2011. Assisted colonization: a question of focal units and recipient localities. Restoration Ecology 19(4): 433-40.

Lacy, R.C. 2000. Considering threats to the viability of small populations. Ecological Bulletin 48: $39-51$.

Lande, R. 1988. Genetics and demography in biological conservation. Science, New Series 241(4872): 1455-60.

Lande, R. 1993. Risks of population extinction from demographic and environmental stochasticity and random catastrophes. The American Naturalist 142(6): 911-27.

Lavergne, S., N. Mouquet, W. Thuiller, \& O. Ronce. 2010. Biodiversity and climate change: integrating evolutionary responses of species and communities. Annual Review of Ecology, Evolution, and Systematics 41: 321-50.

Lawler, J.J. \& J.D. Olden. 2011. Reframing the debate over assisted colonisation. Frontiers in Ecology and Environment 9(10): 569-74.

Lebreton, J.-D., K.P. Burnham, J. Clobert, \& D.R. Anderson. 1992. Modelling survival and testing biological hypotheses with marked animals: a unified approach with case studies. Ecological Monographs 62: 67-118.

Ledig, F.T., G.E. Rehfeldt, \& B. Jaquish. 2012. Projections of suitable habitat under climate change scenarios: implications for trans-boundary assisted colonization. American Journal of Botany 99(7): 1217-30.

Lee, T.M. \& W. Jetz. 2008. Future battlegrounds for conservation under global change. Proceedings of the Royal Society B 275(1640): 1261-70.

Le Gouar, P., J.-B. Mihoub, \& François Sarrazin. 2012. Dispersal and habitat selection: behavioural and spatial constraints for animal translocations. In: J.G. Ewen, D.P. Armstrong, K.A. Parker, \& P.J. Seddon, Eds. Reintroduction biology: integrating science and management. West Sussex, UK: Wiley-Blackwell, pp. 138-164. 
Liebezeit, J.R. \& T.L. George. 2002. Nest predators, nest-site selection, and nesting success of the dusky flycatcher in a managed ponderosa pine forest. Condor 104: 507-17.

Lindeman, P.V. 1992. Nest-site fixity among painted turtles (Chrysemys picta) in northern Idaho. Northwest Naturalist 73: 27-30.

Loiselle, B.A., C.A. Howell, C.H. Graham, J.M. Goerck, T. Brooks, K.G. Smith, \& P.H. Williams. 2003. Avoiding pitfalls of using species distribution models in conservation planning. Conservation Biology 17(6): 1591-1600.

Lomolino, M.W. \& R. Channell. 1998. Range collapse, re-introductions, and biogeographic guidelines for conservation. Conservation Biology 12: 481-4.

Lopes, A.M.G., A.C.M. Sousa, \& D.X. Viegas. 1995. Numerical simulation of turbulent flow and fire propagation in complex terrain. Numerical Heat Transfer Part A 27: 229-53.

Lopes, A.M.G. 2003. WindStation: a software for the simulation of atmospheric flows over complex topography. Environmental Modelling and Software 18: 81-96.

Lopes, A.M.G. 2011. WindStation v 2.0.7 user's manual. 49 pp.

Loss, S.R., L.A. Terwilliger, \& A.C. Peterson. 2011. Assisted colonization: integrating conservation strategies in the face of climate change. Biological Conservation 144(1): 92-100.

Lui, B.Y.H. \& R.C. Jordan. 1960. The interrelationship and characteristic distribution of direct, diffuse and total solar radiation. Solar Energy 4 (3): 1-19.

Lund, U. \& C. Agostinelli. 2013. circular: circular statistics from Topics in Circular Statistics by S. Rao Jammalamadaka and A. Sengupta, World Scientific. R package version 0.4-7. http://cran.r-project.org/package=circular.

Lunt, I.D., M. Byrne, J.J. Hellmann, N.J. Mitchell, S.T. Garnett, M.W. Hayward, T.G. Martin, E. McDonald-Madden, S.E. Williams, \& K.K. Zander. 2013. Using assisted colonisation to conserve biodiversity and restore ecosystem function under climate change. Biological Conservation 157: 172-7. 
Lyons, J.E., M.C. Runge, H.P. Laskowski, \& W.L. Kendall. 2008. Monitoring in the context of structured decision-making and adaptive management. The Journal of Wildlife Management 72(8): 1683-92.

Macdonald, D.W., F.H. Tattersal, S. Ruston, A.B. South, S. Rao, P. Maitland, \& R. Strachan. 2000. Reintroducing the beaver (Castor fiber) to Scotland: a protocol for identifying and assessing suitable release sites. Animal Conservation 3: 125-33.

Mace, G.M., P.H. Harvey, \& T.H. Clutton-Brock. 1983. Vertebrate home range size and metabolic requirements. In: I. Swingland \& P.J. Woods, Eds. The ecology of animal movement. Oxford, UK: Clarendon Press, 311 pp.

Madsen T. \& R. Shine. 1999. Life history consequences of nest-site variation in tropical pythons (Liasis fuscus). Ecology 80: 989-97.

Manzato, A. 2007. A note on the maximum Peirce skill score. Weather and Forecasting 22: 114854.

Maschinski, J., J.E. Baggs, P.F. Quintana-Ascencio, \& E.S. Menges. 2006. Effects of climate change on the extinction risk of an endangered limestone endemic shrub, Arizona cliffrose. Conservation Biology 20: 218-28.

Mata-González, R., R.D. Pieper, \& M.M. Cárdenas. 2002. Vegetation patterns as affected by aspect and elevation in small desert mountains. The Southwestern Naturalist 47(3): 440-8.

Matsushima N. \& M. Kawata. 2005. The choice of oviposition site and the effects of density and oviposition timing on survivorship in Rana japonica. Ecology Research 20: 81-6.

Mazaris, A.D., A.S. Kallimanis, J.D. Pantis, \& G.C. Hays. 2012. Phenological response of sea turtles to environmental variation across a species' northern range. Proceedings of the Royal Society B 280: doi: 10.1098/rspb.2012.2397.

McCallum, J. \& F.R. Harker. 1982. Reptiles of Little Barrier Island. Tane 28: 21-7.

McCullough, E.C. \& W.P. Porter. 1971. Computing clear day solar radiation spectra for the terrestrial ecological environment. Ecology 52(6): 1008-15. 
McGaugh, S.E. \& F.J. Janzen. 2011. Effective heritability of targets of sex-ratio selection under environmental sex determination. Journal of Evolutionary Biology 24: 784-94.

McGaugh, S.E., R.M. Bowden, C.-H. Kuo, \& F.J. Janzen. 2011. Field-measured narrow sense heritability of the threshold for sex determination in a turtle with temperature dependent sex-determination. Evolutionary Ecology Research 13: 75-90.

McIntyre, M.E. 1988. Management implications if habitat use and dispersal of juvenile tuatara, Sphenodon punctatus, on Stephens Island. School of Biological Sciences, Victoria University of Wellington: Unpublished management report.

McNab, B.K. 1963. Bioenergetics and the determination of home range size. The American Naturalist 97(894): 133-40.

Miller, K.A., H.C. Miller, J.A. Moore, N.J. Mitchell, A. Cree, F.W. Allendorf, S.D. Sarre, S.N. Keall, \& N.J. Nelson 2012. Securing the demographic and genetic future of tuatara through assisted colonisation. Conservation Biology 26(5): 790-8.

Millett, J. \& S. Edmondson. 2013. The impact of 36 years of grazing management on vegetation dynamics in dune slacks. Journal of Applied Ecology 50: 1367-76.

Mills, J.A., R.B. Lavers, \& W.G. Lee. 1984. The takahe- a relic of the Pleistocene grassland avifauna in New Zealand. New Zealand Journal of Ecology 7: 55-70.

[MFE] Ministry for the Environment (New Zealand). 2008. Climate change effects and impacts assessment: a guidance manual for local government in New Zealand. 2nd Ed. Mullan B., D. Wratt, S. Dean, M. Hollis, S. Allan, T. Williams, G. Kenny, \& MFE. Wellington, NZ: Ministry for the Environment. $167 \mathrm{pp}$.

Mitchell, N.J., F.W. Allendorf, S.N. Keall, C.H. Daugherty, \& N.J. Nelson. 2009. Demographic effects of temperature-dependent sex determination: will tuatara survive global warming? Global Change Biology 16: 60-72. 
Mitchell, N.J., M.R. Hipsey, S. Arnall, G. McGrath, H.B. Tareque, G. Kuchling, R. Vogwill, M. Sivapalan, W.P. Porter, \& M.R. Kearney. 2013a. Linking eco-energetics and eco-hydrology to select sites for the assisted colonization of Australia's rarest reptile. Biology 2: 1-25.

Mitchell, N.J. \& F.J. Janzen. 2010. Temperature-dependent sex determination and contemporary climate change. Sexual Development 4: 129-40.

Mitchell, N.J., T. Jones, \& G. Kuchling. 2012. Simulated climate change increases juvenile growth in a critically endangered tortoise. Endangered Species Research 17: 73-82.

Mitchell, N.J., M.R. Kearney, N.J. Nelson, \& W.P. Porter. 2008. Predicting the fate of a living fossil: how will global warming affect sex determination and hatching phenology in tuatara? Proceedings of the Royal Society B 275: 2185-93.

Mitchell, N.J., N.J. Nelson, A. Cree, S. Pledger, S.N. Keall, \& C.H. Daugherty. 2006. Support for a rare pattern of temperature-dependent sex determination in archaic reptiles: evidence from two species of tuatara (Sphenodon). Frontiers in Zoology 3: 9.

Mitchell, N.J. \& R.S. Seymour. 2000. Effects of temperature on the energy cost and timing of embryonic and larval development of the terrestrially breeding moss frog, Bryobatrachus nimbus. Physiological and Biochemical Zoology 73: 829-40.

Mitchell, N.J. \& R.S. Seymour. 2003. The effects of nest temperature, nest substrate and clutch size on the oxygenation of embryos and larvae of the Australian moss frog, Bryobatrachus nimbus. Physiological and Biochemical Zoology 76: 60-71.

Mitchell, T.S., J.A. Maciel, \& F. Janzen. 2013b. Does sex ratio selection influence nest-site choice in a reptile with temperature-dependent sex determination? Proceedings of the Royal Society B 280: 20132460.

Monahan, W.B. 2009. A mechanistic niche model for measuring species' distributional responses to seasonal temperature gradients. PloS ONE 4(11): 1-7.

Moore, J.A. 2008. Fitness implications of the mating system and reproductive ecology of tuatara. Unpublished Ph.D. thesis. Victoria University of Wellington, New Zealand. 142 pp. 
Moore, J.A., C.H. Daugherty, \& N.J. Nelson. 2009. Large male advantage: phenotypic and genetic correlates of territoriality in tuatara. Journal of Herpetology 43(4): 570-8.

Moore, J.A., J.M. Hoare, C.H. Daugherty, \& N.J. Nelson. 2007. Waiting reveals waning weight: monitoring over 54 years shows a decline in body condition of a long-lived reptile (tuatara, Sphenodon punctatus). Biological Conservation: 135: 181-8.

Moore, J.A., H.C. Miller, C.H. Daugherty, \& N.J. Nelson. 2008. Fine-scale genetic structure of a longlived reptile reflects recent habitat modification. Molecular Ecology 17: 4630-41.

Moore, J.A., N.J. Nelson, S.N. Keall, \& C.H. Daugherty. 2008. Implications of social dominance and multiple paternity for the genetic diversity of a captive-bred reptile population (tuatara). Conservation Genetics 9: 1243-51.

Morin, X. \& M.J. Lechowicz. 2008. Contemporary perspectives on the niche that can improve models of species range shifts under climate change. Biology Letters 4: 573-6.

Moritz C., G. Langham, M. Kearney, A. Krockenberger, J. VanDerWal, \& S. Williams. 2012. Integrating phylogeography and physiology reveals divergence of thermal traits between central and peripheral lineages of tropical rainforest lizards. Philosophical Transactions of the Royal Society B 367: 1680-7.

Morjan, C.L. 2003a. How rapidly can maternal behavior affecting primary sex ratio evolve in a reptile with environmental sex determination? The American Naturalist 162(2): 205-19.

Morjan, C.L. 2003b. Variation in nesting patterns affecting nest temperatures in two populations of painted turtles (Chrysemys picta) with temperature-dependent sex determination. Behavioral Ecology and Sociobiology 53: 254-61.

Mosblech, N.A.S., M.B. Bush, \& R. van Woesik. 2011. On metapopulations and microrefugia: paleoecological insights. Journal of Biogeography 38(30): 419-29.

Mpelasoka, F.S., A.B. Mullan, \& R.G. Heerdegen. 2001. New Zealand climate change information derived by multivariate statistical and artificial neural network approaches. International Journal of Climatology 21: 1415-33. 
Mrosovsky, N. \& C. Pieau. 1991. Transitional range of temperature, pivotal temperatures and thermosensitive stages for sex determination in reptiles. Amphibia-Reptilia 12: 169-79.

Mueller, J.M. \& J.J. Hellmann. 2008. An assessment of invasion risk from assisted migration. Conservation Biology 22: 562-7.

Murcia, C. 1995. Edge effects in fragmented forests: implications for conservation. Trends in Ecology and Evolution 10(2): 60-2.

National Institute of Water and Atmospheric Research Ltd. [NIWA]. 2013. State of the climate 2013: a snapshot of recent climate in New Zealand (2010-2013). A. Semadeni-Davies, Ed. NIWA Science and Technology Series No. 57.109 pp.

Nelson, N.J., A. Cree, M.B. Thompson, S.N. Keall, \& C.H. Daugherty. 2004a. Temperaturedependent sex determination in tuatara. In: N. Valenzuela \& V. Lance, Eds. Temperaturedependent sex determination in vertebrates. Washington, DC, USA: Smithsonian, pp 53-8.

Nelson, N.J., S.N. Keall, D.Brown, \& C.H. Daugherty. 2002a. Establishing a new wild population of tuatara. Conservation Biology 16(4): 887-94.

Nelson, N.J., S.N. Keall, S. Pledger, \& C.H. Daugherty. 2002b. Male-biased sex ratio in a small tuatara population. Journal of Biogeography 29: 633-40.

Nelson, N.J., J.A. Moore, S. Pillai, \& S.N. Keall. 2010. Thermosensitive period for sex determination in the tuatara. Herpetological Conservation and Biology 5(2): 324-9.

Nelson, N.J., M.B. Thompson, S. Pledger, S.N. Keall, \& C.H. Daugherty. 2004b. Do TSD, sex ratios, and nest characteristics influence the vulnerability of tuatara to global warming? International Congress Series 1275: 250-7.

Nelson, N.J., M.B. Thompson, S. Pledger, S.N. Keall, \& C.H. Daugherty. 2004c. Induction of oviposition produces smaller eggs in tuatara (Sphenodon punctatus). New Zealand Journal of Zoology 31: 283-9. 
Nelson, N.J., M.B. Thompson, S. Pledger, S.N. Keall, \& C.H. Daugherty. 2006. Performance of juvenile tuatara depends on age, clutch, and incubation regime. Journal of Herpetology 40(3): 399-403.

Neuwald, J.L. \& N. Valenzuela. 2009. The lesser known challenge of climate change: thermal variance and sex-reversal in vertebrates with temperature-dependent sex determination. PLOS ONE 6(3): 1-9.

New, M., D. Lister, M. Hulme, \& I. Makin. 2002. A high-resolution data set of surface climate over global land areas. Climate Research 21: 1-25.

Newman, D.G. 1982. Tuatara, Sphenodon punctatus, and burrows, Stephens Island. In: D.G. Newman, Ed. New Zealand Herpetology, New Zealand Wildlife Service Occasional Publication No. 2: 213-21.

Newman, D.G. 1987. Burrow use and population densities of tuatara (Sphenodon punctatus) and how they are influenced by fairy prions (Pachyptila turtur) on Stephen's Island, New Zealand. Herpetologica 43: 336-44.

New Zealand Climate Change Office. 2004. Climate change effects and impacts assessment: a guidance manual for local government in New Zealand. Wellington, New Zealand: Ministry for the Environment.

Nussey, D.H., A.J. Wilson, \& J.E. Brommer. 2007. The evolutionary ecology of individual phenotypic plasticity in wild populations. European Society for Evolutionary Biology Journal Compilation 831-44.

Ochsner, T.E., R. Horton, \& T. Ren. 2001. A new perspective on soil thermal properties. Soil Science Society of America Journal 65: 1641-7.

O'Donnell, J.A., V.E. Romanovsky, J.W. Harden, \& A.D. McGuire. 2009. The effect of moisture content on the thermal conductivity of moss and organic soil horizons from black spruce ecosystems in interior Alaska. Soil Science 174(12): 646-51. 
Oliver, S.A., H.R. Oliver, J.S. Wallace, \& A.M. Roberts. 1987. Soil heat flux and temperature variation with vegetation, soil type, and climate. Agricultural and Forest Meteorology 39: 257-69.

Osborne, P.E. \& P.J. Seddon. 2012. Selecting suitable habitats for reintroduction: variation, change, and the role of species distribution modelling. In: J.G. Ewen, D.P. Armstrong, K.A. Parker, \& P.J. Seddon, Eds. Reintroduction biology: integrating science and management. West Sussex, UK: Wiley-Blackwell, pp 73-104.

Papaionnou, G., G. Nikolidakis, D. Asimakopoulos, \& D. Retalis. 1996. Photosynthetically active radiation in Athens. Agricultural and Forest Meteorology 81: 287-98.

Parmesan, C. 2007. Influences of species, latitudes and methodologies on estimates of phenological response to global warming. Global Change Biology 13: 1860-72.

Pearson, R.G. \& T.P. Dawson. 2003. Predicting the impacts of climate change on the distribution of species: are bioclimate envelope models useful? Global Ecology \& Biogeography 12: 36171.

Peirce, C.S. 1884. A numerical measure of the success of predictions. Science 4(93): 453-54.

Perry, M. \& D. Hollis. 2005. The generation of monthly gridded datasets for a range of climatic variables over the UK. International Journal of Climatology 25: 1041-54.

Pigliucci, M. 2001. Phenotypic plasticity: beyond nature and nurture. Baltimore, MD, USA: Johns Hopkins University Press. 328 pp.

Pigliucci, M. 2005. Evolution of phenotypic plasticity: where are we going now? Trends in Ecology and Evolution 20(9): 481-6.

Pimm, S.L., G.J. Russell, J.L. Gittleman, \& T.M. Brooks. 1995. The future of biodiversity. Science 269(5222): 347-50.

Pinheiro, J. \& D. Bates. 2013. nlme: linear and nonlinear mixed-effects models. R package version 1.3-311. http://CRAN.R-project.org/package=nlme. 
Piper, W.H., M.W. Palmer, N. Banfield, \& M.W. Meyer. 2013. Can settlement in natal-like habitat explain maladaptive habitat selection? Proceedings of the Royal Society B 280, doi: 10.1098/rspb.2013.0979.

Pledger, S., K.H. Pollock, \& J.L. Norris. 2003. Open capture-recapture models with heterogeneity: I. Cormack-Jolly-Seber model. Biometrics 59: 786-94.

Pontius Jr., R.G. 2000. Quantification error versus location error in comparison of categorical maps. Photogrammetric Engineering \& Remote Sensing 66(8): 1011-6.

Pontius Jr., R.G. 2002. Statistical methods to partition effects of quantity and location during comparison of categorical maps at multiple resolutions. Photogrammetric Engineering \& Remote Sensing 68(10): 1041-9.

Pontius Jr., R.G., W. Boersma, J.-C. Castella, K. Clarke, T. de Nijs, C. Dietzel, Z. Duan, E. Fotsing, N. Goldstein. K. Kok, E. Koomen, C.D. Lippitt, W. McConnell, A.M. Sood, B. Pijanowski, S. Pithadia, S. Sweeney, T.N. Trung, A.T. Veldkamp, \& P.H. Verburg. 2008. Comparing the input, output, and validation maps for several models of land change. Annals of Regional Science 42: 11-37.

Pontius Jr., R.G., D. Huffaker, \& K. Denman. 2004. Useful techniques of validation for spatially explicit land-change models. Ecological Modelling 129: 445-61.

Pontius Jr., R.G. \& C.D. Lippitt. 2006. Can error explain map differences over time? Cartography and Geographic Information Science 33(2): 159-71.

Pontius Jr., R.G. \& M. Millones. 2011. Death to kappa: birth of quantity disagreement and allocation disagreement for accuracy assessment. International Journal of Remote Sensing 32(15): 4407-29.

Pontius Jr., R.G. \& S.H. Petrova. 2010. Assessing a predictive model of land change use using uncertain data. Environmental Modelling \& Software 25: 299-309.

Pontius Jr., R.G., E. Shusas, \& M. McEachern. 2004. Detecting important categorical land changes while accounting for persistence. Agriculture, Ecosystems and Environment 101: 251-68. 
Porter, W.P., S. Budaraju, W.E. Stewart, \& N. Ramankutty. 2000. Calculating climate effects on birds and mammals: impacts on biodiversity, conservation, population parameters, and global community structure. American Zoologist 40: 597-630.

Porter, W.P. \& D.M. Gates. 1969. Thermodynamic equilibria of animals with environment. Ecological Monographs 39(3): 227-44.

Porter, W.P. \& J.W. Mitchell. 2006. Method and system for calculating the spatio-temporal effects of climate and other environmental conditions on animals. USA Patent No. 7155377 B2. 38 pp.

Porter, W.P., J.W. Mitchell, W.A. Beckman, \& C.B. DeWitt. 1973. Behavioral implications of mechanistic ecology: thermal and behavioral modeling of desert ectotherms and their microenvironment. Oecologia 13(1): 1-54.

Porter, W.P., S. Ostrowski, \& J.B. Williams. 2010. Modeling animal landscapes. Physiological and Biochemical Zoology 83(5): doi: 10.1086/656181.

Porter, W.P., J.L. Sabo, C.R. Tracy, O.J. Reichman, \& N. Ramankutty. 2002. Physiology on a landscape scale: plant-animal interactions. Integrative and Comparative Biology 42: 43153.

Porter, W.P. \& C.R. Tracy. 1983. Biophysical analyses of energetics, time-space utilization, and distributional limits. In: Lizard ecology: studies of a model organism. Huey, R.B., E.R. Pianka, \& T.W. Schoener, Eds. Cambridge, MA, USA: Harvard University Press, pp. 55-83.

Porter, W.P., N. Vakharia, W.D. Klousie, \& D. Duffy. 2006. Po'ouli landscape bioinformatics models predict energetics, behavior, diets, and distribution on Maui. Integrative and Comparative Biology 46(6): 1143-58.

Post, D.F., A. Fimbres, A.D. Matthias, E.E. Sano, L. Accioly, A.K. Batchily, \& L.G. Ferreira. 2000. Predicting soil albedo from soil color and spectral reflectance data. Soil Science Society of America Journal 64(3): 1027-34. 
Potter, K.A., H.A. Woods, \& S. Pincebourde. 2013. Microclimatic challenges in global change biology. Global Change Biology 19: 2932-9.

Quinn, A.E., S.D. Sarre, T. Ezaz, J.A.M. Graves, \& A. Georges. 2011. Evolutionary transitions between mechanisms of sex determination in vertebrates. Biology Letters 7: 443-8.

Rand, A.S. \& B. Dugan. 1983. Structure of complex iguana nests. Copeia 1983: 705-11.

Randin, C.F., T. Dirnbock, S. Dullinger, N.E. Zimmerman, M. Zappa, \& A. Guisan. 2006. Are nichebased species distribution models transferable in space? Journal of Biogeography 33: $1689-1703$.

Rao, J.S. 1969. Some contributions to the analysis of circular data. Unpublished Ph.D. thesis. Indian Statistical Institute, Calcutta, India. 222 pp.

Rao, J.S. 1972. Some variants of chi-square for testing uniformity on the circle. Zeitschrift für wahrscheinlichkeitstheorie und verwandte gebiete 22: 33-44.

Ratterman, R.J. \& R.A. Ackerman. 1989. The water exchange and hydric microclimate of painted turtle (Chrysemys picta) eggs incubating in field nests. Physiological Zoology 62(5): 105979.

R Development Core Team. 2008. R: A language and environment for statistical computing. $R$ Foundation for Statistical Computing, Vienna, Austria: http://www.R-project.org.

Refsnider, J.M. 2012. Effects of climate change on reptiles with temperature-dependent sex determination and potential adaptation via maternal nest-site choice. Unpublished Ph.D. thesis. Iowa State University, USA: Paper 12570, 180 pp.

Refsnider, J.M. \& F.J. Janzen. 2010. Putting eggs in one basket: ecological and evolutionary hypotheses for variation in oviposition-site choice. Annual Review of Ecological, Evolution, and Systematics 41: 39-57.

Refsnider, J.M., S.N. Keall, C.H. Daugherty, \& N.J. Nelson. 2009. Does nest guarding in female tuatara (Sphenodon punctatus) reduce nest destruction by conspecific females? Journal of Herpetology 43(2): 294-9. 
Refsnider, J.M., C.H. Daugherty, S.N. Keall, \& N.J. Nelson. 2010. Nest site choice and fidelity in tuatara on Stephens Island, New Zealand. Journal of Zoology 280: 396-402.

Refsnider, J.M. \& F.J. Janzen. 2010. Putting eggs in one basket: ecological and evolutionary hypotheses for variation in oviposition-site choice. Annual Review of Ecology, Evolution and Systematics 41: 39-57.

Refsnider, J.M. \& F.J. Janzen. 2012. Behavioural plasticity may compensate for climate change in a long-lived reptile with temperature-dependent sex determination. Biological Conservation 152: $90-5$.

Regan, H.M., A.D. Syphard, J. Franklin, R.M. Swab, L. Markovchick, A.L. Flint, L.E. Flint, \& P.H. Zedler. 2012. Evaluation of assisted colonization strategies under global change for a rare, fire-dependent plant. Global Change Biology 18: 936-47.

Reinhold, K. 1998. Nest-site philopatry and selection for environmental sex determination. Evolutionary Ecology 12: 245-50.

Reischek, A. 1885. Observations on Sphenodon punctatum, fringe-back lizard (tuatara). Transactions and Proceedings of the New Zealand Institute 18: 108-10.

Reside, A.E., J. VanDerWal, B.L. Phillips, L.P. Shoo, D.F. Rosauer, B.J. Anderson, J.A. Welbergen, C. Moritz, S. Ferrier, T.D. Harwood, K.J. Williams, B. Mackey, S. Hugh, Y.M. Williams, \& S.E. Williams. 2013. Climate change refugia for terrestrial biodiversity: defining areas that promote species persistence and ecosystem resilience in the face of global climate change. Gold Coast, Australia: National Climate Change Adaptation Research Facility. 216 pp.

Reside, A.E., I. Watson, J. VanDerWal, \& A.S. Kutt. 2011. Incorporating low-resolution historic species location data decreases performance of distribution models. Ecological Modelling 222(18): 3444-8.

Resetarits, W.J., Jr. 1996. Oviposition site choice and life history evolution. American Zoologist 36: 205-15. 
Ricciardi, A. \& D. Simberloff. 2009a. Assisted colonisation is not a viable conservation strategy. Trends in Ecology and Conservation 24(5): 248-53.

Ricciardi, A. \& D. Simberloff. 2009b. Assisted colonisation: good intentions and dubious risk assessment. Trends in Ecology and Conservation 24(9): 476-7.

Robert, K., M. Thompson, \& F. Seebacher. 2003. Facultative sex allocation in the viviparous lizard Eulamprus tympanum, a species with temperature-dependent sex determination. Australian Journal of Zoology 51: 367-70.

Roosenburg, W. M. \& Niewiarowski, P. E. 1998. Maternal effects and the maintenance of environmental sex determination. In: Maternal effects as adaptations. T.A. Mousseau \& C.W. Fox, Eds. Oxford, U.K.: Oxford University Press, pp. 307-22.

Ross, D.G., I.N. Smith, P.C. Manins, \& D.G. Fox. 1988. Diagnostic wind field modeling for complex terrain: model development and testing. Journal of Applied Meteorology 27: 785-96.

Roughgarden, J., S.W. Running, \& P.A. Matson. 1991. What does remote sensing do for ecology? Ecology 72(6): 1918-22.

Rout, T.M., E. McDonald-Madden, T.G. Martin, N.J. Mitchell, H.P. Possingham, \& D.P. Armstrong. 2013. How to decide whether to move species threatened by climate change. PLoS ONE 8(10): 1-7.

Rull, V. 2009. Microrefugia. Journal of Biogeography 36(3): 481-4.

Saint-Girons, H. 1980. Thermoregulation in reptiles with special reference to the tuatara and its ecophysiology. Tuatara 24(2): 59-79.

Sakamoto, Y., M. Ishiguro, \& G. Kitagawa. 1986. Akaike information criterion statistics. Tokyo: D. Reidel Publishing, 320 pp.

Sangermano, F., J.R. Eastman, \& H. Zhu. 2010. Similarity weighted instance based learning for the generation of transition potentials in land change modeling. Transactions in GIS 14(5): $569-80$. 
Sax, D.F., K.F. Smith, \& A.R. Thompson. 2009. Managed relocation: a nuanced evaluation is needed. Trends in Ecology and Evolution, doi:10.1016/j.tree.2009.05.004.

Scheirs J., L. De Bruyn, \& R. Verhagen. 2000. Optimization of adult performance determines host choice in a grass miner. Proceedings of the Royal Society London B 267: 2065-9.

Schwanz, L.E. \& F. Janzen. 2008. Climate change and temperature-dependent sex determination: can individual plasticity in nesting phenology prevent extreme sex ratios? Physiological and Biochemical Zoology 81(6): 826-34.

Schwanz, L.E. \& S.R. Proulx. 2008. Mutual information reveals variation in temperaturedependent sex determination in response to environmental fluctuation, lifespan and selection. Proceedings of the Royal Society of London B 282(1803): 2441-8.

Schwanz L.E., R.J. Spencer, R.M. Bowden, \& F.J. Janzen. 2010. Climate and predation dominate early life demography and adult recruitment in a turtle with temperature-dependent sex determination. Ecology 91(10): 3016-26.

Seddon, P.J. 1999. Persistence without intervention: assessing success in wildlife reintroductions. Trends in Ecology and Evolution 14(12): 503.

Seddon, P.J. 2010. From reintroduction to assisted colonization: moving along the conservation translocation spectrum. Restoration Ecology 18(6): 796-802.

Seddon, P.J., D.P. Armstrong, \& R.F. Maloney. 2007. Developing the science of reintroduction biology. Conservation Biology 21(2): 303-12.

Seddon, P.J., D.P. Armstrong, P.Soorae, F. Launay, S. Walker, C.R. Ruiz-Miranda, S. Molur, H. Koldewey, \& D.G. Kleiman. 2009. The risks of assisted colonisation. Conservation Biology 23: 788-9.

Seddon, P.J., W.M. Strauss, \& J. Innes. 2012. Animal translocations: what are they and why do we do them? In: J.G. Ewen, D.P. Armstrong, K.A. Parker, \& P.J. Seddon, Eds. Reintroduction biology: integrating science and management. West Sussex, UK: Wiley-Blackwell, pp 1-32. 
Sekhon, J.S. 2011. Multivariate and propensity score matching software with automated balance optimization: the Matching package for R. Journal of Statistical Software 42(7): 1-52.

Sekhon, J.S. 2013. Matching: multivariate and propensity score matching with balance. R package version 4.8-3.4. http://cran.r-project.org/package=Matching.

Sharpe, P.J.H. \& D.W. DeMichele. 1977. Reaction kinetics of poikilotherm development. Journal of Theoretical Biology 64: 649-70.

Sheppard, S.C. \& J.A. Addison. 2006. Soil sample handling and storage. In: Soil sampling and methods of analysis. 2nd Ed. Carter, M.R. \& E.G. Gregorich, Eds. Boca Raton, FL, USA: Taylor \& Francis. pp. 64-73.

Shine, R. 1999a. Why is sex determined by nest temperature in many reptiles? Trends in Ecology and Evolution 14: 186-9.

Shine, R. 1999b. Reply from R. Shine (Girondot, M. \& C. Pieau, A fifth hypothesis for the evolution of TSD in reptiles.) Trends in Ecology and Evolution 14: 360.

Shine, R. 1999c. Egg-laying reptiles in cold climates: determinants and consequences of nest temperatures in montane lizards. Journal of Evolutionary Biology 12(5): 918-26.

Shine, R. 2002. Eggs in autumn: responses to declining incubation temperatures by the eggs of montane lizards. Biological Journal of the Linnaean Society 76: 71-7.

Shine, R. \& P.S. Harlow. 1996. Maternal manipulation of offspring phenotypes via nest-site selection in an oviparous lizard. Ecology 77: 1808-17.

Shirazi, M.A. \& L. Boersma. 1984. A unifying quantitative analysis of soil texture. Soil Science Society of America Journal 48: 142-7.

Shou-Li, L., Y. Fei-Hai, M.J.A. Werger, D. Ming, S. Ramula, \& P.A. Zuidema. 2013. Understanding the effects of a new grazing policy: the impact of seasonal grazing on shrub demography in the Inner Mongolian steppe. Journal of Applied Ecology 50: 1377-86. 
Silber, S., J.H. Geisler, \& M. Bolortsetseg. 2010. Unexpected resilience of species with temperature-dependent sex determination at the Cretaceous-Palaeogene boundary. Biology Letters, doi:10.1098/rsbl.2010.0882.

Smith, B. 2001. LPJ-Guess: an ecosystem modelling framework. Unpublished software manual. $19 \mathrm{pp}$.

Smith, D.M., S. Cusack, A.W. Colman, C.K. Folland, G.R. Harris, \& J.M. Murphy. 2007. Improved surface temperature prediction for the coming decade from a global climate model. Science 317(5839): 796-9.

Smith, B., I.C. Prentice, \& M.T. Sykes. 2001. Representation of vegetation dynamics in the modelling of terrestrial ecosystems: comparing two contrasting approaches within European climate space. Global Ecology and Biogeography 10(6): 621-37.

Soberón, J. \& A.T. Peterson. 2005. Interpretation of models of fundamental ecological niches and species' distributional areas. Biodiversity Informatics 2: 1-10.

South, A., S. Ruston, \& D. Macdonald. 2000. Simulating the proposed reintroduction of the European beaver (Castor fiber) to Scotland. Biological Conservation 93: 103-16.

Spencer, R.-J. 2002. Experimentally testing nest site selection: fitness trade-offs and predation risk in turtles. Ecology 83(8): 2136-44.

Spencer, R.-J. \& M.B. Thompson. 2003. The significance of predation in nest site selection of turtles: an experimental consideration of macro- and microhabitat preferences. Oikos 102: 592-600.

Spiess, A.-N. 2013. qpcR: Modelling and analysis of real-time PCR data. R package version 1.3-7.1. http://CRAN.R-project.org/package=qpcR

Standora, E.A. \& J.R. Spotila. 1985. Temperature dependent sex determination in sea turtles. Copeia 3: 711-22.

Stockwell, D.R.B. \& A.T. Peterson. 2002. Effects of sample size on accuracy of species distribution models. Ecological Modelling 148: 1-13. 
Sultan, S.E. \& H.G. Spencer. 2002. Metapopulation structure favors plasticity over local adaptation. American Naturalist 160(2): 271-83.

Suttle, K.B., M.A. Thomsen, \& M.E. Power. 2007. Species interactions reverse grassland responses to changing climate. Science $315:$ 640-2.

Swart, D., H. Rahn, \& J. de Kock. 1987. Nest microclimate and incubation water loss of eggs of the African ostrich (Struthio camelus var. domesticus). Journal of Experimental Zoology 1: 23946.

Tait, A., R. Henderson, R. Turner, \& X.G. Zheng. 2006. Thin plate smoothing spline interpolation of daily rainfall for New Zealand using a climatological rainfall surface. International Journal of Climatology 26(14): 2097-115.

Tait, A., J. Sturman, \& M. Clark. 2012. An assessment of the accuracy of interpolated daily rainfall for New Zealand. Journal of Hydrology (NZ), 51(1): 25-44.

Telemeco, R.S., M.J. Elphick, \& R. Shine. 2009. Nesting lizards (Bassiana duperreyi) compensate partly, but not completely, for climate change. Ecology 90(1): 17-22.

Telemeco, R.S., K.C. Abbot, \& F.J. Janzen. 2013. Modeling the effects of climate change-induced shifts in reproductive phenology on temperature-dependent traits. The American Naturalist 181(5): 637-48.

Thompson, M.B. 1990. Incubation of eggs of tuatara, Sphenodon punctatus. Journal of the Zoological Society of London 222: 303-18.

Thompson, M.B. \& C.H. Daugherty. 1998. Metabolism of tuatara (Sphenodon punctatus). Comparative Biochemistry and Physiology A 119(2): 519-22.

Thompson, M.B., C.H. Daugherty, \& A. Cree. 1989. Management recommendations for Stephens Island. Department of Zoology, Victoria University of Wellington: Unpublished report to Department of Conservation.

Thompson, M.B., C.H. Daugherty, A. Cree, D.C. French, J.C. Gillinghamd, \& R.E. Barwick. 1992. Status and longevity of the tuatara, Sphenodon guntheri, and Duvaucel's gecko, 
Hoplodactylus duvaucelli, on North Brother Island, New Zealand. Journal of the Royal Society of New Zealand 22: 123-30.

Thompson, M.B., G.C. Packard, M.J. Packard, \& B. Rose. 1996. Analysis of the nest environment of tuatara Sphenodon punctatus. Journal of the Zoological Society of London 238: 239-51.

Thuiller, W. 2004. Patterns and uncertainties of species' range shifts under climate change. Global Change Biology 10: 2020-7.

Thuiller, W., C. Albert, M.B. Araújo, P.M. Berry, M. Cabeza, A. Guisan, T. Hickler, G.F. Midgley, J. Paterson, F.M. Shurr, M.T. Sykes, \& N.E. Zimmerman. 2008. Predicting global change impacts on plant species distributions: future challenges. Perspectives in Plant Ecology, Evolution, \& Systematics 9: 137-52.

Towns, D.R. 2011. Eradications of vertebrate pests from islands around New Zealand: what have we delivered and what have we learned? In: C.R. Veitch, M.N. Clout, \& D.R. Towns, Eds. Island invasives: eradication and management. Gland, Switzerland: IUCN, pp. 364-71.

Towns, D.R., C.H. Daugherty, \& A. Cree. 2001. Raising the prospects for a forgotten fauna: a review of 10 years of conservation effort for New Zealand reptiles. Biological Conservation 99: 3-16.

Towns, D.R., G.R. Parrish, C.L. Tyrrell, G.T. Ussher, A. Cree, D.G. Newman, A.H. Whitaker, \& I. Westbrooke. 2007. Responses of tuatara (Sphenodon punctatus) to removal of introduced Pacific rats from islands. Conservation Biology 21: 1021-31.

Tracy, C.R. 1982. Biophysical modelling in reptilian physiology and ecology. In: Physiology C: Physiological Ecology. Gans, C. \& F.H. Pough, Eds. London, UK: Academic Press, pp. 275321.

Tucker, J.K., C.R. Dolan, J.T. Lamer, \& E.A. Dustman. 2008. Climatic warming, sex ratios, and redeared sliders (Trachemys scripta elegans). Chelonian Conservation and Biology 7(1): 60-9. 
Tyrrell, C.L., A. Cree, \& D.R. Towns. 2000. Variation in reproduction and condition of northern tuatara (Sphenodon punctatus punctatus) in the presence and absence of kiore. Science for Conservation 153, Department of Conservation, Wellington, New Zealand.

Valenzuela, N. 2001. Constant, shift, and natural temperature effects on sex determination in Podocnemus expansa turtles. Ecology 82: 3010-24.

van Dooren, T.J.M. \& O. Leimar. 2003. The evolution of environmental and genetic sex determination in fluctuating environments. Evolution 57(12): 2667-77.

Vasseur, D.A., J.P. DeLong, B. Gilbert, H.S. Greig, C.D.G. Harley, K.S. McCann, V. Savage, T.D. Tunney, \& M.I. O'Conner. 2014. Increased temperature variation poses a greater risk to species than climate warming. Proceedings of the Royal Society B 281: 20132612.

Venables, W.N. \& B.D. Ripley. 2002. Modern applied statistics with S. $4^{\text {th }}$ Ed. New York: Springer, $497 \mathrm{pp}$.

Versace, V.L., D. Ierodiaconou, F. Stagnitti, \& A.J. Hamilton. 2008. Appraisal of random and systematic land cover transitions for regional water balance and revegetation strategies. Agriculture, Ecosystems and Environment 123: 328-36.

Via, S., R. Gomulkiewicz, G. de Jong, S.M. Scheiner, C.D. Schlichting, \& P.H. van Tienderen. 1995. Adaptive phenotypic plasticity: consensus and controversy. Trends in Ecology and Evolution 10(5): 212-7.

Visser, M.E. \& C. Both. 2005. Shifts in phenology due to global climate change: the need for a yardstick. Proceedings of the Royal Society B 272: 2561-9.

Visser, M.E. \& L.J.M. Holleman. 2001. Warmer springs disrupt the synchrony of oak and winter moth phenology. Proceedings of the Royal Society of London B 268: 289-94.

Voltz, M. \& Webster, R. 1990. A comparison of kriging, cubic splines and classification for predicting soil properties from sample information. Journal of Soil Science 41: 473-90.

Wackernagel, H. 1998. Multivariate geostatistics. Berlin: Springer Verlag. 291 pp. 
Walls, G.Y. 1976. Vegetation of Stephens Island, Cook Strait. Nelson: Department of Scientific and Industrial Research [DSIR], Botany Division. Unpublished report. 11 pp.

Walls, G.Y. 1983. Activity of the tuatara and its relationships to weather conditions on Stephens Island, Cook Strait, with observations on geckos and invertebrates. New Zealand Journal of Zoology 10: 309-18.

Walls, G.Y., S. Courtney, \& P. Williams. 1988. Vegetation and flora of Stephens Island (Takapourewa) with management suggestions. Nelson: Department of Scientific and Industrial Research [DSIR], Botany Division. Unpublished report. 22 pp.

Walters, C.J. 1986. Adaptive management of renewable resources. New York, NY, USA: MacMillan Publishing Co. 374 pp.

Walters, R.J., W.U. Blanckenhorn, \& D. Berger. 2012. Forecasting extinction risk of ectotherms under climate warming: an evolutionary perspective. Functional Ecology, doi: 10.1111/j.1365-2435.2012.02045.x.

Ward, W.T. 1961. Soils of Stephens Island. New Zealand Journal of Science 4: 493-505.

Warner, D.A. \& R.M. Andrews. 2002. Nest-site selection in relation to temperature and moisture by the lizard Sceloporus undulatus. Herpetologica 58(4): 399-407.

Warner, D.A. \& R. Shine. 2008. The adaptive significance of temperature-dependent sex determination in a reptile. Nature 451: 566-8.

Warren, D.L., M. Cardillo, D.F. Rosauer, \& D.I. Bolnick. 2014. Mistaking geography for biology: inferring processes from species distributions. Trends in Ecology and Evolution, doi: 10.1016/j.tree.2014.08.003.

Watt, M.S., M.U.F. Kirschbaum, T.S.H. Paul, A. Tait, H.G. Pearce, E.G. Brockerhoff, J.R. Moore, L.S. Bulman, \& D.J. Kriticos. 2008: The effect of climate change on New Zealand's planted forests: impacts, risks and opportunities. Report for the Ministry of Agriculture and Fisheries. Christchurch, New Zealand: Scion. 148 p. 
Weber, S.B., A.C. Broderick, T.G.G. Groothuis, J. Ellick, B.J. Godley, \& J.D. Blount. 2012. Fine-scale thermal adaptation in a green turtle nesting population. Proceedings of the Royal Society $B$ 279: 1077-84.

West-Eberhard, M.J. 1989. Phenotypic plasticity and the origins of diversity. Annual Review of Ecology and Systematics 20: 249-78.

Whitaker, A.H. 1993. Research on the tuatara (Sphenodon punctatus) of Little Barrier Island, 612 October 1992. Unpublished report. New Zealand Department of Conservation [DOC], Auckland Conservancy. 52 pp.

Whitaker, A.H. \& C.H. Daugherty. 1991. Research on the tuatara (Sphenodon punctatus) of Little Barrier Island, 5-12 February 1991. Unpublished report. Threatened Species Unit, New Zealand Department of Conservation [DOC], Wellington, New Zealand. 55 pp.

Whittaker, R.J., K.J. Willis, \& R. Field. 2001. Scale and species richness: towards a general, hierarchical theory of species diversity. Journal of Biogeography 28: 453-70.

Wickham, H. \& W. Chang. 2014. ggplot2: An implementation of the Grammar of Graphics. R package version 1.0.0. http://cran.r-project.org/package=ggplot2.

Williams, B.K. 2001. Uncertainty, learning, and the optimal management of wildlife. Environmental and Ecological Statistics 8: 269-88.

Williams, K.S. \& L.E. Gilbert. 1981. Insects as selective agents on plant vegetative morphology: egg mimicry reduces egg laying by butterflies. Science 212: 467-9.

Williams, J., S. Ostrowski, E. Bedin, \& K. Ismail. 2001. Seasonal variation in energy expenditure, water flux and food consumption of Arabian oryx Oryx leucoryx. Journal of Experimental Biology 204: 2301-11.

Williams, S.E., L.P. Shoo, J.L. Isaac, A.A. Hoffmann, \& G. Langham. 2008. Towards an integrated framework for assessing the vulnerability of species to climate change. PLoS Biology 6: 2621-6. 
Willis, S.G., J.K. Hill, C.D. Thomas, D.B. Roy, R. Fox, D.S. Blakeley, \& B. Huntley. 2009. Assisted colonization in a changing climate: a test-study using two U.K. butterflies. Conservation Letters 2: 45-51.

Willis, K.J. \& R.J. Whittaker. 2002. Species diversity- scale matters. Science 295: 1245-8.

Willson, R.C. \& H.S. Hudson. 1991. The sun's luminosity over a complete solar cycle. Nature 351(6321): 42-4.

Wilson, D. 1998. Nest-site selection: microhabitat variation and its effects on the survival of turtle embryos. Ecology 79: 1884-92.

Wintle, B., S.A. Bekessy, L.A. Venier, J.L. Pearce, \& R.A. Chisholm. 2005. Utility of dynamiclandscape metapopulation models for sustainable forest management. Conservation Biology 19: 1930-43.

Wisz, M.S., R.J. Hijmans, J. Li, A.T. Peterson, C.H Graham, A. Guisan, \& NCEAS Predicting Species Distributions Working Group. 2008. Effects of sample size of the performance of species distribution models. Diversity and Distributions 14: 763-73.

Wood, A.W., L.R. Leung, V. Sridhar, \& D.P. Lettenmaier. 2004. Hydrologic Implications of dynamical and statistical approaches to downscaling climate model outputs. Climatic Change 62: 189-216.

Wood, J. R. 2009. Two late quaternary avifaunal assemblages from the Dunback district, eastern Otago, South Island, New Zealand. Notornis 56: 154-7.

Woods, H.A. \& R.T. Bonnecaze. 2006. Insect eggs at a transition between diffusion and reaction limitation: temperature, oxygen, and water. Journal of Theoretical Biology 243: 483-92.

Woolgar L., S. Trocini, \& N. Mitchell. 2013. Key parameters describing temperature-dependent sex determination in the southernmost population of loggerhead sea turtles. Journal of Experimental Marine Biology and Ecology 449: 77-84.

Worthy, T.H. \& R.N. Holdaway. 2002. The lost world of the moa: prehistoric life of New Zealand. Bloomington, Indiana: Indiana University Press. 718 pp. 
Wright, L.I., K.L. Stokes, W.J. Fuller, B.J. Godley, A. McGowan, R. Snape, T. Tregenza, \& A.C. Broderick. 2012. Turtle mating patterns buffer against disruptive effects of climate change. Proceedings of the Royal Society B 279: 2122-7.

Yang, L.H. \& V.H.W. Rudolf. 2010. Phenology, ontogeny and the effects of climate change on the timing of species interactions. Ecology Letters 13: 1-10.

Zachos, M. Pagani, L. Sloan, E. Thomas, \& K. Billups. 2001. Trends, rhythms, and aberrations in global climate 65 Ma to present. Science 292: 686-93.

Zambrano-Bigiarini, M. 2014. hydroGOF: goodness-of-fit functions for comparison of simulated and observed hydrological time series. $\mathrm{R}$ package version 0.3-8. http://cran.rproject.org/package=hydroGOF .

Zhao, B., T. Li, R. Shine, \& W.-G. Du. 2013. Turtle embryos move to optimal thermal environments within the egg. Biology Letters 9: 20130337.

Zhuang J., K. Nakayama, G.R. Yu, \& T. Urushisaki. 2001. Estimation of root water uptake of maize: an ecophysiological perspective. Field Crops Research 69(4): 201-13. 



\section{Appendices}

\section{Chapter 2}

Appendix 2.1 Cross-tabulated error matrices used to calculate accuracy of categorical land cover maps developed for Takapourewa. Cells along the diagonal contain the total number of correctly classified pixels. Darker shading indicates a larger proportion, relative to the size of the 'true class,' of pixels binned into each class by the classification algorithm.

\begin{tabular}{|c|c|c|c|c|c|c|c|}
\hline True class & rock & pasture & $\begin{array}{l}\text { Number of pix } \\
\text { tall grasses }\end{array}$ & $\begin{array}{l}\text { Is classifiec } \\
\text { vineland }\end{array}$ & $\begin{array}{l}00 \text { map } \\
\text { scrub }\end{array}$ & forest & Total \\
\hline rock & 44 & 0 & 3 & 0 & 2 & 0 & 49 \\
\hline pasture & 1 & 62 & 30 & 0 & 1 & 0 & 94 \\
\hline tall grasses & 3 & 2 & 134 & 0 & 2 & 0 & 141 \\
\hline vineland & 0 & 1 & 2 & 18 & 0 & 0 & 21 \\
\hline scrub & 1 & 0 & 1 & 1 & 123 & 0 & 126 \\
\hline forest & 0 & 0 & 0 & 0 & 7 & 62 & 69 \\
\hline Total & 49 & 65 & 170 & 19 & 135 & 62 & 500 \\
\hline
\end{tabular}




\begin{tabular}{rrrrrrrr}
\hline & \multicolumn{7}{c}{ Number of pixels classified in 2007 map } \\
True class & rock & pasture & tall grasses & vineland & scrub & forest & Total \\
\hline rock & 67 & 0 & 0 & 0 & 0 & 0 & 67 \\
pasture & 0 & 26 & 14 & 0 & 2 & 0 & 42 \\
tall grasses & 0 & 0 & 84 & 1 & 4 & 0 & 89 \\
vineland & 2 & 0 & 3 & 79 & 3 & 0 & 87 \\
scrub & 1 & 0 & 0 & 0 & 102 & 0 & 103 \\
forest & 0 & 0 & 0 & 0 & 22 & 90 & 112 \\
Total & 70 & 26 & 101 & 80 & 133 & 90 & $\mathbf{5 0 0}$ \\
\hline
\end{tabular}

\begin{tabular}{rrrrrrrr}
\hline & \multicolumn{8}{c}{ Number of pixels classified in 2011 map } & & \\
True class & rock & pasture & tall grasses & vineland & scrub & forest & Total \\
\hline $\begin{array}{r}\text { rock } \\
\text { pasture }\end{array}$ & 18 & 0 & 0 & 0 & 0 & 0 & 18 \\
tall grasses & 0 & 0 & 0 & 0 & 0 & 0 & 0 \\
vineland & 4 & 0 & 90 & 13 & 6 & 4 & 117 \\
scrub & 2 & 0 & 2 & 92 & 1 & 0 & 97 \\
forest & 0 & 0 & 0 & 0 & 117 & 0 & 117 \\
Total & 0 & 0 & 2 & 8 & 24 & 117 & 151 \\
& 24 & 0 & 94 & 113 & 148 & 121 & $\mathbf{5 0 0}$ \\
\hline
\end{tabular}


Appendix 2.2 Methods for development of a land cover classification map for Takapourewa for the year 2012

Change analysis was carried out on two pairs of classifications, 2000/2007 and 2000/2011, using the Land Change Modeler in Idrisi Selva ${ }^{\circledR}$ (Clark Labs, Clark University: Worcester, MA, USA, 2013) to determine whether land cover changes differed between the two time periods and, if so, which pair best reflected the changes most relevant to tuatara nesting behaviour. Net change and persistence (Pontius et al. 2004) of each land cover class and change between each pair of classes were determined using per-pixel cross tabulation. Only transition types comprised of at least 10,000 pixels $\left(40,000 \mathrm{~m}^{2}\right)$ were subsequently included as sub-models for predicting land cover transition potentials. Selecting only the 'major' transition types minimised errors introduced as a result of imperfect classifications of the original photos due to user error, spectral similarity among classes, or spatial incongruities between pairs of original images digitised using different methods (Green \& Hartley 2000; Pontius et al. 2004; Pontius \& Petrova 2010).

Producing land cover transition models required a set of driver variables to predict the observed land cover transitions. While environmental variables that directly influence vegetation growth were more likely be useful, transition models relied only on the ability of a particular variable to differentiate between pixels that underwent a particular transition versus those that did not. Selection of potential explanatory variables for this study focused primarily on geospatial drivers of vegetation growth for three reasons: (1) the transitions examined in this study were the effects of habitat restoration rather than degradation, so large-scale anthropogenic drivers normally considered in land change analysis (e.g., road construction, forestry, human population density; Gao 2009) were not factors in this study; (2) the 2011 classification map was used, albeit indirectly, to inform an island-wide microclimate model (Chapters 3, 4). As a 
result, while temperature and soil moisture, for example, are important drivers of vegetation growth, they would not be independent of vegetation composition in this study; and (3) geospatial data (e.g., elevation, aspect) are highly correlated with vegetation dynamics (Mata-González et al. 2002) and can be used to derive other variables (de Smith et al. 2007), particularly wind exposure and nutrient runoff patterns, which may constrain vegetation composition in the harsh maritime environment of Takapourewa (Brown 2000).

All driver variables were generated in ArcGIS as individual geospatial datasets with spatial orientation and dimensions identical to the classified images (although land cover classification horizontal resolution was $6 \mathrm{~m}^{2}$, map resolution was not itself decreased from $2 \mathrm{~m}^{2}$ ). Per-pixel values for elevation, slope, and aspect (maximum rate of change in slope from each pixel to its eight nearest neighbors) were calculated from a $2 \mathrm{~m}^{2}$ gridded digital elevation model. Raw aspect values were binned into eight categories representing cardinal and ordinal directions (Burrough \& McDonell 1998).

Watershed analysis was used to calculate the distribution of local topographical high and low points. The potential of points to act as nutrient sinks for vegetation growth was then represented by a variable 'gully,' calculated as the Euclidean (i.e., straight-line) distance of each pixel from its nearest low point (the nearest pixel in a gully). Wind exposure is an important variable for vegetation dynamics, particularly in a maritime environment, and may have accelerated deforestation on Takapourewa in the past (DOCa; Brown 2000). To quantify potential wind exposure at each location, the variable 'ridge' was calculated as the Euclidean distance from each pixel to the nearest high point (the nearest pixel on a ridgeline). A categorical map of island soils was manually digitised and used to create the variable 'soil.'

Two anthropogenic drivers were also examined for modeling transition potentials. Throughout the ecological restoration of the island, both temporary and permanent fences were installed, initially to prevent remaining livestock from 
destroying newly planted areas and then to prevent access roads through replanted areas from being overgrown (DOCa). The final fence line delineates fragments of different vegetation types, and points nearer the fence may have been more likely to transition to an adjacent land cover type than points farther away from the fence. The distance of a point on the island from a fence line, therefore, was used as a proxy for the vulnerability of a pixel to edge effects of the interaction between adjacent fragments (Murcia 1995). The variable 'fences' was created by digitising a map of fences (DOCb) and calculating Euclidean distance from each point on the island to the nearest point on the fence line.

Finally, the island vegetation restoration was represented by the variable 'replants,' generated using a hard-copy map of restoration plantings (DOCc). Each revegetated area was categorised by the number of years since replanting had been undertaken in that area. Three locations had undergone multiple plantings but were not treated separately from areas that had only been planted once. A small patch near the Winch House rookery had been replanted in both 1993 and 1994, but the growth rates of the two groups of seedlings should not have been so different as to impact assessment of transitions starting from 2000. A subsection of Keeper's Bush underwent seven plantings between 1987 and 2000; likely for that very reason, the entire area persisted as forest in both change analyses and so was not included in transition modelling at all. Finally, a large area below the Light House rookery had been first replanted in 2008 and again in 2012; however, since the second replanting occurred after the final aerial photo used in land cover classification had been obtained in 2011, the subsequent planting was not relevant for modelling transitions in this study. Variables that were developed using scanned, hard-copy maps (soil, fences, replants) were georeferenced to the 2011 aerial imagery using a 2nd-order polynomial transformation (RMS error <0.0001) and manually digitised. 
The three distance-decay variables (ridge, gully, fences) were natural logtransformed (REF). The three categorical variables (aspect, soil, replants) were transformed using an evidence likelihood measure of association between each variable category and each transition type (Eastman 2012). All potential driver variables (Fig. 2.2.1) were weighted for modelling by comparing the standard deviation of each variable within areas that had undergone each transition to the standard deviation of that variable in the total study area (Sangermano et al. 2010):

$$
\text { relevance weight }=1-\left(\frac{S D_{\text {change area }}}{S D_{\text {study area }}}\right)
$$

Relevance weights simply quantified the likelihood that a pixel of a given class, which itself either transitioned or persisted, could be differentiated from other pixels of the same initial class using the associated values of a particular driver variable. With the exception of pixels with an initial class of tall grasses (G), 'elevation' and 'slope' were best able to differentiate between transitioned and persisted pixels. Variables with calculated relevance weights $<0.05$ were not included in the final models (Table 2.2.1). Each transition type was modelled separately. 

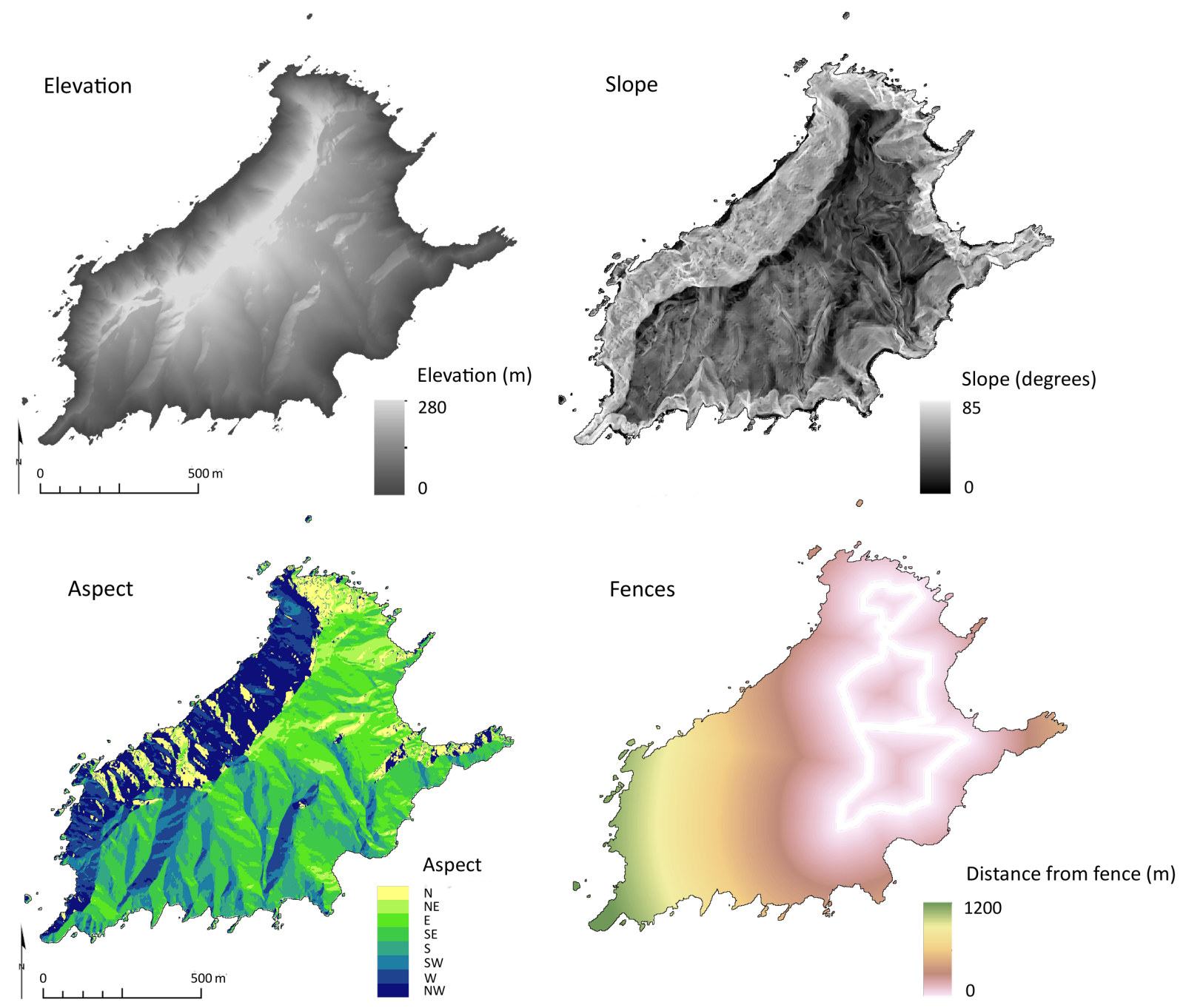


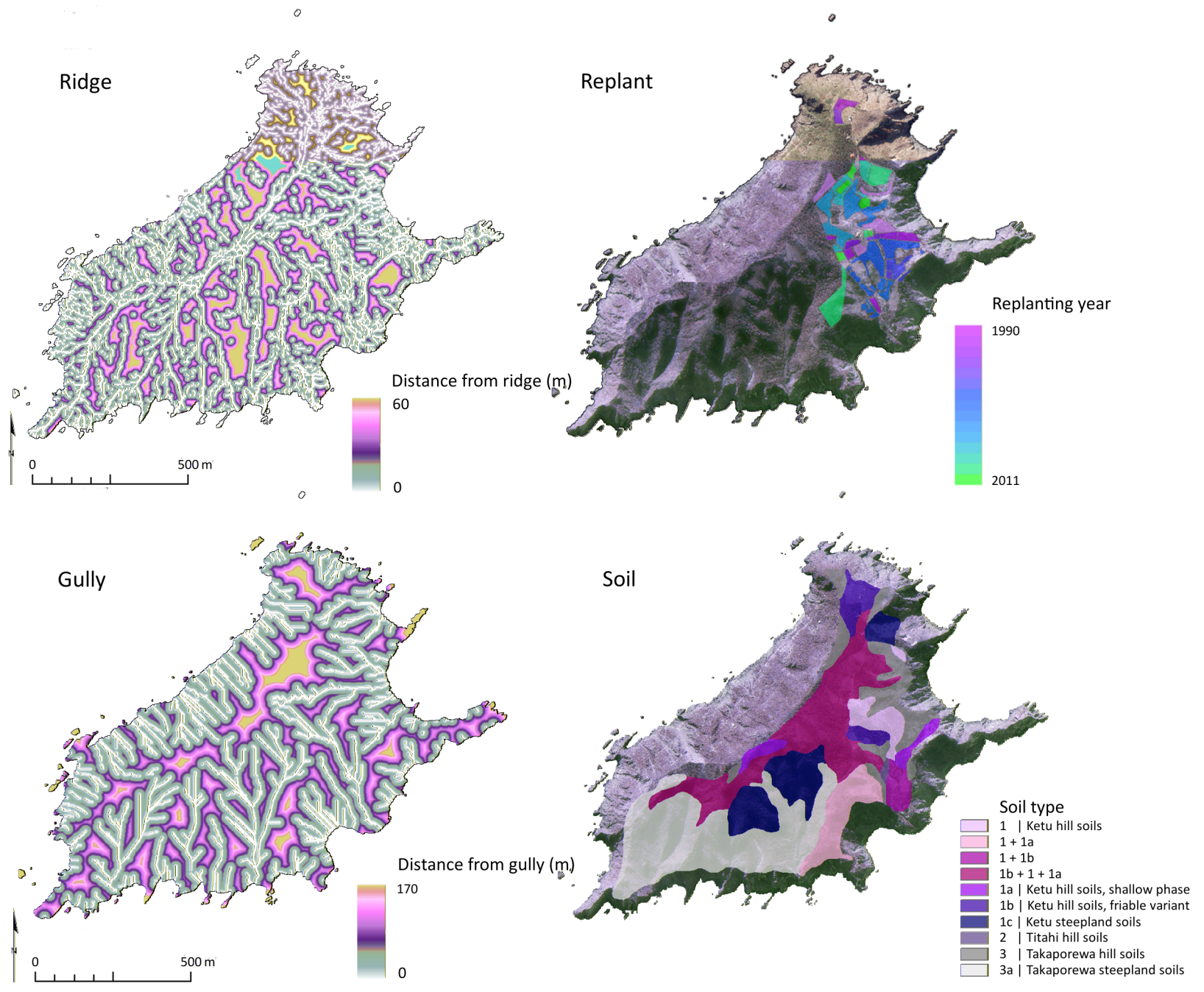

Figure 2.2.1. Spatial distributions of driver variables examined for transition potential modelling of land cover change on Takapourewa. 'Replant' and 'soil' are overlaid onto an aerial photo of the island to visualise variable coverage. The spelling of 'Takaporewa' in the 'Soil type' key is retained from Ward (1961). 


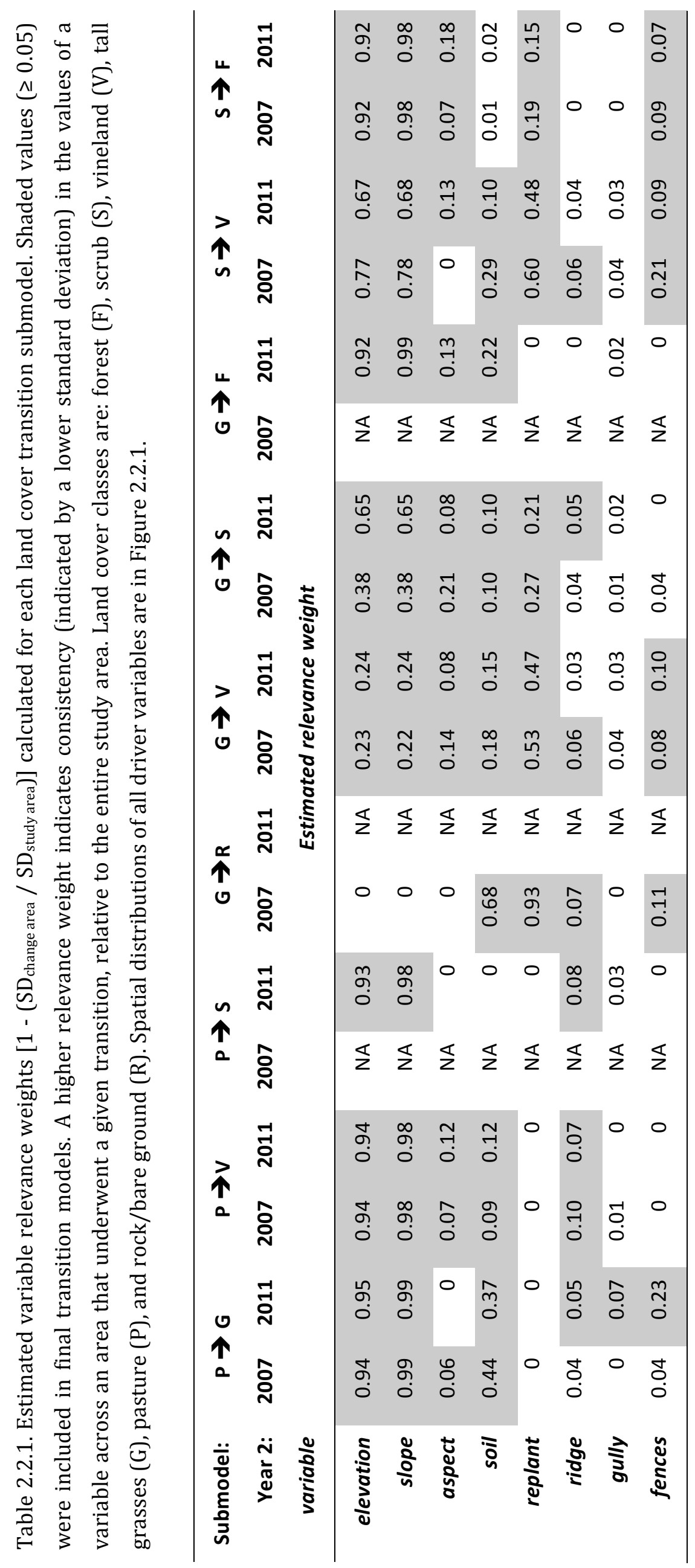


Unlike instances of change, class persistence between any two time points only expresses that a location did not transition between those points. In contrast with 'hard' classification models that bin locations into definitive classifications, consequently losing information on persistence, transition potential models are fuzzy classifications that predict the vulnerability of a location to change (Eastman et al. 2005). In land change analyses, the vulnerability to change is synonymous with the probability that a pixel will transition from its initial class within the given time frame. Transition potential maps are generally preferable for ecological applications, since they capture information on both change and persistence (Eastman 2012).

Transition potentials were modeled using SimWeight, a similarity-weighted instance-based machine-learning algorithm based on k-nearest neighbours (Fix \& Hodges 1951). SimWeight predicts transitions by calculating the distance of each pixel in a training set to k-nearest pixels with the same initial class membership, which are categorised as 'change' pixels in the second classified image (Pontius 2002). Only pixels that underwent change have membership in $\mathrm{k}$, since persistence between times $t_{0}$ and $t_{1}$ does not ensure persistence between times $t_{1}$ and $t_{2}$ (Sangermano et al. 2010). Larger values of k consequently produce more generalised models (e.g., for landscapes with relatively well-defined land class boundaries), while smaller values can more effectively model smaller areas or those with a 'patchy' distribution of land classes (Sangermano et al. 2010; Eastman 2012). Sample sizes of 1000 pixels were extracted for each transition model, with half used for training and half for validation (Sangermano et al. 2010). Since learning algorithms used in land cover change analyses require instances of both change and persistence to model either (Pontius 2002), the near-total reduction of pixels classified as pasture in the 2011 image required reduction of the sample size to 100 pixels for modelling transitions from pasture. The ability of models to successfully differentiate between 'change' and 'persistence' pixels in the validation set was 
measured using the Peirce skill score (Table 3; Peirce 1884; Manzato 2007), the difference between the mean transition potential of 'change' pixels correctly predicted as change (true change) and the mean transition potential of 'persistence' pixels predicted as change (false change; Sangermano et al. 2010). The highest overall model performance across all transition types for the study area was obtained with $\mathrm{k}=12$.

Since transition models are not absolute classifications, their accuracy cannot be directly assessed; however, 'hard' classifications predicted using Markov chain analysis of transition maps can be compared to actual land cover maps, if the latter are available (Pontius et al. 2008; Pontius \& Petrova 2010; Eastman 2012). For the purpose of land cover prediction, Markov chain analysis predicts the quantity of pixels that will have transitioned between classes at some time, $t_{j}$, given (1) the classification of pixels at $t_{1}$ and (2) a matrix of probabilities of pixel membership in every possible class at $t_{j-1}$, given the classification at $t_{1}$. For this study, the actual 2011 classification map was used to assess accuracy of the transition models for 2000/2007; however, no post-2011 imagery was available with which to compare predictions made using transition models for $2000 / 2011$. Since the aim of this study was to explain changes in vegetation across a single location over a finite study period and not to extrapolate predictions past 2012 or to generate predictions for novel locations, the best model for land cover in 2012 was likely to be over-fitted to the classification data.

Markov matrices were calculated using the 2007 and 2011 classification maps as $t_{1}$ states. Predicted quantities of land cover change were generated for regular intervals post- $t_{1}$ using multiple calculation iterations, the first of which was roughly equal to 2011. A prediction from 2011 to 2021 calculated through 10 iterations, for example, would have allocated $10 \%$ of the total change to each year between 2011 and 2021 . Predicted first-iteration classifications were compared with the actual 2011 land cover map using cross-tabulation. Pixels that both transitioned and were correctly classified 
were categorised as 'true change;' persistence pixels that were incorrectly classified as change were categorised as 'false change;' pixels that either (1) were successfully predicted to transition but were misclassified or (2) were predicted to be persistence but actually transitioned were categorised as 'incorrect change.' The actual 2007 land cover map was likewise compared with the first iteration of a $2007 / 2011$ prediction. The best transition model for $2000 / 2011$ was used to generate a categorical land cover map for 2012 and to hypothesize transitions through 2100 for examining possible longterm effects of vegetation on soil temperature cycles (Chapter 4).

Appendix 2.3 Results of land cover change analysis used to predict 2012 vegetation coverage for Takapourewa

The total pixels gained and lost by each land cover category were similar between the two pairs of analysed images; however, no additional pixels were classified as pasture in 2011 from 2000. Differences in quantities of all land cover types between the two pairs of analysed images are shown in the plot of net changes in quantities of classes (Fig. 2.5.1). Most land cover types experienced a net loss in area; only vineland and forest gained area from 2000 to both 2007 and 2011. Persistence was apparent in every land cover class with the exception of pasture, which experienced a near-total loss of area from 2000 to 2011 (Fig. 2.3.2). Examination of all transitions (Fig. 2.3.3-4) identified seven between $2000 / 2007$ and eight between $2000 / 2011$ of at least 10,000 pixels $\left(40,000 \mathrm{~m}^{2}\right)$ that were further assessed through transition modelling (Fig. 2.3.58).

Land cover change analysis found that the 2000/2007 model had higher predictive abilities across individual transitions between vegetation types. Consolidation of individual models, however, showed that the 2000/2011 model estimated consistently higher transition probabilities for pixels classified as pasture in 
2000, suggesting that the $2000 / 2011$ model would generate, overall, a more accurate land cover map for 2012. Three 'hard' classification maps were generated for 2011 to more clearly assess the accuracy of transition models (Fig. 2.3.9-10). The use of 'first iteration' land cover maps (essentially over-fitted models) ignored extrapolative power in order to describe vegetation changes during the study period (Pontius et al. 2004). Even so, the 2000/2007 model was unable to predict the loss of pasture areas evident in the actual 2011 classification map. The best model was the 2000/2011 model, which was used to generate land cover classification maps for 2012 (Chapter 2) and 2100 (Chapter 4). 


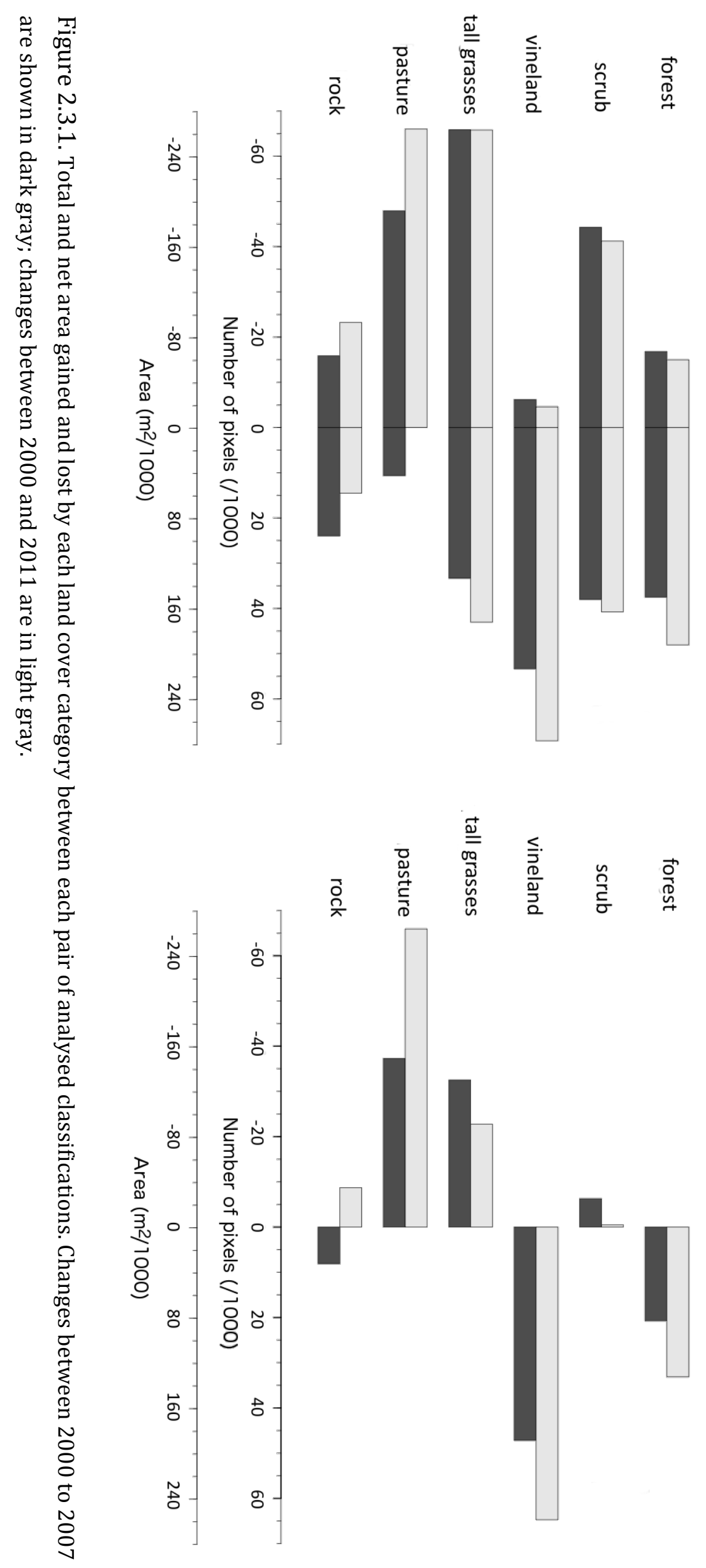




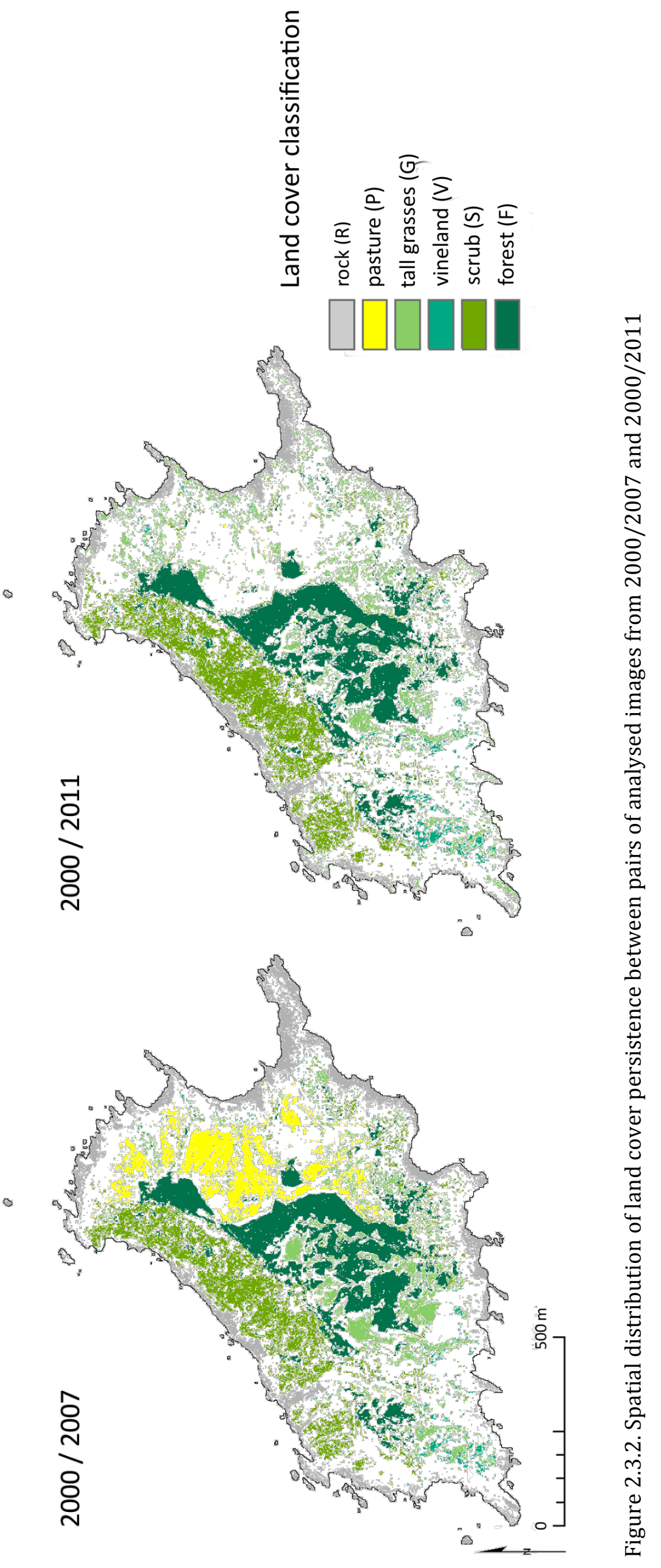




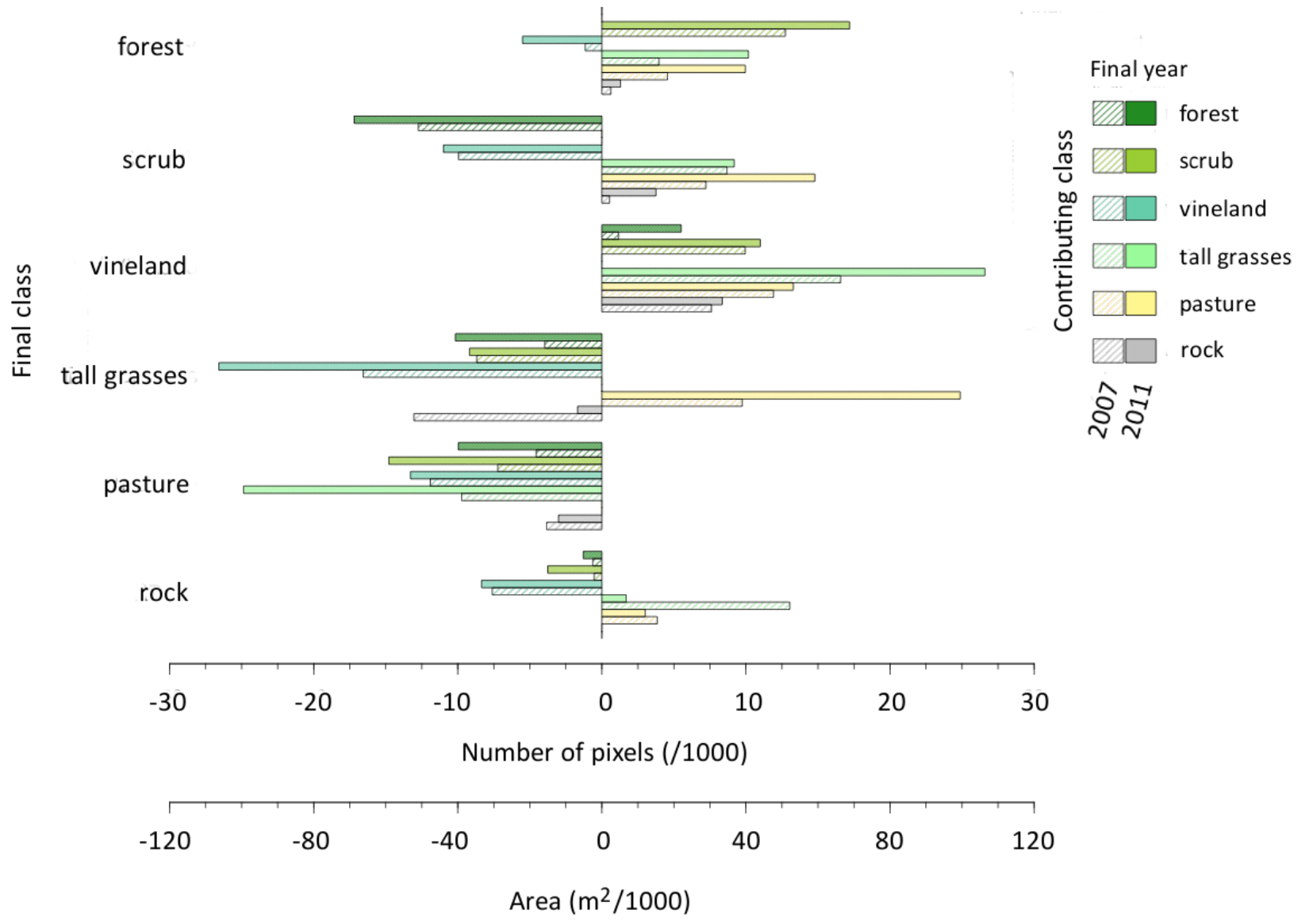

Figure 2.3.3. Contribution of each land cover class to net changes in the area of every other class (i.e., all possible transitions between classes) between 2000 and images from 2007 and 2011. Positive contributions indicate a gain in the final class from the contributing class; negative contributions indicate a loss in the final class to the contributing class. 


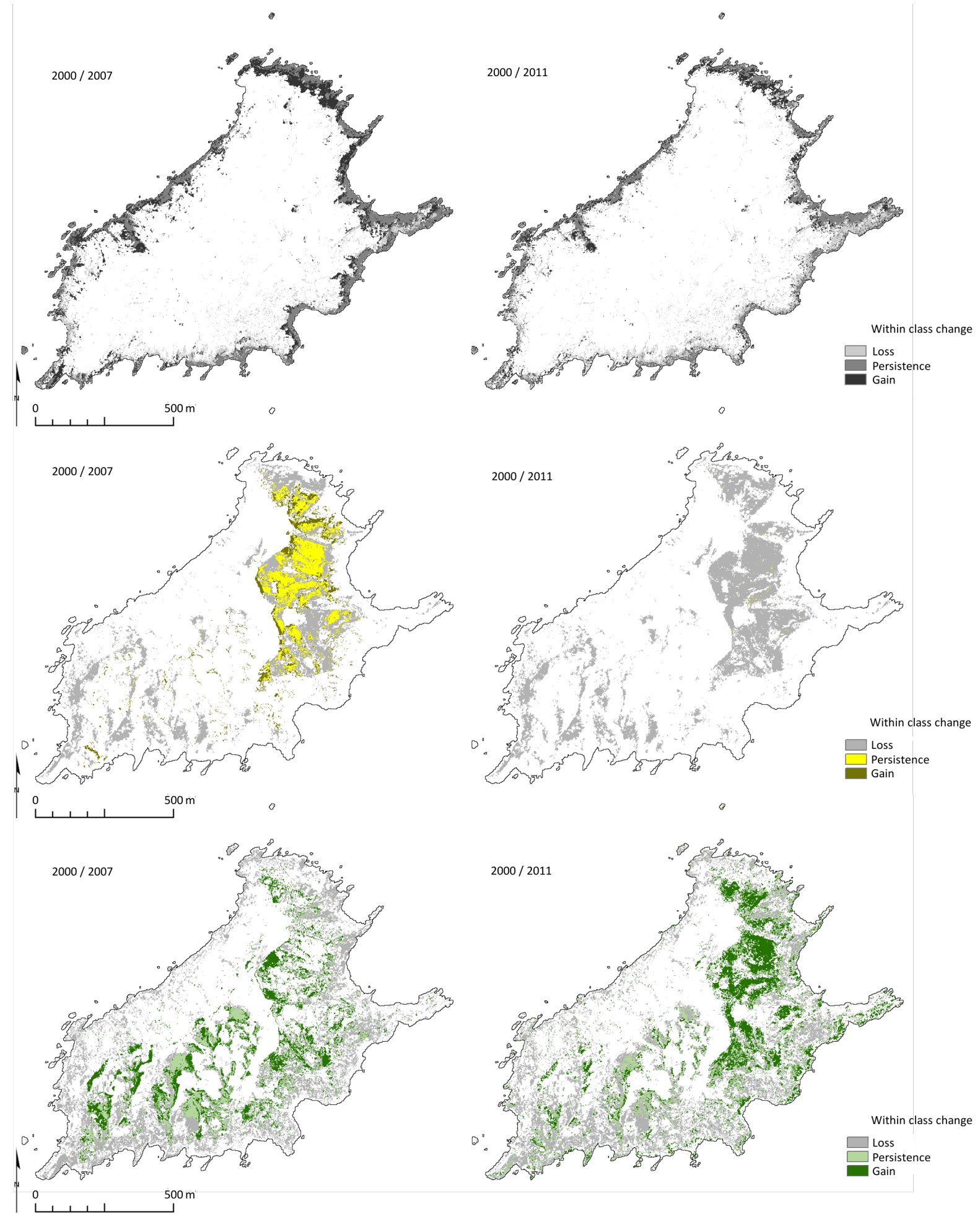



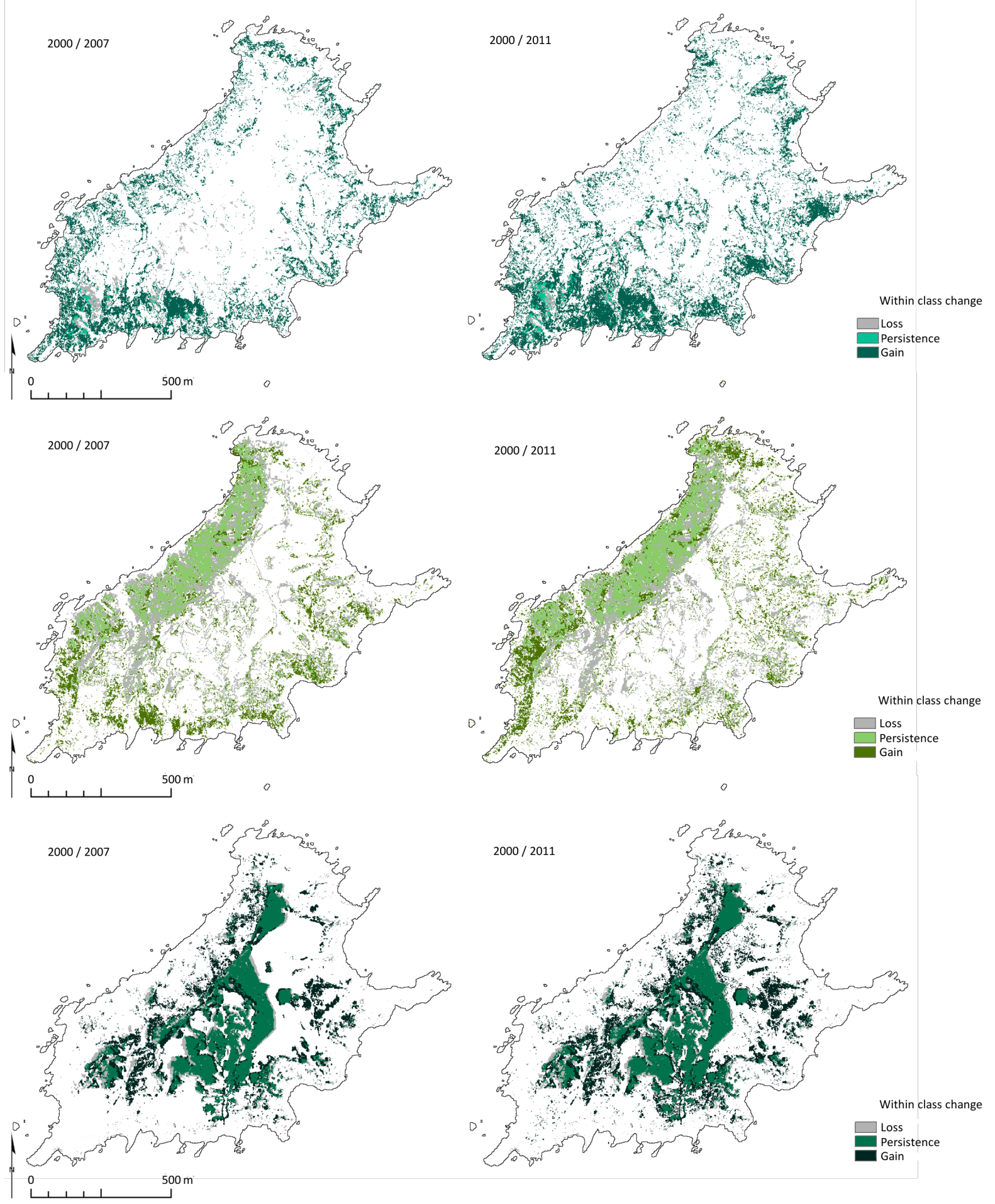

Figure 2.3.4. Spatial distributions of gains, losses, and persistence for individual land cover types on Takapourewa from 2000/2007 and 2000/2011 


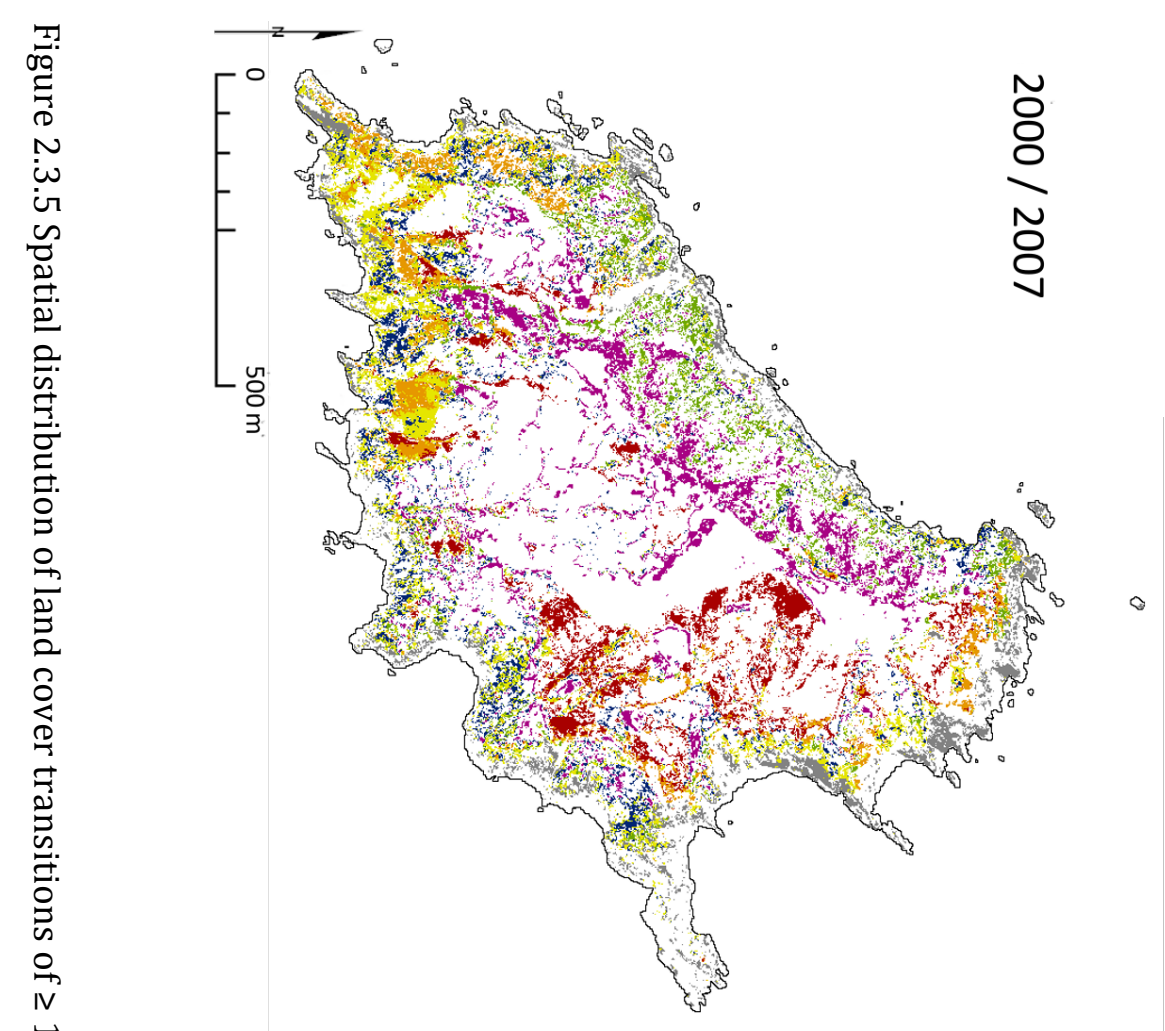

5
0
0
8
0
0
$\times$
0
0
0
0
0
0
0
0
0
0
0
0
0
0
0
0
0
0
0
0
0
0
0
0
0
0
0
0
0
0
5
0
0
0
0
0
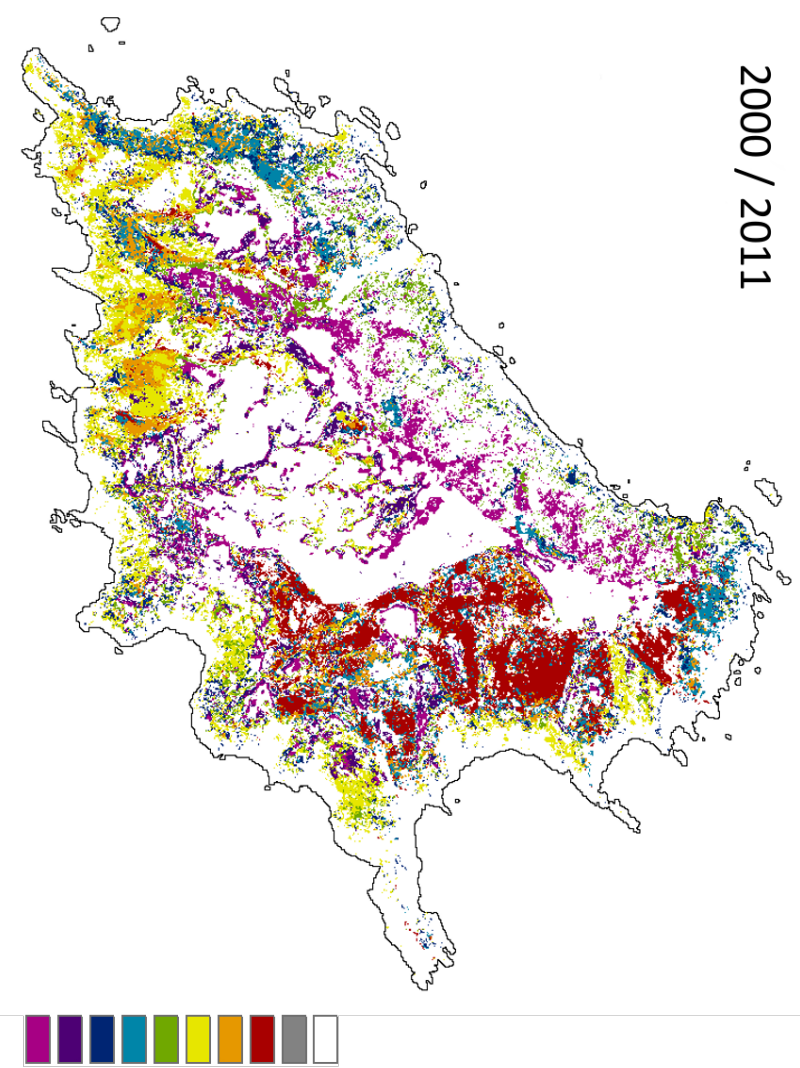

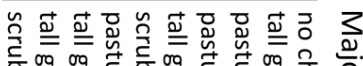

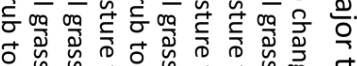

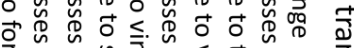

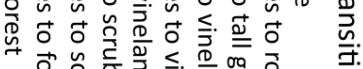

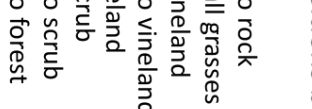


Table 2.3.1. Predictive abilities of individual land cover transition models. The Peirce skill score (PSS) is the difference between the mean transition potentials of pixels in the validation set correctly predicted as 'change' (true change) and the mean transition potentials of 'persistence' pixels incorrectly predicted as 'change' (false change). Models with higher PSS values were better able to differentiate between change and persistence using the included explanatory variables.

\begin{tabular}{|c|c|c|c|c|c|c|}
\hline \multirow[b]{2}{*}{ Transition } & \multicolumn{3}{|c|}{$2000 / 2007$} & \multicolumn{3}{|c|}{$2000 / 2011$} \\
\hline & true change & false change & PSS & true change & false change & PSS \\
\hline$P \rightarrow G$ & 0.62 & 0.36 & 0.26 & 0.48 & 0.32 & 0.16 \\
\hline$P \rightarrow V$ & 0.79 & 0.17 & 0.62 & 0.77 & 0.21 & 0.56 \\
\hline$P \rightarrow S$ & NA & NA & NA & 0.52 & 0.28 & 0.24 \\
\hline$G \rightarrow R$ & 0.88 & 0.13 & 0.75 & NA & NA & NA \\
\hline$G \rightarrow V$ & 0.72 & 0.26 & 0.45 & 0.63 & 0.39 & 0.23 \\
\hline$G \rightarrow S$ & 0.69 & 0.30 & 0.40 & 0.66 & 0.30 & 0.36 \\
\hline$G \rightarrow F$ & NA & NA & NA & 0.62 & 0.31 & 0.31 \\
\hline$S \rightarrow V$ & 0.58 & 0.43 & 0.15 & 0.69 & 0.32 & 0.37 \\
\hline$S \rightarrow F$ & 0.63 & 0.35 & 0.28 & 0.67 & 0.30 & 0.37 \\
\hline
\end{tabular}




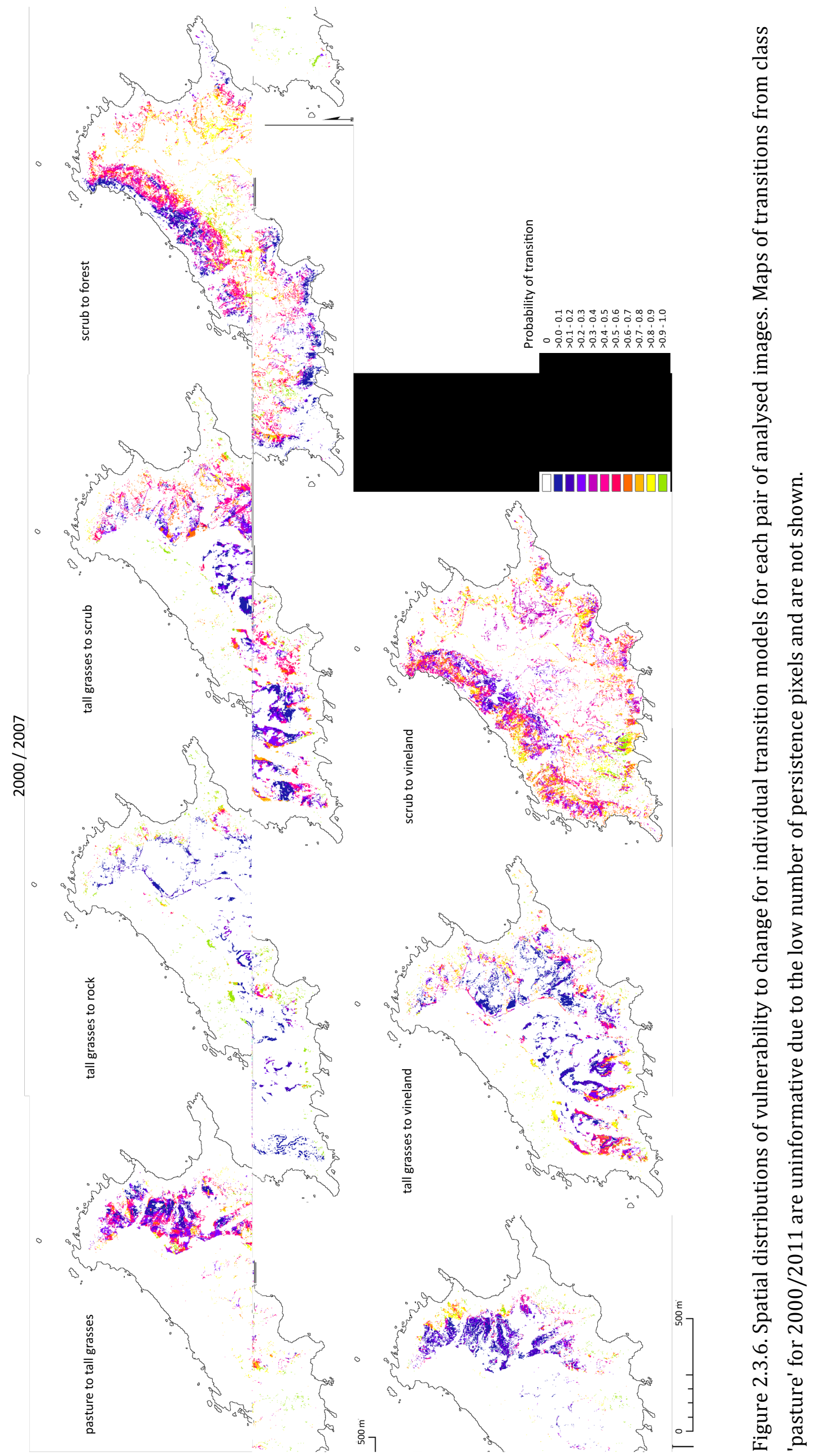




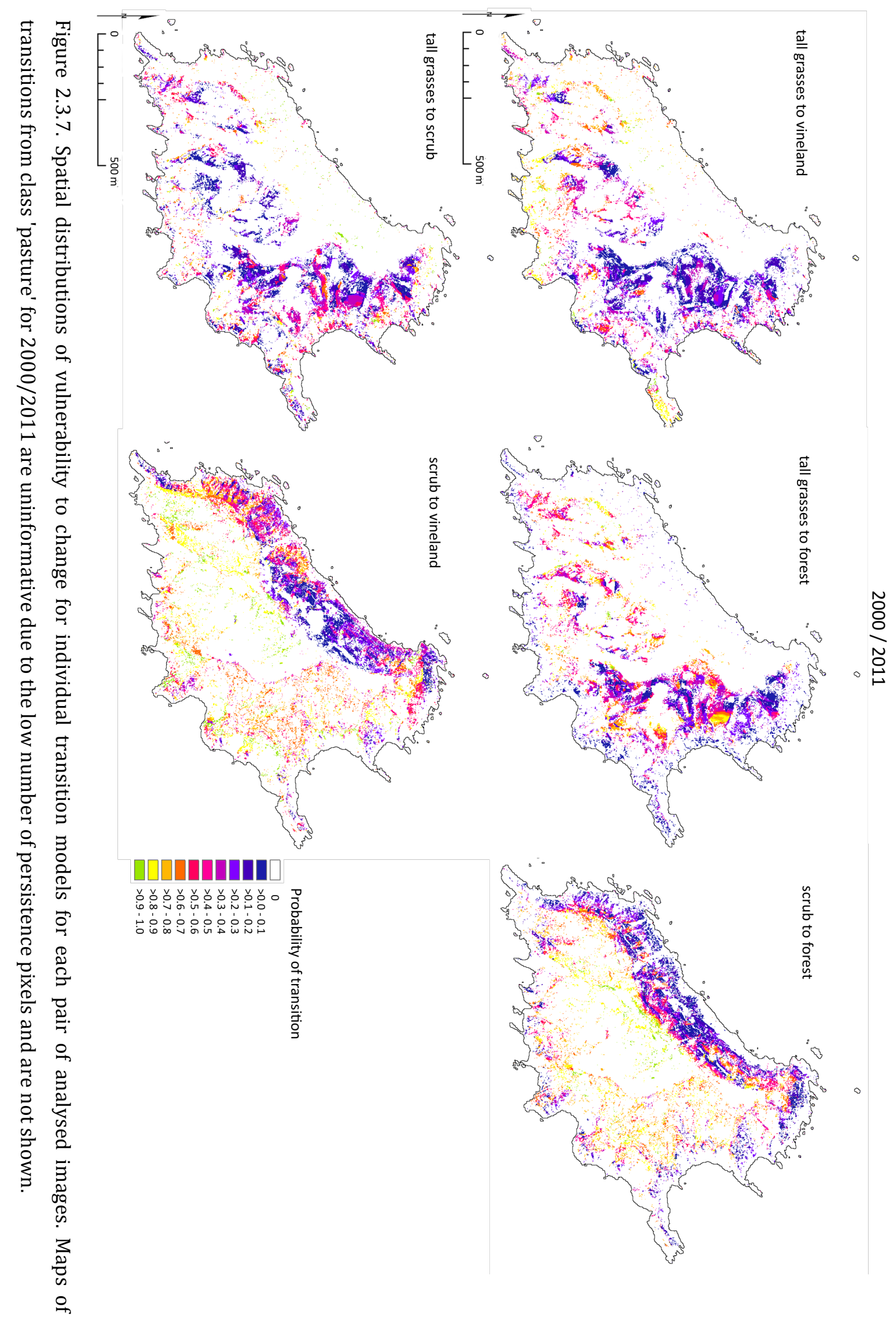




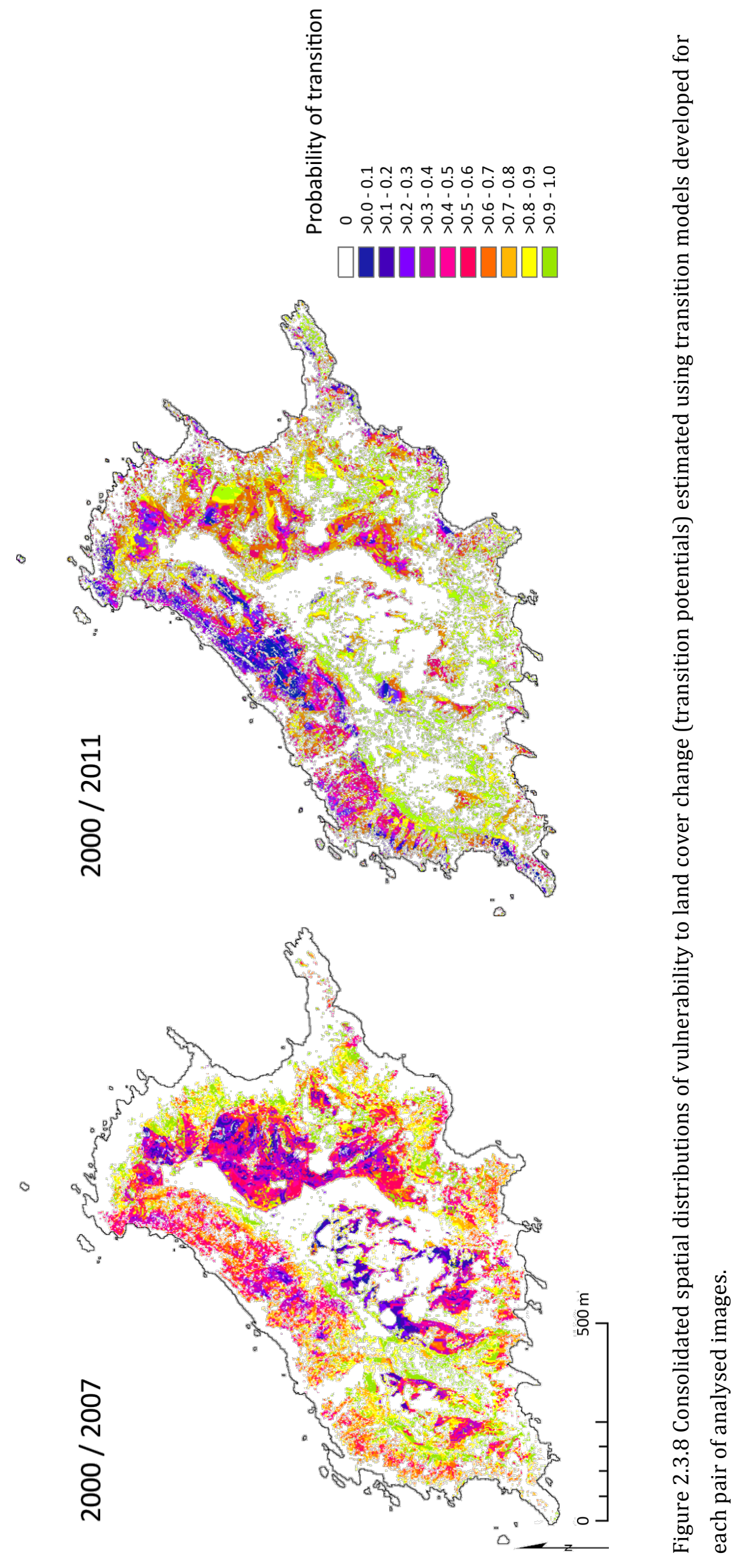


a.

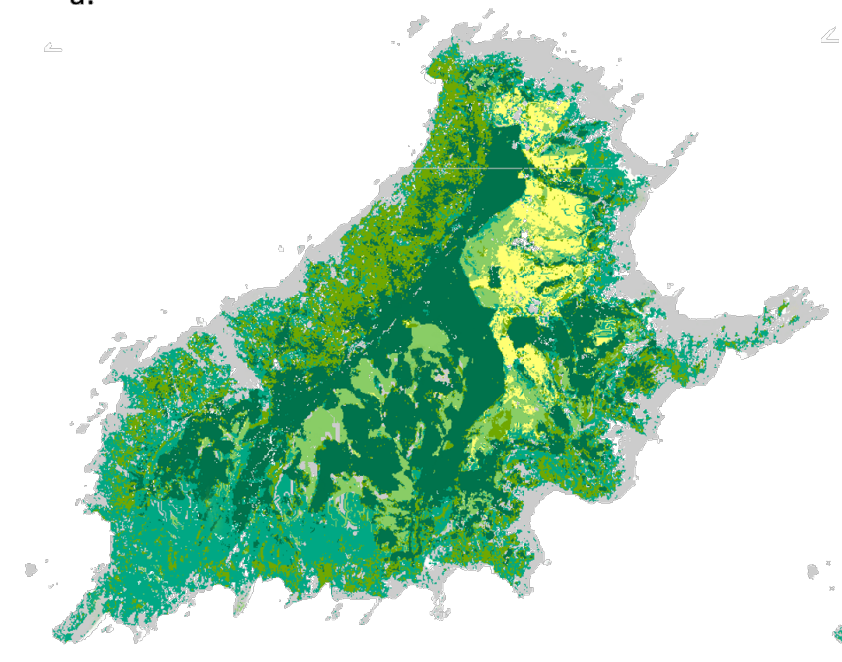

c.

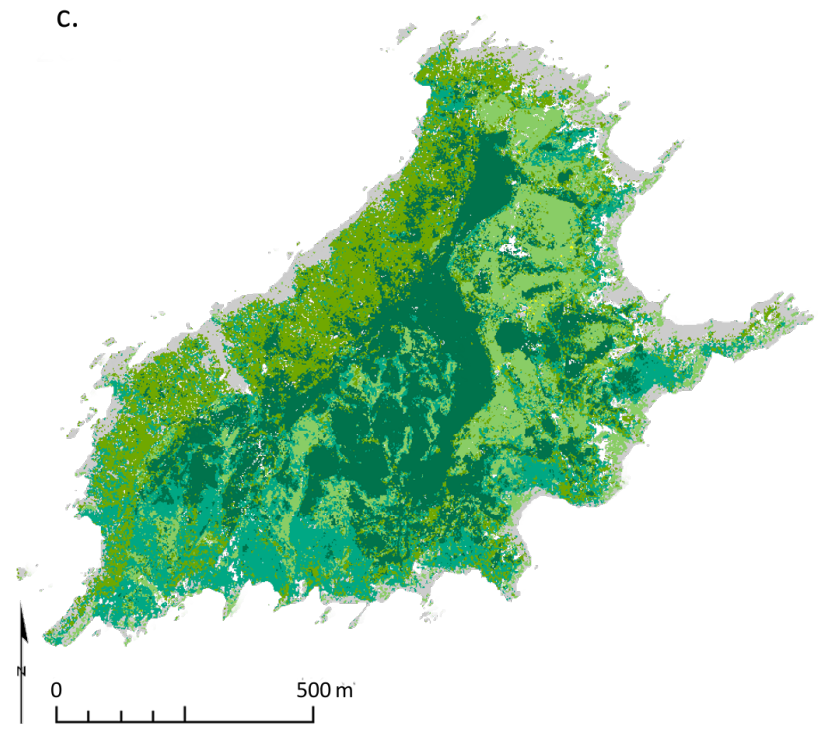

b.

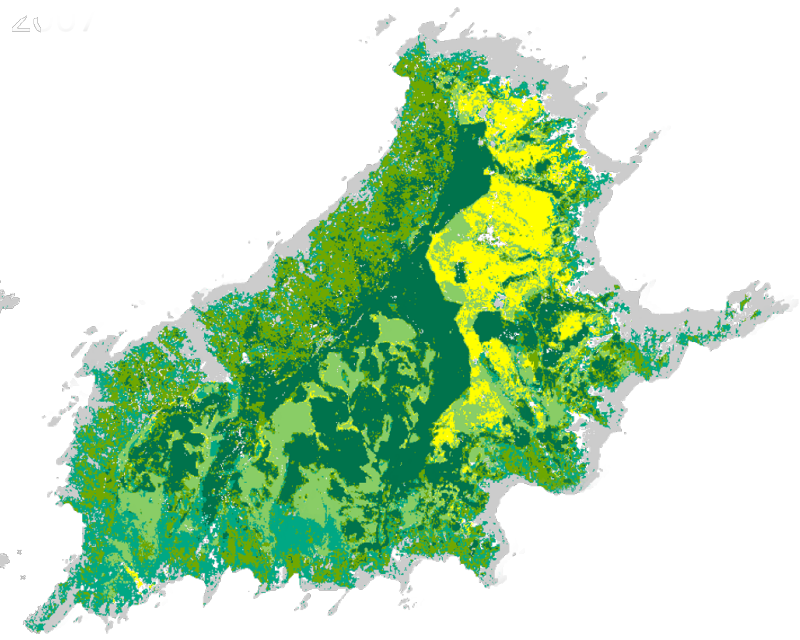

Land cover classification

$\operatorname{rock}(\mathrm{R})$

pasture $(\mathrm{P})$

tall grasses $(\mathrm{G})$

vineland $(\mathrm{V})$

scrub (S)

forest (F)

Figure 2.3.9. Spatial distribution of 'hard' land cover class predictions for 2011 used to assess accuracy of transition models for Takapourewa Predictions are for a) 2011 using transition models for $2000 / 2007$, b) the first $10 \%$ of change from 2007 to 2017 (year 1 of 10 years) using transition models for 2000/2007, and c) the first 2\% of change from 2011 to 2050 (year 1 of 50 years) using transition models for 2000/2011. 

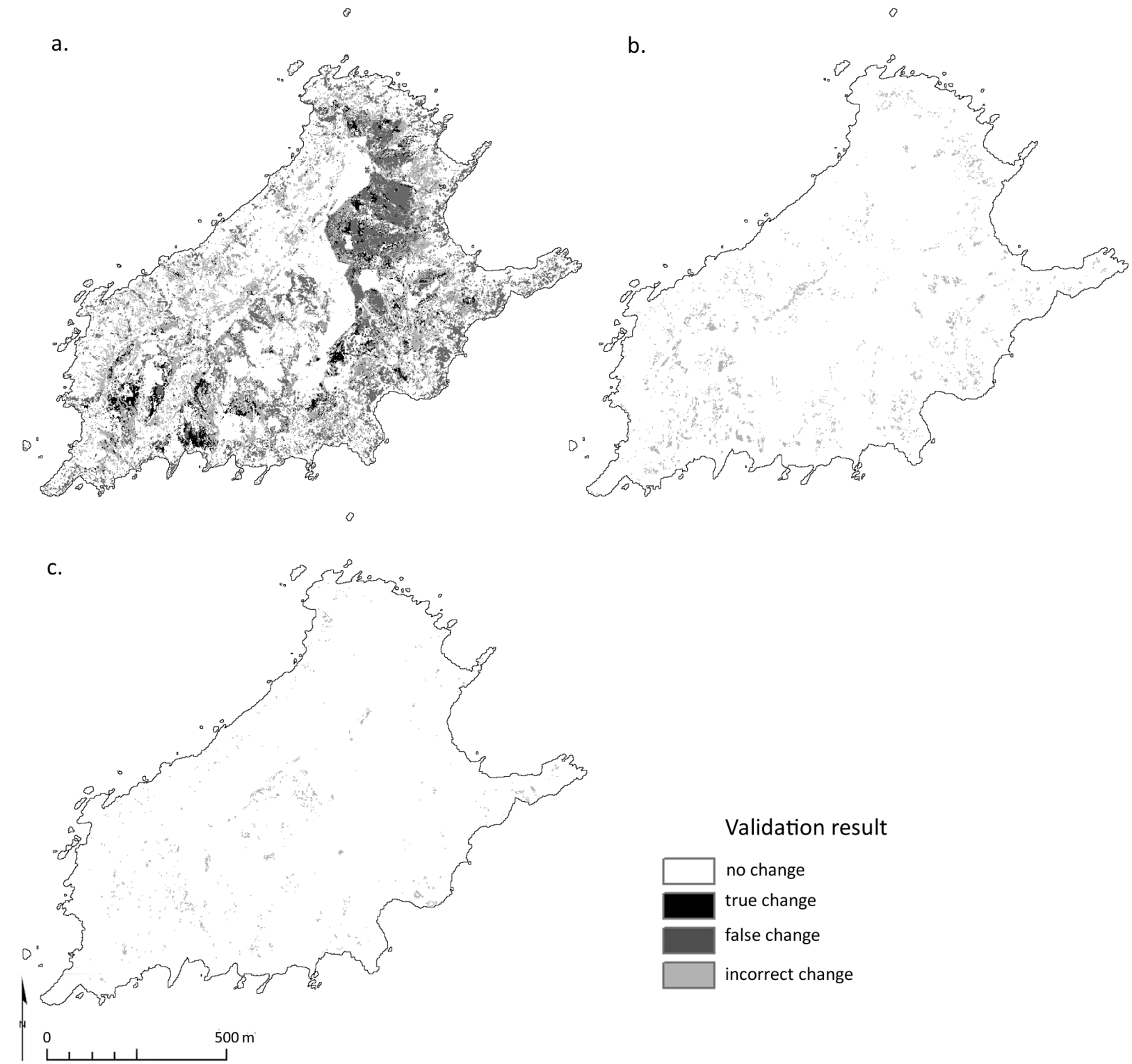

Figure 2.3.10. Cross-tabulated distributions of correctly and incorrectly predicted change for (a) 2011 using transition models for 2000/2007, (b) the first 10\% of change from 2007 to 2017 (year 1 of 10 years) using transition models for 2000/2007, and (c) the first $2 \%$ of change from 2011 to 2051 (year 1 of 50 years) using transition models for 2000/2011. 


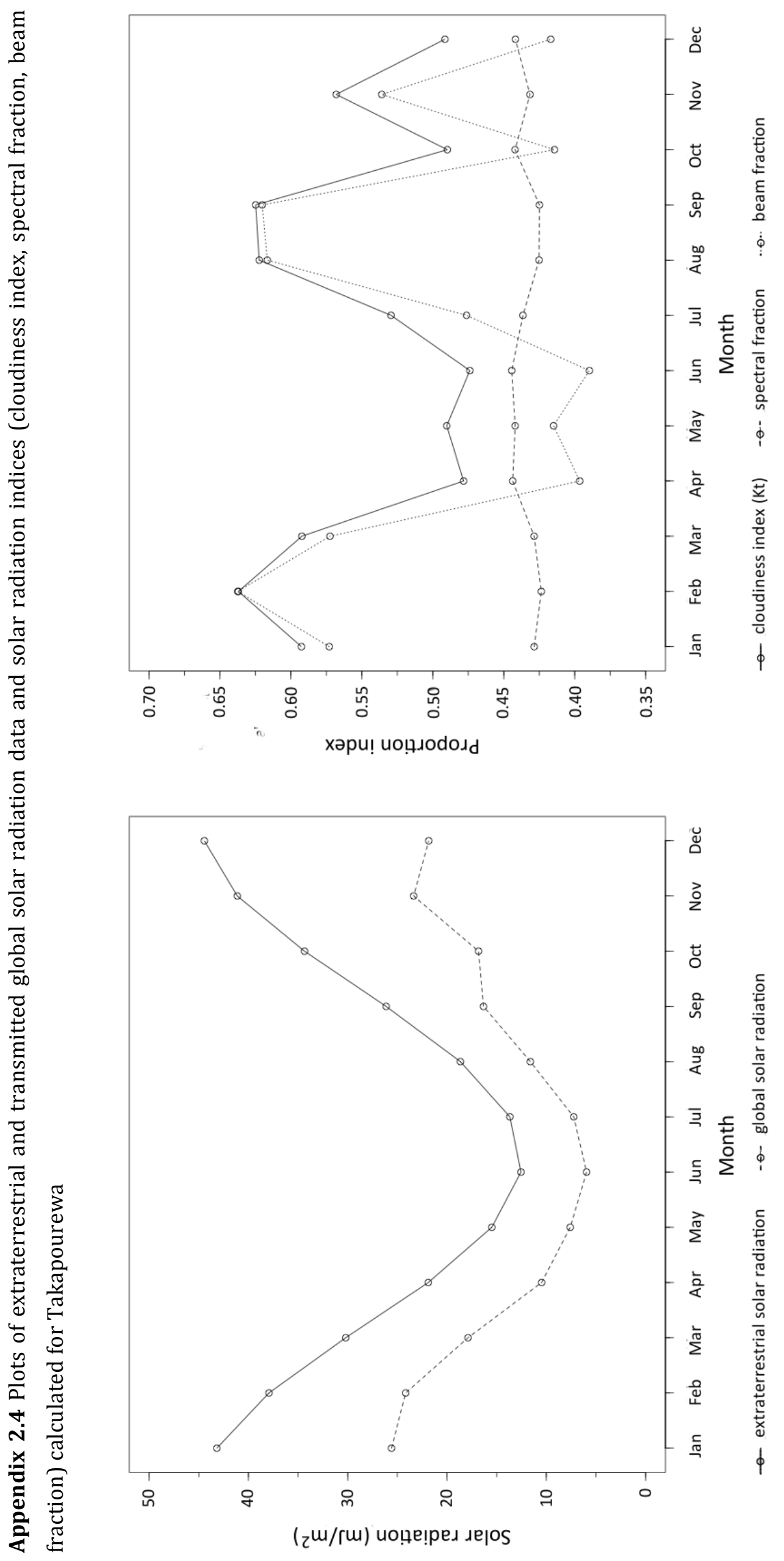


Appendix 2.5 Comparisons among 91 candidate multinomial models that examined the effects of years, initial rookery choice, and interpolated proportions of land cover types on subsequent rookery choice, ordered by increasing complexity. Variable codes are first and second rookery choices (rook1, rook2); first and second segment years (year1, year2); a binary indicator of same vs. different choice between years (stay); number of years between year1 and year2 (interval); the proportion of each land cover type $\mathrm{X}$ in the first rookery in the first year (r1y1.X); the proportion of each land cover type $\mathrm{X}$ in the first rookery in the second year (r1y2.X); and the proportion of each land cover type X in the second rookery in the second year (r2y2.X).

\begin{tabular}{|c|c|c|c|c|c|c|}
\hline \multicolumn{7}{|c|}{ Null model: rook2 } \\
\hline 0 & 1 & 1847.84 & 1865.87 & 1665.16 & 0 & 0 \\
\hline \multicolumn{7}{|c|}{ Single-predictor models: rook2 } \\
\hline 1 & rook1 & 568.78 & 714.22 & 513.51 & $1.05 e^{-112}$ & $3.11 e^{-112}$ \\
\hline 2 & year1 & 1799.35 & 1835.44 & 1634.73 & 0 & 0 \\
\hline 3 & year2 & 1768.37 & 1804.46 & 1603.75 & 0 & 0 \\
\hline 4 & stay & 1310.47 & 1346.56 & 1145.85 & $5.12 e^{-250}$ & $1.52 e^{-249}$ \\
\hline 5 & interval & 1769.72 & 1805.82 & 1605.11 & 0 & 0 \\
\hline 6 & r1y1.R & 1386.63 & 1422.73 & 1222.02 & $1.48 e^{-266}$ & $4.38 \mathrm{e}^{-266}$ \\
\hline 7 & r1y1.P & 1648.35 & 1684.44 & 1483.73 & $1.98 e^{-323}$ & $6.42 e^{-323}$ \\
\hline 8 & r1y1.G & 1546.19 & 1582.28 & 1381.57 & $3.33 e^{-301}$ & $9.91 e^{-301}$ \\
\hline 9 & r1y1.v & 1202.28 & 1238.37 & 1037.66 & $1.59 e^{-226}$ & $4.73 e^{-226}$ \\
\hline 10 & r1y1.s & 1566.34 & 1602.43 & 1401.72 & $1.40 \mathrm{e}^{-305}$ & $4.17 e^{-305}$ \\
\hline 11 & r1y1.F & 1833.52 & 1869.61 & 1668.90 & 0 & 0 \\
\hline 12 & r1y2.R & 1606.78 & 1642.87 & 1442.16 & $2.32 \mathrm{e}^{-314}$ & $6.90 \mathrm{e}^{-314}$ \\
\hline 13 & r1y2.P & 1622.52 & 1658.61 & 1457.90 & $8.87 e^{-318}$ & $2.63 e^{-317}$ \\
\hline 14 & r1y2.G & 1694.61 & 1730.70 & 1529.99 & 0 & 0 \\
\hline 15 & r1y2.V & 1407.07 & 1443.16 & 1242.45 & $5.40 e^{-271}$ & $1.60 e^{-270}$ \\
\hline 16 & r1y2.s & 1632.49 & 1668.58 & 1467.87 & $6.06 e^{-320}$ & $1.80 e^{-319}$ \\
\hline 17 & r1y2.F & 1655.01 & 1691.10 & 1490.39 & 0 & 0 \\
\hline 18 & $\mathrm{r} 2 \mathrm{y} 2 . \mathrm{R}$ & 1350.28 & 1386.39 & 1185.68 & $1.15 e^{-258}$ & $3.41 \mathrm{e}^{-258}$ \\
\hline 19 & r2y2.P & 1770.96 & 1807.05 & 1606.34 & 0 & 0 \\
\hline 20 & r2y2.G & 1592.23 & 1628.32 & 1427.61 & $3.35 \mathrm{e}^{-311}$ & $9.96 e^{-311}$ \\
\hline 21 & r2y2.V & 670.90 & 707.00 & 506.29 & $3.87 e^{-111}$ & $1.15 \mathrm{e}^{-110}$ \\
\hline 22 & $\mathrm{r} 2 \mathrm{y} 2 . \mathrm{s}$ & 1632.49 & 1668.58 & 1467.87 & $6.07 e^{-320}$ & $1.80 \mathrm{e}^{-319}$ \\
\hline 23 & $\mathrm{r} 2 \mathrm{y} 2 . \mathrm{F}$ & 1655.01 & 1691.10 & 1490.39 & 0 & 0 \\
\hline \multicolumn{7}{|c|}{ Two-predictor models: rook2 rook1 + } \\
\hline 24 & year1 & 483.57 & 647.39 & 446.68 & $3.40 e^{-98}$ & $1.01 \mathrm{e}^{-97}$ \\
\hline 25 & year2 & 464.49 & 628.31 & 427.60 & $4.73 e^{-94}$ & $1.41 \mathrm{e}^{-93}$ \\
\hline
\end{tabular}




$\begin{array}{rrrrrll}26 & \text { stay } & 417.52 & 581.33 & 380.62 & 7.52 \mathrm{e}^{-84} & 2.24 \mathrm{e}^{-83} \\ 27 & \text { interval } & 489.41 & 653.23 & 452.52 & 1.83 \mathrm{e}^{-99} & 5.45 \mathrm{e}^{-99} \\ 28 & \text { r1y1.R } & 491.58 & 655.40 & 454.69 & 6.20 \mathrm{e}^{-100} & 1.84 \mathrm{e}^{-99} \\ 29 & \text { r1y1.P } & 496.67 & 660.48 & 459.77 & 4.89 \mathrm{e}^{-101} & 1.45 \mathrm{e}^{-100} \\ 30 & \text { r1y1.G } & 518.96 & 682.78 & 482.07 & 7.03 \mathrm{e}^{-106} & 2.09 \mathrm{e}^{-105} \\ 31 & \text { r1y1.V } & 522.25 & 686.07 & 485.36 & 1.36 \mathrm{e}^{-106} & 4.03 \mathrm{e}^{-106} \\ 32 & \text { r1y1.S } & 516.56 & 680.37 & 479.66 & 2.35 \mathrm{e}^{-105} & 6.97 \mathrm{e}^{-105} \\ 33 & \text { r1y1.F } & 564.44 & 728.26 & 527.55 & 9.35 \mathrm{e}^{-116} & 2.78 \mathrm{e}^{-115} \\ 34 & \text { r1y2.R } & 454.90 & 618.72 & 418.01 & 5.72 \mathrm{e}^{-92} & 1.70 \mathrm{e}^{-91} \\ 35 & \text { r1y2.P } & 462.06 & 625.87 & 425.16 & 1.60 \mathrm{e}^{-93} & 4.76 \mathrm{e}^{-93} \\ 36 & \text { r1y2.G } & 466.62 & 630.43 & 429.72 & 1.64 \mathrm{e}^{-94} & 4.87 \mathrm{e}^{-94} \\ 37 & \text { r1y2.V } & 552.22 & 716.03 & 515.32 & 4.23 \mathrm{e}^{-113} & 1.26 \mathrm{e}^{-112} \\ 38 & \text { r1y2.S } & 486.48 & 650.29 & 449.58 & 7.98 \mathrm{e}^{-99} & 2.37 \mathrm{e}^{-98} \\ 39 & \text { r1y2.F } & 507.52 & 671.33 & 470.62 & 2.15 \mathrm{e}^{-103} & 6.40 \mathrm{e}^{-103} \\ 40 & \text { r2y2.R } & 366.58 & 530.39 & 329.68 & 8.67 \mathrm{e}^{-73} & 2.58 \mathrm{e}^{-72} \\ 41 & \text { r2y2.P } & 464.53 & 628.35 & 427.64 & 4.64 \mathrm{e}^{-94} & 1.38 \mathrm{e}^{-93} \\ 42 & \text { r2y2.G } & 346.64 & 510.46 & 309.75 & 1.84 \mathrm{e}^{-68} & 5.48 \mathrm{e}^{-68} \\ 43 & \text { r2y2.V } & 88.91 & 252.72 & 52.01 & 1.71 \mathrm{e}^{-12} & 5.08 \mathrm{e}^{-12} \\ 44 & \text { r2y2.S } & 450.96 & 614.78 & 414.07 & 4.10 \mathrm{e}^{-91} & 1.22 \mathrm{e}^{-90} \\ 45 & \text { r2y2.F } & 297.12 & 460.94 & 260.23 & 1.04 \mathrm{e}^{-57} & 3.10 \mathrm{e}^{-57}\end{array}$

Three-predictor models: rook2 rook1 + r2y2.V +

\begin{tabular}{|c|c|c|c|c|c|c|}
\hline 46 & year1 & 89.43 & 271.67 & 70.96 & $1.31 \mathrm{e}^{-16}$ & $3.90 \mathrm{e}^{-16}$ \\
\hline 47 & year2 & 89.63 & 271.88 & 71.17 & $1.18 \mathrm{e}^{-16}$ & $3.51 e^{-16}$ \\
\hline 48 & stay & 80.27 & 262.52 & 61.81 & $1.27 \mathrm{e}^{-14}$ & $3.79 \mathrm{e}^{-14}$ \\
\hline 49 & interval & 69.70 & 251.95 & 51.24 & $2.51 \mathrm{e}^{-12}$ & $7.47 e^{-12}$ \\
\hline 50 & r1y1.R & 85.35 & 267.59 & 66.88 & $1.01 \mathrm{e}^{-15}$ & $3.00 e^{-15}$ \\
\hline 51 & r1y1.P & 70.61 & 252.85 & 52.14 & $1.60 \mathrm{e}^{-12}$ & $4.76 \mathrm{e}^{-12}$ \\
\hline 52 & r1y1.G & 89.57 & 271.81 & 71.10 & $1.22 \mathrm{e}^{-16}$ & $3.64 \mathrm{e}^{-16}$ \\
\hline 53 & r1y1.v & 79.60 & 261.84 & 61.13 & $1.79 \mathrm{e}^{-14}$ & $5.32 e^{-14}$ \\
\hline 54 & r1y1.s & 77.05 & 259.29 & 58.58 & $6.41 \mathrm{e}^{-14}$ & $1.90 \mathrm{e}^{-13}$ \\
\hline 55 & r1y1.F & 89.10 & 271.34 & 70.63 & $1.55 \mathrm{e}^{-16}$ & $4.60 \mathrm{e}^{-16}$ \\
\hline 56 & r1y2.R & 84.93 & 267.17 & 66.46 & $1.25 \mathrm{e}^{-15}$ & $3.70 \mathrm{e}^{-15}$ \\
\hline 57 & r1y2.P & 63.79 & 246.03 & 45.32 & $4.85 e^{-11}$ & $1.44 \mathrm{e}^{-10}$ \\
\hline 58 & r1y2.G & 60.24 & 242.48 & 41.77 & $2.86 \mathrm{e}^{-10}$ & $8.51 \mathrm{e}^{-10}$ \\
\hline 59 & r1y2.v & 81.12 & 263.36 & 62.65 & $8.37 e^{-15}$ & $2.49 \mathrm{e}^{-14}$ \\
\hline 60 & r1y2.s & 59.24 & 241.48 & 40.77 & $4.72 \mathrm{e}^{-10}$ & $1.40 \mathrm{e}^{-09}$ \\
\hline 61 & r1y2.F & 52.21 & 234.46 & 33.75 & $1.58 \mathrm{e}^{-08}$ & $4.69 \mathrm{e}^{-08}$ \\
\hline 62 & r2y2.R & 75.63 & 257.87 & 57.16 & $1.30 \mathrm{e}^{-13}$ & $3.87 e^{-13}$ \\
\hline 63 & r2y2.P & 50.39 & 232.63 & 31.92 & $3.94 e^{-08}$ & $1.17 \mathrm{e}^{-07}$ \\
\hline 64 & r2y2.G & 29.53 & 211.77 & 11.06 & $1.33 \mathrm{e}^{-03}$ & $3.97 \mathrm{e}^{-03}$ \\
\hline 65 & r2y2.s & 48.84 & 231.08 & 30.37 & $8.56 \mathrm{e}^{-08}$ & $2.54 \mathrm{e}^{-07}$ \\
\hline 66 & $\mathrm{r} 2 \mathrm{y} 2 . \mathrm{F}$ & 71.97 & 254.21 & 53.50 & $8.12 \mathrm{e}^{-13}$ & $2.41 e^{-12}$ \\
\hline \multicolumn{7}{|c|}{ Four-predictor models: rook2 rook1 + r2y2.V+r2y2.G + } \\
\hline 67 & year1 & 28.83 & 229.55 & 28.84 & $1.84 \mathrm{e}^{-07}$ & $5.46 \mathrm{e}^{-07}$ \\
\hline 68 & year2 & 29.01 & 229.72 & 29.01 & $1.69 \mathrm{e}^{-07}$ & $5.02 e^{-07}$ \\
\hline 69 & stay & 12.06 & 212.77 & 12.06 & $8.10 \mathrm{e}^{-04}$ & $2.41 e^{-03}$ \\
\hline 70 & interval & 0.08 & 200.80 & 0.09 & $3.22 \mathrm{e}^{-01}$ & $9.56 \mathrm{e}^{-01}$ \\
\hline
\end{tabular}




$\begin{array}{llrllll}71 & \text { r1y1.R } & 49.80 & 250.51 & 49.80 & 5.17 \mathrm{e}^{-12} & 1.53 \mathrm{e}^{-11} \\ 72 & \text { r1y1.P } & 28.28 & 228.99 & 28.28 & 2.43 \mathrm{e}^{-07} & 7.23 \mathrm{e}^{-07} \\ 73 & \text { r1y1.G } & 41.40 & 242.11 & 41.40 & 3.44 \mathrm{e}^{-10} & 1.02 \mathrm{e}^{-09} \\ 74 & \text { r1y1.V } & 33.04 & 233.75 & 33.04 & 2.25 \mathrm{e}^{-08} & 6.69 \mathrm{e}^{-08} \\ 75 & \text { r1y1.S } & 28.22 & 228.94 & 28.23 & 2.49 \mathrm{e}^{-07} & 7.41 \mathrm{e}^{-07} \\ 76 & \text { r1y1.F } & 29.53 & 230.24 & 29.53 & 1.30 \mathrm{e}^{-07} & 3.87 \mathrm{e}^{-07} \\ 77 & \text { r1y2.R } & 47.91 & 248.62 & 47.91 & 1.33 \mathrm{e}^{-11} & 3.95 \mathrm{e}^{-11} \\ 78 & \text { r1y2.P } & 13.60 & 214.31 & 13.60 & 3.74 \mathrm{e}^{-04} & 1.11 \mathrm{e}^{-03} \\ 79 & \text { r1y2.G } & 35.24 & 235.95 & 35.24 & 7.50 \mathrm{e}^{-09} & 2.23 \mathrm{e}^{-08} \\ 80 & \text { r1y2.V } & 54.48 & 255.19 & 54.48 & 4.98 \mathrm{e}^{-13} & 1.48 \mathrm{e}^{-12} \\ 81 & \text { r1y2.S } & 26.12 & 226.84 & 26.13 & 7.13 \mathrm{e}^{-07} & 2.12 \mathrm{e}^{-06} \\ 82 & \text { r1y2.F } & 21.46 & 222.17 & 21.46 & 7.36 \mathrm{e}^{-06} & 2.19 \mathrm{e}^{-05} \\ 83 & \text { r2y2.R } & 23.04 & 223.76 & 23.05 & 3.33 \mathrm{e}^{-06} & 9.88 \mathrm{e}^{-06} \\ 84 & \text { r2y2.P } & 9.83 & 210.54 & 9.83 & 2.47 \mathrm{e}^{-03} & 7.34 \mathrm{e}^{-03} \\ 85 & \text { r2y2.S } & <0.01 & 200.71 & 0.00 & 3.37 \mathrm{e}^{-01} & 1.00 \\ 86 & \text { r2y2.F } & <0.01 & 200.71 & 0.00 & 3.37 \mathrm{e}^{-01} & 1.00\end{array}$

Five-predictor models: rook2 rook1 + r2y2.V + r2y2.G + r2y2.F +

$\begin{array}{rrrrrrr}87 & \text { interval } & <0.01 & 219.23 & 18.52 & 3.20 \mathrm{e}^{-05} & 9.52 \mathrm{e}^{-05} \\ 88 & \text { r1y2.P } & <0.01 & 219.23 & 18.52 & 3.20 \mathrm{e}^{-05} & 9.52 \mathrm{e}^{-05} \\ 89 & \text { r2y2.P } & 0.26 & 219.49 & 18.78 & 2.81 \mathrm{e}^{-05} & 8.36 \mathrm{e}^{-05} \\ 90 & \text { r2y2.S } & <0.01 & 219.23 & 18.52 & 3.20 \mathrm{e}^{-05} & 9.52 \mathrm{e}^{-05}\end{array}$




\section{Chapter 3}

Appendix 3.1 Line plots comparing mean monthly climate data obtained from the weather station on Takapourewa for the year 2011 and interpolated averages based on the 1961-1990 standard normal period downloaded from the CRU CL 2.0 global climate database (New et al. 2002). With the exception of cloud cover, all local climate data were input as daily values.
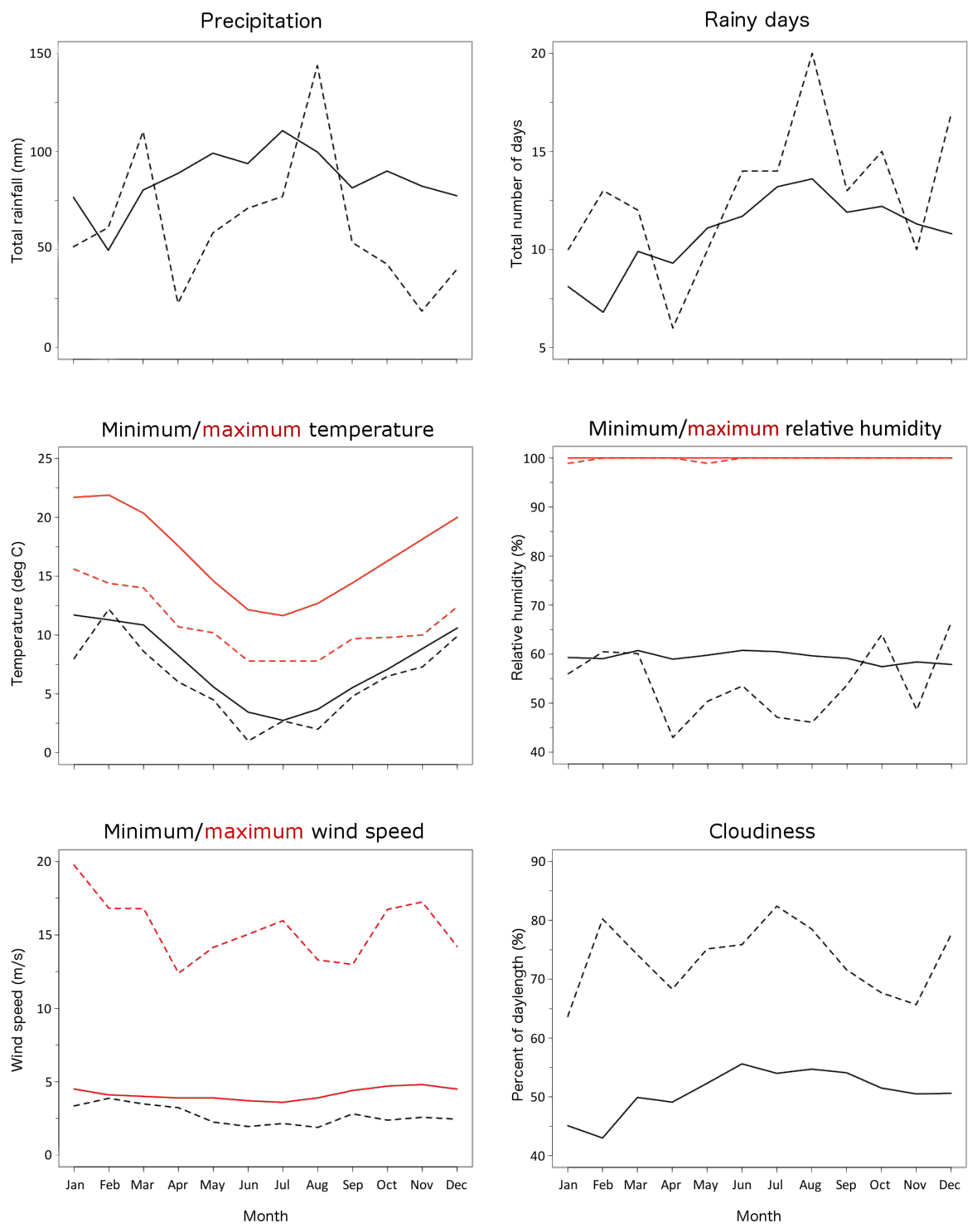

----- local weather station

_ global climate database 
Appendix 3.2 Geospatial parameters derived from a high-resolution gridded digital elevation map of Takapourewa
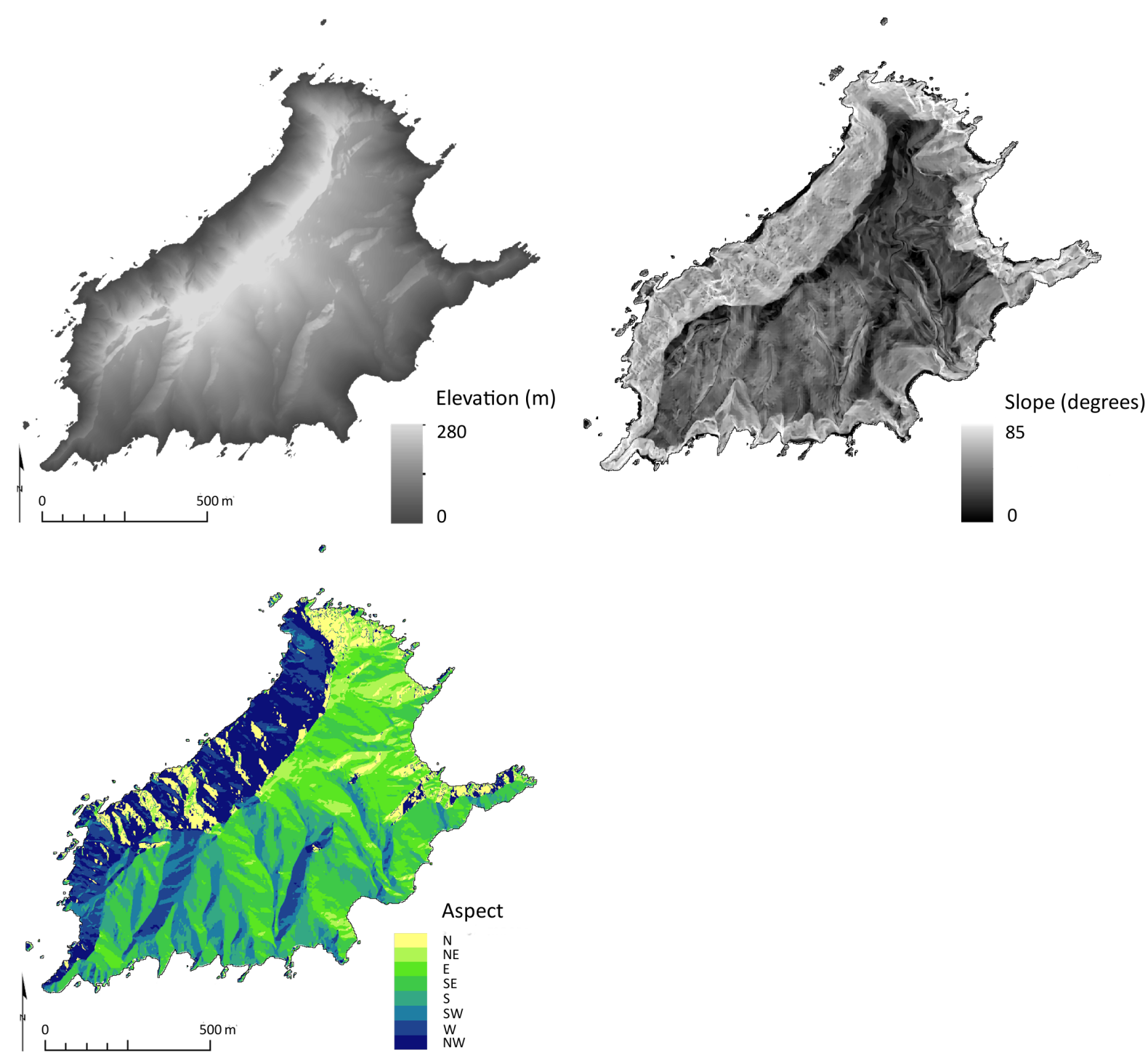
Appendix 3.3 Spatial distributions of experimentally-determined physical and thermal properties of the ten soil types and type-composites present on Takapourewa, superimposed on an aerial photograph of the island. Cliff areas surrounding the island were assigned mean values. Clay content was estimated as $20 \%$ for all sites. Volumetric water content at saturation was estimated as $0.26 \mathrm{~m}^{3} / \mathrm{m}^{3}$, and surface roughness was estimated as $0.004 \mathrm{~m}$ for all simulations (Kearney et al. 2014a). Labeled points approximate the sampling location within each soil polygon. The spelling of Takaporewa is maintained from Ward (1961).

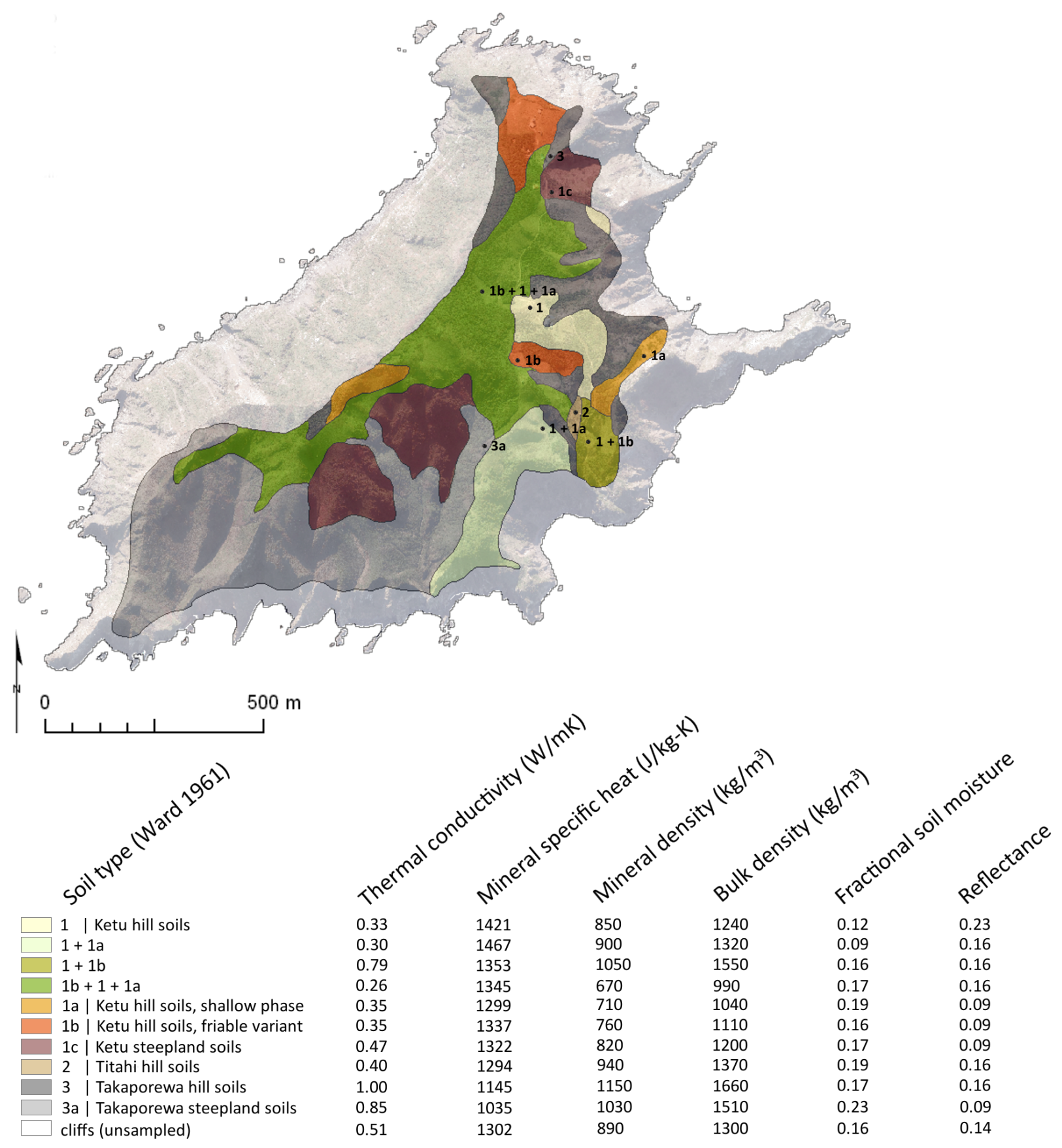


Appendix 3.4 Spatial distributions of maximum monthly wind speeds on Takapourewa, calculated at a $25 \mathrm{~m}$ horizontal resolution. Gridded wind speed data were input into the microclimate model as site-specific, daily maximum and minimum values. The location of the weather station is approximated by the black point.
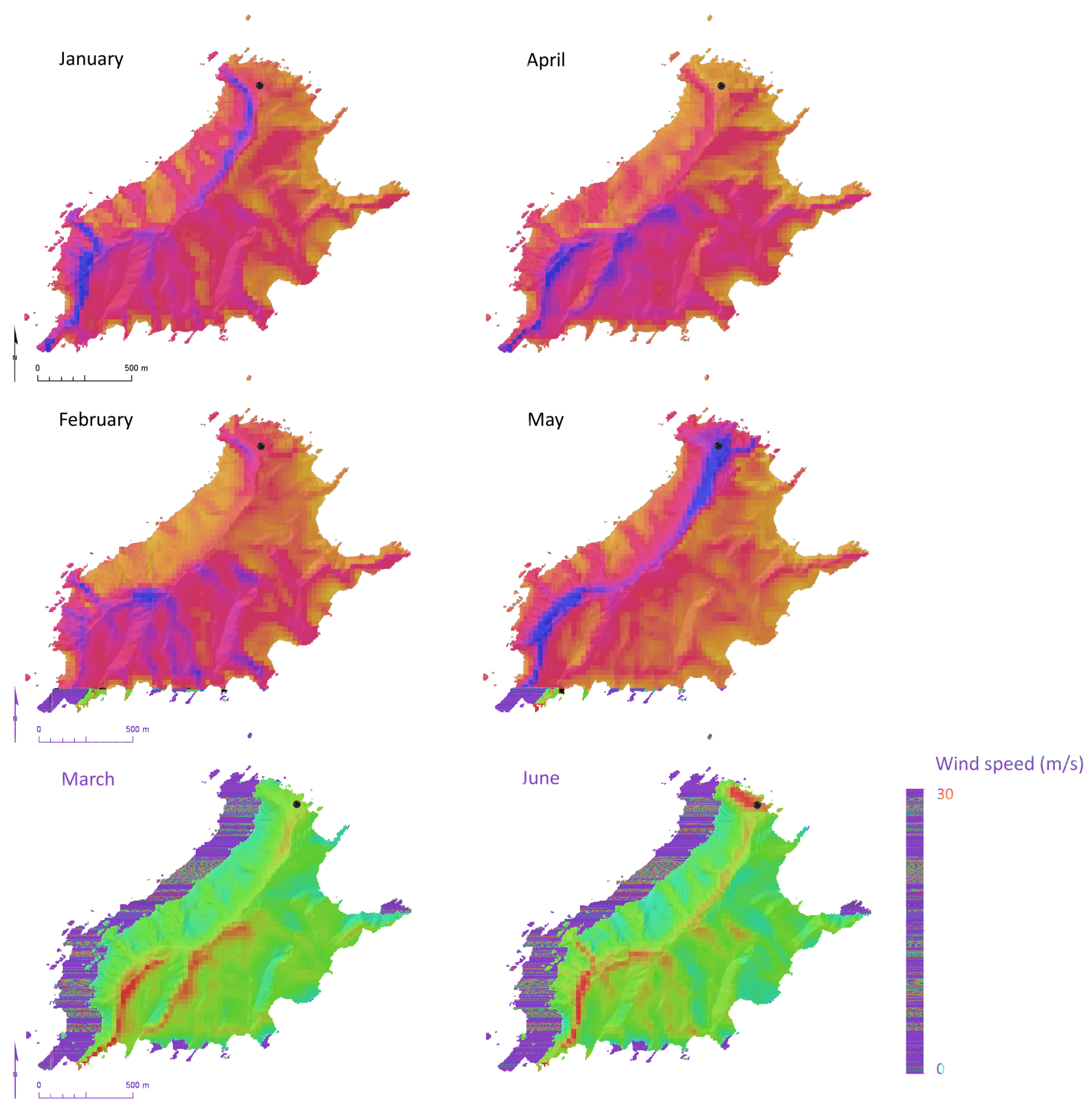

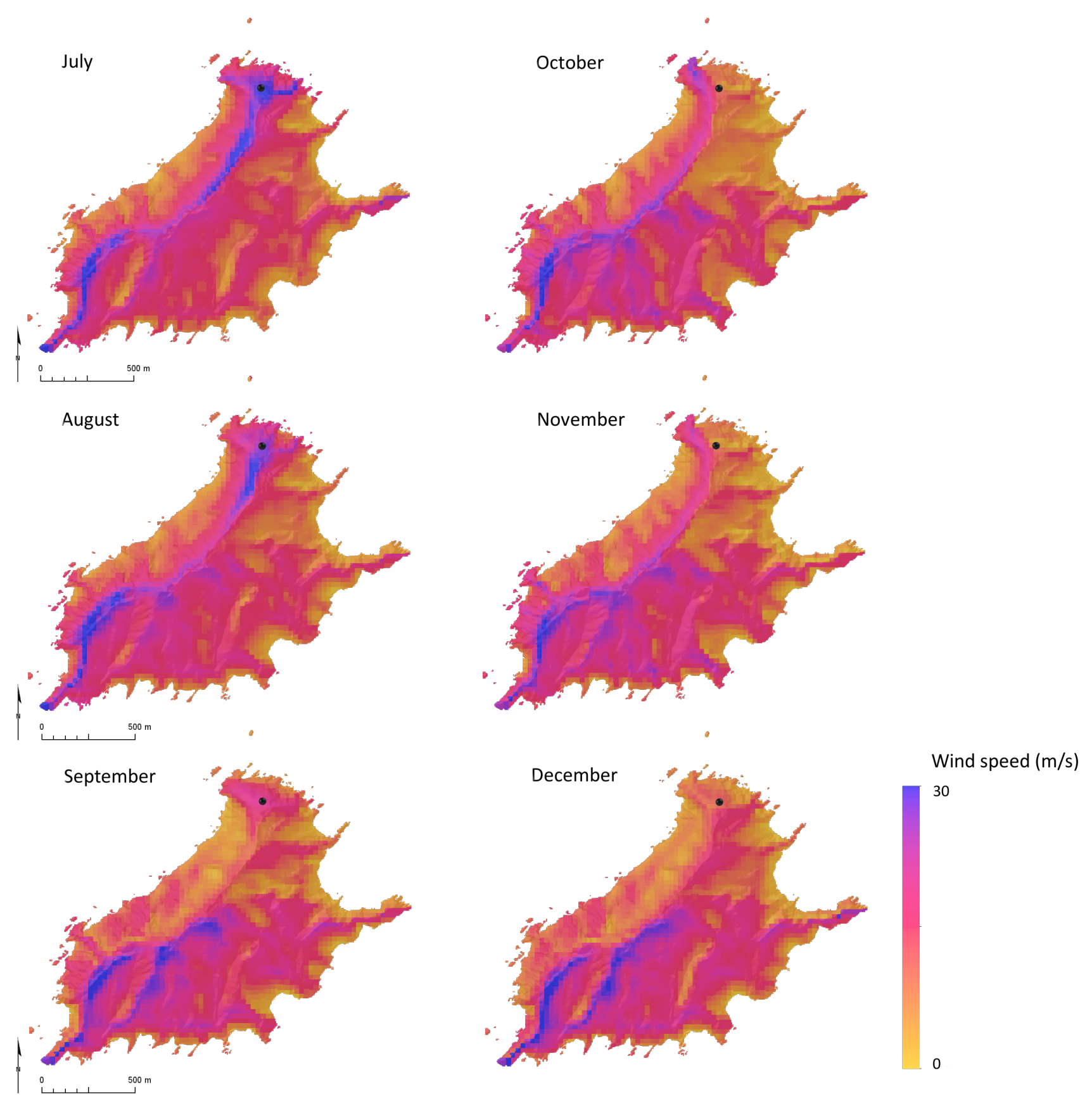
Appendix 3.5 Spatial distributions of minimum monthly wind speeds on Takapourewa, calculated at a $25 \mathrm{~m}$ horizontal resolution. Gridded wind speed data were input into the microclimate model as site-specific, daily maximum and minimum values. The location of the weather station is approximated by the black point.
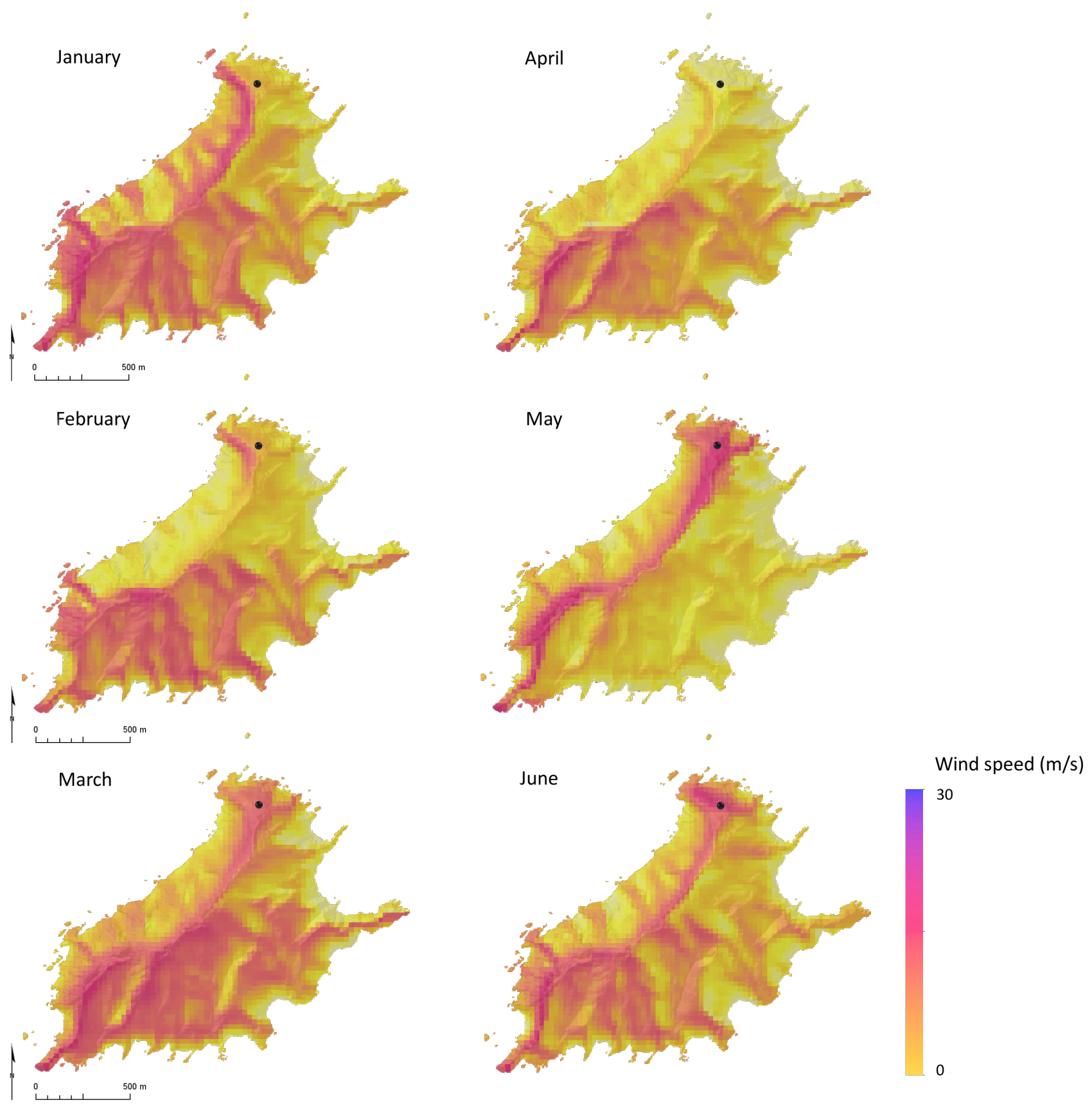

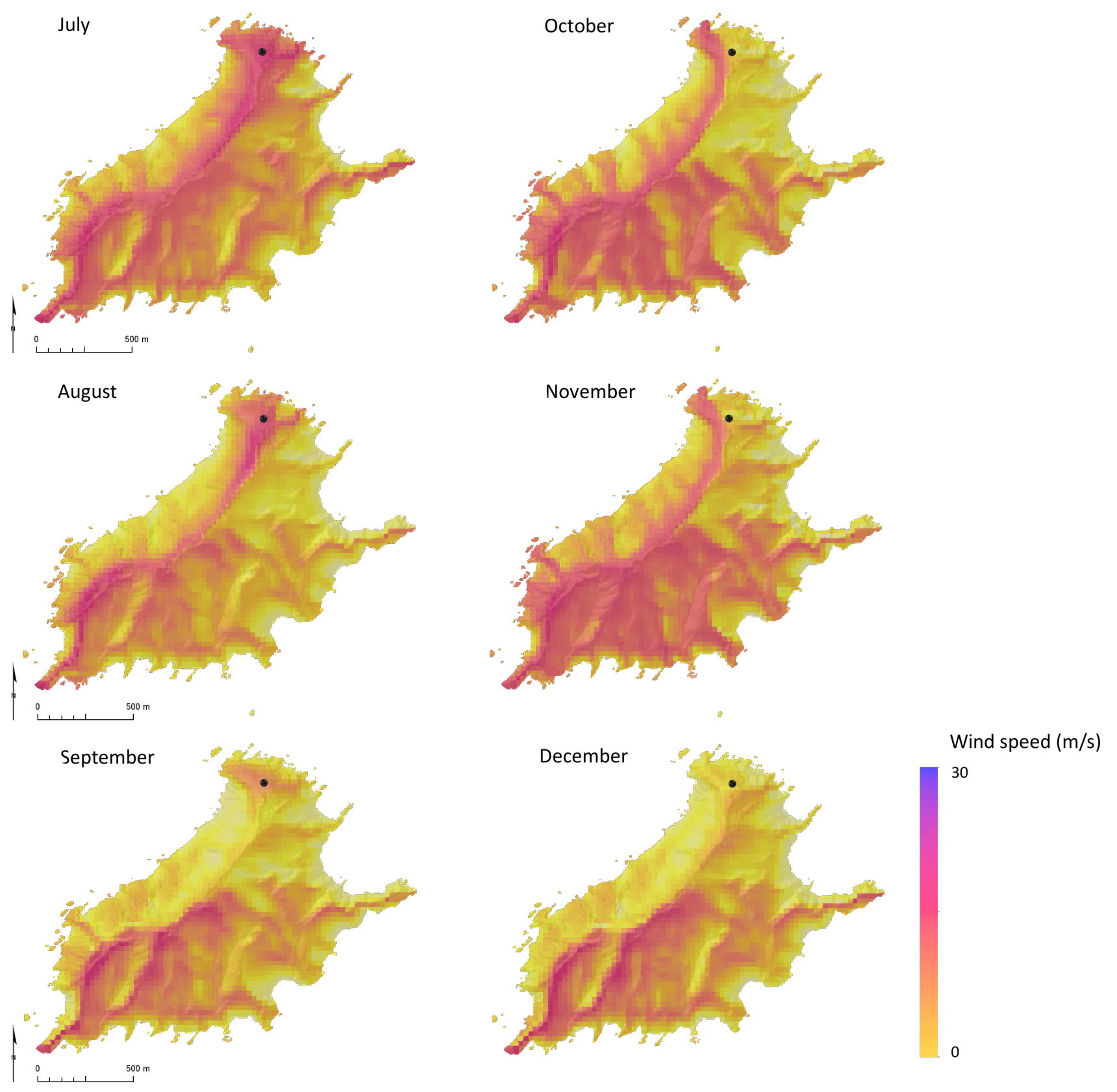


\section{Chapter 4}

Appendix 4.1 Standard error in island-wide constant incubation temperatures (CTEs) predicted for Takapourewa based on leave-one-out cross-validation. Confidence that the true CTE is within $+/$ - SE of interpolated values is $95.5 \%$.
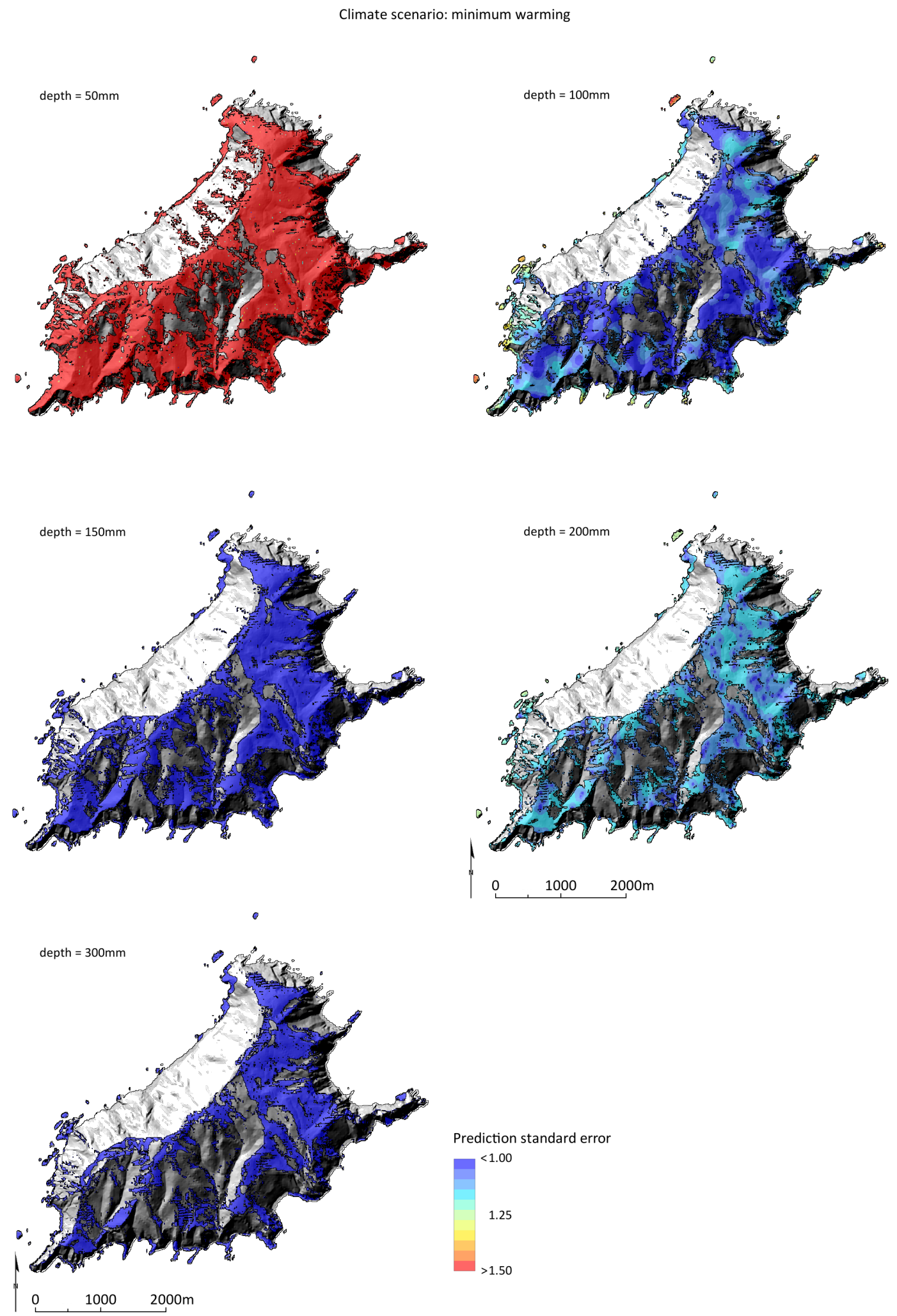

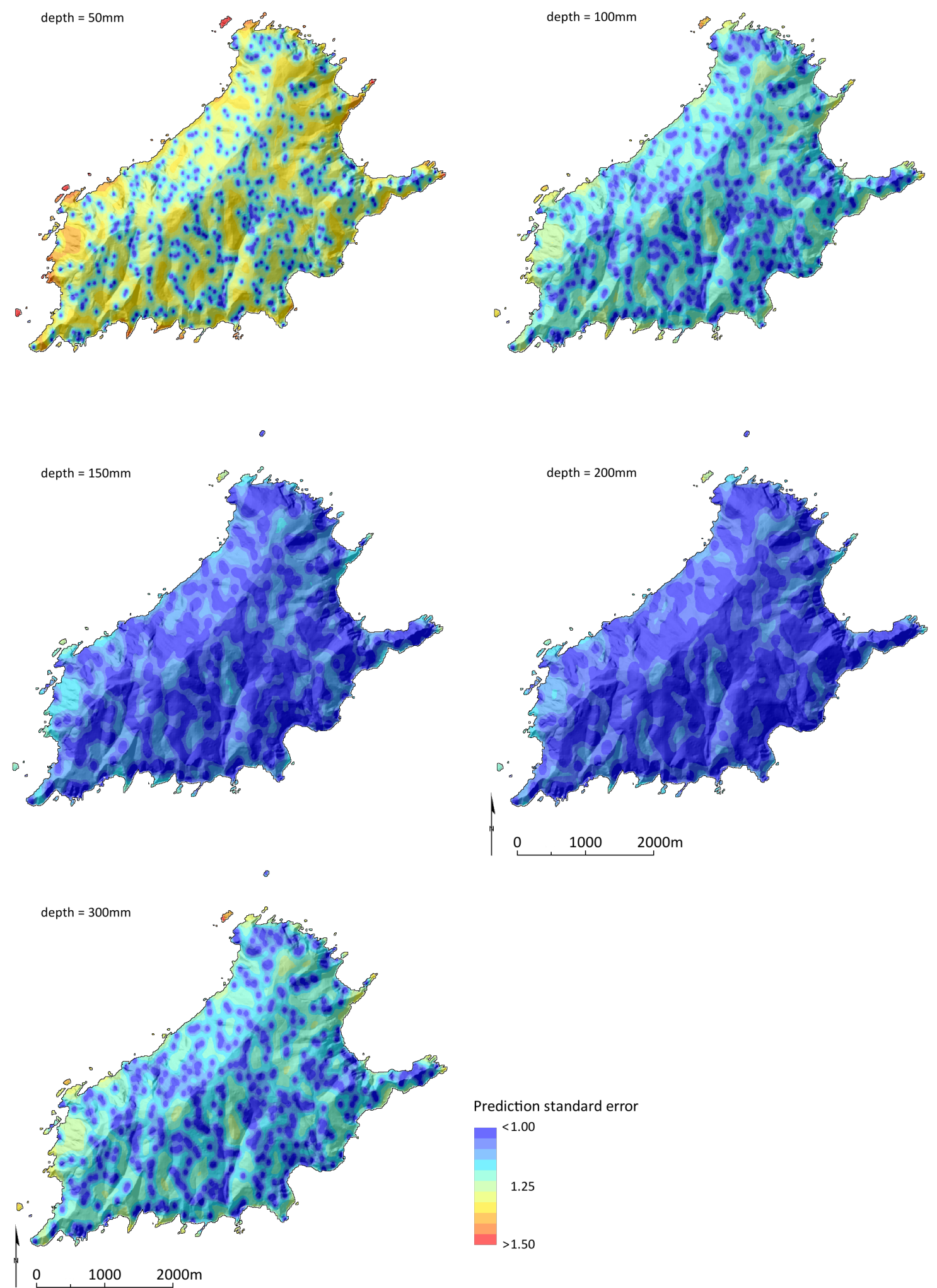

Prediction standard error

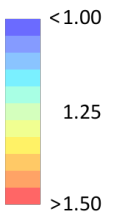




\section{Chapter 5}

Appendix 5.1 Geospatial parameters derived from a high-resolution gridded digital elevation map of Hauturu
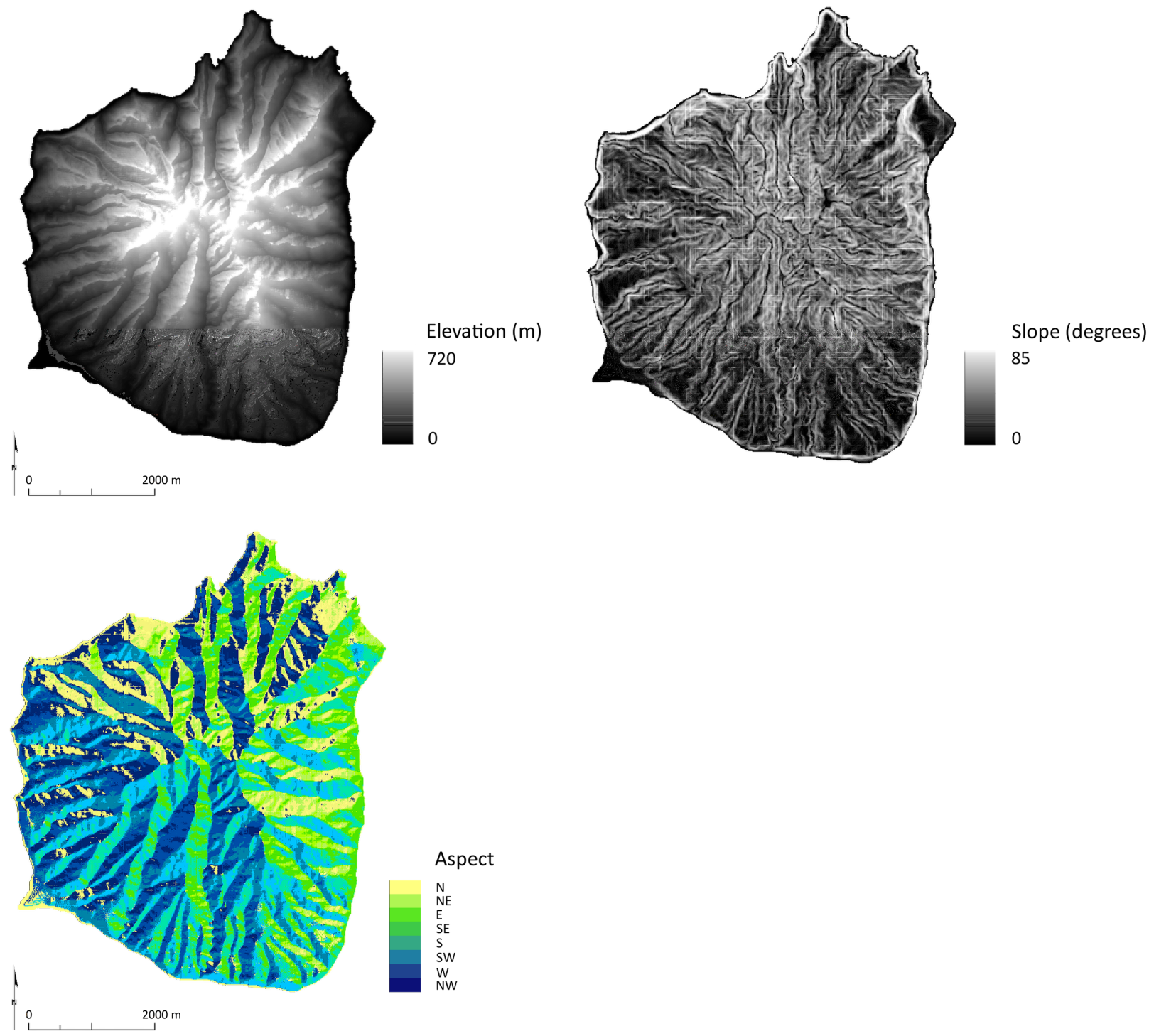

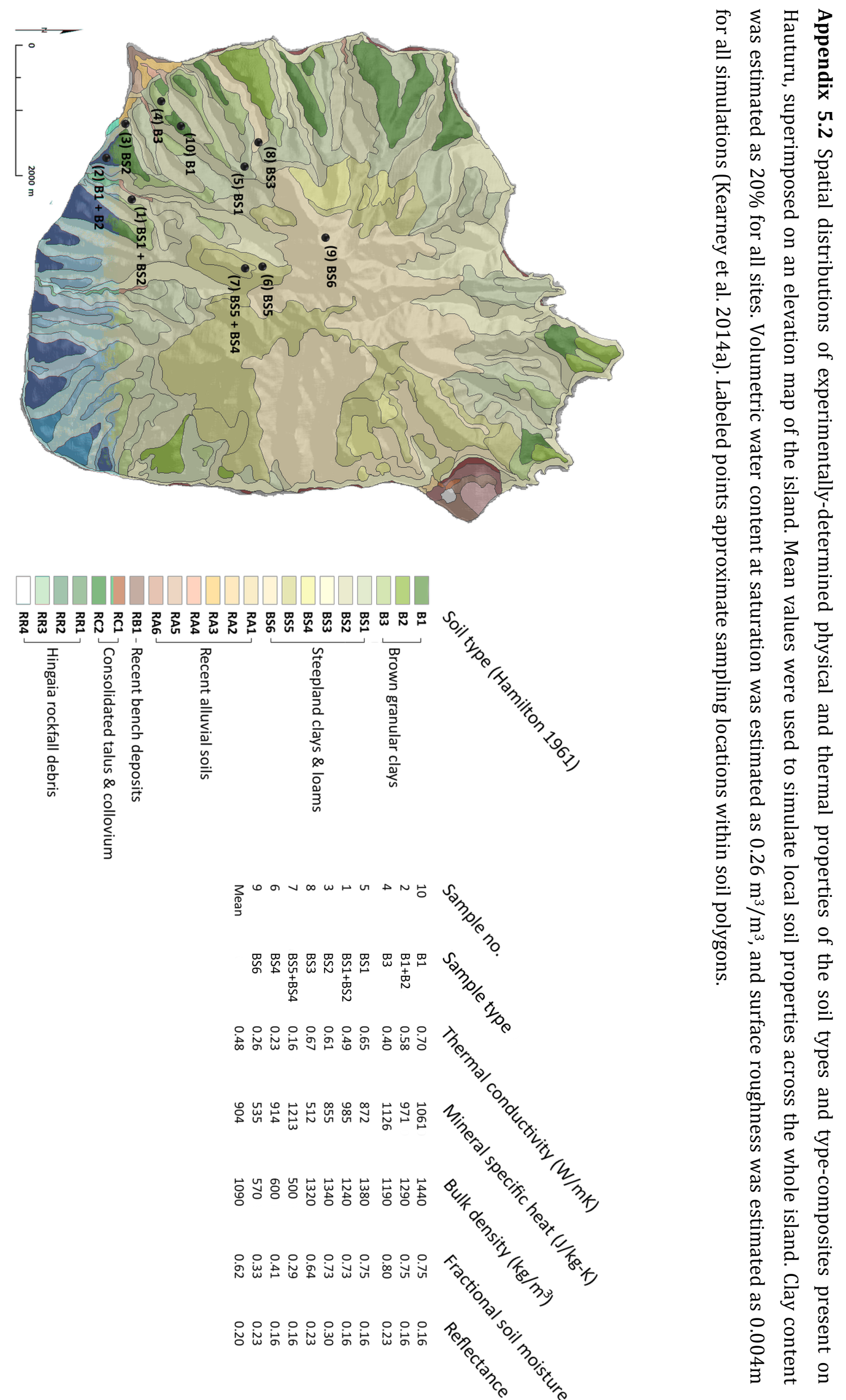
Appendix 5.3 Line plots showing local, daily climate data from 2011 used to parameterise the microclimate model for Hauturu. Total rainfall for the year was approximately $1600 \mathrm{~mm}$.
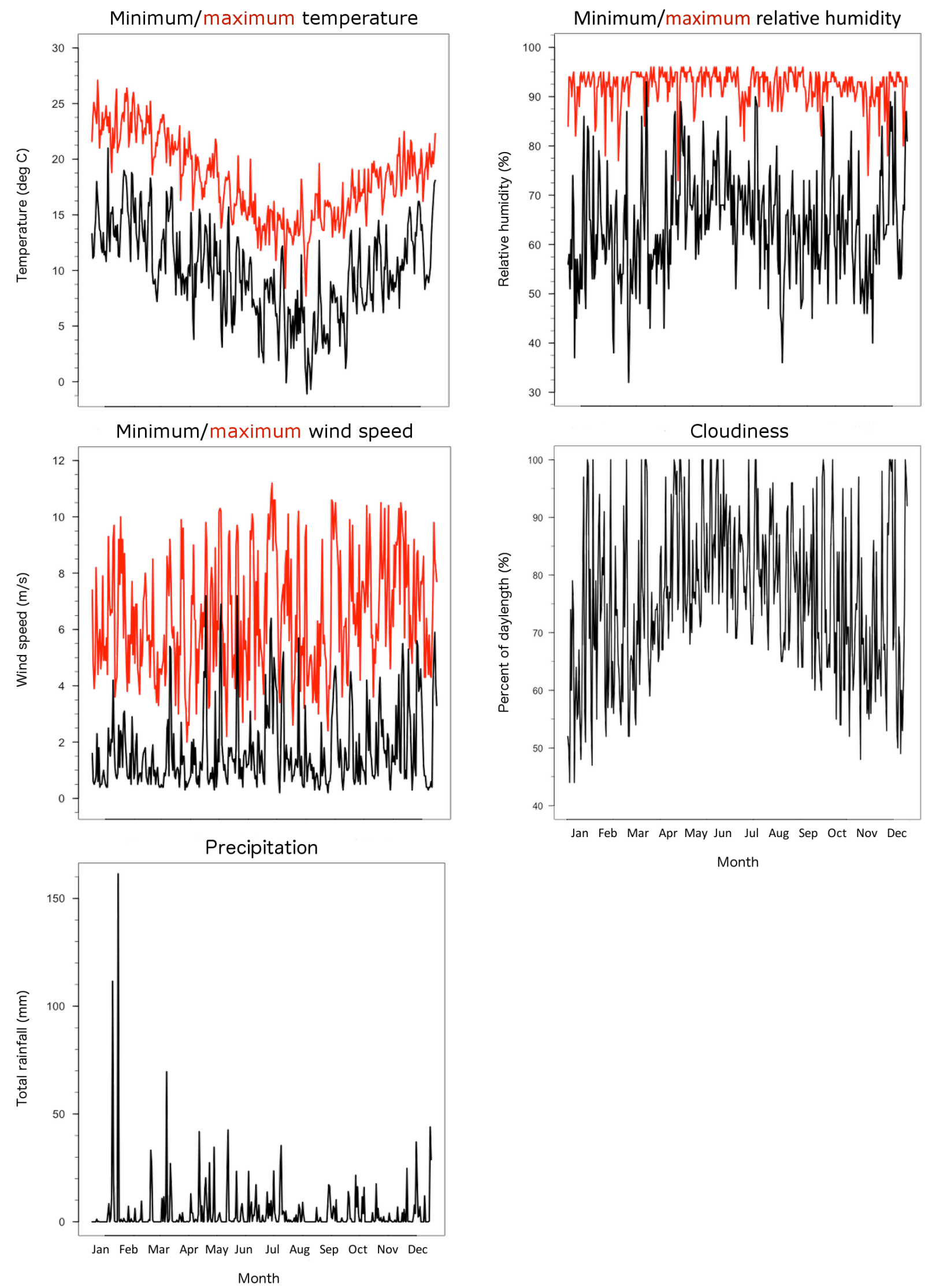
Appendix 5.4 Island-wide constant incubation temperatures (CTEs) predicted for Hauturu, irrespective of whether total embryonic development reached $100 \%$. White pixels represent sites at which predicted development did not reach at least 35\%.

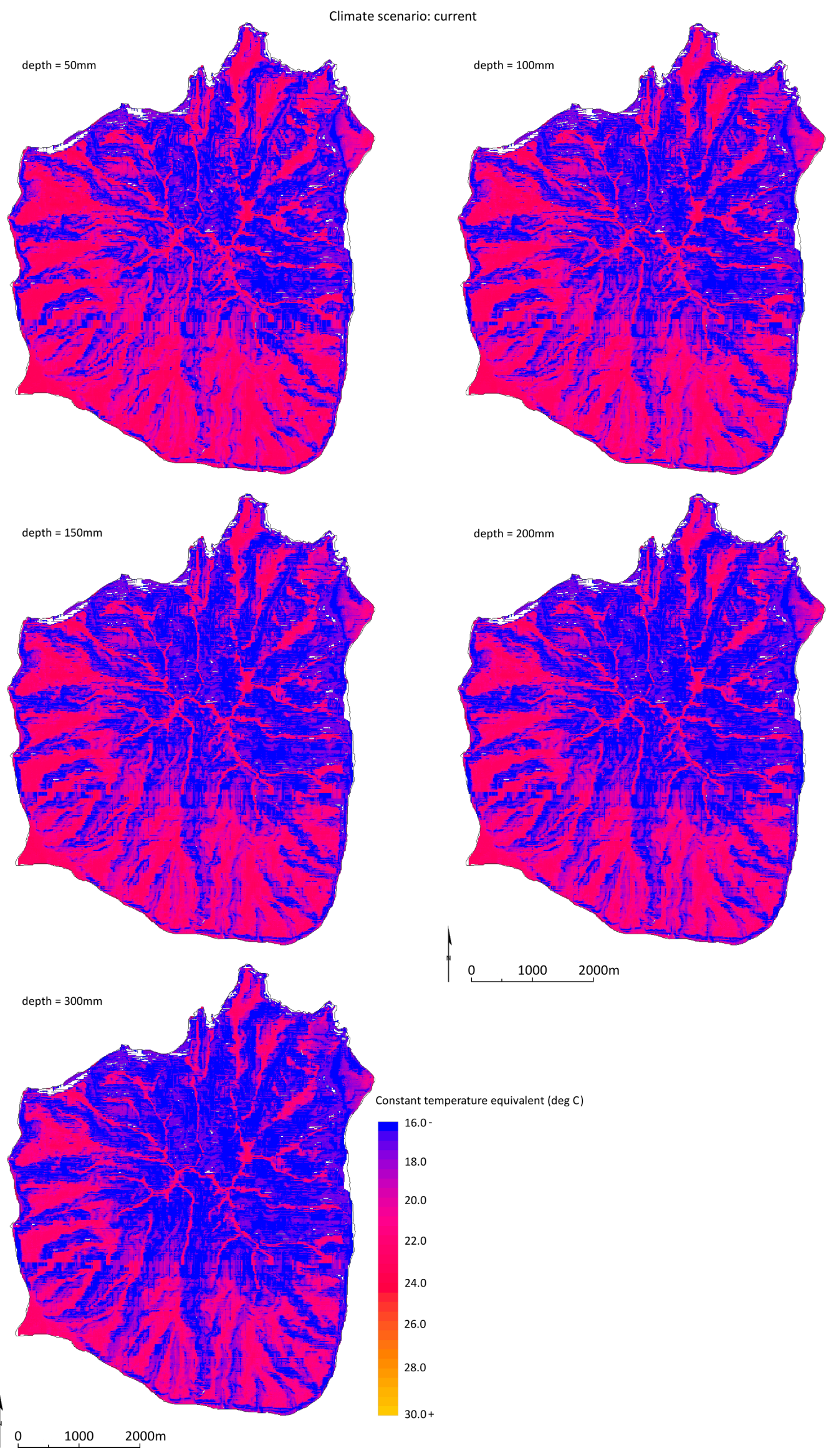




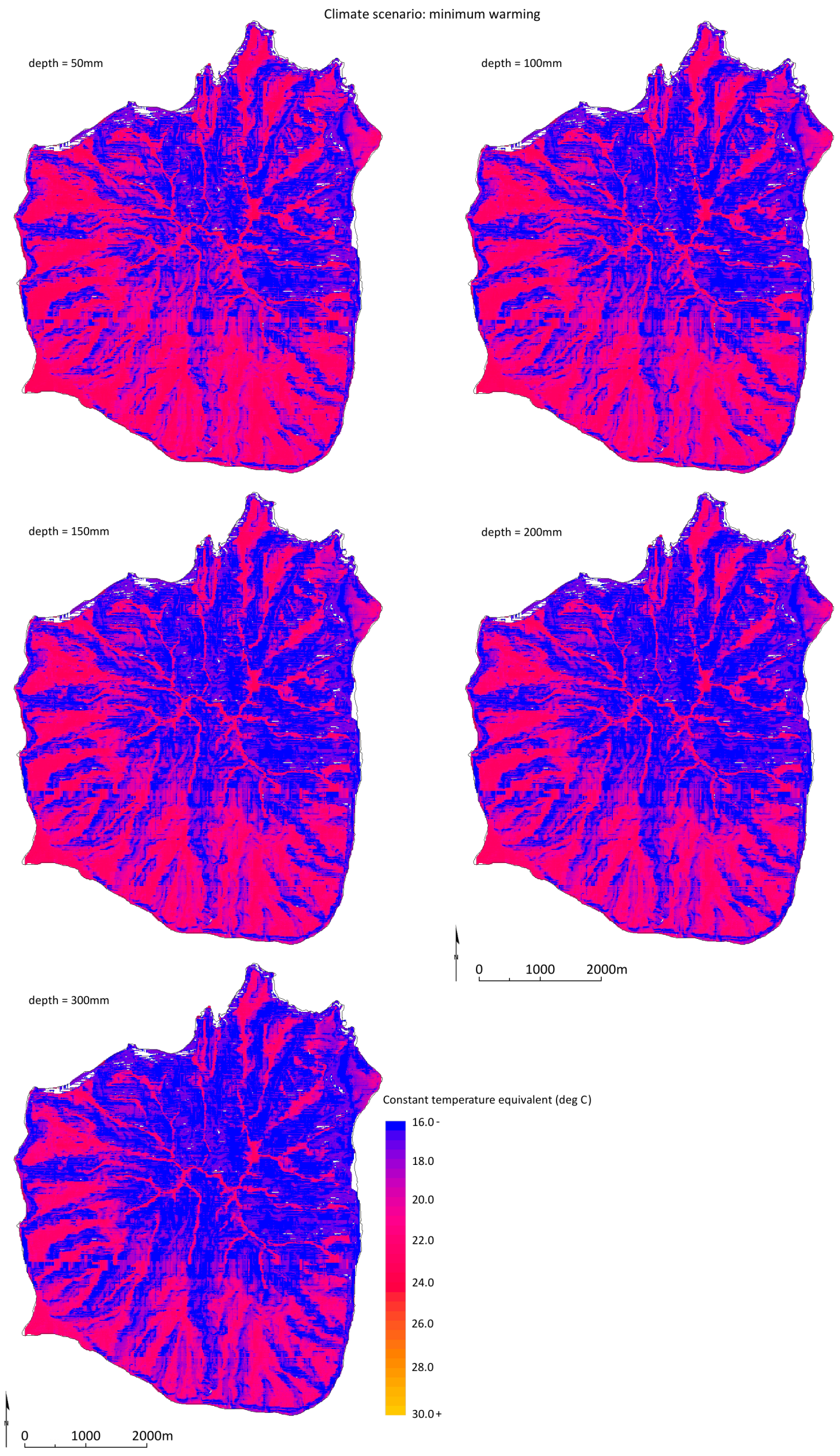




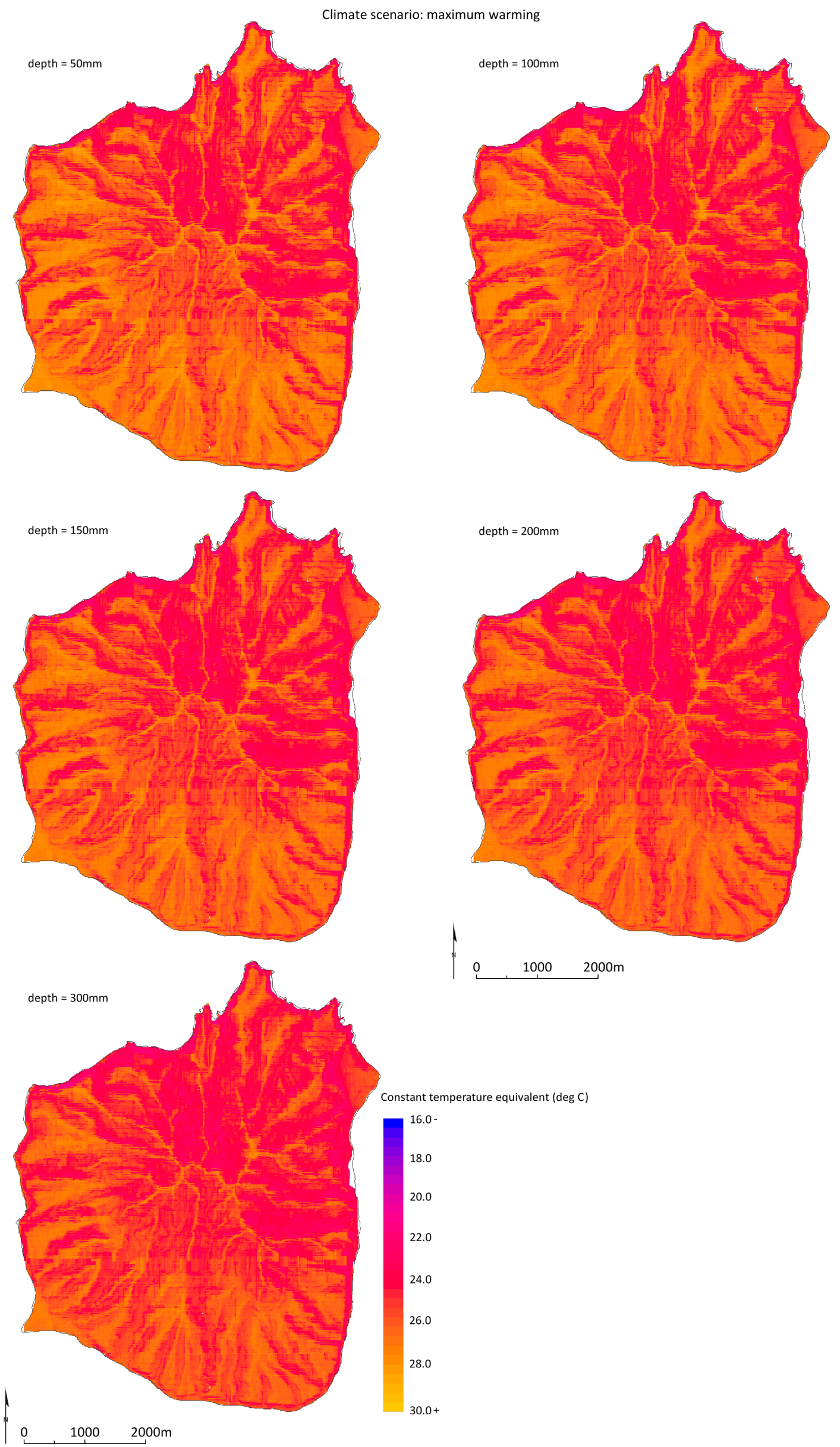

